\title{
PHYSICAL, CHEMICAL, AND BIOLOGICAL DATA FOR DETAILED STUDY OF IRRIGATION DRAINAGE IN THE SALTON SEA AREA, CALIFORNIA, $1988-90$
}

By Roy A. Schroeder', Mick Rivera, ${ }^{2}$ and others

'U.S. Geological Survey

${ }^{2}$ U.S. Fish and Wildlife Service

U.S. GEOLOGICAL SURVEY

Open-File Report 93-83

U.S. GEOLOGICAL SURVEY

U.S. FISH AND WILDLIFE SERVICE

U.S. BUREAU OF RECLAMATION

U.S. BUREAU OF INDIAN AFFAIRS

and in cooperation with

CALIFORNIA REGIONAL WATER QUALITY

CONTROL BOARD--COLORADO RIVER BASIN REGION

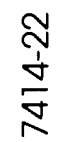




\section{U.S. DEPARTMENT OF THE INTERIOR BRUCE BABBITT, Secretary}

\section{U.S. GEOLOGICAL SURVEY}

Dallas L. Peck, Director

Any use of trade, product, or firm names in this publication is for descriptive purposes only and does not imply endorsement by the U.S. Government.

For sale by the Books and Open-File Reports Section U.S. Geological Survey Federal Center, Box 25425

Denver, CO 80225

For additional information write to:

District Chief

U.S. Geological Survey

Federal Building, Room W-2233

2800 Cottage Way

Sacramento, CA 95825 


\section{PREFACE}

The following individuals (agency affiliations are at the time of this study) participated in the collection and analysis of samples, compilation of data, and preparation of this report.

Roy A. Schroeder, U.S. Geological Survey, San Diego, California, participated in most field operations for the geochemical aspects of the study, did the laboratory experiments for tables 17 and 18, and prepared that part of this report related to tables 1-18 done by the U.S. Geological Survey.

Mick Rivera, U.S. Fish and Wildlife Service, Carlsbad, California, participated in field operations for the biological aspects of the study and prepared that part of this report related to tables 19-24 done by the U.S. Fish and Wildlife Service.

Brenda J. Redfield, U.S. Geological Survey, San Diego, California, compiled and reformatted data tables received in various forms from other agencies, offices, and individuals and assisted in the data-table verification process.

Jill N. Densmore, U.S. Geological Survey, San Diego, California, collected surface-water and ground-water quality data that are presented in tables $2,4,5$, and 10 and that also were used by her to prepare an M.S. thesis at San Diego State University.

Robert L. Michel, U.S. Geological Survey, Reston, Virginia, obtained the tritium data presented in tables 6 and 16 and included elsewhere with additional water-quality data in various other tables.

Daniel R. Norton, U.S. Geological Survey, Denver, Colorado, analyzed soils and soil extracts that provided the data presented in tables 11-15.

Daniel J. Audet, U.S. Fish and Wildlife Service, Carlsbad, California, was the co-principal investigator with the project chief (Steven L. Goodbred) for collection of the biological data presented in tables 22 and 23.

James G. Setmire, U.S. Geological Survey, San Diego, California, served as project chief for the U.S. Geological Survey's part of this study, collected sediment and water-quality data from the Alamo River delta presented in table 7 and figure 4, and had primary responsibility for preparation of that agency's contribution to the interpretive report.

Steven L. Goodbred, U.S. Fish and Wildlife Service, Carlsbad, California, was project chief for the U.S. Fish and Wildlife Service's part of this study and had primary responsibility for preparation of that agency's contribution to the interpretive report. 



\title{
CONTENTS
}

\author{
Preface III \\ Abstract 1 \\ Introduction 1 \\ Irrigation and drainage systems in the Imperial Valley 2 \\ Purpose and scope 2 \\ Types and numbers of samples $\mathbf{3}$ \\ Analytical laboratories and methods 3 \\ Acknowledgments 6 \\ Drainwater data 6 \\ 1988 synoptic sampling at 108 sites 7 \\ 1988-89 monthly monitoring at 15 sites 7 \\ Surface-water data $\mathbf{1 0}$ \\ Monthly monitoring at six locations 10 \\ Historical tritium concentrations in the Colorado River 10 \\ Alamo River delta sampling $\mathbf{1 0}$ \\ Selenium redox speciation $\mathbf{1 1}$ \\ Lithology and ground-water data $\mathbf{1 1}$ \\ Lithology at three drilling sites $\mathbf{1 1}$ \\ Ground-water quality 12 \\ Soils and porewater data 12 \\ Shallow soils 13 \\ Cores 13 \\ Tritium in shallow soils $\mathbf{1 3}$ \\ Laboratory experimental data 13 \\ Evaporations of irrigation water $\mathbf{1 3}$ \\ Dilutions of seawater and Salton Sea water 14 \\ Biological data 14 \\ Background 14 \\ Sampling sites 14 \\ Sample collection and preservation 14 \\ Chemical concentrations 15 \\ References cited 15
}

\section{FIGURES}

1-4. Maps showing:

1. Location of study area 2

2. Water-sampling sites in the study area 4

3. Water- and sediment-sampling locations, and selenium concentration in surficial sediments, in the Alamo River delta 6

4. Biological sampling sites in the study area $\mathbf{8}$

5. Graph showing historical increase in number of drainwater sumps installed in the Imperial Valley 10 


\section{TABLES}

1. Data from Imperial Valley drainwater samples collected by the California Regional Water Quality Control Board, June 24-July 10, 198618

2. Data from Imperial Valley drainwater samples collected by the U.S. Geological Survey, May 16-20, 198823

3. Comparison of data for drainwater samples collected at selected locations in the Imperial Valley by the U.S. Geological Survey in 1986 and $1988 \mathbf{3 2}$

4. Data from periodic sampling of drainwater at 15 Imperial Valley sites at fields, May 1988-August 198934

5. Data from periodic sampling of streams, East Highline Canal, and Trifolium Drain 1, August 1988August $1989 \mathbf{5 4}$

6. Annual average tritium concentrations in the lower Colorado River at Imperial Dam, 1965-88 72

7. Depth-profile data along a transect between the mouth of the Alamo River and the Salton Sea, August $1988 \mathbf{7 2}$

8. Particle-size distribution in cores from the Imperial Valley $\mathbf{7 3}$

9. Lithologic description and perforated intervals for piezometers in the Imperial Valley $\mathbf{7 4}$

10. Data from lysimeters and piezometers at three sites in the Imperial Valley $\mathbf{7 5}$

11. Selenium and water-extractable sulfate, chloride, and selenium concentrations in soils from 15 fields

12. Arsenic, selenium, and boron, and water-extractable sulfate, chloride, and selenium concentrations in soils from 15 fields 87

13. Concentrations of selected constituents in near-surface soils from 15 fields $\mathbf{8 8}$

14. Arsenic and selenium, and water-extractable sulfate, chloride, and selenium concentrations in cores

15. Concentrations of selected elements in cores 94

16. Tritium concentration in soil moisture from eight fields in the Imperial Valley, August 198897

17. Analyses of untreated and acidified irrigation water from the East Highline Canal concentrated by evaporation in the laboratory $\mathbf{9 8}$

18. Analyses of undiluted and diluted (with deionized water) water samples from the Salton Sea and the Pacific Ocean 100

19. Biological sampling sites for the detailed study of the Salton Sea area 104

20. Biotic samples collected from sites in the study area 105

21. Chemicals analyzed for in biota in the detailed study of the Salton Sea area $\mathbf{1 0 6}$

22. Inorganic chemical analysis and moisture content for biotic samples collected during 1988-90 from the Salton Sea and associated drainwaters and rivers 107

23. Organic chemical analysis, moisture content, and lipid content for biotic samples collected during 1986-90 from the Salton Sea and associated drainwaters and rivers 137

24. Polycyclic aromatic hydrocarbon concentration, moisture content, and lipid content for single samples of crayfish and tilapia collected in the study area in $1987 \mathbf{1 7 9}$ 


\section{Conversion Factors, Vertical Datum, and Abbreviations}

\section{Conversion Factors}

\begin{tabular}{rcl}
\hline Multiply & By & To obtain \\
\hline acre & 0.4047 & hectare \\
acre & 4,047 & square meter \\
cubic foot per second (ft $\left.{ }^{3} / \mathrm{s}\right)$ & 0.02832 & cubic meter per second \\
foot (ft) & 0.3048 & meter \\
gallon (gal) & 3.785 & liter \\
gallon per minute (gal/min) & 0.06308 & liter per second \\
inch (in.) & 25.4 & millimeter \\
mile (mi) & 1.609 & kilometer \\
\hline
\end{tabular}
equation:

Temperature is given in degrees Celsius $\left({ }^{\circ} \mathrm{C}\right)$, which can be converted to degrees Fahrenheit $\left({ }^{\circ} \mathrm{F}\right)$ by the following

$$
{ }^{\circ} \mathrm{F}=1.8\left({ }^{\circ} \mathrm{C}\right)+32
$$

Isotope composition is expressed in permil (parts per thousand).

\section{Vertical Datum}

Sea level: In this report, "sea level" refers to the National Geodetic Vertical Datum of 1929--a geodetic datum derived from a general adjustment of the first-order level nets of the United States and Canada, formerly called Sea Level Datum of 1929.
Abbreviations
$\mathrm{L} / \mathrm{min}$ - liter per minute
$\mathrm{L} / \mathrm{hr}$ - liter per hour
$\mu \mathrm{g} / \mathrm{L} \quad$ - microgram per liter
$\mu \mathrm{g} / \mathrm{g}$ - microgram per gram
$\mu \mathrm{S} / \mathrm{cm}$ - microsiemen per centimeter at $25^{\circ} \mathrm{C}$
$\mu \mathrm{m} \quad$ - micrometer
$\mathrm{mg} / \mathrm{L}$ - milligram per liter
$\mathrm{mL}$ - milliliter
$\mathrm{mm}$ - millimeter
PCB - polychlorinated biphenyl
$\mathrm{pCi} / \mathrm{L}$ - picocurie per liter
PVC - polyvinyl chloride





\title{
PHYSICAL, CHEMICAL, AND BIOLOGICAL DATA FOR DETAILED STUDY OF IRRIGATION DRAINAGE IN THE SALTON SEA AREA,
}

\section{CALIFORNIA, 1988-90}

\author{
By Roy A. Schroeder, ${ }^{1}$ Mick Rivera, ${ }^{2}$ and others
}

\begin{abstract}
This report contains physical, chemical, and biological data associated with irrigation drainage in the Salton Sea area collected during the late 1980 's. The data were collected in support of the U.S. Department of the Interior's National Irrigation Water Quality Program in the Western United States to evaluate effects on the environment from potential toxins in irrigation-induced drainage. The data have been used to support interpretations in several recent publications. This data report is the companion to a comprehensive U.S. Geological Survey interpretive report that describes the geochemical and biological pathways of potential toxins, especially selenium, in the study area.
\end{abstract}

The report contains data on concentrations of a broad suite of trace elements in soil, irrigation (Colorado River) water, drainwater, surface water (including the Salton Sea), ground water, aquatic plants, invertebrates, amphibians, reptiles, fish, birds, bird eggs, and turtle eggs. Included, also, are light stable isotope (hydrogen, oxygen, carbon, nitrogen, and sulfur), tritium, and radiocarbon data for selected aqueous samples and organochlorine-pesticide concentrations in biota.

Geochemical samples were collected from more than 100 drainwater-collection sites, several surface-water locations, 15 fields, 3 multipledepth lysimeter and piezometer installations, and the Alamo River delta on the southeastern shore of the Salton Sea, and from laboratory evaporations of Colorado River water.

Biological samples were collected from 39 sites, including 16 Salton Sea shore locations, 5 streams, 7 freshwater impoundments, 11 drainwater ditches, and 2 additional locations in the Imperial Valley.

\section{INTRODUCTION}

In response to concerns about irrigation-induced water-quality problems and their potential effects on biota, the U.S. Department of the Interior (DOI) began a study in the Salton Sea area, California, in 1986. The study was part of the DOI National Irrigation Water Quality Program (NIWQP). The initial phase of the Salton Sea area investigation was a reconnaissance, completed in 1987 , that led to a decision to do a more detailed study in the Imperial Valley. The results of the reconnaissance were reported by Setmire and others (1990b).

This report presents the data from a detailed study done during 1988-90. The detailed study was a joint effort conducted by scientists from the U.S. Geological Survey (USGS) and the U.S. Fish and Wildlife Service (USFWS). The USGS was responsible for determining the hydrologic and geochemical factors affecting concentrations of irrigation-induced contaminants, particularly selenium, and the USFWS was responsible for identifying pathways of contaminant accumulation in biota. Results of the detailed study will be used as part of the information needed for

${ }^{1}$ U.S. Geological Survey

${ }^{2}$ U.S. Fish and Wildlife Service 


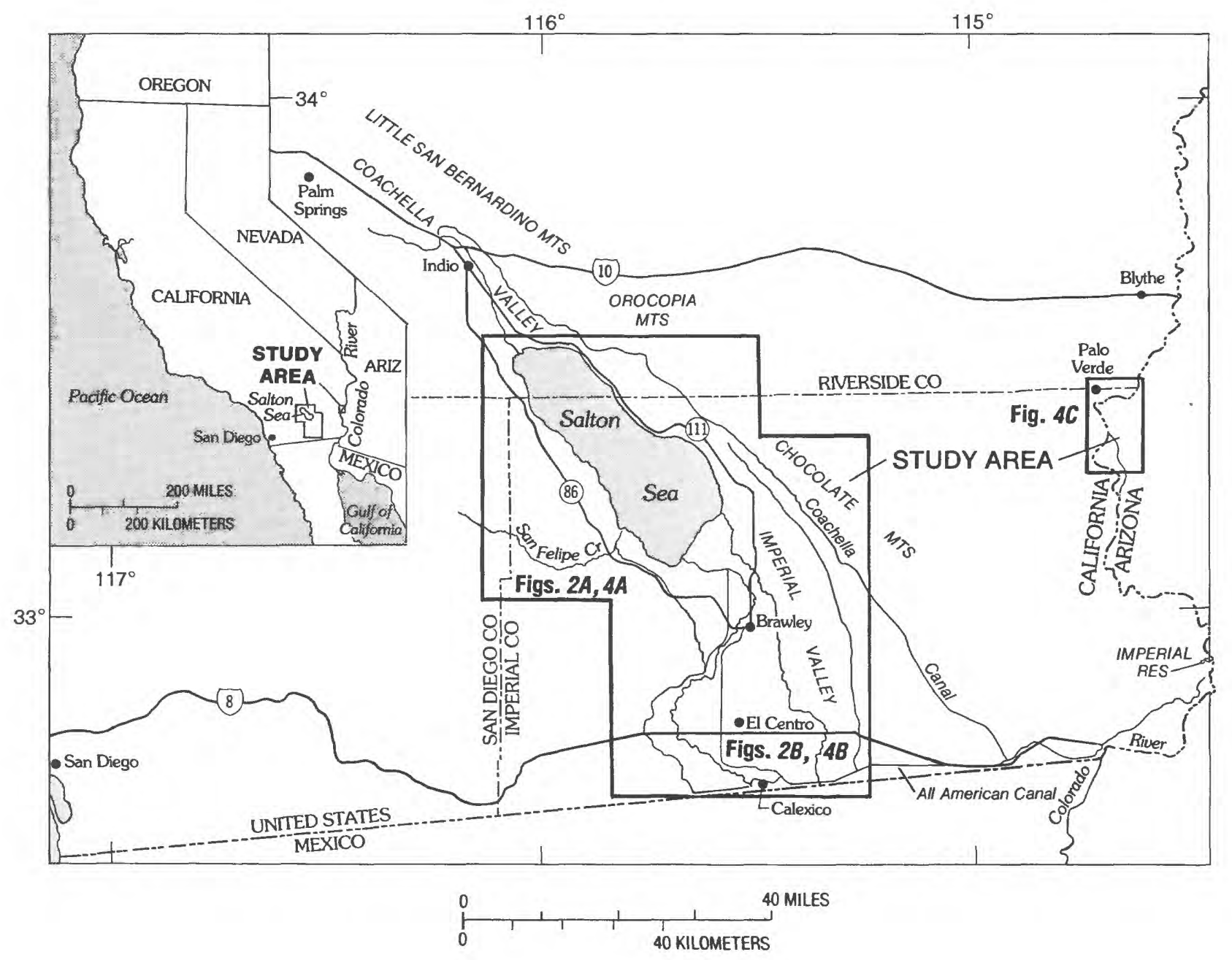

Figure 1. Location of study area.

planning and conducting remediation efforts under direction of the U.S. Bureau of Reclamation.

\section{IRRIGATION AND DRAINAGE SYSTEMS IN THE IMPERIAL VALLEY}

The central part of the Imperial Valley consists of 500,000 acres of irrigated and drained farmland in the desert area of southeastern California (fig. 1). Irrigation water is obtained by gravity diversion of water from the Colorado River, via the All-American Canal, to (from east to west) the East Highline, Central Main, and Westside Main (and its Trifolium Extension) Canals (fig. $2 B$ ). These canals supply numerous smaller canals throughout the valley. Drains at a depth of 6 to $10 \mathrm{ft}$ carry subsurface water containing dissolved salts to sumps at the tail end of selected fields or discharge directly to drainage ditches. All drainwater ultimately is discharged to the Salton Sea, either directly from drainage ditches or by way of the New and Alamo Rivers.

The Alamo River discharges to the Salton Sea at the northern unit (Unit 2) of the Salton Sea National Wildlife Refuge (SSNWR), and the New River and Trifolium Drain 1 discharge at the southern unit (Unit 1) of the refuge (fig. 2A). The proportions of total discharge that originates from use within the Imperial Valley - for the New River, Alamo River, and Trifolium Drain 1-are about 50, 99, and 100 percent, respectively. Sources of discharge to San Felipe Creek (fig. $2 A$ ) are precipitation in mountains to the west and ground water.

\section{PURPOSE AND SCOPE}

The purpose of this report is to present all data collected during the 1988-90 U.S. Department of the 
Interior detailed study of irrigation-induced contamination problems in the Salton Sea area, along with a brief description of methods, principal reasons for collecting each of the various kinds of data, and unusual or special field observations. Also included are the results from pesticide and polycyclic aromatic hydrocarbon analyses of biological tissues collected during 1986-87 that were not published (analyses had not been completed) in the reconnaissance (Setmire and others, 1990b) report. This report is intended to serve as a companion and supplement to the interpretive report of the detailed study by Setmire and others (1993) that is based on these data. Other reports and abstracts published to date on this study include Schroeder and others $(1988,1989,1991)$, Michel and others (1988), Michel and Schroeder (1989), Setmire and others (1990a, b), and Densmore (1991).

\section{TYPES AND NUMBERS OF SAMPLES}

Analytical data for the detailed study (samplingsite locations shown in figs. 2,3 , and 4) include the following:

1. A single synoptic sampling of drainwater from 108 sites in May 1988.

2. Monthly monitoring at 15 of the 108 drainwater sites for 1 year.

3. Monthly monitoring at six surface-water locations for 1 year.

4. Single collections of surface-water samples from several sources, including the Salton Sea.

5. Ground water from multiple-depth lysimeters and piezometers at three sites.

6. Soils and water extracts of soils (soil extracts) from fields at the 15 drainwater-monitoring sites and from cores at the 3 ground-water monitoring sites.

7. Evaporation and dilution laboratory experiments.

8. Collection of 276 biological samples from 29 sites for analysis of 67 organic analytes.

9. Collection of 363 biological samples from 36 sites for analysis of 23 inorganic analytes.

\section{ANALYTICAL LABORATORIES AND METHODS}

Aqueous chemical analyses were done at the USGS National Water Quality Laboratory (NWQL) in Arvada, Colorado, using methods described by Fishman and Friedman (1989) and Pritt and Jones (1989). These references also give general information on analytical sensitivity and precision for the laboratory analyses. Stable hydrogen- and oxygen-isotopes and tritium were analyzed at the USGS Isotope Laboratory in Reston, Virginia. The stable-isotope ratios were determined by mass-spectrometry, as concentration ratios for hydrogen and as activity ratios for oxygen. Tritium was analyzed by liquid-scintillation counting following electrolytic enrichment. Other isotopes of carbon, nitrogen, and sulfur were analyzed by NWQL-contract laboratories. Precision $(1 \sigma)$ is 0.1 to 0.2 permil for $\delta^{13} \mathrm{C}, \delta^{15} \mathrm{~N}$, and $\delta^{18} \mathrm{O} ; 1$ to 2 permil for $\delta \mathrm{D}$ and $\delta^{34} \mathrm{~S}$; about 1 percent modern carbon for ${ }^{14} \mathrm{C}$ near the reporting limit; and ranges from about 1 $\mathrm{pCi} / \mathrm{L}$ for ${ }^{3} \mathrm{H}$ at concentrations near zero to about 3 percent on the basis of counting statistics at concentrations above ambient irrigation-water (Colorado River water) concentration. Stable-isotope ratios are reported in standard notation relative to ratios in commonly used standards (Fritz and Fontes, 1980).

Chemical analyses of soils and soil extracts were done by the USGS Branch of Geochemistry in Denver, Colorado. Methods for analysis of soil extracts were similar to those used by the NWQL. Analysis of extractable (readily soluble) salts was done by overnight extraction with a 5-to-1 weight ratio of deionized water to soil. Shallow-soil and core samples were stored and shipped in wide-mouth plastic containers to the laboratory, where they were prepared for analysis by drying and grinding (to pass an 80 mesh [0.18-mm] sieve), followed by complete dissolution with an oxidizing agent and strong mineral acids. Methods for analysis of soils were identical to those used by Severson and others (1987) in the reconnaissance phase of this study, and qualityassurance and analytical precision standards are given by Arbogast (1990). Forty-element scans were done by inductively coupled argon-plasma atomic emission spectrometry (ICP). Arsenic and selenium were analyzed by hydride-generation atomic absorption, and boron concentrations were determined in hot-water extracts.

Grain-size analyses of cores and shallow soils were done at the USGS Sediment Laboratory in Salinas, California, using sieves for the coarse fraction and hydrometers for the fine fraction as described by Guy (1969).

Chemical analyses of biological samples were done at contract laboratories. The contract was administered by the USFWS Patuxent Analytical Control Facility in Laurel, Maryland, which also was responsible for ensuring quality control and quality assurance. Methods used for analysis of biological samples are those prescribed in a USFWS procedures 


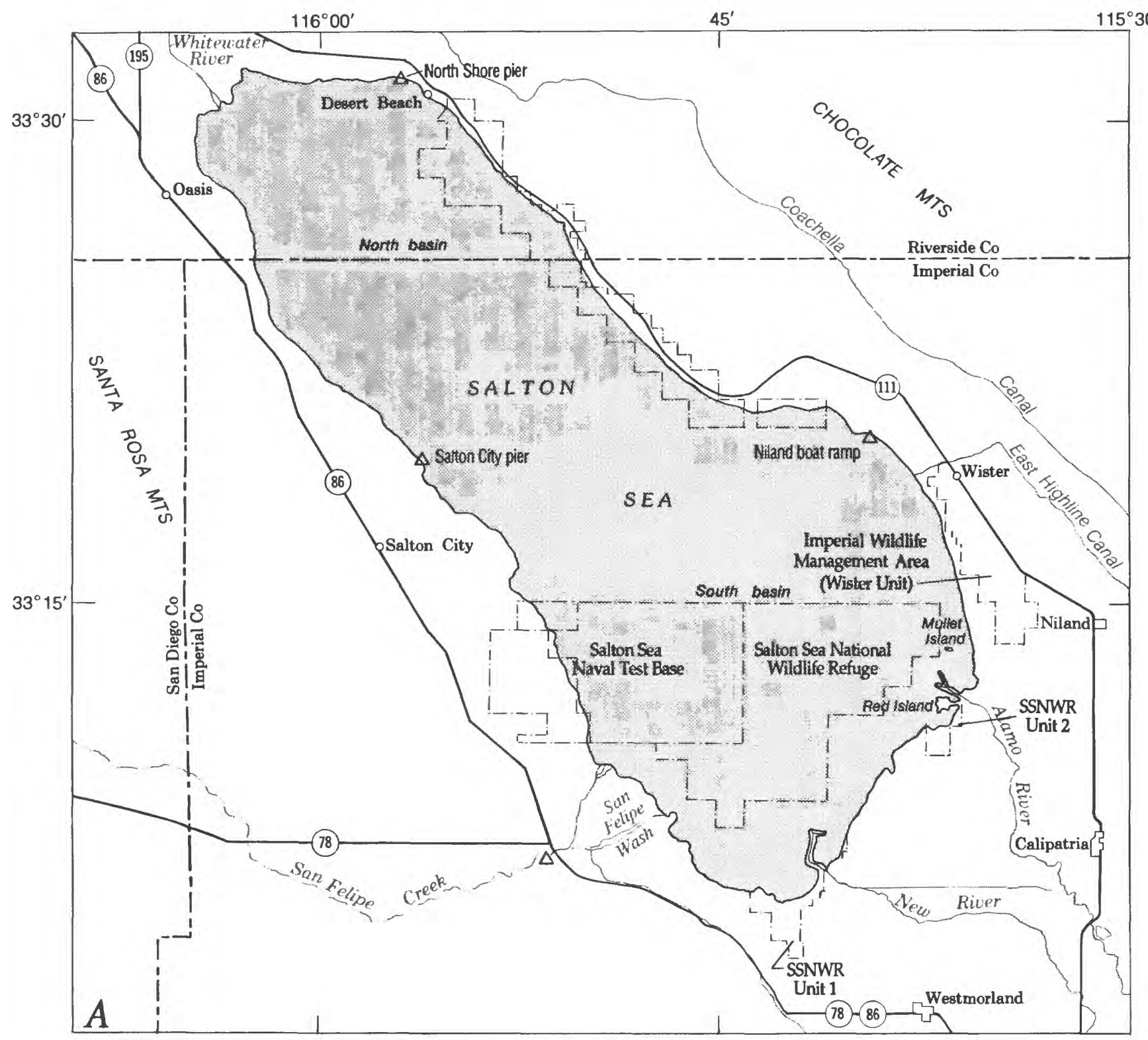

EXPLANATION

Sampling site and number--

$\nabla_{97}$ Subsurface-drainwater site

$\triangle$ Surface-water site

Figure 2. Water-sampling sites in the study area. A, San Felipe Creek and Salton Sea (August 1988 sampling). $B$, Imperial Valley. Location of map areas shown in figure 1.

manual (USFWS, 1985a). Instruments and methods used for trace-element analysis (Lowe and others, 1985 ) are the same as those used by the USGS (Fishman and Friedman, 1989). Mercury, which was determined in biological tissues only (not in soil and water) for this study, was analyzed by cold-vapor atomic absorption. Gas-liquid chromatographic methods used to analyze organochlorine compounds also are similar to those in use by the USGS (Wershaw and others, 1987). Polycyclic aromatic hydrocarbons on crayfish and tilapia collected in 1987 during the reconnaissance were analyzed by gas chromatography-mass spectrometry. Concentrations of constituents in biota are reported on a dry-weight basis for trace elements 


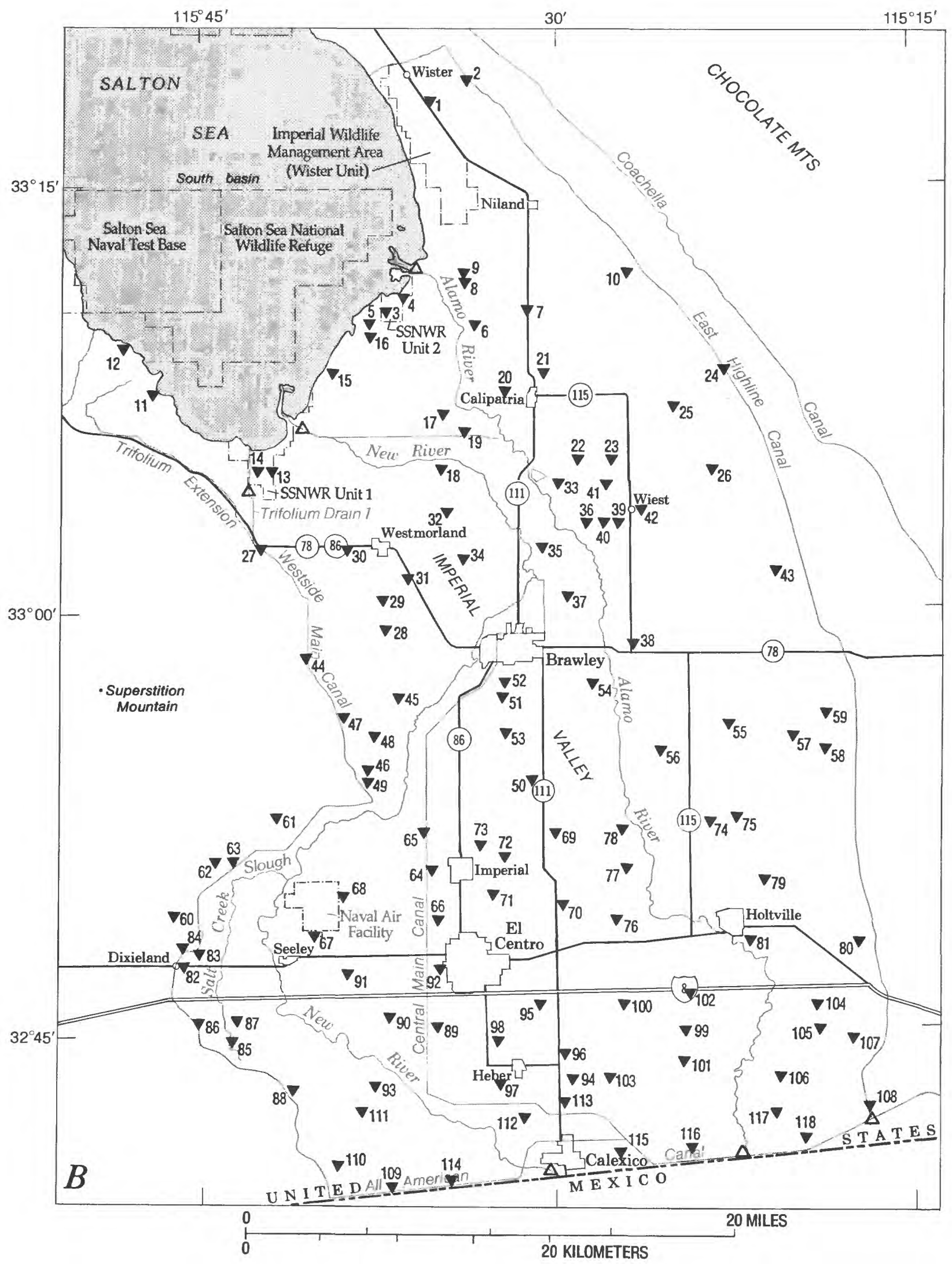

Figure 2. Continued. 


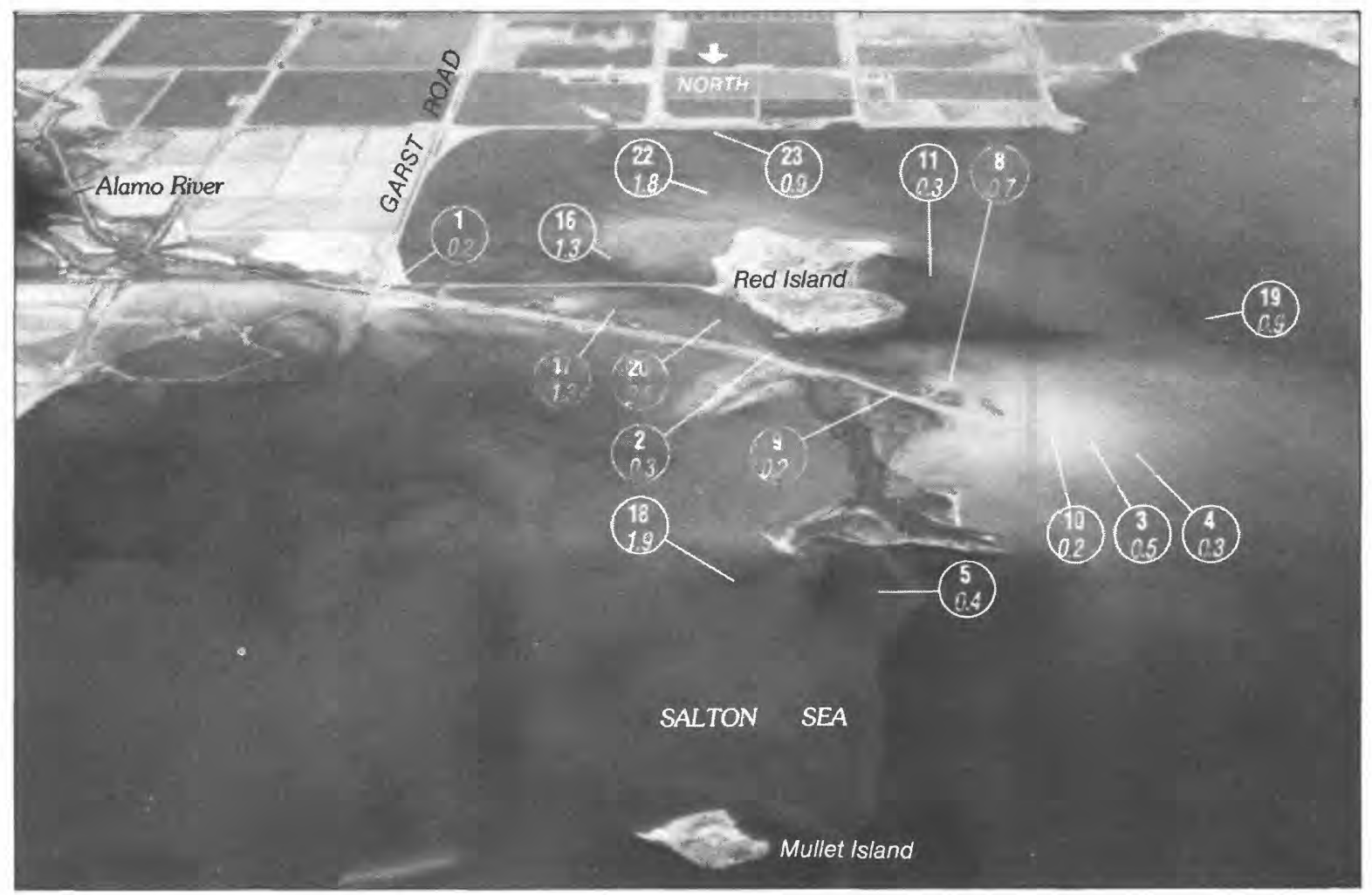

EXPLANATION

5. Site number
0.4 Selenium concentration-In micrograms per gram

Figure 3. Water- and sediment-sampling locations, and selenium concentration in surficial sediments, in the Alamo River delta.

and on a wet-weight basis for organochlorine and polycyclic aromatic hydrocarbon compounds.

To maintain internal consistency within the entire data set, results are reported herein without correction for unknown analytical or occasional transcription errors that may occur during transfer of the data into permanent computer storage. When large, such errors usually are apparent as data outliers (especially when multiple samples were collected over a period of time) and from comparisons between constituents; on the basis of cursory inspection of the data, such errors are believed to represent less than 1 percent of the data set.

\section{ACKNOWLEDGMENTS}

The U.S. Department of the Interior provided most of the support for this study. The authors also thank the California Regional Water Quality Control Board, Colorado River Basin Region, for additional financial support and for field assistance with the May 1988 sampling of drainwater sites, and the Imperial Irrigation District for procuring access to sites and for assistance in the coring of shallow soils.

\section{DRAINWATER DATA}

The Imperial Irrigation District uses letters followed by numerals to designate drainwater-discharge sites in the Imperial Valley. The letter designations are "TD" for subsurface drains that discharge directly to a drainage ditch, "S" for sumps that collect drainwater from a network of subsurface drains at the corner of a field and then discharge to ditches, and "SS" for sumps that are adjacent to the Salton Sea. (Some sumps designated "S" also are near the Salton Sea, and a few even discharge directly to the Salton Sea.) 
As of 1990, when this study was completed, 532 drainwater sumps ( 9 of which were subsequently removed or placed out of service) had been installed in the Imperial Valley. Thirty of the sumps are designated "SS." In addition, there are about 3,700 drains which are designated "TD" and which discharge directly to ditches. The first subsurface drains were installed in the Imperial Valley in 1928, and the first sump was installed in 1947. The historical pattern of sump installation is depicted in figure 5 (S.R. Knell, Imperial Irrigation District, oral and written commun., 1991). The historical pattern of subsurface-drain installation (Moore, 1991, fig. 19, p. 88) is similar to that of sump installation. The annual rates of installation are highest from the early 1950's to the early 1970 's. More than $30,000 \mathrm{mi}$ of subsurface drains had been installed by 1990 (Moore, 1991).

It should be noted that the term "drainwater," used during this study to refer to water in the sumps, includes not only subsoil moisture from interior parts of the field, but also rapid-percolation irrigation water directly above the subsurface drains; ground water; leakage from nearby canals, ditches, and ponds; and possibly even stormwater and tailwater in unusual circumstances.

Water samples from drains that discharge directly to a ditch were obtained in a 5-gallon plastic bucket held beneath the lip of the drain. Instantaneous discharge was measured by timing the rate at which the bucket filled. Water samples from sumps were obtained in a similar manner after manually activating the pump, causing the sump to discharge its contents through a pipe into a nearby ditch. Instantaneous discharge to the sump then was calculated as the sump refilled by multiplying sump diameter (90 in.) and rate at which the water level rose in the sump as measured on a vertical stem attached to the float. Accuracy of discharge measurements is estimated to be about 20 percent at most sites, and somewhat poorer at a few sites where flow to the sump was either extremely high or near zero.

Most drainwater samples were free of visible particulates. Occasional samples contained a few large particles derived from encrusted mineral deposits and soils, either in the sump itself or on the lip of the discharge pipe; such particles were readily removed from the sample by rapid gravitational settling.

\section{SYNOPTIC SAMPLING AT 108 SITES}

Drainwater samples were collected from 108 sites in the Imperial Valley during May 16-20, 1988, to determine spatial variation in drainwater quality and to compare with and expand on results of a similar synoptic sampling done by the California Regional Water Quality Control Board during June 24-July 10, 1986. The 1986 sampling included 119 sites that subsequently were located with the aid of Imperial Irrigation District's canal and drainage maps and USGS quadrangle maps. Eleven of the 119 sites were not resampled in the 1988 synoptic because they were no longer in service, contained no water, or could not be found by field personnel. Results from the 1986 synoptic sampling (P.A. Gruenberg, California Regional Water Quality Control Board, written commun., 1987) are given in table 1, and results from the 1988 synoptic sampling are given in table 2 . The location of drainwater sampling sites is shown in figure $2 B$.

All drainwater samples collected from the 108 sites in May 1988 were analyzed for selenium and a few other constituents. In addition, about one-third of the samples were analyzed for a broad suite of chemical and isotopic constituents. These data are the principal basis for the conclusion that evaporation of irrigation water supplied by the Colorado River is the primary geochemical mechanism controlling selenium concentrations in Imperial Valley drainwater (Schroeder and others, 1991, and Setmire and others, 1993). Eighty percent of the sumps sampled had a Se/Cl ratio ranging from 0.5 to 2 times the ratio (of $2.2 \times 10^{-5}$ ) in Colorado River water (refer to the section "Evaporations of Irrigation Water" presented later in this report). For those sumps in which the ratio lies above this range, leaching of selenium from the soil is thought to be the predominant geochemical mechanism controlling selenium concentration (Schroeder and others, 1991). Only 5 percent of the sumps had a $\mathrm{Se} / \mathrm{Cl}$ ratio greater than $4.4 \times 10^{-5}$, and the highest ratio measured exceeds the ratio in irrigation water by only a factor of 3. Fifteen percent of the sumps had a $\mathrm{Se} / \mathrm{Cl}$ ratio less than $1.1 \times 10^{-5}$, and the ratios for half of these were about one-tenth the ratio in irrigation water, or less. At these sites, there exists a substantial sink for selenium believed to be the result of microbial reduction within the local field and (or) intrusion of deeper selenium-deficient ground water into shallow drains (Schroeder and others, 1991).

Drainwater samples were collected from eight sites (three sumps and five tile drains) in 1986 during the reconnaissance study. Six of the eight sites were resampled in August 1988, and results from both years are given in table 3 .

\section{8-89 MONTHLY MONITORING AT 15 SITES}

Samples from 15 of the drainwater-collection sites were collected monthly during $1988-89$ to check for 


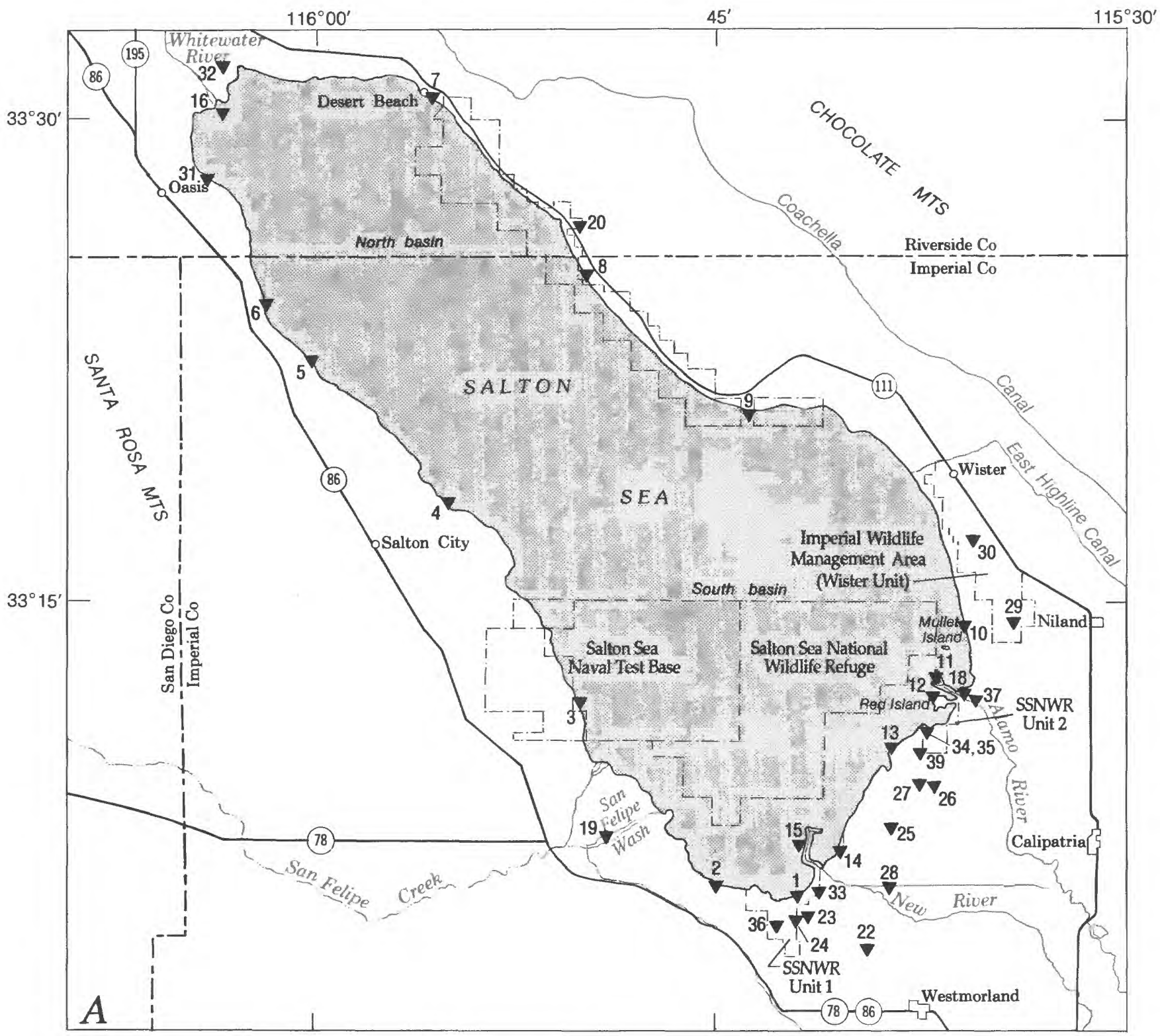

EXPLANATION

${ }_{36} \nabla$ Biological sampling site and number--In tables site numbers are preceded by the letter " $\mathrm{B}$ "

Figure 4. Biological sampling sites in the study area. A, Salton Sea. B, Imperial Valley. C, Colorado River near Palo Verde, California. Location of map areas shown in figure 1.

temporal variations in drainwater quality and to compare the results with soils and soil-extract data from fields that drain to these sumps. The 15 sites were chosen with the intent of obtaining good areal coverage over the valley and to represent sites with moderate to high concentrations of selenium. Results from the monthly monitoring are given in table 4.
To document the expected minimal effect of filtration on most constituent concentrations, the last monthly sample, collected in August 1989, was filtered through a $0.45-\mu \mathrm{m}$ membrane. The results can be compared with those of unfiltered samples collected in the prior months. In addition, concentrations of selenium (and a few other constituents) were deter- 


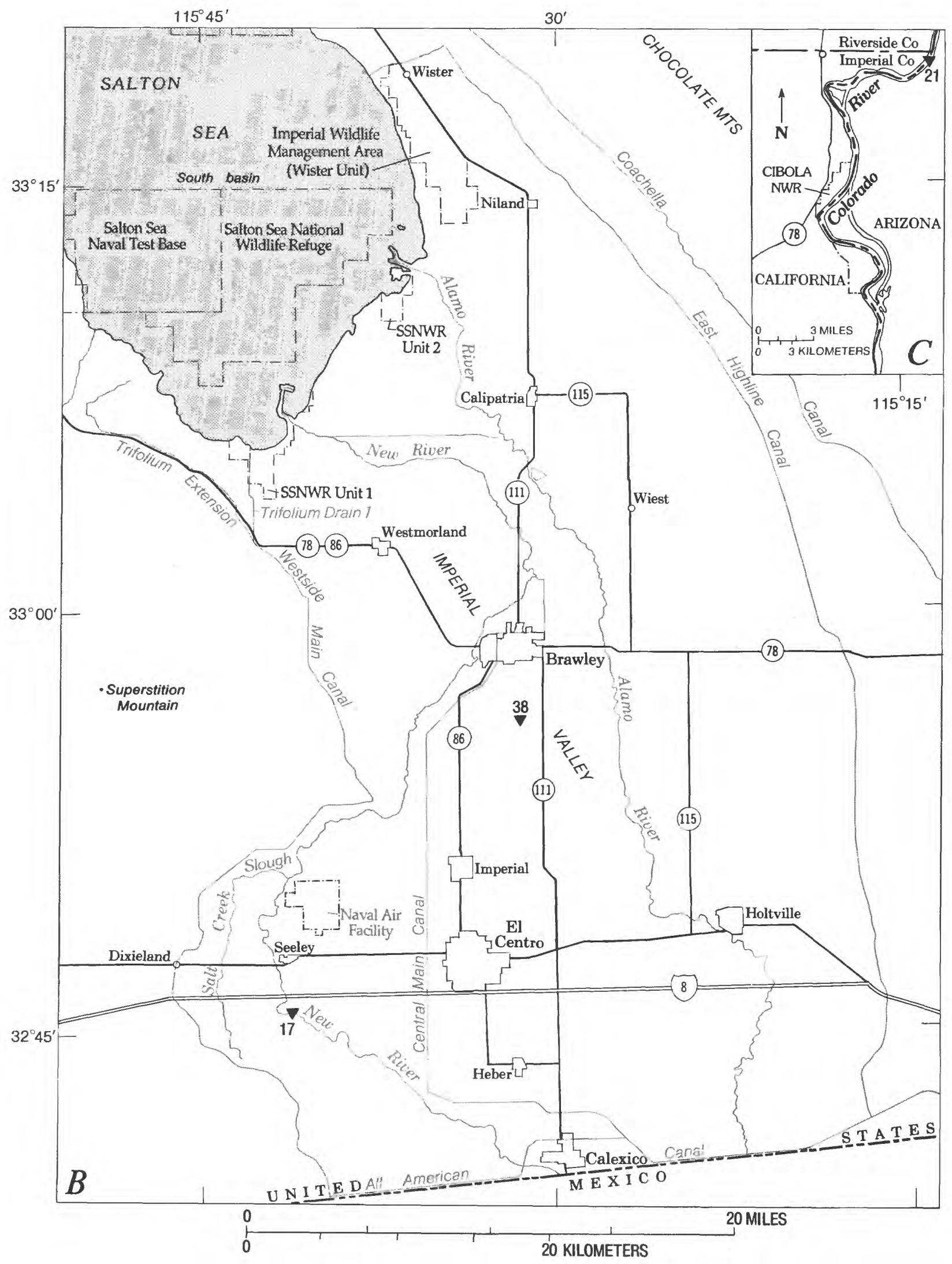

Figure 4. Continued. 


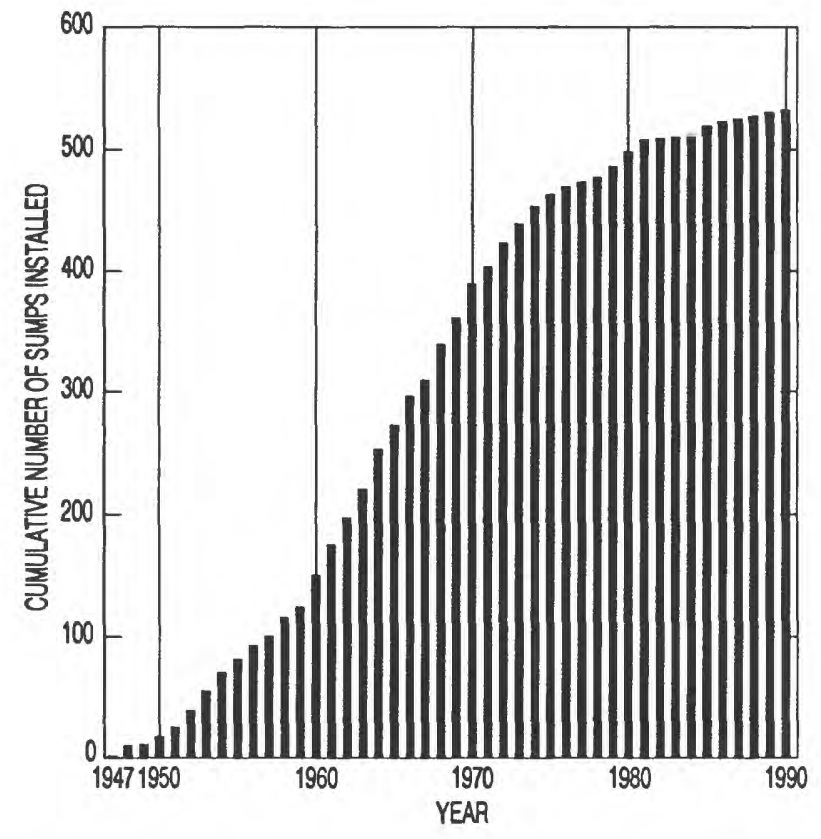

Figure 5. Historical increase in number of drainwater sumps installed in the Imperial Valley.

mined for unfiltered samples from the last collection, and these data are given in the bottom row for each site in table 4. Data from the filtered sample are given in the preceding row.

Of the 15 sites, 14 showed visible evidence of recent irrigation or had measurable drainwater discharge during the period of monitoring. Sump S-142, at site 33, was not receiving drainwater flow during any of the sampling dates, and the soil's surface frequently showed no visible evidence of recent irrigation. Isotopic data for the winter period are believed to be anomalous-possibly owing to evaporation from the sump and absence of irrigation during this period until the sump was once again completely flushed prior to the April 10 collection.

\section{SURFACE-WATER DATA}

\section{MONTHLY MONITORING AT SIX LOCATIONS}

Surface-water samples from six locations were collected and analyzed in conjunction with monthly monitoring at the 15 drainwater sites. The data are given in table 5. The East Highline Canal represents irrigation water that is supplied to the Imperial Valley, and the New and Alamo Rivers and Trifolium Drain 1, where they discharge to the Salton Sea, represent the accumulated input of agricultural drainage in the Imperial Valley. All samples from the monthly collections were filtered. Quarterly data also include analyses of unfiltered samples that were collected using the equal-width-increment method and composited in a churn splitter.

Chemical data for a single sample from San Felipe Creek also are given in table 5. The sample from San Felipe Creek was a grab sample collected immediately west of Highway 78 just south of the junction of Highways 78 and 86 . This collection site is upstream from the cultivated part of the San Felipe Creek subbasin that is irrigated with Colorado River water. The channel contained extremely shallow flows and isolated pools when the sample was collected.

Comprehensive data for water samples from the Salton Sea are given and described in a later section of this report.

\section{HISTORICAL TRITIUM CONCENTRATIONS IN THE COLORADO RIVER}

Tritium concentrations in surface water, ground water, and shallow soils were determined during this study to distinguish post-1950 irrigation water from older water in the Imperial Valley and to place time constraints on the percolation of irrigation water to subsurface drains. Historical tritium concentration data for the lower Colorado River at Imperial Dam (table 6) are essential to both objectives. The historical data were obtained from a national monitoring network operated by the USGS (Michel, 1989). All tritium concentrations are reported in picocuries per liter $(\mathrm{pCi} / \mathrm{L})$ - obtained by multiplying concentrations reported by the analyst in tritium units by 3.2 . Annual average tritium concentrations, and concentrations adjusted for radioactive decay to 1988 (the year in which most drainwater, surface-water, and groundwater samples were collected for this study), are given in table 6. The average concentration of 90 $\mathrm{pCi} / \mathrm{L}$ in 1988 , which is based on the USGS monitoring of the Colorado River at Imperial Dam, can be compared with values of $82,88,90$, and $96 \mathrm{pCi} / \mathrm{L}$ obtained for this study during August 1988 on water samples from the All-American and East Highline Canals.

\section{ALAMO RIVER DELTA SAMPLING}

Aqueous-profile and surficial-sediment chemical data were obtained along a transect across the Alamo 
River delta (figs. $2 A, 3$ ) from the mouth of the river to the Salton Sea to determine the location of the mixing zone between freshwater and saltwater and to ascertain any areal trends in selenium concentration in the sediment. The location of sample-collection sites and the selenium-concentration data from sediment samples are shown in figure 3 . Sediment samples were collected, using either a small Ekman dredge or a piston corer, in August 1988 and February 1989. See Stewart and others (1992) for data from samples collected in February 1989 and for a description of analytical methods, precision, and reporting limits.

Water samples were collected from an airboat; a Van Dorn sampler was used in deep water, and a wide-mouth glass bottle was used in embayments where water depths were less than about $1 \mathrm{ft}$. In addition, specific conductance, temperature, $\mathrm{pH}$, and dissolved oxygen were measured in the field with a multi-parameter probe. Sampling was done in August 1988, February 1989, and August 1989. Results of these field measurements for selected (1988) samples are given in table 7 . The freshwater/saltwater interface occurs over a very narrow horizontal distance. The interface is shown by the vertical profile to be at delta site 10 , about $200 \mathrm{ft}$ from the levee on the left bank of the Alamo River, where freshwater is present above the more dense Salton Sea water.

\section{SELENIUM REDOX SPECIATION}

Previous studies showed that a high proportion of selenium in the low-selenium water (that is, low in comparison with drainwater) from the Salton Sea was in highly reduced (negative oxidation) states (Cooke and Bruland, 1987). Also shown were high selenium concentrations (also, likely in reduced state) in surficial sediments from the Salton Sea (Schroeder and others, 1988), and high concentrations in drainwater that are consistent with selenium in the highly soluble oxidized $(+6)$ state. These studies indicated the need for selenium redox-speciation analyses of water, especially near the Salton Sea. Several aqueous samples were analyzed for selenate $(+6)$, for the less highly oxidized and less soluble selenite $(+4)$, and for total selenium; selenium in negative and zero oxidation states was not determined. The proportion as highly oxidized selenate $(+6)$ decreased from 60 percent on the freshwater side at the mouth of the Alamo River to below detection on the saltwater side in the Alamo River delta. At drilling site 8 (see next section of this report), ground water from piezometers in which selenium was present, albeit at low concentrations, contained small amounts of selenite $(+4)$ but no detectable selenate-in contrast to drainwater from the sump (S-417) at this site, in which 98 percent of the total selenium content (concentration was 275 $\mu \mathrm{g} / \mathrm{L}$ ) was selenate (Ann Maest, USGS, written commun., 1990).

\section{LITHOLOGY AND GROUND-WATER DATA}

\section{LITHOLOGY AT THREE DRILLING SITES}

Holes were drilled for installation of multipledepth piezometers and suction-cup lysimeters at three sites in the Imperial Valley. The sites are located in the northern, middle, and southern parts of the valley (sites 8,50 , and 98 in fig. $2 B$ ) and are near the valley's topographic axis. The purpose of the drilling and sampling program was to determine changes in ground-water quality with depth and to ascertain the possible influence of shallow, regional ground water on drainwater quality.

A deep hole (depth of about 100-200 ft) was drilled at each location with a mud-rotary rig using irrigation water from nearby canals to make up the drilling fluid. Several 2-inch-diameter PVC piezometers with 5-foot perforated intervals were installed at each location. The annulus was backfilled with sand opposite the perforations, with bentonite pellets between the perforations, with grout above the shallowest perforation to within 3 to $5 \mathrm{ft}$ of land surface, and with cement from above the grout to land surface. At the middle and southern sites, where spacing of perforated intervals was thought to be too close to permit installation of a good bentonite seal, a second, shallower hole was drilled and one piezometer was installed at a preselected depth.

The near-surface soils in Imperial Valley are fine grained; clay (particle size less than $0.004 \mathrm{~mm}$ ) content ranged from about 70 percent at the northern drilling site to about 20 percent at the southern drilling site (table 8). The lithologic description in table 9 was prepared from the drill operator's observations of drilling characteristics, field observations on cuttings brought to the surface in the mud, grainsize analysis of cores shown in table 8 , and geophysical logs (caliper, gamma ray, spontaneous potential, and single-point resistivity). A 2-foot split-spoon core was collected whenever field observations, including a perceived change in drill characteristics, indicated a possible significant change in texture from that of the overlying material.

The objective of drilling was to penetrate at least one thick clay-rich, low-permeability zone and place 
piezometers in the more permeable material above and below this zone, thereby increasing the likelihood that the deepest piezometer tapped the shallow, regional ground-water system. This objective was readily achieved at the northern site, where the two deepest piezometers were separated from each other by a clay-rich zone extending from 85 to $181 \mathrm{ft}$ below land surface (table 9). At the middle site, and especially at the southern site, numerous clay-rich layers were penetrated that are much thinner than those at the northern site. As a result, one or more piezometers were installed within the shallow, regional groundwater system at the middle and southern sites.

Water levels in the shallowest piezometers from the northern and middle sites were below land surface, and close to the altitude of the subsurface drains. However, in the 75- and 199-foot piezometers at the northern site and in the 71- and 95-foot piezometers at the middle site, ground water flowed at $1 \mathrm{~L} / \mathrm{min}$ or more from the casing that extended about $2 \mathrm{ft}$ above land surface. At both of the flowing (artesian) piezometers at the northern site, the ground water was heavily charged with carbon dioxide and had temperatures indicative of a higher than normal geothermal gradient. Numerous mudpots with escaping carbon dioxide are present beneath nearby parts of the Salton Sea and the Imperial Wildlife Management Area, and geothermal-resource development is prevalent in the area. The 75-foot piezometer always "resealed" itself a few weeks after sampling, but it could be induced to erupt again, several feet into the air, with minimal pumping and physical agitation of the water in the well casing. Water levels in all piezometers at the southern site, unlike conditions at the northern and middle sites, were near or a few feet below the depth of the drains.

A few piezometers were placed opposite clayrich, low-permeability zones (as designated in table 9). After complete evacuation of the casing, recovery from these piezometers was very slow (about $1 \mathrm{~L} / \mathrm{hr}$ ); hence, it is believed that they were never completely purged (during the time of this study) of drilling fluid. The presence of small quantities of drilling fluid mixed with formation water in these samples is supported by reversal of the general pattern of decreasing tritium with increasing depth, and may be the indirect cause (by stimulating biological activity) of a slight odor of hydrogen sulfide that was observed when these wells were first pumped to remove stagnant water from the casing and annulus.

The holes for installation of as many as four multiple-depth ceramic-cup suction lysimeters (not all the lysimeters produced water) were drilled using an air-rotary technique that eliminated the need for extensive development prior to sample collection. A very fine crystal-silica sand was placed opposite the cups, and coarse sand was placed immediately above and below each lysimeter. A bentonite seal was placed between each of the lysimeters. Installation of lysimeters at the southern site was unusually difficult. Collapse of the hole occurred during drilling because of the presence of thin, fine-sand and silt lenses and of wetness owing to nearby canals, ditches, a tailwater channel, and the sump. As a result, the chemical data from shallow depths at this location may be unduly influenced by the direct infiltration of comparatively fresh irrigation water, and thus the data are not representative of drainwater conditions in interior parts of the field.

The lysimeters are located next to the sump at a corner of the field at the southern and middle sites and along the edge of a field at the northern site. Partly because of access constraints and partly to avoid localized influences from irrigation, the piezometers were installed along elevated dirt roads a short distance from the fields.

\section{GROUND-WATER QUALITY}

Ground-water-quality data from the lysimeters and piezometers in Imperial Valley are given in table 10. Water sampled from the lysimeters was effectively filtered during passage of the water from the soil through the ceramic cup. Samples from piezometers were filtered through a $0.45-\mu \mathrm{m}$ membrane in the field as the ground water was pumped directly from the casing. Noteworthy is the fact that a large quantity of carbonate precipitated almost immediately in the collection bottle as the supersaturated solutions from the two deepest piezometers at site 8 (the northern drilling site) degassed carbon dioxide. Much smaller quantities of carbonate also precipitated as deep ground-water samples were brought to the surface at site 50 (the middle drilling site).

\section{SOILS AND POREWATER DATA}

Soil samples for chemical and tritium analyses were obtained by manually augering to depths of about 3 and $6 \mathrm{ft}$ in fields at the 15 monthly drainwater-monitoring sites. These depths correspond to the maximum depth of cultivation (ripping by chisel) and to a common depth for subsurface-drain installation, respectively. Soils were recovered at the 
head $(\mathrm{H})$, middle $(\mathrm{M})$, and tail $(\mathrm{T})$ of the field-from directly above, midway between, and one-quarter the distance between drain laterals. Hence, S-417-T-1850-6 designates a sample from the tail end of the field, at $S-417,50 \mathrm{ft}$ from subsurface drain lateral 18 , at a depth of $6 \mathrm{ft}$.

Cores from the three drilling sites are designated by depth below land surface. All solid-phase and soils-extraction data (tables 11-15) include the laboratory identification number used by the USGS to designate the sample analyzed.

\section{SHALLOW SOILS}

Chemical analysis of soils from fields at the 15 sites was done to establish range in constituent concentrations and any possible relation between concentrations in soil and drainwater. Deionized-water extracts of soils (using a water-to-soil ratio of 5 to 1) were analyzed to ascertain the possible relation between extract and drainwater chemistry, and between extract and soil concentrations. Data are presented in tables 11,12 , and 13 . The 40 samples in table 12 are laboratory splits (samples reprocessed and analyzed separately) for selected samples in table 11, and results can be compared between the tables.

\section{CORES}

Cores were analyzed for comparison of concentrations of constituents (especially selenium) with concentrations in shallow soils. Data are presented in tables 14 and 15.

In addition, deionized-water extracts were obtained to provide a quality-assurance check on the entire extraction procedure by comparison to known concentrations in ground water. Comparison of the extract data in table 14 with the ground-water data in table 10 confirms the presence of some aqueous selenium in core extracts from depths below which selenium in ground water is absent (less than $1 \mu \mathrm{g} / \mathrm{L}$ ). This indicates that a significant portion of the aqueous selenium extracted from the solid phase is an artifact of the storage and (or) extraction procedure itself, and this fact must be considered when interpreting the extract data from shallow soils in tables 11 and 12 .

\section{TRITIUM IN SHALLOW SOILS}

Tritium concentrations were measured in soil moisture (porewater), which ranged from 18 to 30 percent of the soil on a wet-weight basis. The analysis was done on porewater baked from the soil at a temperature of about $80^{\circ} \mathrm{C}$. The purposes of these analyses were to determine possible spatial variation within fields and to estimate the rate at which shallow, subsurface water moves to the drains. The soils that were sampled were obtained from 8 of the 15 fields where drainwater was monitored monthly for 1 year. The results, which are given in table 16 , can be compared with historical tritium concentration in irrigation water from the Colorado River (table 6 ) and with drainwater tritium concentrations (table 4).

\section{LABORATORY EXPERIMENTAL DATA}

\section{EVAPORATIONS OF IRRIGATION WATER}

In order to determine more accurately the selenium-to-chloride ratio in irrigation water, periodic samples from the East Highline Canal at its diversion from the All-American Canal were concentrated by evaporation in the laboratory and then analyzed. Water was reduced to as little as 2 percent of its original volume (final volume approximately $500 \mathrm{~mL}$ ) by evaporation at $30-35^{\circ} \mathrm{C}$, with continuous stirring, from large glass chromatography jars; the concentrated sample then was centrifuged prior to analysis. About 1 month was required to complete each evaporation sequence. Evaporations were carried out using both raw water and water acidified to a $\mathrm{pH}$ of approximately 3 by addition of nitric acid (except for the January 1989 evaporation sequence, in which hydrobromic acid was used but found to be contaminated by a small but significant quantity of chloride) prior to evaporative concentration. Low $\mathrm{pH}$ was maintained in the acid-treated water to prevent formation of calcium carbonate, which began to appear in the untreated water soon after evaporation commenced. Calcium sulfate precipitated during evaporation of both the acidified and untreated waters.

Calculated mean selenium-to-chloride weight (gravimetric) ratio for the most concentrated sample from eight evaporation studies during April-December 1989 (on the basis of analyses reported in table 17) is $2.2 \times 10^{-5}$ in the nitric-acid-acidified concentrates (range 1.7-2.6 $\times 10^{-5}$ ) and $1.7 \times 10^{-5}$ in the untreated concentrates (range 1.4-2.3 $\times 10^{-5}$ ). Presence of some selenium in the calcium carbonate (dissolved with nitric acid and diluted by addition of about $100 \mathrm{~mL}$ of deionized water) that formed during evaporation of the untreated water is indicated by data in table 17 . However, mass-balance calculations to quantify the apportionment of selenium between aqueous and solid 
phases should not be attempted because aqueous volumes (and precipitate weight) were only estimated, and the precipitate itself was only partially rinsed with deionized water prior to its dissolution with acid.

Analysis of both precipitates from one evaporation sequence using complete-mineral digestion techniques demonstrates that incorporation of selenium in the laboratory experiment is by calcium carbonate but not by calcium sulfate. Results of the comparison were as follows:

\begin{tabular}{lcc}
\hline \multicolumn{1}{c}{ Element } & $\begin{array}{c}\text { Untreated } \\
\text { evaporation }\end{array}$ & $\begin{array}{c}\text { Acidified } \\
\text { evaporation }\end{array}$ \\
\hline Calcium (percent) & 14 & 23 \\
Sulfur (percent) & 11 & 18 \\
Selenium $(\mu \mathrm{g} / \mathrm{g})$ & 6 & $<0.1$ \\
\hline
\end{tabular}

Note that other minerals, in addition to calcium sulfate and calcium carbonate, also are present (silica, for example) as indicated by lower calcium percentages than would be present in a pure phase of either of these two calcium-containing minerals.

\section{DILUTIONS OF SEAWATER AND SALTON SEA WATER}

Results of chemical analyses of water samples from the Salton Sea and the Pacific Ocean (near the San Diego coast), and of samples from both sources diluted by addition of deionized water are given in table 18 . These data were obtained to provide quality assurance for chloride and bromide analyses over the range in concentrations present in the study area. The published average oceanic chloride-to-bromide weight ratio of 288 (Riley and Skirrow, 1975) can be compared with mean ratios of 317 in undiluted and 307 in diluted seawater calculated from the data in table 18. The measured chloride-to-bromide ratio in Salton Sea water was about 1,300 in both diluted and undiluted samples. The data in table 18 are from analysis of samples that were collected and archived for periodic later analysis; the dates given in the table correspond to the approximate periodic monthly spacing of the evaporation experiments described in the preceding section rather than the actual date of sample collection.

Analyses of major and selected minor constituents in filtered samples collected in October 1989 provide data for a comprehensive chemical comparison of seawater and water from the Salton Sea. Comparison between published and measured chemical concentrations in seawater provides an indication of the accuracy of saline-water analyses for this study.
Isotope data on grab samples collected during August 1988 from nearshore areas (fig. 2A) around the Salton Sea-at the Niland boat ramp, North Shore pier, Salton City pier, center of the south Salton Sea basin (the only site not close to shore), and between the Alamo and New River deltas-are given (in temporal order of collection) in table 18 .

\section{BIOLOGICAL DATA}

\section{BACKGROUND}

Increased concern about trace-element and organic contaminant loading to the Salton Sea and nearby areas from the associated drainwater system led to this examination of possible contamination of these habitats. Sampling was done in 1986-87 during a reconnaissance investigation to assess concentrations of trace elements and selected pesticides. Chemical data and interpretations from the reconnaissance were published by Setmire and others (1990b). Elevated levels of selenium, boron, and DDT metabolites were found during the reconnaissance. These findings led to the decision to do a more detailed study during the following 3 years, at which time additional biological samples were collected to determine contaminant pathways and levels of exposure of organisms to contaminants.

\section{SAMPLING SITES}

The location of sampling sites for collection of biological data is shown in figure 4 , and sites are listed in table 19. The sites were chosen on the basis of ecological importance to fish and wildlife, relevance to exposure pathways, and strategic importance to tracking of contaminant sources. Priority was given to areas with high resource use, areas adjacent to high pesticide use, and areas of known high traceelement concentrations.

\section{SAMPLE COLLECTION AND PRESERVATION}

The biological samples that were collected include 31 diverse species (or genera) representing all the trophic levels present in the Salton Sea area. The samples include vegetation, invertebrates, fish, amphibians, reptiles and reptile eggs, and birds and bird eggs. The species collected and the locations from which they were taken are listed in table 20 . The procedures followed for collection of biological samples are those specified by USFWS (1985b). All bio- 
logical samples were frozen after collection and stored frozen until analysis.

Vegetation was collected by hand from agricultural drainage ditches and from various locations in the Salton Sea where suitable habitat was provided by a rocky substrate. Algae were collected from several shore locations around the Salton Sea. Excess debris was removed from the algae by washing with Salton Sea water, after which the algae sample was placed in chemically cleansed jars. Cattail samples were collected from agricultural drainage ditches. Excess debris was removed from cattails by washing at the collection site with drainwater before placement of the sample in polyethylene bags for frozen storage.

Invertebrates, such as waterboatmen and amphipods, were collected using lighted activity traps. Pileworms were collected by washing sediment onto fine-meshed screens. Crayfish were collected using small seines. Some Asiatic river clams that were retrieved from rivers and drainage ditches (drains) were specimens transplanted from a section of the Colorado River in plastic-mesh cages; the others were native Asiatic river clams that were collected from rivers and drains by sifting sediment through a sieve. All clams were shucked and their contents were placed in chemically cleansed jars. All other invertebrate samples were composited whole and placed in chemically cleansed jars.

Small fish, including both predator and prey species, were collected using long-handled dip nets or seines. Mosquitofish and sailfin mollies were obtained from rivers, creeks, and drains. Longjaw mudsuckers were obtained from the Salton Sea. Bairdiella were salvaged soon after they had washed ashore from the Salton Sea near Salton Sea NWR Unit 1 (the southern unit). Orangemouth corvina were collected from the Salton Sea using gill nets. Bairdiella and corvina were wrapped in aluminum foil and placed in polyethylene bags before freezing. All other fish species were placed in chemically cleansed jars.

Bullfrogs were collected from the Alamo River, and spiny softshell turtles were collected at Vail 4 Drain and at Hazard Pond. Frogs were speared and placed in aluminum foil. Turtles were collected using fish-baited hoop nets. Samples of liver and fat were dissected from the turtles and placed in chemically cleansed jars. Egg contents from one of the female turtles were composited and placed in chemically cleansed jars.
Several water-bird species were collected from the Salton Sea itself and from various locations throughout the Imperial Valley including rivers, creeks, drainwater ditches, and freshwater impoundments. The birds were shot using a 12-gauge shotgun and steel shot. Livers and breast-muscle tissue were dissected from birds of the following species and placed in chemically cleansed jars: ruddy duck, northern shoveler, black-necked stilt, American coot, eared grebe, and white-faced ibis. Carcasses from the black-necked stilts were wrapped in aluminum foil and placed in polyethylene bags or in chemically cleansed jars. Black-necked stilt eggs were collected from nesting sites near the Salton Sea. Eggs were harvested and their contents placed in chemically cleansed jars. One Yuma clapper rail was salvaged from the Wister Unit of the Imperial Wildlife Management Area; its carcass was wrapped in aluminum foil and placed in a polyethylene bag.

\section{CHEMICAL CONCENTRATIONS}

The inorganic and organic analytes determined in biological samples are listed in table 21. Inorganic concentrations are given in table 22. Organochlorineinsecticide and polychlorinated biphenyl (PCB) concentrations are given in table 23. The PCB data include total-PCB concentrations and concentrations for categories based on the number of chlorine atoms present and on the four-digit Arochlor number used to designate commercial mixtures of the PCB congeners. Polycyclic aromatic hydrocarbon concentrations are given in table 24 .

\section{References Cited}

Arbogast, B.F., ed., 1990, Quality assurance manual for the Branch of Geochemistry: U.S. Geological Survey Open-File Report 90-668, 183 p.

Cooke, T.D., and Bruland, K.W., 1987, Aquatic chemistry of selenium: Evidence of biomethylation: Environmental Science and Technology, v. 21, no. 12, p. 1214-1219.

Densmore, J.N., 1991, Water quality conditions in shallow subsurface waters, Imperial Valley, California: unpublished M.S. thesis, San Diego State University, 178 p.

Fishman, M.J., and Friedman, L.C., eds., 1989, Methods for determination of inorganic substances in water and fluvial sediments: U.S. Geological Survey Techniques of Water-Resources Investigations, Book 5, Chapter A1, $545 \mathrm{p}$. 
Fritz, P., and Fontes, J., Ch., eds., 1980, Handbook of Environmental Isotope Geochemistry, v. 1, The Terrestrial Environment, A: Ellsevier, 1980, 545 p.

Guy, H.P., 1969, Laboratory theory and methods for sediment analysis: U.S. Geological Survey Techniques of Water-Resources Investigations, Book 5, Chapter $\mathrm{C} 1$, $58 \mathrm{p}$.

Lowe, T.P., May, T.W., Brumbaugh, W.G., and Kane, D.A., 1985, National contaminant biomonitoring program: Concentration of seven elements in freshwater fish, 1979-1981: Archives of Environmental Contamination and Toxicology, v. 14, p. 363-388.

Michel, R.L., 1989, Tritium deposition in the continental United States, 1953-83: U.S. Geological Survey Water-Resources Investigations Report 89-4072, 46 p.

Michel, R. L., and Schroeder, R.A., 1989, Isotopic composition of drainwaters in the Imperial Valley, California: Eos Transactions of the American Geophysical Union, Abstract, v. 70, no. 43, p. 1379.

Michel, R.L., Schroeder, R.A., Setmire, J.G., and Hall, S.S., 1988, Soluble salts and tritium concentrations in irrigation drainwaters from the Imperial Valley, California: Eos Transactions of the American Geophysical Union, Abstract, v. 69, no. 44, p. 1181.

Moore, S.L., 1991, The use of tile drainage in the reclamation of the Imperial Valley: unpublished M.A. thesis, University of San Diego, 113 p.

Pritt, Jeffrey, and Jones, B.E., 1989, eds., 1990 National Water Quality Laboratory services catalog: U.S. Geological Survey Open-File Report 89-386, variously paged.

Riley, J.P., and Skirrow, G., eds., 1975, Chemical Oceanography, v. 1: Academic Press, 2d ed., 606 p.

Schroeder, R.A., Setmire, J.G., and Densmore, J.N., 1989, Controls on drainwater composition in the Imperial Valley, California, in Pederson, G.L., and Smith, M.M., compilers, U.S. Geological Survey Water Resources Division National Symposium on Water Quality, Orlando, Florida, November 12-17, 1989: U.S. Geological Survey Open-File Report 89-409, Abstract, p. 85.

1991, Use of stable isotopes, tritium, soluble salts, and redox-sensitive elements to distinguish ground water from irrigation water in the Salton Sea basin, in W.F. Ritter, ed., Proceedings of the 1991 National Conference: American Society of Civil Engineers, Irrigation and Drainage Division, Honolulu, Hawaii, July 22-26, 1991, p. 524-530.
Schroeder, R.A., Setmire, J.G., and Wolfe, J.C., 1988, Trace elements and pesticides in the Salton Sea area, California, in Proceedings on Planning for Irrigation and Drainage: American Society of Civil Engineers, Irrigation and Drainage Division, Lincoln, Nebraska, July 19-21, 1988, p. 700-707.

Setmire, J.G., Goodbred, S.L., Audet, D.J., Schroeder, R.A., Radke, W.R., and Densmore, J.N., 1993, Detailed study of water quality, bottom sediment, and biota associated with irrigation drainage in the Salton Sea area, California, 1988-90: U.S. Geological Survey Water-Resources Investigations Report 93-4014.

Setmire, J.G., Schroeder, R.A., and Densmore, J.N., 1990a, Shallow drainwater chemistry in the Salton Sea area, Imperial County, California: Selenium V Symposium, University of California (Berkeley), March 30-31, 1990, Abstract, 2 p.

Setmire, J.G., Wolfe, J.C., and Stroud, R.K., 1990b, Reconnaissance investigation of water quality, bottom sediment, and biota associated with irrigation drainage in the Salton Sea area, California, 1986-87: U.S. Geological Survey Water-Resources Investigations Report 89-4102, 68 p.

Severson, R.C., Wilson, S.A., and McNeal, J.J., 1987, Analyses of bottom material collected at nine areas in the Western United States for the DOI irrigation drainage task group: U.S. Geological Survey OpenFile Report 87-490, 24 p.

Stewart, K.C., Fey, D.L., Hageman, P.L., Kennedy, K.R., Love, A.H., McGregor, R.E., Papp, C.S.E., Peacock, T.R., Sharkey, J.D., Vaughn, R.B., and Welsch, E.P., 1992, Results of chemical analysis for sediments from Department of the Interior National Irrigation Water Quality Program studies, 1988-1990: U.S. Geological Survey Open-File Report 92-443, 38 p.

Stumm, Werner, and Morgan, J.J., 1970, Aquatic chemistry: an introduction emphasizing chemical equilibria in natural waters: New York, John Wiley, 583 p.

U.S. Fish and Wildlife Service, 1985a, Procedures for resource contaminant assessment contract analytical work: Habitat Resources Instructional Memorandum, $203 \mathrm{p}$

$1985 \mathrm{~b}$, Field operations manual for resource contaminant assessment: Habitat Resources Instructional Memorandum, Chapter 1.5, $48 \mathrm{p}$.

Wershaw, R.L., Fishman, M.G. Grabbe, R.R., and Lowe, L.E., eds., 1987, Methods for the determination of organic substances in water and fluvial sediments: U.S. Geological Survey Techniques of WaterResources Investigations, Book 5, Chapter A3, 80 p. 


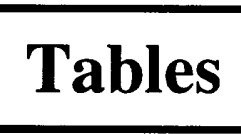

Tables 


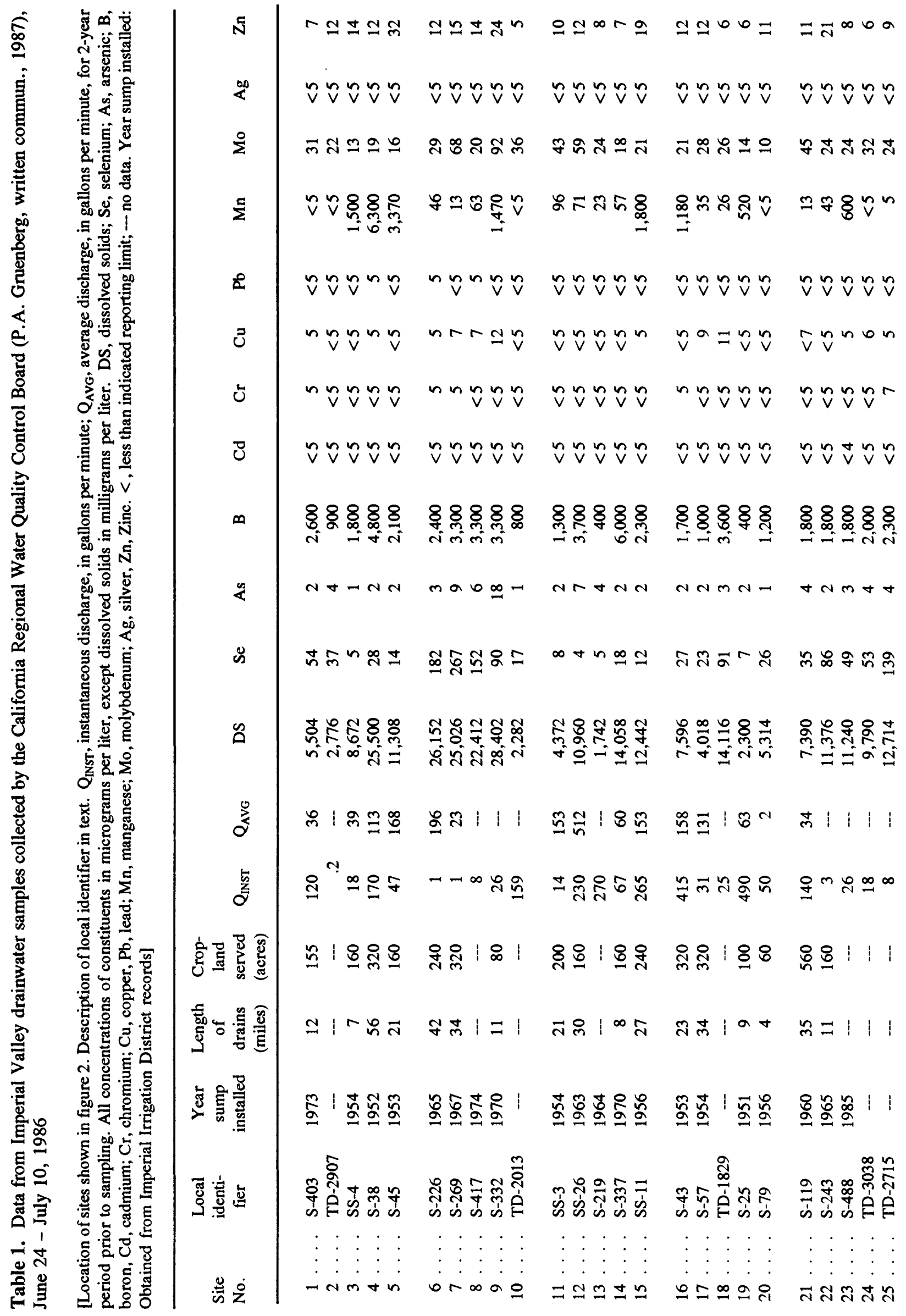




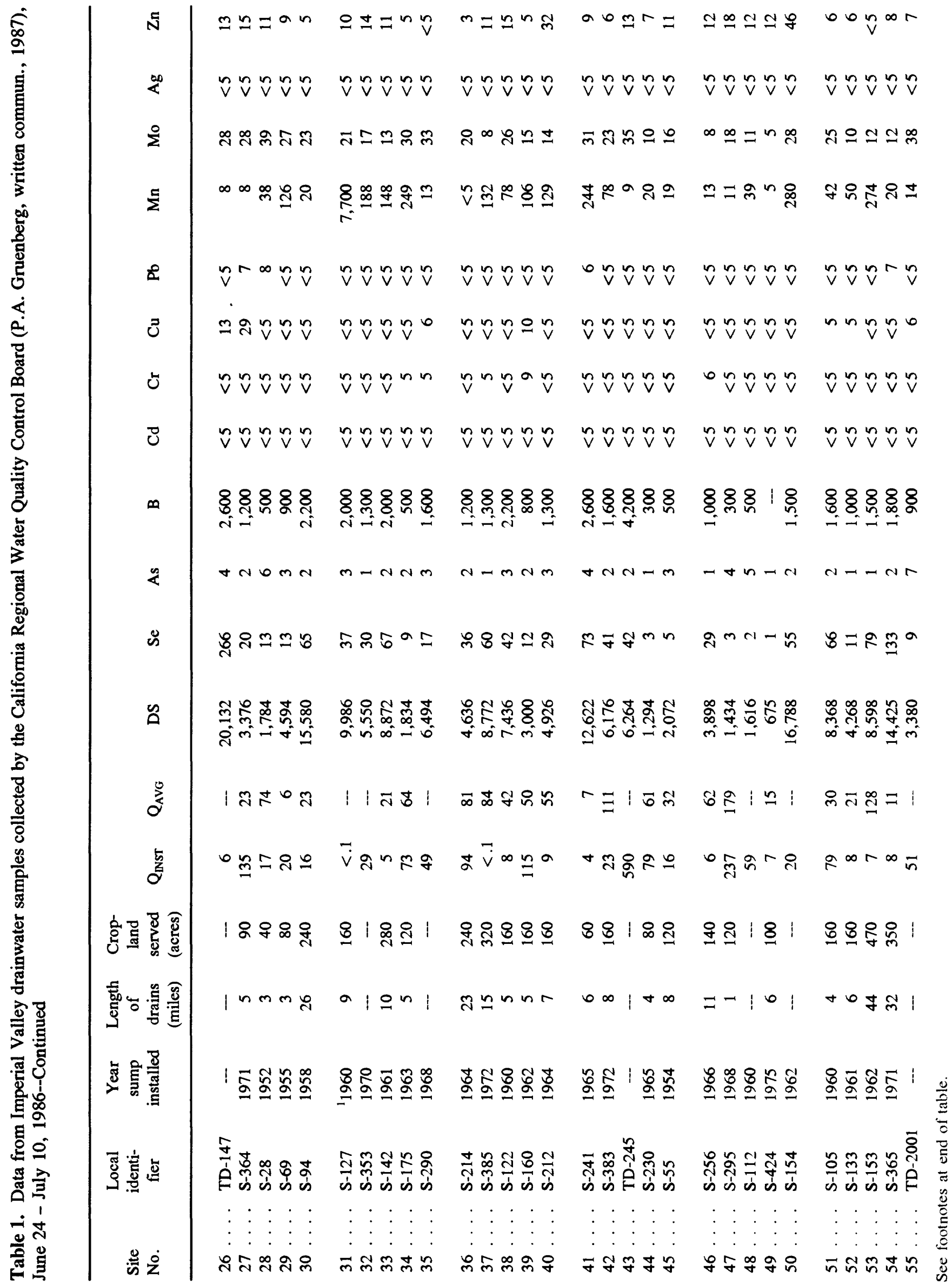




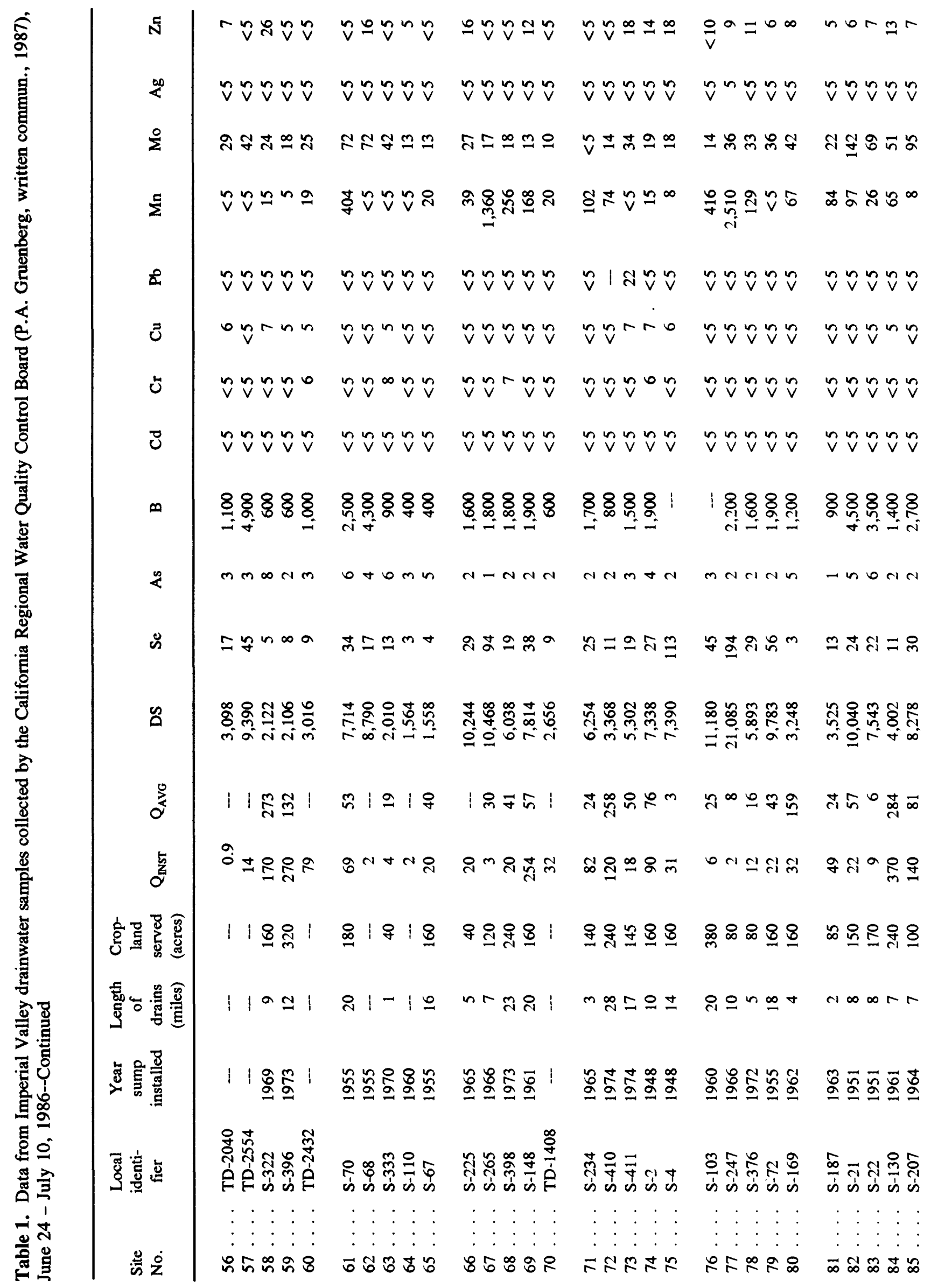




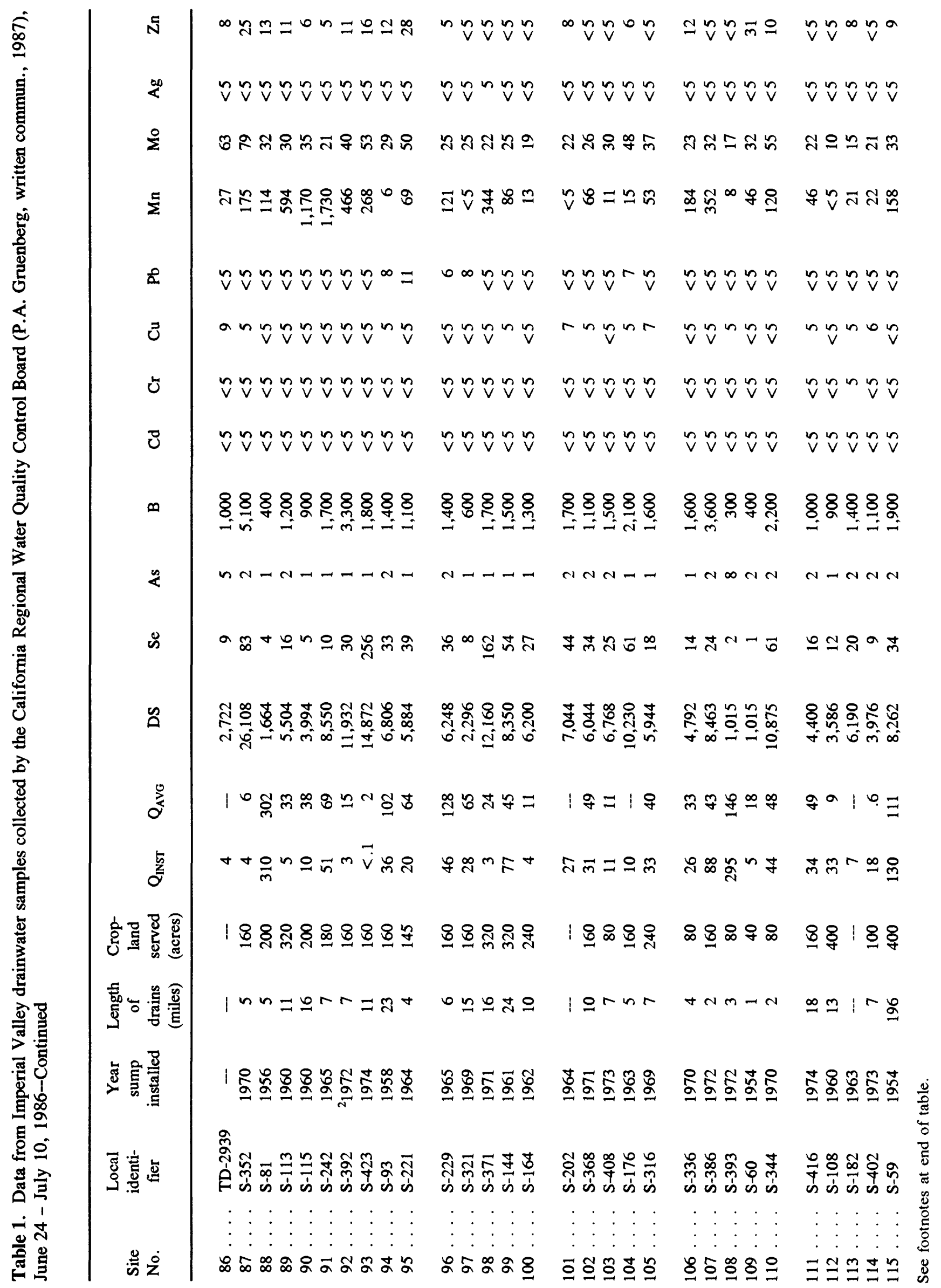




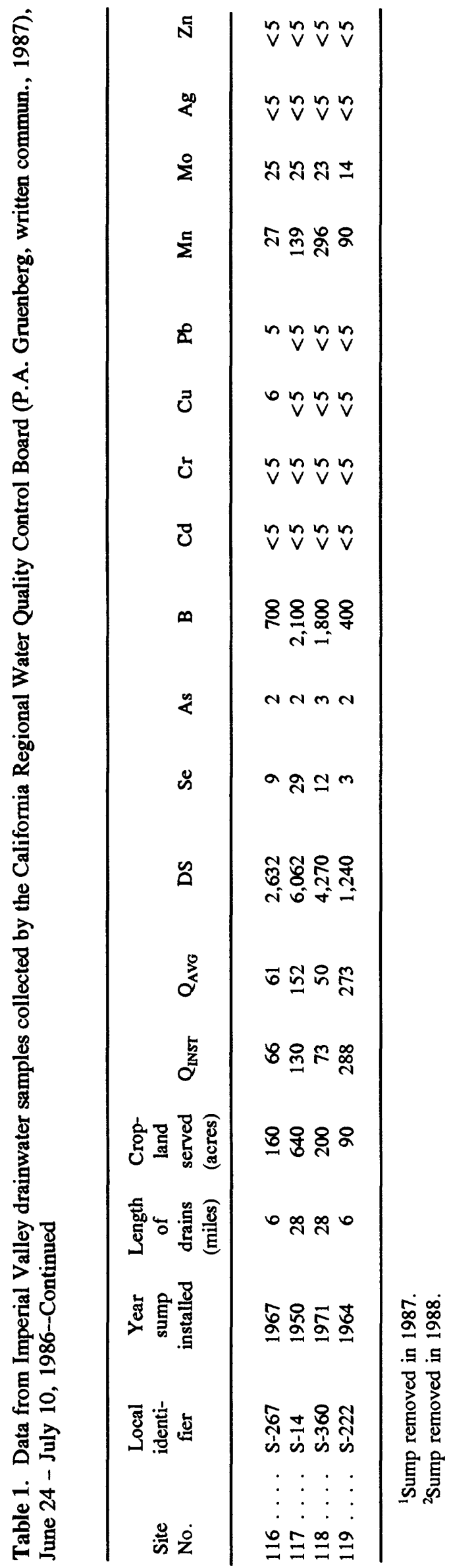


Table 2. Data from Imperial Valley drainwater samples collected by the U.S. Geological Survey, May 16-20, 1988

[Location of sites shown in figure 2. Date: Eleven sites with no date shown were sampled in 1986 (see table 1 for results) but not sampled in 1988; Altitude of land surface in feet above or below (-) sea level. $\mathrm{ft}^{3} / \mathrm{s}$, cubic foot per second; $\mu \mathrm{S} / \mathrm{cm}$, microsiemen per centimeter at $25^{\circ} \mathrm{C} ;{ }^{\circ} \mathrm{C}$, degree Celsius; $\mathrm{mg} / \mathrm{L}$, milligram per liter; $\mu \mathrm{g} / \mathrm{L}$, microgram per liter; ---, no data. The analysis for each sample is displayed as one line on three consecutive pages]

\begin{tabular}{|c|c|c|c|c|c|c|c|c|c|}
\hline $\begin{array}{c}\text { Site } \\
\text { number }\end{array}$ & $\begin{array}{c}\text { Local } \\
\text { identifier }\end{array}$ & Latitude & Longitude & Date & Time & $\begin{array}{c}\text { Altitude } \\
\text { of } \\
\text { land } \\
\text { surface }\end{array}$ & $\begin{array}{c}\text { Discharge, } \\
\text { instanta- } \\
\text { neous } \\
\left(\mathrm{ft}^{3} / \mathrm{s}\right)\end{array}$ & $\begin{array}{c}\text { Specific } \\
\text { conductance } \\
(\mu \mathrm{S} / \mathrm{cm})\end{array}$ & $\begin{array}{c}\mathrm{pH} \\
\text { (standard } \\
\text { units) }\end{array}$ \\
\hline
\end{tabular}

\begin{tabular}{|c|c|c|c|c|c|c|c|c|c|}
\hline 1 & $\ldots$ S-403 & $33^{\circ} 18^{\prime} 02^{\prime \prime} \mathrm{N}$ & $115^{\circ} 35^{\prime} 22^{\prime \prime} \mathrm{W}$ & $5-16-88$ & 1045 & -195 & 0.018 & 11,300 & 7.4 \\
\hline 2 & . . . TD-2907 & $33^{\circ} 18^{\prime} 55^{\prime \prime} \mathrm{N}$ & $115^{\circ} 33^{\prime} 40^{\prime \prime} \mathrm{W}$ & --- & --- & --- & --- & --- & --- \\
\hline 3 & $\ldots$ SS-4 & $33^{\circ} 10^{\prime} 33^{\prime \prime} \mathrm{N}$ & $115^{\circ} 37^{\prime} 09^{\prime \prime} \mathrm{W}$ & $5-16-88$ & 1230 & -227 & .012 & 8,640 & 7.0 \\
\hline 4 & . . S-38 & $33^{\circ} 10^{\prime} 58^{\prime \prime} \mathrm{N}$ & $115^{\circ} 36^{\prime} 15^{\prime \prime} \mathrm{W}$ & $5-16-88$ & 1255 & -228 & -- & 38,200 & 6.9 \\
\hline 5 & $\ldots$ S-45 & $33^{\circ} 10^{\prime} 10^{\prime \prime} \mathrm{N}$ & $115^{\circ} 37^{\prime} 50^{\prime \prime} \mathrm{W}$ & $5-16-88$ & 1320 & --- & .070 & 13,900 & 7.1 \\
\hline 6 & . . . S-226 & $33^{\circ} 10^{\prime} 06^{\prime \prime} \mathrm{N}$ & $115^{\circ} 33^{\prime} 21^{\prime \prime W}$ & $5-16-88$ & 1415 & -212 & .17 & 36,100 & 7.7 \\
\hline 7 & . . S S-269 & $33^{\circ} 10^{\prime} 37^{\prime \prime} \mathrm{N}$ & $115^{\circ} 31^{\prime} 03^{\prime \prime W}$ & $5-16-88$ & 1430 & -183 & .079 & 28,200 & 7.1 \\
\hline 8 & . . S S-417 & $33^{\circ} 11^{\prime} 30^{\prime \prime} \mathrm{N}$ & $115^{\circ} 33^{\prime} 40^{\prime \prime} \mathrm{W}$ & $5-16-88$ & 1450 & -217 & .0028 & 44,000 & 7.3 \\
\hline 9 & $\ldots \mathrm{S}-332$ & $33^{\circ} 11^{\prime} 57^{\prime \prime} \mathrm{N}$ & $115^{\circ} 33^{\prime} 39^{\prime \prime} \mathrm{W}$ & $5-16-88$ & 1510 & -216 & .0028 & 14,100 & 6.8 \\
\hline 10 & . . . TD-2013 & $33^{\circ} 11^{\prime} 55^{\prime \prime} \mathrm{N}$ & $115^{\circ} 26^{\prime} 55^{\prime \prime} \mathrm{W}$ & --- & --- & -.. & - & ... & $\cdots$ \\
\hline 11 & $\ldots$ SS-3 & $33^{\circ} 07^{\prime} 30^{\prime \prime} \mathrm{N}$ & $115^{\circ} 46^{\prime} 58^{\prime \prime W}$ & $5-17-88$ & 1140 & -230 & .054 & 7,280 & 7.2 \\
\hline 12 & . . . SS-26 & $33^{\circ} 09^{\prime} 15^{\prime \prime} \mathrm{N}$ & $115^{\circ} 48^{\prime} 15^{\prime \prime W}$ & $5-17-88$ & 1210 & -225 & .86 & 13,000 & 7.3 \\
\hline 13 & . . S S-219 & $33^{\circ} 04^{\prime} 53^{\prime \prime} \mathrm{N}$ & $115^{\circ} 41^{\prime} 57^{\prime \prime} \mathrm{W}$ & $5-17-88$ & 1305 & -219 & .15 & 3,890 & 7.3 \\
\hline 14 & . . S s-337 & $33^{\circ} 04^{\prime} 55^{\prime \prime} \mathrm{N}$ & $115^{\circ} 42^{\prime} 30^{\prime \prime W}$ & $5-17-88$ & 1250 & -- & -- & 39,700 & 7.2 \\
\hline 15 & . . . SS-11 & $33^{\circ} 08^{\prime} 23^{\prime \prime} \mathrm{N}$ & $115^{\circ} 39^{\prime} 19^{\prime \prime W}$ & $5-17-88$ & 1705 & -228 & .12 & 33,800 & 7.0 \\
\hline 16 & $\ldots \mathrm{s}-43$ & $33^{\circ} 09^{\prime} 42^{\prime \prime} \mathrm{N}$ & $115^{\circ} 37^{\prime} 48^{\prime \prime W}$ & $5-17-88$ & 1645 & -224 & .52 & 6,370 & 7.4 \\
\hline 17 & $\ldots$ S-57 & $33^{\circ} 06^{\prime} 53^{\prime \prime} \mathrm{N}$ & $115^{\circ} 34^{\prime} 42^{\prime \prime W}$ & $5-17-88$ & 1605 & -198 & .22 & 6,240 & 7.5 \\
\hline 18 & . . TD-1829 & $33^{\circ} 04^{\prime} 55^{\prime \prime} \mathrm{N}$ & $115^{\circ} 34^{\prime} 40^{\prime \prime} \mathrm{W}$ & --- & -- & -.- &.- & $\ldots$ & --- \\
\hline 19 & $\ldots$ S-25 & $33^{\circ} 06^{\prime} 15^{\prime \prime} \mathrm{N}$ & $115^{\circ} 33^{\prime} 39^{\prime \prime W}$ & $5-17-88$ & 1545 & -189 & .19 & 2,780 & 7.1 \\
\hline 20 & . . S S-79 & $33^{\circ} 07^{\prime} 30^{\prime \prime} \mathrm{N}$ & $115^{\circ} 32^{\prime} 33^{\prime \prime} \mathrm{W}$ & $5-17-88$ & 1530 & -190 & .11 & 7,480 & 7.1 \\
\hline 21 & $\ldots$ S-119 & $33^{\circ} 08^{\prime} 22^{\prime \prime} \mathrm{N}$ & $115^{\circ} 30^{\prime} 26^{\prime \prime W}$ & $5-17-88$ & 1510 & -182 & .079 & 6,700 & 7.3 \\
\hline 22 & $\ldots$ S-243 & $33^{\circ} 05^{\prime} 18^{\prime \prime} \mathrm{N}$ & $115^{\circ} 29^{\prime} 00^{\prime \prime} \mathrm{W}$ & $5-17-88$ & 1415 & -161 & .047 & 16,500 & 7.2 \\
\hline 23 & $\ldots$ S-488 & $33^{\circ} 05^{\prime} 18^{\prime \prime} \mathrm{N}$ & $115^{\circ} 27^{\prime} 26^{\prime \prime W}$ & $5-17-88$ & 1445 & -148 & .0052 & 16,900 & 7.5 \\
\hline 24 & $\ldots \mathrm{TD}-3038$ & $33^{\circ} 08^{\prime} 30^{\prime \prime} \mathrm{N}$ & $115^{\circ} 22^{\prime} 55^{\prime \prime W}$ & $5-16-88$ & 1605 & -.. & .0060 & 16,600 & 7.1 \\
\hline 25 & $\ldots$ TD-2715 & $33^{\circ} 07^{\prime} 10^{\prime \prime} \mathrm{N}$ & $115^{\circ} 24^{\prime} 55^{\prime \prime} \mathrm{W}$ & -- & -- & --- & -- & -- & $\ldots$ \\
\hline 26 & $\ldots$ TD -147 & $33^{\circ} 04^{\prime} 55^{\prime \prime} \mathrm{N}$ & $115^{\circ} 23^{\prime} 20^{\prime \prime} \mathrm{W}$ & $5-16-88$ & 1650 & --- & .016 & 21,000 & 7.1 \\
\hline 27 & $\ldots$ s-364 & $33^{\circ} 02^{\prime} 03^{\prime \prime} \mathrm{N}$ & $115^{\circ} 42^{\prime} 19^{\prime \prime} \mathrm{W}$ & $5-17-88$ & 1030 & -148 & .46 & 3,990 & 7.4 \\
\hline 28 & $\ldots$ S-28 & $32^{\circ} 59^{\prime} 18^{\prime \prime} \mathrm{N}$ & $115^{\circ} 37^{\prime} 07^{\prime \prime} \mathrm{W}$ & $5-17-88$ & 0910 & -121 & .084 & 2,520 & 7.7 \\
\hline 29 & . . S S-69 & $33^{\circ} 00^{\prime} 23^{\prime \prime} \mathrm{N}$ & $115^{\circ} 37^{\prime} 13^{\prime \prime W}$ & $5-17-88$ & 0900 & -139 & .014 & 4,430 & 7.3 \\
\hline 30 & . . S S-94 & $33^{\circ} 02^{\prime} 12^{\prime \prime} \mathrm{N}$ & $115^{\circ} 38^{\prime} 45^{\prime \prime} \mathrm{W}$ & $5-17-88$ & 0955 & -172 & .053 & 19,800 & 7.1 \\
\hline 31 & $\ldots$ S-127 & $33^{\circ} 01^{\prime} 15^{\prime \prime} \mathrm{N}$ & $115^{\circ} 36^{\prime} 10^{\prime \prime} \mathrm{W}$ & -- & +- & -- & --- & $-\cdots$ & --- \\
\hline 32 & $\ldots$. S-353 & $33^{\circ} 03^{\prime} 28^{\prime \prime} \mathrm{N}$ & $115^{\circ} 34^{\prime} 36^{\prime \prime W}$ & $5-17-88$ & 0935 & -167 & .0061 & 10,600 & 7.2 \\
\hline 33 & . . S S-142 & $33^{\circ} 04^{\prime} 26^{\prime \prime} \mathrm{N}$ & $115^{\circ} 29^{\prime} 39^{\prime \prime} \mathrm{W}$ & $5-18-88$ & 0800 & -159 & .0042 & 2,620 & 7.1 \\
\hline 34 & . . S S-175 & $33^{\circ} 01^{\prime} 45^{\prime \prime} \mathrm{N}$ & $115^{\circ} 33^{\prime} 52^{\prime \prime} \mathrm{W}$ & $5-17-88$ & 0825 & -142 & .15 & 4,310 & 7.4 \\
\hline 35 & . . S S-290 & $33^{\circ} 02^{\prime} 12^{\prime \prime} \mathrm{N}$ & $115^{\circ} 30^{\prime} 29^{\prime \prime} \mathrm{W}$ & $5-17-88$ & 0800 & -151 & .0036 & 9,580 & 7.0 \\
\hline 36 & . . S S-214 & $33^{\circ} 03^{\prime} 04^{\prime \prime} \mathrm{N}$ & $115^{\circ} 28^{\prime} 44^{\prime \prime W}$ & $5-17-88$ & 1805 & -150 & .064 & 7,480 & 7.0 \\
\hline 37 & . . . S-385 & $33^{\circ} 00^{\prime} 27^{\prime \prime} \mathrm{N}$ & $115^{\circ} 29^{\prime} 26^{\prime \prime W}$ & $5-17-88$ & 0740 & -141 & .049 & 12,200 & 7.1 \\
\hline 38 & . . S S-122 & $32^{\circ} 58^{\prime} 52^{\prime \prime} \mathrm{N}$ & $115^{\circ} 26^{\prime} 51^{\prime \prime W}$ & $5-18-88$ & 0730 & -127 & .092 & 10,400 & 7.2 \\
\hline 39 & . . S S-160 & $33^{\circ} 03^{\prime} 05^{\prime \prime} \mathrm{N}$ & $115^{\circ} 27^{\prime} 25^{\prime \prime} \mathrm{W}$ & $5-17-88$ & 1830 & -144 & .20 & 3,890 & 7.3 \\
\hline 40 & $\ldots$ s-212 & $33^{\circ} 03^{\prime} 04^{\prime \prime} \mathrm{N}$ & $115^{\circ} 27^{\prime} 53^{\prime \prime} \mathrm{W}$ & $5-17-88$ & 1820 & -147 & .053 & 7,430 & 7.3 \\
\hline 41 & $\ldots s-241$ & $33^{\circ} 04^{\prime} 26^{\prime \prime} \mathrm{N}$ & $115^{\circ} 27^{\prime} 53^{\prime \prime} \mathrm{W}$ & $5-17-88$ & 1900 & -151 & .014 & 10,800 & 7.3 \\
\hline 42 & $\ldots \mathrm{S}-383$ & $33^{\circ} 03^{\prime} 33^{\prime \prime} \mathrm{N}$ & $115^{\circ} 26^{\prime} 21^{\prime \prime W}$ & $5-17-88$ & 1845 & -134 & .067 & 6,710 & 7.0 \\
\hline 43 & $\ldots$ TD -245 & $33^{\circ} 01^{\prime} 20^{\prime \prime} \mathrm{N}$ & $115^{\circ} 20^{\prime} 40^{\prime \prime} \mathrm{W}$ & -- & -- & -- & --- & -- & $\cdots$ \\
\hline 44 & $\ldots \mathrm{S}-230$ & $32^{\circ} 58^{\prime} 23^{\prime \prime} \mathrm{N}$ & $115^{\circ} 40^{\prime} 31^{\prime \prime W}$ & $5-18-88$ & 1625 & -75 & .50 & 2,000 & 7.4 \\
\hline 45 & $\ldots S-55$ & $32^{\circ} 57^{\prime} 00^{\prime \prime} \mathrm{N}$ & $115^{\circ} 36^{\prime} 40^{\prime \prime} \mathrm{W}$ & -- & -- & --- & --- & -- & --- \\
\hline
\end{tabular}


Table 2. Data from Imperial Valley drainwater samples collected by the U.S. Geological Survey, May 16-20, 1988--Continued

\begin{tabular}{|c|c|c|c|c|c|c|c|c|c|c|c|}
\hline $\begin{array}{l}\text { Local } \\
\text { identifier }\end{array}$ & $\begin{array}{c}\text { Temper- } \\
\text { ature, } \\
\text { water } \\
\left({ }^{\circ} \mathrm{C}\right)\end{array}$ & $\begin{array}{c}\text { Calcium } \\
(\mathrm{mg} / \mathrm{L}) \\
\text { as } \mathrm{Ca})\end{array}$ & $\begin{array}{c}\text { Magne- } \\
\text { sium } \\
\text { (mg/L } \\
\text { as } \mathrm{Mg} \text { ) }\end{array}$ & $\begin{array}{l}\text { Sodium } \\
(\mathrm{mg} / \mathrm{L} \\
\text { as } \mathrm{Na})\end{array}$ & $\begin{array}{c}\text { Potas- } \\
\text { sium } \\
\text { (mg/L } \\
\text { as K) }\end{array}$ & $\begin{array}{c}\text { Alka- } \\
\text { linity, } \\
\text { lab } \\
(\mathrm{mg} / \mathrm{L} \\
\text { as } \\
\left.\mathrm{CaCO}_{3}\right)\end{array}$ & $\begin{array}{c}\text { Sulfate } \\
(\mathrm{mg} / \mathrm{L} \\
\left.\text { as } \mathrm{SO}_{4}\right)\end{array}$ & $\begin{array}{l}\text { Chlo- } \\
\text { ride } \\
(\mathrm{mg} / \mathrm{L} \\
\text { as } \mathrm{Cl})\end{array}$ & $\begin{array}{l}\text { Fluo- } \\
\text { ride } \\
\text { (mg/L) } \\
\text { as F) }\end{array}$ & $\begin{array}{c}\text { Silica } \\
(\mathrm{mg} / \mathrm{L} \\
\text { as } \\
\left.\mathrm{SiO}_{2}\right)\end{array}$ & $\begin{array}{c}\text { Solids, } \\
\text { residue } \\
\text { at } \\
180^{\circ} \mathrm{C} \\
(\mathrm{mg} / \mathrm{L})\end{array}$ \\
\hline$S-403$ & 22.0 & 300 & 330 & 2,000 & 20 & 270 & 2,600 & 2,500 & 0.9 & 21 & --- \\
\hline TD-2907 & -- & --- & -- & -. & -- & - & -- & -- & -- & $\ldots$ & -- \\
\hline SS-4 & 22.0 & - & -- & -- & -- & 343 & -.- & 2,400 & $\ldots$ & -.. & 6,270 \\
\hline S-38 & 24.0 & 590 & 970 & 7,500 & 41 & 425 & 4,400 & 14,000 & .2 & 17 & -.- \\
\hline$S-45$ & 21.5 & -.- & - & - & -- & 386 & -- & 3,900 & --- & -- & 9,480 \\
\hline S-226 & 22.5 & 640 & 1,100 & 6,000 & 45 & 216 & 3,800 & 11,000 & .5 & 12 & --- \\
\hline S-269 & 22.0 & 260 & 830 & 5,400 & 56 & 490 & 5,700 & 7,900 & .7 & 17 & -- \\
\hline S-417 & 23.0 & -- & $\ldots$ & $\ldots$ & --- & 444 & -- & --- & --- & --- & 32,500 \\
\hline S-332 & 24.5 & 180 & 480 & 2,300 & 32 & 654 & 2,300 & 3,700 & 1.0 & 19 & - \\
\hline TD-2013 & --- & -- & -- & -- & -- &.- & -- & --- & -- & --- & --- \\
\hline SS-3 & 21.0 & 300 & 170 & 1,2000 & 16 & 376 & 1,600 & 1,200 & 1.3 & 18 & -- \\
\hline SS-26 & 22.5 & 320 & 250 & 2,400 & 26 & 223 & 2,700 & 3,100 & 1.1 & 16 & --- \\
\hline S-219 & 21.5 & - & $\ldots$ & -- & $\ldots$ & 371 & --- & 640 & --- & -- & 2,570 \\
\hline S-337 & -- & 180 & 1,100 & 8,100 & 64 & 1,150 & 7,100 & 12,000 & .6 & 19 & --- \\
\hline SS-11 & 23.5 & 340 & 810 & 6,800 & 47 & 343 & 4,700 & 11,000 & .4 & 15 & -- \\
\hline$S-43$ & 23.0 & 310 & 160 & 920 & 8.1 & 221 & 1,500 & 1,200 & .6 & 12 & --- \\
\hline$S-57$ & 21.0 & -- & -- & -- & --- & 377 & -.- & 690 & --- & --- & 4,850 \\
\hline TD-1829 & -- & -- & -- & --- & -- & -- & --- & -- & --- & --- & --- \\
\hline S-25 & 22.0 & -- & --- & -- & -- & 305 & --- & 460 & -- & -- & 1,880 \\
\hline S-79 & 22.0 & - & -- & -- & -- & 454 & --- & 910 & -- & -.- & 5,890 \\
\hline S-119 & 23.0 & -- & -- & -.. & $\ldots$ & 291 & -- & 930 & -- & --- & 4,860 \\
\hline$S-243$ & 22.0 & 300 & 420 & 2,900 & 21 & 421 & 4,200 & 3,900 & .4 & 12 & -- \\
\hline S-488 & 22.5 & -- & -- & -.. & $\ldots$ & 377 & -.- & 4,400 & --- & -- & 11,700 \\
\hline TD-3038 & 25.0 & -- & --- & --- & -- & 488 & -- & 3,300 & --- & --- & 13,700 \\
\hline TD-2715 & -- & - & -- & -- & -- & -. & -- & -- & -- & -- & -- \\
\hline TD-147 & 21.5 & 260 & 570 & 3,200 & 37 & 437 & 3,800 & 6,100 & .3 & 12 & -- \\
\hline S-364 & 21.5 & - & --- & -- & -- & 314 & ... & 500 & --- & -- & 2,910 \\
\hline S-28 & 21.0 & $\ldots$ & -- & --- &.- & 258 & -- & 240 & -- & - & 1,700 \\
\hline S-69 & 18.5 & - & -- & --- & --- & 503 & -- & 580 & -.- & $\ldots$ & 3,190 \\
\hline S-94 & 19.5 & 420 & 530 & 2,900 & 20 & 451 & 2,800 & 5,500 & .4 & 15 & -- \\
\hline S-127 & - & $\ldots$ & $\ldots$ & -- & --- & -- & -- & -- & -- & --- & -- \\
\hline S-353 & 21.0 & --- & $\ldots$ & $\ldots$ & -- & 512 & -- & 1,200 & -- & --- & 8,420 \\
\hline S-142 & 19.5 & -- & -- & - & -- & 364 & -.- & 220 & --- & --- & 1,590 \\
\hline S-175 & 20.0 & -.. & $\ldots$ & -- & --- & 332 & --- & 430 & --- & -.. & 3,240 \\
\hline S-290 & 20.5 & --- & - & -- & -- & 515 & -- & 1,400 & -- & --. & 7,540 \\
\hline S-214 & 20.0 & -- & -- & -- & $\ldots$ & 591 & -- & 610 & --- & -- & 6,230 \\
\hline S-385 & 19.5 & 460 & 380 & 2,100 & 15 & 416 & 3,800 & 1,900 & .4 & 15 & -.- \\
\hline S-122 & 20.5 & --- & $\ldots$ & $\ldots$ & -. & 475 & -.. & 1,400 & --- & --- & 8,530 \\
\hline S-160 & 18.5 & - & - & -- & - & 503 & --- & 350 & --- & --- & 3,020 \\
\hline$S-212$ & 19.5 & 310 & 210 & 1,400 & 16 & 361 & 2,300 & 1,000 & .5 & 15 & -- \\
\hline S-241 & 19.5 & 180 & 330 & 1,800 & 24 & 497 & 3,300 & 1,200 & .6 & 14 & -- \\
\hline S-383 & 20.0 & -- & $\ldots$ & $\ldots$ & -- & 406 & -- & 1,500 & --- & --- & 5,100 \\
\hline TD-245 & -- & --- & -- & -- & --- & --- &.- & --- & --- & --- & -.- \\
\hline$S-230$ & 21.0 & 140 & 53 & 200 & 4.4 & 313 & 540 & 160 & .8 & 16 & --- \\
\hline S-55 & -- & --- & -- & -- & $\ldots$ & --- & --- & -- & --- & --- & $-\ldots$ \\
\hline
\end{tabular}


Table 2. Data from Imperial Valley drainwater samples collected by the U.S. Geological Survey, May 16-20, 1988--Continued

\begin{tabular}{|c|c|c|c|c|c|c|c|c|c|c|c|c|c|}
\hline $\begin{array}{l}\text { Local } \\
\text { ident- } \\
\text { ifier }\end{array}$ & $\begin{array}{c}\text { Nitro- } \\
\text { gen, } \\
\mathrm{NO}_{2}+ \\
\mathrm{NO}_{3} \\
(\mathrm{mg} / \mathrm{L} \\
\text { as } \mathrm{N})\end{array}$ & $\begin{array}{l}\text { Nitro- } \\
\text { gen, } \\
\text { am- } \\
\text { monia } \\
\text { (mg/L } \\
\text { as } \mathrm{N} \text { ) }\end{array}$ & $\begin{array}{c}\text { Arsenic } \\
(\mu \mathrm{g} / \mathrm{L} \\
\text { as As) }\end{array}$ & $\begin{array}{l}\text { Boron } \\
(\mu \mathrm{g} / \mathrm{L} \\
\text { as B) }\end{array}$ & $\begin{array}{c}\text { Iron } \\
(\mu \mathrm{g} / \mathrm{L} \\
\text { as } \mathrm{Fe})\end{array}$ & $\begin{array}{c}\text { Manga- } \\
\text { nese } \\
(\mu \mathrm{g} / \mathrm{L} \\
\text { as } \mathrm{Mn})\end{array}$ & $\begin{array}{l}\text { Molyb- } \\
\text { denum } \\
(\mu \mathrm{g} / \mathrm{L} \\
\text { as Mo })\end{array}$ & $\begin{array}{c}\text { Sele- } \\
\text { nium } \\
(\mu \mathrm{g} / \mathrm{L} \\
\text { as } \mathrm{Se})\end{array}$ & $\begin{array}{l}\text { Vana- } \\
\text { dium } \\
(\mu \mathrm{g} / \mathrm{L}) \\
\text { as } \mathrm{V})\end{array}$ & $\begin{array}{c}\text { Tritium } \\
\text { total } \\
(\mathrm{pCi} / \mathrm{L})\end{array}$ & $\begin{array}{l}\begin{array}{l}\text { Stable } \\
\text { ratio }\end{array} \\
{ }^{2} \underline{\mathrm{H} /{ }^{1} \mathrm{H}}\end{array}$ & $\begin{array}{l}\text {-isotope } \\
\frac{\text { (permil) }}{{ }^{18} \mathrm{O} /{ }^{16} \mathrm{O}}\end{array}$ & $\begin{array}{c}\text { Selenium/ } \\
\text { chloride } \\
\text { (weight } \\
\text { ratio } \\
\text { times } \\
10^{-5} \text { ) }\end{array}$ \\
\hline S-403 & 23 & 0.09 & 2 & 2,900 & 40 & 20 & 32 & 130 & 31 & 103 & -93.0 & -10.75 & 5.2 \\
\hline TD-2907 & --- & -- & -- & --- & --- & --- & --- & 3 & --- & --- & --- & -- & --- \\
\hline SS-4 & - & -- & -- & -- & -- & --- & -- & --- & --- & -- & --- & -- & .1 \\
\hline S-38 & 1.0 & 3.2 & 2 & 5,600 & 300 & 9,500 & 12 & 15 & 170 & 111 & -72.0 & -7.30 & .1 \\
\hline S-45 & -- & -- & -- & -- & --- & --- & -- & --- & --- & --- & --- & --- & --- \\
\hline S-226 & 45 & 1.2 & 3 & 2,200 & 210 & 70 & 33 & 250 & 170 & 96 & -81.5 & -9.00 & 2.3 \\
\hline S-269 & 34 & .44 & 6 & 3,300 & 140 & 40 & 37 & 230 & 99 & 117 & -89.0 & -10.20 & 2.9 \\
\hline S-417 & -- & --- & --- & -- & --- & -- & -- & 300 & --- & --- & --- & -- & -- \\
\hline S-332 & 7.6 & 6.0 & 6 & 1,900 & 120 & 170 & 17 & 37 & 44 & 88 & -85.0 & -9.05 & 1.0 \\
\hline TD-2013 & -- & -- & -- & --- & --- & --- & $\cdots$ & --- & --- & -- & --- & --- & --- \\
\hline SS-3 & 8.4 & .14 & 2 & 1,300 & 80 & 120 & 40 & 14 & 28 & 110 & -95.0 & -11.70 & 1.2 \\
\hline SS-26 & 18 & .51 & 3 & 3,100 & 130 & 460 & 51 & 5 & 55 & 71 & -80.0 & -9.45 & .2 \\
\hline S-219 & $\cdots$ & -- & -- & -- & --- & --. & --- & 5 & -- & --- & --- & --- & .8 \\
\hline S-337 & 1.1 & .40 & 2 & 17,000 & 290 & 70 & 19 & 17 & 200 & 104 & -82.0 & -8.65 & .1 \\
\hline SS-11 & 7.4 & 10 & 2 & 5,500 & 1,500 & 5,800 & 12 & 3 & 140 & 91 & -68.0 & -6.75 & .03 \\
\hline$S-43$ & 4.2 & .14 & 2 & 930 & 300 & 540 & 16 & 14 & 20 & 121 & -79.5 & -8.00 & 1.2 \\
\hline S-57 & - & $-\cdot-$ & $\cdots$ & -- & -- & -- & --- & 30 & --- & --- & --- & --- & 4.3 \\
\hline TD-1829 & -- & $\cdots$ & $\cdots$ & -- & -- & --- & $\cdots$ & --- &.- & -- & -.. & $\ldots$ & --- \\
\hline S-25 & - & $--\cdot$ & --- & --- & -- & --- & - & 3 & -- & --- & --- & --- & .7 \\
\hline S-79 & $\cdots$ & -- & --- & -- & --- & --- & -- & 28 & -- & --- & -- & --- & 3.1 \\
\hline S-119 & --- & --- & --- & -- & --- & --- & - & 20 & $\cdots$ & -- & --- & --- & 2.2 \\
\hline S-243 & 22 & .20 & 2 & 1,700 & 110 & 100 & 20 & 89 & 42 & 160 & -91.0 & -10.80 & 2.3 \\
\hline S-488 & $\cdots$ & -- & $\cdots$ & --- & -- & --- & -- & 100 & --- & --- & --- & -- & 2.3 \\
\hline TD-3038 & -- & --- & -- & --- & -- & $\cdots$ & --- & 100 & --- & -- & -- & --- & 3.0 \\
\hline TD-2715 & - & --- & --- & -- & --- & -- & $\cdots$ & --- & $\cdots$ & --- & --- & $-\cdots$ & --- \\
\hline TD-147 & 50 & .40 & 3 & 2,000 & 160 & 40 & 18 & 210 & 64 & 122 & -90.5 & -10.70 & 3.4 \\
\hline S-364 & -- & --- & $\cdots$ & --- & -- & --- & -- & 18 & -- & --- & --- & --- & 3.6 \\
\hline$S-28$ & --- & -- & $\cdots$ & --- & --- & -- & --- & 7 & --- & -- & -- & -- & 2.9 \\
\hline$S-69$ & -- & $\cdots$ & -- & -- & --- & --- & $\cdots$ & 3 & -- & -- & --- & --- & .5 \\
\hline S-94 & 4.4 & .36 & 1 & 1,800 & 90 & 170 & 16 & 51 & 68 & --- & -91.5 & -10.85 & .9 \\
\hline S-127 & - & --- & -- & -- & --- & -- & -- & --- & $\ldots$ & -- & +- & -.- & -- \\
\hline S-353 & --- & -- & -- & --- & --- & -- & --- & 48 & --- & --- & -- & --- & 4.0 \\
\hline S-142 & - & --- & -- & --- & -- & -- & --- & 16 & --- & --- & -- & --- & 7.3 \\
\hline S-175 & --- & -- & --- & --- & -- & -- & --- & 12 & --- & --- & -- & --- & 2.8 \\
\hline S-290 & -- & -- & -- & -- & -- & -- & -- & 40 & -- & --- & --- & --- & 2.9 \\
\hline S-214 & --- & -- & --- & --- & -- & --- & -- & 40 & --- & --- & --- & --- & 6.6 \\
\hline S-385 & 14 & .19 & 1 & 1,700 & 40 & 30 & 14 & 98 & 43 & - & -95.5 & -11.45 & 5.2 \\
\hline S-122 & -- & -- & -- & --- & -- & --- & -- & 43 & --- & --- & --- & --- & 3.1 \\
\hline S-160 & -- & - & -- & --- & -- & --- & -- & 10 & $-\cdots$ & --- & --- & --- & 2.9 \\
\hline S-212 & 8.2 & .20 & 4 & 1,300 & 30 & 250 & 16 & 37 & 21 & 118 & -97.5 & -12.05 & 3.7 \\
\hline S-241 & 4.9 & .13 & 3 & 2,000 & 120 & 40 & 14 & 30 & 38 & 131 & -97.0 & -11.90 & 2.5 \\
\hline S-383 & & -- & -- & --- & --- & -- & --- & 29 & --- & --- & --- & --- & 1.9 \\
\hline TD-245 & --- & -- & $\cdots$ & -- & --- & --- & --- & --- & --- & --- & --- & --- & --- \\
\hline S-230 & 12 & .06 & 2 & 320 & 60 & 30 & 13 & 7 & 6 & 110 & -102.5 & -12.60 & 4.4 \\
\hline S-55 & -- & -- & -- & - & -- & --- & -- & -- & -- & --- & --- & --- & --- \\
\hline
\end{tabular}


Table 2. Data from Imperial Valley drainwater samples collected by the U.S. Geological Survey, May 16-20, 1988--Continued

\begin{tabular}{|c|c|c|c|c|c|c|c|c|c|c|}
\hline \multicolumn{2}{|c|}{$\begin{array}{c}\text { Site } \\
\text { number }\end{array}$} & \multirow{2}{*}{$\begin{array}{c}\begin{array}{c}\text { Local } \\
\text { identifier }\end{array} \\
S-256\end{array}$} & \multirow{2}{*}{$\begin{array}{c}\text { Latitude } \\
32^{\circ} 54^{\prime} 25^{\prime \prime} \mathrm{N}\end{array}$} & \multirow{2}{*}{$\begin{array}{l}\text { Longitude } \\
115^{\circ} 38^{\prime} 00^{\prime \prime} \mathrm{W}\end{array}$} & \multirow{2}{*}{$\begin{array}{c}\text { Date } \\
5-18-88\end{array}$} & \multirow{2}{*}{$\begin{array}{r}\text { Time } \\
1445\end{array}$} & \multirow{2}{*}{$\begin{array}{c}\begin{array}{c}\text { Altitude } \\
\text { of } \\
\text { land } \\
\text { surface }\end{array} \\
-72\end{array}$} & \multirow{2}{*}{$\begin{array}{c}\begin{array}{c}\text { Discharge, } \\
\text { instanta- } \\
\text { neous } \\
\left(\mathrm{ft}^{3} / \mathrm{s}\right)\end{array} \\
.035\end{array}$} & \multirow{2}{*}{$\begin{array}{c}\begin{array}{c}\text { Specific } \\
\text { conductance } \\
(\mu \mathrm{S} / \mathrm{cm})\end{array} \\
2,770\end{array}$} & \multirow{2}{*}{$\begin{array}{c}\begin{array}{c}\mathrm{pH} \\
\text { (standard } \\
\text { units) }\end{array} \\
7.0\end{array}$} \\
\hline 46 & . & & & & & & & & & \\
\hline 47 & $\ldots$ & S-295 & $32^{\circ} 56^{\prime} 17^{\prime \prime} \mathrm{N}$ & $115^{\circ} 38^{\prime} 58^{\prime \prime W}$ & $5-18-88$ & 1520 & -67 & --- & 1,650 & 7.5 \\
\hline 48 & . & S-112 & $32^{\circ} 55^{\prime} 33^{\prime \prime} \mathrm{N}$ & $115^{\circ} 37^{\prime} 43^{\prime \prime W}$ & $5-18-88$ & 1430 & -80 & 0.31 & 2,760 & 7.5 \\
\hline 49 & $\ldots$ & $S-424$ & $32^{\circ} 53^{\prime} 55^{\prime \prime N}$ & $115^{\circ} 38^{\prime} 00^{\prime \prime} \mathrm{W}$ & $5-18-88$ & 1500 & -67 & -- & 1,540 & 7.7 \\
\hline 50 & . & S-154 & $32^{\circ} 53^{\prime} 54^{\prime \prime} \mathrm{N}$ & $115^{\circ} 31^{\prime} 01^{\prime \prime W}$ & $5-18-88$ & 1310 & -142 & -- & 15,700 & 7.1 \\
\hline 51 & $\ldots$ & S-105 & $32^{\circ} 56^{\prime} 55^{\prime \prime} \mathrm{N}$ & $115^{\circ} 32^{\prime} 20^{\prime \prime} \mathrm{W}$ & $5-18-88$ & 1205 & -116 & .028 & 12,100 & 6.9 \\
\hline 52 & $\ldots$ & $S-133$ & $32^{\circ} 57^{\prime} 25^{\prime \prime} \mathrm{N}$ & $115^{\circ} 32^{\prime} 10^{\prime \prime} \mathrm{W}$ & $5-18-88$ & 1150 & -. & .0072 & - & 7.2 \\
\hline 53 & . & $S-153$ & $32^{\circ} 55^{\prime} 39^{\prime \prime} \mathrm{N}$ & $115^{\circ} 32^{\prime} 07^{\prime \prime W}$ & $5-18-88$ & 1255 & -133 & .080 & 13,600 & 7.3 \\
\hline 54 & $\ldots$ & S-365 & $32^{\circ} 57^{\prime} 21^{\prime \prime} \mathrm{N}$ & $115^{\circ} 28^{\prime} 25^{\prime \prime W}$ & $5-18-88$ & 1130 & -136 & .0000 & 17,900 & 7.0 \\
\hline 55 & . & TD-2001 & $32^{\circ} 55^{\prime} 55^{\prime \prime} \mathrm{N}$ & $115^{\circ} 22^{\prime} 50^{\prime \prime} \mathrm{W}$ & --- & --- & -- & --- & -- & --- \\
\hline 56 & . & TD-2040 & $32^{\circ} 55^{\prime} 00^{\prime \prime} \mathrm{N}$ & $115^{\circ} 25^{\prime} 40^{\prime \prime} \mathrm{W}$ & -- & -.. & - & --- & --- & --- \\
\hline 57 & . & TD-2554 & $32^{\circ} 55^{\prime} 25^{\prime \prime} \mathrm{N}$ & $115^{\prime} 20^{\prime} 05^{\prime \prime W}$ & $5-18-88$ & 1000 & $\cdots$ & .032 & 10,300 & 6.8 \\
\hline 58 & $\ldots$ & $S-322$ & $32^{\circ} 54^{\prime} 57^{\prime \prime} \mathrm{N}$ & $115^{\circ} 18^{\prime} 36^{\prime \prime} \mathrm{W}$ & $5-18-88$ & 0930 & -11 & .85 & 2,670 & 7.3 \\
\hline 59 & $\ldots$ & S-396 & $32^{\circ} 56^{\prime} 16^{\prime \prime} \mathrm{N}$ & $115^{\circ} 18^{\prime} 36^{\prime \prime W}$ & $5-18-88$ & 0905 & -8 & .21 & 2,850 & 7.0 \\
\hline 60 & $\ldots$ & TD-2432 & $32^{\circ} 49^{\prime} 12^{\prime \prime} \mathrm{N}$ & $115^{\circ} 46^{\prime} 09^{\prime \prime} \mathrm{W}$ & $5-19-88$ & 0850 & -32 & .18 & 5,180 & 7.2 \\
\hline 61 & $\ldots$ & $S-70$ & $32^{\circ} 52^{\prime} 43^{\prime \prime N}$ & $115^{\circ} 41^{\prime} 46^{\prime \prime} \mathrm{W}$ & $5-18-88$ & 1800 & -54 & .24 & 8,030 & 7.3 \\
\hline 62 & . & $S-68$ & $32^{\circ} 51^{\prime} 07^{\prime \prime N} \mathrm{~N}$ & $115^{\circ} 44^{\prime} 23^{\prime \prime W}$ & $5-18-88$ & 1725 & -45 & .012 & 13,400 & 7.5 \\
\hline 63 & $\ldots$ & S-333 & $32^{\circ} 51^{\prime} 06^{\prime \prime} \mathrm{N}$ & $115^{\circ} 43^{\prime} 35^{\prime \prime W}$ & $5-18-88$ & 1830 & -49 & .012 & 2,060 & 7.3 \\
\hline 64 & . & $S-110$ & $32^{\circ} 50^{\prime} 48^{\prime \prime} \mathrm{N}$ & $115^{\circ} 35^{\prime} 17^{\prime \prime} \mathrm{W}$ & $5-19-88$ & 1330 & -60 & .034 & 12,000 & 7.1 \\
\hline 65 & . & $S-67$ & $32^{\circ} 52^{\prime} 07^{\prime \prime} \mathrm{N}$ & $115^{\circ} 35^{\prime} 35^{\prime \prime} \mathrm{W}$ & $5-19-88$ & 1350 & -66 & .26 & 7,460 & 7.1 \\
\hline \multirow[t]{2}{*}{66} & $\ldots$ & $S-225$ & $32^{\circ} 49^{\prime} 04^{\prime \prime} \mathrm{N}$ & $115^{\circ} 35^{\prime} 09^{\prime \prime} \mathrm{W}$ & $5-19-88$ & 1410 & -52 & .0091 & 17,000 & 7.1 \\
\hline & & & & & $5-19-88$ & 0845 & -52 & .0067 & 17,100 & 7.1 \\
\hline 67 & . & $S-265$ & $32^{\circ} 48^{\prime} 26^{\prime \prime} \mathrm{N}$ & $115^{\circ} 40^{\prime} 12^{\prime \prime} \mathrm{W}$ & $5-19-88$ & 0815 & -43 & .12 & 10,200 & 7.3 \\
\hline 68 & . & S-398 & $32^{\circ} 49^{\prime} 56^{\prime \prime} \mathrm{N}$ & $115^{\circ} 39^{\prime} 11^{\prime \prime W}$ & $5-19-88$ & 1430 & -50 & .045 & 10,600 & 7.2 \\
\hline 69 & $\ldots$ & $S-148$ & $32^{\circ} 52^{\prime} 06^{\prime \prime} \mathrm{N}$ & $115^{\circ} 30^{\prime} 03^{\prime \prime} \mathrm{W}$ & $5-19-88$ & 0910 & -118 & .019 & 12,900 & 7.2 \\
\hline 70 & $\ldots$ & TD-1408 & $32^{\circ} 49^{\prime} 30^{\prime \prime} \mathrm{N}$ & $115^{\circ} 29^{\prime} 46^{\prime \prime} \mathrm{W}$ & $5-20-88$ & 0930 & -68 & .35 & 2,940 & 7.4 \\
\hline 71 & $\ldots$ & S-234 & $32^{\circ} 49^{\prime} 55^{\prime \prime} \mathrm{N}$ & $115^{\circ} 32^{\prime} 37^{\prime \prime} \mathrm{W}$ & $5-20-88$ & 0810 & -62 & .086 & 7,360 & 7.4 \\
\hline 72 & $\ldots$ & S-410 & $32^{\circ} 51^{\prime} 15^{\prime \prime} \mathrm{N}$ & $115^{\circ} 32^{\prime} 10^{\prime \prime} \mathrm{W}$ & $5-20-88$ & 0830 & -.. & .20 & 5,190 & 7.3 \\
\hline 73 & $\ldots$ & S-411 & $32^{\circ} 51^{\prime} 40^{\prime \prime} \mathrm{N}$ & $115^{\circ} 33^{\prime} 10^{\prime \prime} \mathrm{W}$ & $5-20-88$ & 0845 & -- & .027 & 6,020 & 7.4 \\
\hline 74 & $\ldots$ & S-2 & $32^{\circ} 52^{\prime} 30^{\prime \prime} \mathrm{N}$ & $115^{\circ} 23^{\prime} 45^{\prime \prime} \mathrm{W}$ & $5-20-88$ & 1025 & -- & .053 & 11,900 & 7.2 \\
\hline 75 & $\ldots$ & S-4 & $32^{\circ} 52^{\prime} 30^{\prime \prime} \mathrm{N}$ & $115^{\circ} 22^{\prime} 30^{\prime \prime} \mathrm{W}$ & $5-19-88$ & 1030 & -57 & .045 & 29,300 & 7.0 \\
\hline 76 & . & S-103 & $32^{\circ} 48^{\prime} 55^{\prime \prime} \mathrm{N}$ & $115^{\circ} 27^{\prime} 23^{\prime \prime W}$ & $5-19-88$ & 1000 & -72 & .041 & 9,160 & 7.0 \\
\hline 77 & . . & S-247 & $32^{\circ} 50^{\prime} 48^{\prime \prime} \mathrm{N}$ & $115^{\circ} 27^{\prime} 04^{\prime \prime W}$ & $5-19-99$ & 0940 & -88 & .0025 & 28,700 & 6.7 \\
\hline 78 & . & S-376 & $32^{\circ} 52^{\prime} 13^{\prime \prime} \mathrm{N}$ & $115^{\circ} 27^{\prime} 19^{\prime \prime} \mathrm{W}$ & $5-20-88$ & 1000 & -150 & .0081 & 7,840 & 7.2 \\
\hline 79 & . & $S-72$ & $32^{\circ} 50^{\prime} 21^{\prime \prime} \mathrm{N}$ & $115^{\circ} 21^{\prime} 16^{\prime \prime} \mathrm{W}$ & $5-20-88$ & 1050 & -22 & .0027 & 13,400 & 7.3 \\
\hline 80 & . . & S-169 & $32^{\circ} 48^{\prime} 09^{\prime \prime} \mathrm{N}$ & $115^{\circ} 17^{\prime} 32^{\prime \prime} \mathrm{W}$ & $5-19-88$ & 1055 & 11 & .15 & 4,980 & 7.4 \\
\hline 81 & . & S-187 & $32^{\circ} 48^{\prime} 12^{\prime \prime} \mathrm{N}$ & $115^{\circ} 21^{\prime} 51^{\prime \prime} \mathrm{W}$ & $5-20-88$ & 1115 & -1 & .71 & 6,720 & 7.1 \\
\hline 82 & . & $S-21$ & $32^{\circ} 47^{\prime} 29^{\prime \prime} \mathrm{N}$ & $115^{\circ} 45^{\prime} 52^{\prime \prime} \mathrm{W}$ & $5-19-88$ & 1520 & -41 & .013 & 11,500 & 7.2 \\
\hline 83 & . & S-22 & $32^{\circ} 47^{\prime} 52^{\prime \prime} \mathrm{N}$ & $115^{\circ} 45^{\prime} 07^{\prime \prime W}$ & $5-19-88$ & 0940 & -42 & .045 & 8,780 & 7.0 \\
\hline 84 & . & $S-130$ & $32^{\circ} 48^{\prime} 02^{\prime \prime} \mathrm{N}$ & $115^{\circ} 45^{\prime} 46^{\prime \prime W}$ & $5-19-88$ & 0910 & -37 & .42 & 4,470 & 7.3 \\
\hline 85 & . & S-207 & $32^{\circ} 44^{\prime} 40^{\prime \prime} \mathrm{N}$ & $115^{\circ} 43^{\prime} 47^{\prime \prime} \mathrm{W}$ & $5-19-88$ & 1020 & -35 & .18 & 12,500 & 7.3 \\
\hline 86 & . & TD-2939 & $32^{\circ} 42^{\prime} 25^{\prime \prime} \mathrm{N}$ & $115^{\circ} 45^{\prime} 10^{\prime \prime} \mathrm{W}$ & --- & --- & --- & --- & --- & --- \\
\hline 87 & . & S-352 & $32^{\circ} 45^{\prime} 28^{\prime \prime} \mathrm{N}$ & $115^{\circ} 43^{\prime} 31^{\prime \prime W}$ & $5-19-88$ & 1540 & -40 & 0 & 22,800 & 7.3 \\
\hline 88 & . . & S-81 & $32^{\circ} 42^{\prime} 59^{\prime \prime} \mathrm{N}$ & $115^{\circ} 41^{\prime} 10^{\prime \prime W}$ & $5-19-88$ & 1055 & -22 & .35 & 2,370 & 7.4 \\
\hline 89 & $\ldots$ & S-113 & $32^{\circ} 45^{\prime} 11^{\prime \prime} \mathrm{N}$ & $115^{\circ} 35^{\prime} 08^{\prime \prime W}$ & $5-19-88$ & 1430 & -26 & .0048 & 8,290 & 7.0 \\
\hline
\end{tabular}


Table 2. Data from Imperial Valley drainwater samples collected by the U.S. Geological Survey, May 16-20, 1988--Continued

\begin{tabular}{|c|c|c|c|c|c|c|c|c|c|c|c|}
\hline $\begin{array}{l}\text { Local } \\
\text { identifier }\end{array}$ & $\begin{array}{c}\text { Temper- } \\
\text { ature, } \\
\text { water } \\
\left({ }^{\circ} \mathrm{C}\right)\end{array}$ & $\begin{array}{c}\text { Calcium } \\
(\mathrm{mg} / \mathrm{L}) \\
\text { as } \mathrm{Ca})\end{array}$ & $\begin{array}{c}\text { Magne- } \\
\text { sium } \\
(\mathrm{mg} / \mathrm{L} \\
\text { as } \mathrm{Mg})\end{array}$ & $\begin{array}{c}\text { Sodium } \\
\text { (mg/L } \\
\text { as } \mathrm{Na} \text { ) }\end{array}$ & $\begin{array}{l}\text { Potas- } \\
\text { sium } \\
(\mathrm{mg} / \mathrm{L} \\
\text { as K) }\end{array}$ & $\begin{array}{c}\text { Alka- } \\
\text { linity, } \\
\text { lab } \\
(\mathrm{mg} / \mathrm{L} \\
\text { as } \\
\left.\mathrm{CaCO}_{3}\right)\end{array}$ & $\begin{array}{c}\text { Sulfate } \\
(\mathrm{mg} / \mathrm{L} \\
\left.\text { as } \mathrm{SO}_{4}\right)\end{array}$ & $\begin{array}{l}\text { Chlo- } \\
\text { ride } \\
(\mathrm{mg} / \mathrm{L} \\
\text { as } \mathrm{Cl})\end{array}$ & $\begin{array}{l}\text { Fluo- } \\
\text { ride } \\
\text { (mg/L) } \\
\text { as F) }\end{array}$ & $\begin{array}{c}\text { Silica } \\
(\mathrm{mg} / \mathrm{L} \\
\text { as } \\
\left.\mathrm{SiO}_{2}\right)\end{array}$ & $\begin{array}{c}\text { Solids, } \\
\text { residue } \\
\text { at } \\
180^{\circ} \mathrm{C} \\
(\mathrm{mg} / \mathrm{L})\end{array}$ \\
\hline S-256 & - & --. & - & -- & -- & 345 & $-\ldots$ & 240 & -- & -- & 1,830 \\
\hline S-295 & - & 110 & 45 & 170 & 4.4 & 204 & 420 & 130 & 0.8 & 17 & - \\
\hline S-112 & -- & -- & -- & -- & --- & 343 & -- & 240 & $\ldots$ & $\ldots$ & 1,880 \\
\hline S-424 & -- & 90 & 42 & 160 & 3.2 & 191 & 430 & 150 & .5 & 12 & --- \\
\hline S-154 & $\ldots$ & 430 & 490 & 2,200 & 26 & 277 & 2,200 & 4,600 & .4 & 11 & -- \\
\hline S-105 & 21.0 & - & -- & - & -- & 559 & -- & 2,200 & --- & --- & 9,690 \\
\hline S-133 & 20.0 & - & -- & 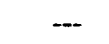 & - & 565 & -- & 940 & -- & --- & 4,400 \\
\hline S-153 & -- & -- & -- & -- & --- & 412 & -- & 3,000 & -- & --- & 10,400 \\
\hline S-365 & 21.5 & 440 & 600 & 2,900 & 29 & 408 & 3,500 & 5,000 & .3 & 12 & -- \\
\hline TD-2001 & -- & - & -- & - & --- & -- & -.- & -- & -- & --- & -- \\
\hline TD-2040 & -- & -- & -- & --- & -- & -- & --- & -- & - & --- & -- \\
\hline TD-2554 & 21.0 & 360 & 300 & 1,900 & 10 & 528 & 4,000 & 1,400 & .4 & 22 & --- \\
\hline S-322 & 20.0 & -- & --- & --- & --- & 335 & --- & 2,200 & -- & -.- & 1,920 \\
\hline S-396 & 19.5 & -- & -- & - & --- & 500 & --- & 220 & -- & -- & 1,970 \\
\hline TD-2432 & 21.5 & -- & -- & -- &.-- & 380 & --- & 920 & $-\ldots$ & -- & 3,840 \\
\hline$S-70$ & 21.5 & $\ldots$ & -- & -- & --- & 337 & --- & 1,300 & --- & -- & 6,060 \\
\hline S-68 & 22.0 &.-- & -- &.-- & --- & 340 & --- & 2,800 & --- & --. & 9,780 \\
\hline S-333 & 21.5 & -- & -- & --- & --- & 241 & --- & 170 & --- & -- & 1,330 \\
\hline S-110 & --- & -- & -- & --- & --- & 370 & -- & 2,800 & --- & -.. & 85,800 \\
\hline S-67 & -- & --- & -- & --- & --- & 408 & --- & 1,000 & -- & -.- & 6,070 \\
\hline \multirow[t]{2}{*}{ S-225 } & -- & --- & - & -- & -.- & 386 & -- & 4,700 & --- & $\ldots$ & 12,100 \\
\hline & -- & 430 & 460 & 2,600 & 16 & 403 & 2,900 & 4,800 & .4 & 16 & -- \\
\hline S-265 & 21.5 & 380 & 270 & 1,600 & 32 & 484 & 3,000 & 1,900 & .4 & 18 & -.- \\
\hline S-398 & -- & --- & -.. & -.- & -- & 473 & -.. & 2,100 & --- & -. & 8,240 \\
\hline S-148 & 20.5 & 270 & 400 & 2,100 & 21 & 418 & 3,500 & 4,300 & .4 & 15 & -.. \\
\hline TD-1408 & 23.0 & -- & --- & -- & -- & 274 & -.- & 410 & --- & $\cdots$ & 1,800 \\
\hline S-234 & 20.5 & - & -- & --- & --- & 458 & --- & 1,300 & -- & $\cdots$ & 5,270 \\
\hline$S-410$ & 21.5 & -- & --- & --. & --- & 315 & --- & 890 & -- & --- & 3,610 \\
\hline S-411 & 21.0 & -- & $\ldots$ & -.. & --- & 322 & --- & 1,000 & -- & $-\ldots$ & 4,420 \\
\hline S-2 & 21.5 & - & -- & --- & --- & 395 & -- & 2,100 & -- & $-\ldots$ & 9,120 \\
\hline$S-4$ & 21.0 & 390 & 910 & 4,900 & 24 & 406 & 4,600 & 9,000 & .3 & 13 & --. \\
\hline S-103 & 20.0 & 360 & 360 & 1,500 & 13 & 544 & 3,200 & 1,300 & .7 & 13 & --- \\
\hline S-247 & 20.0 & 700 & 1,000 & 3,600 & 34 & 378 & 3,500 & 9,500 & .3 & 13 & $\ldots$ \\
\hline S-376 & 22.5 & - & -- & -- & $\ldots$ & 384 & --- & 1,400 & --- & -.. & 5,710 \\
\hline S-72 & 21.5 & -- & -- & - & -- & 437 & -.- & 2,500 & -- & $\ldots$ & 10,100 \\
\hline S-169 & 21.0 & 180 & 98 & 770 & 5.5 & 337 & 980 & 870 & 1.5 & 25 & -- \\
\hline S-187 & 22.5 & -- & - & -- & --- & 475 & --. & 800 & -- & --- & 5,300 \\
\hline$S-21$ & 22.0 & 280 & 250 & 2,100 & 10 & 338 & 3,600 & 1,900 & 1.6 & & -- \\
\hline S-22 & 22.0 & -- & -- & - & -- & 312 & -.. & 1,700 & - & - & 6,560 \\
\hline S-130 & 22.5 & - & -- & -- & 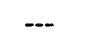 & 307 &.- & 600 & -- & $\ldots$ & 3,210 \\
\hline S-207 & 21.5 & -- & -- & -- & -- & 282 &.-- & 2,700 & -- & $\cdots$ & 9,150 \\
\hline TD-2939 & --- & -- & -- & --- & $\cdots$ & --. & -- & -- & -- & -- & --- \\
\hline S-352 & 21.5 & 280 & 480 & 4,300 & 20 & 407 & 4,100 & 6,000 & .5 & 11 & --- \\
\hline$S-81$ & 24.0 & --- & -- & -. & $\ldots$ & 252 & -- & 250 & -- & -- & 1,670 \\
\hline$S-113$ & 21.5 & 230 & 260 & 1,300 & 8.5 & 434 & 2,700 & 1,100 & .8 & 17 & $\ldots$ \\
\hline
\end{tabular}


Table 2. Data from Imperial Valley drainwater samples collected by the U.S. Geological Survey, May 16-20, 1988--Continued

\begin{tabular}{|c|c|c|c|c|c|c|c|c|c|c|c|c|c|}
\hline $\begin{array}{l}\text { Local } \\
\text { ident- } \\
\text { ifier }\end{array}$ & $\begin{array}{l}\text { Nitro- } \\
\text { gen, } \\
\mathrm{NO}_{2}+ \\
\mathrm{NO}_{3} \\
(\mathrm{mg} / \mathrm{L} \\
\text { as } \mathrm{N})\end{array}$ & $\begin{array}{l}\text { Nitro- } \\
\text { gen, } \\
\text { am- } \\
\text { monia } \\
\text { (mg/L } \\
\text { as N) }\end{array}$ & $\begin{array}{c}\text { Arsenic } \\
(\mu \mathrm{g} / \mathrm{L} \\
\text { as As) }\end{array}$ & $\begin{array}{l}\text { Boron } \\
(\mu \mathrm{g} / \mathrm{L} \\
\text { as B) }\end{array}$ & $\begin{array}{c}\text { Iron } \\
(\mu \mathrm{g} / \mathrm{L} \\
\text { as } \mathrm{Fe})\end{array}$ & $\begin{array}{c}\text { Manga- } \\
\text { nese } \\
(\mu \mathrm{g} / \mathrm{L} \\
\text { as } \mathrm{Mn})\end{array}$ & $\begin{array}{c}\text { Molyb- } \\
\text { denum } \\
(\mu \mathrm{g} / \mathrm{L} \\
\text { as Mo) }\end{array}$ & $\begin{array}{c}\text { Sele- } \\
\text { nium } \\
(\mu \mathrm{g} / \mathrm{L} \\
\text { as } \mathrm{Se})\end{array}$ & $\begin{array}{l}\text { Vana- } \\
\text { dium } \\
(\mu g / L) \\
\text { as } V)\end{array}$ & $\begin{array}{l}\text { Tritium } \\
\text { total } \\
(\mathrm{pCi} / \mathrm{L})\end{array}$ & $\begin{array}{l}\begin{array}{c}\text { Stable- } \\
\text { ratio }\end{array} \\
{ }^{2} \mathrm{H} /{ }^{1} \mathrm{H}\end{array}$ & $\begin{array}{l}\text {-isotope } \\
\frac{\text { (permil) }}{{ }^{18} \mathrm{O} /{ }^{16} \mathrm{O}}\end{array}$ & $\begin{array}{c}\text { Selenium/ } \\
\text { chloride } \\
\text { (weight } \\
\text { ratio } \\
\text { times } \\
10^{-5} \text { ) }\end{array}$ \\
\hline S-256 & -- & - & - & $\ldots$ & --- & --- & $\ldots$ & 7 & $\cdots$ & -- & -- & -- & 2.9 \\
\hline S-295 & 26 & 0.02 & 4 & 230 & 10 & 10 & 12 & 5 & 9 & 110 & -102.0 & -12.70 & 3.8 \\
\hline$S-112$ & - & -- & --- & - & --- & -- & -- & 4 & -- & --- & --- & --- & 1.7 \\
\hline$S-424$ & 1.3 & .01 & 1 & 230 & 110 & 20 & 9 & 8 & 3 & 104 & -103.5 & -12.95 & 5.3 \\
\hline S-154 & 7.1 & .34 & 2 & 1,300 & 150 & 40 & 14 & 15 & 59 & 110 & -92.0 & -11.20 & .3 \\
\hline S-105 & - & -- & -- & ... & -- & --- & --- & 65 & --- & --- & -.. & --- & 3.0 \\
\hline$S-133$ & $\cdots$ & -- & -- & --- & $-\cdots$ & -- & $\cdots$ & 21 & -- & -- & --- & --- & 2.2 \\
\hline S-153 & -- & -- & -- & -- & -- & -- & --- & 110 & $\cdots$ & -- & -.- & --- & 3.7 \\
\hline S-365 & 28 & .40 & 2 & 1,400 & 90 & 50 & 11 & 130 & 53 & 129 & -92.0 & -11.05 & 2.6 \\
\hline TD-2001 & - & -- & -- & - & -- & -- & --- & -- & --- & --- & --- & --- & --- \\
\hline TD-2040 & - & -- & --- & --- & -- & -- & $\ldots$ & -- & -- & -- & --- & --- & --- \\
\hline TD-2554 & 9.1 & .11 & 3 & 4,300 & 40 & 20 & 23 & 41 & 31 & 169 & -99.0 & -12.05 & 2.9 \\
\hline S-322 & - & -- & --. & -- & $\ldots$ & $\ldots$ & - & 5 & $\ldots$ & $\ldots$ & --- & --- & .2 \\
\hline S-396 & - & - & --- & -- & -- & - & - & 10 & -- & --- & --- & --- & 4.5 \\
\hline TD-2432 & - & -- & -- & -- & -- & - & -- & 10 & --- & -- & --- & -- & 1.1 \\
\hline$S-70$ & -- & -- & -- & -- & --- &.-- & $\ldots$ & 22 & --- & -- & --- & --- & 1.7 \\
\hline S-68 & - & - & - & - & $\ldots$ & --- & -- & 22 & --- & --- & --- & -.- & .8 \\
\hline S-333 & - & -- & - & - & -- & --- & -- & 6 & -- & --- & -- & --- & 3.5 \\
\hline$S-110$ & -- & -- & -- & - & --- &.-- & $\ldots$ & 31 & --- & --- &.-- & $-\ldots$ & 1.1 \\
\hline S-67 & - & -- & -- & - & -- & --- & --- & 31 & $\ldots$ & --- & --- & --- & 3.1 \\
\hline \multirow[t]{2}{*}{ S-225 } & $-\cdots$ & $\ldots$ & $\ldots$ & -.- & --- & --- & --- & 47 & -- & --- & --- & --- & 1.0 \\
\hline & 4.4 & 3.9 & 1 & 1,800 & 80 & 70 & 18 & 44 & 56 & 143 & -93.5 & -11.50 & .9 \\
\hline S-265 & 33 & .18 & 2 & 1,400 & 70 & 250 & 14 & 76 & 32 & 127 & -96.0 & -11.60 & 4.0 \\
\hline S-398 & -- & -- & -- & -.- & -- & $\ldots$ & $\ldots$ & 62 & -- & -.. & $\ldots$ & --- & 3.0 \\
\hline S-148 & 9.0 & .22 & 1 & 1,900 & 230 & 380 & 16 & 48 & 61 & 140 & -91.0 & -11.00 & 1.1 \\
\hline TD-1408 & -- & $\ldots$ & $\cdots$ & -- & --- & -- & -- & 6 & $\ldots$ & -- & --- & -- & 1.5 \\
\hline S-234 & --- & - & -- & --- & --- & -- & $\ldots$ & 24 & -- & --- & --- & --- & 1.8 \\
\hline$S-410$ & -- & - & -- & --- & --- & --- & --- & 13 & $\ldots$ & -- & --- & --- & 1.5 \\
\hline$S-411$ & -- & $\ldots$ & -- & $-\cdots$ & --- & -- & --- & 24 & $\ldots$ & --- & --- & --- & 2.4 \\
\hline S-2 & -- & -- & $\cdots$ & -.. & -- & -- & --- & 50 & - & -- & -- & --- & 2.4 \\
\hline$S-4$ & 9.8 & .60 & 2 & 2,200 & 140 & 40 & 21 & 170 & 120 & 103 & -89.0 & -10.50 & 1.9 \\
\hline S-103 & 6.6 & .14 & 2 & 1,400 & 200 & 70 & 17 & 51 & 29 & -. & -97.5 & -11.90 & 3.9 \\
\hline S-247 & 33 & 1.3 & 2 & 2,000 & 160 & 2,600 & 19 & 230 & 160 & 111 & -90.0 & -10.30 & 2.4 \\
\hline S-376 & -- & -- & -- & - & - & - & -- & 30 & -- & -- & -- & --- & 2.1 \\
\hline S-72 & -- & -- & -- & -.- & -- & --- & --- & 68 & -- & - & $\ldots$ & --. & 2.7 \\
\hline S-169 & 20 & .05 & 4 & 1,000 & 60 & 120 & 19 & 4 & 21 & 189 & -96.5 & -11.60 & .5 \\
\hline S-187 & - & -- & -- & - & $\ldots$ & --- & -- & 16 & $\ldots$ & -.. & $\ldots$ & -.- & 2.0 \\
\hline$S-21$ & 5.9 & .11 & 4 & 3,500 & 120 & 160 & 65 & 19 & 41 & 105 & -97.0 & -11.75 & 1.0 \\
\hline S-22 & - & $\ldots$ & -- & -.. & -- & --- & -- & 22 & - & -- & --- & --. & 1.3 \\
\hline S-130 & - & - & -- & $\ldots$ & $\ldots$ & --- & $\ldots$ & 9 & -- & --- & --- & --- & 1.5 \\
\hline S-207 & - & - & -- & -- & $\ldots$ & -- & $\ldots$ & 41 & -- & -- &.- & -- & 1.5 \\
\hline TD-2939 & $\cdots$ & -- & - & -- & -- & -- & -- & -- & -- & --- & --- & -- & -- \\
\hline S-352 & 16 & .17 & 2 & 3,000 & 110 & 80 & 25 & 65 & 10 & --- & -94.0 & -11.15 & 1.1 \\
\hline S-81 & - & $\ldots$ & -- & --- & -- & - & - & 5 & -- & --- & --- & --- & 2.0 \\
\hline$S-113$ & 19 & .15 & 2 & 1,300 & 50 & 80 & 18 & 39 & 21 & 134 & -100.0 & -12.45 & 3.5 \\
\hline
\end{tabular}


Table 2. Data from Imperial Valley drainwater samples collected by the U.S. Geological Survey, May 16-20, 1988--Continued

\begin{tabular}{|c|c|c|c|c|c|c|c|c|c|c|}
\hline \multicolumn{2}{|c|}{$\begin{array}{c}\text { Site } \\
\text { number }\end{array}$} & \multirow{2}{*}{$\begin{array}{c}\begin{array}{c}\text { Local } \\
\text { identifier }\end{array} \\
\text { S-115 }\end{array}$} & \multirow{2}{*}{$\begin{array}{r}\text { Latitude } \\
32^{\circ} 45^{\prime} 33^{\prime \prime} \mathrm{N}\end{array}$} & \multirow{2}{*}{$\begin{array}{c}\text { Longitude } \\
115^{\circ} 37^{\prime} 10^{\prime \prime} \mathrm{W}\end{array}$} & \multirow{2}{*}{$\begin{array}{c}\text { Date } \\
5-19-88\end{array}$} & \multirow{2}{*}{$\begin{array}{l}\text { Time } \\
1500\end{array}$} & \multirow{2}{*}{$\begin{array}{c}\begin{array}{c}\text { Altitude } \\
\text { of } \\
\text { land } \\
\text { surface }\end{array} \\
-29\end{array}$} & \multirow{2}{*}{$\begin{array}{c}\begin{array}{c}\text { Discharge, } \\
\text { instanta- } \\
\text { neous } \\
\left(\mathrm{ft}^{3} / \mathrm{s}\right)\end{array} \\
0.38\end{array}$} & \multirow{2}{*}{$\begin{array}{c}\begin{array}{c}\text { Specific } \\
\text { conductance } \\
(\mu \mathrm{S} / \mathrm{cm})\end{array} \\
3,880\end{array}$} & \multirow{2}{*}{$\begin{array}{c}\mathrm{pH} \\
\text { (standard } \\
\text { units) }\end{array}$} \\
\hline 90 & $\ldots$ & & & & & & & & & \\
\hline 91 & . . & S-242 & $32^{\circ} 47^{\prime} 05^{\prime \prime} \mathrm{N}$ & $115^{\circ} 38^{\prime} 45^{\prime \prime} \mathrm{W}$ & $5-19-88$ & 1140 & -40 & .092 & 15,800 & 7.1 \\
\hline 92 & .. & S-392 & $32^{\circ} 47^{\prime} 20^{\prime \prime} \mathrm{N}$ & $115^{\circ} 35^{\prime} 00^{\prime \prime} \mathrm{W}$ & -- & --- & --- & -- & --- & - \\
\hline 93 & $\ldots$ & $S-423$ & $32^{\circ} 42^{\prime} 59^{\prime \prime} \mathrm{N}$ & $115^{\circ} 37^{\prime} 38^{\prime \prime W}$ & $5-19-88$ & 1620 & -21 & 0 & 20,200 & 6.9 \\
\hline 94 & $\ldots$ & S-93 & $32^{\circ} 43^{\prime} 20^{\prime \prime} \mathrm{N}$ & $115^{\circ} 29^{\prime} 30^{\prime \prime} \mathrm{W}$ & $5-19-88$ & 1715 & -3 & .23 & 6,150 & 7.1 \\
\hline 95 & $\ldots$ & S-221 & $32^{\circ} 45^{\prime} 58^{\prime \prime} \mathrm{N}$ & $115^{\circ} 30^{\prime} 43^{\prime \prime} \mathrm{W}$ & $5-19-88$ & 1745 & -37 & .052 & 8,310 & 7.2 \\
\hline 96 & $\ldots$ & S-229 & $32^{\circ} 44^{\prime} 14^{\prime \prime} \mathrm{N}$ & $115^{\circ} 29^{\prime} 43^{\prime \prime W}$ & $5-19-88$ & 1730 & -16 & .42 & 8,290 & 7.2 \\
\hline 97 & $\ldots$ & $S-321$ & $32^{\circ} 43^{\prime} 22^{\prime \prime} \mathrm{N}$ & $115^{\circ} 32^{\prime} 30^{\prime \prime} \mathrm{W}$ & $5-19-88$ & 1655 & -12 & --- & 3,200 & 7.1 \\
\hline 98 & $\ldots$ & S-371 & $32^{\circ} 44^{\prime} 42^{\prime \prime} \mathrm{N}$ & $115^{\circ} 32^{\prime} 35^{\prime \prime W}$ & $5-19-88$ & 1410 & -20 & .10 & 11,300 & 7.2 \\
\hline 99 & ... & S-144 & $32^{\circ} 45^{\prime} 04^{\prime \prime} \mathrm{N}$ & $115^{\circ} 24^{\prime} 43^{\prime \prime W}$ & $5-20-88$ & 1300 & -2 & .021 & 8,850 & 7.1 \\
\hline 100 & $\ldots$ & S-164 & $32^{\circ} 45^{\prime} 58^{\prime \prime} \mathrm{N}$ & $115^{\circ} 27^{\prime} 19^{\prime \prime} \mathrm{W}$ & $5-20-88$ & 1415 & -18 & .26 & 6,300 & 7.2 \\
\hline 101 & $\ldots$ & S-202 & $32^{\circ} 43^{\prime} 46^{\prime \prime} \mathrm{N}$ & $115^{\circ} 24^{\prime} 44^{\prime \prime} \mathrm{W}$ & $5-20-88$ & 1330 & 11 & -- & 3,350 & 7.0 \\
\hline 102 & .. & S-368 & $32^{\circ} 46^{\prime} 19^{\prime \prime} \mathrm{N}$ & $115^{\circ} 24^{\prime} 41^{\prime \prime W}$ & $5-20-88$ & 1315 & -17 & .18 & 5,940 & 7.3 \\
\hline 103 & . . & S-408 & $32^{\circ} 43^{\prime} 20^{\prime \prime} \mathrm{N}$ & $115^{\circ} 27^{\prime} 50^{\prime \prime} \mathrm{W}$ & $5-20-88$ & 1355 & -1 & .027 & 10,000 & 7.2 \\
\hline 104 & . . & S-176 & $32^{\circ} 45^{\prime} 55^{\prime \prime} \mathrm{N}$ & $115^{\circ} 19^{\prime} 10^{\prime \prime} \mathrm{W}$ & $5-19-88$ & 1125 & 8 & .16 & 11,800 & 7.3 \\
\hline 105 & $\cdots$ & S-316 & $32^{\circ} 45^{\prime} 04^{\prime \prime} \mathrm{N}$ & $115^{\circ} 18^{\prime} 59^{\prime \prime W}$ & $5-19-88$ & 1135 & 12 & .17 & 7,570 & 7.2 \\
\hline 106 & . . & S-336 & $32^{\circ} 43^{\prime} 20^{\prime \prime} \mathrm{N}$ & $115^{\circ} 20^{\prime} 40^{\prime \prime} \mathrm{W}$ & $5-20-88$ & 0950 & 25 & .16 & 6,620 & 7.3 \\
\hline 107 & . . & S-386 & $32^{\circ} 44^{\prime} 38^{\prime \prime} \mathrm{N}$ & $115^{\circ} 17^{\prime} 34^{\prime \prime} \mathrm{W}$ & $5-20-88$ & 1015 & 21 & .22 & 8,300 & 7.4 \\
\hline 108 & $\ldots$ & S-393 & $32^{\circ} 44^{\prime} 02^{\prime \prime} \mathrm{N}$ & $115^{\circ} 16^{\prime} 15^{\prime \prime} \mathrm{W}$ & $5-20-88$ & 1050 & 34 & .38 & 1,700 & 7.5 \\
\hline 109 & $\ldots$ & $S-60$ & $32^{\circ} 39^{\prime} 26^{\prime \prime} \mathrm{N}$ & $115^{\circ} 37^{\prime} 10^{\prime \prime} \mathrm{W}$ & $5-19-88$ & 1650 & -8 & .070 & 3,890 & 7.4 \\
\hline 110 & . & S-344 & $32^{\circ} 40^{\prime} 18^{\prime \prime} \mathrm{N}$ & $115^{\circ} 39^{\prime} 16^{\prime \prime} \mathrm{W}$ & $5-19-88$ & 1635 & -18 & .045 & 16,000 & 7.3 \\
\hline 111 & . . & S-416 & $32^{\circ} 42^{\prime} 16^{\prime \prime} \mathrm{N}$ & $115^{\circ} 38^{\prime} 16^{\prime \prime} \mathrm{W}$ & $5-19-88$ & 1530 & -25 & .016 & 4,420 & 7.4 \\
\hline 112 & . . & S-108 & $32^{\circ} 41^{\prime} 58^{\prime \prime} \mathrm{N}$ & $115^{\circ} 31^{\prime} 25^{\prime \prime} \mathrm{W}$ & $5-19-88$ & 1615 & -4 & .055 & 6,230 & 7.3 \\
\hline 113 & . . & S-182 & $32^{\circ} 39^{\prime} 29^{\prime \prime} \mathrm{N}$ & $115^{\circ} 29^{\prime} 48^{\prime \prime W}$ & $5-19-88$ & 1630 & -2 & .020 & 9,510 & 7.2 \\
\hline 114 & . . & $S-402$ & $32^{\circ} 39^{\prime} 38^{\prime \prime} \mathrm{N}$ & $115^{\circ} 34^{\prime} 33^{\prime \prime} \mathrm{W}$ & $5-19-88$ & 1555 & -7 & .078 & 3,260 & 7.1 \\
\hline 115 & . . & S-59 & $32^{\circ} 40^{\prime} 27^{\prime \prime} \mathrm{N}$ & $115^{\circ} 27^{\prime} 21^{\prime \prime W}$ & $5-19-88$ & 1730 & 10 & -- & 7,770 & 7.3 \\
\hline 116 & . . & S-267 & $32^{\circ} 40^{\prime} 45^{\prime \prime} \mathrm{N}$ & $115^{\circ} 24^{\prime} 26^{\prime \prime} \mathrm{W}$ & $5-20-88$ & 0915 & 21 & .069 & 3,950 & 7.3 \\
\hline 117 & ... & $S-14$ & $32^{\circ} 42^{\prime} 03^{\prime \prime} \mathrm{N}$ & $115^{\circ} 20^{\prime} 57^{\prime \prime} \mathrm{W}$ & $5-19-88$ & 1755 & 25 & .77 & 8,720 & 7.1 \\
\hline 118 & .. & S-360 & $32^{\circ} 41^{\prime} 19^{\prime \prime} \mathrm{N}$ & $115^{\circ} 29^{\prime} 40^{\prime \prime} \mathrm{W}$ & $5-20-88$ & 0850 & 35 & .084 & 5,690 & 7.3 \\
\hline 119 & . . & S-222 & $32^{\circ} 42^{\prime} 19^{\prime \prime} \mathrm{N}$ & $115^{\circ} 15^{\prime} 58^{\prime \prime W}$ & $5-19-88$ & 1815 & 35 & .55 & 1,850 & 7.1 \\
\hline
\end{tabular}


Table 2. Data from Imperial Valley drainwater samples collected by the U.S. Geological Survey, May 16-20, 1988--Continued

\begin{tabular}{|c|c|c|c|c|c|c|c|c|c|c|c|}
\hline $\begin{array}{c}\text { Local } \\
\text { identifier }\end{array}$ & $\begin{array}{l}\text { Temper- } \\
\text { ature, } \\
\text { water } \\
\left({ }^{\circ} \mathrm{C}\right)\end{array}$ & $\begin{array}{c}\text { Calcium } \\
(\mathrm{mg} / \mathrm{L}) \\
\text { as Ca) }\end{array}$ & $\begin{array}{c}\text { Magne- } \\
\text { sium } \\
(\mathrm{mg} / \mathrm{L} \\
\text { as } \mathrm{Mg})\end{array}$ & $\begin{array}{c}\text { Sodium } \\
(\mathrm{mg} / \mathrm{L} \\
\text { as } \mathrm{Na})\end{array}$ & $\begin{array}{l}\text { Potas- } \\
\text { sium } \\
(\mathrm{mg} / \mathrm{L} \\
\text { as } \mathrm{K})\end{array}$ & $\begin{array}{c}\text { Alka- } \\
\text { linity, } \\
\text { lab } \\
(\mathrm{mg} / \mathrm{L} \\
\text { as } \\
\left.\mathrm{CaCO}_{3}\right)\end{array}$ & $\begin{array}{c}\text { Sulfate } \\
(\mathrm{mg} / \mathrm{L} \\
\left.\text { as } \mathrm{SO}_{4}\right)\end{array}$ & $\begin{array}{l}\text { Chlo- } \\
\text { ride } \\
(\mathrm{mg} / \mathrm{L} \\
\text { as } \mathrm{Cl})\end{array}$ & $\begin{array}{l}\text { Fluo- } \\
\text { ride } \\
(\mathrm{mg} / \mathrm{L}) \\
\text { as } \mathrm{F})\end{array}$ & $\begin{array}{c}\text { Silica } \\
(\mathrm{mg} / \mathrm{L} \\
\text { as } \\
\left.\mathrm{SiO}_{2}\right)\end{array}$ & $\begin{array}{c}\text { Solids, } \\
\text { residue } \\
\text { at } \\
180^{\circ} \mathrm{C} \\
(\mathrm{mg} / \mathrm{L})\end{array}$ \\
\hline S-115 & -- & - & -- & --- & --- & 390 & -- & 370 & -- & --- & 2,870 \\
\hline S-242 & 22.5 & -- & - & -- & -- & 341 & $-\cdots$ & 4,600 & -- & -- & 10,900 \\
\hline S-392 & - & - & - & --- & - & - & --- & --- & --- & --- & -.- \\
\hline$S-423$ & 20.5 & 580 & 510 & 2,900 & 25 & 299 & 2,500 & 6,300 & 0.3 & 14 & -.- \\
\hline$S-93$ & 22.0 & --- & -- & --- & -- & 383 & --- & 1,000 & -- & -- & 4,850 \\
\hline S-221 & 20.0 & -- & -- & -- & -- & 358 & --- & 2,200 & -. & -. & 6,150 \\
\hline S-229 & 21.0 & -- & - & -- & $\ldots$ & 392 & -- & 1,800 & -.. & -- & 6,560 \\
\hline S-321 & 23.0 & -- & --- & -- & - & 395 & --- & 260 & -.- & -- & 2,360 \\
\hline S-371 & 22.0 & 560 & 350 & 1,600 & 7.6 & 360 & 2,300 & 2,600 & .5 & 18 & -- \\
\hline S-144 & 22.0 & --. & -.. & -- & $\ldots$ & 434 & -.- & 2,400 & --- & -- & 6,300 \\
\hline S-164 & 20.5 & -- & - & -- & - & 343 & -- & 1,000 & -- & -- & 4,820 \\
\hline S-202 & -- & -- & -- & -- & -- & 413 & -- & 260 & -- & --- & 2,370 \\
\hline S-368 & 20.5 & $-\cdots$ & - & -- & --- & 382 & -- & 1,000 & -- & -- & 4,130 \\
\hline$S-408$ & 21.0 & -.- & -- & --- & --- & 441 & --- & 2,800 & --- & --- & 7,150 \\
\hline S-176 & 19.0 & 390 & 330 & 1,900 & 7.6 & 484 & 3,300 & 2,200 & .5 & 18 & --- \\
\hline S-316 & -- & 400 & 220 & 1,100 & 5.8 & 370 & 2,100 & 1,200 & .8 & 15 & --- \\
\hline S-336 & 20.0 & - & - & --- & --. & 334 & --- & 1,200 & -- & --- & 4,760 \\
\hline S-386 & 20.5 & -- &.- & -.. & --. & 318 & $\ldots$ & 1,800 & --- & --- & 5,920 \\
\hline S-393 & 20.0 & --- & -- & --- & --- & 282 & -.- & 130 & -- & -- & 1,100 \\
\hline S-60 & 21.0 & 250 & 110 & 530 & 5.4 & 355 & 1,200 & 480 & .6 & 16 & --- \\
\hline S-344 & 21.5 & 250 & 320 & 2,800 & 19 & 319 & 3,800 & 3,800 & .4 & 15 & --- \\
\hline S-416 & 22.0 & -- & --. & --- & --- & 397 & --- & 540 & --- & -- & 3,330 \\
\hline S-108 & 22.0 & -- & --- & -- & --- & 410 & -.- & 770 & --- & -- & 4,960 \\
\hline S-182 & 21.5 & - & --- & -- & --- & 450 & --- & 2,200 & -- & --- & 7,540 \\
\hline S-402 & -- & -- & --- & --- & -- & 584 & --- & 310 & -- & --- & 2,260 \\
\hline S-59 & 21.0 & 290 & 220 & 1,300 & 7.3 & 419 & 3,000 & 810 & .7 & 17 & -- \\
\hline S-267 & 20.0 & - & -- & --- & --- & 336 & --- & 430 & --- & -- & 2,840 \\
\hline S-14 & 20.5 & 390 & 290 & 1,400 & 8.2 & 446 & 3,800 & 1,300 & .7 & 17 & -. \\
\hline S-360 & 20.0 & -- & --- & --- & -- & 347 & --- & 870 & --- & --- & 4,280 \\
\hline S-222 & 21.0 & 90 & 46 & 260 & 3.5 & 225 & 470 & 200 & .8 & 19 & -- \\
\hline
\end{tabular}


Table 2. Data from Imperial Valley drainwater samples collected by the U.S. Geological Survey, May 16-20, 1988--Continued

\begin{tabular}{|c|c|c|c|c|c|c|c|c|c|c|c|c|c|}
\hline $\begin{array}{l}\text { Local } \\
\text { ident- } \\
\text { ifier }\end{array}$ & $\begin{array}{c}\text { Nitro- } \\
\text { gen, } \\
\mathrm{NO}_{2}+ \\
\mathrm{NO}_{3} \\
(\mathrm{mg} / \mathrm{L} \\
\text { as } \mathrm{N})\end{array}$ & $\begin{array}{l}\text { Nitro- } \\
\text { gen, } \\
\text { am- } \\
\text { monia } \\
(\mathrm{mg} / \mathrm{L} \\
\text { as } \mathrm{N})\end{array}$ & $\begin{array}{c}\text { Arsenic } \\
(\mu \mathrm{g} / \mathrm{L} \\
\text { as As })\end{array}$ & $\begin{array}{l}\text { Boron } \\
(\mu \mathrm{g} / \mathrm{L} \\
\text { as } \mathrm{B})\end{array}$ & $\begin{array}{c}\text { Iron } \\
(\mu \mathrm{g} / \mathrm{L} \\
\text { as } \mathrm{Fe})\end{array}$ & $\begin{array}{c}\text { Manga- } \\
\text { nese } \\
(\mu \mathrm{g} / \mathrm{L} \\
\text { as } \mathrm{Mn})\end{array}$ & $\begin{array}{c}\text { Molyb- } \\
\text { denum } \\
(\mu \mathrm{g} / \mathrm{L} \\
\text { as Mo) }\end{array}$ & $\begin{array}{l}\text { Sele- } \\
\text { nium } \\
(\mu \mathrm{g} / \mathrm{L} \\
\text { as Se })\end{array}$ & $\begin{array}{c}\text { Vana- } \\
\text { dium } \\
(\mu \mathrm{g} / \mathrm{L}) \\
\text { as } \mathrm{V})\end{array}$ & $\begin{array}{c}\text { Tritium } \\
\text { total } \\
(\mathrm{pCi} / \mathrm{L})\end{array}$ & $\begin{array}{l}\text { Stable- } \\
\text { ratio } \\
{ }^{2} \mathrm{H} /{ }^{1} \mathrm{H}\end{array}$ & $\begin{array}{l}\text {-isotope } \\
\text { permil) } \\
\frac{{ }^{18} \mathrm{O} /{ }^{16} \mathrm{O}}{}\end{array}$ & $\begin{array}{c}\text { Selenium/ } \\
\text { chloride } \\
\text { (weight } \\
\text { ratio } \\
\text { times } \\
10^{-5} \text { ) }\end{array}$ \\
\hline S-115 & - & --- & --- & -- & -- & -- & --- & 14 & -- & --- & --- & -- & 3.8 \\
\hline S-242 & - & - & -- & -- & -- & -- & -- & 8 & -- & -- & --- & -- & .2 \\
\hline S-392 & - & $\ldots$ & -- & --- & --- & $\ldots$ & --- & -- & --- & --- & $\ldots$ & --- & --- \\
\hline S-423 & 97 & 0.62 & 1 & 1,800 & 180 & 270 & 19 & 240 & 74 & 91 & -90.5 & -10.15 & 3.8 \\
\hline S-93 & - & $\ldots$ & -- & -- & --. & --- & --. & 23 & $\ldots$ & -- & -.. & --. & 2.3 \\
\hline S-221 & - & --- & -- & --- & --- & --- & $\ldots$ & 33 & --- & --. & --- & $\ldots$ & 1.5 \\
\hline S-229 & - & -- & --- & -- & -- & -- & --- & 40 & --- & --- & --- & --- & 2.2 \\
\hline S-321 & -- & --- & -- & --- & -- & -- & $\cdots$ & 9 & --- & -- & -- & -- & 3.5 \\
\hline S-371 & 9.5 & .20 & 2 & 1,300 & 340 & 170 & 20 & 76 & 48 & 132 & -96.0 & -11.80 & 2.9 \\
\hline S-144 & --- & - & -- & -- & -- & --- & -- & 31 & --- & --- & --- & -- & 1.3 \\
\hline S-164 & --- & -- & -- & -- & -- & -- & $\cdots$ & 22 & $\cdots$ & --- & --- & $\cdots$ & 2.2 \\
\hline S-202 & $\ldots$ & --- & $\ldots$ & $\ldots$ & -.. &.-- & $\ldots$ & 12 & $\ldots$ & --- & --- & $\ldots$ & 4.6 \\
\hline S-368 & - & --- & --- & $\ldots$ & --- & -- & --- & 32 & -- & $\ldots$ & $\ldots$ & $\ldots$ & 3.2 \\
\hline S-408 & --- & -.- & -- & $\ldots$ & -- & -- &.-- & 7 & $\ldots$ & --- & --- & --- & .2 \\
\hline S-176 & 9.9 & .17 & 1 & 1,700 & 80 & 190 & 17 & 51 & 37 & --- & -96.5 & -11.60 & 2.3 \\
\hline S-316 & 19 & .14 & 1 & 1,100 & 80 & 300 & 16 & 28 & 24 & 132 & -100.0 & -12.15 & 2.3 \\
\hline S-336 & --- & -- & -- & -- & -- & --- & $\ldots$ & 17 & --- & -.. & --- & -- & 1.4 \\
\hline S-386 & $\ldots$ & -- & $\ldots$ & --. & --- & --- & $\ldots$ & 24 & $\ldots$ & $\ldots$ & --- & --- & 1.3 \\
\hline S-393 & - & -- & --. & --- & -- & -.- & $\ldots$ & 3 & -.. & --- & --- & --- & 2.3 \\
\hline S-60 & 13 & .05 & 1 & 540 & 40 & 60 & 15 & 6 & 9 & 113 & -102.0 & -12.50 & 1.2 \\
\hline S-344 & 37 & .21 & 2 & 2,300 & 90 & 70 & 33 & 60 & 36 & 85 & -94.5 & -11.15 & 1.6 \\
\hline S-416 & --- & -- & --- & -- & --- & -.- & --- & 17 & --- & --- & --- & -- & 3.1 \\
\hline S-108 & -- & -- & --- & --- & -- & --- & --- & 27 & --- & ... & --- & --. & 3.5 \\
\hline S-182 & -- & --. & -- & --- & --. & $\ldots$ & --- & 52 & $\ldots$ & $\ldots$ & --. & -.. & 2.4 \\
\hline S-402 & -- & -- & -- & -- & --- & -- & --- & 4 & --- & -.. & -- & -- & 1.3 \\
\hline S-59 & 16 & .12 & 2 & 1,400 & 60 & 20 & 17 & 27 & 15 & 113 & -100.5 & -12.35 & 3.3 \\
\hline S-267 & -- & - & --- & -- & --- & --- & - & 7 & --- & --- & --- & -- & 1.6 \\
\hline S-14 & 23 & .13 & 1 & 1,800 & 90 & 130 & 20 & 35 & 27 & 113 & -97.5 & -11.90 & 2.7 \\
\hline S-360 & --- & - & $\cdots$ & --- & --- & --- & -- & 14 & --- & --- & --- & --- & 1.6 \\
\hline S-222 & 1.2 & .03 & 2 & 400 & 10 & 70 & 10 & 3 & 7 & 135 & -103.0 & -12.80 & 1.5 \\
\hline
\end{tabular}


Table 3. Comparison of data for drainwater samples collected at selected locations in the Imperial Valley by the [1986 data are from three sumps and five tile drains (published by Setmire and others, 1990b). $\mu \mathrm{S} / \mathrm{cm}$, microsiemen $<$, actual value is less than value shown; --- no data]

\begin{tabular}{|c|c|c|c|c|c|c|}
\hline & \multicolumn{2}{|c|}{ Location 1} & \multicolumn{2}{|c|}{ Location 2} & \multicolumn{2}{|c|}{ Location 3} \\
\hline Local identifier & \multicolumn{2}{|c|}{ SS-18 } & \multicolumn{2}{|c|}{ Unknown } & \multicolumn{2}{|c|}{ S-462 } \\
\hline Latitude . & \multicolumn{2}{|c|}{$\begin{array}{l}33^{\circ} 10^{\prime} 33^{\prime \prime N} \\
11^{\circ} 26^{\circ} 15 \mathrm{w}\end{array}$} & \multicolumn{2}{|c|}{$33^{\circ} 03^{\prime} 04 " \mathrm{~N}$} & \multirow{2}{*}{\multicolumn{2}{|c|}{$\begin{array}{c}32^{\circ} 51^{\prime} 15^{\prime \prime N} \\
115^{\circ} 37^{\prime} 45^{\prime \prime} \mathrm{W}\end{array}$}} \\
\hline Longitude $\ldots \ldots \ldots \ldots \ldots \ldots \ldots$ & $115^{\circ} 3$ & $15 " \mathrm{~W}$ & $115^{\circ} 37^{\prime} 1$ & & & \\
\hline Altitude of land surface (ft below sea level) & \multicolumn{2}{|c|}{226} & \multicolumn{2}{|c|}{172} & \multicolumn{2}{|c|}{56} \\
\hline Date $\ldots \ldots \ldots \ldots \ldots \ldots \ldots$ & $7-14-86$ & $8-20-88$ & $7-14-86$ & -..- & $7-14-86$ & 8-19-88 \\
\hline Specific conductance $(\mu \mathrm{S} / \mathrm{cm}) \ldots \ldots \ldots$ & 28,400 & 30,900 & 7,120 & --- & 32,200 & 15,240 \\
\hline $\mathrm{pH}$ (standard units) $\ldots \ldots \ldots \ldots \ldots \ldots$ & 7.1 & 7.3 & 7.3 & --- & 6.8 & 6.9 \\
\hline Calcium $(\mathrm{mg} / \mathrm{L}$ as $\mathrm{Ca}) \ldots$ & 1,300 & 1,600 & 370 & $\cdots$ & 1,800 & 820 \\
\hline Magnesium $(\mathrm{mg} / \mathrm{L}$ as $\mathrm{Mg}) \ldots \ldots \ldots \ldots$ & 810 & 960 & 220 & --- & 820 & 360 \\
\hline Sodium $(\mathrm{mg} / \mathrm{L}$ as $\mathrm{Na}) \ldots \ldots \ldots \ldots \ldots$ & 4,400 & 4,400 & 1,100 & --- & 3,800 & 2,100 \\
\hline Potassium $(\mathrm{mg} / \mathrm{L}$ as $\mathrm{K}) \ldots \ldots \ldots \ldots \ldots$ & 38 & 37 & 15 & --- & 35 & 16 \\
\hline Alkalinity $\left(\mathrm{mg} / \mathrm{L}\right.$ as $\left.\mathrm{CaCO}_{3}\right)$ & 282 & 312 & 475 & $\cdots$ & 240 & 213 \\
\hline Sulfate $\left(\mathrm{mg} / \mathrm{L}\right.$ as $\left.\mathrm{SO}_{4}\right) \ldots$ & 3,700 & 2,900 & 2,800 & -.- & 2,000 & 1,400 \\
\hline Chloride $(\mathrm{mg} / \mathrm{L}$ as $\mathrm{Cl})$ & 9,400 & 10,000 & 780 & --- & 11,000 & 4,600 \\
\hline Fluoride $(\mathrm{mg} / \mathrm{L}$ as $\mathrm{F})$. & 0.4 & 0.3 & 0.8 & -.- & 0.2 & 0.6 \\
\hline Silica $\left(\mathrm{mg} / \mathrm{L}\right.$ as $\left.\mathrm{SiO}_{2}\right) \ldots \ldots \ldots \ldots$ & 14 & 17 & 20 & $\cdots$ & 12 & 13 \\
\hline Nitrogen, $\mathrm{NO}_{2}+\mathrm{NO}_{3}(\mathrm{mg} / \mathrm{L}$ as $\mathrm{N}) \ldots \ldots$ & -- & 8.2 & --- & --- & $\cdots$ & 29 \\
\hline Nitrogen, ammonia $(\mathrm{mg} / \mathrm{L}$ as $\mathrm{N}) \ldots \ldots$ & -- & 1.1 & -. & --- & --- & 4.2 \\
\hline Arsenic ( $\mu \mathrm{g} / \mathrm{L}$ as As) . & 1 & 1 & 3 & --- & 1 & 4 \\
\hline Boron $(\mu \mathrm{g} / \mathrm{L}$ as $\mathrm{B}) \ldots$ & 3,400 & 3,700 & 1,800 & --- & 3,100 & 920 \\
\hline Iron $(\mu \mathrm{g} / \mathrm{L}$ as $\mathrm{Fe}) \ldots \ldots$ & 100 & 160 & 50 & -- & 140 & 250 \\
\hline Manganese $(\mu \mathrm{g} / \mathrm{L}$ as $\mathrm{Mn})$ & 2,900 & 2,700 & 540 & --- & 130 & 580 \\
\hline Molybdenum ( $\mu \mathrm{g} / \mathrm{L}$ as $\mathrm{Mo})$ & 25 & 11 & 30 & -- & 20 & 24 \\
\hline Selenium $(\mu \mathrm{g} / \mathrm{L}$ as $\mathrm{Se}) \ldots \ldots \ldots \ldots$ & 55 & 20 & 24 & -- & 120 & 61 \\
\hline Vanadium $(\mu \mathrm{g} / \mathrm{L}$ as $\mathrm{V}) \ldots \ldots \ldots \ldots$ & 40 & 87 & 20 & -- & 100 & 41 \\
\hline${ }^{2} \mathrm{H} /{ }^{1} \mathrm{H}$ isotope ratio (permil) . & -- & -89.5 & --- & -- & -- & -95.5 \\
\hline${ }^{18} \mathrm{O} /{ }^{16} \mathrm{O}$ isotope ratio (permil) $\ldots \ldots \ldots \ldots$ & -_ & -9.95 & --- & -- & -- & -11.30 \\
\hline
\end{tabular}


U.S. Geological Survey in 1986 and 1988

per centimeter at $25^{\circ} \mathrm{C} ;{ }^{\circ} \mathrm{C}$, degree Celsius; $\mathrm{mg} / \mathrm{L}$, milligram per liter; $\mu \mathrm{g} / \mathrm{L}$, microgram per liter; $\mathrm{ft}$, foot;

\begin{tabular}{|c|c|c|c|c|c|c|c|c|c|}
\hline \multicolumn{2}{|c|}{ Location 4} & \multicolumn{2}{|c|}{ Location 5} & \multicolumn{2}{|c|}{ Location 6} & \multicolumn{2}{|c|}{ Location 7} & \multicolumn{2}{|c|}{ Location 8} \\
\hline \multicolumn{2}{|c|}{$\mathrm{S}-113$} & \multicolumn{2}{|c|}{ Unknown } & \multicolumn{2}{|c|}{ Unknown } & \multicolumn{2}{|c|}{ Unknown } & \multicolumn{2}{|c|}{ Unknown } \\
\hline \multirow{2}{*}{\multicolumn{2}{|c|}{$\begin{array}{c}32^{\circ} 45^{\prime} 07^{\prime \prime} \mathrm{N} \\
\end{array}$}} & \multicolumn{2}{|c|}{$33^{\circ} 10^{\prime} 37^{\prime \prime N}$} & \multicolumn{2}{|c|}{$32^{\circ} 05^{\prime} 20^{\prime \prime} \mathrm{N}$} & \multicolumn{2}{|c|}{$32^{\circ} 58^{\prime} 00^{\prime \prime} \mathrm{N}$} & \multicolumn{2}{|c|}{$32^{\circ} 49^{\prime} 31^{\prime \prime N}$} \\
\hline & $09 " \mathrm{~W}$ & $115^{\circ} 2$ & '12"W & $115^{\circ} 2$ & $45 " \mathrm{~W}$ & $115^{\circ} 2$ & $52 " \mathrm{~W}$ & $115^{\circ} 21^{\prime}$ & \\
\hline \multicolumn{2}{|c|}{24} & \multicolumn{2}{|c|}{130} & \multicolumn{2}{|c|}{108} & \multicolumn{2}{|c|}{116} & \multicolumn{2}{|c|}{17} \\
\hline $7-14-86$ & $8-19-88$ & $7-14-86$ & $8-20-88$ & $7-14-86$ & $8-20-88$ & $7-14-86$ & $8-20-88$ & $7-14-86$ & -...- \\
\hline 5,800 & 5,570 & 5,610 & 5,460 & 35,100 & 36,400 & 6,640 & 4,660 & 8,290 & --- \\
\hline 7.4 & 7.3 & 7.2 & 7.9 & 7.1 & 7.4 & 7.3 & 8.0 & 7.9 & \\
\hline 370 & 340 & 310 & 310 & 920 & 940 & 290 & 230 & 440 & --- \\
\hline 150 & 170 & 160 & 170 & 1,300 & 1,400 & 160 & 150 & 250 & -.- \\
\hline 850 & 840 & 810 & 790 & 5,800 & 6,800 & 970 & 730 & 1,300 & -.. \\
\hline 13 & 10 & 7 & 10 & 65 & 66 & 20 & 11 & 10 & -- \\
\hline 386 & 457 & 366 & 270 & 350 & 445 & 310 & 279 & 530 & -.- \\
\hline 2,200 & 2,000 & 1,900 & 1,600 & 5,700 & 6,100 & 2,000 & 1,200 & 3,500 & ... \\
\hline 660 & 620 & 740 & 770 & 12,000 & 11,000 & 990 & 720 & 820 & ... \\
\hline 0.5 & 0.9 & 1.4 & 0.6 & 0.3 & 0.2 & 1.1 & 0.7 & 0.5 & -.. \\
\hline 20 & 18 & 26 & 23 & 15 & 7.7 & 18 & 22 & 22 & -- \\
\hline-- & 27 & $-\cdots$ & 31 & --. & 18 & --. & 8.4 & -.- & -.- \\
\hline--- & 0.10 & $\ldots$ & 0.07 & -- & 0.65 & $-\cdots$ & 1.2 & --. & $\ldots$ \\
\hline 1 & 1 & 1 & 1 & 4 & 5 & 2 & 4 & 1 & ... \\
\hline 1,100 & 980 & 1,200 & 1,100 & 3,500 & 4,000 & 2,400 & 880 & 1,700 & -.- \\
\hline 30 & 80 & 30 & 30 & 140 & 270 & 40 & 190 & 50 & $\ldots$ \\
\hline 340 & 20 & 40 & $<10$ & 50 & 50 & 50 & $<10$ & 20 & --- \\
\hline 28 & 16 & 36 & 20 & 35 & 14 & 58 & 16 & 15 & -.- \\
\hline 14 & 18 & 7 & 13 & 300 & 260 & 25 & 16 & 26 & $\ldots$ \\
\hline 17 & 12 & 17 & 12 & 100 & 98 & 38 & 17 & 22 & -- \\
\hline--- & -101.0 & $\ldots$ & -100.5 & $\ldots$ & -78.5 & $\ldots$ & -97.0 & $\cdots$ & -.. \\
\hline--- & -12.50 & $\cdots$ & -12.40 & -- & -8.10 & $\cdots$ & -12.10 & $\cdots$ & $\cdots$ \\
\hline
\end{tabular}


Table 4. Data from periodic sampling of drainwater at 15 Imperial Valley sites at fields, May 1988-August 1989

[Location of sites shown in figure 2. Data from May 1988 also given in table $2 . \mathrm{ft}^{3} / \mathrm{s}$, cubic foot per second; $\mu \mathrm{S} / \mathrm{cm}$, microsiemen per centimeter at $25^{\circ} \mathrm{C} ;{ }^{\circ} \mathrm{C}$, degree Celsius; $\mathrm{mg} / \mathrm{L}$, milligram per liter; $\mu \mathrm{g} / \mathrm{L}$, microgram per liter; $\mathrm{pCi} / \mathrm{L}$, picocurie per liter; <, actual value less than value shown; --- no data. The analysis for each sample is displayed as one line on four consecutive pages. Nitrate (plus nitrite) analyzed twice on many samples; results of both analyses are listed]

\begin{tabular}{|c|c|c|c|c|c|c|c|c|c|c|c|}
\hline $\begin{array}{c}\text { Site } \\
\text { number }\end{array}$ & $\begin{array}{c}\text { Local } \\
\text { identi- } \\
\text { fier }\end{array}$ & Date & Time & $\begin{array}{c}\text { Dis } \\
\text { charge, } \\
\text { instan- } \\
\text { taneous } \\
\left(\mathrm{ft}^{3} / \mathrm{s}\right)\end{array}$ & $\begin{array}{l}\text { Spe- } \\
\text { cific } \\
\text { con- } \\
\text { duct- } \\
\text { ance } \\
(\mu \mathrm{S} / \mathrm{cm})\end{array}$ & $\begin{array}{c}\mathrm{pH} \\
\text { (stand- } \\
\text { ard } \\
\text { units) }\end{array}$ & $\begin{array}{l}\text { Temper- } \\
\text { ature, } \\
\text { water } \\
\left({ }^{\circ} \mathrm{C}\right)\end{array}$ & $\begin{array}{l}\text { Calcium } \\
(\mathrm{mg} / \mathrm{L} \\
\text { as Ca) }\end{array}$ & $\begin{array}{l}\text { Magne- } \\
\text { sium } \\
(\mathrm{mg} / \mathrm{L} \\
\text { as } \mathrm{Mg})\end{array}$ & $\begin{array}{l}\text { Sodium } \\
(\mathrm{mg} / \mathrm{L} \\
\text { as } \mathrm{Na})\end{array}$ & $\begin{array}{c}\text { Potas- } \\
\text { sium } \\
(\mathrm{mg} / \mathrm{L} \\
\text { as K) }\end{array}$ \\
\hline \multirow[t]{14}{*}{6} & \multirow[t]{14}{*}{ S-226 } & $5-16-88$ & 1415 & 0.1700 & 36,100 & 7.7 & 22.5 & 640 & 1,100 & 6,000 & 45 \\
\hline & & $8-23-88$ & 0855 & -- & 50,000 & 7.6 & $\ldots$ & --- & -.- & -.. & $\ldots$ \\
\hline & & $10-20-88$ & 1405 & .0520 & 36,700 & 7.4 & 27.0 & 830 & 790 & 7,300 & 72 \\
\hline & & $11-14-88$ & 1240 & .0118 & 33,600 & 7.4 & 23.5 & 820 & --- & -- & -- \\
\hline & & $12-16-88$ & 1350 & .1930 & 31,100 & 7.0 & -- & 880 & -- & -- & -- \\
\hline & & $1-19-89$ & 1640 & .0000 & 38,400 & 7.6 & 18.0 & 1,200 & 1,300 & 7,100 & 48 \\
\hline & & $2-16-89$ & 0950 & .2460 & 26,300 & 7.4 & 19.0 & 890 & --- & -- & -- \\
\hline & & $3-15-89$ & 1340 & .0432 & 32,600 & 7.5 & 22.0 & 1,200 & --- & ... & --- \\
\hline & & $4-10-89$ & 1750 & .0490 & 32,800 & 7.1 & 20.0 & 1,200 & 1,100 & 5,300 & 39 \\
\hline & & $5-22-89$ & 1630 & .0685 & 39,500 & 7.2 & 22.0 & 1,500 & 1,200 & 6,000 & 50 \\
\hline & & $6-20-89$ & 1210 & .0137 & 39,900 & 7.2 & 24.0 & 1,600 & 1,300 & 6,700 & 18 \\
\hline & & $7-19-89$ & 1820 & .0288 & 18,200 & 7.4 & 25.0 & 820 & 500 & 2,600 & 37 \\
\hline & & $8-27-89^{1}$ & 1600 & .1030 & 30,600 & 7.3 & 26.5 & 1,100 & 820 & 4,800 & 61 \\
\hline & & $8-27-89$ & 1601 & --- & 30,600 & 7.2 & -- & -- & --- & -- & -- \\
\hline \multirow[t]{14}{*}{7} & \multirow[t]{14}{*}{ S-269 } & $5-16-88$ & 1430 & .0790 & 28,200 & 7.1 & 22.0 & 260 & 830 & 5,400 & 56 \\
\hline & & 8-23-88 & 1030 & .0360 & 32,800 & 7.1 & --- & -- & --- & --- & -- \\
\hline & & $10-20-88$ & 1330 & .0110 & 34,000 & 7.6 & 26.0 & 740 & 1,100 & 6,000 & 60 \\
\hline & & $11-14-88$ & 1325 & .0061 & 20,000 & 7.9 & 24.0 & 560 & -- & --- & $\cdots$ \\
\hline & & $12-16-88$ & 1415 & .0000 & 32,800 & 7.6 & -- & 910 & -- & --- & -- \\
\hline & & $1-19-89$ & 1655 & .0036 & 32,700 & 7.7 & 18.0 & 780 & 1,200 & 5,300 & 71 \\
\hline & & $2-16-89$ & 0920 & .0000 & 31,900 & 7.9 & 18.5 & 820 & --- & --- & $\cdots$ \\
\hline & & $3-15-89$ & 1510 & .0012 & 33,100 & 7.3 & 20.0 & 820 & --- & -.. & --- \\
\hline & & $4-10-89$ & 1830 & .0535 & 28,600 & 7.0 & 20.5 & 780 & 980 & 5,000 & 48 \\
\hline & & $5-22-89$ & 1745 & .0400 & 24,000 & 7.1 & 22.0 & 590 & 730 & 3,900 & 58 \\
\hline & & $6-20-89$ & 1300 & .0517 & 34,500 & 7.0 & 23.5 & 900 & 1,200 & 6,000 & 85 \\
\hline & & $7-19-89$ & 1840 & .0287 & 31,500 & 7.0 & 24.0 & 900 & 1,100 & 5,500 & 85 \\
\hline & & $8-27-89^{1}$ & 1700 & 1.1640 & 33,200 & 6.9 & 26.0 & 850 & 1,100 & 5,700 & 95 \\
\hline & & $8-27-89$ & 1701 & -- & 33,200 & 6.8 & $\cdots$ & -- & -- & -- & $\cdots$ \\
\hline \multirow[t]{14}{*}{8} & \multirow[t]{14}{*}{ S-417 } & $5-16-88$ & 1450 & .0028 & 44,000 & 7.3 & 23.0 & --- & --- & -- & -- \\
\hline & & $8-18-88$ & 1400 & --- & 42,300 & 7.4 & -- & --- & --- & --- & -- \\
\hline & & $10-20-88$ & 1425 & .0050 & 34,300 & 7.3 & 26.0 & 820 & 920 & 6,500 & 59 \\
\hline & & $11-14-88$ & 1305 & .0198 & 32,300 & 7.6 & 24.0 & 830 & --- & --- & --- \\
\hline & & $12-16-88$ & 1415 & .0147 & 33,100 & 7.4 & 22.0 & 760 & --- & --- & -- \\
\hline & & $1-18-89$ & 1600 & --- & 19,700 & 7.6 & 15.0 & 630 & 620 & 3,200 & 39 \\
\hline & & $2-16-89$ & 0930 & .2190 & 32,600 & 7.4 & 18.5 & 900 & -- & -.- & --- \\
\hline & & $3-15-89$ & 1425 & .1090 & 12,000 & 7.4 & 18.5 & 410 & --- & --- & --- \\
\hline & & $4-10-89$ & 1810 & .2670 & 10,400 & 7.5 & 18.5 & 350 & 320 & 1,600 & 18 \\
\hline & & $5-22-89$ & 1715 & .0066 & 38,000 & 7.2 & 21.0 & 1,200 & 1,400 & 5,700 & 30 \\
\hline & & $6-20-89$ & 1245 & .0330 & 39,400 & 7.2 & 23.0 & 1,200 & 1,500 & 6,400 & 86 \\
\hline & & $7-19-89$ & 1900 & -- & 12,000 & 7.3 & 26.0 & 630 & 370 & 1,700 & 28 \\
\hline & & $8-27-89^{1}$ & 1620 & .0078 & 35,400 & 7.3 & 25.0 & 1,100 & 1,200 & 5,600 & 89 \\
\hline & & $8-27-89$ & 1621 & --- & 35,500 & 7.3 & -- & --- & -- & --- & --- \\
\hline
\end{tabular}

See footnote at end of table. 
Table 4. Data from periodic sampling of drainwater at 15 Imperial Valley sites at fields, May 1988-August 1989-Continued

\begin{tabular}{|c|c|c|c|c|c|c|c|c|c|c|}
\hline $\begin{array}{l}\text { Local } \\
\text { identi- } \\
\text { fier }\end{array}$ & Date & $\begin{array}{c}\text { Alka- } \\
\text { linity, } \\
\text { lab } \\
(\mathrm{mg} / \mathrm{L} \\
\text { as } \\
\left.\mathrm{CaCO}_{3}\right)\end{array}$ & $\begin{array}{c}\text { Sulfate } \\
(\mathrm{mg} / \mathrm{L} \\
\left.\text { as } \mathrm{SO}_{4}\right)\end{array}$ & $\begin{array}{l}\text { Chlo- } \\
\text { ride } \\
(\mathrm{mg} / \mathrm{L} \\
\text { as } \mathrm{Cl})\end{array}$ & $\begin{array}{c}\text { Fluo- } \\
\text { ride } \\
(\mathrm{mg} / \mathrm{L} \\
\text { as F) }\end{array}$ & $\begin{array}{c}\text { Bromide } \\
(\mathrm{mg} / \mathrm{L} \\
\text { as } \mathrm{Br})\end{array}$ & $\begin{array}{c}\text { Silica } \\
(\mathrm{mg} / \mathrm{L} \\
\text { as } \\
\left.\mathrm{SiO}_{2}\right)\end{array}$ & $\begin{array}{l}\text { Solids, } \\
\text { residue } \\
\text { at } 180 \\
{ }^{\circ} \mathrm{C} \\
(\mathrm{mg} / \mathrm{L})\end{array}$ & $\begin{array}{c}\text { Nitro- } \\
\text { gen, } \\
\mathrm{NO}_{2}+ \\
\mathrm{NO}_{3} \\
(\mathrm{mg} / \mathrm{L} \\
\text { as } \mathrm{N})\end{array}$ & $\begin{array}{c}\text { Nitro- } \\
\text { gen, } \\
\mathrm{NO}_{2}+ \\
\mathrm{NO}_{3} \\
(\mathrm{mg} / \mathrm{L} \\
\text { as } \mathrm{N})\end{array}$ \\
\hline \multirow[t]{14}{*}{ S-226 } & $5-16-88$ & 216 & 3,800 & 11,000 & 0.5 & - & 12 & -- & 45 & -- \\
\hline & $8-23-88$ & 173 & 2,800 & 19,000 & --. & -- & -- & 35,300 & -.. & -- \\
\hline & $10-20-88$ & 203 & 5,200 & 12,000 & .7 & -- & 10 & 27,900 & 190 & 170 \\
\hline & $11-14-88$ & 269 & 4,900 & 10,000 & -- & 7.3 & -- & 25,000 & 140 & -- \\
\hline & $12-16-88$ & 300 & 4,900 & 9,500 & -- & 6.8 & $\cdots$ & 22,900 & 110 & $\ldots$ \\
\hline & $1-19-89$ & 349 & 4,800 & 12,000 & - & 9.2 & 12 & 28,200 & 120 & 120 \\
\hline & $2-16-89$ & 304 & 3,900 & 7,700 & -- & 5.2 & -- & 19,200 & 94 & - \\
\hline & $3-15-89$ & 250 & 4,500 & 10,000 & -- & 6.9 & --- & 23,500 & 77 & --- \\
\hline & $4-10-89$ & 323 & 3,900 & 11,000 & -- & 6.6 & 9.2 & 23,500 & 100 & 100 \\
\hline & $5-22-89$ & 262 & 3,700 & 14,000 & .5 & 9.8 & 13 & 28,400 & 66 & 66 \\
\hline & $6-20-89$ & 274 & 3,700 & 14,000 & .5 & 9.9 & 13 & 28,400 & 63 & 63 \\
\hline & $7-19-89$ & 289 & 3,400 & 4,700 & $\ldots$ & 2.8 & 13 & 13,300 & -- & 36 \\
\hline & $8-27-89^{1}$ & 235 & 3,900 & 9,400 & $\ldots$ & 6.8 & 13 & 21,500 & --- & 57 \\
\hline & $8-27-89$ & 233 & 3,900 & 9,500 & -- & 7.1 & -- & -.- & -- & -- \\
\hline \multirow[t]{14}{*}{ S-269 } & $5-16-88$ & 490 & 5,700 & 7,900 & .7 & $\ldots$ & 17 & $\ldots$ & 34 & -.- \\
\hline & $8-23-88$ & 475 & 5,800 & 9,500 & --- & --- & --- & 25,200 & -- & --- \\
\hline & $10-20-88$ & 444 & 5,400 & 11,000 & .7 & $\cdots$ & 17 & 26,000 & 49 & 50 \\
\hline & $11-14-88$ & 239 & 4,000 & 4,800 & -- & 4.0 & $\cdots$ & 15,100 & 140 & -- \\
\hline & $12-16-88$ & 509 & 5,100 & 11,000 & - & 6.9 & --- & 24,700 & 57 & -- \\
\hline & $1-19-89$ & 410 & 4,900 & 10,000 & -- & 8.0 & 16 & 24,300 & 51 & 51 \\
\hline & $2-16-89$ & 427 & 4,800 & 13,000 & -- & 7.4 & -- & 23,900 & 42 & -- \\
\hline & $3-15-89$ & 455 & 5,100 & 10,000 & $\cdots$ & 7.5 & -.- & 25,300 & 37 & -- \\
\hline & $4-10-89$ & 499 & 5,100 & 8,000 & -- & 6.1 & 14 & 21,500 & 79 & 79 \\
\hline & $5-22-89$ & 440 & 4,300 & 6,800 & .9 & 5.0 & 18 & 17,700 & 26 & 26 \\
\hline & $6-20-89$ & 491 & 5,300 & 10,000 & .7 & 8.1 & 18 & 22,000 & 38 & 38 \\
\hline & $7-19-89$ & 512 & 5,500 & 9,200 & -- & 7.0 & 18 & 23,400 & --- & 28 \\
\hline & $8-27-89^{1}$ & 508 & 5,400 & 10,000 & -- & 7.0 & 19 & 25,100 & --- & 27 \\
\hline & $8-27-89$ & 491 & 5,700 & 10,000 & - & 7.7 & -- & -- & -- & --- \\
\hline \multirow[t]{14}{*}{ S-417 } & $5-16-88$ & 444 & - & -. & $\ldots$ & --- & -- & 32,500 & -- & $\cdots$ \\
\hline & $8-18-88$ & 472 & 4,300 & 15,000 & $\ldots$ & $\cdots$ & --- & 30,700 & --- & -- \\
\hline & $10-20-88$ & 465 & 4,900 & 11,000 & .7 & --- & 19 & 25,800 & 36 & 33 \\
\hline & $11-14-88$ & 468 & 5,100 & 9,400 & -- & 6.6 & $\ldots$ & 24,200 & 34 & --- \\
\hline & $12-16-88$ & 419 & 5,500 & 10,000 & -- & 7.7 & -- & 25,000 & 32 & $\cdots$ \\
\hline & $1-18-89$ & 438 & 4,900 & 4,500 & - & 2.7 & 15 & 15,300 & 21 & 21 \\
\hline & $2-16-89$ & 548 & 4,800 & 10,000 & -- & 6.8 & - & 24,200 & 23 & $\ldots$ \\
\hline & $3-15-89$ & 407 & 3,300 & 2,600 & -- & 1.3 & --- & 9,080 & 3.9 & --- \\
\hline & $4-10-89$ & 394 & 2,700 & 2,000 & --- & 0.99 & 15 & 7,610 & 3.3 & 3.3 \\
\hline & $5-22-89$ & 580 & 4,500 & 13,000 & .7 & 8.9 & 19 & 27,700 & 14 & 14 \\
\hline & $6-20-89$ & 530 & 4,200 & 13,000 & .7 & 9.1 & 19 & 28,900 & 30 & 30 \\
\hline & $7-19-89$ & 345 & 4,300 & 2,100 & -- & .86 & 17 & 9,720 & -- & .93 \\
\hline & $8-27-89^{1}$ & 465 & 4,500 & 12,000 & -- & 8.1 & 20 & 25,200 & --- & 21 \\
\hline & $8-27-89$ & 378 & 4,600 & 12,000 & - & 7.9 & -- & -- & - & -- \\
\hline
\end{tabular}

See footnote at end of table. 
Table 4. Data from periodic sampling of drainwater at 15 Imperial Valley sites at fields, May 1988-August 1989-Continued

\begin{tabular}{|c|c|c|c|c|c|c|c|c|c|c|c|}
\hline $\begin{array}{l}\text { Local } \\
\text { identi- } \\
\text { fier }\end{array}$ & Date & $\begin{array}{c}\text { Nitro- } \\
\text { gen, } \\
\text { ammonia } \\
(\mathrm{mg} / \mathrm{L} \\
\text { as } \mathrm{N})\end{array}$ & $\begin{array}{c}\text { Nitro- } \\
\text { gen, am- } \\
\text { monia + } \\
\text { organic, } \\
\text { dis. } \\
\text { (mg/L } \\
\text { as N) }\end{array}$ & $\begin{array}{c}\text { Phos- } \\
\text { phorus, } \\
\text { hydro. } \\
+ \text { ortho } \\
\text { (mg/L } \\
\text { as P) }\end{array}$ & $\begin{array}{l}\text { Alum- } \\
\text { inum } \\
(\mu \mathrm{g} / \mathrm{L} \\
\text { as } \mathrm{Al})\end{array}$ & $\begin{array}{l}\text { Anti- } \\
\text { mony } \\
(\mu \mathrm{g} / \mathrm{L} \\
\text { as Sb) }\end{array}$ & $\begin{array}{c}\text { Arsenic } \\
(\mu \mathrm{g} / \mathrm{L} \\
\text { as } \mathrm{As})\end{array}$ & $\begin{array}{l}\text { Boron } \\
(\mu \mathrm{g} / \mathrm{L} \\
\text { as B) }\end{array}$ & $\begin{array}{c}\text { Iron } \\
(\mu \mathrm{g} / \mathrm{L}) \\
\text { as } \mathrm{Fe})\end{array}$ & $\begin{array}{c}\text { Lithium } \\
(\mu \mathrm{g} / \mathrm{L} \\
\text { as } \mathrm{Li})\end{array}$ & $\begin{array}{c}\text { Manga- } \\
\text { nese } \\
(\mu \mathrm{g} / \mathrm{L} \\
\text { as Mn) }\end{array}$ \\
\hline \multirow[t]{14}{*}{ S-226 } & $5-16-88$ & 1.2 & -- & -- & --- & -- & 3 & 2,200 & 210 & -- & 70 \\
\hline & $8-23-88$ & -- & -- & -- & --- & --- & 2 & 2,600 & -- & $\ldots$ & -- \\
\hline & $10-20-88$ & .80 & -- & -- & 50 & $<1$ & 4 & 2,100 & 200 & - & 60 \\
\hline & $11-14-88$ & .75 & -- & -- & -- & -- & -- & 2,400 & 140 & -- & 40 \\
\hline & $12-16-88$ & .54 & -- & -- & -- & - & -- & 2,000 & 130 & - & 50 \\
\hline & $1-19-89$ & 1.1 & - & $\ldots$ & 40 & -- & 4 & 2,100 & 170 & 980 & 50 \\
\hline & $2-16-89$ & .44 & $\cdots$ & -- & -- & -- & -- & 1,500 & $<90$ & -- & 20 \\
\hline & $3-15-89$ & .40 & -- & -- & --- & $\ldots$ & --- & 1,700 & 140 & --- & 40 \\
\hline & $4-10-89$ & .50 & -- & -- & 210 & -- & 3 & 2,000 & 130 & 790 & 40 \\
\hline & $5-22-89$ & .81 & -- & -- & $<50$ & --- & 2 & 2,200 & 220 & 900 & 70 \\
\hline & $6-20-89$ & .71 & -- & -- & 30 & --- & 2 & 2,500 & 220 & 960 & 60 \\
\hline & $7-19-89$ & -- & -- & -- & 30 & - & 3 & 2,200 & 70 & -.. & 40 \\
\hline & $8-27-89^{1}$ & .35 & 0.3 & 0.10 & 40 & -- & 4 & 2,500 & 130 & -- & 50 \\
\hline & $8-27-89$ & - & --- & -- & $\cdots$ & -- & --- & -- & -. & --- & -- \\
\hline \multirow[t]{14}{*}{ S-269 } & $5-16-88$ & .44 & $\ldots$ & -- & --- & --- & 6 & 3,300 & 140 & --- & 40 \\
\hline & $8-23-88$ & --- & $-\ldots$ & --- & -- & -- & 7 & 3,600 & -.. & -- & -. \\
\hline & $10-20-88$ & .63 & $\cdots$ & -- & 20 & $<1$ & 10 & 3,600 & 160 & $\cdots$ & 60 \\
\hline & $11-14-88$ & .28 & ... & - & $\ldots$ & -- & --- & 2,600 & 80 & --- & 20 \\
\hline & $12-16-88$ & .63 & - & -- & - & -- & -- & 3,600 & 130 & $\cdots$ & 310 \\
\hline & $1-19-89$ & .70 & - & -- & 20 & -.. & 8 & 2,700 & 160 & 1,100 & 40 \\
\hline & $2-16-89$ & .44 & - & -- & - & -- & -- & 2,600 & 100 & -.. & 40 \\
\hline & $3-15-89$ & .31 & - & -- & -- & -- & -- & 2,900 & 150 & --- & 40 \\
\hline & $4-10-89$ & .34 & - & --- & 60 & -- & 8 & 2,900 & 120 & 1,800 & 40 \\
\hline & $5-22-89$ & .18 & - & --- & 30 & -- & 8 & 2,600 & 140 & 870 & 40 \\
\hline & $6-20-89$ & .37 & $\cdots$ & -- & 20 & -- & 9 & 3,300 & 190 & 1,600 & 50 \\
\hline & 7-19-89 & .29 & -- & -- & 70 & -- & 10 & 3,600 & 130 & -- & 50 \\
\hline & $8-27-89^{1}$ & .29 & .2 & .12 & 80 & -- & 10 & 4,000 & 160 & $\cdots$ & 40 \\
\hline & $8-27-89$ & -- & $\cdots$ & -- & -- & -- & -- & -- & - & -- & -- \\
\hline \multirow[t]{14}{*}{ S-417 } & $5-16-88$ & - & -- & --- & -- & -- & --- & -- & -- & -- & -- \\
\hline & $8-18-88$ & -- & - & --- & -- & -- & 4 & 4,300 & -- & --- & --- \\
\hline & $10-20-88$ & .79 & - & -- & 30 & $<1$ & 5 & 4,000 & 140 & $\cdots$ & 230 \\
\hline & $11-14-88$ & .65 & - & - & --- & --- & --- & 4,000 & 120 & --- & 250 \\
\hline & $12-16-88$ & .68 & - & -- & -- & -- & -- & 3,200 & 170 & $\cdots$ & 300 \\
\hline & $1-18-89$ & .24 & $\ldots$ & --- & 30 & -- & 4 & 2,100 & 120 & 840 & 100 \\
\hline & $2-16-89$ & .60 & -- & -- & -- & --- & --- & 2,900 & $<110$ & -.. & 150 \\
\hline & $3-15-89$ & .06 & - & --- & --- & --- & --- & 1,500 & 50 & -- & 110 \\
\hline & $4-10-89$ & .09 & - & - & 30 & -- & 3 & 1,500 & 40 & 510 & 90 \\
\hline & $5-22-89$ & .87 & -- & --- & $<50$ & -- & 4 & 3,700 & 200 & 1,100 & 470 \\
\hline & $6-20-89$ & .76 & -- & --- & 40 & --- & 5 & 4,000 & 240 & 1,900 & 230 \\
\hline & $7-19-89$ & .24 & -- & -- & 40 & -- & 4 & 2,300 & 130 & -- & 1,300 \\
\hline & $8-27-89^{1}$ & .60 & 1.2 & .13 & 80 & -- & 7 & 4,000 & 180 & $\cdots$ & 270 \\
\hline & $8-27-89$ & -- & -- & -- & -- & -- & -- & - & -.- & -- & --- \\
\hline
\end{tabular}

See footnote at end of table. 
Table 4. Data from periodic sampling of drainwater at 15 Imperial Valley sites at fields, May 1988-August 1989-Continued

\begin{tabular}{|c|c|c|c|c|c|c|c|c|c|c|}
\hline \multirow{2}{*}{$\begin{array}{l}\text { Local } \\
\text { identi- } \\
\text { fier }\end{array}$} & \multirow[t]{2}{*}{ Date } & \multirow{2}{*}{$\begin{array}{l}\text { Molyb- } \\
\text { denum } \\
(\mu \mathrm{g} / \mathrm{L} \\
\text { as Mo) }\end{array}$} & \multirow{2}{*}{$\begin{array}{l}\text { Sele- } \\
\text { nium } \\
(\mu \mathrm{g} / \mathrm{L} \\
\text { as Se) }\end{array}$} & \multirow{2}{*}{$\begin{array}{l}\text { Stron- } \\
\text { tium } \\
(\mu \mathrm{g} / \mathrm{L} \\
\text { as } \mathrm{Sr})\end{array}$} & \multirow{2}{*}{$\begin{array}{l}\text { Vana- } \\
\text { dium } \\
(\mu \mathrm{g} / \mathrm{L} \\
\text { as V) }\end{array}$} & \multirow{2}{*}{$\begin{array}{c}\text { Uranium, } \\
\text { natural } \\
(\mu \mathrm{g} / \mathrm{L} \\
\text { as } U)\end{array}$} & \multirow{2}{*}{$\begin{array}{c}\text { Carbon, } \\
\text { organic } \\
(\mathrm{mg} / \mathrm{L} \\
\text { as C) }\end{array}$} & \multirow{2}{*}{$\begin{array}{l}\text { Tritium, } \\
\text { total } \\
\text { (pCi/L) }\end{array}$} & \multicolumn{2}{|c|}{$\begin{array}{c}\text { Stable-isotope } \\
\text { ratio (permil) }\end{array}$} \\
\hline & & & & & & & & & ${ }^{2} \mathrm{H} /{ }^{1} \mathrm{H}$ & ${ }^{18} \mathrm{O} /{ }^{16} \mathrm{O}$ \\
\hline \multirow[t]{14}{*}{ S-226 } & $5-16-88$ & 33 & 250 & $\cdots$ & 170 & - & --- & 96 & -81.5 & -9.00 \\
\hline & $8-23-88$ & -- & 280 & -- & -- & - & --- & 72 & --- & --- \\
\hline & $10-20-88$ & 78 & 340 & -- & - & -- & -- & -- & -83.0 & -9.25 \\
\hline & $11-14-88$ & -- & 360 & --- & -- & -- & --- & --- & -86.0 & -9.45 \\
\hline & $12-16-88$ & -- & 300 & -- & - & -- & --- & -- & -86.0 & -9.85 \\
\hline & $1-19-89$ & 32 & 340 & 33,000 & -- & -- & 10 & -- & -84.0 & -9.40 \\
\hline & $2-16-89$ & $\ldots$ & 280 & --. & $\cdots$ & $\cdots$ & $\cdots$ & -- & -81.0 & -10.35 \\
\hline & $3-15-89$ & -- & 280 & -.- & -.- & --- & -- & --- & -85.5 & -9.90 \\
\hline & $4-10-89$ & 24 & 240 & 26,000 & $\cdots$ & $\cdots$ & $\cdots$ & -- & -87.5 & -9.90 \\
\hline & $5-22-89$ & 15 & 300 & 35,000 & $\cdots$ & --- & --- & --- & -84.5 & -9.50 \\
\hline & $6-20-89$ & 17 & 310 & 34,000 & $\cdots$ & --- & -- & $\cdots$ & -88.0 & -9.60 \\
\hline & 7-19-89 & 20 & 71 & 14,000 & -- & --- & --- & --- & -86.0 & -9.75 \\
\hline & $8-27-89^{1}$ & 30 & 190 & 18,000 & -- & 190 & 15 & --- & -86.0 & -9.85 \\
\hline & $8-27-89$ & -- & 200 & --- & --- & --- & -- & --- & --- & --- \\
\hline \multirow[t]{14}{*}{ S-269 } & $5-16-88$ & 37 & 230 & -- & 99 & --- & $\cdots$ & 117 & -89.0 & -10.20 \\
\hline & $8-23-88$ & -- & 290 & $\cdots$ & -- & --- & -- & 115 & --- & $\cdots$ \\
\hline & $10-20-88$ & 55 & 310 & -- & -.- & --- & --- & --- & -85.5 & -9.70 \\
\hline & $11-14-88$ & - & 270 & --- & -- & --- & -- & --- & -90.5 & -9.95 \\
\hline & $12-16-88$ & - & 260 & --- & --- & --- & -- & --- & -83.4 & -9.55 \\
\hline & $1-19-89$ & 47 & 290 & 25,000 & -- & -- & 9.9 & --- & -91.0 & -9.80 \\
\hline & $2-16-89$ & - & 260 & --- & $\cdots$ & --- & --- & --- & -87.0 & -9.85 \\
\hline & $3-15-89$ & -- & 270 & -- & -- & --- & $\cdots$ & --- & -86.5 & -9.75 \\
\hline & $4-10-89$ & 29 & 360 & 21,000 & -- & -- & -- & -.- & -87.0 & -9.80 \\
\hline & $5-22-89$ & 34 & 180 & 16,000 & -- & --- & -- & --- & -88.5 & -10.55 \\
\hline & $6-20-89$ & 38 & 280 & 22,000 & -- & -- & $\cdots$ & --- & -89.5 & -9.80 \\
\hline & $7-19-89$ & 39 & 230 & 14,000 & $\cdots$ & --- & -- & -- & -89.0 & -9.90 \\
\hline & $8-27-89^{1}$ & 49 & 240 & 17,000 & -- & 140 & 12 & $\ldots$ & -88.0 & -9.85 \\
\hline & $8-27-89$ & - & 47 & -- & -- & -.. & -- & --- & --- & --- \\
\hline \multirow[t]{14}{*}{$S-417$} & $5-16-88$ & -- & 300 & $\cdots$ & --- & --- & -- & --- & -- & -- \\
\hline & $8-18-88$ & -- & 340 & --- & -- & -- & - & 93 & --- & --- \\
\hline & $10-20-88$ & 41 & 230 & --- & -- & -- & -- & --- & -86.5 & -9.90 \\
\hline & $11-14-88$ & - & 190 & -- & $\cdots$ & --- & -- & --- & -88.0 & -10.00 \\
\hline & $12-16-88$ & 26 & 180 & -- & -- & --- & - & --- & -86.0 & -9.95 \\
\hline & $1-18-89$ & - & 100 & 17,000 & -- & $-\cdots$ & $\cdots$ & --- & -94.5 & -10.60 \\
\hline & $2-16-89$ & -- & 230 & --- & --- & -- & $\cdots$ & --- & -88.0 & -10.10 \\
\hline & $3-15-89$ & - & 46 & -- & -- & --- & -- & -.. & -97.0 & -11.60 \\
\hline & $4-10-89$ & 20 & 35 & 8,200 & --- & --- & -- & --. & -97.5 & -11.75 \\
\hline & $5-22-89$ & 28 & 210 & 32,000 & --- & --- & -- & --- & -84.5 & -9.70 \\
\hline & $6-20-89$ & 29 & 280 & 32,000 & -- & -- & -- & --- & -86.0 & -9.60 \\
\hline & $7-19-89$ & 55 & 19 & 5,000 & -- & - & - & --. & -92.0 & -11.20 \\
\hline & $8-27-89^{1}$ & 41 & 200 & 26,000 & --- & 680 & 11 & -.- & -89.0 & -9.90 \\
\hline & $8-27-89$ & $\cdots$ & 210 & --. & -- & - & -- & - & --- & --- \\
\hline
\end{tabular}

See footnote at end of table. 
Table 4. Data from periodic sampling of drainwater at 15 Imperial Valley sites at fields, May 1988-August 1989-Continued

\begin{tabular}{|c|c|c|c|c|c|c|c|c|c|c|c|}
\hline $\begin{array}{c}\text { Site } \\
\text { number }\end{array}$ & $\begin{array}{c}\text { Local } \\
\text { identi- } \\
\text { fier }\end{array}$ & Date & Time & $\begin{array}{c}\text { Dis- } \\
\text { charge, } \\
\text { instan- } \\
\text { taneous } \\
\left(\mathrm{ft}^{3} / \mathrm{s}\right)\end{array}$ & $\begin{array}{l}\text { Spe- } \\
\text { cific } \\
\text { con- } \\
\text { duct- } \\
\text { ance } \\
(\mu \mathrm{S} / \mathrm{cm})\end{array}$ & $\begin{array}{l}\mathrm{pH} \\
\text { (stand- } \\
\text { ard } \\
\text { units) }\end{array}$ & $\begin{array}{l}\text { Temper- } \\
\text { ature, } \\
\text { water } \\
\left({ }^{\circ} \mathrm{C}\right)\end{array}$ & $\begin{array}{l}\text { Calcium } \\
(\mathrm{mg} / \mathrm{L} \\
\text { as Ca) }\end{array}$ & $\begin{array}{c}\text { Magne- } \\
\text { sium } \\
(\mathrm{mg} / \mathrm{L} \\
\text { as } \mathrm{Mg})\end{array}$ & $\begin{array}{l}\text { Sodium } \\
(\mathrm{mg} / \mathrm{L} \\
\text { as } \mathrm{Na})\end{array}$ & $\begin{array}{c}\text { Potas- } \\
\text { sium } \\
(\mathrm{mg} / \mathrm{L} \\
\text { as K) }\end{array}$ \\
\hline \multirow[t]{14}{*}{30} & S-94 & $5-17-88$ & 0955 & 0.0527 & 19,800 & 7.1 & 19.5 & 420 & 530 & 2,900 & 20 \\
\hline & & $8-26-88$ & 0640 & $\ldots$ & 6,620 & 7.2 & -- & --- & --. & -. & --- \\
\hline & & $10-25-88$ & 1000 & .0690 & 10,400 & 7.4 & 25.0 & 540 & 310 & 1,600 & 16 \\
\hline & & $11-13-88$ & 1325 & .0147 & 17,200 & 7.6 & 24.0 & 770 & --. & -- & -.- \\
\hline & & $12-16-88$ & 1155 & .0131 & 13,700 & 7.5 & 22.0 & 630 & -- & -- & -- \\
\hline & & $1-19-89$ & 1515 & .0631 & 6,760 & 7.4 & 18.0 & 310 & 160 & 1,200 & 13 \\
\hline & & $2-16-89$ & 1230 & 1.0385 & 6,870 & 7.6 & 18.0 & 340 & -.. & --- & --- \\
\hline & & $3-15-89$ & 1100 & .0588 & 10,400 & 7.3 & 19.0 & 420 & --- & -.. & --- \\
\hline & & $4-10-89$ & 1400 & .0260 & 14,300 & 7.3 & 19.5 & 730 & 490 & 1,900 & 16 \\
\hline & & $5-22-89$ & 1320 & .0432 & 17,500 & 7.3 & 21.5 & 870 & 560 & 2,500 & 19 \\
\hline & & $6-20-89$ & 0930 & .0169 & 24,300 & 7.2 & 23.0 & 1,200 & 790 & 3,400 & 35 \\
\hline & & $7-19-89$ & 2000 & .0095 & 25,100 & 7.1 & 26.0 & 1,200 & 740 & 3,500 & 37 \\
\hline & & $8-27-89^{1}$ & 1300 & .1110 & 11,900 & 7.1 & 26.0 & 560 & 320 & 1,700 & 24 \\
\hline & & $8-27-89$ & 1301 & --- & 11,800 & 7.2 & -- & -- & -- & -- & -- \\
\hline \multirow[t]{14}{*}{33} & S-142 & $5-18-88$ & 0800 & .0042 & 2,620 & 7.1 & 19.5 & --- & --- & ... & -.- \\
\hline & & $8-18-88$ & 1320 & --- & 2,620 & 7.2 & --- & $\ldots$ & --- & -- & $\ldots$ \\
\hline & & $10-25-88$ & 0905 & .0000 & 3,070 & 7.5 & 24.5 & 140 & 78 & 450 & 31 \\
\hline & & $11-13-88$ & 1250 & .0000 & 2,820 & 7.4 & 21.5 & 130 & -- & -.. & --- \\
\hline & & $12-16-88$ & 1510 & .0000 & 3,410 & 7.7 & 15.0 & 160 & -- & $\cdots$ & -- \\
\hline & & $1-20-89$ & 1010 & .0000 & 3,440 & 7.7 & 11.0 & 150 & 77 & 500 & 10 \\
\hline & & $2-16-89$ & 0830 & .0000 & 3,360 & 7.9 & 11.5 & 170 & --- & -- & --- \\
\hline & & $3-15-89$ & 1540 & .0000 & 3,110 & 7.3 & 16.5 & 150 & --- & --- & --- \\
\hline & & $4-10-89$ & 1855 & .0000 & 2,630 & 7.4 & 19.0 & 160 & 76 & 360 & 8.1 \\
\hline & & $5-22-89$ & 1820 & .0000 & 3,130 & 7.9 & 22.0 & 160 & 77 & 420 & 9.3 \\
\hline & & $6-20-89$ & 1330 & .0000 & 2,550 & 7.8 & 24.0 & 150 & 71 & 340 & 9.5 \\
\hline & & $7-19-89$ & 1625 & .0000 & 2,200 & 6.9 & 26.5 & 160 & 55 & 260 & 9.0 \\
\hline & & $8-27-89^{1}$ & 1740 & .0000 & 2,910 & 7.1 & 24.0 & 160 & 69 & 380 & 10 \\
\hline & & $8-27-89$ & 1741 & --- & 2,910 & 7.0 & -- & --- & -- & -- & -- \\
\hline \multirow[t]{14}{*}{41} & S-241 & $5-17-88$ & 1900 & .0140 & 10,800 & 7.3 & 19.5 & 180 & 330 & 1,800 & 24 \\
\hline & & $8-26-88$ & 1100 & --- & 9,300 & 7.1 & -- & --- & --- & --- & $\ldots$ \\
\hline & & $10-25-88$ & 0930 & .0410 & 15,700 & 7.3 & 24.0 & 580 & 510 & 2,600 & 28 \\
\hline & & $11-13-88$ & 1240 & .0511 & 15,700 & 7.3 & 23.5 & 550 & --- & --- & -. \\
\hline & & $12-16-88$ & 1525 & .0152 & 17,100 & 7.3 & 21.0 & 640 & -- & -- & -- \\
\hline & & $1-20-89$ & 1030 & .0103 & 17,000 & 7.3 & 18.5 & 600 & 570 & 2,700 & 44 \\
\hline & & $2-16-89$ & 0850 & .0597 & 16,700 & 7.4 & 18.0 & 670 & --- & --- & -- \\
\hline & & $3-15-89$ & 1550 & .1330 & 9,560 & 7.2 & 19.0 & 390 & --- & -.. & -- \\
\hline & & $4-10-89$ & 1910 & .0316 & 16,400 & 7.2 & 19.0 & 620 & 580 & 2,600 & 40 \\
\hline & & $5-22-89$ & 1830 & .0390 & 15,600 & 7.3 & 21.0 & 600 & 520 & 2,400 & 37 \\
\hline & & $6-20-89$ & 1345 & .1370 & 9,530 & 7.4 & 23.0 & 440 & 330 & 1,500 & 24 \\
\hline & & $7-19-89$ & 1655 & .0542 & 14,800 & 7.1 & 23.5 & 590 & 470 & 2,300 & 46 \\
\hline & & $8-27-89^{1}$ & 1815 & .0389 & 16,000 & 7.0 & 25.0 & 610 & 520 & 2,500 & 56 \\
\hline & & $8-27-89$ & 1816 & --- & 16,100 & 7.0 & --- & --- & --- & --- & --- \\
\hline
\end{tabular}

See footnote at end of table. 
Table 4. Data from periodic sampling of drainwater at 15 Imperial Valley sites at fields, May 1988-August 1989-Continued

\begin{tabular}{|c|c|c|c|c|c|c|c|c|c|c|}
\hline $\begin{array}{c}\text { Local } \\
\text { identi- } \\
\text { fier }\end{array}$ & Date & $\begin{array}{c}\text { Alka- } \\
\text { linity, } \\
\text { lab } \\
(\mathrm{mg} / \mathrm{L} \\
\text { as } \\
\left.\mathrm{CaCO}_{3}\right)\end{array}$ & $\begin{array}{c}\text { Sulfate } \\
(\mathrm{mg} / \mathrm{L} \\
\left.\text { as } \mathrm{SO}_{4}\right)\end{array}$ & $\begin{array}{l}\text { Chlo- } \\
\text { ride } \\
\text { (mg/L } \\
\text { as } \mathrm{Cl} \text { ) }\end{array}$ & $\begin{array}{l}\text { Fluo- } \\
\text { ride } \\
\text { (mg/L } \\
\text { as } \mathrm{F} \text { ) }\end{array}$ & $\begin{array}{c}\text { Bromide } \\
\text { (mg/L } \\
\text { as } \mathrm{Br})\end{array}$ & $\begin{array}{c}\text { Silica } \\
(\mathrm{mg} / \mathrm{L} \\
\text { as } \\
\left.\mathrm{SiO}_{2}\right)\end{array}$ & $\begin{array}{l}\text { Solids, } \\
\text { residue } \\
\text { at } 180 \\
{ }^{\circ} \mathrm{C} \\
(\mathrm{mg} / \mathrm{L})\end{array}$ & $\begin{array}{c}\text { Nitro- } \\
\text { gen, } \\
\mathrm{NO}_{2}+ \\
\mathrm{NO}_{3} \\
(\mathrm{mg} / \mathrm{L} \\
\text { as } \mathrm{N})\end{array}$ & $\begin{array}{c}\text { Nitro- } \\
\text { gen, } \\
\mathrm{NO}_{2}+ \\
\mathrm{NO}_{3} \\
(\mathrm{mg} / \mathrm{L} \\
\text { as } \mathrm{N})\end{array}$ \\
\hline \multirow[t]{14}{*}{ S-94 } & $5-17-88$ & 451 & 2,800 & 5,500 & 0.4 & -- & 15 & -- & 4.4 & - \\
\hline & $8-26-88$ & 435 & 2,000 & 930 & -- & -- & -- & 4,980 & -- & -- \\
\hline & $10-25-88$ & 433 & 2,400 & 2,300 & .8 & -- & 14 & 7,570 & 9.9 & 9.5 \\
\hline & $11-13-88$ & 413 & 2,800 & 4,700 & -- & 4.4 & --- & 12,200 & 7.2 & -.. \\
\hline & $12-16-88$ & 454 & 2,900 & 3,500 & -- & 2.9 & -- & 10,000 & 6.5 & -- \\
\hline & $1-19-89$ & 478 & 2,200 & 870 & -- & .57 & 16 & 5,170 & 13 & 13 \\
\hline & $2-16-89$ & 463 & 2,100 & 1,000 & -- & .72 & -- & 5,230 & 7.7 & --. \\
\hline & $3-15-89$ & 437 & 2,600 & 2,100 & -- & 1.6 & --. & 7,750 & 12 & -- \\
\hline & $4-10-89$ & 466 & 2,800 & 3,600 & -- & 3.0 & 14 & 10,300 & 5.8 & 5.8 \\
\hline & $5-22-89$ & 470 & 3,000 & 4,700 & .5 & 4.4 & 15 & 12,500 & 3.0 & 3.0 \\
\hline & $6-20-89$ & 390 & 2,800 & 7,600 & .3 & 7.4 & 15 & 18,200 & 5.0 & 5.0 \\
\hline & $7-19-89$ & 356 & 3,100 & 8,000 & -- & 7.1 & 17 & 16,900 & --- & 4.5 \\
\hline & $8-27-89^{1}$ & 354 & 2,700 & 2,700 & --- & 2.3 & 14 & 8,350 &.- & 3.2 \\
\hline & $8-27-89$ & 342 & 2,700 & 2,600 & -- & 2.3 & --- & --. & --- & --- \\
\hline \multirow[t]{14}{*}{ S-142 } & $5-18-88$ & 364 & --- & 220 & --- & -- & -- & 1,590 & --- & -- \\
\hline & 8-18-88 & 386 & 690 & 230 & -- & --- & -- & 1,780 & --- & -- \\
\hline & $10-25-88$ & 402 & 810 & 340 & .7 & -- & 16 & 2,100 & 3.9 & 3.9 \\
\hline & $11-13-88$ & 380 & 730 & 290 & --- & .24 & $\ldots$ & 1,880 & 3.7 & --- \\
\hline & $12-16-88$ & 401 & 940 & 400 & -- & .31 & --- & 2,410 & 4.3 & -- \\
\hline & $1-20-89$ & 391 & 940 & 400 & --- & .30 & 15 & 2,400 & 6.2 & 6.2 \\
\hline & $2-16-89$ & 389 & 920 & 380 & -- & .25 & - & 2,350 & 7 & $\cdots$ \\
\hline & $3-15-89$ & 396 & 860 & 340 & -- & .20 & -- & 2,180 & 4.9 & -- \\
\hline & $4-10-89$ & 406 & 660 & 260 & $\ldots$ & .21 & 3.9 & 1,810 & 3.7 & 3.7 \\
\hline & $5-22-89$ & 395 & 810 & 360 & .6 & .28 & 11 & 2.170 & 4.0 & 4.0 \\
\hline & $6-20-89$ & 374 & 670 & 270 & .7 & .20 & 3.9 & 1,760 & 3.5 & 3.5 \\
\hline & $7-19-89$ & 355 & 580 & 190 & --- & .16 & 11 & 1,470 & -- & 2.6 \\
\hline & $8-27-89^{1}$ & 413 & 780 & 290 & --- & .25 & 15 & 1,880 & --- & .84 \\
\hline & $8-27-89$ & 315 & 790 & 260 & --- & .23 & --- & --- & -- & - - \\
\hline \multirow[t]{14}{*}{ S-241 } & $5-17-88$ & 497 & 3,300 & 1,200 & .6 & $\cdots$ & 14 & --. & 4.9 & $\cdots$ \\
\hline & $8-26-88$ & 599 & 2,800 & 1,500 & --- & -- & --- & 7,260 & $\ldots$ & -- \\
\hline & $10-25-88$ & 627 & 4,200 & 2,600 & .5 & -- & 15 & 12,400 & 12 & 11 \\
\hline & $11-13-88$ & 621 & 4,500 & 3,200 & --- & 2.2 & -- & 12,400 & 12 & - \\
\hline & $12-16-88$ & 608 & 4,400 & 3,900 & -- & 2.5 & -- & 13,300 & 18 & -- \\
\hline & $1-20-89$ & 603 & 4,000 & 4,000 & -- & 2.6 & 15 & 13,200 & 21 & -.. \\
\hline & $2-16-89$ & 593 & 4,100 & 4,100 & -- & 2.6 & -- & 12,800 & 19 & --- \\
\hline & $3-15-89$ & 557 & 3,000 & 1,600 & --- & .90 & -- & 7,520 & 10 & --- \\
\hline & $4-10-89$ & 596 & 4,000 & 3,600 & -- & 2.3 & 14 & 12,700 & 18 & 18 \\
\hline & $5-22-89$ & 582 & 4,200 & 3,400 & .5 & 2.2 & 14 & 12,100 & 18 & 18 \\
\hline & $6-20-89$ & 543 & 2,800 & 1,600 & .7 & 1.0 & 14 & 7,510 & 11 & 11 \\
\hline & $7-19-89$ & 580 & 4,200 & 3,100 & $\ldots$ & 2.1 & 15 & 11,400 & --- & 13 \\
\hline & $8-27-89^{1}$ & 559 & 4,500 & 3,500 & -- & 2.1 & 15 & 12,300 & --- & 15 \\
\hline & $8-27-89$ & 425 & 4,500 & 3,500 & -- & 2.4 & --- & --- & --- & --- \\
\hline
\end{tabular}

See footnote at end of table. 
Table 4. Data from periodic sampling of drainwater at 15 Imperial Valley sites at fields, May 1988-August 1989-Continued

\begin{tabular}{|c|c|c|c|c|c|c|c|c|c|c|c|}
\hline $\begin{array}{l}\text { Local } \\
\text { identi- } \\
\text { fier }\end{array}$ & Date & $\begin{array}{l}\text { Nitro- } \\
\text { gen, } \\
\text { ammonia } \\
(\mathrm{mg} / \mathrm{L} \\
\text { as } \mathrm{N})\end{array}$ & $\begin{array}{c}\text { Nitro- } \\
\text { gen, am- } \\
\text { monia + } \\
\text { organic, } \\
\text { dis. } \\
\text { (mg/L } \\
\text { as } \mathrm{N})\end{array}$ & $\begin{array}{l}\text { Phos- } \\
\text { phorus, } \\
\text { hydro. } \\
\text { + ortho } \\
\text { (mg/L } \\
\text { as P) }\end{array}$ & $\begin{array}{l}\text { Alum- } \\
\text { inum } \\
(\mu \mathrm{g} / \mathrm{L} \\
\text { as } \mathrm{Al})\end{array}$ & $\begin{array}{l}\text { Anti- } \\
\text { mony } \\
(\mu \mathrm{g} / \mathrm{L} \\
\text { as Sb) }\end{array}$ & $\begin{array}{c}\text { Arsenic } \\
(\mu \mathrm{g} / \mathrm{L} \\
\text { as As) }\end{array}$ & $\begin{array}{l}\text { Boron } \\
(\mu \mathrm{g} / \mathrm{L} \\
\text { as B) }\end{array}$ & $\begin{array}{c}\text { Iron } \\
(\mu \mathrm{g} / \mathrm{L}) \\
\text { as } \mathrm{Fe})\end{array}$ & $\begin{array}{l}\text { Lithium } \\
(\mu \mathrm{g} / \mathrm{L} \\
\text { as } \mathrm{Li})\end{array}$ & $\begin{array}{c}\text { Manga- } \\
\text { nese } \\
(\mu \mathrm{g} / \mathrm{L} \\
\text { as } \mathrm{Mn})\end{array}$ \\
\hline \multirow[t]{14}{*}{ S-94 } & $5-17-88$ & 0.36 & -- & --- & --- & -- & 1 & 1,800 & 90 & -- & 170 \\
\hline & $8-26-88$ & - & -- & -- & --- & -- & 2 & 1,300 & -- &.-- & -.. \\
\hline & $10-25-88$ & .08 & -- & -- & 40 & $<1$ & 2 & 1,500 & 50 & -- & 100 \\
\hline & $11-13-88$ & .33 & - & --- & -- & -- & -- & 2,000 & 70 & -- & 210 \\
\hline & $12-16-88$ & .20 & - & -- & -- & --- & -- & 1,800 & 60 & -- & 180 \\
\hline & $1-19-89$ & .08 & -- & - & 10 & -- & 1 & 1,300 & 30 & 400 & 40 \\
\hline & $2-16-89$ & .07 & - & -- & -- & --- & --- & 1,200 & 20 & --- & $<10$ \\
\hline & $3-15-89$ & .07 & - & -- & -.- & --- & -- & 1,600 & 50 & --- & 40 \\
\hline & $4-10-89$ & .19 & -- & --- & $<20$ &.-- & 1 & 1,600 & 40 & 570 & 100 \\
\hline & $5-22-89$ & .25 & -- & -- & $<20$ & $\ldots$ & 1 & 1,800 & 80 & 600 & 150 \\
\hline & $6-20-89$ & .38 & --- & -- & 30 & --- & 2 & 2,200 & 110 & 660 & 70 \\
\hline & $7-19-89$ & .40 & -- & -- & 80 & --- & 2 & 2,500 & 90 & -.- & 210 \\
\hline & $8-27-89^{1}$ & .14 & 1.1 & 0.04 & 60 & --- & 2 & 1,700 & 40 & -- & 50 \\
\hline & $8-27-89$ & -- & -- & -- & -- & -- & --- & -- & --- & --- & --- \\
\hline \multirow[t]{14}{*}{ S-142 } & $5-18-88$ & -- & $\cdots$ & -- & -- & $\ldots$ & --- & --- & $\ldots$ & --- & -- \\
\hline & 8-18-88 & -- & -- & -- & - & --- & 1 & 570 & -.- & -.. & --- \\
\hline & $10-25-88$ & .01 & -- & -- & 90 & $<1$ & 2 & 800 & 150 & --- & 20 \\
\hline & $11-13-88$ & .03 & -- & -- & -- & --- & --- & 710 & 80 & -- & 20 \\
\hline & $12-16-88$ & .05 & -- & -- & -- & --- & $\ldots$ & 800 & 100 & -- & 30 \\
\hline & $1-20-89$ & .11 & -- & -- & 20 & -- & 2 & 760 & 80 & 130 & 20 \\
\hline & $2-16-89$ & .03 & -- & -- & -- & --- & -- & 700 & $<10$ & --- & $<10$ \\
\hline & $3-15-89$ & .06 & -- & - & --- & --- & --- & 650 & 90 & -- & 30 \\
\hline & $4-10-89$ & .06 & -- & -- & 20 & --- & 1 & 550 & 40 & 170 & 50 \\
\hline & $5-22-89$ & .02 & -- & -- & 10 & - & 1 & 640 & 30 & 200 & 10 \\
\hline & $6-20-89$ & .08 & -- & -- & 10 & -- & $<1$ & 530 & 20 & 170 & 10 \\
\hline & $7-19-89$ & .04 & --- & -- & 20 & $-\cdots$ & 1 & 480 & 20 & -- & 20 \\
\hline & $8-27-89^{1}$ & .04 & .50 & .02 & 20 & - & 1 & 740 & 20 & $\cdots$ & 20 \\
\hline & $8-27-89$ & -- & -- & -- & -- & - & -- & -- & $\cdots$ & -- & -- \\
\hline \multirow[t]{14}{*}{$S-241$} & $5-17-88$ & .13 & - & -- & -- & -- & 3 & 2,000 & 120 & --- & 40 \\
\hline & $8-26-88$ & - & -- & -- & -- & -- & 2 & 1,800 & -.. & -- & -- \\
\hline & $10-25-88$ & .15 & -- & -- & 10 & $<1$ & 4 & 2,900 & 50 & --- & 90 \\
\hline & $11-13-88$ & .22 & -- & -- & -- & -- & --- & 3,000 & 60 & --- & 100 \\
\hline & $12-16-88$ & .23 & - & -- & -- & -- & $\cdots$ & 2,800 & 60 & -- & 140 \\
\hline & $1-20-89$ & .19 & -- & -- & 20 & -- & 3 & 2,600 & 80 & 910 & 140 \\
\hline & $2-16-89$ & .20 & -- & -- & - & -- & --- & 2,300 & 60 & --- & 80 \\
\hline & $3-15-89$ & .08 & -- & -- & -- & -- & -- & 1,600 & 40 & --- & 150 \\
\hline & $4-10-89$ & .17 & -- & -- & 30 & --- & 3 & 2,600 & 50 & 860 & 250 \\
\hline & $5-22-89$ & .17 & - & $\cdots$ & $<20$ & -- & 3 & 2,500 & 70 & 800 & 310 \\
\hline & $6-20-89$ & .11 & -- & --- & 30 & -- & 2 & 1,700 & 50 & 550 & 140 \\
\hline & $7-19-89$ & .14 & -- & -- & 50 & -- & 3 & 2,700 & 60 & -- & 340 \\
\hline & $8-27-89^{1}$ & .15 & 1.7 & .07 & 20 & -- & 5 & 2,900 & 60 & --- & 250 \\
\hline & $8-27-89$ & -- & - & -- & -- & -- & $\ldots$ & --- & -- & --- & --- \\
\hline
\end{tabular}

See footnote at end of table. 
Table 4. Data from periodic sampling of drainwater at 15 Imperial Valley sites at fields, May 1988-August 1989-Continued

\begin{tabular}{|c|c|c|c|c|c|c|c|c|c|c|}
\hline \multirow{2}{*}{$\begin{array}{l}\text { Local } \\
\text { identi- } \\
\text { fier }\end{array}$} & \multirow[t]{2}{*}{ Date } & \multirow{2}{*}{$\begin{array}{l}\text { Molyb- } \\
\text { denum } \\
(\mu \mathrm{g} / \mathrm{L} \\
\text { as Mo) }\end{array}$} & \multirow{2}{*}{$\begin{array}{l}\text { Sele- } \\
\text { nium } \\
(\mu \mathrm{g} / \mathrm{L} \\
\text { as Se })\end{array}$} & \multirow{2}{*}{$\begin{array}{l}\text { Stron- } \\
\text { tium } \\
(\mu \mathrm{g} / \mathrm{L} \\
\text { as } \mathrm{Sr})\end{array}$} & \multirow{2}{*}{$\begin{array}{l}\text { Vana- } \\
\text { dium } \\
(\mu g / L \\
\text { as V) }\end{array}$} & \multirow{2}{*}{$\begin{array}{c}\text { Uranium, } \\
\text { natural } \\
(\mu \mathrm{g} / \mathrm{L} \\
\text { as } \mathrm{U})\end{array}$} & \multirow{2}{*}{$\begin{array}{c}\text { Carbon, } \\
\text { organic } \\
\text { (mg/L } \\
\text { as C) }\end{array}$} & \multirow{2}{*}{$\begin{array}{l}\text { Tritium, } \\
\text { total } \\
(\mathrm{pCi} / \mathrm{L})\end{array}$} & \multicolumn{2}{|c|}{$\begin{array}{c}\text { Stable-isotope } \\
\text { ratio (permil) } \\
\end{array}$} \\
\hline & & & & & & & & & ${ }^{2} \mathrm{H} /{ }^{1} \mathrm{H}$ & ${ }^{18} \mathrm{O} /{ }^{16} \mathrm{O}$ \\
\hline \multirow[t]{14}{*}{ S-94 } & $5-17-88$ & 16 & 51 & - & 68 & -- & --- & -- & -91.5 & -10.85 \\
\hline & $8-26-88$ & -- & 20 & -- & --- & -- & --- & 110 & --- & --- \\
\hline & $10-25-88$ & 20 & 23 & -- & -- & -- & -- & -- & -96.0 & -11.65 \\
\hline & $11-13-88$ & -- & 37 & -- & --- & $\ldots$ & --- & $\cdots$ & -93.5 & -11.05 \\
\hline & $12-16-88$ & -- & 31 & -- & --- & -.. & --- & --- & -96.0 & -11.30 \\
\hline & $1-19-89$ & 19 & 21 & 5,000 & -- & -- & --- & --- & -99.5 & -11.95 \\
\hline & $2-16-89$ & -- & 17 & --- & -- & --- & --- & --- & -97.0 & -11.95 \\
\hline & $3-15-89$ & -- & 26 & - & --- & -- & --- & -- & -96.0 & -11.55 \\
\hline & $4-10-89$ & 15 & 32 & 13,000 & $\ldots$ & -.. & --- & --- & -95.5 & -11.35 \\
\hline & $5-22-89$ & 14 & 42 & 16,000 & $\ldots$ & --. & --- & --- & -92.0 & -11.10 \\
\hline & $6-20-89$ & 13 & 64 & 22,000 & --- & -- & --- & --- & -95.0 & -10.40 \\
\hline & $7-19-89$ & 14 & 67 & 14,000 & -- & -. & --- & -.- & -90.0 & -10.30 \\
\hline & $8-27-89^{1}$ & 28 & 33 & 7,400 & --- & 190 & 9.8 & --- & -93.5 & -11.55 \\
\hline & $8-27-89$ & -. & 28 & --- & --- & -.. & --- & $-\cdots$ & -- & -.. \\
\hline \multirow[t]{14}{*}{ S-142 } & $5-18-88$ & -- & 16 & -- & --- & $\ldots$ & --- & --- & --- & -- \\
\hline & 8-18-88 & -- & 12 & -- & --- & --- & -- & 99 & --- & --- \\
\hline & $10-25-88$ & 12 & 12 & -- & --- & -- & --- & -- & -97.0 & -11.70 \\
\hline & $11-13-88$ & - & 12 & - & --- & -- & --- & --- & -99.0 & -11.90 \\
\hline & $12-16-88$ & -- & 14 & - & -- & - & --- & -- & -95.5 & -11.20 \\
\hline & $1-20-89$ & 33 & 13 & 2,600 & -.. & -- & $\cdots$ & -- & -87.0 & -9.50 \\
\hline & $2-16-89$ & -- & 13 & -.- & -- & $\ldots$ & --- & -- & -83.0 & -8.85 \\
\hline & $3-15-89$ & -- & 14 & --- & -- & --- & --- & -- & -82.0 & -8.90 \\
\hline & $4-10-89$ & 9 & 17 & 2,400 & -- & -- & --- & -- & -97.0 & -11.75 \\
\hline & $5-22-89$ & 12 & 17 & 2,900 & -- & -- & -- & --- & -97.0 & -11.75 \\
\hline & $6-20-89$ & 8 & 13 & 2,500 & -- & -- & --- & --- & -97.0 & -11.45 \\
\hline & $7-19-89$ & 10 & 11 & 24,000 & -- & -- & -- & -- & -97.5 & -11.65 \\
\hline & $8-27-89^{1}$ & 15 & 12 & 2,300 & -- & 21 & 4.1 & -- & -97.5 & -11.45 \\
\hline & $8-27-89$ & -- & 13 & -- & -- & -- & --- & -- & -- & --- \\
\hline \multirow[t]{14}{*}{ S-241 } & $5-17-88$ & 14 & 30 & -- & 38 & --- & --- & 131 & -97.0 & -11.90 \\
\hline & $8-26-88$ & -- & 13 & -- & -- & -- & --- & 110 & --- & --- \\
\hline & $10-25-88$ & 19 & 42 & -- & -- & -- & $\ldots$ & --- & -96.5 & -11.55 \\
\hline & $11-13-88$ & -- & 43 & -- & -- & -- & $\ldots$ & --- & -97.0 & -11.65 \\
\hline & $12-16-88$ & -- & 54 & -- & $\cdots$ & -- & -- & $\ldots$ & -95.0 & -11.30 \\
\hline & $1-20-89$ & 25 & 62 & 15,000 & --- & $\ldots$ & -- & --- & -94.0 & -11.25 \\
\hline & $2-16-89$ & $\cdots$ & 70 & -.- & --- & --- & -- & --- & -97.0 & -11.40 \\
\hline & $3-15-89$ & $\cdots$ & 30 & --- & -- & --- & --- & --- & -98.5 & -11.90 \\
\hline & $4-10-89$ & 23 & 53 & 14,000 & --- & -.. & --- & --- & -98.0 & -11.45 \\
\hline & $5-22-89$ & 27 & 55 & 13,000 & --- & -- & $\ldots$ & -- & -96.0 & -11.55 \\
\hline & $6-20-89$ & 20 & 26 & 7,800 & $\ldots$ & -- & --- & -- & -100.5 & -12.00 \\
\hline & $7-19-89$ & 27 & 47 & 8,700 & -- & -- & --- & --. & -96.5 & -11.55 \\
\hline & $8-27-89^{1}$ & 34 & 54 & 10,000 & -- & $<170$ & 10 & -- & -97.5 & -11.50 \\
\hline & $8-27-89$ & - & 52 & -- & -- & - & --- & $\ldots$ & --- & -- \\
\hline
\end{tabular}

See footnote at end of table. 
Table 4. Data from periodic sampling of drainwater at 15 Imperial Valley sites at fields, May 1988-August 1989-Continued

\begin{tabular}{|c|c|c|c|c|c|c|c|c|c|c|c|}
\hline $\begin{array}{c}\text { Site } \\
\text { number }\end{array}$ & $\begin{array}{l}\text { Local } \\
\text { identi- } \\
\text { fier }\end{array}$ & Date & Time & $\begin{array}{c}\text { Dis- } \\
\text { charge, } \\
\text { instan- } \\
\text { taneous } \\
\left(\mathrm{ft}^{3} / \mathrm{s}\right)\end{array}$ & $\begin{array}{c}\text { Spe- } \\
\text { cific } \\
\text { con- } \\
\text { duct- } \\
\text { ance } \\
(\mu S / c m)\end{array}$ & $\begin{array}{l}\mathrm{pH} \\
\text { (stand- } \\
\text { ard } \\
\text { units) }\end{array}$ & $\begin{array}{c}\text { Temper- } \\
\text { ature, } \\
\text { water } \\
\left({ }^{\circ} \mathrm{C}\right)\end{array}$ & $\begin{array}{c}\text { Calcium } \\
(\mathrm{mg} / \mathrm{L} \\
\text { as Ca })\end{array}$ & $\begin{array}{c}\text { Magne- } \\
\text { sium } \\
(\mathrm{mg} / \mathrm{L} \\
\text { as } \mathrm{Mg})\end{array}$ & $\begin{array}{l}\text { Sodium } \\
\text { (mg/L } \\
\text { as } \mathrm{Na} \text { ) }\end{array}$ & $\begin{array}{c}\text { Potas- } \\
\text { sium } \\
(\mathrm{mg} / \mathrm{L} \\
\text { as K) }\end{array}$ \\
\hline \multirow[t]{16}{*}{50} & S-154 & $5-18-88$ & 1310 & -- & 15,700 & 7.1 & --- & 430 & 490 & 2,200 & 26 \\
\hline & & $3-23-88$ & 1200 & -- & 17,900 & 7.6 & -- & --- & --- & - & --- \\
\hline & & $10-25-88$ & 0825 & 0.0130 & 15,600 & 7.2 & 24.0 & 800 & 470 & 2,200 & 4.7 \\
\hline & & $11-13-88$ & 1400 & .0137 & 19,100 & 7.3 & 23.5 & --- & -- & --- & --- \\
\hline & & $12-16-88$ & 1640 & -- & 15,000 & 7.2 & 20.0 & 810 & -- & -- & -- \\
\hline & & $1-18-89$ & 1450 & .0032 & 24,700 & 7.2 & 18.0 & 1,300 & 840 & 3,200 & 39 \\
\hline & & $2-15-89$ & 1700 & .0122 & 24,800 & 7.1 & 16.5 & 1,100 & --- & --- & --- \\
\hline & & $3-16-89$ & 0920 & .0021 & 24,400 & 6.8 & 18.0 & 1,400 & --- & -- & -- \\
\hline & & $4-11-89$ & 0945 & .0493 & 13,800 & 7.1 & 19.5 & 750 & 470 & 1,600 & 23 \\
\hline & & $5-23-89$ & 0735 & .0053 & 19,600 & 7.1 & 20.0 & 1,100 & 700 & 2,700 & 41 \\
\hline & & $6-20-89$ & 1530 & .0010 & 19,000 & 7.0 & 23.0 & 1,100 & 680 & 2,500 & 52 \\
\hline & & $7-19-89$ & 0920 & .0073 & 16,600 & 7.1 & 23.5 & 920 & 560 & 2,300 & 42 \\
\hline & & $8-28-89^{1}$ & 0645 & .0090 & 24,400 & 7.1 & 25.0 & 1,400 & 810 & 2,800 & 54 \\
\hline & & $8-28-89$ & 0646 & -- & 24,400 & 6.9 & -- & --- & -.. & -- & -.- \\
\hline & & $8-30-89$ & 1120 & -- & 24,600 & 6.8 & 25.5 & --- & -- & -- & --- \\
\hline & & $8-30-89$ & 1121 & -- & 24,600 & 6.9 & 25.5 & -- & --- & -- & $\cdots$ \\
\hline \multirow[t]{14}{*}{67} & S-265 & $5-19-88$ & 0815 & .1180 & 10,200 & 7.3 & 21.5 & 380 & 270 & 1,600 & 32 \\
\hline & & $8-22-88$ & 1600 & 1.6070 & 12,800 & 7.2 & --- & --- & --- & -.- & --- \\
\hline & & $10-24-88$ & 0850 & .0500 & 12,300 & 7.3 & 25.5 & 680 & 360 & 2,000 & 19 \\
\hline & & $11-13-88$ & 1445 & .2010 & 12,300 & 7.4 & 23.5 & 620 & --- & -- & --- \\
\hline & & $12-17-88$ & 1350 & .1080 & 9,910 & 7.4 & 22.0 & 540 & -- & - & $\cdots$ \\
\hline & & $1-20-89$ & 1500 & .0335 & 12,000 & 7.3 & 20.0 & 610 & 320 & 1,800 & 24 \\
\hline & & $2-15-89$ & 1740 & .1310 & 10,800 & 7.6 & 18.0 & 600 & -- & --- & --- \\
\hline & & $3-15-89$ & 1020 & .4540 & 11,200 & 7.3 & 19.0 & 590 & --- & -- & -- \\
\hline & & $4-10-89$ & 1300 & .3700 & 11,900 & 7.0 & 19.5 & 610 & 350 & 1,600 & 35 \\
\hline & & $5-23-89$ & 1500 & .2740 & 12,200 & 7.2 & 21.0 & 650 & 340 & 1,900 & 19 \\
\hline & & $6-20-89$ & 0835 & .4680 & 11,900 & 7.2 & 23.0 & 670 & 370 & 1,800 & 250 \\
\hline & & $7-19-89$ & 1015 & .2130 & 13,200 & 7.2 & 23.5 & 720 & 360 & 2,000 & 39 \\
\hline & & $8-27-89^{1}$ & 1115 & -- & 11,200 & 7.1 & 25.0 & 580 & 300 & 1,600 & 64 \\
\hline & & $8-27-89$ & 1116 & -- & 11,100 & 7.1 & -- & -- & - & -- & -- \\
\hline \multirow[t]{14}{*}{75} & $S-4$ & $5-19-88$ & 1030 & .0449 & 29,300 & 7.0 & 21.0 & 390 & 910 & 4,900 & 24 \\
\hline & & $3-23-88$ & 1125 & .3570 & 22,400 & 7.0 & -- & --- & --- & --- & --- \\
\hline & & $10-25-88$ & 0800 & .0200 & 30,600 & 7.1 & 24.5 & 790 & 910 & 5,300 & 45 \\
\hline & & $11-13-88$ & 1200 & .0126 & 31,200 & 7.5 & 25.0 & 800 & --- & -- & -- \\
\hline & & $12-16-88$ & 1610 & .0808 & 22,900 & 7.3 & 22.0 & 720 & $\ldots$ & -- & -- \\
\hline & & $1-20-89$ & 1110 & .0254 & 28,700 & 7.2 & 19.0 & 910 & 1,100 & 4,500 & 29 \\
\hline & & $2-15-89$ & 1640 & .3490 & 28,800 & 7.3 & 18.0 & 940 & --- & --- & -- \\
\hline & & $3-15-89$ & 1720 & .0929 & 28,600 & 7.1 & 18.0 & 930 & -. & --- & -- \\
\hline & & $4-11-89$ & 0915 & .0438 & 28,900 & 7.4 & 19.0 & 1,000 & 1,100 & 5,000 & 26 \\
\hline & & $5-23-89$ & 0810 & .0330 & 28,200 & 7.1 & 20.0 & 940 & 978 & 4,300 & 34 \\
\hline & & $6-20-89$ & 1815 & .0560 & 26,500 & 7.2 & 23.0 & 970 & 940 & 4,200 & 25 \\
\hline & & $7-19-89$ & 0815 & .0530 & 28,000 & 7.0 & 25.0 & 880 & 990 & 4,600 & 32 \\
\hline & & $8-27-89^{1}$ & 1900 & .0528 & 26,100 & 6.9 & 25.0 & 780 & 890 & 4,000 & 35 \\
\hline & & $8-27-89$ & 1901 & -- & 26,100 & 6.8 & --- & --- & --- & --- & --- \\
\hline
\end{tabular}

See footnote at end of table. 
Table 4. Data from periodic sampling of drainwater at 15 Imperial Valley sites at fields, May 1988-August 1989-Continued

\begin{tabular}{|c|c|c|c|c|c|c|c|c|c|c|}
\hline $\begin{array}{l}\text { Local } \\
\text { identi- } \\
\text { fier }\end{array}$ & Date & $\begin{array}{c}\text { Alka- } \\
\text { linity, } \\
\text { lab } \\
(\mathrm{mg} / \mathrm{L} \\
\text { as } \\
\left.\mathrm{CaCO}_{3}\right)\end{array}$ & $\begin{array}{c}\text { Sulfate } \\
(\mathrm{mg} / \mathrm{L} \\
\left.\text { as } \mathrm{SO}_{4}\right)\end{array}$ & $\begin{array}{l}\text { Chlo- } \\
\text { ride } \\
(\mathrm{mg} / \mathrm{L} \\
\text { as } \mathrm{Cl})\end{array}$ & $\begin{array}{l}\text { Fluo- } \\
\text { ride } \\
\text { (mg/L } \\
\text { as F) }\end{array}$ & $\begin{array}{c}\text { Bromide } \\
\text { (mg/L } \\
\text { as } \mathrm{Br})\end{array}$ & $\begin{array}{c}\text { Silica } \\
(\mathrm{mg} / \mathrm{L} \\
\text { as } \\
\left.\mathrm{SiO}_{2}\right)\end{array}$ & $\begin{array}{l}\text { Solids, } \\
\text { residue } \\
\text { at } 180 \\
{ }^{\circ} \mathrm{C} \\
(\mathrm{mg} / \mathrm{L})\end{array}$ & $\begin{array}{c}\text { Nitro- } \\
\text { gen, } \\
\mathrm{NO}_{2}+ \\
\mathrm{NO}_{3} \\
(\mathrm{mg} / \mathrm{L} \\
\text { as } \mathrm{N})\end{array}$ & $\begin{array}{c}\text { Nitro- } \\
\text { gen, } \\
\mathrm{NO}_{2}+ \\
\mathrm{NO}_{3} \\
\text { (mg/L } \\
\text { as } \mathrm{N} \text { ) }\end{array}$ \\
\hline \multirow[t]{16}{*}{ S-154 } & $5-18-88$ & 277 & 2,200 & 4,600 & 0.4 & -- & 11 & -- & 7.1 & $\cdots$ \\
\hline & $3-23-88$ & 336 & 2,500 & 5,200 & --- & - & - & 12,500 & --- & -- \\
\hline & $10-25-88$ & 341 & 2,700 & 4,200 & .4 & -- & 12 & 11,300 & 4.5 & 4.5 \\
\hline & $11-13-88$ & -- & -- & -- & -- & -- & -- & -- & 6.3 & -- \\
\hline & $12-16-88$ & 323 & 2,300 & 4,400 & - & 6.2 & --- & 10,600 & 4.9 & -- \\
\hline & $1-18-89$ & 320 & 2,500 & 8,200 & -- & 13 & 11 & 16,200 & 9.1 & 9.1 \\
\hline & $2-15-89$ & 333 & 2,600 & 8,200 & -- & 13 & -- & 17,200 & 8.2 & --- \\
\hline & $3-16-89$ & 366 & 2,600 & 8,700 & -- & 12 & -- & 16,600 & 5.5 & -- \\
\hline & 4-11-89 & 342 & 2,200 & 3,800 & -- & 5.2 & 11 & 9,270 & 2.7 & 2.7 \\
\hline & $5-23-89$ & 371 & 2,600 & 6,000 & .3 & .44 & 13 & 13,400 & 4.6 & 4.6 \\
\hline & $6-20-89$ & 370 & 2,400 & 5,600 & .4 & 8.4 & 13 & 14,000 & 4.7 & 4.7 \\
\hline & $7-19-89$ & 381 & 2,600 & 4,600 & -- & 7.0 & 13 & 10,900 & --- & 3.5 \\
\hline & $8-28-89^{1}$ & 333 & 2,600 & 7,800 & -- & 12 & 14 & 16,600 & $\cdots$ & 3.8 \\
\hline & $8-28-89$ & 304 & 2,600 & 7,800 & -- & 13 & --- & --- & $\cdots$ & -- \\
\hline & $8-30-89$ & 373 & 2,600 & 8,100 & -- & 13 & --- & --- & $\cdots$ & -- \\
\hline & $8-30-89$ & 193 & 2,600 & 8,100 & -- & 13 & -- & --- & -- & -- \\
\hline \multirow[t]{14}{*}{ S-265 } & $5-19-88$ & 484 & 3,000 & 1,900 & .4 & -- & 18 & --- & 33 & -- \\
\hline & $8-22-88$ & 589 & 3,600 & 2,400 & -- & --. & -- & 9,890 & --- & -- \\
\hline & $10-24-88$ & 475 & 3,300 & 2,600 & .3 & -- & 19 & 9,550 & 22 & 21 \\
\hline & $11-13-88$ & 458 & 3,400 & 2,600 & -- & 2.5 & --- & 9,500 & 19 & -- \\
\hline & $12-17-88$ & 476 & 3,000 & 1,800 & -- & 2.2 & $\cdots$ & 7,900 & 34 & $\cdots$ \\
\hline & $1-20-89$ & 442 & 2,800 & 2,700 & -- & 2.3 & 18 & 8,960 & 22 & 22 \\
\hline & $2-15-89$ & 462 & 2,600 & 2,200 & -- & 1.9 & -- & 7,990 & 26 & -- \\
\hline & $3-15-89$ & 520 & 3,300 & 2,100 & --- & 1.4 & -- & 8,810 & 24 & -- \\
\hline & $4-10-89$ & 622 & 3,500 & 2,200 & -- & 1.5 & 17 & 9,180 & 41 & 41 \\
\hline & 5-23-89 & 484 & 3,300 & 2,500 & .3 & 2.2 & 19 & 9,380 & 21 & 21 \\
\hline & $6-20-89$ & 491 & 3,500 & 2,200 & .3 & 1.7 & 19 & 9,190 & 42 & 42 \\
\hline & $7-19-89$ & 502 & 3,400 & 2,900 & -- & 2.4 & 18 & 9,890 & --- & 21 \\
\hline & $8-27-89^{1}$ & 353 & 2,500 & 1,600 & -- & 1.4 & -.- & --- & --- & -- \\
\hline & $8-27-89$ & 538 & 3,800 & 1,600 & -- & 1.2 & 19 & 8,820 & -- & 24 \\
\hline \multirow[t]{14}{*}{$S-4$} & $5-19-88$ & 406 & 4,600 & 9,000 & .3 &.- & 13 & --- & 9.8 & --- \\
\hline & $3-23-88$ & 539 & 4,700 & 6,000 & --- & -- & -- & 17,100 & --- & -- \\
\hline & $10-25-88$ & 408 & 4,900 & 9,200 & .3 & -- & 14 & 23,100 & 11 & 9.6 \\
\hline & $11-13-88$ & 393 & 4,800 & 9,200 & --- & 12 & -- & 23,200 & 7.9 & -- \\
\hline & $12-16-88$ & 468 & 4,600 & 6,300 & --- & 7.1 & -- & 17,200 & 13 & -- \\
\hline & $1-20-89$ & 413 & 4,900 & 8,900 & -- & 10 & 13 & 21,400 & 9.5 & 9.5 \\
\hline & $2-15-89$ & 410 & 4,600 & 8,100 & --- & 10 & -- & 21,600 & 11 & $-\ldots$ \\
\hline & $3-15-89$ & 434 & 4,600 & 9,100 & -- & 8.0 & -- & 20,100 & 17 & -- \\
\hline & $4-11-89$ & 417 & 4,900 & 8,700 & -- & 9.8 & 12 & 21,300 & 17 & 17 \\
\hline & $5-23-89$ & 430 & 4,900 & 8,500 & .4 & 9.2 & 13 & 21,000 & 15 & 15 \\
\hline & $6-20-89$ & 393 & 4,500 & 7,600 & .4 & 8.7 & 14 & 19,700 & 24 & 24 \\
\hline & $7-19-89$ & 395 & 4,800 & 8,400 & -- & 9.1 & 14 & 20,600 & -- & 34 \\
\hline & $8-27-89^{1}$ & 414 & 4,800 & 7,200 & -- & 8.7 & 15 & 19,800 & -- & 26 \\
\hline & $8-27-89$ & 343 & 4,800 & 7,500 & -- & 8.3 & -- & $\ldots$ & -- & -- \\
\hline
\end{tabular}

See footnote at end of table. 
Table 4. Data from periodic sampling of drainwater at 15 Imperial Valley sites at fields, May 1988-August 1989-Continued

\begin{tabular}{|c|c|c|c|c|c|c|c|c|c|c|c|}
\hline $\begin{array}{l}\text { Local } \\
\text { identi- } \\
\text { fier }\end{array}$ & Date & $\begin{array}{c}\text { Nitro- } \\
\text { gen, } \\
\text { ammonia } \\
(\mathrm{mg} / \mathrm{L} \\
\text { as N) }\end{array}$ & $\begin{array}{c}\text { Nitro- } \\
\text { gen, am- } \\
\text { monia }+ \\
\text { organic, } \\
\text { dis. } \\
(\mathrm{mg} / \mathrm{L} \\
\text { as } \mathrm{N})\end{array}$ & $\begin{array}{l}\text { Phos- } \\
\text { phorus, } \\
\text { hydro. } \\
+ \text { ortho } \\
\text { (mg/L } \\
\text { as P) }\end{array}$ & $\begin{array}{l}\text { Alum- } \\
\text { inum } \\
(\mu \mathrm{g} / \mathrm{L} \\
\text { as } \mathrm{Al})\end{array}$ & $\begin{array}{l}\text { Anti- } \\
\text { mony } \\
(\mu \mathrm{g} / \mathrm{L} \\
\text { as } \mathrm{Sb})\end{array}$ & $\begin{array}{c}\text { Arsenic } \\
(\mu \mathrm{g} / \mathrm{L} \\
\text { as As })\end{array}$ & $\begin{array}{l}\text { Boron } \\
(\mu \mathrm{g} / \mathrm{L} \\
\text { as B })\end{array}$ & $\begin{array}{c}\text { Iron } \\
(\mu \mathrm{g} / \mathrm{L}) \\
\text { as } \mathrm{Fe})\end{array}$ & $\begin{array}{c}\text { Lithium } \\
(\mu \mathrm{g} / \mathrm{L} \\
\text { as } \mathrm{Li})\end{array}$ & $\begin{array}{c}\text { Manga- } \\
\text { nese } \\
(\mu \mathrm{g} / \mathrm{L} \\
\text { as } \mathrm{Mn})\end{array}$ \\
\hline \multirow[t]{16}{*}{ S-154 } & $5-18-88$ & 0.34 & --- & --- & -- & - & 2 & 1,300 & 150 & - & 40 \\
\hline & 3-23-88 & -- & -- & $\ldots$ & - & -_- & 2 & 1,700 & - & --- & --- \\
\hline & $10-25-88$ & .28 & --. & --- & 20 & $<1$ & 3 & 1,800 & 50 & $\ldots$ & 30 \\
\hline & $11-13-88$ & .49 & --. & ... & $\ldots$ & - & $\ldots$ & -.. & $\ldots$ & --- & -.- \\
\hline & $12-16-88$ & .30 & --- & --- & - & --- & --- & 1,300 & 80 & --- & 20 \\
\hline & $1-18-89$ & .63 & --- & --. & 30 & -.- & 2 & 1,300 & 150 & 650 & 90 \\
\hline & $2-15-89$ & .70 & -.. & --. & $\ldots$ & -.- & --- & 1,300 & 90 & --- & 70 \\
\hline & 3-16-89 & .40 & --- & --- & -.- & -- & -- & 1,400 & 140 & --. & 80 \\
\hline & $4-11-89$ & .19 & --- & -- & $<20$ & $-\ldots$ & 3 & 1,400 & 30 & 500 & 10 \\
\hline & $5-23-89$ & .35 & -- & --- & 20 & --- & 3 & 1,600 & 26 & 780 & 27 \\
\hline & 6-20-89 & .33 & -- & ... & 20 & --- & 2 & 1,900 & 100 & 570 & 50 \\
\hline & $7-19-89$ & -- & --- & --- & 30 & $\ldots$ & 3 & 2,000 & 60 & -.. & 40 \\
\hline & $8-28-89^{1}$ & .71 & 1.0 & 0.05 & 80 & $\ldots$ & 3 & 2,000 & 120 & -.- & 160 \\
\hline & $8-28-89$ & -- & --- & -- & - &.-- & -- & --- & --- & $\cdots$ & --- \\
\hline & $8-30-89$ & -- & -- & $\cdots$ & -- & --- & --- & $--\cdot$ & $--\cdot$ & --- & --- \\
\hline & $8-30-89$ & - & -- & - & --- & --- & --- & --- & --- & --- & --- \\
\hline \multirow[t]{14}{*}{ S-265 } & $5-19-88$ & .18 & -.. & $\ldots$ & --- & --- & 2 & 1,400 & 70 & --- & 250 \\
\hline & $8-22-88$ & --- & -- & -- & --. & --- & 2 & 1,600 & --- & --- & --- \\
\hline & $10-24-88$ & .16 & -- & $\ldots$ & 40 & $<1$ & 2 & 1,900 & 40 & .-. & 550 \\
\hline & $11-13-88$ & .25 & -- & --- & - & - & - & 1,900 & 60 & --- & 340 \\
\hline & $12-17-88$ & .22 & -- & - & -- & --- & --- & 1,500 & 40 & --- & 350 \\
\hline & $1-20-89$ & .16 & - & $-\ldots$ & 10 & --- & 1 & 1,600 & 40 & 450 & 440 \\
\hline & $2-15-89$ & .16 & $\ldots$ & --- & --- & -.- & $\ldots$ & 1,600 & 20 & $\ldots$ & 510 \\
\hline & 3-15-89 & .14 & -- & -.- & --- & --- & --- & 1,600 & 50 & --- & 500 \\
\hline & $4-10-89$ & .20 & - & - & 50 & $\ldots$ & 1 & 1,500 & 50 & 490 & 260 \\
\hline & 5-23-89 & .18 & - & -- & $<20$ & --- & 1 & 1,600 & 50 & 470 & 400 \\
\hline & $6-20-89$ & .31 & - & --- & 20 & --- & 2 & 1,600 & 70 & 500 & 1,000 \\
\hline & $7-19-89$ & $\ldots$ & -- & - & 30 & -.. & 1 & 1,900 & 60 & -.. & 950 \\
\hline & $8-27-89^{1}$ & .14 & 1.8 & .10 & 40 & --- & 2 & 1,800 & 50 & --- & 240 \\
\hline & $8-27-89$ & -- & - & -- & -- & --- & -- & -- & $\cdots$ & --- & -- \\
\hline \multirow[t]{14}{*}{$S-4$} & $5-19-88$ & .60 & -- & - & --- & --- & 2 & 2,200 & 140 & --. & 40 \\
\hline & $3-23-88$ & -- & -- & -- & --- & --- & 2 & 2,700 & -- & --- & -- \\
\hline & $10-25-88$ & .58 & -.. & - & 10 & $<1$ & 3 & 2,700 & 130 & --- & 40 \\
\hline & $11-13-88$ & .62 & - & -.. & --- & --- & $\ldots$ & 2,700 & 140 & --- & 80 \\
\hline & $12-16-88$ & .34 & - & --- & $\ldots$ & --- & --- & 2,200 & 100 & --- & 40 \\
\hline & $1-20-89$ & .47 & -- &.-- & 20 & -.- & 2 & 2,100 & 100 & 680 & 30 \\
\hline & $2-15-89$ & .41 & --. & -.. & --. & ... & $\ldots$ & 2,000 & 90 & -.- & 30 \\
\hline & 3-15-89 & .25 & - & -.. & $\ldots$ & --- & --- & 2,200 & 100 & --- & 40 \\
\hline & $4-11-89$ & .49 & -- & --- & 30 & --- & 2 & 2,300 & 90 & 700 & 30 \\
\hline & 5-23-89 & .34 & -- & --- & $<50$ & --- & 2 & 2,400 & 160 & 660 & 50 \\
\hline & $6-20-89$ & .26 & - & ... & 30 & --- & 2 & 2,400 & 120 & 620 & 40 \\
\hline & $7-19-89$ & --- & --- & -- & 30 & --- & 2 & 2,800 & 100 & 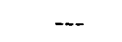 & 50 \\
\hline & $8-27-89^{1}$ & .23 & .40 & .07 & 50 & -.- & 2 & 2,700 & 100 & -.- & 30 \\
\hline & $8-27-89$ & -- & -- & --- & --- & -.. & $\cdots$ & --- & --- & --. & --- \\
\hline
\end{tabular}

See footnote at end of table. 
Table 4. Data from periodic sampling of drainwater at 15 Imperial Valley sites at fields, May 1988-August 1989-Continued

\begin{tabular}{|c|c|c|c|c|c|c|c|c|c|c|}
\hline \multirow{3}{*}{$\begin{array}{l}\text { Local } \\
\text { identi- } \\
\text { fier }\end{array}$} & \multirow[t]{3}{*}{ Date } & \multirow{3}{*}{$\begin{array}{l}\text { Molyb- } \\
\text { denum } \\
\text { ( } \mu \mathrm{g} / \mathrm{L} \\
\text { as Mo) }\end{array}$} & \multirow{3}{*}{$\begin{array}{l}\text { Sele- } \\
\text { nium } \\
(\mu \mathrm{g} / \mathrm{L} \\
\text { as } \mathrm{Se})\end{array}$} & \multirow{3}{*}{$\begin{array}{l}\text { Stron- } \\
\text { tium } \\
\text { ( } \mu \mathrm{g} / \mathrm{L} \\
\text { as Sr) }\end{array}$} & \multirow{3}{*}{$\begin{array}{l}\text { Vana- } \\
\text { dium } \\
(\mu \mathrm{g} / \mathrm{L} \\
\text { as V) }\end{array}$} & \multirow{3}{*}{$\begin{array}{c}\text { Uranium, } \\
\text { natural } \\
(\mu \mathrm{g} / \mathrm{L} \\
\text { as U) }\end{array}$} & \multirow{3}{*}{$\begin{array}{c}\text { Carbon, } \\
\text { organic } \\
\text { (mg/L } \\
\text { as C) }\end{array}$} & \multirow{3}{*}{$\begin{array}{c}\text { Tritium, } \\
\text { total } \\
(\mathrm{pCi} / \mathrm{L})\end{array}$} & \multicolumn{2}{|c|}{$\begin{array}{l}\text { Stable-isotope } \\
\text { ratio (permil) }\end{array}$} \\
\hline & & & & & & & & & ${ }^{2} \mathrm{H} /{ }^{1} \mathrm{H}$ & ${ }^{18} \mathrm{O} /{ }^{16} \mathrm{O}$ \\
\hline & & & & & & & & & , & \\
\hline \multirow[t]{16}{*}{ S-154 } & $5-18-88$ & 14 & 15 & -- & 59 & - & -- & 110 & -92.0 & -11.20 \\
\hline & $3-23-88$ & - & 12 & -- & --- & -- & -- & 107 & -- & --- \\
\hline & $10-25-88$ & 17 & 14 & -. & -- & --. & -- & -.- & -94.5 & -11.25 \\
\hline & $11-13-88$ & - & $\ldots$ & -- & -- & - & -- & --- & -91.5 & -11.00 \\
\hline & $12-16-88$ & -- & 19 & -- & --- & --- & -- & --- & -93.0 & -11.35 \\
\hline & $1-18-89$ & 13 & 28 & 26,000 & -- & -- & --- & -- & -89.0 & -10.55 \\
\hline & $2-15-89$ & -- & 29 & - & -- & $\ldots$ & - & --- & -88.0 & -10.45 \\
\hline & $3-16-89$ & -- & 26 & -- & --- & -- & $\cdots$ & --- & -86.5 & -10.50 \\
\hline & $4-11-89$ & 15 & 18 & 13,000 & --- & -- & $\ldots$ & -- & -95.0 & -11.45 \\
\hline & 5-23-89 & 15 & 18 & 18,000 & --- & -.. & $\ldots$ & -- & -90.5 & -10.95 \\
\hline & $6-20-89$ & 13 & 20 & 17,000 & --- & --- & -- & -- & -92.5 & -10.95 \\
\hline & $7-19-89$ & 16 & 2 & 11,000 & --- & -- & --- & --- & -93.5 & -11.05 \\
\hline & $8-28-89^{1}$ & 17 & 21 & 18,000 & $\ldots$ & 290 & 7.7 & -- & -90.0 & -10.65 \\
\hline & $8-28-89$ & -- & 22 & --- & -- & -.- & --- & $\ldots$ & -- & -- \\
\hline & $8-30-89$ & -- & 19 & --- & --- & -- & --- & --- & --- &.- \\
\hline & $8-30-89$ & $\cdots$ & 22 & --- & --- & -- & --- & -- & --- & -- \\
\hline \multirow[t]{14}{*}{ S-265 } & $5-19-88$ & 14 & 76 & --- & 32 & -- & $\cdots$ & 127 & -96.0 & -11.60 \\
\hline & $8-22-88$ & -- & 99 & --- & -- & -- & --- & 123 & -- & $\ldots$ \\
\hline & $10-24-88$ & 14 & 85 & -- & -- & --- & -- & -- & -96.0 & -11.70 \\
\hline & $11-13-88$ & - & 88 & --- & -- & --- & -- & -- & -97.0 & -11.75 \\
\hline & $12-17-88$ & -- & 50 & --- & -- & -- & -- & -- & -97.0 & -11.75 \\
\hline & $1-20-89$ & 3 & 69 & 9,000 & -- & --- & -- & -- & -97.0 & -11.70 \\
\hline & $2-15-89$ & -- & 50 & -.. & -- & --- & -- & -- & -96.5 & -11.70 \\
\hline & $3-15-89$ & -- & 53 & --- & -- & --- & --- & -- & -97.0 & -11.60 \\
\hline & $4-10-89$ & 12 & 95 & 9,400 & --- & --- & --- & --- & -96.0 & -11.40 \\
\hline & $5-23-89$ & 13 & 81 & 8,800 & -- & - & -- & -- & -96.0 & -11.70 \\
\hline & $6-20-89$ & 17 & 74 & 8,300 & -- & - & - & --- & -100.0 & -11.50 \\
\hline & $7-19-89$ & 16 & 78 & 5,700 & -- & -- & --- & -- & -95.0 & -11.55 \\
\hline & $8-27-89^{1}$ & 21 & 93 & 5,300 & -- & 94 & 11 & -- & -97.5 & -11.60 \\
\hline & $8-27-89$ & -- & 96 & --- & $\cdots$ & -- & --- & -- & --- & --- \\
\hline \multirow[t]{14}{*}{$S-4$} & $5-19-88$ & 21 & 170 & -- & 120 & -- & --- & 103 & -89.0 & -10.50 \\
\hline & $3-23-88$ & -- & 91 & -- & $\ldots$ & - & $\cdots$ & 104 & --- & --- \\
\hline & $10-25-88$ & 23 & 140 & - & $\ldots$ & -- & -- & - & -89.0 & -10.20 \\
\hline & $11-13-88$ & -- & 150 & $\ldots$ & $\ldots$ & - & $\cdots$ & -- & -88.5 & -10.10 \\
\hline & $12-16-88$ & -- & 95 & -.. & --- & $\cdots$ & -- & -- & -93.0 & -11.05 \\
\hline & $1-20-89$ & 21 & 130 & 23,000 & -- & $\ldots$ & -- & -- & -92.5 & -10.35 \\
\hline & $2-15-89$ & -- & 120 & -- & --- & -- &.- & --- & -90.0 & -10.50 \\
\hline & $3-15-89$ & -- & 120 & -- & $\ldots$ & $\ldots$ & --- & --- & -92.0 & -10.60 \\
\hline & $4-11-89$ & 18 & 170 & 23,000 & $\ldots$ & $\ldots$ & -- & --- & -91.5 & -10.45 \\
\hline & $5-23-89$ & 18 & 160 & 22,000 & -- & - & --- & -- & -90.0 & -10.55 \\
\hline & $6-20-89$ & 21 & 130 & 20,000 & $\cdots$ & $\ldots$ & --- & -- & -92.5 & -10.70 \\
\hline & $7-19-89$ & 24 & 120 & 16,000 & $\ldots$ & $\ldots$ & -- & --- & -91.5 & -10.50 \\
\hline & $8-27-89^{1}$ & 32 & 110 & 13,000 & -- & 84 & 11 & -- & -90.5 & -10.65 \\
\hline & $8-27-89$ & -- & 110 & --- & -- & - & --- & $\ldots$ & --- & --. \\
\hline
\end{tabular}

See footnote at end of table. 
Table 4. Data from periodic sampling of drainwater at 15 Imperial Valley sites at fields, May 1988-August 1989-Continued

\begin{tabular}{|c|c|c|c|c|c|c|c|c|c|c|c|}
\hline $\begin{array}{c}\text { Site } \\
\text { number }\end{array}$ & $\begin{array}{l}\text { Local } \\
\text { identi- } \\
\text { fier }\end{array}$ & Date & Time & $\begin{array}{c}\text { Dis- } \\
\text { charge, } \\
\text { instan- } \\
\text { taneous } \\
\left(\mathrm{ft}^{3} / \mathrm{s}\right)\end{array}$ & $\begin{array}{l}\text { Spe- } \\
\text { cific } \\
\text { con- } \\
\text { duct- } \\
\text { ance } \\
(\mu S / \mathrm{cm})\end{array}$ & $\begin{array}{l}\mathrm{pH} \\
\text { (stand- } \\
\text { ard } \\
\text { units) }\end{array}$ & $\begin{array}{c}\text { Temper- } \\
\text { ature, } \\
\text { water } \\
\left({ }^{\circ} \mathrm{C}\right)\end{array}$ & $\begin{array}{c}\text { Calcium } \\
\text { (mg/L } \\
\text { as Ca) }\end{array}$ & $\begin{array}{c}\text { Magne- } \\
\text { sium } \\
(\mathrm{mg} / \mathrm{L} \\
\text { as } \mathrm{Mg})\end{array}$ & $\begin{array}{c}\text { Sodium } \\
\text { (mg/L } \\
\text { as } \mathrm{Na})\end{array}$ & $\begin{array}{c}\text { Potas- } \\
\text { sium } \\
(\mathrm{mg} / \mathrm{L} \\
\text { as K) }\end{array}$ \\
\hline \multirow[t]{14}{*}{79} & \multirow[t]{14}{*}{ S-72 } & $5-20-88$ & 1050 & 0.0027 & 13,400 & 7.3 & 21.5 & -- & -- & -- & - \\
\hline & & $8-15-88$ & 1125 & .8930 & 11,300 & 7.7 & --. & --- & --- & -- & $\ldots$ \\
\hline & & $10-24-88$ & 1435 & .0450 & 12,700 & 7.3 & 24.5 & 600 & 380 & 2,100 & 15 \\
\hline & & $11-13-88$ & 1145 & .7290 & 7,170 & 7.5 & 25.0 & 370 & --- & --- & -- \\
\hline & & $12-17-88$ & 0815 & .2460 & 11,400 & 7.4 & 20.0 & 590 & -- & -- & --- \\
\hline & & $1-20-89$ & 1125 & .2340 & 8,680 & 7.4 & 17.0 & 470 & 300 & 1,300 & 10 \\
\hline & & $2-15-89$ & 1620 & .0782 & 12,300 & 7.6 & 17.0 & 640 & --. & -- & $\ldots$ \\
\hline & & $3-15-89$ & 1740 & .8390 & 9,130 & 7.2 & 16.0 & 470 & -- & -- & $\ldots$ \\
\hline & & $4-11-89$ & 0855 & .0435 & 11,800 & 7.4 & 18.0 & 620 & 400 & 1,700 & 10 \\
\hline & & 5-23-89 & 0830 & .0012 & 9,380 & 7.6 & -- & 490 & 280 & 1,400 & 11 \\
\hline & & $6-20-89$ & 1840 & .0030 & 9,470 & 8.0 & 23.5 & 470 & 290 & 1,500 & 18 \\
\hline & & $7-19-89$ & 0745 & .0022 & 7,040 & 7.9 & 25.0 & 370 & 200 & 1,100 & 16 \\
\hline & & $8-27-89^{1}$ & 1930 & .0763 & 6,250 & 7.2 & 27.0 & 330 & 180 & 820 & 13 \\
\hline & & $8-27-89$ & 1931 & -- & 6,260 & 7.2 & -- & --- & -- & --- & --- \\
\hline \multirow[t]{14}{*}{87} & \multirow[t]{14}{*}{ S-352 } & $5-19-88$ & 1540 & 0 & 22,800 & 7.3 & 21.5 & 280 & 480 & 4,300 & 20 \\
\hline & & $8-22-88$ & 1630 & 0 & 4,270 & 7.4 & --- & --. & --- & --- & -- \\
\hline & & $10-24-88$ & 0920 & 0 & 6,000 & 7.9 & 25.0 & 300 & 170 & 890 & 21 \\
\hline & & $11-13-88$ & 0650 & .0187 & 18,600 & 7.6 & 24.0 & 530 & --- & -- & --- \\
\hline & & $12-17-88$ & 1245 & .0147 & 17,900 & 7.5 & 21.5 & 520 & --- & -- & -- \\
\hline & & $1-20-89$ & 1430 & .0185 & 15,800 & 7.4 & 19.0 & 490 & 370 & 2,800 & 22 \\
\hline & & $2-15-89$ & 1200 & .4540 & 12,700 & 7.6 & 18.0 & 480 & -.. & -- & $-\ldots$ \\
\hline & & $3-16-89$ & 1510 & .0242 & 15,800 & 7.4 & 18.5 & 500 & --- & -- & --- \\
\hline & & $4-14-89$ & 0910 & .0427 & 15,000 & 7.6 & 19.5 & 480 & 380 & 2,500 & 18 \\
\hline & & $5-23-89$ & 1410 & .0170 & 21,500 & 7.5 & 22.5 & 630 & 520 & 3,900 & 11 \\
\hline & & $6-21-89$ & 1120 & .0170 & 24,800 & 7.5 & 24.0 & 660 & 560 & 4,600 & 31 \\
\hline & & $7-19-89$ & 1045 & .0134 & 20,700 & 7.4 & 25.0 & 590 & 440 & 3,800 & 29 \\
\hline & & $8-30-89^{1}$ & 0915 & 0 & 3,680 & 7.4 & 27.5 & 220 & 99 & 460 & 16 \\
\hline & & $8-30-89$ & 0916 & - & 3,690 & 7.4 & - & --- & --- & --- & --- \\
\hline \multirow[t]{14}{*}{93} & \multirow[t]{14}{*}{ S-423 } & $5-19-88$ & 1620 & 0 & 20,200 & 6.9 & 20.5 & 580 & 510 & 2,900 & 25 \\
\hline & & $8-18-88$ & 1800 & .0160 & 19,800 & 7.1 & -- & -- & --- & -.. & -- \\
\hline & & $10-24-88$ & 1020 & 0 & 12,300 & 7.2 & 24.0 & 670 & 350 & 1,900 & 13 \\
\hline & & $11-13-88$ & 0730 & .0170 & 10,400 & 7.2 & 22.0 & 540 & --- & - & --- \\
\hline & & $12-17-88$ & 1315 & .0064 & 14,100 & 7.3 & 21.5 & 710 & -- & $\ldots$ & $\ldots$ \\
\hline & & $1-20-89$ & 1340 & 0 & 18,100 & 7.4 & 17.5 & 970 & 470 & 2,500 & 26 \\
\hline & & $2-15-89$ & 1250 & .0131 & 15,600 & 7.5 & 18.5 & 780 & --- & -.- & --- \\
\hline & & $3-16-89$ & 1430 & .0040 & 14,700 & 7.3 & 19.0 & 620 & --- & --- & --- \\
\hline & & $4-14-89$ & 0955 & .0032 & 14,600 & 7.5 & 20.0 & 680 & 460 & 2,300 & 10 \\
\hline & & $5-23-89$ & 1315 & .0015 & 16,700 & 7.3 & 22.0 & 950 & 460 & 2,400 & 23 \\
\hline & & $6-21-89$ & 1035 & 0 & 19,900 & 7.0 & 22.0 & 1,200 & 540 & 2,700 & 36 \\
\hline & & $7-19-89$ & 1145 & .0018 & 18,200 & 7.0 & 23.0 & 1,100 & 490 & 2,500 & 30 \\
\hline & & $8-28-89^{1}$ & 1045 & 0 & 14,800 & 7.1 & 24.0 & 800 & 390 & 2,100 & 23 \\
\hline & & $8-28-89$ & 1046 & --- & 14,900 & 7.2 & -- & -. & -.. & + & $\ldots$ \\
\hline
\end{tabular}

See footnote at end of table. 
Table 4. Data from periodic sampling of drainwater at 15 Imperial Valley sites at fields, May 1988-August 1989-Continued

\begin{tabular}{|c|c|c|c|c|c|c|c|c|c|c|}
\hline $\begin{array}{l}\text { Local } \\
\text { identi- } \\
\text { fier }\end{array}$ & Date & $\begin{array}{c}\text { Alka- } \\
\text { linity, } \\
\text { lab } \\
(\mathrm{mg} / \mathrm{L} \\
\text { as } \\
\left.\mathrm{CaCO}_{3}\right)\end{array}$ & $\begin{array}{c}\text { Sulfate } \\
(\mathrm{mg} / \mathrm{L} \\
\left.\text { as } \mathrm{SO}_{4}\right)\end{array}$ & $\begin{array}{l}\text { Chlo- } \\
\text { ride } \\
(\mathrm{mg} / \mathrm{L} \\
\text { as } \mathrm{Cl})\end{array}$ & $\begin{array}{l}\text { Fluo- } \\
\text { ride } \\
(\mathrm{mg} / \mathrm{L} \\
\text { as } \mathrm{F})\end{array}$ & $\begin{array}{c}\text { Bromide } \\
(\mathrm{mg} / \mathrm{L} \\
\text { as } \mathrm{Br})\end{array}$ & $\begin{array}{c}\text { Silica } \\
(\mathrm{mg} / \mathrm{L} \\
\text { as } \\
\left.\mathrm{SiO}_{2}\right)\end{array}$ & $\begin{array}{l}\text { Solids, } \\
\text { residue } \\
\text { at } 180 \\
{ }^{\circ} \mathrm{C} \\
(\mathrm{mg} / \mathrm{L})\end{array}$ & $\begin{array}{c}\text { Nitro- } \\
\text { gen, } \\
\mathrm{NO}_{2}+ \\
\mathrm{NO}_{3} \\
(\mathrm{mg} / \mathrm{L} \\
\text { as } \mathrm{N})\end{array}$ & $\begin{array}{c}\text { Nitro- } \\
\text { gen, } \\
\mathrm{NO}_{2}+ \\
\mathrm{NO}_{3} \\
(\mathrm{mg} / \mathrm{L} \\
\text { as } \mathrm{N})\end{array}$ \\
\hline \multirow[t]{14}{*}{$S-72$} & $5-20-88$ & 437 & --- & 2,500 & $\ldots$ & --- & --- & 10,100 & -- & $\ldots$ \\
\hline & $8-15-88$ & 379 & 3,700 & 1,800 & -- & --- & $\cdots$ & 9,120 & $\cdots$ & -- \\
\hline & $10-24-88$ & 412 & 4,000 & 2,200 & 0.4 & --- & 16 & 10,300 & 50 & 38 \\
\hline & $11-13-88$ & 386 & 3,000 & 820 & --- & 0.71 & -- & 5,980 & 19 & $\ldots$ \\
\hline & $12-17-88$ & 422 & 3,700 & 2,000 & -- & 2.5 & -- & 9,240 & 34 & -- \\
\hline & $1-20-89$ & 423 & 2,900 & 1,400 & -.. & 1.7 & 14 & 6,990 & 25 & 25 \\
\hline & $2-15-89$ & 489 & 4,100 & 2,100 & 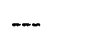 & 2.7 & $\cdots$ & 10,100 & 11 & -- \\
\hline & $3-15-89$ & 444 & 3,000 & 1,500 & -- & 1.8 & --- & 7,280 & 21 & $\ldots$ \\
\hline & $4-11-89$ & 493 & 3,500 & 2,200 & -- & 2.5 & 11 & 9,230 & 14 & 14 \\
\hline & $5-23-89$ & 504 & 3,100 & 1,500 & .5 & 1.6 & 16 & 7,470 & 15 & 15 \\
\hline & $6-20-89$ & 399 & 3,400 & 1,400 & .4 & 1.3 & 17 & 7,430 & 18 & 18 \\
\hline & $7-19-89$ & 419 & 2,400 & 930 &.-- & .57 & 21 & 5,280 & --- & 12 \\
\hline & $8-27-89^{1}$ & 326 & 2,000 & 900 & --- & .72 & 23 & 4,670 & --. & 7.9 \\
\hline & $8-27-89$ & 327 & 2,000 & 890 & --- & .71 & $\cdots$ & -.- & -- & - \\
\hline \multirow[t]{14}{*}{$S-352$} & $5-19-88$ & 407 & 4,100 & 6,000 & .5 & $\ldots$ & 11 &.- & 16 & -- \\
\hline & $8-22-88$ & 356 & 1,400 & 440 & --- & -- & -- & 3,180 & --- & -- \\
\hline & $10-24-88$ & 411 & 2,200 & 660 & .7 & -- & 14 & 4,670 & 21 & 18 \\
\hline & $11-13-88$ & 446 & 4,200 & 4,200 & -.. & 3.8 & -- & 13,600 & 21 & -- \\
\hline & $12-17-88$ & 436 & 4,000 & 4,400 & -- & 3.9 & -- & 13,500 & 17 & --- \\
\hline & $1-20-89$ & 436 & 3,700 & 3,700 & --- & 3.1 & 11 & 11,900 & 17 & 17 \\
\hline & $2-15-89$ & 444 & 3,300 & 2,600 & --- & 2.2 & -- & 9,860 & 16 & --- \\
\hline & $3-16-89$ & 418 & 3,700 & 3,800 & $\cdots$ & 3.2 & -- & 11,800 & 15 & -- \\
\hline & $4-14-89$ & 462 & 3,600 & 3,400 & --- & 2.9 & 11 & 11,000 & 17 & 17 \\
\hline & $5-23-89$ & 441 & 4,300 & 5,300 & .5 & 5.1 & 12 & 15,800 & 18 & 18 \\
\hline & $6-21-89$ & 416 & 4,400 & 6,800 & .5 & 6.3 & 13 & 17,500 & 17 & 17 \\
\hline & $7-19-89$ & 419 & 4,100 & 5,200 & -- & 4.8 & 12 & 14,900 & $\ldots$ & 15 \\
\hline & $8-30-89^{1}$ & 374 & 1,200 & 280 & $\ldots$ & .25 & 14 & 2,670 & $\ldots$ & 11 \\
\hline & $8-30-89$ & 390 & 1,300 & 320 & --- & .22 & -- & -- & -- & -- \\
\hline \multirow[t]{14}{*}{$S-423$} & $5-19-88$ & 299 & 2,500 & 6,300 & .3 & - & 14 & --- & 97 & -- \\
\hline & $8-18-88$ & 340 & 2,700 & 5,900 & -- & -- & -- & 13,700 & - & -- \\
\hline & $10-24-88$ & 561 & 3,400 & 2,200 & .4 & --- & 14 & 9,720 & 28 & 28 \\
\hline & $11-13-88$ & 473 & 3,600 & 1,500 &.- & 1.3 & $\ldots$ & 8,440 & 16 & -- \\
\hline & $12-17-88$ & 454 & 4,100 & 3,000 & -- & 2.6 & - & 11,300 & 39 & --- \\
\hline & $1-20-89$ & 378 & 2,800 & 5,000 & -- & 5.5 & 13 & 13,400 & 77 & 77 \\
\hline & $2-15-89$ & 505 & 3,900 & 3,500 & -- & 2.9 & $\ldots$ & 12,200 & 37 & --- \\
\hline & $3-16-89$ & 505 & 4,100 & 3,200 & --- & 2.6 & --- & 11,500 & 30 & -- \\
\hline & 4-14-89 & 550 & 4,300 & 3,000 & --- & 2.1 & 12 & 11,400 & 29 & 29 \\
\hline & $5-23-89$ & 426 & 3,100 & 4,300 & .2 & 4.4 & 14 & 12,100 & 61 & 61 \\
\hline & $6-21-89$ & 304 & 2,200 & 5,400 & .2 & 7.0 & 15 & 14,500 & 64 & 64 \\
\hline & $7-19-89$ & 334 & 2,500 & 5,100 & --- & 5.4 & 14 & 12,800 & $\ldots$ & 85 \\
\hline & $8-28-89^{1}$ & 418 & 3,300 & 3,300 & -- & 3.6 & 15 & 11,000 & --- & 37 \\
\hline & $8-28-89$ & 307 & 3,300 & 3,500 & -- & 3.6 & -- & -- & $\cdots$ & - \\
\hline
\end{tabular}

See footnote at end of table. 
Table 4. Data from periodic sampling of drainwater at 15 Imperial Valley sites at fields, May 1988-August 1989-Continued

\begin{tabular}{|c|c|c|c|c|c|c|c|c|c|c|c|}
\hline $\begin{array}{l}\text { Local } \\
\text { identi- } \\
\text { fier }\end{array}$ & Date & $\begin{array}{c}\text { Nitro- } \\
\text { gen, } \\
\text { ammonia } \\
(\mathrm{mg} / \mathrm{L} \\
\text { as } \mathrm{N})\end{array}$ & $\begin{array}{c}\text { Nitro- } \\
\text { gen, am- } \\
\text { monia }+ \\
\text { organic, } \\
\text { dis. } \\
(\mathrm{mg} / \mathrm{L} \\
\text { as } \mathrm{N})\end{array}$ & $\begin{array}{l}\text { Phos- } \\
\text { phorus, } \\
\text { hydro. } \\
+ \text { ortho } \\
\text { (mg/L } \\
\text { as P) }\end{array}$ & $\begin{array}{l}\text { Alum- } \\
\text { inum } \\
(\mu \mathrm{g} / \mathrm{L} \\
\text { as } \mathrm{Al})\end{array}$ & $\begin{array}{l}\text { Anti- } \\
\text { mony } \\
(\mu \mathrm{g} / \mathrm{L} \\
\text { as } \mathrm{Sb})\end{array}$ & $\begin{array}{c}\text { Arsenic } \\
(\mu \mathrm{g} / \mathrm{L} \\
\text { as As) }\end{array}$ & $\begin{array}{l}\text { Boron } \\
(\mu \mathrm{g} / \mathrm{L} \\
\text { as B) }\end{array}$ & $\begin{array}{c}\text { Iron } \\
(\mu \mathrm{g} / \mathrm{L}) \\
\text { as } \mathrm{Fe})\end{array}$ & $\begin{array}{c}\text { Lithium } \\
(\mu \mathrm{g} / \mathrm{L} \\
\text { as } \mathrm{Li})\end{array}$ & $\begin{array}{c}\text { Manga- } \\
\text { nese } \\
(\mu \mathrm{g} / \mathrm{L} \\
\text { as } \mathrm{Mn})\end{array}$ \\
\hline \multirow[t]{14}{*}{ S-72 } & $5-20-88$ & - & -- & --- & -- & -- & --- & $\cdots$ & -- & --- & -- \\
\hline & $8-15-88$ & - & $\ldots$ & $\ldots$ & -.. & $\ldots$ & 2 & 1,600 & $\ldots$ & --- & $\ldots$ \\
\hline & $10-24-88$ & 0.12 & -- & -- & $<10$ & $<1$ & 2 & 2,000 & 50 & --- & 20 \\
\hline & $11-13-88$ & .16 & - & -- & -- & -- & $\cdots$ & 1,500 & 90 & -- & $<10$ \\
\hline & $12-17-88$ & .20 & -- & -- & -- & -- & -- & 1,600 & 60 & --- & 20 \\
\hline & $1-20-89$ & .12 & -- & -- & 20 & -.- & 2 & 1,200 & 40 & 370 & 20 \\
\hline & $2-15-89$ & .19 & -.- & -- & --- & --- & -- & 1,600 & 40 & --- & 20 \\
\hline & $3-15-89$ & .08 & -.. & -- & -.- & -- & --- & 1,200 & 50 & --- & 20 \\
\hline & $4-11-89$ & .16 & --- & -- & 20 & -- & 1 & 1,600 & 30 & 440 & 10 \\
\hline & 5-23-89 & .12 & --- & -- & $<10$ & -- & 2 & 1,500 & 50 & 370 & 20 \\
\hline & $6-20-89$ & .13 & --- & -- & 20 & --- & 2 & 1,700 & 50 & 910 & 20 \\
\hline & 7-19-89 & -- & --- & -- & 10 & $-\ldots$ & 4 & 1,300 & 50 & --- & 10 \\
\hline & $8-27-89^{1}$ & .08 & 0.70 & 0.84 & 20 & -- & 3 & 1,100 & 30 & --- & 30 \\
\hline & 8-27-89 & -- & -- & -- & $\cdots$ & $\ldots$ & -- & -- & -- & $\cdots$ & -- \\
\hline \multirow[t]{14}{*}{ S-352 } & $5-19-88$ & .17 & -- & -- & -- & --- & 2 & 3,000 & 110 & --- & 80 \\
\hline & $8-22-88$ & --- & - & -. & -.- & $\ldots$ & 3 & 870 & --- & -- & --- \\
\hline & $10-24-88$ & .19 & -- & - & 30 & $<1$ & 2 & 1,300 & 40 & --- & 50 \\
\hline & $11-13-88$ & .18 & $-\ldots$ & --. & - & --- & --- & 3,000 & 80 & --- & 70 \\
\hline & $12-17-88$ & .18 & - & -- & -- & -- & --- & 2,700 & 60 & -- & 70 \\
\hline & $1-20-89$ & .12 & -- & -- & 10 & -- & 2 & 2,100 & 40 & 680 & 60 \\
\hline & 2-15-89 & .11 & -- & --- & --- & -- & --- & 1,700 & 40 & -- & 50 \\
\hline & $3-16-89$ & .08 & $-\ldots$ & $\ldots$ & $\ldots$ & -- & -- & 2,100 & 70 & -- & 60 \\
\hline & $4-14-89$ & .12 & --- & -- & 30 & -- & 2 & 2,100 & 40 & 660 & 60 \\
\hline & $5-23-89$ & .15 & $\ldots$ & - & 10 & -- & 2 & 2,900 & 100 & 810 & 100 \\
\hline & $6-21-89$ & .20 & --- & $\cdots$ & 30 & - & 1 & 3,500 & 110 & 880 & 110 \\
\hline & $7-19-89$ & .14 & $\ldots$ & -- & 40 & -- & 3 & 3,300 & 100 & -.- & 100 \\
\hline & $8-30-89^{1}$ & .09 & .40 & .05 & $<10$ & -- & 3 & 880 & 20 & -- & 280 \\
\hline & $8-30-89$ & -- & -- & -- & -- & -- & --- & -- & --- & -- & -- \\
\hline \multirow[t]{14}{*}{ S-423 } & $5-19-88$ & .62 & -- & -- & --- & -- & 1 & 1,800 & 180 & --- & 270 \\
\hline & 8-18-88 & -- & -- & - & --- & - & 2 & 1,900 & --- & --- & -- \\
\hline & $10-24-88$ & .24 & --- & --- & 70 & $<1$ & 2 & 2,100 & 100 & $\cdots$ & 70 \\
\hline & $11-13-88$ & .21 & --- & $-\ldots$ & -.. & --- & -. & 1,900 & 170 & -.- & 30 \\
\hline & $12-17-88$ & .27 & -- & -- & -- & --- & - & 1,900 & 80 & --- & 60 \\
\hline & $1-20-89$ & .41 & --- & -- & 50 & -- & 1 & 1,800 & 120 & 540 & 110 \\
\hline & $2-15-89$ & .18 & --- & - & -- & -- & -- & 1,800 & 50 & -- & 50 \\
\hline & $3-16-89$ & .14 & -- & - & -- & --- & -- & 1,800 & 110 & --- & 60 \\
\hline & $4-14-89$ & .22 & - & - & 30 & -- & 1 & 1,900 & 60 & 500 & 40 \\
\hline & $5-23-89$ & .28 & -- & - & $<20$ & -- & 1 & 1,900 & 110 & 500 & 120 \\
\hline & $6-21-89$ & .41 & --- & --- & 10 & -- & 1 & 1,900 & 170 & 510 & 160 \\
\hline & 7-19-89 & - & -- & -- & 20 & - & 1 & 1,900 & 90 & --- & 120 \\
\hline & $8-28-89^{1}$ & .20 & $<.20$ & .04 & 50 & --- & 1 & 2,100 & 80 & -- & 70 \\
\hline & $8-28-89$ & - & -- & -- & -- & --- & -- & -- & --- & -- & - \\
\hline
\end{tabular}

See footnote at end of table. 
Table 4. Data from periodic sampling of drainwater at 15 Imperial Valley sites at fields, May 1988-August 1989-Continued

\begin{tabular}{|c|c|c|c|c|c|c|c|c|c|c|}
\hline \multirow{2}{*}{$\begin{array}{l}\text { Local } \\
\text { identi- } \\
\text { fier }\end{array}$} & \multirow[t]{2}{*}{ Date } & \multirow{2}{*}{$\begin{array}{l}\text { Molyb- } \\
\text { denum } \\
(\mu \mathrm{g} / \mathrm{L} \\
\text { as Mo) }\end{array}$} & \multirow{2}{*}{$\begin{array}{c}\text { Sele- } \\
\text { nium } \\
(\mu \mathrm{g} / \mathrm{L} \\
\text { as Se) }\end{array}$} & \multirow{2}{*}{$\begin{array}{c}\text { Stron- } \\
\text { tium } \\
(\mu \mathrm{g} / \mathrm{L} \\
\text { as Sr) }\end{array}$} & \multirow{2}{*}{$\begin{array}{l}\text { Vana- } \\
\text { dium } \\
(\mu g / L \\
\text { as V) }\end{array}$} & \multirow{2}{*}{$\begin{array}{c}\text { Uranium, } \\
\text { natural } \\
(\mu \mathrm{g} / \mathrm{L} \\
\text { as } \mathrm{U})\end{array}$} & \multirow{2}{*}{$\begin{array}{c}\text { Carbon, } \\
\text { organic } \\
\text { (mg/L } \\
\text { as C) }\end{array}$} & \multirow{2}{*}{$\begin{array}{c}\text { Tritium, } \\
\text { total } \\
(\mathrm{pCi} / \mathrm{L})\end{array}$} & \multicolumn{2}{|c|}{$\begin{array}{l}\text { Stable-isotope } \\
\text { ratio (permil) } \\
\end{array}$} \\
\hline & & & & & & & & & ${ }^{2} \mathrm{H} /{ }^{1} \mathrm{H}$ & ${ }^{18} \mathrm{O} /{ }^{16} \mathrm{O}$ \\
\hline \multirow[t]{14}{*}{ S-72 } & $5-20-88$ & -- & 68 & -- & -- & -- & -- & -- & -- & --- \\
\hline & $8-15-88$ & -- & 83 & --- & -- & -- & --- & 140 & -- & -- \\
\hline & $10-24-88$ & 15 & 88 & -- & -- & -- & -- & --- & -98.0 & -11.85 \\
\hline & $11-13-88$ & -- & 28 & --- & -- & -- & -- & -- & -100.0 & -12.30 \\
\hline & $12-17-88$ & -- & 81 & -- & -- & -- & -- & -- & -97.5 & -11.80 \\
\hline & $1-20-89$ & 16 & 57 & 9,400 & - & -- & -- & -- & -98.0 & -12.00 \\
\hline & $2-15-89$ & - & 71 & - & -- & -- & -- & -- & -94.0 & -11.85 \\
\hline & $3-15-89$ & -- & 59 & -- & -- & -- & --- & -- & -99.0 & -12.00 \\
\hline & $4-11-89$ & 11 & 56 & 11,000 & -- & -- & -- & --- & -97.5 & -11.85 \\
\hline & $5-23-89$ & 21 & 62 & 8,200 & --- & -- & -- & --- & -97.0 & -11.95 \\
\hline & $6-20-89$ & 31 & 83 & 8,600 & -- & -- & --- & --- & -99.5 & -12.15 \\
\hline & $7-19-89$ & 19 & 51 & 4,300 & -- & -- & --- & -- & -99.5 & -12.05 \\
\hline & $8-27-89^{1}$ & 11 & 39 & 3,900 & -.. & 120 & 16 & -- & -98.0 & -11.70 \\
\hline & $8-27-89$ & -- & 36 & -- & --- & --- & --- & --- & -- & --- \\
\hline \multirow[t]{14}{*}{ S-352 } & $5-19-88$ & 25 & 65 & -- & 10 & -- & -- & -- & -94.0 & -11.15 \\
\hline & $8-22-88$ & -- & 12 & -- & -- & -- & --- & 146 & -- & -. \\
\hline & $10-24-88$ & 21 & 24 & -- & -- & -- & -- & --- & -97.0 & -11.65 \\
\hline & $11-13-88$ & - & 60 & -- & -- & -- & --- & --- & -95.5 & -11.30 \\
\hline & $12-17-88$ & -- & 54 & -- & -- & $\cdots$ & $--\infty$ & --- & -95.0 & -11.50 \\
\hline & $1-20-89$ & 29 & 48 & 10,000 & -- & -- & --- & --- & -97.0 & -11.60 \\
\hline & $2-15-89$ & -- & 43 & -- & -- & --- & -- & --. & -89.0 & -11.95 \\
\hline & $3-16-89$ & -- & 44 & - & - & --- & -- & -- & -95.0 & -11.55 \\
\hline & 4-14-89 & 27 & 44 & 10,000 & --- & --- & -- & --- & -96.5 & -11.70 \\
\hline & 5-23-89 & 37 & 61 & 12,000 & -- & $\cdots$ & $\cdots$ & --- & -93.5 & -11.20 \\
\hline & $6-21-89$ & 47 & 61 & 14,000 & $-\ldots$ & $\ldots$ & -- & --- & -93.0 & -11.00 \\
\hline & $7-19-89$ & 36 & 48 & 9,100 & --- & -- & $\ldots$ & --- & -95.5 & -11.25 \\
\hline & $8-30-89^{1}$ & 54 & 15 & 3,400 & -- & 26 & 4.5 & --- & -99.5 & -12.05 \\
\hline & $8-30-89$ & - & 14 & -- & -- & -- & $-\cdots$ & -- & -- & --- \\
\hline \multirow[t]{14}{*}{$S-423$} & $5-19-88$ & 19 & 240 & --- & 74 & -- & --- & 91 & -90.5 & -10.15 \\
\hline & 8-18-88 & -- & 220 & -- & --- & --- & -- & 94 &.-- & -.- \\
\hline & $10-24-88$ & 18 & 88 & -- & -- & --- & -- & $\cdots$ & -96.0 & -11.65 \\
\hline & $11-13-88$ & -- & 68 & --- & $\cdots$ & --- & -- & -.- & -99.0 & -12.10 \\
\hline & $12-17-88$ & -- & 110 & --- & --- & -.. & -- & -- & -95.0 & -11.55 \\
\hline & $1-20-89$ & 21 & 220 & 19,000 & --- & --- & -- & --- & -94.0 & -10.40 \\
\hline & $2-15-89$ & -- & 130 & -- & --- & --- & -- & --- & -93.0 & -11.40 \\
\hline & $3-16-89$ & $\ldots$ & 120 & -- & --- & -- & -- & --. & -96.0 & -11.45 \\
\hline & $4-14-89$ & 16 & 120 & 12,000 & --- & -- & --- &.- & -98.5 & -11.70 \\
\hline & $5-23-89$ & 18 & 180 & 15,000 & -- & -- & -- & -- & -93.0 & -11.05 \\
\hline & $6-21-89$ & 19 & 230 & 19,000 & -- & --- & -- & -- & -92.0 & -10.25 \\
\hline & $7-19-89$ & 20 & 200 & 11,000 & -- & -- & --- & -- & -92.5 & -10.45 \\
\hline & $8-28-89^{1}$ & 26 & 140 & 8,800 & -- & 77 & 8.8 & --- & -95.0 & -11.35 \\
\hline & $8-28-89$ & -- & 140 & -- & -- & --- & --- & --- & -.- & --- \\
\hline
\end{tabular}

See footnote at end of table. 
Table 4. Data from periodic sampling of drainwater at 15 Imperial Valley sites at fields, May 1988-August 1989-Continued

\begin{tabular}{|c|c|c|c|c|c|c|c|c|c|c|c|}
\hline $\begin{array}{c}\text { Site } \\
\text { number }\end{array}$ & $\begin{array}{l}\text { Local } \\
\text { identi- } \\
\text { fier }\end{array}$ & Date & Time & $\begin{array}{c}\text { Dis- } \\
\text { charge, } \\
\text { instan- } \\
\text { taneous } \\
\left(\mathrm{ft}^{3} / \mathrm{s}\right)\end{array}$ & $\begin{array}{l}\text { Spe- } \\
\text { cific } \\
\text { con- } \\
\text { duct- } \\
\text { ance } \\
(\mu \mathrm{S} / \mathrm{cm})\end{array}$ & $\begin{array}{l}\mathrm{pH} \\
\text { (stand- } \\
\text { ard } \\
\text { units) }\end{array}$ & $\begin{array}{l}\text { Temper- } \\
\text { ature, } \\
\text { water } \\
\left({ }^{\circ} \mathrm{C}\right)\end{array}$ & $\begin{array}{c}\text { Calcium } \\
(\mathrm{mg} / \mathrm{L} \\
\text { as } \mathrm{Ca})\end{array}$ & $\begin{array}{c}\text { Magne- } \\
\text { sium } \\
(\mathrm{mg} / \mathrm{L} \\
\text { as } \mathrm{Mg})\end{array}$ & $\begin{array}{l}\text { Sodium } \\
(\mathrm{mg} / \mathrm{L} \\
\text { as } \mathrm{Na})\end{array}$ & $\begin{array}{l}\text { Potas- } \\
\text { sium } \\
\text { ( } \mathrm{mg} / \mathrm{L} \\
\text { as } \mathrm{K})\end{array}$ \\
\hline \multirow[t]{14}{*}{98} & S-371 & $5-19-88$ & 1410 & 0.0995 & 11,300 & 7.2 & 22.0 & 560 & 350 & 1,600 & 7.6 \\
\hline & & $8-16-88$ & 1145 & .3320 & 15,900 & 7.5 & --- & $\cdots$ & -- & -- & --- \\
\hline & & $10-24-88$ & 1050 & .0260 & 13,600 & 7.5 & 26.5 & 800 & 400 & 1,900 & 11 \\
\hline & & $11-13-88$ & 0750 & .0630 & 16,900 & 7.5 & 24.5 & 910 & --. & -- & --- \\
\hline & & $12-17-88$ & 1140 & .1160 & 9,560 & 7.5 & 24.0 & 490 & - & $\cdots$ & -- \\
\hline & & $1-19-89$ & 0750 & .0440 & 15,700 & 7.5 & 20.5 & 790 & 450 & 2,200 & 15 \\
\hline & & $2-15-89$ & 1320 & .2730 & 12,500 & 7.6 & 20.0 & 700 & - & -.. & --- \\
\hline & & $3-16-89$ & 1245 & .2270 & 9,100 & 7.3 & 21.0 & 430 &.-- & -- & -- \\
\hline & & 4-11-89 & 0800 & .0732 & 12,300 & 7.9 & 20.5 & 600 & 360 & 1,700 & 10 \\
\hline & & $5-23-89$ & 0940 & .2710 & 9,480 & 7.4 & 22.0 & 470 & 260 & 1,400 & 11 \\
\hline & & $6-21-89$ & 1015 & .0400 & 14,100 & 7.4 & 23.0 & 760 & 440 & 2,000 & 15 \\
\hline & & $7-19-89$ & 0640 & .0225 & 14,900 & 7.0 & 23.5 & 820 & 420 & 2,000 & 15 \\
\hline & & $8-28-89^{1}$ & 1015 & .0484 & 8,760 & 7.2 & 26.0 & 470 & 230 & 1,300 & 12 \\
\hline & & $8-28-89$ & 1016 & -- & 8,780 & 7.2 & --- & --- & --- & -- & $\cdots$ \\
\hline \multirow[t]{14}{*}{104} & S-176 & $5-19-88$ & 1125 & .1620 & 11,800 & 7.3 & 19.0 & 390 & 330 & 1,900 & 7.6 \\
\hline & & $8-16-88$ & 1210 & .0290 & 11,400 & 7.4 & --- & -.- & --- & -- & -.- \\
\hline & & $10-24-88$ & 1405 & .0120 & 9,790 & 7.4 & 26.5 & 560 & 300 & 1,600 & 11 \\
\hline & & $11-13-88$ & 1105 & .2890 & 10,200 & 7.5 & 25.5 & 500 & ..- & -. & -- \\
\hline & & $12-17-88$ & 0840 & .0484 & 11,400 & 7.5 & 21.0 & 560 & --- & --- & $\cdots$ \\
\hline & & $1-20-89$ & 1145 & -- & 14,400 & 7.6 & 16.0 & 620 & 390 & 2,500 & 15 \\
\hline & & $2-15-89$ & 1600 & .4770 & 10,900 & 7.5 & 17.5 & 580 & --- & --- & $\cdots$ \\
\hline & & $3-16-89$ & 1025 & .2880 & 9,680 & 7.3 & 18.0 & 460 & --- & --- & --- \\
\hline & & 4-11-89 & 0830 & .1720 & 10,700 & 7.4 & 18.0 & 580 & 350 & 1,500 & 11 \\
\hline & & $5-23-89$ & 1025 & --- & 10,500 & 7.8 & 19.5 & 520 & 310 & 1,500 & 11 \\
\hline & & $6-20-89$ & 0730 & .2850 & 10,000 & 7.5 & 21.0 & 550 & 300 & 1,600 & 14 \\
\hline & & $7-19-89$ & 0725 & .5580 & 9,770 & 7.3 & 23.0 & 540 & 280 & 1,600 & 13 \\
\hline & & $8-28-89^{1}$ & 0730 & - & 9,050 & 7.0 & 25.0 & 460 & 250 & 1,400 & 11 \\
\hline & & $8-28-89$ & 0731 & -- & 9,070 & 7.1 & --- & --- & --- & --- & --- \\
\hline \multirow[t]{14}{*}{110} & $S-344$ & $5-19-88$ & 1635 & .0453 & 16,000 & 7.3 & 21.5 & 250 & 320 & 2,800 & 19 \\
\hline & & 8-18-88 & 0640 & --- & 15,100 & 7.6 &.-- & --- & $\cdots$ & -- & --- \\
\hline & & $10-24-88$ & 0955 & .0360 & 15,400 & 7.4 & 25.0 & 560 & 350 & 2,600 & 20 \\
\hline & & $11-13-88$ & 0620 & .0377 & 15,400 & 7.5 & 24.0 & 550 & --- & -- & --- \\
\hline & & $12-17-88$ & 1220 & .0506 & 13,700 & 7.4 & 24.0 & 540 & $\cdots$ & -- & -- \\
\hline & & $1-20-89$ & 1400 & .0377 & 14,700 & 7.4 & -- & 540 & 340 & 2,400 & 23 \\
\hline & & $2-15-89$ & 1230 & .2340 & 14,600 & 7.6 & 21.0 & 570 & --- & -- & -- \\
\hline & & $3-16-89$ & 1445 & .0470 & 15,200 & --- & 20.5 & 580 & --- & -. & -- \\
\hline & & 4-14-89 & 0930 & .0460 & 14,600 & 7.4 & 21.5 & 600 & 370 & 2,500 & 17 \\
\hline & & $5-23-89$ & 1340 & .0697 & 14,800 & 7.5 & 22.0 & 590 & 350 & 2,300 & 27 \\
\hline & & $6-21-89$ & 1050 & .0440 & 16,400 & 7.6 & 23.5 & 660 & 400 & 2,700 & 30 \\
\hline & & $7-19-89$ & 1120 & .0410 & 16,000 & 7.3 & 24.0 & 630 & 360 & 2,700 & 28 \\
\hline & & $8-30-89^{1}$ & 1000 & .1620 & 11,500 & 7.2 & 25.5 & 510 & 270 & 1,900 & 28 \\
\hline & & $8-30-89$ & 1001 & --- & 11,600 & 7.2 & --- & -- & -- & -. & $\ldots$ \\
\hline
\end{tabular}

See footnote at end of table. 
Table 4. Data from periodic sampling of drainwater at 15 Imperial Valley sites at fields, May 1988-August 1989-Continued

\begin{tabular}{|c|c|c|c|c|c|c|c|c|c|c|}
\hline $\begin{array}{l}\text { Local } \\
\text { identi- } \\
\text { fier }\end{array}$ & Date & $\begin{array}{l}\text { Alka- } \\
\text { linity, } \\
\text { lab } \\
(\mathrm{mg} / \mathrm{L} \\
\text { as } \\
\left.\mathrm{CaCO}_{3}\right)\end{array}$ & $\begin{array}{c}\text { Sulfate } \\
(\mathrm{mg} / \mathrm{L} \\
\left.\text { as } \mathrm{SO}_{4}\right)\end{array}$ & $\begin{array}{l}\text { Chlo- } \\
\text { ride } \\
(\mathrm{mg} / \mathrm{L} \\
\text { as } \mathrm{Cl})\end{array}$ & $\begin{array}{l}\text { Fluo- } \\
\text { ride } \\
(\mathrm{mg} / \mathrm{L} \\
\text { as F) }\end{array}$ & $\begin{array}{c}\text { Bromide } \\
(\mathrm{mg} / \mathrm{L} \\
\text { as } \mathrm{Br})\end{array}$ & $\begin{array}{c}\text { Silica } \\
(\mathrm{mg} / \mathrm{L} \\
\text { as } \\
\left.\mathrm{SiO}_{2}\right)\end{array}$ & $\begin{array}{l}\text { Solids, } \\
\text { residue } \\
\text { at } 180 \\
{ }^{\circ} \mathrm{C} \\
(\mathrm{mg} / \mathrm{L})\end{array}$ & $\begin{array}{c}\text { Nitro- } \\
\text { gen, } \\
\mathrm{NO}_{2}+ \\
\mathrm{NO}_{3} \\
(\mathrm{mg} / \mathrm{L} \\
\text { as } \mathrm{N})\end{array}$ & $\begin{array}{c}\text { Nitro- } \\
\text { gen, } \\
\mathrm{NO}_{2}+ \\
\mathrm{NO}_{3} \\
(\mathrm{mg} / \mathrm{L} \\
\text { as } \mathrm{N})\end{array}$ \\
\hline \multirow[t]{14}{*}{ S-371 } & $5-19-88$ & 360 & 2,300 & 2,600 & 0.5 & -- & 18 & -- & 9.5 & $\cdots$ \\
\hline & $8-16-88$ & 407 & 3,100 & 3,800 & $\ldots$ & -- & -- & 11,600 & --- & $-\cdots$ \\
\hline & $10-24-88$ & 345 & 1,800 & 4,200 & .4 & - & 20 & 9,130 & 14 & 13 \\
\hline & $11-13-88$ & 375 & 2,500 & 4,600 & -- & 6.3 & -- & 11,900 & 29 & --- \\
\hline & $12-17-88$ & 255 & 2,600 & 2,000 & -- & 1.8 & -- & 7,190 & 16 & -- \\
\hline & $1-19-89$ & 391 & 2,900 & 4,200 & -- & 5.0 & 18 & 11,300 & 21 & 21 \\
\hline & $2-15-89$ & 377 & 2,300 & 3,200 & --- & 3.8 & -- & 8,790 & 12 & --- \\
\hline & $3-16-89$ & 395 & 2,500 & 1,700 & --- & 2.6 & --- & 6,940 & 13 & --- \\
\hline & $4-11-89$ & 341 & 2,800 & 2,900 & --- & 2.8 & 16 & 8,960 & 13 & 13 \\
\hline & $5-23-89$ & 358 & 2,500 & 1,800 & .6 & 1.6 & 17 & 7,110 & 10 & 10 \\
\hline & $6-21-89$ & 374 & 2,800 & 3,500 & .4 & 4.2 & 19 & 10,300 & 11 & 11 \\
\hline & $7-19-89$ & 372 & 2,800 & 4,000 & --- & 4.8 & 19 & 10,500 & --- & 14 \\
\hline & $8-28-89^{1}$ & 593 & 2,400 & 1,500 & --- & 1.6 & 21 & 6,280 & --- & 2.6 \\
\hline & $8-28-89$ & 341 & 2,500 & 1,500 & -- & 1.7 & -- & -.- & -- & --- \\
\hline \multirow[t]{14}{*}{ S-176 } & $5-19-88$ & 484 & 3,300 & 2,200 & .5 & -- & 18 & --- & 9.9 & -- \\
\hline & $8-16-88$ & 464 & 3,400 & 1,900 & --- & -- & -- & 8,970 & --- & -- \\
\hline & $10-24-88$ & 441 & 3,000 & 1,700 & .5 & - & 20 & 7,630 & 11 & 8.8 \\
\hline & $11-13-88$ & 433 & 3,200 & 1,800 & --- & 2.6 & -- & 8,060 & 15 & $\ldots$ \\
\hline & $12-17-88$ & 437 & 3,500 & 2,100 & --- & 2.3 & - & 8,940 & 13 & --- \\
\hline & $1-20-89$ & 451 & 3,600 & 3,400 & -- & 3.2 & 19 & 11,200 & 18 & 18 \\
\hline & $2-15-89$ & 435 & 3,400 & 2,000 & -- & .80 & $\ldots$ & 8,570 & 16 & -- \\
\hline & $3-16-89$ & 440 & 3,300 & 1,600 & $\cdots$ & 1.5 & -- & 7,790 & 12 & --- \\
\hline & $4-11-89$ & 437 & 3,300 & 2,000 & --- & 1.7 & 17 & 6,990 & 16 & 16 \\
\hline & $5-23-89$ & 415 & 3,200 & 1,800 & .5 & 1.8 & 18 & 8,040 & 13 & 13 \\
\hline & $6-20-89$ & 454 & 3,200 & 1,700 & .6 & 1.7 & 19 & 7,250 & 12 & 12 \\
\hline & $7-19-89$ & 461 & 3,200 & 1,600 & --- & 1.6 & 19 & 7,540 &.- & 10 \\
\hline & $8-28-89^{1}$ & 480 & 3,200 & 1,300 & $\cdots$ & 1.3 & 19 & 7,060 & -- & 11 \\
\hline & $8-28-89$ & 379 & 3,200 & 1,300 & --- & 1.3 & -- & -- & -- & -- \\
\hline \multirow[t]{14}{*}{ S-344 } & $5-19-88$ & 319 & 3,800 & 3,800 & .4 & -- & 15 & ... & 37 & -- \\
\hline & 8-18-88 & 314 & 3,900 & 3,200 & -- & --- & - & 11,400 & -- & --- \\
\hline & $10-24-88$ & 398 & 3,800 & 3,400 & .4 & -- & 16 & 11,700 & 40 & 32 \\
\hline & $11-13-88$ & 293 & 3,900 & 3,400 & --- & 3.6 & -- & 11,700 & 33 & -- \\
\hline & $12-17-88$ & 315 & 3,700 & 2,900 & -- & 2.8 & -- & 10,500 & 32 & $\cdots$ \\
\hline & $1-20-89$ & 297 & 3,600 & 3,200 & --- & 3.2 & 15 & 11,200 & 33 & 33 \\
\hline & $2-15-89$ & 293 & 3,800 & 3,200 & -- & 3.2 & --- & 11,300 & 29 & -- \\
\hline & $3-16-89$ & 305 & 3,900 & 3,500 & --- & 3.2 & $\cdots$ & 10,200 & 36 & -- \\
\hline & $4-14-89$ & 315 & 3,700 & 3,300 & -- & 3.0 & 15 & 10,900 & 32 & 32 \\
\hline & $5-23-89$ & 317 & 3,500 & 3,400 & .3 & 3.3 & 16 & 11,100 & 32 & 32 \\
\hline & $6-21-89$ & 300 & 3,600 & 3,900 & .3 & 4.0 & 16 & 12,200 & 38 & 38 \\
\hline & $7-19-89$ & 299 & 3,800 & 3,800 & -- & 3.7 & 16 & 12,100 & --- & 34 \\
\hline & $8-30-89^{1}$ & 339 & 3,600 & 2,000 & -- & 2.2 & 16 & 8,810 & $\cdots$ & 29 \\
\hline & $8-30-89$ & 317 & 3,600 & 2,000 & -- & 2.0 & -- & -- & --- & -- \\
\hline
\end{tabular}

See footnote at end of table. 
Table 4. Data from periodic sampling of drainwater at 15 Imperial Valley sites at fields, May 1988-August 1989-Continued

\begin{tabular}{|c|c|c|c|c|c|c|c|c|c|c|c|}
\hline $\begin{array}{l}\text { Local } \\
\text { identi- } \\
\text { fier }\end{array}$ & Date & $\begin{array}{c}\text { Nitro- } \\
\text { gen, } \\
\text { ammonia } \\
\text { (mg/L } \\
\text { as N) }\end{array}$ & $\begin{array}{c}\text { Nitro- } \\
\text { gen, am- } \\
\text { monia + } \\
\text { organic, } \\
\text { dis. } \\
(\mathrm{mg} / \mathrm{L} \\
\text { as } \mathrm{N})\end{array}$ & $\begin{array}{l}\text { Phos- } \\
\text { phorus, } \\
\text { hydro. } \\
+ \text { ortho } \\
\text { (mg/L } \\
\text { as P) }\end{array}$ & $\begin{array}{l}\text { Alum- } \\
\text { inum } \\
(\mu \mathrm{g} / \mathrm{L} \\
\text { as Al) }\end{array}$ & $\begin{array}{l}\text { Anti- } \\
\text { mony } \\
(\mu \mathrm{g} / \mathrm{L} \\
\text { as } \mathrm{Sb})\end{array}$ & $\begin{array}{c}\text { Arsenic } \\
(\mu \mathrm{g} / \mathrm{L} \\
\text { as As })\end{array}$ & $\begin{array}{l}\text { Boron } \\
(\mu \mathrm{g} / \mathrm{L} \\
\text { as B) }\end{array}$ & $\begin{array}{c}\text { Iron } \\
(\mu \mathrm{g} / \mathrm{L}) \\
\text { as } \mathrm{Fe})\end{array}$ & $\begin{array}{l}\text { Lithium } \\
(\mu \mathrm{g} / \mathrm{L} \\
\text { as } \mathrm{Li})\end{array}$ & $\begin{array}{c}\text { Manga- } \\
\text { nese } \\
(\mu \mathrm{g} / \mathrm{L} \\
\text { as } \mathrm{Mn})\end{array}$ \\
\hline \multirow[t]{14}{*}{ S-371 } & $5-19-88$ & 0.20 & -- & --- & -- & -- & 2 & 1,300 & 340 & --- & 170 \\
\hline & $8-16-88$ & --- & --- & --- & -.- & --- & 1 & 1,800 & --- & --- & $\ldots$ \\
\hline & $10-24-88$ & .23 & -- & --- & 110 & $<1$ & 2 & 1,500 & 160 & --- & 320 \\
\hline & $11-13-88$ & .42 & -- & -- & --- & -- & -- & 1,800 & 70 & --- & 330 \\
\hline & $12-17-88$ & .47 & -- & -- & --- & - & -- & 1,100 & 40 & --- & 70 \\
\hline & $1-19-89$ & .31 & -- & --- & 20 & -- & 1 & 1,600 & 60 & 470 & 310 \\
\hline & $2-15-89$ & .17 & --- & --- & --- & --- & --- & 1,300 & 40 & --. & 10 \\
\hline & $3-16-89$ & .07 & --- & --- & -- & --- & --- & 1,200 & 60 & --- & 110 \\
\hline & $4-11-89$ & .16 & --- & -- & 30 & --- & 1 & 1,400 & 40 & 420 & 150 \\
\hline & $5-23-89$ & .11 & -- & -- & 20 & --- & 1 & 1,300 & 60 & 350 & 100 \\
\hline & $6-21-89$ & .19 & - & -- & 40 & --- & 1 & 1,700 & 80 & 430 & 180 \\
\hline & $7-19-89$ & .21 & -- & --- & 50 & --- & 1 & 1,700 & 50 & --- & 260 \\
\hline & $8-28-89^{1}$ & .11 & 0.20 & 0.03 & 10 & --- & 2 & 1,500 & 40 & -- & 130 \\
\hline & $8-28-89$ & -- & -- & -- & --- & --- & --- & --- & --- & -- & -- \\
\hline \multirow[t]{14}{*}{ S-176 } & $5-19-88$ & .17 & -- & --- & -.- & --- & 1 & 1,700 & 80 & --- & 190 \\
\hline & $8-16-88$ & - & --- & --- & -.. & --- & 2 & 1,800 & --- & --. & -- \\
\hline & $10-24-88$ & .07 & -- & -- & 10 & $<1$ & 2 & 1,700 & 30 & -- & 10 \\
\hline & $11-13-88$ & .18 & --- & -- & --- & --- & --- & 1,800 & 50 & -- & 40 \\
\hline & $12-17-88$ & .18 & -- & -- & --- & -- & -- & 1,800 & 50 & -- & 30 \\
\hline & $1-20-89$ & .26 & -- & --- & 30 & --. & 2 & 1,900 & 80 & 450 & 40 \\
\hline & $2-15-89$ & .13 & --- & -- & --- & --- & --- & 1,500 & 30 & --- & 40 \\
\hline & $3-16-89$ & .07 & - & --- & -- & -.. & $\ldots$ & 1,500 & 40 & --- & 40 \\
\hline & $4-11-89$ & .13 & -- & --- & $<20$ & --- & 1 & 1,500 & 30 & 380 & 40 \\
\hline & $5-23-89$ & .13 & -- & --- & 20 & --- & 1 & 1,500 & 50 & 380 & 50 \\
\hline & 6-20-89 & .14 & - & --- & 10 & --- & 1 & 1,600 & 50 & 370 & 50 \\
\hline & $7-19-89$ & .13 & -- & --- & 40 & --- & 1 & 1,700 & 40 & --- & 60 \\
\hline & $8-28-89^{1}$ & .11 & 1.5 & .02 & 20 & --- & 1 & 1,700 & 70 & --- & 60 \\
\hline & $8-28-89$ & -- & -- & -- & -- & -- & --- & -- & -- & --- & -- \\
\hline \multirow[t]{14}{*}{ S-344 } & $5-19-88$ & .21 & --- & --- & --- & --- & 2 & 2,300 & 90 & $\ldots$ & 70 \\
\hline & $8-18-88$ & --- & --- & --- & --- & -- & 2 & 2,700 & --- & --- & --- \\
\hline & $10-24-88$ & .14 & -- & -- & $<10$ & $<1$ & 4 & 3,000 & 50 & --- & 30 \\
\hline & $11-13-88$ & .19 & --- & -- & --- & --- & --- & 3,000 & 70 & --- & 30 \\
\hline & $12-17-88$ & .17 & --- & --- & --- & -- & -- & 2,500 & 50 & -- & 40 \\
\hline & $1-20-89$ & .15 & --- & --- & --- & 10 & 2 & 2,500 & .60 & 800 & 40 \\
\hline & $2-15-89$ & .13 & - & --- & --- & -- & -- & 2,500 & 50 & --- & 30 \\
\hline & $3-16-89$ & .10 & -- & --- & -- & -- & -- & 2,400 & 80 & --- & 40 \\
\hline & $4-14-89$ & .15 & -- & --- & -- & 30 & 2 & 2,200 & 40 & 700 & 30 \\
\hline & $5-23-89$ & .15 & --- & --- & --- & $<20$ & 2 & 2,300 & 70 & 680 & 40 \\
\hline & $6-21-89$ & .18 & -- & --- & --- & 30 & 2 & 2,500 & 70 & 730 & 30 \\
\hline & $7-19-89$ & .16 & -- & --- & --- & 60 & 2 & 2,700 & 60 & --- & 20 \\
\hline & $8-30-89^{1}$ & .09 & $<.20$ & .03 & -- & $\ldots$ & 3 & 2,300 & 40 & --- & 30 \\
\hline & $8-30-89$ & -- & $\cdots$ & --- & -- & --- & --- & -- & --- & --- & -- \\
\hline
\end{tabular}

See footnote at end of table. 
Table 4. Data from periodic sampling of drainwater at 15 Imperial Valley sites at fields, May 1988-August 1989-Continued

\begin{tabular}{|c|c|c|c|c|c|c|c|c|c|c|}
\hline \multirow{2}{*}{$\begin{array}{l}\text { Local } \\
\text { identi- } \\
\text { fier }\end{array}$} & \multirow[t]{2}{*}{ Date } & \multirow{2}{*}{$\begin{array}{l}\text { Molyb- } \\
\text { denum } \\
(\mu \mathrm{g} / \mathrm{L} \\
\text { as Mo) }\end{array}$} & \multirow{2}{*}{$\begin{array}{l}\text { Sele- } \\
\text { nium } \\
(\mu \mathrm{g} / \mathrm{L} \\
\text { as Se) }\end{array}$} & \multirow{2}{*}{$\begin{array}{l}\text { Stron- } \\
\text { tium } \\
(\mu \mathrm{g} / \mathrm{L} \\
\text { as } \mathrm{Sr})\end{array}$} & \multirow{2}{*}{$\begin{array}{l}\text { Vana- } \\
\text { dium } \\
(\mu \mathrm{g} / \mathrm{L} \\
\text { as V) }\end{array}$} & \multirow{2}{*}{$\begin{array}{c}\text { Uranium, } \\
\text { natural } \\
\text { ( } \mu \mathrm{g} / \mathrm{L} \\
\text { as } U \text { ) }\end{array}$} & \multirow{2}{*}{$\begin{array}{c}\text { Carbon, } \\
\text { organic } \\
(\mathrm{mg} / \mathrm{L} \\
\text { as C) }\end{array}$} & \multirow{2}{*}{$\begin{array}{c}\text { Tritium, } \\
\text { total } \\
\text { (pCi/L) }\end{array}$} & \multicolumn{2}{|c|}{$\begin{array}{l}\text { Stable-isotope } \\
\text { ratio (permil) }\end{array}$} \\
\hline & & & & & & & & & ${ }^{2} \mathrm{H} /{ }^{1} \mathbf{H}$ & ${ }^{18} \mathrm{O} /{ }^{16} \mathrm{O}$ \\
\hline \multirow[t]{14}{*}{ S-371 } & $5-19-88$ & 20 & 76 & - & 48 & -.- & -.. & 132 & -96.0 & -11.80 \\
\hline & $8-16-88$ & -- & 120 & - & --. & -- & --- & 125 & $\cdots$ & --- \\
\hline & $10-24-88$ & 24 & 120 & -- & -- & --- & $\ldots$ & --- & -98.0 & -11.70 \\
\hline & $11-13-88$ & -- & 140 & -- & --- & -- & -- & $\cdots$ & -96.5 & -11.35 \\
\hline & $12-17-88$ & -- & 98 & -- & -- & -- & -- & -- & -96.0 & -11.70 \\
\hline & $1-19-89$ & 18 & 120 & 17,000 & --- & --- & -..- & -- & -99.0 & -11.50 \\
\hline & $2-15-89$ & - & 99 & --- & -- & --- & $\cdots$ & --- & -99.0 & -11.80 \\
\hline & $3-16-89$ & --- & 56 & -- & --- & --. & -- & -.. & -98.5 & -11.85 \\
\hline & $4-11-89$ & 19 & 94 & 11,000 & --- & --- & -- & --- & -98.5 & -11.95 \\
\hline & $5-23-89$ & 20 & 68 & 7,700 & -- & --- & - & -- & -97.0 & -11.80 \\
\hline & $6-21-89$ & 16 & 120 & 13,000 & $\cdots$ & --- & $\ldots$ & $\ldots$ & -101.0 & -11.60 \\
\hline & $7-19-89$ & 18 & 110 & 8,000 & --- & --- & --- & $\cdots$ & -97.0 & -11.65 \\
\hline & $8-28-89^{1}$ & 22 & 43 & 5,300 & -- & 230 & 0.4 & -- & -100.0 & -12.10 \\
\hline & $8-28-89$ & $\ldots$ & 53 & -. & --- & --- & --- & -. & --- & --- \\
\hline \multirow[t]{14}{*}{ S-176 } & $5-19-88$ & 17 & 51 & --- & 37 & -- & -- & --- & -96.5 & -11.60 \\
\hline & $8-16-88$ & - & 44 & -- & -- & $-\cdots$ & -- & 126 & $\cdots$ & -- \\
\hline & $10-24-88$ & 22 & 41 & -.- & -.- & $\ldots$ & -.. & --- & -97.5 & -11.75 \\
\hline & $11-13-88$ & -- & 49 & -.. & --- & --. & --- & --- & -97.5 & -11.70 \\
\hline & $12-17-88$ & -- & 51 & $\cdots$ & $\cdots$ & --- & $-\ldots$ & --- & -97.0 & -11.60 \\
\hline & $1-20-89$ & 18 & 86 & 12,000 & -- & -.- & --- & $-\cdots$ & -94.0 & -10.70 \\
\hline & $2-15-89$ & - & 51 & --. & --- & --- & --- & --- & -93.0 & -11.65 \\
\hline & $3-16-89$ & -- & 44 & -- & -- & --- & --- & -- & -99.0 & -11.75 \\
\hline & $4-11-89$ & 15 & 52 & 9,100 &.-- & --- & --- & --- & -97.5 & -11.65 \\
\hline & $5-23-89$ & 17 & 46 & 7,700 & --- & -.- & --- & -.- & -97.0 & -11.65 \\
\hline & $6-20-89$ & 17 & 48 & 7,900 & -- & $\cdots$ & -- & --- & -98.5 & -11.70 \\
\hline & $7-19-89$ & 18 & 41 & 5,200 & -- & $\cdots$ & -- & -- & -97.5 & -11.80 \\
\hline & $8-28-89^{1}$ & 18 & 45 & 5,200 & -- & 65 & 7.6 & - & -98.5 & -11.95 \\
\hline & $8-28-89$ & - & 46 & -- & $-\cdots$ & --- & -- & --- & -- & -- \\
\hline \multirow[t]{14}{*}{ S-344 } & $5-19-88$ & 33 & 60 & $\cdots$ & 36 & -- & -- & 85 & -94.5 & -11.15 \\
\hline & 8-18-88 & - & 44 & $\cdots$ & --- & $\cdots$ & --- & 82 & -- & --- \\
\hline & $10-24-88$ & 46 & 52 & -- & --- & $\cdots$ & $-\cdots$ & $\cdots$ & -95.0 & -11.35 \\
\hline & $11-13-88$ & - & 51 & -- & --- & $\cdots$ & -- & --- & -95.5 & -11.30 \\
\hline & $12-17-88$ & -- & 42 & --- & -- & --- & -- & -- & -95.0 & -11.40 \\
\hline & $1-20-89$ & 45 & 48 & 12,000 & --- & --- & --- & $\cdots$ & -98.0 & -11.40 \\
\hline & $2-15-89$ & - & 45 & --- & --- & --- & --- & ... & -94.0 & -11.55 \\
\hline & $3-16-89$ & -. & 49 & - & -- & -- & -- & --- & -95.5 & -11.20 \\
\hline & $4-14-89$ & 35 & 54 & 12,000 & -- & --- & -- & -- & -94.5 & -11.20 \\
\hline & $5-23-89$ & 35 & 66 & 12,000 & -- & --- & -- & -- & -93.0 & -11.10 \\
\hline & $6-21-89$ & 36 & 68 & 13,000 & -- & -- & -- & --- & -89.0 & -11.15 \\
\hline & $7-19-89$ & 37 & 60 & 7,400 & -- & --- & -- & $\ldots$ & -94.5 & -11.15 \\
\hline & $8-30-89^{1}$ & 42 & 36 & 7,500 & -- & 250 & 4.6 & -- & 97.5 & 11.65 \\
\hline & $8-30-89$ & - & 39 & --- & $\ldots$ & --- & -.- & -- & -- & --- \\
\hline
\end{tabular}

'Filtered sample. 
Table 5. Data from periodic sampling of streams, East Highline Canal, and Trifolium Drain 1, August 1988-August 1989

In sampling-site designations, numbers in parentheses are U.S. Geological Survey streamflow-gaging station numbers (8-digit numbers) or latitude-longitude (15-digit numbers). Location of sites shown in figure $2 . \mu \mathrm{S} / \mathrm{cm}$, microsiemen per centimeter at $25^{\circ} \mathrm{C}$; ${ }^{\circ} \mathrm{C}$, degree Celsius; $\mathrm{mg} / \mathrm{L}$, milligram per liter; $\mu \mathrm{g} / \mathrm{L}$, microgram per liter; $\mathrm{ft}$, foot; mm, millimeter; $\mathrm{pCi} / \mathrm{L}, \mathrm{picocurie}$ per liter. --, no data. The analysis for each sample is displayed as one line on six consecutive pages]

\begin{tabular}{|c|c|c|c|c|c|c|c|c|c|}
\hline Date & Time & $\begin{array}{c}\text { Gage } \\
\text { height } \\
\text { (ft) }\end{array}$ & $\begin{array}{c}\text { Dis- } \\
\text { charge, } \\
\text { mean } \\
\text { daily } \\
\left(\mathrm{ft}^{3} / \mathrm{s}\right)\end{array}$ & $\begin{array}{c}\text { Spe- } \\
\text { cific } \\
\text { con- } \\
\text { duct- } \\
\text { tance } \\
(\mu \mathrm{S} / \mathrm{cm})\end{array}$ & $\begin{array}{l}\text { pH } \\
\text { (stand- } \\
\text { ard } \\
\text { units) }\end{array}$ & $\begin{array}{c}\text { Temper- } \\
\text { ature, } \\
\text { water } \\
\left({ }^{\circ} \mathrm{C}\right)\end{array}$ & $\begin{array}{c}\text { Calcium, } \\
\text { total } \\
\text { recov- } \\
\text { erable } \\
(\mathrm{mg} / \mathrm{L} \\
\text { as } \mathrm{Ca})\end{array}$ & $\begin{array}{l}\text { Calcium, } \\
\text { dis- } \\
\text { solved } \\
\text { (mg/L } \\
\text { as } \mathrm{Ca} \text { ) }\end{array}$ & $\begin{array}{c}\text { Magne- } \\
\text { sium, } \\
\text { total } \\
\text { recov- } \\
\text { erable } \\
(\mathrm{mg} / \mathrm{L} \\
\text { as } \mathrm{Mg} \text { ) }\end{array}$ \\
\hline \multicolumn{10}{|c|}{ Alamo River at Outlet (10254730) } \\
\hline $8-17-88$ & . 1300 & -- & 823 & 3,170 & $\ldots$ & -- & $\cdots$ & -- & -- \\
\hline $10-20-88$ & . 1605 & 3.98 & 827 & 3,470 & 7.70 & 26.0 & -- & - & --- \\
\hline $10-20-88$ & . 1606 & 3.98 & 827 & 3,480 & 7.90 & 26.0 & 190 & --- & 93 \\
\hline $11-14-88$ & . 1200 & -- & 827 & 3,530 & 8.10 & 19.0 & -- & 170 & -- \\
\hline $12-16-88$ & . . 1325 & - & 738 & 3,190 & 7.80 & 15.0 & - & 170 & $-\cdots$ \\
\hline $1-19-89$ & .1100 & 3.64 & 631 & 3,320 & 8.10 & 12.0 & -- & 150 & --- \\
\hline $1-19-89$ & .. 1101 & 3.64 & 631 & 3,360 & 7.60 & 12.0 & 99 & -- & 50 \\
\hline $2-16-89$ & . . 1010 & $\ldots$ & 818 & 3,140 & 8.10 & 12.0 & $\cdots$ & 160 & $\ldots$ \\
\hline $3-15-89$ & . . 1305 & 4.26 & 884 & 3,260 & 7.90 & 20.0 & - & 150 & -- \\
\hline $4-11-89$ & . . 1120 & 4.65 & 1,040 & 3,110 & 7.79 & 24.0 & -- & 160 & -- \\
\hline $4-11-89$ & . 1125 & 4.65 & 1,040 & 3,120 & 7.62 & 24.0 & 180 & -- & 86 \\
\hline $5-22-89$ & . 1600 & 4.36 & 809 & 3,120 & 7.90 & 25.5 & --. & 150 & -- \\
\hline $6-20-89$ & . 1145 & 4.12 & 875 & 3,090 & 7.60 & 28.5 & --- & 140 & -- \\
\hline $7-27-89$ & . . 1815 & - & 747 & 3,260 & 7.96 & 31.0 & --- & 160 & -- \\
\hline $7-27-89$ & . . 1816 & -- & 747 & 3,270 & 7.52 & 31.0 & 170 & - & 86 \\
\hline $8-27-89$ & . 1520 & 2.96 & 764 & 3,670 & 7.94 & 27.5 & - & 180 & - \\
\hline $8-27-89$ & . 1521 & - & 764 & 3,670 & 7.61 & -- & - & -- & -- \\
\hline \multicolumn{10}{|c|}{ Alamo River at Border (324032115220501) } \\
\hline $8-17-88$ & . . 0930 & - & - & 2,790 & - & --- & --- & -- & -- \\
\hline $10-21-88$ & . . 1200 & - & -- & 4,160 & 7.60 & --- & --- & --- & --- \\
\hline $10-21-88$ & . . 1201 & -- & - & 4,200 & 7.90 & --- & 200 & --- & 100 \\
\hline $11-13-88$ & .0930 & - & -- & 3,630 & 7.50 & 19.5 & -- & 180 & -.. \\
\hline $12-17-88$ & . . 0940 & -- & -- & 4,940 & 7.80 & 15.0 & --- & 230 & -- \\
\hline $1-18-89$ & . 1215 & 0.36 & $\cdots$ & 5,930 & 7.90 & 12.5 & -- & 210 & --- \\
\hline $1-18-89$ & . 1216 & .36 & - & 5,960 & 7.70 & 12.5 & 210 & --- & 120 \\
\hline $2-15-89$ & . 1500 & - & -- & 5,170 & 7.90 & 14.5 & --- & 240 & --- \\
\hline $3-16-89$ & . . 1125 & .42 & - & 5,020 & 7.90 & 20.5 & -- & 200 & -- \\
\hline 4-14-89 & . 1100 & - & -- & 5,580 & 7.90 & -- & -- & 210 & -- \\
\hline $5-23-89$ & . . 1140 & - & -- & 4,740 & 7.90 & 25.0 & -- & 190 & -- \\
\hline $6-21-89$ & $\ldots 0845$ & - & -- & 5,950 & 7.80 & 24.0 & -- & 230 & $\ldots$ \\
\hline $7-28-89$ & . . 1305 & -- & - & 5,840 & 7.99 & 30.0 & --- & 210 & --- \\
\hline $7-28-89$ & . 1306 & - & -- & 5,840 & 7.81 & 30.0 & 200 & --- & 110 \\
\hline $8-28-89$ & . . 0845 & - & -- & 5,670 & 7.94 & 23.0 & --- & 200 & -.. \\
\hline $8-28-89$ & . . 0846 & - & - & 5,680 & 7.90 & -- & -- & --- & -- \\
\hline \multicolumn{10}{|c|}{ San Felipe Creek West of Highway 78 (330722115511201) } \\
\hline $8-20-88$ & . 1150 & -- & - & 13,170 & 8.00 & -- & -- & 350 & -- \\
\hline
\end{tabular}


Table 5. Data from periodic sampling of streams, East Highline Canal, and Trifolium Drain 1, August 1988-August 1989--Continued

\begin{tabular}{|c|c|c|c|c|c|c|c|c|c|}
\hline Date & $\begin{array}{l}\text { Magne- } \\
\text { sium, } \\
\text { dis- } \\
\text { solved } \\
\text { (mg/L } \\
\text { as } \mathrm{Mg} \text { ) }\end{array}$ & $\begin{array}{c}\text { Sodium, } \\
\text { total } \\
\text { recov- } \\
\text { erable } \\
(\mathrm{mg} / \mathrm{L} \\
\text { as } \mathrm{Na})\end{array}$ & $\begin{array}{c}\text { Sodium, } \\
\text { dis- } \\
\text { solved } \\
(\mathrm{mg} / \mathrm{L} \\
\text { as } \mathrm{Na})\end{array}$ & $\begin{array}{l}\text { Potas- } \\
\text { sium, } \\
\text { total } \\
\text { recov- } \\
\text { erable } \\
\text { (mg/L } \\
\text { as } \mathrm{K} \text { ) }\end{array}$ & $\begin{array}{l}\text { Potas- } \\
\text { sium, } \\
\text { dis- } \\
\text { solved } \\
(\mathrm{mg} / \mathrm{L} \\
\text { as K) }\end{array}$ & $\begin{array}{c}\text { Alka- } \\
\text { linity, } \\
\text { lab } \\
(\mathrm{mg} / \mathrm{L} \\
\text { as } \\
\left.\mathrm{CaCO}_{3}\right)\end{array}$ & $\begin{array}{c}\text { Sulfate, } \\
\text { dis- } \\
\text { solved } \\
(\mathrm{mg} / \mathrm{L} \\
\left.\text { as } \mathrm{SO}_{4}\right)\end{array}$ & $\begin{array}{l}\text { Chlo- } \\
\text { ride, } \\
\text { dis- } \\
\text { solved } \\
(\mathrm{mg} / \mathrm{L} \\
\text { as } \mathrm{Cl})\end{array}$ & $\begin{array}{l}\text { Fluo- } \\
\text { ride, } \\
\text { dis- } \\
\text { solved } \\
(\mathrm{mg} / \mathrm{L} \\
\text { as F) }\end{array}$ \\
\hline
\end{tabular}

$\begin{array}{rlc} & & \\ 8-17-88 & \ldots & - \\ 10-20-88 & \ldots & - \\ 10-20-88 & \ldots & -- \\ 11-14-88 & \ldots & - \\ 12-16-88 & \ldots & -- \\ & & \\ 1-19-89 & \ldots & 79 \\ 1-19-89 & \ldots & - \\ 2-16-89 & \ldots & -- \\ 3-15-89 & \ldots & -- \\ 4-11-89 & \ldots & 92 \\ 4-11-89 & \ldots & \ldots \\ 5-22-89 & \ldots & 78 \\ 6-20-89 & \ldots & 81 \\ 7-27-89 & \ldots & 91 \\ 7-27-89 & \ldots & -- \\ 8-27-89 & \ldots & 100 \\ 8-27-89 & \ldots & -- \\ & & \end{array}$

Alamo River at Outlet (10254730)

-
420
--

---

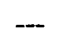

430

-.-

$\cdots$

---

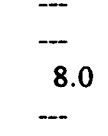

$\begin{array}{rr}-- & -- \\ -- & - \\ -- & 218 \\ -- & 220\end{array}$

--- $\quad-\cdots$

-.- $\quad 209$

$\begin{array}{rr}-- & - \\ --- & -- \\ --- & -- \\ 910 & 530 \\ 800 & 560\end{array}$

--
--
---
--
--
--
--
--
--

\begin{tabular}{|c|c|c|c|c|c|c|c|}
\hline 350 & --. & 9.3 & -- & 208 & --- & -- & --- \\
\hline-- & 400 & --- & 10 & 211 & 750 & 460 & 0.5 \\
\hline- & 410 & -- & 11 & 202 & 720 & 520 & .5 \\
\hline- & 420 & --- & 11 & 201 & 830 & 480 & --- \\
\hline 380 & -- & 13 & -- & 199 & --- & --- & -- \\
\hline --- & 430 & -- & 12 & 212 & 910 & 580 & --- \\
\hline --- & --- & --- & --- & 212 & 940 & 520 & -- \\
\hline
\end{tabular}

$\begin{array}{rlr}8-17-88 & \ldots & -- \\ 10-21-88 & \ldots & -- \\ 10-21-88 & \ldots & -- \\ 11-13-88 & \ldots & -- \\ 12-17-88 & \ldots & -- \\ & & \\ 1-18-89 & \ldots & 120 \\ 1-18-89 & \ldots & -- \\ 2-15-89 & \ldots & - \\ 3-16-89 & \ldots & -- \\ 4-14-89 & \ldots & 120 \\ & & \\ 5-23-89 & \ldots & 98 \\ 6-21-89 & \ldots & 130 \\ 7-28-89 & \ldots & 120 \\ 7-28-89 & \ldots & -- \\ 8-28-89 & \ldots & 110 \\ 8-28-89 & \ldots & --\end{array}$

$8-20-88 \ldots 220$

\section{Alamo River at Border (324032115220501)}

\begin{tabular}{|c|c|c|c|c|c|c|c|}
\hline-- & -- & --- & --- & $\cdots$ & $\cdots$ & --- & --- \\
\hline --- & --- & --- & --- & --- & --- & --- & --- \\
\hline 560 & - & 5.3 & --- & 286 & --- & --- & --- \\
\hline --. & -- & --- & --. & 255 & 930 & 520 & --- \\
\hline -.- & -- & --- & --- & 307 & 1,100 & 890 & --- \\
\hline$\cdots$ & 910 & --- & 15 & 318 & 1,100 & 1,300 & --- \\
\hline 940 & --. & 10 & --- & 309 & -. & --- & --- \\
\hline--- & --- & -- & --- & 299 & 1,100 & 960 & -.. \\
\hline-- & --- & --- & --- & 284 & 990 & 980 & $\ldots$ \\
\hline-- & 840 & --- & 13 & 304 & 1,000 & 710 & --- \\
\hline-- & 720 & --- & 11 & 270 & 910 & 940 & 0.8 \\
\hline --- & 960 & -.- & 12 & 301 & 1,200 & 1,300 & .8 \\
\hline$\ldots$ & 870 & --. & 12 & 299 & 1,200 & 1,200 & --- \\
\hline 860 & -- & 11 & --- & 297 & --- & --- & --- \\
\hline --. & 830 & --- & 11 & 294 & 1,100 & 1,100 & --- \\
\hline-- & --- & --- & --- & 295 & 1,200 & 1,100 & --. \\
\hline
\end{tabular}

San Felipe Creek West of Highway 78 (330722115511201)

$\begin{array}{llllllll}- & 2,700 & -- & 11 & 127 & 3,600 & 2,800 & 1.3\end{array}$


Table 5. Data from periodic sampling of streams, East Highline Canal, and Trifolium Drain 1, August 1988-August 1989--Continued

\begin{tabular}{|c|c|c|c|c|c|c|c|c|c|c|}
\hline Date & & $\begin{array}{c}\text { Bromide, } \\
\text { dis- } \\
\text { solved } \\
(\mathrm{mg} / \mathrm{L} \\
\text { as } \mathrm{Br})\end{array}$ & $\begin{array}{l}\text { Silica } \\
\text { dis- } \\
\text { solved } \\
(\mathrm{mg} / \mathrm{L} \\
\text { as } \\
\left.\mathrm{SiO}_{2}\right)\end{array}$ & $\begin{array}{c}\text { Solids, } \\
\text { residue } \\
\text { at } 180 \\
{ }^{\circ} \mathrm{C}, \\
\text { dis- } \\
\text { solved } \\
(\mathrm{mg} / \mathrm{L})\end{array}$ & $\begin{array}{l}\text { Solids, } \\
\text { residue } \\
\text { at } 105 \\
{ }^{\circ} \mathrm{C}, \\
\text { total } \\
(\mathrm{mg} / \mathrm{L})\end{array}$ & $\begin{array}{c}\text { Nitro- } \\
\text { gen, } \\
\mathrm{NO}_{2}+ \\
\mathrm{NO}_{3}, \\
\text { total } \\
(\mathrm{mg} / \mathrm{L} \\
\text { as } \mathrm{N})\end{array}$ & $\begin{array}{c}\text { Nitro- } \\
\text { gen, } \\
\mathrm{NO}_{2}+ \\
\mathrm{NO}_{3}, \\
\text { dis- } \\
\text { solved } \\
(\mathrm{mg} / \mathrm{L} \\
\text { as } \mathrm{N})\end{array}$ & $\begin{array}{l}\text { Nitro- } \\
\text { gen, am- } \\
\text { monia } \\
\text { total } \\
(\mathrm{mg} / \mathrm{L} \\
\text { as } \mathrm{N})\end{array}$ & $\begin{array}{l}\text { Nitro- } \\
\text { gen, am- } \\
\text { monia, } \\
\text { dis- } \\
\text { solved } \\
\text { (mg/L } \\
\text { as } \mathrm{N} \text { ) }\end{array}$ & $\begin{array}{c}\text { Nitro- } \\
\text { gen, am- } \\
\text { monia }+ \\
\text { organic, } \\
\text { dis. } \\
(\mathrm{mg} / \mathrm{L} \\
\text { as } \mathrm{N})\end{array}$ \\
\hline \multicolumn{11}{|c|}{ Alamo River at Outlet $(\mathbf{1 0 2 5 4 7 3 0 )}$} \\
\hline $8-17-88$ & . & -- & -- & --- & -.. & -- & $-\cdots$ & --- & $\ldots$ & --- \\
\hline $10-20-88$ & . & -- & - & -- &.- & -- & 9.5 & $\ldots$ & 0.39 & --- \\
\hline $10-20-88$ & . & -- & -- &.-- & 2,840 & 8.6 & --- & 0.37 & --- & $-\cdots$ \\
\hline $11-14-88$ & . & 0.42 & -- & 2,450 & -- & -- & 11 & -- & .52 & --- \\
\hline $12-16-88$ & . & .20 & -- & 2,240 & -- & -- & 8.4 & -- & 1.3 & $\cdots$ \\
\hline $1-19-89$ & $\ldots$ & .39 & 12 & 2,200 & -- & --- & 7.7 & --- & 2.3 & --- \\
\hline 1-19-89 & $\ldots$ & - & -- & --- & 2,680 & 10 & -- & 2.9 & $\ldots$ & -- \\
\hline $2-16-89$ & . & .33 & --- & 2,070 & --- & -- & 8.4 & --- & 4.4 & --- \\
\hline $3-15-89$ & . & .34 & --- & 2,140 & --- & -- & 9.4 & -- & 4.2 & --- \\
\hline 4-11-89 & . & .36 & 13 & 2,070 & -- & -- & 9.5 & -- & 1.3 & -- \\
\hline $4-11-89$ & . & - &.- & -+- & 2,910 & 8.7 & -- & 1.3 & $\ldots$ & --- \\
\hline $5-22-89$ & . & .29 & 13 & 2,050 & --- & -- & 6.1 & --- & .65 & --- \\
\hline $6-20-89$ & $\ldots$ & .36 & 13 & 2,090 & --- & -- & 6.3 &.- & .94 & $\ldots$ \\
\hline $7-27-89$ & . & .31 & 14 & 2,230 & -- & -- & 6.0 & --- & .54 & --- \\
\hline $7-27-89$ & $\cdots$ & -- & -- & -- & 2,310 & 6.2 & --- & .70 & --- & -- \\
\hline $8-27-89$ & $\ldots$ & .40 & 13 & 2,500 & --- & -- & 5.9 & -- & .83 & 1.8 \\
\hline $8-27-89$ & . & 2.0 & -- & --- & --- & $\cdots$ & --- & --- & -- & --- \\
\hline \multicolumn{11}{|c|}{ Alamo River at Border (324032115220501) } \\
\hline 8-17-88 & . & - & -- & -.- & -.- & -- & $-\cdots$ & --- & --- & -- \\
\hline $10-21-88$ & . & $\ldots$ & --- & -- & --- & $\ldots$ & 15 & $-\cdots$ & 0.14 & --- \\
\hline $10-21-88$ & . & -- & --- & -- & 1,300 & 16 & -- & 0.12 & --- & $\cdots$ \\
\hline $11-13-88$ & . & .49 & --- & 2,540 & --- & -- & 13 & --- & .73 & --- \\
\hline $12-17-88$ & $\cdots$ & .80 & --- & 3,490 & --- & --- & 13 & --- & .12 & -- \\
\hline $1-18-89$ & .. & 1.0 & 15 & 3,940 & --. & -- & 1.7 & -- & 1.0 & --- \\
\hline $1-18-89$ & .. & - & --- & -- & 4,120 & 1.7 & -- & 1.0 & --- & --- \\
\hline $2-15-89$ & . & .78 & -- & 3,580 & -- & --- & 8.0 & -- & .10 & --- \\
\hline $3-16-89$ & . & .75 & -.- & -- & -- & $\cdots$ & 1.3 & --- & .14 & -- \\
\hline $4-14-89$ & . & .87 & 20 & 3,690 & --- & --. & 1.1 & --- & .42 & --- \\
\hline $5-23-89$ & $\ldots$ & .59 & 18 & 3,140 & -.- & --- & .91 & --- & .19 & --- \\
\hline $6-21-89$ & $\ldots$ & .95 & 20 & 4,260 & $\ldots$ & --- & 1.4 & -- & .28 & -- \\
\hline $7-28-89$ & . & 1.0 & 22 & 3,900 & -.. & -.- & 1.6 & -- & .17 & --- \\
\hline $7-28-89$ & . & - & $\ldots$ & -.. & 3,990 & 1.7 & --- & .11 & $\ldots$ & --- \\
\hline $8-28-89$ & . & .90 & 20 & 3,720 & -.- & -- & 1.3 & --- & .13 & .4 \\
\hline $8-28-89$ & . & 1.5 & -- & --- & --- & --- & --- & $\cdots$ & -- & --- \\
\hline \multicolumn{11}{|c|}{ San Felipe Creek West of Highway 78 (330722115511201) } \\
\hline $8-20-88$ & . & -- & 25 &.-- & --- & - & $<.1$ & 0.13 & --- & -- \\
\hline
\end{tabular}


Table 5. Data from periodic sampling of streams, East Highline Canal, and Trifolium Drain 1, August 1988-August 1989--Continued

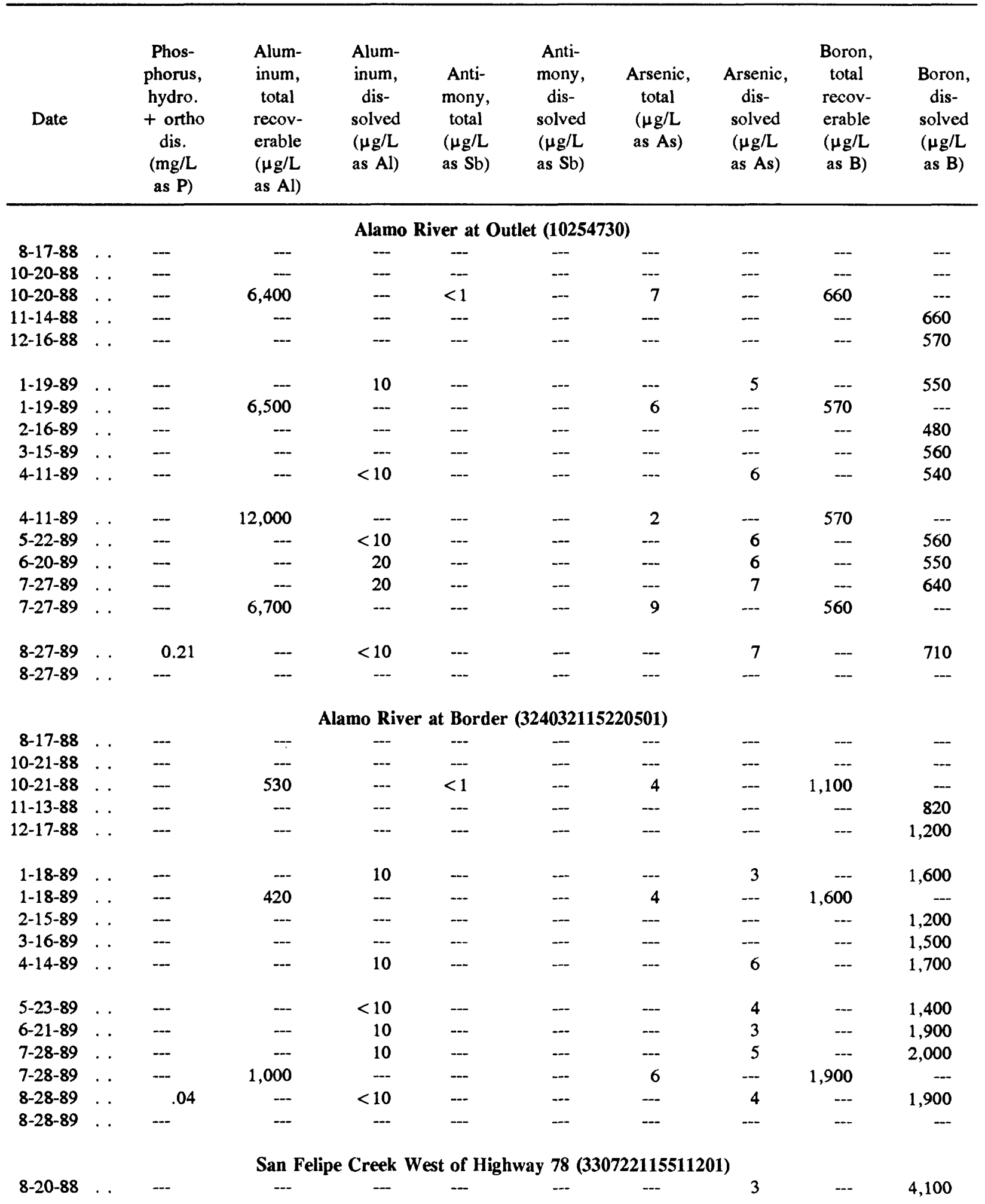


Table 5. Data from periodic sampling of streams, East Highline Canal, and Trifolium Drain 1, August 1988-August 1989--Continued

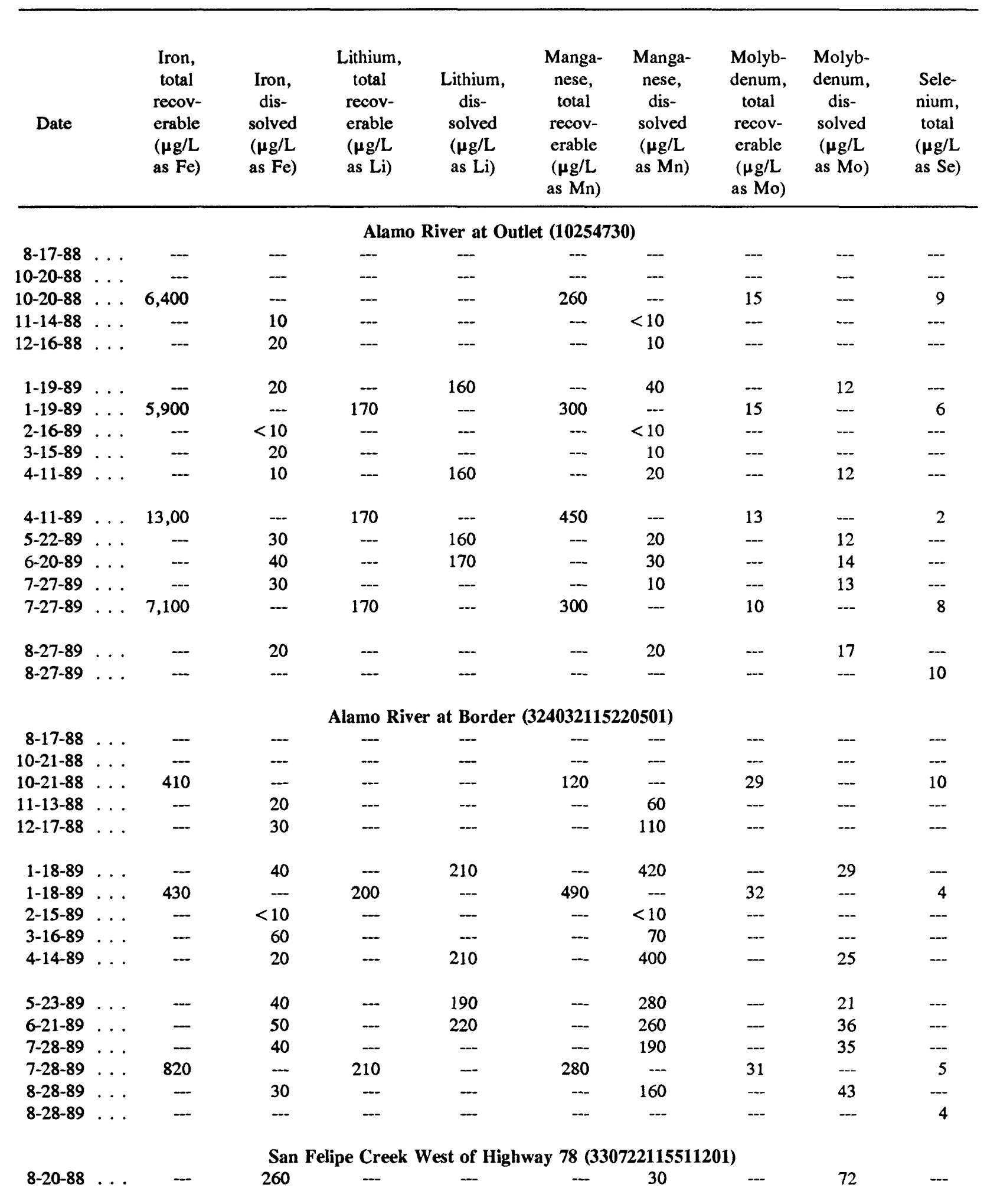


Table 5. Data from periodic sampling of streams, East Highline Canal, and Trifolium Drain 1, August 1988-August 1989--Continued

\begin{tabular}{|c|c|c|c|c|c|c|c|c|c|c|}
\hline \multirow[t]{3}{*}{ Date } & \multirow{3}{*}{$\begin{array}{c}\text { Sele- } \\
\text { nium, } \\
\text { dis- } \\
\text { solved } \\
(\mu \mathrm{g} / \mathrm{L} \\
\text { as } \mathrm{Se})\end{array}$} & \multirow{3}{*}{$\begin{array}{l}\text { Stron- } \\
\text { tium, } \\
\text { total } \\
\text { recov- } \\
\text { erable } \\
(\mu \mathrm{g} / \mathrm{L} \\
\text { as } \mathrm{Sr})\end{array}$} & \multirow{2}{*}{$\begin{array}{c}\text { Stron- } \\
\text { tium, } \\
\text { dis- } \\
\text { solved } \\
\text { ( } \mu \mathrm{g} / \mathrm{L} \\
\text { as } \mathrm{Sr})\end{array}$} & \multirow{2}{*}{$\begin{array}{c}\text { Uran- } \\
\text { ium, } \\
\text { natural } \\
\text { dis- } \\
\text { solved } \\
(\mu \mathrm{g} / \mathrm{L} \\
\text { as } \mathrm{U})\end{array}$} & \multirow{2}{*}{$\begin{array}{c}\text { Carbon, } \\
\text { organic } \\
\text { total } \\
(\mathrm{mg} / \mathrm{L} \\
\text { as C) }\end{array}$} & \multirow{2}{*}{$\begin{array}{c}\text { Sedi- } \\
\text { ment, } \\
\text { sus- } \\
\text { pended } \\
(\mathrm{mg} / \mathrm{L})\end{array}$} & \multirow{3}{*}{$\begin{array}{c}\text { Sedi- } \\
\text { ment, } \\
\text { sus- } \\
\text { pended } \\
\text { (\% finer } \\
\text { than } \\
0.062 \\
\text { mm) }\end{array}$} & \multirow{3}{*}{$\begin{array}{c}\text { Tri- } \\
\text { tium, } \\
\text { total } \\
(\mathrm{pCi} / \mathrm{L})\end{array}$} & \multicolumn{2}{|c|}{$\begin{array}{l}\text { Stable-isotope } \\
\text { ratio (permil) }\end{array}$} \\
\hline & & & & & & & & & ${ }^{2} \mathrm{H} /{ }^{1} \mathrm{H}$ & ${ }^{18} \mathrm{O} /{ }^{16} \mathrm{O}$ \\
\hline & & & \multicolumn{4}{|c|}{ Alamo River at Outlet (10254730) } & & & & \\
\hline $8-17-88$ & -- & $\ldots$ & $\ldots$ & -- & $\ldots$ & --- & $-\ldots$ & 100 & -99.0 & -12.15 \\
\hline 10-20-88 & --- & -- & $\ldots$ & --- & $\ldots$ & --- & -.. & -.. & -96.5 & -11.90 \\
\hline $10-20-88$ & -- & -_- & --- & -- & -_ & 557 & 69 & -.. & --- & --- \\
\hline 11-14-88 & 10 & -- & -- & -.- & -- & -- & -- & --- & -97.5 & -12.15 \\
\hline $12-16-88$ & 8 & --- & --- & -.- & -- & --- & --- & --- & -100.5 & -12.35 \\
\hline $1-19-89$ & 6 & -- & 2,800 & -- & -.. & --- & --- & --. & -101.0 & -12.00 \\
\hline $1-19-89$ & -.- & 3,000 & -. & $\ldots$ & -. & 389 & 94 & -.. & --- & -- \\
\hline 2-16-89 & 7 & -. & -- & -.. & -- & --- & -.- & --- & -88.9 & -11.10 \\
\hline 3-15-89 & 8 & $\ldots$ & $\ldots$ & $\ldots$ & $\ldots$ & -.- & $\ldots$ & --- & -97.5 & -12.00 \\
\hline $4-11-89$ & 8 & -- & 2,800 & --- & - & --- & -- & --- & -99.0 & -12.05 \\
\hline $4-11-89$ & --- & 2,800 & -.- & --- & --. & 849 & 74 & -.. & --- & --- \\
\hline $5-22-89$ & 8 & -- & 2,800 & --- & - & --- & --- & --- & -98.0 & -11.95 \\
\hline $6-20-89$ & 6 & $\ldots$ & 2,700 & -.- & -- & -.. & -.- & --- & -101.0 & -11.90 \\
\hline 7-27-89 & 8 & --- & 2,700 & -.. & -.. & --- & -.. & --- & -97.5 & -11.50 \\
\hline $7-27-89$ & -- & 2,900 & -.- & -.- & --. & 543 & 76 & --- & --- & $-\cdots$ \\
\hline 8-27-89 & 10 & -.- & 3,100 & 17 & 9.3 & -.- & -.- & --- & -96.5 & -11.80 \\
\hline $8-27-89$ & --. & - & -- & -- & -- & -- & -- & --- & --- & --- \\
\hline \multicolumn{11}{|c|}{ Alamo River at Border (324032115220501) } \\
\hline $8-17-88$ & $\ldots$ & --. & -- & ... & --- & - & -.. & 96 & -102.5 & -12.35 \\
\hline $10-21-88$ & -.. & - & -- & --- & -- & $\ldots$ & --- & --- & -98.0 & -11.80 \\
\hline $10-21-88$ & - & -- & --- & --- & --- & -- & -- & --- & --- & --- \\
\hline $11-13-88$ & 8 & -.. & $\ldots$ & ... & --- & -.- & ... & --- & --. & --- \\
\hline $12-17-88$ & 10 & $\cdots$ & - & - & -- & - & --- & -- & -98.0 & -11.70 \\
\hline $1-18-89$ & 3 & - & 4,000 & --- & $\ldots$ & --- & --- & --- & -95.0 & -11.30 \\
\hline $1-18-89$ & - & 4,000 & -- & $\ldots$ & --- & 90 & 58 & --- & $\ldots$ & -.. \\
\hline $2-15-89$ & 8 & - & $\ldots$ & -.- & $\ldots$ & --. & --. & --- & -101.0 & -11.70 \\
\hline $3-16-89$ & 3 & --- & -- & --- & -- & --- & -- & --- & -96.5 & -11.50 \\
\hline $4-14-89$ & 3 & - & 3,900 & --- & $\ldots$ & - & --- & --- & -94.0 & -10.85 \\
\hline $5-23-89$ & 3 & --- & 3,400 & --- & --- & -.- & -- & --- & -92.5 & -10.65 \\
\hline $6-21-89$ & 4 & -- & 4,100 & --- & --- & --- & - & -.. & -93.0 & -10.55 \\
\hline $7-28-89$ & 5 & -- & 3,600 & --- & -- & --- & -.- & --- & -91.5 & -10.55 \\
\hline $7-28-89$ & $\ldots$ & 3,600 & -. & ..- & - & 74 & 58 & --- & $\ldots$ & $\ldots$ \\
\hline $8-28-89$ & 5 & --. & 3,700 & 21 & 7.6 & $\cdots$ & -. & --- & -93.0 & -10.80 \\
\hline $8-28-89$ & - & --. & -- & --- & --- & -- & -- & --- & --- & --- \\
\hline \multicolumn{11}{|c|}{ San Felipe Creek West of Highway 78 (330722115511201) } \\
\hline $8-20-88$ & 17 & --. & - & -- & $\ldots$ & -- & -- & -.- & -59.0 & -6.05 \\
\hline
\end{tabular}


Table 5. Data from periodic sampling of streams, East Highline Canal, and Trifolium Drain 1, August 1988-August 1989--Continued

\begin{tabular}{|c|c|c|c|c|c|c|c|c|c|}
\hline Date & Time & $\begin{array}{c}\text { Gage } \\
\text { height } \\
\text { (ft) }\end{array}$ & $\begin{array}{c}\text { Dis- } \\
\text { charge, } \\
\text { mean } \\
\text { daily } \\
\left(\mathrm{ft}^{3} / \mathrm{s}\right)\end{array}$ & $\begin{array}{l}\text { Spe- } \\
\text { cific } \\
\text { con- } \\
\text { duct- } \\
\text { tance } \\
(\mu \mathrm{S} / \mathrm{cm})\end{array}$ & $\begin{array}{l}\mathrm{pH} \\
\text { (stand- } \\
\text { ard } \\
\text { units) }\end{array}$ & $\begin{array}{l}\text { Temper- } \\
\text { ature, } \\
\text { water } \\
\left({ }^{\circ} \mathrm{C}\right)\end{array}$ & $\begin{array}{c}\text { Calcium, } \\
\text { total } \\
\text { recov- } \\
\text { erable } \\
(\mathrm{mg} / \mathrm{L} \\
\text { as } \mathrm{Ca})\end{array}$ & $\begin{array}{c}\text { Calcium, } \\
\text { dis- } \\
\text { solved } \\
(\mathrm{mg} / \mathrm{L} \\
\text { as } \mathrm{Ca})\end{array}$ & $\begin{array}{c}\text { Magne- } \\
\text { sium, } \\
\text { total } \\
\text { recov- } \\
\text { erable } \\
(\mathrm{mg} / \mathrm{L} \\
\text { as } \mathrm{Mg})\end{array}$ \\
\hline \multicolumn{10}{|c|}{ East Highline Canal (324152115165501) } \\
\hline $10-24-88$ & 1310 & $--\cdot$ & $\cdots$ & 1,120 & 8.00 & 25.0 & $\cdots$ & 76 & $\cdots$ \\
\hline $11-13-88$ & 1005 & --- & --- & 1,010 & 8.10 & 20.0 & -.. & 75 & -.. \\
\hline $12-17-88$ & 0910 & --- & --- & 1,010 & 8.30 & 14.0 & -- & 74 & --- \\
\hline $1-18-89$ & 1300 & -- & --. & 1,250 & --- & 11.0 & -. & 87 & -.. \\
\hline $1-18-89$ & 1301 & --- & -- & 1,240 & 7.70 & 11.0 & 76 & --- & 32 \\
\hline $2-15-89$ & 1520 & $\cdots$ & -- & 1,070 & 8.40 & 13.5 & -- & 77 & -- \\
\hline $3-16-89$ & 1055 & $\ldots$ & --- & 947 & 8.30 & 19.0 & ... & 71 & --- \\
\hline $4-11-89$ & 1340 & - & --- & 1,060 & 8.23 & 23.0 & -- & 77 & -- \\
\hline $4-11-89$ & 1354 & -- & -- & 1,060 & 8.33 & 23.0 & 73 & --. & 29 \\
\hline $5-23-89$ & 1050 & - & -- & 1,080 & -- & 25.0 & -- & 82 & $\cdots$ \\
\hline $6-21-89$ & 0800 & -- & $\cdots$ & 1,020 & 8.30 & 26.0 & $\cdots$ & 74 & --- \\
\hline $7-28-89$ & 1420 & $\cdots$ & - & 1,050 & 8.33 & $\cdots$ & --- & 74 & $\cdots$ \\
\hline $7-28-89$ & 1421 & - & - & 1,060 & 8.27 & 30.0 & 65 & --- & 27 \\
\hline $8-28-89$ & 0800 & - & - & 1,100 & 8.31 & 26.0 & -- & 77 & -- \\
\hline \multicolumn{10}{|c|}{ New River at Border (10254970) } \\
\hline $8-17-88$ & 1020 & -- & 301 & 4,590 & -- & -- & -- & --- & --- \\
\hline $10-21-88$ & 1030 & 9.63 & 239 & 4,300 & 7.30 & 26.0 & --- & -- & -- \\
\hline $10-21-88$ & 1031 & 9.63 & 239 & 4,340 & 7.40 & 26.0 & 170 & -.. & 77 \\
\hline 11-13-88 & 0825 & -- & 215 & 4,130 & 7.40 & 22.0 & --- & 160 & -- \\
\hline 12-17-88 & 1030 & -- & 296 & 3,690 & 7.70 & 16.0 & -- & 160 & --- \\
\hline $1-18-89$ & 1030 & 9.37 & 257 & 4,210 & 7.80 & 15.0 & -- & 160 & --- \\
\hline $1-18-89$ & 1031 & 9.37 & 257 & 4,260 & 7.50 & 15.0 & 160 & --- & 80 \\
\hline $2-15-89$ & 1345 & 9.34 & 242 & 4,300 & 7.90 & 15.5 & --- & 190 &.- \\
\hline $3-16-89$ & 1200 & 9.24 & 242 & 4,920 & 7.90 & 20.0 & --- & 190 & -- \\
\hline 4-11-89 & 1510 & 9.08 & 225 & 5,420 & 7.92 & 27.5 & -- & 220 & -- \\
\hline $4-11-89$ & 1515 & 9.08 & 225 & 5,440 & 7.34 & 27.5 & 190 & --- & 96 \\
\hline $5-23-89$ & .1230 & 9.94 & 212 & 5,230 & 7.90 & 25.0 & --- & 190 & -- \\
\hline $6-21-89$ & 0930 & 8.76 & 194 & 4,750 & 7.60 & 28.0 & --- & 190 & $\cdots$ \\
\hline $7-28-89$ & 1045 & 8.62 & 192 & 4,980 & 7.71 & 30.5 & $\cdots$ & 190 & - \\
\hline $7-28-89$ & 1046 & -- & 192 & 5,000 & 7.29 & 30.5 & 180 & --- & 96 \\
\hline $8-28-89$ & 0920 & 9.15 & 221 & 4,390 & 7.79 & --. & -- & 170 & -- \\
\hline $8-28-89$ & 0921 & - & 221 & 4,400 & 7.62 & --- & --- & --- & -- \\
\hline
\end{tabular}


Table 5. Data from periodic sampling of streams, East Highline Canal, and Trifolium Drain 1, August 1988-August 1989--Continued

\begin{tabular}{|c|c|c|c|c|c|c|c|c|c|c|}
\hline Date & & $\begin{array}{c}\text { Magne- } \\
\text { sium, } \\
\text { dis- } \\
\text { solved } \\
(\mathrm{mg} / \mathrm{L} \\
\text { as } \mathrm{Mg})\end{array}$ & $\begin{array}{c}\text { Sodium, } \\
\text { total } \\
\text { recov- } \\
\text { erable } \\
(\mathrm{mg} / \mathrm{L} \\
\text { as } \mathrm{Na})\end{array}$ & $\begin{array}{c}\text { Sodium, } \\
\text { dis- } \\
\text { solved } \\
(\mathrm{mg} / \mathrm{L} \\
\text { as } \mathrm{Na})\end{array}$ & $\begin{array}{l}\text { Potas- } \\
\text { sium, } \\
\text { total } \\
\text { recov- } \\
\text { erable } \\
\text { (mg/L } \\
\text { as } \mathrm{K})\end{array}$ & $\begin{array}{c}\text { Potas- } \\
\text { sium, } \\
\text { dis- } \\
\text { solved } \\
(\mathrm{mg} / \mathrm{L} \\
\text { as } \mathrm{K})\end{array}$ & $\begin{array}{c}\text { Alka- } \\
\text { linity, } \\
\text { lab } \\
(\mathrm{mg} / \mathrm{L} \\
\text { as } \\
\left.\mathrm{CaCO}_{3}\right)\end{array}$ & $\begin{array}{c}\text { Sulfate, } \\
\text { dis- } \\
\text { solved } \\
(\mathrm{mg} / \mathrm{L} \\
\left.{\left.\text { as } \mathrm{SO}_{4}\right)}\right)\end{array}$ & $\begin{array}{l}\text { Chlo- } \\
\text { ride, } \\
\text { dis- } \\
\text { solved } \\
(\mathrm{mg} / \mathrm{L} \\
\text { as } \mathrm{Cl})\end{array}$ & $\begin{array}{c}\text { Fluo- } \\
\text { ride, } \\
\text { dis- } \\
\text { solved } \\
(\mathrm{mg} / \mathrm{L} \\
\text { as } \mathrm{F})\end{array}$ \\
\hline \multicolumn{11}{|c|}{ East Highline Canal (324152115165501) } \\
\hline $10-24-88$ & $\ldots$ & 31 & $\ldots$ & 110 & -. & 4.1 & 152 & 290 & 100 & 0.3 \\
\hline $11-13-88$ & . & --. & -.. & -.. & -.- & ... & 140 & 260 & 84 & --- \\
\hline $12-17-88$ & .. & -.- & $\ldots$ & --- & -.- & -.- & 145 & 260 & 88 & ... \\
\hline $1-18-89$ & $\ldots$ & 31 & - & 130 & --- & 4.4 & 162 & 300 & 120 & --- \\
\hline $1-18-89$ & . & -- & 130 & - & 2.8 & -- & 163 & --- & -- & -- \\
\hline $2-15-89$ & $\ldots$ & -- & -.. & --- & --- & ... & 146 & 280 & 93 & --- \\
\hline $3-16-89$ & $\ldots$ & - & --- & --- & --- & --- & 139 & 250 & 72 & --- \\
\hline $4-11-89$ & $\ldots$ & 29 & ..- & 110 & $\ldots$ & 3.9 & 147 & 260 & 92 & ... \\
\hline $4-11-89$ & $\ldots$ & --- & 100 & --. & 2.9 & --- & 147 & -.- & --. & --- \\
\hline $5-23-89$ & . & 30 & $\ldots$ & 100 & -- & 4.3 & 152 & 270 & 93 & .3 \\
\hline $6-21-89$ & $\ldots$ & 27 & - & 93 & -.- & 4.3 & 143 & 260 & 84 & .3 \\
\hline $7-28-89$ & $\ldots$ & 28 & $\ldots$ & 100 & $\ldots$ & 4.5 & 143 & 270 & 90 & -- \\
\hline $7-28-89$ & $\ldots$ & -.. & 91 & $\ldots$ & 3.6 & $\ldots$ & 143 & $\ldots$ & --- & --- \\
\hline $8-28-89$ & . & 29 & $\ldots$ & 110 & -- & 4.4 & 139 & 280 & 97 & -- \\
\hline \multicolumn{11}{|c|}{ New River at Border (10254970) } \\
\hline $8-17-88$ & $\ldots$ & -.. & -.. & -.. & -.. & -.. & --- & -.- & -.. & $\ldots$ \\
\hline $10-21-88$ & $\ldots$ & $\ldots$ & ... & - & --- & -.- & -.- & -.- & --- & -.- \\
\hline $10-21-88$ & $\ldots$ & --- & 610 & --. & 19 & $\ldots$ & 224 & --- & -- & --. \\
\hline $11-13-88$ & $\ldots$ & -.. & --. & -..- & ... & -.- & 248 & 620 & 930 & -.. \\
\hline $12-17-88$ & . & -- & --- & --. & --- & --- & 236 & 570 & 780 & --- \\
\hline $1-18-89$ & . & 79 & -.. & 580 & --- & 16 & 266 & 630 & 880 & --- \\
\hline $1-18-89$ & . & --. & 590 & ... & 16 & --- & 247 & --- & $\ldots$ & -.- \\
\hline $2-15-89$ & . & -.. & -.. & -.. & -.- & --- & 248 & 660 & 950 & --.- \\
\hline $3-16-89$ & . & $\ldots$ & $\ldots$ & -..- & ... & -.- & 250 & 740 & 1,100 & -.- \\
\hline $4-11-89$ & . & 120 & -.- & 800 & --- & 13 & 253 & 750 & 1,300 & -- \\
\hline $4-11-89$ & $\ldots$ & -- & 680 & --- & 29 & --. & 249 & --- & --- & $\ldots$ \\
\hline $5-23-89$ & . . & 110 & -.- & 770 & -.. & 30 & 251 & 730 & 1,200 & .6 \\
\hline $6-21-89$ & $\ldots$ & 99 & --. & 700 & -.. & 27 & 238 & 660 & 1,100 & .5 \\
\hline $7-28-89$ & $\ldots$ & 100 & -.- & 740 & ... & 24 & 229 & 760 & 1,100 & .-. \\
\hline $7-28-89$ & $\ldots$ & --- & 680 & --. & 24 & -.. & 225 & -.. & --- & --- \\
\hline $8-28-89$ & $\ldots$ & 85 & --- & 600 & --- & 19 & 232 & 680 & 870 & ..- \\
\hline $8-28-89$ & $\ldots$ & -- & --. & -.. &.-- & -.. & 226 & 690 & 850 & ---- \\
\hline
\end{tabular}


Table 5. Data from periodic sampling of streams, East Highline Canal, and Trifolium Drain 1, August 1988-August 1989--Continued

\begin{tabular}{|c|c|c|c|c|c|c|c|c|c|}
\hline Date & $\begin{array}{c}\text { Bromide, } \\
\text { dis- } \\
\text { solved } \\
(\mathrm{mg} / \mathrm{L} \\
\text { as } \mathrm{Br})\end{array}$ & $\begin{array}{c}\begin{array}{c}\text { Silica, } \\
\text { dis- } \\
\text { solved }\end{array} \\
(\mathrm{mg} / \mathrm{L} \\
\text { as } \\
\left.\mathrm{SiO}_{2}\right)\end{array}$ & $\begin{array}{l}\text { Solids, } \\
\text { residue } \\
\text { at } 180 \\
{ }^{\circ} \mathrm{C}, \\
\text { dis- } \\
\text { solved } \\
(\mathrm{mg} / \mathrm{L})\end{array}$ & $\begin{array}{l}\text { Solids, } \\
\text { residue } \\
\text { at } 105 \\
{ }^{\circ} \mathrm{C} \text {, } \\
\text { total } \\
(\mathrm{mg} / \mathrm{L})\end{array}$ & $\begin{array}{c}\text { Nitro- } \\
\text { gen, } \\
\mathrm{NO}_{2}+ \\
\mathrm{NO}_{3}, \\
\text { total } \\
\text { (mg/L } \\
\text { as } \mathrm{N})\end{array}$ & $\begin{array}{c}\text { Nitro- } \\
\text { gen, } \\
\mathrm{NO}_{2}+ \\
\mathrm{NO}_{3} \text {, } \\
\text { dis- } \\
\text { solved } \\
(\mathrm{mg} / \mathrm{L} \\
\text { as } \mathrm{N})\end{array}$ & $\begin{array}{c}\text { Nitro- } \\
\text { gen, am- } \\
\text { monia, } \\
\text { total } \\
(\mathrm{mg} / \mathrm{L} \\
\text { as } \mathrm{N})\end{array}$ & $\begin{array}{c}\text { Nitro- } \\
\text { gen, am- } \\
\text { monia, } \\
\text { dis- } \\
\text { solved } \\
\text { (mg/L } \\
\text { as } \mathrm{N})\end{array}$ & $\begin{array}{l}\text { Nitro- } \\
\text { gen, am- } \\
\text { monia + } \\
\text { organic, } \\
\text { dis. } \\
(\mathrm{mg} / \mathrm{L} \\
\text { as } \mathrm{N})\end{array}$ \\
\hline \multicolumn{10}{|c|}{ East Highline Canal (324152115165501) } \\
\hline 10-24-88 & -.- & 11 & 737 & --- & -.- & 0.17 & -.. & 0.02 & --- \\
\hline $11-13-88$ & 0.06 & --- & 653 & --- & --- & .18 & --- & .04 & --- \\
\hline $12-17-88$ & .06 & --- & 678 & -.. & --- & .24 & -.- & .03 & --- \\
\hline $1-18-89$ & .07 & 11 & 800 & --- & -.- & .20 & --. & .04 & --- \\
\hline $1-18-89$ & - & -- & --- & 837 & 0.20 & --- & $<0.01$ & --- & -- \\
\hline $2-15-89$ & .06 & --- & 698 & -.. & --- & .31 & --. & .03 & -.. \\
\hline $3-16-89$ & $<.05$ & --- & -.. & --- & -.. & .27 & --- & .04 & -.- \\
\hline $4-11-89$ & .06 & 10 & 687 & --- & --- & .31 & -.- & .04 & --- \\
\hline $4-11-89$ & $\ldots$ & ... & --. & 742 & .30 & $\ldots$ & .03 & --- & --- \\
\hline $5-23-89$ & .06 & 9.8 & 700 & --- & --- & .27 & --- & .02 & --- \\
\hline $6-21-89$ & .06 & 9.3 & 674 & --- & $\ldots$ & .22 & --. & .05 & --- \\
\hline $7-28-89$ & .06 & 10 & 683 & $\ldots$ & --- & .20 & -.. & .01 & -.- \\
\hline $7-28-89$ & -- & -. & --- & 697 & .20 & --- & $<.01$ & --- & --- \\
\hline $8-28-89$ & .07 & 10 & 685 & --- & -- & .15 & --- & .02 & 0.2 \\
\hline \multicolumn{10}{|c|}{ New River at Border (10254970) } \\
\hline $8-17-88$ & -- & -_ & --- & -.. & -.. & -.- & -.- & --- & -.- \\
\hline $10-21-88$ & -.- & -- & --. & --- & $\ldots$ & 0.27 & --- & 3.7 & -.- \\
\hline $10-21-88$ & -.- & -- & -- & 2,760 & 0.30 & $\ldots$ & 4.9 & $\ldots$ & ... \\
\hline $11-13-88$ & 1.2 & -. & 2,580 & --- & $\ldots$ & .50 & --- & 3.8 & --- \\
\hline $12-17-88$ & .91 & - & 2,350 & $\ldots$ & --- & .73 & --- & 4.2 & --- \\
\hline $1-18-89$ & 1.0 & 16 & 2,630 & --- & ... & .70 & --- & 5.8 & ... \\
\hline $1-18-89$ & -.- & --- & -.. & 2,790 & .70 & $\ldots$ & 5.9 & -.- & --- \\
\hline $2-15-89$ & 1.1 &.-- & 2,670 & --- & --- & .84 & --- & 7.6 & --- \\
\hline $3-16-89$ & 1.4 & -.- & -.. & -.- & -.- & .50 & ---- & 5.2 & --. \\
\hline $4-11-89$ & 1.6 & 20 & 3,370 & --- & --- & .34 & -.- & 5.5 & $\ldots$ \\
\hline $4-11-89$ & $\ldots$ & --- & -.. & 3,620 & .40 & --- & 4.9 & --- & -.. \\
\hline $5-23-89$ & .45 & 16 & 3,270 & -.- & $\ldots$ & .23 & --- & 3.0 & --- \\
\hline $6-21-89$ & 1.3 & 17 & 3,050 & --- &.-- & .19 & --- & 3.3 &.-- \\
\hline $7-28-89$ & 1.4 & 20 & 3,150 & ..- & -.- & $<.1$ & -.. & 1.9 & $\ldots$ \\
\hline $7-28-89$ & - & -- & -- & 3,280 & $<.1$ & --- & 3.2 & -- & --- \\
\hline $8-28-89$ & 1.0 & 20 & 2,590 & --- & --- & .19 & -.. & 2.4 & 1.9 \\
\hline $8-28-89$ & 1.2 & $\ldots$ & -.- & -.. & $\ldots$ & -.- & --- & --- & --- \\
\hline
\end{tabular}


Table 5. Data from periodic sampling of streams, East Highline Canal, and Trifolium Drain 1, August 1988-August 1989--Continued

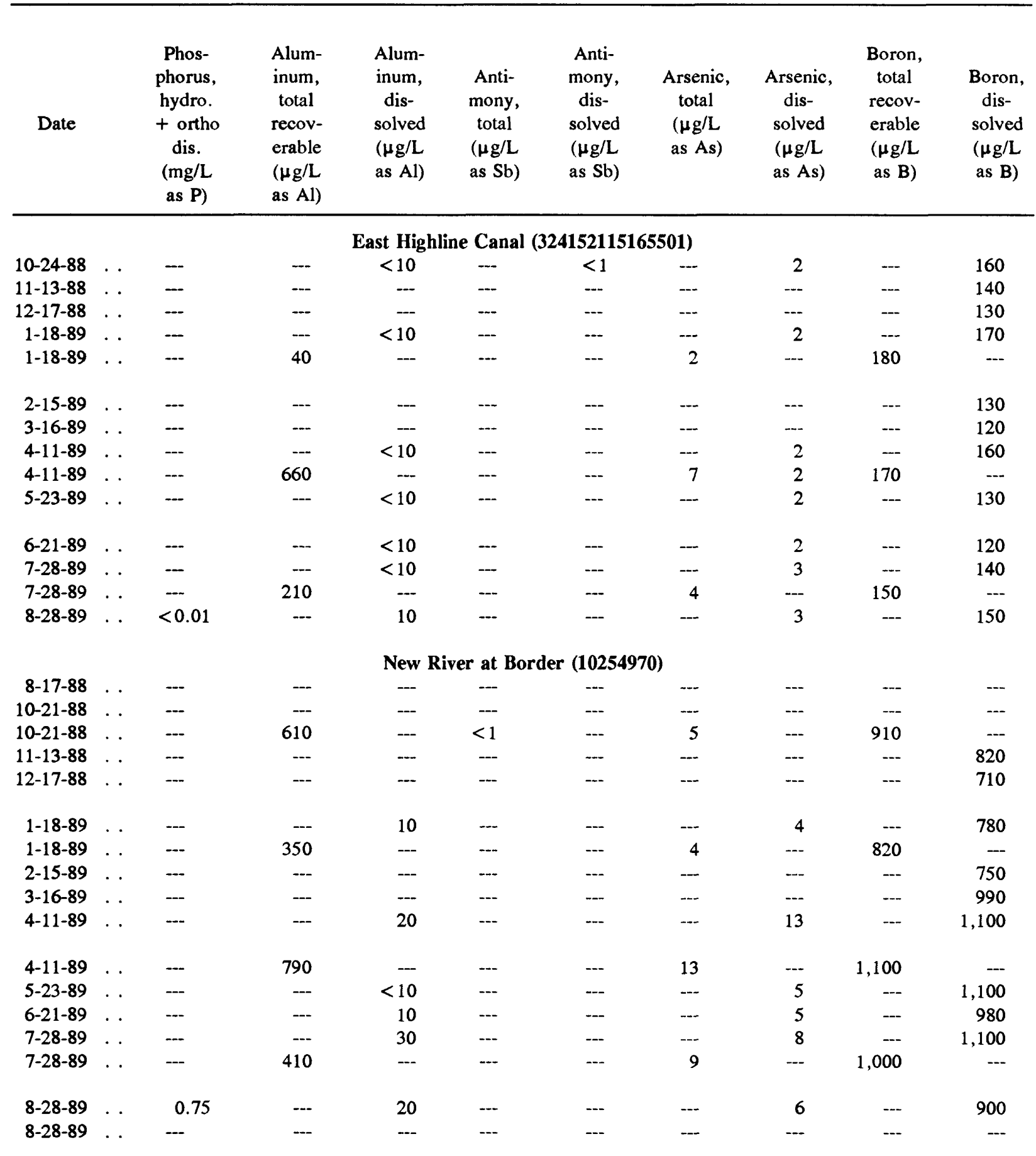


Table 5. Data from periodic sampling of streams, East Highline Canal, and Trifolium Drain 1, August 1988-August 1989--Continued

\begin{tabular}{|c|c|c|c|c|c|c|c|c|c|}
\hline Date & $\begin{array}{l}\text { Iron, } \\
\text { total } \\
\text { recov- } \\
\text { erable } \\
(\mu \mathrm{g} / \mathrm{L} \\
\text { as } \mathrm{Fe})\end{array}$ & $\begin{array}{c}\text { Iron, } \\
\text { dis- } \\
\text { solved } \\
(\mu \mathrm{g} / \mathrm{L} \\
\text { as } \mathrm{Fe})\end{array}$ & $\begin{array}{c}\text { Lithium, } \\
\text { total } \\
\text { recov- } \\
\text { erable } \\
(\mu \mathrm{g} / \mathrm{L} \\
\text { as } \mathrm{Li})\end{array}$ & $\begin{array}{c}\text { Lithium, } \\
\text { dis- } \\
\text { solved } \\
(\mu \mathrm{g} / \mathrm{L} \\
\text { as } \mathrm{Li})\end{array}$ & $\begin{array}{c}\text { Manga- } \\
\text { nese, } \\
\text { total } \\
\text { recov- } \\
\text { erable } \\
(\mu \mathrm{g} / \mathrm{L} \\
\text { as } \mathrm{Mn})\end{array}$ & $\begin{array}{l}\text { Manga- } \\
\text { nese, } \\
\text { dis- } \\
\text { solved } \\
(\mu \mathrm{g} / \mathrm{L} \\
\text { as Mn) }\end{array}$ & $\begin{array}{c}\text { Molyb- } \\
\text { denum, } \\
\text { total } \\
\text { recov- } \\
\text { erable } \\
(\mu \mathrm{g} / \mathrm{L} \\
\text { as Mo) }\end{array}$ & $\begin{array}{c}\text { Molyb- } \\
\text { denum, } \\
\text { dis- } \\
\text { solved } \\
(\mu \mathrm{g} / \mathrm{L} \\
\text { as Mo) }\end{array}$ & $\begin{array}{l}\text { Sele- } \\
\text { nium, } \\
\text { total } \\
(\mu \mathrm{g} / \mathrm{L} \\
\text { as } \mathrm{Se})\end{array}$ \\
\hline \multicolumn{10}{|c|}{ East Highline Canal (324152115165501) } \\
\hline $10-24-88$ & $\ldots$ & 3 & ... & $\ldots$ & -.. & $<10$ &.-- & 4 & -.- \\
\hline $11-13-88$ & -- & 13 & --- & --- & -.. & 3 & --- & -.. & --- \\
\hline $12-17-88$ & - & $<3$ & ... & ... & ... & 1 & --- & --- & ... \\
\hline $1-18-89$ & - & 3 & --. & 58 & --- & 4 & --- & 7 & --- \\
\hline $1-18-89$ & 50 & $\ldots$ & 60 & -- & 20 & - & 14 & -- & 8 \\
\hline $2-15-89$ & --- & $<3$ & --- & -- & --- & 1 & -- & --- & --- \\
\hline $3-16-89$ & $\ldots$ & 210 & -.. & $\ldots$ & --- & 33 & --- & --- & --. \\
\hline $4-11-89$ & -- & 5 & --- & 49 & --- & 2 & --- & 6 & --- \\
\hline $4-11-89$ & 630 & --- & 50 & -.- & 60 & --- & 8 & --- & 8 \\
\hline 5-23-89 & --- & 4 & -- & 47 & -- & 2 & -- & 4 & -- \\
\hline $6-21-89$ & ...- & 8 & $\ldots$ & 43 & $\ldots$ & 2 & $\ldots$ & 5 & $\ldots$ \\
\hline $7-28-89$ & $\ldots$ & 5 & ... & --- & --- & $<1$ & --- & 4 & $\ldots$ \\
\hline $7-28-89$ & 200 & $\ldots$ & 50 & $\ldots$ & 30 & -.. & 4 & $\ldots$ & 2 \\
\hline $8-28-89$ & -- & 10 & -- & -- & -- & $<10$ & -- & 6 & 2 \\
\hline \multicolumn{10}{|c|}{ New River at Border (10254970) } \\
\hline 8-17-88 & $\ldots$ & --- & $\ldots$ & -.. & -- & --- & -- & --- & --- \\
\hline $10-21-88$ & -.- & -.- & -.- & -- & -- & --- & -- & --- & --- \\
\hline $10-21-88$ & 560 & -.- & --- & --- & 100 & -.- & 14 & -.. & 2 \\
\hline $11-13-88$ & -.. & 20 & --- & -- & -_ & 50 & -.- & $\ldots$ & --- \\
\hline $12-17-88$ & -- & 30 & $-\ldots$ & $\ldots$ & $\ldots$ & 60 & $\ldots$ & --- & --- \\
\hline $1-18-89$ & -. & 30 & -.- & 300 & -- & 90 & $\ldots$ & 5 & $\ldots$ \\
\hline $1-18-89$ & 480 & --- & 290 & --- & 130 & -- & 9 & -- & 2 \\
\hline $2-15-89$ & -.- & $<10$ & --- & --. & --- & $<10$ & -- & -.- & --- \\
\hline $3-16-89$ & $\ldots$ & 60 & $\ldots$ & --. & $\ldots$ & 160 & --- & --- & --- \\
\hline 4-11-89 & -- & 30 & - & 540 & - & 170 & -- & 5 & -- \\
\hline $4-11-89$ & 1,000 & -.. & 510 & -- & 220 & -.. & 11 & --- & 2 \\
\hline $5-23-89$ & -.. & 40 & --- & 480 & -- & 160 & -.- & 4 & -- \\
\hline $6-21-89$ & --- & 40 & -.- & 440 & ... & 130 & ... & 5 & -- \\
\hline $7-28-89$ & --- & 50 & --- & -.- & --- & 110 & --- & 6 & --- \\
\hline $7-28-89$ & 720 & - & 380 & $\cdots$ & 160 & --- & 9 & $\cdots$ & 2 \\
\hline $8-28-89$ & -.- & 30 & $\ldots$ & -.. & --- & 70 & -.. & 8 & ... \\
\hline $8-28-89$ & --- & -- & --- & -- & -.. & -.. & --- & --- & 1 \\
\hline
\end{tabular}


Table 5. Data from periodic sampling of streams, East Highline Canal, and Trifolium Drain 1, August 1988-August 1989--Continued

\begin{tabular}{|c|c|c|c|c|c|c|c|c|c|c|c|}
\hline \multirow[t]{2}{*}{ Date } & & \multirow{2}{*}{$\begin{array}{c}\text { Sele- } \\
\text { nium, } \\
\text { dis- } \\
\text { solved } \\
(\mu \mathrm{g} / \mathrm{L} \\
\text { as } \mathrm{Se})\end{array}$} & \multirow{2}{*}{$\begin{array}{l}\text { Stron- } \\
\text { tium, } \\
\text { total } \\
\text { recov- } \\
\text { erable } \\
(\mu \mathrm{g} / \mathrm{L} \\
\text { as } \mathrm{Sr})\end{array}$} & \multirow{2}{*}{$\begin{array}{l}\text { Stron- } \\
\text { tium, } \\
\text { dis- } \\
\text { solved } \\
(\mu \mathrm{g} / \mathrm{L} \\
\text { as } \mathrm{Sr})\end{array}$} & \multirow{2}{*}{$\begin{array}{l}\text { Uran- } \\
\text { ium, } \\
\text { natural } \\
\text { dis- } \\
\text { solved } \\
(\mu \mathrm{g} / \mathrm{L} \\
\text { as } \mathrm{U})\end{array}$} & \multirow{2}{*}{$\begin{array}{c}\text { Carbon, } \\
\text { organic } \\
\text { total } \\
\text { (mg/L } \\
\text { as C) }\end{array}$} & \multirow{2}{*}{$\begin{array}{l}\text { Sedi- } \\
\text { ment, } \\
\text { sus- } \\
\text { pended } \\
\text { (mg/L) }\end{array}$} & \multirow{3}{*}{$\begin{array}{l}\text { Sedi- } \\
\text { ment, } \\
\text { sus- } \\
\text { pended } \\
\text { (\% finer } \\
\text { than } \\
0.062 \\
\mathrm{~mm} \text { ) }\end{array}$} & \multirow{3}{*}{$\begin{array}{c}\text { Tri- } \\
\text { tium, } \\
\text { total } \\
(\mathrm{pCi} / \mathrm{L})\end{array}$} & \multicolumn{2}{|c|}{$\begin{array}{l}\text { Stable-isotope } \\
\text { ratio (permil) } \\
\end{array}$} \\
\hline & & & & & & & & & & ${ }^{2} \mathrm{H} /{ }^{1} \mathrm{H}$ & ${ }^{18} \mathrm{O} /{ }^{16} \mathrm{O}$ \\
\hline \multicolumn{10}{|c|}{ East Highline Canal (324152115165501) } & & \\
\hline $10-24-88$ & . & 3 & - & --- & - & -- & --- & -- & -- & -102.0 & -13.00 \\
\hline $11-13-88$ & . & 2 & $\ldots$ & -- & - & $\ldots$ & --- & -- & $\ldots$ & -103.0 & -12.80 \\
\hline $12-17-88$ & . & 2 & - & $\ldots$ & -- & --- & --- & -- & -- & -104.5 & -13.10 \\
\hline $1-18-89$ & . & 2 & -- & 1,200 & -- & --- & --- & -- & --- & -102.0 & -12.70 \\
\hline $1-18-89$ & . & - & 1,200 & -- & --- & --- & -- & --- & --- & --- & -- \\
\hline $2-15-89$ & . & 2 & -- & -- & --- &.-- & --- & --- & $\ldots$ & -100.0 & -12.90 \\
\hline $3-16-89$ & . & 2 & -- & - & -- & --- & --- & -- & --- & -103.0 & -13.10 \\
\hline 4-11-89 & . & 2 & -- & 1,100 & --- & --- & --- & -- & --- & -103.5 & -12.95 \\
\hline 4-11-89 & . & -- & 1,100 & -- & -- & -- & 86 & 76 & --- & --- & --- \\
\hline $5-23-89$ & . & 2 & - & 1,100 & -- & --- & -- & -- & --- & -102.5 & -12.80 \\
\hline $6-21-89$ & . . & 2 & --- & 1,000 & --- & --- & --- & --- & $\ldots$ & -101.0 & -13.00 \\
\hline $7-28-89$ & $\ldots$ & 2 & -- & 1,100 & --- & --- & $\cdots$ & -- & --- & -103.0 & -12.80 \\
\hline $7-28-89$ & . & --. & 1,000 & -- & -- & --- & --- & -- & -- & --- & --- \\
\hline $8-28-89$ & . & 2 & -- & 1,000 & 22 & 3.2 & --- & -- & --- & -102.5 & -12.65 \\
\hline \multicolumn{12}{|c|}{ New River at Border (10254970) } \\
\hline $8-17-88$ & . & -- & - & -- & -- &.-- &.-- & -- & 88 & -96.5 & -11.55 \\
\hline $10-21-88$ & . & -- & -- & -- & - & --- &.$-->$ & --- & -- & -96.5 & -11.75 \\
\hline $10-21-88$ & . & -- & - & -- & - & -- & 48 & 63 & --- & --- & $-\ldots$ \\
\hline $11-13-88$ & . & 2 & -- & -- & -- & -- & --- & -- & -- & -96.5 & -11.65 \\
\hline $12-17-88$ & . & 2 & - & -- & -- & -- & --- & -- & -- & -99.0 & -12.10 \\
\hline $1-18-89$ & . & 2 & -. & 2,900 & --- & --- & --- & --- & --- & -99.0 & -11.85 \\
\hline $1-18-89$ & . & -- & 2,900 & --- & -- & --- & 34 & 59 & -- & --- & -.- \\
\hline $2-15-89$ & . & 1 & -- & --- & - & -- & -- & -- & --- & -85.0 & -10.35 \\
\hline $3-16-89$ & . & 2 & - & -- & -- & --- & --- & --- & --- & -98.0 & -11.65 \\
\hline $4-11-89$ & . & 2 & -- & 3,700 & --- & $-\cdots$ & $\cdots$ & -- & -- & -97.0 & -11.35 \\
\hline $4-11-89$ & . . & - & 3,300 & -.. & $\ldots$ & $\ldots$ & 49 & 71 & -- & --- & --- \\
\hline $5-23-89$ & $\ldots$ & 1 & - & 3,700 & --- & $\cdots$ & -- & -- & -- & -94.0 & -10.85 \\
\hline $6-21-89$ & . & 1 &.-- & 3,200 & -- & --- & --- & -- & -- & -97.0 & -10.80 \\
\hline $7-28-89$ & . & 2 & -- & 3,300 & -- & --- & --- & -- & -.. & -89.5 & -10.25 \\
\hline $7-28-89$ & . & -- & 3,400 & -- & --- & -- & 41 & 54 & -- & -- & $\cdots$ \\
\hline $8-28-89$ & . & 1 & -- & 2,800 & 8.4 & 17 & --- & -- &.- & -94.0 & -11.15 \\
\hline 8-28-89 & . & -- & - & - & -- & -- & --- & --- & -- & --- & --- \\
\hline
\end{tabular}


Table 5. Data from periodic sampling of streams, East Highline Canal, and Trifolium Drain 1, August 1988-August 1989--Continued

\begin{tabular}{|c|c|c|c|c|c|c|c|c|c|}
\hline Date & Time & $\begin{array}{c}\text { Gage } \\
\text { height } \\
\text { (ft) }\end{array}$ & $\begin{array}{c}\text { Dis- } \\
\text { charge, } \\
\text { mean } \\
\text { daily } \\
\left(\mathrm{ft}^{3} / \mathrm{s}\right)\end{array}$ & $\begin{array}{l}\text { Spe- } \\
\text { cific } \\
\text { con- } \\
\text { duct- } \\
\text { tance } \\
(\mu \mathrm{S} / \mathrm{cm})\end{array}$ & $\begin{array}{l}\mathrm{pH} \\
\text { (stand- } \\
\text { ard } \\
\text { units) }\end{array}$ & $\begin{array}{l}\text { Temper- } \\
\text { ature, } \\
\text { water } \\
\left({ }^{\circ} \mathrm{C}\right)\end{array}$ & $\begin{array}{c}\text { Calcium, } \\
\text { total } \\
\text { recov- } \\
\text { erable } \\
(\mathrm{mg} / \mathrm{L} \\
\text { as Ca) }\end{array}$ & $\begin{array}{l}\text { Calcium, } \\
\text { dis- } \\
\text { solved } \\
(\mathrm{mg} / \mathrm{L} \\
\text { as Ca) }\end{array}$ & $\begin{array}{c}\text { Magne- } \\
\text { sium, } \\
\text { total } \\
\text { recov- } \\
\text { erable } \\
\text { (mg/L } \\
\text { as } \mathrm{Mg})\end{array}$ \\
\hline \multicolumn{10}{|c|}{ New River at Outlet (1025550) } \\
\hline $8-17-88$ & 1225 & -- & 655 & 4,500 & $\cdots$ & -- & -- & --- & -- \\
\hline $10-20-88$ & 1730 & --- & 647 & 4,360 & 7.70 & 25.5 & -.- & --- & -- \\
\hline $10-20-88$ & 1731 & -- & 647 & 4,380 & 7.60 & 25.5 & 190 & -.. & 90 \\
\hline $11-14-88$ & 1120 & -..- & 580 & 4,590 & 7.80 & --- & -- & 180 & --- \\
\hline $12-16-88$ & 1225 & -- & 673 & 4,180 & 7.80 & 15.5 & -- & 180 & -- \\
\hline $1-19-89$ & .1315 & $\ldots$ & 580 & 4,900 & 7.50 & 15.0 & 200 & -.- & 180 \\
\hline $1-19-89$ & 1316 & 5.55 & 580 & 4,850 & 7.90 & 15.0 & --. & 170 & -.. \\
\hline $2-16-89$ & 1100 & 5.92 & 643 & 4,250 & 7.80 & 14.0 & -- & 180 & --- \\
\hline $3-15-89$ & 1220 & 6.02 & 662 & 4,350 & 7.70 & 23.0 & --- & 180 & --- \\
\hline $4-10-89$ & 1545 & - & 694 & 4,700 & 7.32 & 28.0 & --- & 190 & -- \\
\hline $4-10-89$ & .1550 & -- & 694 & 4,820 & 7.51 & 28.0 & 200 & --- & 94 \\
\hline $5-22-89$ & .1515 & 5.68 & 608 & 4,280 & 7.60 & 26.0 & --. & 180 & --. \\
\hline $6-20-89$ & .1100 & 5.61 & 592 & 4,250 & 7.60 & 29.0 & -- & 160 & -- \\
\hline 7-28-89 & 0725 & 5.42 & 600 & 4,610 & 7.88 & 29.0 & --- & 200 & --- \\
\hline 7-28-89 & .0726 & -- & 600 & 4,630 & 7.34 & 29.0 & 200 & --- & 96 \\
\hline $8-27-89$ & 1430 & 5.56 & 598 & 4,440 & 7.82 & 27.5 & $\ldots$ & 180 & ... \\
\hline 8-27-89 & .1431 & $-\ldots$ & 598 & 4,400 & 7.60 & -- & -- & --- & --- \\
\hline \multicolumn{10}{|c|}{ Trifolium Drain 1 (330459115430101) } \\
\hline $10-21-88$ & .0830 & 1.26 & --- & 3,590 & 7.40 & 22.0 & -- & $\cdots$ & -- \\
\hline $10-21-88$ & .0831 & 1.26 & $\ldots$ & 3,590 & 7.40 & 22.0 & 170 & --- & 96 \\
\hline $11-14-88$ & .1025 & - & $\ldots$ & 3,320 & 7.80 & 18.0 & -.. & 140 & --- \\
\hline $12-16-88$ & .1040 & $\ldots$ & - & 2,560 & 7.80 & 15.0 & --- & 130 & --- \\
\hline $1-19-89$ & 1540 & .55 & -- & 3,930 & 7.90 & 15.0 & -- & 140 & --- \\
\hline $1-19-89$ & .1541 & .55 & $\ldots$ & 3,960 & 7.80 & 15.0 & 150 & --- & 98 \\
\hline $2-16-89$ & .1145 & - & $\ldots$ & 5,170 & 8.20 & 15.0 & --- & 190 & --- \\
\hline $3-15-89$ & .1130 & .84 & -- & 2,950 & 8.10 & 22.0 & --- & 120 & --. \\
\hline $4-10-89$ & .1420 & .82 & -.- & -- & --- & 28.0 & --- & --- & -.- \\
\hline $4-10-89$ & .1425 & .82 & -- & -- & --- & 28.0 & -- & --- & --- \\
\hline $5-02-89$ & .1050 & 1.05 & $\ldots$ & 3,030 & 7.89 & 28.0 & 110 & -.. & 80 \\
\hline $5-02-89$ & 1055 & 1.05 & -- & 2,980 & 7.67 & 28.0 & -..- & 130 & -.. \\
\hline $5-22-89$ & 1430 & .53 & $\ldots$ & 5,930 & 7.80 & 30.0 & --- & 200 & $\ldots$ \\
\hline $6-20-89$ & 0955 & .82 & $\ldots$ & 3,410 & 7.30 & 27.0 & --- & 150 & --- \\
\hline $7-28-89$ & .0900 & .76 & - & 3,610 & 8.04 & 26.5 & -- & 140 & --- \\
\hline $7-28-89$ & 0901 & -.. & ... & 3,620 & 7.10 & 26.5 & 130 & -- & 91 \\
\hline $8-27-89$ & .1345 & .68 & - & 5,200 & 8.08 & 30.0 & -- & 200 & $\ldots$ \\
\hline $8-27-89$ & .1346 & -- & -- & 5,200 & 8.04 & --- & --- & --- & --. \\
\hline
\end{tabular}


Table 5. Data from periodic sampling of streams, East Highline Canal, and Trifolium Drain 1, August 1988-August 1989--Continued

\begin{tabular}{|c|c|c|c|c|c|c|c|c|c|c|}
\hline Date & & $\begin{array}{l}\text { Magne- } \\
\text { sium, } \\
\text { dis- } \\
\text { solved } \\
\text { (mg/L } \\
\text { as } \mathrm{Mg} \text { ) }\end{array}$ & $\begin{array}{c}\text { Sodium, } \\
\text { total } \\
\text { recov- } \\
\text { erable } \\
(\mathrm{mg} / \mathrm{L} \\
\text { as } \mathrm{Na})\end{array}$ & $\begin{array}{l}\text { Sodium, } \\
\text { dis- } \\
\text { solved } \\
(\mathrm{mg} / \mathrm{L} \\
\text { as } \mathrm{Na})\end{array}$ & $\begin{array}{l}\text { Potas- } \\
\text { sium, } \\
\text { total } \\
\text { recov- } \\
\text { erable } \\
(\mathrm{mg} / \mathrm{L} \\
\text { as } \mathrm{K})\end{array}$ & $\begin{array}{l}\text { Potas- } \\
\text { sium, } \\
\text { dis- } \\
\text { solved } \\
(\mathrm{mg} / \mathrm{L} \\
\text { as K) }\end{array}$ & $\begin{array}{c}\text { Alka- } \\
\text { linity, } \\
\text { lab } \\
(\mathrm{mg} / \mathrm{L} \\
\text { as } \\
\left.\mathrm{CaCO}_{3}\right)\end{array}$ & $\begin{array}{c}\text { Sulfate, } \\
\text { dis- } \\
\text { solved } \\
(\mathrm{mg} / \mathrm{L} \\
\left.\text { as } \mathrm{SO}_{4}\right)\end{array}$ & $\begin{array}{l}\text { Chlo- } \\
\text { ride, } \\
\text { dis- } \\
\text { solved } \\
(\mathrm{mg} / \mathrm{L} \\
\text { as } \mathrm{Cl})\end{array}$ & $\begin{array}{l}\text { Fluo- } \\
\text { ride, } \\
\text { dis- } \\
\text { solved } \\
\text { (mg/L } \\
\text { as F) }\end{array}$ \\
\hline \multicolumn{11}{|c|}{ New River at Outlet (1025550) } \\
\hline $8-17-88$ & . & - & $\cdots$ & -- &.- & -.. & -- & -- & --- & -- \\
\hline $10-20-88$ & . & -- & --- & --- & -- & --- & --- & -- & --- & -- \\
\hline $10-20-88$ & . & -- & 610 & --- & 10 & $-\cdots$ & 225 & -- & -- & -- \\
\hline $11-14-88$ & . & -- & --- & --- &.-- & -- & 240 & 780 & 990 & $-\ldots$ \\
\hline $12-16-88$ & . & -- & -- & --- & -- & --- & 237 & 720 & 870 & --- \\
\hline $1-19-89$ & . . &.- & 700 & -.- & 12 & --- & 227 & -- & -- & --- \\
\hline $1-19-89$ & . . & 87 & -- & 700 & -- & 14 & 248 & 750 & 1,000 & --- \\
\hline $2-16-89$ & . & -- & --- & --- & --- & -- & 207 & 730 & 870 & --- \\
\hline $3-15-89$ & . . & - & --- & -.- & -- & -- & 210 & 770 & 900 & --- \\
\hline $4-10-89$ & . & 99 & -- & 640 & -- & 29 & 197 & 710 & 1,000 & $\ldots$ \\
\hline $4-10-89$ & . & $\cdots$ & 690 & -- & 2.5 & --- & 202 & --- & --- & --- \\
\hline $5-22-89$ & $\ldots$ & 98 & -.- & 600 & -- & 16 & 226 & 720 & 910 & 0.5 \\
\hline $6-20-89$ & $\ldots$ & 83 &.-- & 610 & --- & 17 & 211 & 710 & 880 & .5 \\
\hline $7-28-89$ & $\ldots$ & 98 & -.. & 630 & -.. & 17 & 229 & 810 & 920 & --- \\
\hline $7-28-89$ & . & -- & 620 & -- & 19 & --- & 224 & -- & -.- & $\ldots$ \\
\hline $8-27-89$ & . . & 90 & -- & 600 & --- & 15 & 227 & 800 & 880 & --- \\
\hline $8-27-89$ & $\ldots$ & -- & -- & -- & --- & -- & 226 & 800 & 840 & $\ldots$ \\
\hline \multicolumn{11}{|c|}{ Trifolium Drain 1 (330459115430101) } \\
\hline $10-21-88$ & . . & $\cdots$ & --- & --- & --- & -- &.- & --- & --- & --- \\
\hline $10-21-88$ & . & $\ldots$ & 450 & -- & 7.1 & --- & 237 & --- & --- & -- \\
\hline $11-14-88$ & . & -- & --- & -- & -- & --- & 242 & 800 & 480 & $\ldots$ \\
\hline $12-16-88$ & . . & 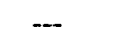 &.- & -.. & --- & --- & 218 & 650 & 340 & --- \\
\hline $1-19-89$ & . & 95 & --- & 550 & --- & 11 & 237 & 880 & 670 & --- \\
\hline $1-19-89$ & . . & -.- & 580 & --- & 7.1 & --- & 237 & -- & -- & --- \\
\hline $2-16-89$ & . & --- & --- & --- & $\ldots$ & --- & 279 & 1,200 & 880 & --- \\
\hline $3-15-89$ & . & --- & -- & $\ldots$ & --- & $\ldots$ & 193 & 740 & 460 &.-- \\
\hline $4-10-89$ & $\ldots$ &.-- & -- & $\cdots$ & --- & $\cdots$ & -- &.- & --- & --- \\
\hline $4-10-89$ & . & -- & - & -- & --- & --- & -- & $\cdots$ & --- & --- \\
\hline $5-02-89$ & . & -- & 4.7 & -.- & 6.7 & -- & 244 & --- & -..-- & \\
\hline $5-02-89$ & . & 72 &..- & 410 & -- & 11 & 243 & 690 & 410 & --- \\
\hline $5-22-89$ & $\ldots$ & 150 & -- & 890 & --- & 16 & 342 & 1,400 & 1,100 & 0.9 \\
\hline $6-20-89$ & $\ldots$ & 91 & -- & 460 & --- & 12 & 244 & 770 & 550 & .6 \\
\hline $7-28-89$ & . & 93 & -- & 490 & -- & 11 & 179 & 790 & 610 & --- \\
\hline $7-28-89$ & $\ldots$ & -. & 450 & --- & 12 & -- & 183 & --- &.- & --- \\
\hline $8-27-89$ & $\ldots$ & 130 & -- & 710 & -- & 16 & 346 & 1,300 & 800 & --- \\
\hline $8-27-89$ & . & -.- & $\ldots$ & --- & --- & -- & 309 & 1,300 & 840 & --- \\
\hline
\end{tabular}


Table 5. Data from periodic sampling of streams, East Highline Canal, and Trifolium Drain 1, August 1988-August 1989--Continued

\begin{tabular}{|c|c|c|c|c|c|c|c|c|c|c|}
\hline Date & & $\begin{array}{c}\text { Bromide, } \\
\text { dis- } \\
\text { solved } \\
(\mathrm{mg} / \mathrm{L} \\
\text { as } \mathrm{Br})\end{array}$ & 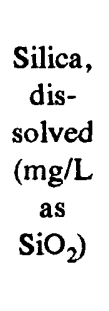 & $\begin{array}{c}\text { Solids, } \\
\text { residue } \\
\text { at } 180 \\
{ }^{\circ} \mathrm{C}, \\
\text { dis- } \\
\text { solved } \\
(\mathrm{mg} / \mathrm{L})\end{array}$ & $\begin{array}{l}\text { Solids, } \\
\text { residue } \\
\text { at } 105 \\
{ }^{\circ} \mathrm{C}, \\
\text { total } \\
(\mathrm{mg} / \mathrm{L})\end{array}$ & $\begin{array}{c}\text { Nitro- } \\
\text { gen, } \\
\mathrm{NO}_{2}+ \\
\mathrm{NO}_{3}, \\
\text { total } \\
(\mathrm{mg} / \mathrm{L} \\
\text { as } \mathrm{N})\end{array}$ & $\begin{array}{c}\text { Nitro- } \\
\text { gen, } \\
\mathrm{NO}_{2}+ \\
\mathrm{NO}_{3} \text {, } \\
\text { dis- } \\
\text { solved } \\
(\mathrm{mg} / \mathrm{L} \\
\text { as } \mathrm{N})\end{array}$ & $\begin{array}{l}\text { Nitro- } \\
\text { gen, am- } \\
\text { monia, } \\
\text { total } \\
(\mathrm{mg} / \mathrm{L} \\
\text { as } \mathrm{N})\end{array}$ & $\begin{array}{c}\text { Nitro- } \\
\text { gen, am- } \\
\text { monia, } \\
\text { dis- } \\
\text { solved } \\
\text { (mg/L } \\
\text { as } \mathrm{N} \text { ) }\end{array}$ & $\begin{array}{c}\text { Nitro- } \\
\text { gen, am- } \\
\text { monia }+ \\
\text { organic, } \\
\text { dis. } \\
(\mathrm{mg} / \mathrm{L} \\
\text { as } \mathrm{N})\end{array}$ \\
\hline \multicolumn{11}{|c|}{ New River at Outlet $(\mathbf{1 0 2 5 5 5 0 )}$} \\
\hline $8-17-88$ & . & - & -- & - & --- & -- & -- & -.. & -- & -- \\
\hline $10-20-88$ & . & -- & --- & - & --- & --- & 4.3 & --- & 2.7 & --- \\
\hline $10-20-88$ & . & -- & --- & -- & 3,130 & 4.3 & -- & 2.8 & -- & --- \\
\hline $11-14-88$ & . & 1.2 & --- & 2,960 & -- & --- & 5.5 & --- & 2.5 & --- \\
\hline $12-16-88$ & . & 1.0 & -- & 2,720 & -- & -- & 4.9 & -- & 2.4 & -- \\
\hline $1-19-89$ & . & --- & -- & 3,350 & 4.3 & --- & 4.9 & --- & --- & \\
\hline $1-19-89$ & . & 1.3 & 15 & 3,050 & -.. & .... & $<.1$ & ... & .01 & ... \\
\hline $2-16-89$ & . & .92 & --- & 2,700 & --. & $\ldots$ & 5.2 & --- & 2.5 & --- \\
\hline $3-15-89$ & . & .99 & --- & 2,820 & --- & -.. & 6.1 & $\ldots$ & 3.8 & --- \\
\hline $4-10-89$ & . & 1.4 & 16 & 2,910 & -- & --- & 6.0 & $-\cdots$ & 3.5 & --- \\
\hline $4-10-89$ & . & -- & -- & - & 4,190 & 6.0 & -- & 3.2 & --- & --- \\
\hline $5-22-89$ & . & .85 & 15 & 2,720 & -- & -- & 4.0 & --- & 1.5 & --- \\
\hline $6-20-89$ & . & 1.0 & 15 & 2,720 & -- & -.. & 3.8 & -.. & 1.6 & --- \\
\hline 7-28-89 & . & 1.1 & 18 & 3,000 & $\ldots$ & --- & 5.3 & --- & 1.2 & --- \\
\hline $7-28-89$ & . & - & -- & -- & 3,080 & 5.4 & $\cdots$ & 1.3 & -- & --- \\
\hline $8-27-89$ & . & 1.3 & 17 & 2,850 & --- & --- & 3.1 & -.. & .72 & 0.9 \\
\hline $8-27-89$ & . . & 1.0 & -- & -- & -- & -- & -- & -- & --- & --- \\
\hline \multicolumn{11}{|c|}{ Trifolium Drain 1 (330459115430101) } \\
\hline $10-21-88$ & . & -- & -.. & -.- & --- & --- & 25 & -..- & 4.1 & -.- \\
\hline $10-21-88$ & . & -- & --- & -.- & 2,690 & 17 & --- & 4.4 & --- & --- \\
\hline 11-14-88 & . & 0.35 & -.. & 2,240 & --- & -.. & 14 & --- & 5.5 & $\ldots$ \\
\hline $12-16-88$ & . . & .09 & -- & 1,780 & $\ldots$ & -.- & 10 & -.. & .12 & --- \\
\hline $1-19-89$ & . . & .37 & 14 & 2,660 & $\ldots$ & --- & 15 & --- & .43 & --- \\
\hline $1-19-89$ & . & --- & -- & --- & 2,970 & 13 & $\cdots$ & .23 & -- & --- \\
\hline $2-16-89$ & . . & .70 & -.. & 3,600 & -- & -.- & 20 & --- & .20 & --- \\
\hline $3-15-89$ & . & .31 & --- & 1,990 & -- & -- & 8.7 & --- & 1.5 & --- \\
\hline $4-10-89$ & . & --- & $\cdots$ & -- & --- & -.. & 13.0 & --- & .57 & --- \\
\hline $4-10-89$ & . & -- & --- & $\cdots$ & --- & 12 & --- & .56 & -- & --- \\
\hline $5-02-89$ & . & -- & -- & -- & 2,160 & 7.0 & --- & .18 & -- & -.- \\
\hline $5-02-89$ & . . & .26 & 15 & 2,010 & -- & $\cdots$ & 6.8 & --- & .18 & -- \\
\hline $5-22-89$ & . & .79 & 21 & 4,070 & --- & -.. & 13 & --- & .85 & -.- \\
\hline $6-20-89$ & . & .33 & 16 & 2,330 & $\cdots$ & $\cdots$ & 6.6 & $\cdots$ & 1.5 & $\cdots$ \\
\hline $7-28-89$ & . . & .38 & 15 & 2,370 & -- & -- & 6.5 & --- & .36 & --- \\
\hline $7-28-89$ & . & -- & -- & -- & 2,400 & 17 & -.. & 8.0 & --- & -.. \\
\hline $8-27-89$ & $\cdots$ & .69 & 24 & 3,580 & ... & -- & 11 & -.- & .06 & 0.4 \\
\hline $8-27-89$ & . . & .71 & -- & -. & $\cdots$ & -.. & -- & $\cdots$ & --- & $\ldots$ \\
\hline
\end{tabular}


Table 5. Data from periodic sampling of streams, East Highline Canal, and Trifolium Drain 1, August 1988-August 1989--Continued

\begin{tabular}{|c|c|c|c|c|c|c|c|c|c|c|}
\hline Date & & $\begin{array}{l}\text { Phos- } \\
\text { phorus, } \\
\text { hydro. } \\
+ \text { ortho } \\
\text { dis. } \\
\text { (mg/L } \\
\text { as P) }\end{array}$ & $\begin{array}{l}\text { Alum- } \\
\text { inum, } \\
\text { total } \\
\text { recov- } \\
\text { erable } \\
(\mu \mathrm{g} / \mathrm{L} \\
\text { as } \mathrm{Al})\end{array}$ & $\begin{array}{l}\text { Alum- } \\
\text { inum, } \\
\text { dis- } \\
\text { solved } \\
(\mu \mathrm{g} / \mathrm{L} \\
\text { as } \mathrm{Al})\end{array}$ & $\begin{array}{c}\text { Anti- } \\
\text { mony, } \\
\text { total } \\
(\mu \mathrm{g} / \mathrm{L} \\
\text { as } \mathrm{Sb})\end{array}$ & $\begin{array}{c}\text { Anti- } \\
\text { mony, } \\
\text { dis- } \\
\text { solved } \\
(\mu \mathrm{g} / \mathrm{L} \\
\text { as Sb) }\end{array}$ & $\begin{array}{c}\text { Arsenic, } \\
\text { total } \\
(\mu \mathrm{g} / \mathrm{L} \\
\text { as As) }\end{array}$ & $\begin{array}{c}\text { Arsenic, } \\
\text { dis- } \\
\text { solved } \\
(\mu \mathrm{g} / \mathrm{L} \\
\text { as As) }\end{array}$ & $\begin{array}{c}\text { Boron, } \\
\text { total } \\
\text { recov- } \\
\text { erable } \\
(\mu \mathrm{g} / \mathrm{L} \\
\text { as B) }\end{array}$ & $\begin{array}{c}\text { Boron, } \\
\text { dis- } \\
\text { solved } \\
(\mu \mathrm{g} / \mathrm{L} \\
\text { as B) }\end{array}$ \\
\hline \multicolumn{11}{|c|}{ New River at Outlet (1025550) } \\
\hline $8-17-88$ & . & -- & --- & --- & --- & --- & --- & -.- & --- & -- \\
\hline $10-20-88$ & . & -- & -- & --- & --- & --- & --- & -- & --- & $\cdots$ \\
\hline $10-20-88$ & . & - & 7,000 & --- & $<1$ & --- & 7 & -- & 980 & -- \\
\hline $11-14-88$ & . . & -- &.-- & --- & --- & --- & -- & $\cdots$ & --- & 1,000 \\
\hline $12-16-88$ & . & -- & --- & --- & --- & $\cdots$ & -- & --- & $\ldots$ & 930 \\
\hline $1-19-89$ & . & -- & 3,500 & --- & --- & --- & 6 & -- & 1,100 & $\ldots$ \\
\hline $1-19-89$ & . & -- & --- & 10 & --- & -- & -- & 4 & --- & 1,100 \\
\hline $2-16-89$ & $\ldots$ & --- & -- & -- & --- & --- & $\ldots$ & -- &.-- & 820 \\
\hline $3-15-89$ & $\ldots$ & -- & --- &.- & --- & -- & --- & -- & --- & 960 \\
\hline $4-10-89$ & . & -- & --- & 10 & -- & -- & -- & 7 & -- & 1,100 \\
\hline $4-10-89$ & $\ldots$ & -- & 9,500 & -. & --. & --- & 10 & --- & 1,200 & -- \\
\hline $5-22-89$ & $\ldots$ & --- & -.- & $<10$ & $-\cdots$ & --- & -- & 6 & -- & 970 \\
\hline $6-20-89$ & . & --- & --- & 20 & -- & -- & --- & 5 & -- & 990 \\
\hline $7-28-89$ & $\ldots$ & - & -- & 20 & -- & -- & --- & 7 & --- & 1,100 \\
\hline $7-28-89$ & . & --- & 6,300 & --- & -- & -- & 10 & -- & 1,100 & --- \\
\hline $8-27-89$ & $\ldots$ & 0.37 & $-\cdots$ & $<10$ & -- & --- & -- & 6 & -- & 1,000 \\
\hline $8-27-89$ & . & -- & --- & -- & --- & -- & -- & --- & --- & -- \\
\hline \multicolumn{11}{|c|}{ Trifolium Drain 1 (330459115430101) } \\
\hline $10-21-88$ & . . & -- & -- & -.. & --- & --- & -- & --- & --- & --- \\
\hline $10-21-88$ & . & -- & 2,200 & -- & $<1$ & $\cdots$ & 3 &.-- & 930 &.- \\
\hline $11-14-88$ & . & -- & -- & -- & -- & --- & $\cdots$ & $\cdots$ &.-- & 770 \\
\hline $12-16-88$ & . & -- & --- & -- & -- & --- & -- & --- & --- & 520 \\
\hline $1-19-89$ & . & $\cdots$ & --- & 20 & $\cdots$ & -- & -- & 2 & $\cdots$ & 980 \\
\hline $1-19-89$ & $\ldots$ & --- & 4,000 & --- & --- & -- & 3 & --- & 1,000 & --- \\
\hline $2-16-89$ & .. & -- & -- & --- & -- & -- & 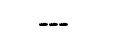 & --- & -.- & 1,300 \\
\hline $3-15-89$ & . & --- & -- & -- & -- & -- &.- & --- & -- & 650 \\
\hline $4-10-89$ & . & --- &.- & --- & -- & - & -- & --- &.- & --- \\
\hline 4-10-89 & . & --- & -- & --- & -- & -- & -- & $\cdots$ & $\cdots$ & -- \\
\hline $5-02-89$ & $\ldots$ & --- & 3,600 & --- & --- & -- & 3 & -- & 720 & --- \\
\hline $5-02-89$ & . & --- & -- & 20 & --- & -- & --- & 3 & --- & 690 \\
\hline $5-22-89$ & . & --- & -- & $<10$ & -- & -- & --- & 2 & -- & 1,700 \\
\hline $6-20-89$ & $\ldots$ & --- & -- & 10 & $\cdots$ & --- & --- & 3 & --- & 840 \\
\hline $7-28-89$ & . & - & -- & $<10$ & -- & -- & -- & 4 & --- & 860 \\
\hline $7-28-89$ & . & -- & 1,600 & --- & --- & -.- & 4 & --- & 840 & --- \\
\hline $8-27-89$ & . & 0.04 & --- & 20 & -- & --- & --- & 3 & --. & 1,500 \\
\hline $8-27-89$ & . & -- & --- & --- & -- & --- & -- & --- & --- & ... \\
\hline
\end{tabular}


Table 5. Data from periodic sampling of streams, East Highline Canal, and Trifolium Drain 1, August 1988-August 1989--Continued

\begin{tabular}{|c|c|c|c|c|c|c|c|c|c|}
\hline Date & $\begin{array}{l}\text { Iron, } \\
\text { total } \\
\text { recov- } \\
\text { erable } \\
(\mu \mathrm{g} / \mathrm{L} \\
\text { as } \mathrm{Fe})\end{array}$ & $\begin{array}{c}\text { Iron, } \\
\text { dis- } \\
\text { solved } \\
(\mu \mathrm{g} / \mathrm{L} \\
\text { as } \mathrm{Fe})\end{array}$ & $\begin{array}{c}\text { Lithium, } \\
\text { total } \\
\text { recov- } \\
\text { erable } \\
(\mu \mathrm{g} / \mathrm{L} \\
\text { as } \mathrm{Li})\end{array}$ & $\begin{array}{c}\text { Lithium, } \\
\text { dis- } \\
\text { solved } \\
(\mu g / L \\
\text { as } \mathrm{Li})\end{array}$ & $\begin{array}{c}\text { Manga- } \\
\text { nese, } \\
\text { total } \\
\text { recov- } \\
\text { erable } \\
(\mu \mathrm{g} / \mathrm{L} \\
\text { as } \mathrm{Mn})\end{array}$ & $\begin{array}{c}\text { Manga- } \\
\text { nese, } \\
\text { dis- } \\
\text { solved } \\
(\mu \mathrm{g} / \mathrm{L} \\
\text { as } \mathrm{Mn})\end{array}$ & $\begin{array}{c}\text { Molyb- } \\
\text { denum, } \\
\text { total } \\
\text { recov- } \\
\text { erable } \\
(\mu \mathrm{g} / \mathrm{L} \\
\text { as Mo) }\end{array}$ & $\begin{array}{c}\text { Molyb- } \\
\text { denum, } \\
\text { dis- } \\
\text { solved } \\
(\mu \mathrm{g} / \mathrm{L} \\
\text { as Mo) }\end{array}$ & $\begin{array}{c}\text { Sele- } \\
\text { nium, } \\
\text { total } \\
(\mu \mathrm{g} / \mathrm{L} \\
\text { as Se) }\end{array}$ \\
\hline \multicolumn{10}{|c|}{ New River at Outlet $(\mathbf{1 0 2 5 5 5 0})$} \\
\hline 8-17-88 & -- & - & --- & -- & --- & --- & --- & $\ldots$ & --- \\
\hline $10-20-88$ & - & -- & -- & --- & --- & -- & --- & --- & --- \\
\hline $10-20-88$ & 5,900 & -- & -.- & -- & 320 & -- & 15 & --- & 4 \\
\hline $11-14-88$ & - & 30 & -- &.- & -.. & 70 & --- & --- & --- \\
\hline $12-16-88$ & -- & 30 & -- & -- & -- & 120 & -- & --- & $-\cdots$ \\
\hline $1-19-89$ & 3,500 & -- & 410 & -.- & 360 & $-\ldots$ & 14 & --- & 5 \\
\hline $1-19-89$ & -- & 20 & -. & 420 & --- & 200 & -- & 8 & --- \\
\hline $2-16-89$ & -- & $<10$ & -.. & ... & -.. & $<10$ & ... & --- & --- \\
\hline $3-15-89$ & -_ & 20 & -- & -.. & --. & 160 & -.. & -.. & -- \\
\hline $4-10-89$ & -- & 20 & -- & 580 &.- & 120 & --- & 9 & - \\
\hline $4-10-89$ & 9,700 & --. & 600 & -- & 490 & -- & 12 & --- & 4 \\
\hline $5-22-89$ & - & 30 & -- & 360 & --- & 90 & --- & 7 & -.- \\
\hline $6-20-89$ & -_ & 40 & -.- & 380 & --- & 110 & --- & 9 & ... \\
\hline $7-28-89$ & -- & 40 & --- & --- & --- & 80 & -.- & 10 & --- \\
\hline $7-28-89$ & 7,200 & -- & 380 & --- & 420 & -- & 11 & --- & 5 \\
\hline $8-27-89$ & - & 20 & --- & -.- & -.- & 60 & -.. & 13 & ... \\
\hline $8-27-89$ & - & -- & --- & -- & --- & -- & -- & --- & 5 \\
\hline \multicolumn{10}{|c|}{ Trifolium Drain 1 (330459115430101) } \\
\hline 10-21-88 & -- & $\cdots$ & -- & -- & --- & -- & --- & --- & -.- \\
\hline 10-21-88 & 1,800 & -- & --- & --- & 90 & -- & 18 & -.- & 10 \\
\hline $11-14-88$ & -- & $<10$ & -- & --- & -- & $<10$ & -- & --- & --- \\
\hline $12-16-88$ & -.- & 20 & -.- & -.- & --- & 30 & --- & -.- & --- \\
\hline $1-19-89$ & - & 20 & --- & 320 & --- & 70 & -- & 13 & -- \\
\hline $1-19-89$ & 3,900 & - & 300 & -- & 200 & -- & 16 & --. & 7 \\
\hline $2-16-89$ & -- & $<10$ & -- & -- & --- & $<10$ & -- & -- & -- \\
\hline $3-15-89$ & -- & $<10$ & --- & -- & --- & 20 & -- & --- & -.. \\
\hline $4-10-89$ & -- & -.. & -- & $\ldots$ & -- & - & $\ldots$ & -- & --- \\
\hline $4-10-89$ & -- & -- & $-\cdots$ & -- & --- & $\cdots$ & -- & --- & -- \\
\hline $5-02-89$ & 3,800 & --- & 200 & -- & 150 & --- & 19 & -- & 6 \\
\hline $5-02-89$ & -- & 30 & --- & 240 & -- & 60 & $\cdots$ & 10 & -.. \\
\hline $5-22-89$ & -- & 20 & -- & 520 & -- & 60 & $\ldots$ & 18 &.-- \\
\hline $6-20-89$ & - & 40 & -- & 270 & -- & 70 & -- & 12 & -- \\
\hline $7-28-89$ & -- & 30 & -- & - & - & 30 & -- & 10 & --- \\
\hline $7-28-89$ & 1,700 & -- & 240 & $\cdots$ & 90 & - & 10 & -- & 8 \\
\hline $8-27-89$ & -.- & 20 & --- & --- & --- & 30 & --- & 24 & -- \\
\hline $8-27-89$ & - & -- & -.. & -.. & $\ldots$ & $\ldots$ & -.. & $\ldots$ & 10 \\
\hline
\end{tabular}


Table 5. Data from periodic sampling of streams, East Highline Canal, and Trifolium Drain 1, August 1988-August 1989--Continued

\begin{tabular}{|c|c|c|c|c|c|c|c|c|c|c|c|}
\hline Date & & $\begin{array}{c}\text { Sele- } \\
\text { nium, } \\
\text { dis- } \\
\text { solved } \\
(\mu \mathrm{g} / \mathrm{L} \\
\text { as } \mathrm{Se})\end{array}$ & $\begin{array}{l}\text { Stron- } \\
\text { tium, } \\
\text { total } \\
\text { recov- } \\
\text { erable } \\
(\mu \mathrm{g} / \mathrm{L} \\
\text { as } \mathrm{Sr})\end{array}$ & $\begin{array}{c}\text { Stron- } \\
\text { tium, } \\
\text { dis- } \\
\text { solved } \\
(\mu \mathrm{g} / \mathrm{L} \\
\text { as } \mathrm{Sr})\end{array}$ & $\begin{array}{c}\text { Uran- } \\
\text { ium, } \\
\text { natural } \\
\text { dis- } \\
\text { solved } \\
\text { ( } \mu \text { g/L } \\
\text { as U) }\end{array}$ & $\begin{array}{c}\text { Carbon, } \\
\text { organic } \\
\text { total } \\
(\mathrm{mg} / \mathrm{L} \\
\text { as C) }\end{array}$ & $\begin{array}{c}\text { Sedi- } \\
\text { ment, } \\
\text { sus- } \\
\text { pended } \\
(\mathrm{mg} / \mathrm{L})\end{array}$ & $\begin{array}{c}\text { Sedi- } \\
\text { ment, } \\
\text { sus- } \\
\text { pended } \\
\text { (\% finer } \\
\text { than } \\
0.062 \\
\text { mm) }\end{array}$ & $\begin{array}{c}\text { Tri- } \\
\text { tium, } \\
\text { total } \\
(\mathrm{pCi} / \mathrm{L})\end{array}$ & $\begin{array}{l}\text { Stable } \\
\text { ratio } \\
{ }^{2} \mathrm{H} /{ }^{l} \mathrm{H}\end{array}$ & $\begin{array}{l}\text { otope } \\
\frac{{ }^{18} \mathrm{O} / \mathrm{I}^{16} \mathrm{O}}{}\end{array}$ \\
\hline \multicolumn{12}{|c|}{ New River at Outlet $(1025550)$} \\
\hline $8-17-88$ & $\ldots$ & --- & --- & -.- & -.- & --- & 505 & 55 & 90 & -97.0 & -11.75 \\
\hline $10-20-88$ & $\ldots$ & --- & --- & --- & --- & --- & --- & --- & -.. & -97.0 & -11.75 \\
\hline $10-20-88$ & . & --- & --. & -- & --- & -..- & --- & --- & --- & --- & $\ldots$ \\
\hline $11-14-88$ & $\cdots$ & 5 & --- & --- & 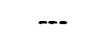 & -.- & --- & --- & -.. & -97.0 & -11.80 \\
\hline $12-16-88$ & $\ldots$ & 4 & --- & --- & -.- & --- & 351 & 59 & --- & -99.0 & -11.95 \\
\hline $1-19-89$ & $\ldots$ & -.. & 3,900 & -.- & $\ldots$ & -.- & $\ldots$ & --- & --- & $\ldots$ & $\ldots$ \\
\hline $1-19-89$ & $\ldots$ & 4 & -- & 3,700 & $-\ldots$ & --- & -.. & --- & --- & -95.0 & -11.45 \\
\hline $2-16-89$ & $\ldots$ & 4 & --- & -.. & --- & --. & -.- & --- & --- & -95.0 & -11.80 \\
\hline $3-15-89$ & . & 4 & --. & --- & --- & --- & --- & --- & --- & -92.5 & -11.15 \\
\hline $4-10-89$ & $\ldots$ & 4 & -- & 3,300 & --- & --- & 613 & 73 & --- & -98.5 & -11.55 \\
\hline 4-10-89 & $\ldots$ & --- & 3,600 & --- & --- & --- & --- & -.. & --- & --- & --- \\
\hline $5-22-89$ & $\ldots$ & 4 & -.- & 3,400 & --- & $\ldots$ & $\cdots$ & -.- & -.. & -96.0 & -11.45 \\
\hline $6-20-89$ & . & 4 & --- & 3,400 & --- & --- & --- & $\ldots$ & --- & -96.0 & -11.40 \\
\hline 7-28-89 & $\ldots$ & 5 & --. & 3,500 & --- & --- & 564 & 66 & $\ldots$ & -95.5 & -11.05 \\
\hline $7-28-89$ & $\ldots$ & --- & 3,300 & -- & --- & --- & -- & -- & -- & --- & --- \\
\hline 8-27-89 & . & 4 & --. & 3,500 & 14 & 12 & --- & -.- & --- & -95.0 & -11.30 \\
\hline $8-27-89$ & $\ldots$ & -- & -- & -.. & --- & --- & --- & --- & $-\cdots$ & -- & --- \\
\hline \multicolumn{12}{|c|}{ Trifolium Drain 1 (330459115430101) } \\
\hline $10-21-88$ & $\ldots$ & -- & --- & -- & --- & --- & 222 & 74 & -- & -99.5 & -12.10 \\
\hline $10-21-88$ & $\ldots$ & -- & -.- & --- & --- & --- & --- & $\cdots$ & --- & --- & --- \\
\hline $11-14-88$ & $\ldots$ & 6 & --- & --- & --- & --- & --- & -- & $\cdots$ & -101.0 & -12.25 \\
\hline $12-16-88$ & $\ldots$ & 6 & --- & -.- & --- & --- & --- & -- & -.. & -100.0 & -12.25 \\
\hline $1-19-89$ & . & 6 & --- & 2,700 & --- & --- & --- & -- & --- & -98.0 & -11.85 \\
\hline $1-19-89$ & $\ldots$ & -- & 2,800 & -- & -- & --- & --- & --- & --- & --- & --- \\
\hline $2-16-89$ & $\ldots$ & 9 & -- & -.- & --- & --- & 243 & 93 & --- & -93.0 & -11.85 \\
\hline $3-15-89$ & $\ldots$ & 6 & ... & $\ldots$ & --. & --- & --- & -.. & $\ldots$ & -99.0 & -12.15 \\
\hline $4-10-89$ & $\ldots$ & $\cdots$ & -- & --- & $\cdots$ & $\cdots$ & -.. & $\cdots$ & --- & -98.5 & -12.10 \\
\hline $4-10-89$ & $\ldots$ & -- & -- & -.- & -- & --- & 246 & 94 & -- & -- & -- \\
\hline $5-02-89$ & $\ldots$ & ... & 2,400 & ... & --. & --- & 243 & 85 & -.- & --- & ... \\
\hline $5-02-89$ & $\ldots$ & 5 & --- & 2,400 & --- & --- & --- & --- & --- & -101.0 & -12.25 \\
\hline $5-22-89$ & $\ldots$ & 10 & -..- & 4,300 & --- & ... & $\ldots$ & --- & -.- & -96.5 & -11.55 \\
\hline $6-20-89$ & $\ldots$ & 5 & --- & 2,800 & --- & --- & $\ldots$ & --- & -- & -99.0 & -12.00 \\
\hline $7-28-89$ & $\ldots$ & 7 & --- & 2,900 & --- & --- & --- & --- & --- & -99.0 & -11.90 \\
\hline $7-28-89$ & . & --. & 2,600 & ..- & --- & --- & --- & --- & --- & --- & --- \\
\hline $8-27-89$ & $\ldots$ & 10 & -.. & 4,100 & 120 & 6.7 & $\ldots$ & $\ldots$ & --- & -96.0 & -11.35 \\
\hline $8-27-89$ & . & -- & --- & -.. & -. & --- & --- & --- & --- & -- & --- \\
\hline
\end{tabular}


Table 6. Annual average tritium concentrations in the lower Colorado River at Imperial Dam, 1965-88

[Concentrations are given, in picocuries per liter, unadjusted and adjusted for radioactive decay $(\lambda=0.0556$ per year) to 1988 ]

\begin{tabular}{|c|c|c|c|c|c|}
\hline Year & Unadjusted & $\begin{array}{l}\text { Decay- } \\
\text { adjusted } \\
\text { to } 1988\end{array}$ & Year & Unadjusted & $\begin{array}{l}\text { Decay- } \\
\text { adjusted } \\
\text { to } 1988\end{array}$ \\
\hline $1965 \ldots \ldots \ldots$ & 1,060 & 293 & 1977 & 422 & 229 \\
\hline $1966 \ldots \ldots \ldots$ & 1,410 & 415 & 1978 & 365 & 209 \\
\hline 1967 & 2,290 & 712 & 1979 & 310 & 188 \\
\hline 1968 & 1,960 & 642 & 1980 & 266 & 170 \\
\hline 1969 . & 1,700 & 589 & 1981 & 253 & 171 \\
\hline $1970 \ldots \ldots \ldots$ & 1,370 & 503 & 1982 & 221 & 158 \\
\hline $1971 \ldots \ldots \ldots$ & 1,110 & 432 & 1983 & 186 & 140 \\
\hline $1972 \ldots \ldots$ & 931 & 382 & 1984 & 154 & 123 \\
\hline $1973 \ldots \ldots$ & 800 & 347 & 1985 & 125 & 106 \\
\hline $1974 \ldots \ldots \ldots$ & 675 & 310 & 1986 & 109 & 97 \\
\hline $1975 \ldots \ldots \ldots$ & 579 & 281 & 1987 & 99 & 94 \\
\hline $1976 \ldots \ldots \ldots$ & 483 & 248 & 1988 & 90 & 90 \\
\hline
\end{tabular}

Table 7. Depth-profile data along a transect between the mouth of the Alamo River and the Salton Sea, August 1988

[Site locations, which are shown in figure 3, are listed in order from the river to the Salton Sea. The deepest sample at each site is immediately above bottom sediment. $\mu \mathrm{S} / \mathrm{cm}$, microsiemen per centimeter at $25^{\circ} \mathrm{C} ;{ }^{\circ} \mathrm{C}$, degree Celsius; $\mathrm{mg} / \mathrm{L}$, milligram per liter; $\mathrm{ft}$, foot]

\begin{tabular}{|c|c|c|c|c|c|c|}
\hline \multirow{2}{*}{$\begin{array}{l}\text { Delta } \\
\text { site } \\
\text { number }\end{array}$} & \multirow{2}{*}{$\begin{array}{l}\text { Water } \\
\text { depth } \\
\text { (ft) }\end{array}$} & \multirow{2}{*}{$\begin{array}{c}\text { Specific } \\
\text { conductance } \\
(\mu \mathrm{S} / \mathrm{cm})\end{array}$} & \multirow{2}{*}{$\begin{array}{c}\text { Water } \\
\text { temperature } \\
\left({ }^{\circ} \mathrm{C}\right)\end{array}$} & \multirow{2}{*}{$\begin{array}{c}\mathrm{pH} \\
\text { (standard } \\
\text { units) }\end{array}$} & \multicolumn{2}{|c|}{ Dissolved oxygen } \\
\hline & & & & & $(\mathrm{mg} / \mathrm{L})$ & $\begin{array}{c}\text { (percent } \\
\text { saturation) }\end{array}$ \\
\hline $1 \ldots \ldots$ & $\begin{array}{l}2.3 \\
8.9\end{array}$ & $\begin{array}{l}2,910 \\
2,900\end{array}$ & $\begin{array}{l}30.3 \\
30.3\end{array}$ & $\begin{array}{l}7.5 \\
7.5\end{array}$ & $\begin{array}{l}4.9 \\
5.0\end{array}$ & $\begin{array}{l}64 \\
66\end{array}$ \\
\hline$\ldots \ldots$ & $\begin{array}{r}.7 \\
3.3 \\
6.6\end{array}$ & $\begin{array}{l}2,840 \\
2,840 \\
2,840\end{array}$ & $\begin{array}{l}30.8 \\
30.8 \\
30.8\end{array}$ & $\begin{array}{l}7.5 \\
7.5 \\
7.5\end{array}$ & $\begin{array}{l}4.9 \\
4.9 \\
4.9\end{array}$ & $\begin{array}{l}65 \\
65 \\
65\end{array}$ \\
\hline $9 \ldots \ldots$ & 1.6 & 3,150 & 30.9 & 7.7 & 4.3 & 57 \\
\hline $10 \ldots \ldots$ & $\begin{array}{l}1.3 \\
2.9\end{array}$ & $\begin{array}{r}5,000 \\
51,000\end{array}$ & $\begin{array}{l}30.9 \\
30.3\end{array}$ & $\begin{array}{l}8.1 \\
8.2\end{array}$ & $\begin{array}{l}4.2 \\
1.2\end{array}$ & $\begin{array}{l}56 \\
18\end{array}$ \\
\hline$\ldots \ldots$ & $\begin{array}{r}.3 \\
1.6\end{array}$ & $\begin{array}{l}52,130 \\
52,180\end{array}$ & $\begin{array}{l}29.9 \\
29.9\end{array}$ & $\begin{array}{l}8.3 \\
8.1\end{array}$ & $\begin{array}{l}4.5 \\
5.0\end{array}$ & $\begin{array}{l}69 \\
77\end{array}$ \\
\hline $4 \ldots \ldots$ & $\begin{array}{l}1.6 \\
3.3 \\
4.9 \\
6.6 \\
8.9\end{array}$ & $\begin{array}{l}47,200 \\
51,500 \\
53,130 \\
53,250 \\
53,250\end{array}$ & $\begin{array}{l}33.8 \\
32.2 \\
30.8 \\
30.2 \\
29.8\end{array}$ & $\begin{array}{l}8.5 \\
8.5 \\
8.5 \\
8.5 \\
8.3\end{array}$ & $\begin{array}{r}9.8 \\
10.0 \\
9.3 \\
9.4 \\
4.7\end{array}$ & $\begin{array}{r}172 \\
167 \\
150 \\
145 \\
72\end{array}$ \\
\hline
\end{tabular}


Table 8. Particle-size distribution in cores from the Imperial Valley

[ft, foot. Location of sites shown in figure 2]

\begin{tabular}{|c|c|c|c|c|c|c|c|c|c|c|}
\hline \multicolumn{11}{|c|}{ Percentage finer than size, in millimeters, indicated } \\
\hline \multirow{2}{*}{$\begin{array}{l}\text { Depth of } \\
\text { core (ft) }\end{array}$} & \multicolumn{4}{|c|}{ Sand } & \multicolumn{4}{|c|}{ Silt } & \multicolumn{2}{|c|}{ Clay } \\
\hline & 1 & 0.5 & 0.25 & 0.125 & 0.062 & 0.031 & 0.016 & 0.008 & 0.004 & 0.002 \\
\hline \multicolumn{11}{|c|}{ S-417 (Northern Site) Lysimeter Hole--(Site 8) } \\
\hline $15-15.5$ & - & - & -- & 100 & 99 & 99 & 98 & 89 & 72 & 61 \\
\hline 20-20.5 & -- & - & 100 & 98 & 86 & 70 & 58 & 47 & 40 & 32 \\
\hline \multicolumn{11}{|c|}{ S-417 (Northern Site) Piezometer Hole } \\
\hline 74-74.7 & - & 100 & 98 & 58 & 6 & 3 & 2 & 2 & 2 & 1 \\
\hline $141-141.5$ & - & -- & -- & 100 & 99 & 98 & 93 & 86 & 68 & 57 \\
\hline 195.7-196.3 & 100 & 90 & 42 & 17 & 7 & 5 & 4 & 3 & 3 & 2 \\
\hline \multicolumn{11}{|c|}{ S-154 (Middle Site) Lysimeter Hole--(Site 50) } \\
\hline $13-13.5$ & -- & - & 100 & 99 & 98 & 94 & 83 & 71 & 57 & 48 \\
\hline \multicolumn{11}{|c|}{ S-154 (Middle Site) Piezometer Hole } \\
\hline $26-26.5$ & - & 100 & 96 & 50 & 38 & 37 & 35 & 31 & 24 & 21 \\
\hline $56-56.5$ & - & - & - & 100 & 94 & 75 & 49 & 37 & 34 & 31 \\
\hline $71-71.5$ & - & - & - & 100 & 89 & 60 & 22 & 12 & 12 & 9 \\
\hline $101-101.5$ & -- & - & - & 100 & 99 & 98 & 95 & 88 & 72 & 60 \\
\hline \multicolumn{11}{|c|}{ S-371 (Southern Site) Lysimeter Hole--(Site 98) } \\
\hline $12-12.5$ & - & -- & 100 & 99 & $96^{\circ}$ & 73 & 41 & 26 & 23 & 22 \\
\hline $13-13.5$ & - & -- & - & 100 & 99 & 98 & 98 & 78 & 59 & 49 \\
\hline $18-18.5$ & -- & -- & -- & 100 & 94 & 69 & 36 & 20 & 18 & 17 \\
\hline $19-19.5$ & - & -- & 100 & 99 & 96 & 91 & 91 & 79 & 63 & 52 \\
\hline \multicolumn{11}{|c|}{ S-371 (Southern Site) Piezometer Hole } \\
\hline $17-17.5$ & -- & - & - & -- & 100 & 99 & 94 & 69 & 47 & 42 \\
\hline $24-24.5$ & - & 100 & 99 & 75 & 44 & 37 & 33 & 27 & 22 & 19 \\
\hline $34-34.5$ & - & -- & 100 & 99 & 98 & 96 & 88 & 72 & 57 & 46 \\
\hline $65-65.5$ & - & -- & 100 & 99 & 97 & 95 & 86 & 73 & 58 & 49 \\
\hline $65.8-66$ & - & 100 & 99 & 28 & 12 & 9 & 7 & 6 & 5 & 4 \\
\hline $81-81.5$ & -- & - & 100 & 99 & 97 & 95 & 89 & 75 & 58 & 47 \\
\hline $106-106.5$ & 100 & 99 & 77 & 32 & 16 & 12 & 11 & 98 & 6 & 5 \\
\hline
\end{tabular}


Table 9. Lithologic description and perforated intervals for piezometers in the Imperial Valley

[Depth of clay-rich, low-permeability zones indicated in italics. Perforated interval: Asterisk indicates that piezometer is placed in a separate hole. $\mathrm{ft}$, foot; $\mathrm{cm}$, centimeter; $\mathrm{mm}$, millimeter]

\begin{tabular}{|c|c|c|}
\hline $\begin{array}{l}\text { Depth } \\
\text { (ft) }\end{array}$ & $\begin{array}{l}\text { Perforated } \\
\text { interval } \\
\text { (ft) }\end{array}$ & Lithologic description \\
\hline \multicolumn{3}{|r|}{ S-417 (Northern Site)--Site 8} \\
\hline $0-69$ & $\begin{array}{l}29-34 \\
49-54\end{array}$ & $\begin{array}{l}\text { Clay and fine silt; increase in silt with depth; invertebrate shells near } 25 \mathrm{ft} \text {; moderate } \\
\text { brown (5YR4/4) trending to dark yellowish brown (10YR4/2) at bottom of interval. }\end{array}$ \\
\hline $69-85$ & $70-75$ & Silt and fine sand; dark-gray or black thin stringers or small blebs in brown matrix. \\
\hline $85-181$ & & $\begin{array}{l}\text { Clay and silt; moderate yellowish brown }(10 \mathrm{YR} 4 / 2) \text { in top } 20 \mathrm{ft} \text { of interval to olive gray } \\
(5 \mathrm{Y} 4 / 1) \text { in bottom } 20 \mathrm{ft} \text {; olive-gray }(5 \mathrm{Y} 4 / 1) \text { to greenish-gray }(5 \mathrm{GY} 6 / 1) \text { thin }(<1 \mathrm{~cm}) \\
\text { horizontal and vertical (up to several centimeters in length and a few millimeters in } \\
\text { diameter) bands in dark-yellowish-brown }(10 \mathrm{YR} 4 / 2) \text { matrix in core from } 140-142 \mathrm{ft} \text {. }\end{array}$ \\
\hline & $194-199$ & Fine to medium sand; numerous small (1-3 mm) Turritella shells in core from $195-197 \mathrm{ft}$. \\
\hline \multicolumn{3}{|r|}{ S-154 (Middle Site)--Site 50} \\
\hline $0-21$ & & $\begin{array}{l}\text { Clay and silt with small amount of fine sand at } 16-18 \mathrm{ft} \text {; dark yellowish brown } \\
(5-10 \text { YR } 4 / 2-4) \text {. }\end{array}$ \\
\hline $21-32$ & $23-28 *$ & Very fine sand, clay, and silt. \\
\hline $32-34$ & & Thin clay layer. \\
\hline $34-42$ & $35-40$ & Very fine sand, clay, and silt similar to material from $20-32 \mathrm{ft}$. \\
\hline $42-64$ & $50-55$ & Silt and clay; moderate yellowish brown (5-10YR4-5/4). \\
\hline $64-76$ & $66-71$ & Silt; dark yellowish brown (5-10YR3-4/2). \\
\hline $76-80$ & & Thin clay layer. \\
\hline $80-96$ & $90-95$ & Silt similar to material from $64-76 \mathrm{ft}$. \\
\hline $96-102$ & & $\begin{array}{l}\text { Clay and fine silt; dark yellowish brown }(5-10 \text { YR } 4 / 2-4) \text {; numerous very small }(<1 \mathrm{~mm}) \\
\text { Turritella shells in core from } 100-102 \mathrm{ft} \text {. }\end{array}$ \\
\hline
\end{tabular}

\section{S-371 (Southern Site)--Site 98}

$0-18$

$18-25$

25-74

$18-23$

34-39*

44-49

$60-65$

Silt and clay with silt increasing with depth; silt and fine sand lens from 7 to $9 \mathrm{ft}$; dark yellowish brown (10YR4/2) at top grading to moderate brown (5-10YR4-5/4) at bottom.

Unsorted fine sand, silt, and clay; moderate brown (5YR4/4).

Numerous thin (up to a few feet thick) strata alternating between silt and clay, clay and silt, and fine sand and silt; dark yellowish brown (10YR4-5/2-4).

74-103

Clay and silt; numerous 1-mm gastropod and Turritella shells in core from $80-82 \mathrm{ft}$; moderate brown (5-10YR4-5/4).

103-114

109-114

Fine to medium sand. 
Table 10. Data from lysimeters and piezometers at three sites in the Imperial Valley

[Perforated interval and water level in feet below land surface (negative water-level values, including $<0$, above land surface). Altitude of land surface in feet above or below (-) sea level. $\mu \mathrm{S} / \mathrm{cm}$, microsiemen per centimeter at $25^{\circ} \mathrm{C}$; ${ }^{\circ} \mathrm{C}$, degree Celsius; $\mathrm{mg} / \mathrm{L}$, milligram per liter, $\mu \mathrm{g} / \mathrm{L}$, microgram per liter; $\mathrm{pCi} / \mathrm{L}$, picocurie per liter; ---, no data; <, actual value less than value shown. The analysis for each sample is displayed as one line on four consecutive pages]

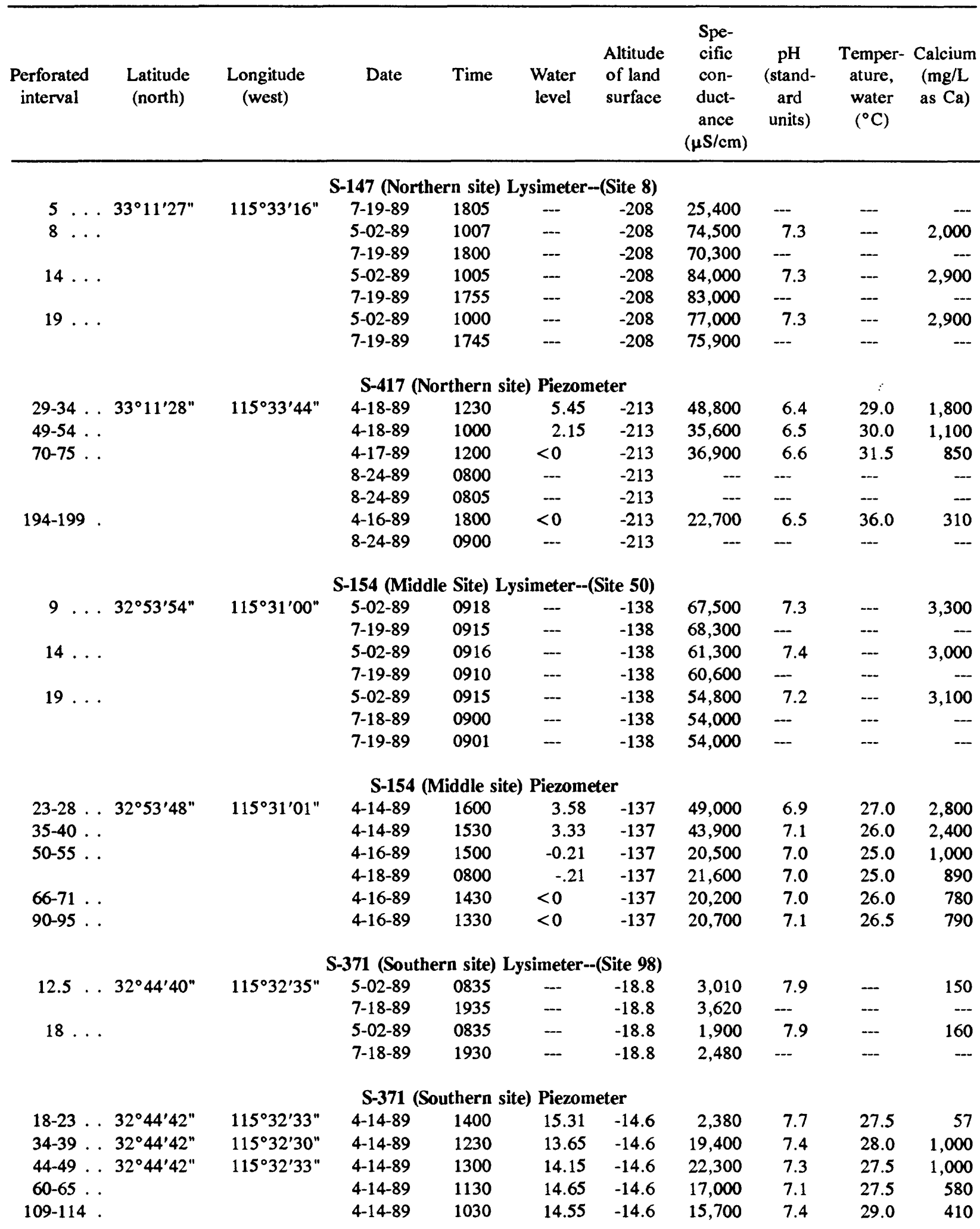


Table 10. Data from lysimeters and piezometers at three sites in the Imperial Valley--Continued

\begin{tabular}{|c|c|c|c|c|c|c|c|c|c|}
\hline $\begin{array}{l}\text { Perforated } \\
\text { interval }\end{array}$ & $\begin{array}{c}\text { Magne- } \\
\text { sium } \\
(\mathrm{mg} / \mathrm{L} \\
\text { as } \mathrm{Mg})\end{array}$ & $\begin{array}{c}\text { Sodium } \\
(\mathrm{mg} / \mathrm{L} \\
\text { as } \mathrm{Na})\end{array}$ & $\begin{array}{c}\text { Potas- } \\
\text { sium } \\
(\mathrm{mg} / \mathrm{L} \\
\text { as } \mathrm{K})\end{array}$ & $\begin{array}{c}\text { Alka- } \\
\text { linity, } \\
\text { lab } \\
(\mathrm{mg} / \mathrm{L} \\
\text { as } \\
\left.\mathrm{CaCO}_{3}\right)\end{array}$ & $\begin{array}{l}\text { Sulfate } \\
(\mathrm{mg} / \mathrm{L} \\
\text { as SO } \\
4)\end{array}$ & $\begin{array}{l}\text { Chlo- } \\
\text { ride } \\
(\mathrm{mg} / \mathrm{L} \\
\text { as } \mathrm{Cl})\end{array}$ & $\begin{array}{c}\text { Fluo- } \\
\text { ride } \\
(\mathrm{mg} / \mathrm{L} \\
\text { as F) }\end{array}$ & $\begin{array}{c}\text { Bromide } \\
\text { (mg/L } \\
\text { as } \mathrm{Br} \text { ) }\end{array}$ & $\begin{array}{c}\text { Silica } \\
(\mathrm{mg} / \mathrm{L} \\
\text { as } \\
\left.\mathrm{SiO}_{2}\right)\end{array}$ \\
\hline \multicolumn{10}{|c|}{ S-147 (Northern site) Lysimeter--(Site 8) } \\
\hline 5 & -- & -_ & -- & -- & -_ & --. & -- & -.. &.-- \\
\hline \multirow[t]{2}{*}{8} & 2,800 & 13,000 & 130 & 435 & 4,400 & 30,000 & 0.5 & 22 & 23 \\
\hline & - & -- & -- & - & -.- & --- & -- & - & --- \\
\hline 14 & 3,900 & 12,000 & 170 & 272 & 3,300 & 31,000 & .5 & 28 & 25 \\
\hline & - & -- & -- & -- & --- & -- & $\cdots$ & -- & --- \\
\hline \multirow[t]{2}{*}{19} & 3,600 & 12,000 & 140 & 242 & 3,300 & 34,000 & .3 & 27 & 31 \\
\hline & - & --- & -- & --- & -- & --- & --- & -- & -- \\
\hline \multicolumn{10}{|c|}{ S-417 (Northern site) Piezometer } \\
\hline $29-34$ & 2,300 & 6,600 & 74 & 644 & 3,400 & 18,000 & 0.1 & 16 & 29 \\
\hline 49-54 & 1,500 & 5,400 & 53 & 782 & 3,800 & 12,000 & .2 & 9.4 & 43 \\
\hline \multirow[t]{3}{*}{$70-75$} & 1,700 & 6,800 & 57 & 889 & 6,800 & 11,000 & .2 & 10 & 56 \\
\hline & -- & -.- & -- & - & --- & --. & -- & --- & --- \\
\hline & - & -- & $\cdots$ & -.. & --- & -- & --- & $\cdots$ & --- \\
\hline \multirow[t]{2}{*}{ 194-199 } & 490 & 3,900 & 77 & 717 & 620 & 6,700 & .6 & 6.6 & 63 \\
\hline & -- & -- & -- & --- & -- & -- & -- & -- & $\cdots$ \\
\hline \multicolumn{10}{|c|}{ S-154 (Middle Site) Lysimeter--(Site 50) } \\
\hline \multirow[t]{2}{*}{9} & 2,200 & 10,000 & 65 & 172 & 2,500 & 28,000 & 0.1 & 45 & 24 \\
\hline & - & -.- & - & $-\cdots$ & --- & -.. & $\cdots$ & --- & -- \\
\hline \multirow[t]{2}{*}{14.} & 2,400 & 8,500 & 83 & 206 & 2,500 & 25,000 & .2 & 42 & 24 \\
\hline & - & -- & --- & --- & -- & --- & -- & $\cdots$ & --- \\
\hline \multirow[t]{3}{*}{19} & 2,400 & 7,300 & 74 & 234 & 2,500 & 21,000 & .2 & 39 & 17 \\
\hline & -- & -- & --- & --- & --- & - & --- & --- & --- \\
\hline & --- & -- & -- & --. & $\ldots$ & $\cdots$ & --- & $\cdots$ & --- \\
\hline \multicolumn{10}{|c|}{ S-154 (Middle site) Piezometer } \\
\hline $23-28$ & 2,000 & 5,500 & 54 & 203 & 2,200 & 19,000 & 0.4 & 37 & 21 \\
\hline $35-40$ & 1,700 & 5,000 & 47 & 202 & 1,800 & 17,000 & .2 & 32 & 20 \\
\hline \multirow[t]{2}{*}{$50-55$} & 650 & 2,400 & 28 & 395 & 260 & 7,500 & .3 & 15 & 16 \\
\hline & 570 & 2,500 & 30 & 383 & 310 & 6,900 & .3 & 14 & 22 \\
\hline $66-71$ & 360 & 2,800 & 14 & 399 & 470 & 6,900 & .3 & 14 & 22 \\
\hline $90-95$ & 470 & 3,000 & 7.0 & 368 & 940 & 6,800 & .3 & 10 & 21 \\
\hline \multicolumn{10}{|c|}{ S-371 (Southern site) Lysimeter--(Site 98) } \\
\hline \multirow[t]{2}{*}{12.5} & 56 & 240 & 5.4 & 355 & 910 & 240 & 0.5 & 0.23 & 21 \\
\hline & $\ldots$ & --- & $\cdots$ & --- & --- & --- & -- & -- & -.- \\
\hline \multirow[t]{2}{*}{18.} & 66 & 450 & 8.6 & 309 & 500 & 180 & .4 & .15 & 20 \\
\hline & - & --- & -- & -- & --- & - & -- & --- & --- \\
\hline \multicolumn{10}{|c|}{ S-371 (Southern site) Piezometer } \\
\hline $18-23$ & . 30 & 390 & 12 & 264 & 480 & 270 & 0.8 & 0.59 & 20 \\
\hline 34-39 & 580 & 2,600 & 21 & 241 & 2,900 & 5,800 & .4 & 8.3 & 17 \\
\hline 44-49 & 580 & 2,900 & 26 & 251 & 2,500 & 6,700 & .7 & 11 & 20 \\
\hline $60-65$ & 290 & 2,500 & 17 & 252 & 750 & 5,200 & .5 & 9.1 & 26 \\
\hline $109-114$ & 210 & 2,400 & 21 & 261 & 510 & 4,900 & .5 & 8.5 & 32 \\
\hline
\end{tabular}


Table 10. Data from lysimeters and piezometers at three sites in the Imperial Valley--Continued

\begin{tabular}{|c|c|c|c|c|c|c|c|c|c|}
\hline $\begin{array}{l}\text { Perforated } \\
\text { interval }\end{array}$ & $\begin{array}{c}\text { Solids, } \\
\text { residue at } \\
180^{\circ} \mathrm{C} \\
(\mathrm{mg} / \mathrm{L})\end{array}$ & $\begin{array}{c}\text { Nitro- } \\
\text { gen, } \\
\mathrm{NO}_{2}+\mathrm{NO}_{3} \\
(\mathrm{mg} / \mathrm{L} \\
\text { as } \mathrm{N})\end{array}$ & $\begin{array}{c}\text { Nitro- } \\
\text { gen, } \\
\text { ammonia } \\
\text { (mg/L } \\
\text { as N) }\end{array}$ & $\begin{array}{c}\text { Alum- } \\
\text { inum } \\
(\mu \mathrm{g} / \mathrm{L} \\
\text { as } \mathrm{Al})\end{array}$ & $\begin{array}{c}\text { Arsenic } \\
(\mu \mathrm{g} / \mathrm{L} \\
\text { as As })\end{array}$ & $\begin{array}{l}\text { Boron } \\
(\mu \mathrm{g} / \mathrm{L} \\
\text { as B) }\end{array}$ & $\begin{array}{c}\text { Iron } \\
(\mu \mathrm{g} / \mathrm{L} \\
\text { as } \mathrm{Fe})\end{array}$ & $\begin{array}{c}\text { Lithium } \\
(\mu \mathrm{g} / \mathrm{L} \\
\text { as } \mathrm{Li})\end{array}$ & $\begin{array}{c}\text { Manga- } \\
\text { nese } \\
(\mu \mathrm{g} / \mathrm{L} \\
\text { as } \mathrm{Mn})\end{array}$ \\
\hline \multicolumn{10}{|c|}{ S-147 (Northern site) Lysimeter--(Site 8) } \\
\hline 5 & ... & 3.30 & 0.04 & -- & -.. & ... & -- & --- & --- \\
\hline \multirow[t]{2}{*}{8} & 57,300 & 7.00 & 1.60 & 60 & 4 & 6,100 & 590 & 3,000 & 390 \\
\hline & -.. & 5.00 & 1.30 & -- & --- & --- & -- & --. & - \\
\hline \multirow[t]{2}{*}{14} & 65,900 & .850 & 1.70 & 90 & 8 & 4,900 & 320 & 3,200 & 11,000 \\
\hline & -..- & .210 & .38 & ... & --. & -- & - & -. & --- \\
\hline \multirow[t]{2}{*}{19} & 57,900 & $<.080$ & 1.60 & 60 & 9 & 2,500 & 490 & 3,000 & 5,100 \\
\hline & -.. & .410 & .98 & -- & -- & -- & -- & -- & -- \\
\hline \multicolumn{10}{|c|}{ S-417 (Northern site) Piezometer } \\
\hline $29-34$ & 33,800 & 0.230 & 2.8 & 60 & 5 & 4,700 & 220 & 2,400 & 8,000 \\
\hline $49-54$ & 25,700 & .160 & 6.7 & 50 & 2 & 5,000 & 340 & 2,600 & 3,600 \\
\hline \multirow[t]{3}{*}{$70-75$} & 31,200 & $<.100$ & 21.0 & 50 & 91 & 6,400 & 23,000 & 5,100 & 400 \\
\hline & ... & $\ldots$ & $\ldots$ & ... & -.. & -.- & -.. & --- & --- \\
\hline & -.- & $\ldots$ & -.- & -.- & -.- & --- & -.. & -.. & -.- \\
\hline \multirow[t]{2}{*}{ 194-199 } & 13,800 & $<.100$ & 28.0 & 30 & 67 & 11,000 & 4,800 & 4,500 & 210 \\
\hline & - & --- & -- & -- & -- & -- & -- & -- & -- \\
\hline \multicolumn{10}{|c|}{ S-154 (Middle Site) Lysimeter--(Site 50) } \\
\hline \multirow[t]{2}{*}{9} & 49,500 & 110 & 1.6 & 80 & 2 & 840 & 340 & 1,000 & 4,600 \\
\hline & - & 120 & .38 & -- & --- & --- & -.- & --- & -.. \\
\hline \multirow[t]{2}{*}{14} & 46,500 & 34.0 & 1.6 & 70 & 5 & 1,400 & 390 & 1,900 & 4,200 \\
\hline & - & 29.0 & 3.7 & ... & ... & $\ldots$ & -.. & --- & --- \\
\hline \multirow[t]{3}{*}{19} & 40,000 & 1.10 & 2.0 & 90 & 6 & 2,500 & 490 & 880 & 5,800 \\
\hline & -. & -. & $\ldots$ & -.- & -- & -- & --- & -- & --- \\
\hline & -- & $<.100$ & .27 & - & --- & - & -- & -- & -- \\
\hline \multicolumn{10}{|c|}{ S-154 (Middle site) Piezometer } \\
\hline $23-28$ & 33,400 & 5.80 & 0.89 & $<50$ & 2 & 3,200 & 220 & 740 & 8,600 \\
\hline $35-40$ & 29,900 & 1.40 & 1.2 & 40 & 2 & 2,600 & 170 & 680 & 14,000 \\
\hline \multirow[t]{2}{*}{$50-55$} & 14,000 & $<.100$ & 1.3 & 50 & 5 & 1,800 & 310 & 440 & 4,600 \\
\hline & 13,600 & $<.100$ & 1.2 & 1,600 & 7 & 1,600 & 730 & 410 & 3,600 \\
\hline $66-71$ & 13,200 & $<.100$ & 5.5 & 20 & 46 & 3,200 & 6,400 & 260 & 1,200 \\
\hline $90-95$ & 13,400 & $<.100$ & 3.6 & 50 & 59 & 3,500 & 6,000 & 730 & 2,400 \\
\hline \multicolumn{10}{|c|}{ S-371 (Southern site) Lysimeter--(Site 98) } \\
\hline \multirow[t]{2}{*}{12.5} & 2,140 & 8.10 & 0.03 & 20 & 1 & 310 & 30 & 140 & 80 \\
\hline & - & 9.40 & .04 & -- & -.- & -.- & -- & -- & -.- \\
\hline \multirow[t]{2}{*}{18} & 1,350 & .470 & .03 & 20 & 2 & 370 & 14 & 140 & 19 \\
\hline & -.. & .320 & .04 & - & - & -- & -- & -- & $\cdots$ \\
\hline \multicolumn{10}{|c|}{ S-371 (Southern site) Piezometer } \\
\hline $18-23$ & . 1,450 & 0.190 & 0.19 & 30 & 13 & 570 & 10 & 70 & 170 \\
\hline $34-39$ & 13,200 & .320 & .36 & $<20$ & 6 & 2,400 & 60 & 540 & 780 \\
\hline $44-49$ & 15,000 & $<.100$ & .53 & 50 & 4 & 2,900 & 80 & 600 & 3,400 \\
\hline $60-65$ & 10,700 & .150 & .08 & 20 & 2 & 3,500 & 80 & 1,500 & 3,000 \\
\hline $109-114$ & 9,620 & $<.100$ & 5.7 & 20 & 3 & 3,600 & 40 & 2,000 & 1,500 \\
\hline
\end{tabular}


Table 10. Data from lysimeters and piezometers at three sites in the Imperial Valley--Continued

\begin{tabular}{|c|c|c|c|c|c|c|c|c|c|c|}
\hline \multirow{2}{*}{$\begin{array}{l}\text { Perforated } \\
\text { interval }\end{array}$} & \multirow{2}{*}{$\begin{array}{l}\text { Molyb- } \\
\text { denum } \\
(\mu \mathrm{g} / \mathrm{L} \\
\text { as Mo) }\end{array}$} & \multirow{2}{*}{$\begin{array}{c}\text { Sele- } \\
\text { nium } \\
(\mu \mathrm{g} / \mathrm{L} \\
\text { as Se })\end{array}$} & \multirow{2}{*}{$\begin{array}{c}\text { Stron- } \\
\text { tium } \\
(\mu \mathrm{g} / \mathrm{L} \\
\text { as } \mathrm{Sr})\end{array}$} & \multirow[t]{2}{*}{$\begin{array}{c}\text { Tritium, } \\
\text { total } \\
(\mathrm{pCi} / \mathrm{L})\end{array}$} & \multicolumn{5}{|c|}{ Stable-isotope ratio (permil) } & \multirow[t]{2}{*}{$\begin{array}{c}\text { Carbon-14 } \\
\text { (percent } \\
\text { modern) }\end{array}$} \\
\hline & & & & & ${ }^{2} \mathrm{H} /{ }^{1} \mathrm{H}$ & ${ }^{18} \mathrm{O}^{16} \mathrm{O}$ & ${ }^{13} \mathrm{C} /{ }^{12} \mathrm{C}$ & ${ }^{15} \mathrm{~N} /{ }^{14} \mathrm{~N}$ & ${ }^{34} S /{ }^{32} S$ & \\
\hline \multicolumn{11}{|c|}{ S-147 (Northern site) Lysimeter--(Site 8) } \\
\hline 5 & -- & -- & --- & - & -- & - & -- & 17.70 & -- & - \\
\hline \multirow[t]{2}{*}{8} & .90 & 24 & 54,000 & 110 & -80.5 & -8.10 & --. & $\ldots$ & --- & -- \\
\hline & - & - & -- & -- & - & -- & --- & 33.10 & -- & - \\
\hline \multirow[t]{2}{*}{14} & 100 & 20 & 79,000 & 21 & -69.5 & -6.50 & $\ldots$ & - & -.- & -- \\
\hline & - & -- & -- & -- & - & -- & --- & -- & -- & -- \\
\hline \multirow[t]{2}{*}{19} & . . 99 & 91 & 71,000 & 15 & -68.0 & -6.25 & $\ldots$ & - & --- & - \\
\hline & - & -- & -- & -- & -- & - & -- & 78.70 & -- & -- \\
\hline \multicolumn{11}{|c|}{ S-417 (Northern site) Piezometer } \\
\hline $29-34$ & 5 & 4 & 48,000 & 6.4 & -59.5 & -5.15 & -- & $\ldots$ & 4.00 & -.. \\
\hline $49-54$ & 6 & 1 & 27,000 & 14 & -60.5 & -5.70 & -.. & - & 7.00 & -. \\
\hline \multirow[t]{3}{*}{$70-75$} & 4 & $<1$ & 22,000 & 2.2 & -67.5 & -6.75 & --- & - & 6.00 & - \\
\hline & - & -- & -- & $\ldots$ & - & $\ldots$ & -3.50 & -- & --- & 2.5 \\
\hline & - & -- & -- & -- & $\ldots$ & -- & -- & -_ & --- & 2.6 \\
\hline \multirow[t]{2}{*}{ 194-199 } & $\ldots 10$ & $<1$ & 11,000 & .3 & -77.5 & -7.95 & --- & -_ & 22.10 & - \\
\hline & - & - & -.. & -- & --- & --- & -4.60 & -- & --- & 1.3 \\
\hline \multicolumn{11}{|c|}{ S-154 (Middle Site) Lysimeter--(Site 50) } \\
\hline \multirow[t]{2}{*}{9} & $\ldots 70$ & 120 & 54,000 & -- & $-75.5^{\circ}$ & -7.85 & --- & - & -.. & -- \\
\hline & -- & -- & -.. & - & -- & - & -- & 67.30 & $\cdots$ & -- \\
\hline \multirow[t]{2}{*}{14} & $\ldots 75$ & 73 & 60,000 & 2.6 & -78.5 & -8.40 & --- & $\ldots$ & -.. & --- \\
\hline & -- & -- & -.. & $\ldots$ & -- & - & $\cdots$ & 102.00 & -- & -- \\
\hline \multirow[t]{3}{*}{19} & .50 & 2 & 55,000 & 1.9 & -83.5 & -8.75 & -- & -- & -- & -- \\
\hline & -- & - & --. & $\ldots$ & -- & $\ldots$ & - & -- & -- & -- \\
\hline & - & - & -- & -- & -- & - & - & -- & -- & -- \\
\hline \multicolumn{11}{|c|}{ S-154 (Middle site) Piezometer } \\
\hline $23-28$ & $\ldots 16$ & 9 & 59,000 & 0.6 & -85.5 & -8.75 & --- & --- & 0.90 & --- \\
\hline $35-40$ & $\ldots 15$ & 4 & 49,000 & 5.5 & -87.0 & -9.10 & $-\ldots$ & -.. & .20 & -.- \\
\hline \multirow[t]{2}{*}{$50-55$} & $\ldots 11$ & $<1$ & 20,000 & 7.3 & -88.5 & -9.55 & --- & -- & 28.00 & -- \\
\hline & 12 & $<1$ & 18,000 & 3.8 & -89.0 & -9.75 & -- & -- & 33.50 & -- \\
\hline $66-71$ & . . 24 & $<1$ & 14,000 & 1.3 & -87.5 & -9.30 & $\ldots$ & --- & 20.80 & --- \\
\hline $90-95$ & $\ldots 15$ & $<1$ & 16,000 & .6 & -87.5 & -9.35 & -- & -- & 9.40 & -- \\
\hline \multicolumn{11}{|c|}{ S-371 (Southern site) Lysimeter-(Site 98) } \\
\hline \multirow[t]{2}{*}{12.5} & .26 & 7 & 2,100 & 95 & -99.5 & -12.20 & -. & -- & -- & -- \\
\hline & - & $\ldots$ & -.. & $\ldots$ & _. & --- & --- & $\left.10.2^{\circ}\right)$ & --- & --- \\
\hline 18 & $\ldots 59$ & 15 & 2,500 & 91 & -102.0 & -12.10 & -- & -- & --- & -- \\
\hline & - & - & -- & - & - & -- & -- & -- & -- & -- \\
\hline & & & & S-371 (Sou & thern sit & Piezomet & & & & \\
\hline $18-23$ & $\ldots 23$ & $<1$ & 980 & 88 & -103.5 & -13.00 & --- & -- & -0.1 & $\cdots$ \\
\hline 34-39 & $\ldots 23$ & 19 & 18,000 & 11 & -96.0 & -11.35 & --- & $-\ldots$ & .30 & --- \\
\hline $44-49$ & .24 & $<1$ & 20,000 & 6.7 & -89.5 & -10.10 & -.- & -- & .70 & -- \\
\hline $60-65$ & $\ldots 31$ & $<1$ & 11,000 & 1.3 & -84.0 & -9.20 & --- & --- & 8.20 & -.. \\
\hline $109-114$ &. .35 & $<1$ & 9,800 & 2.6 & -84.0 & -9.05 & --- & -- & 13.20 & --- \\
\hline
\end{tabular}


Table 11. Selenium and water-extractable sulfate, chloride, and selenium concentrations in soils from 15 fields

[Soils data on dry-weight basis; aqueous data are from analysis of extract using 5 to 1 ratio of water to soil. See text (p. 12-13) for description of soil identifier. Concentrations in ppm (parts per million) and ppb (parts per billion). Laboratory standard is a soil from the San Joaquin Valley. <, less than indicated reporting limit; --- designates insufficient sample for analysis]

\begin{tabular}{|c|c|c|c|c|c|}
\hline \multirow{2}{*}{$\begin{array}{c}\text { Soil } \\
\text { identifier }\end{array}$} & \multirow{2}{*}{$\begin{array}{l}\text { Laboratory } \\
\text { number }\end{array}$} & \multirow{2}{*}{$\frac{\text { Soil }}{\begin{array}{c}\text { Selenium } \\
(\mathrm{ppm})\end{array}}$} & \multicolumn{3}{|c|}{ Water extract } \\
\hline & & & $\begin{array}{l}\text { Sulfate } \\
\text { (ppm) }\end{array}$ & $\begin{array}{c}\text { Chloride } \\
\text { (ppm) }\end{array}$ & $\begin{array}{l}\text { Selenium } \\
(\mathrm{ppb})\end{array}$ \\
\hline \multicolumn{6}{|c|}{ Field at S-226 (Site 6) } \\
\hline H-53-0-3 & D-322562 & 0.5 & 5,200 & 2,900 & 63 \\
\hline H-53-0-6 & D-322563 & .3 & 2,400 & 4,500 & 76 \\
\hline H-53-25-3 & D-322564 & .5 & 11,000 & 2,300 & 73 \\
\hline H-53-25-6 & $D-322565$ & .5 & 8,800 & 4,500 & 76 \\
\hline H-53-50-3 & D-322566 & .4 & 12,000 & 1,900 & 91 \\
\hline H-53-50-6 & D-322567 & .3 & 1,300 & 4,200 & 70 \\
\hline$M-41-0-3$ & D-322568 & .6 & 5,200 & 3,500 & 130 \\
\hline$M-41-0-6$ & D-322569 & .3 & 1,300 & 4,900 & 90 \\
\hline$M-41-25-3$ & D-322570 & .3 & 6,600 & 2,400 & 76 \\
\hline$M-41-25-6$ & D-322571 & .3 & 1,200 & 4,200 & 73 \\
\hline M-41-50-3 & D-322572 & .4 & 10,000 & 3,100 & 97 \\
\hline$M-41-50-6$ & D-322573 & .6 & 4,800 & 4,900 & 90 \\
\hline$T-7-0-3$ & D-322574 & .3 & 12,000 & 2,700 & 53 \\
\hline$T-7-0-6$ & D-322575 & .6 & 4,700 & 4,200 & 54 \\
\hline $\mathrm{T}-7-25-3$ & D-322576 & .4 & 11,000 & 2,800 & 51 \\
\hline$T-7-25-6$ & D-322577 & .7 & 4,700 & 4,200 & 74 \\
\hline T-7-50-3 & D-322578 & .3 & 8,200 & 2,500 & 52 \\
\hline $\mathrm{T}-7-50-6$ & D-322579 & .3 & 3,200 & 4,000 & 46 \\
\hline H-53-0-3 duplicate & D-322580 & .5 & 5,200 & 2,700 & 88 \\
\hline Laboratory standard & D-322581 & 1.1 & 1,000 & 240 & 10 \\
\hline \multicolumn{6}{|c|}{ Field at S-269 (Site 7) } \\
\hline H-12-0-3 & D-322582 & 0.1 & 9,600 & 400 & 46 \\
\hline H-12-0-6 & D-322583 & .6 & 1,500 & 420 & 35 \\
\hline H-12-12-3 & D-322584 & .1 & 3,500 & 410 & 46 \\
\hline$H-12-16-6$ & D-322585 & .9 & 4,900 & 520 & 53 \\
\hline $\mathrm{H}-12-25-3$ & D-322586 & .4 & 10,000 & 330 & 110 \\
\hline$H-12-25-6$ & D-322587 & .8 & 2,300 & 460 & 39 \\
\hline T-2A-0-3 & D-322588 & .1 & 11,000 & 820 & 11 \\
\hline T-2A-0-6 & D-322589 & .1 & 5,200 & 1,300 & 28 \\
\hline T-2A-12-3 & D-322590 & .1 & 11,000 & 840 & 14 \\
\hline$T-2 A-12-6$ & D-322591 & .1 & 4,700 & 1,700 & 47 \\
\hline T-2A-25-3 & D-322592 & .1 & 11,000 & 1,300 & 17 \\
\hline T-2A-25-6 & D-322593 & .1 & 4,800 & 1,800 & 47 \\
\hline H-12-0-3 duplicate & D-322594 & .1 & 9,600 & 390 & 46 \\
\hline Laboratory standard & D-322595 & 1.1 & 1,000 & 270 & 10 \\
\hline
\end{tabular}


Table 11. Selenium and water-extractable sulfate, chloride, and selenium concentrations in soils from 15 fields--Continued

\begin{tabular}{|c|c|c|c|c|c|}
\hline \multirow{2}{*}{$\begin{array}{c}\text { Soil } \\
\text { identifier }\end{array}$} & \multirow{2}{*}{$\begin{array}{l}\text { Laboratory } \\
\text { number }\end{array}$} & \multirow{2}{*}{$\frac{\text { Soil }}{\begin{array}{c}\text { Selenium } \\
(\mathrm{ppm})\end{array}}$} & \multicolumn{3}{|c|}{ Water extract } \\
\hline & & & $\begin{array}{c}\text { Sulfate } \\
(\mathrm{ppm})\end{array}$ & $\begin{array}{c}\text { Chloride } \\
\text { (ppm) }\end{array}$ & $\begin{array}{c}\text { Selenium } \\
\text { (ppb) }\end{array}$ \\
\hline \multicolumn{6}{|c|}{ Field at S-417 (Site 8) } \\
\hline $\mathrm{H}-32-0-3$ & D-322462 & 0.3 & 8,100 & 340 & 22 \\
\hline $\mathrm{H}-32-0-6$ & D-322463 & .1 & 11,000 & 2,500 & 79 \\
\hline $\mathrm{H}-32-25-3$ & D-322464 & .2 & 7,400 & 1,800 & 41 \\
\hline H-32-25-6 & $D-322465$ & .2 & 7,800 & 2,600 & 63 \\
\hline $\mathrm{H}-32-50-3$ & D-322466 & .3 & 11,000 & 2,000 & 68 \\
\hline H-32-50-6 & D-322467 & .2 & 11,000 & 2,800 & 89 \\
\hline M-24-0-3 & D-322468 & .2 & 12,000 & 780 & 40 \\
\hline M-24-0-6 & D-322469 & .7 & 9,800 & 1,800 & 100 \\
\hline$M-24-25-3$ & D-322470 & .3 & 12,000 & 1,800 & 36 \\
\hline$M-24-25-6$ & D-322471 & .4 & 11,000 & 2,300 & 37 \\
\hline$M-24-50-3$ & D-322472 & .4 & 8,400 & 1,700 & 23 \\
\hline M-24-50-6 & D-322473 & .5 & 5,400 & 2,400 & 200 \\
\hline$T-18-0-3$ & $\mathrm{D}-322474$ & .2 & 10,000 & 450 & 34 \\
\hline$T-18-0-6$ & D-322475 & .2 & 11,000 & 640 & 27 \\
\hline$T-18-25-3$ & D-322476 & .2 & 12,000 & 1,000 & 26 \\
\hline$T-18-25-6$ & D-322477 & .1 & 10,000 & 1,200 & 23 \\
\hline$T-18-50-3$ & D-322478 & .3 & 10,000 & 1,500 & 20 \\
\hline$T-18-50-6$ & D-322479 & 3 & 5,000 & 1,600 & 26 \\
\hline H-32-0-3 duplicate & D-322480 & .3 & 7,000 & 380 & 20 \\
\hline Laboratory standard & D-322481 & .9 & 1,200 & 320 & 9 \\
\hline \multicolumn{6}{|c|}{ Field at S-94 (Site 30) } \\
\hline$H-13-0-3$ & D-322596 & 0.3 & 1,000 & 180 & 14 \\
\hline H-13-0-6 & D-322597 & .2 & 11,000 & 45 & 18 \\
\hline $\mathrm{H}-13-23-3$ & D-322598 & .3 & 7,200 & 110 & 14 \\
\hline $\mathrm{H}-13-23-6$ & D-322599 & .2 & 10,000 & 300 & 28 \\
\hline $\mathrm{H}-13-46-3$ & D-322600 & .4 & 6,900 & 230 & 16 \\
\hline H-13-46-6 & D-322601 & .2 & 11,000 & 180 & 14 \\
\hline$M-11-0-3$ & D-322602 & .5 & 4,200 & 380 & 15 \\
\hline M-11-0-6 & D-322603 & .4 & 3,500 & 890 & 18 \\
\hline M-11-23-3 & D-322604 & .3 & 5,400 & 450 & 10 \\
\hline M-11-23-6 & D-322605 & .2 & 2,300 & 670 & 8 \\
\hline M-11-46-3 & D-322606 & .2 & 4,300 & 260 & 8 \\
\hline M-11-46-6 & D-322607 & .3 & 62 & 780 & 12 \\
\hline$T-9-0-3$ & D-322608 & .3 & 6,700 & 800 & 7 \\
\hline$T-9-0-6$ & D-322609 & .4 & 5,400 & 970 & 12 \\
\hline$T-9-23-3$ & D-322610 & .2 & 11,000 & 540 & 7 \\
\hline T-9-23-6 & D-322611 & .3 & 9,600 & 1,200 & 14 \\
\hline$T-9-46-3$ & D-322612 & .1 & 1,700 & 74 & 3 \\
\hline$T-9-46-6$ & D-322613 & .3 & 6,300 & 980 & 13 \\
\hline H-13-0-3 duplicate & D-322614 & .4 & 1,200 & 170 & 13 \\
\hline Laboratory standard & D-322615 & 1.1 & 1,100 & 270 & 11 \\
\hline
\end{tabular}


Table 11. Selenium and water-extractable sulfate, chloride, and selenium concentrations in soils from 15 fields--Continued

\begin{tabular}{|c|c|c|c|c|c|}
\hline \multirow{2}{*}{$\begin{array}{c}\text { Soil } \\
\text { identifier }\end{array}$} & \multirow{2}{*}{$\begin{array}{l}\text { Laboratory } \\
\text { number }\end{array}$} & \multirow{2}{*}{$\frac{\text { Soil }}{\begin{array}{c}\text { Selenium } \\
(\mathrm{ppm})\end{array}}$} & \multicolumn{3}{|c|}{ Water extract } \\
\hline & & & $\begin{array}{l}\text { Sulfate } \\
\text { (ppm) }\end{array}$ & $\begin{array}{l}\text { Chloride } \\
\text { (ppm) }\end{array}$ & $\begin{array}{c}\text { Selenium } \\
\text { (ppb) }\end{array}$ \\
\hline \multicolumn{6}{|c|}{ Field at S-142 (Site 33) } \\
\hline H- $6 A-0-3$ & D-322482 & 0.3 & 8,500 & 560 & 17 \\
\hline $\mathrm{H}-6 \mathrm{~A}-0-6$ & D-322483 & .1 & 1,000 & 220 & 11 \\
\hline H-6A-33-3 & D-322484 & .4 & 5,000 & 320 & 10 \\
\hline H-6A-33-6 & D-322485 & .1 & 1,500 & 250 & 11 \\
\hline H-6A-66-3 & $D-322486$ & .3 & 4,300 & 440 & 7 \\
\hline$H-6 A-66-6$ & D-322487 & .2 & 2,200 & 300 & 11 \\
\hline M-4B-0-3 & D-322488 & .5 & 3,700 & 440 & 21 \\
\hline M-4B-0-6 & D-322489 & .1 & 1,000 & 220 & 10 \\
\hline M-4B-33-3 & D-322490 & .5 & 11,000 & 800 & 70 \\
\hline M-4B-33-6 & D-322491 & .2 & 2,500 & 420 & 40 \\
\hline$M-4 B-66-3$ & D-322492 & .2 & 4,800 & 320 & 11 \\
\hline$M-4 B-66-6$ & D-322493 & .2 & 1,800 & 700 & 7 \\
\hline$T-2 B-0-3$ & D-322494 & .2 & 3,800 & 820 & 22 \\
\hline$T-2 B-0-6$ & D-322495 & .2 & 2,600 & 640 & 13 \\
\hline $\mathrm{T}-2 \mathrm{~B}-33-3$ & D-322496 & .3 & 12,000 & 920 & 61 \\
\hline T-2B-33-6 & D-322497 & .2 & 4,500 & 650 & 14 \\
\hline $\mathrm{T}-2 \mathrm{~B}-66-3$ & D-322498 & .2 & 5,600 & 680 & 33 \\
\hline T-2B-66-6 & D-322499 & .2 & 2,200 & 580 & 13 \\
\hline H-6A-0-3 duplicate & D-322500 & .3 & 8,500 & 550 & 19 \\
\hline Laboratory standard & D-322501 & 1.1 & 1,200 & 290 & 8 \\
\hline \multicolumn{6}{|c|}{ Field at S-241 (Site 41) } \\
\hline $\mathrm{H}-20-0-3$ & D-322616 & 0.5 & 15,000 & 2,400 & 34 \\
\hline $\mathrm{H}-20-0-6$ & D-322617 & .4 & 12,000 & 2,200 & 31 \\
\hline$H-20-62-3$ & D-322618 & .5 & 14,000 & 1,800 & 24 \\
\hline H-20-62-6 & D-322619 & .3 & 6,400 & 2,000 & 35 \\
\hline $\mathrm{H}-20-124-3$ & $D-322620$ & .4 & 13,000 & 1,800 & 21 \\
\hline $\mathrm{H}-20-124-6$ & D-322621 & .2 & 11,000 & 2,000 & 21 \\
\hline M-18-0-3 & D-322622 & .4 & 13,000 & 690 & 23 \\
\hline$M-18-0-6$ & $D-322623$ & .3 & 6,400 & 1,600 & 25 \\
\hline$M-18-62-3$ & D-322624 & .3 & 10,000 & 290 & 11 \\
\hline$M-18-62-6$ & D-322625 & .4 & 13,000 & 1,400 & 28 \\
\hline M-18-124-3 & D-322626 & .4 & 12,000 & 160 & 17 \\
\hline$M-18-124-6$ & D-322627 & .3 & 11,000 & 420 & 27 \\
\hline$T-14-0-3$ & D-322628 & .4 & 14,000 & 570 & 60 \\
\hline$T-14-0-6$ & D-322629 & --- & -.. & -.. & -.- \\
\hline$T-14-62-3$ & $D-322630$ & .2 & 11,000 & 610 & 29 \\
\hline$T-14-62-6$ & D-322631 & .2 & 12,000 & 1,900 & 17 \\
\hline T-14-124-3 & D-322632 & .2 & 7,600 & 530 & 18 \\
\hline T-14-124-6 & D-322633 & .2 & 12,000 & 2,100 & 13 \\
\hline H-20-0-3 duplicate & D-322634 & .5 & 14,000 & 2,400 & 35 \\
\hline Laboratory standard & D-322635 & 1.2 & 990 & 240 & 10 \\
\hline
\end{tabular}


Table 11. Selenium and water-extractable sulfate, chloride, and selenium concentrations in soils from 15 fields--Continued

\begin{tabular}{|c|c|c|c|c|c|}
\hline \multirow{2}{*}{$\begin{array}{c}\text { Soil } \\
\text { identifier }\end{array}$} & \multirow{2}{*}{$\begin{array}{l}\text { Laboratory } \\
\text { number }\end{array}$} & \multirow{2}{*}{$\frac{\text { Soil }}{\begin{array}{c}\text { Selenium } \\
\text { (ppm) }\end{array}}$} & \multicolumn{3}{|c|}{ Water extract } \\
\hline & & & $\begin{array}{l}\text { Sulfate } \\
(\mathrm{ppm})\end{array}$ & $\begin{array}{c}\text { Chloride } \\
\text { (ppm) }\end{array}$ & $\begin{array}{c}\text { Selenium } \\
\text { (ppb) }\end{array}$ \\
\hline \multicolumn{6}{|c|}{ Field at S-154 (Site 50) } \\
\hline H-23-0-3 & D-325043 & 0.6 & 720 & 120 & 11 \\
\hline $\mathrm{H}-23-0-6$ & D-325044 & .4 & 1,000 & 290 & 6 \\
\hline $\mathrm{H}-23-44-3$ & D-325045 & .4 & 9,700 & 390 & 9 \\
\hline H-23-44-6 & D-325046 & .4 & 2,700 & 2,000 & 16 \\
\hline H-23-89-3 & D-325047 & .2 & 2,500 & 500 & 4 \\
\hline H-23-89-6 & D-325048 & .3 & 4,000 & 2,300 & 35 \\
\hline M-17-0-3 & D-325049 & .2 & 4,000 & 330 & 7 \\
\hline M-17-0-6 & $D-325050$ & .2 & 1,900 & 2,800 & 8 \\
\hline$M-17-49-3$ & D-325051 & .3 & 4,200 & 470 & 8 \\
\hline$M-17-49-6$ & D-325052 & .5 & 4,400 & 3,600 & 15 \\
\hline M-17-98-3 & D-325053 & .3 & 8,800 & 680 & 10 \\
\hline M-17-98-6 & D-325054 & .5 & 5,000 & 3,700 & 16 \\
\hline$T-5-0-3$ & D-325055 & .3 & 10,000 & 1,400 & 12 \\
\hline$T-5-0-6$ & D-325056 & .4 & 1,600 & 3,900 & 9 \\
\hline$T-5-49-3$ & D-325057 & .2 & 10,000 & 1,200 & 12 \\
\hline$T-5-49-6$ & D-325058 & .4 & 5,600 & 3,700 & 12 \\
\hline$T-5-98-3$ & D-325059 & .4 & 10,000 & 1,700 & 17 \\
\hline T-5-98-6 & D-325060 & .4 & 2,500 & 3,500 & 11 \\
\hline H-23-0-3 duplicate & D-325061 & .2 & 10,000 & 200 & 7 \\
\hline Laboratory standard & D-325062 & 1.0 & 1,100 & 280 & 11 \\
\hline \multicolumn{6}{|c|}{ Field at S-265 (Site 67) } \\
\hline $\mathrm{H}-30-0-3$ & D-322522 & 0.1 & 7,500 & 82 & 3 \\
\hline$H-30-0-6$ & D-322523 & .1 & 6,600 & 160 & 6 \\
\hline H-30-25-3 & D-322524 & .1 & 6,800 & 320 & $<3$ \\
\hline$H-30-25-6$ & D-322525 & .1 & 2,700 & 360 & 4 \\
\hline H-30-50-3 & D-322526 & .1 & 4,300 & 45 & 3 \\
\hline$H-30-50-6$ & D-322527 & .2 & 6,300 & 560 & 15 \\
\hline M-30-0-3 & D-322528 & .1 & 530 & 51 & 3 \\
\hline$M-40-0-6$ & D-322529 & .1 & 550 & 82 & 3 \\
\hline$M-30-25-3$ & D-322530 & .1 & 560 & 140 & 6 \\
\hline M-30-25-6 & D-322531 & .1 & 3,100 & 230 & 12 \\
\hline M-30-50-3 & D-322532 & .1 & 260 & 97 & 3 \\
\hline M-30-50-6 & D-322533 & .1 & 320 & 44 & 3 \\
\hline T-30-0-3 & D-322534 & .2 & 990 & 130 & 9 \\
\hline$T-30-0-6$ & D-322535 & .1 & 1,900 & 200 & 6 \\
\hline$T-30-25-3$ & D-322536 & .2 & 7,700 & 330 & 18 \\
\hline$T-30-25-6$ & D-322537 & .1 & 1,400 & 340 & 10 \\
\hline$T-30-50-3$ & D-322538 & .1 & 10,000 & 180 & 10 \\
\hline$T-30-50-6$ & D-322539 & .2 & 2,200 & 720 & 34 \\
\hline H-30-0-3 duplicate & D-322540 & .1 & 9,400 & 100 & 3 \\
\hline Laboratory standard & D-322541 & 1.0 & 920 & 250 & 9 \\
\hline
\end{tabular}


Table 11. Selenium and water-extractable sulfate, chloride, and selenium concentrations in soils from 15 fields--Continued

\begin{tabular}{|c|c|c|c|c|c|}
\hline \multirow{2}{*}{$\begin{array}{c}\text { Soil } \\
\text { identifier }\end{array}$} & \multirow{2}{*}{$\begin{array}{l}\text { Laboratory } \\
\text { number }\end{array}$} & \multirow{2}{*}{$\frac{\text { Soil }}{\substack{\text { Selenium } \\
(\mathrm{ppm})}}$} & \multicolumn{3}{|c|}{ Water extract } \\
\hline & & & $\begin{array}{c}\text { Sulfate } \\
\text { (ppm) }\end{array}$ & $\begin{array}{c}\text { Chloride } \\
\text { (ppm) }\end{array}$ & $\begin{array}{c}\text { Selenium } \\
\text { (ppb) }\end{array}$ \\
\hline \multicolumn{6}{|c|}{ Field at $S-4$ (Site 75) } \\
\hline $\mathrm{H}-12 \mathrm{C}-0-3$ & D-322502 & 0.2 & 5,400 & 140 & 4 \\
\hline$H-12 C-0-6$ & D-322503 & .2 & 5,400 & 790 & 15 \\
\hline $\mathrm{H}-12 \mathrm{C}-18-3$ & D-322504 & .1 & 9,200 & 380 & 7 \\
\hline $\mathrm{H}-12 \mathrm{C}-18-6$ & D-322505 & .2 & 2,800 & 790 & 25 \\
\hline $\mathrm{H}-12 \mathrm{C}-36-3$ & D-322506 & .1 & 7,200 & 380 & 4 \\
\hline$H-12 C-36-6$ & D-322507 & .2 & 4,300 & 1,200 & 25 \\
\hline$M-9 C-0-3$ & D-322508 & .1 & 3,800 & 140 & $<3$ \\
\hline$M-9 C-0-6$ & D-322509 & .1 & 3,500 & 790 & 12 \\
\hline M-9C-18-3 & D-322510 & .1 & 12,000 & 290 & 7 \\
\hline M-9C-18-6 & D-322511 & .2 & 2,400 & 1,400 & 40 \\
\hline$M-9 C-36-3$ & D-322512 & .1 & 5,000 & 310 & 4 \\
\hline M-9C-36-6 & D-322513 & .1 & 4,000 & 780 & 25 \\
\hline$T-6 C-0-3$ & D-322514 & .1 & 9,600 & 500 & 13 \\
\hline$T-6 C-0-6$ & D-322515 & .2 & 4,500 & 1,100 & 22 \\
\hline$T-6 C-18-3$ & D-322516 & .2 & 8,200 & 360 & 4 \\
\hline$T-6 C-18-6$ & D-322517 & .2 & 1,200 & 1,600 & 65 \\
\hline$T-6 C-36-3$ & D-322518 & .1 & 7,800 & 1,000 & 22 \\
\hline$T-6 C-36-6$ & D-322519 & .2 & 4,500 & 1,700 & 50 \\
\hline H-12C-0-3 duplicate & D-322520 & .2 & 5,400 & 150 & 3 \\
\hline Laboratory standard & D-322521 & 1.1 & 950 & 200 & 8 \\
\hline \multicolumn{6}{|c|}{ Field at S-72 (Site 79) } \\
\hline $\mathrm{H}-8-0-3$ & D-322382 & 0.3 & 7,700 & 200 & 18 \\
\hline H-8-0-6 & D-322383 & .1 & 2,600 & 270 & 11 \\
\hline$H-8-11-3$ & D-322384 & .2 & 10,000 & 120 & 4 \\
\hline H-8-11-6 & D-322385 & .1 & 3,600 & 270 & 12 \\
\hline$H-8-23-3$ & D-322386 & .2 & 3,500 & 110 & 3 \\
\hline$H-8-23-6$ & D-322387 & .1 & 2,800 & 170 & 9 \\
\hline M-5-0-3 & D-322388 & .2 & 7,600 & 140 & 8 \\
\hline M-5-0-6 & D-322389 & .1 & 2,900 & 130 & 7 \\
\hline$M-5-11-3$ & D-322390 & .2 & 3,600 & 140 & 5 \\
\hline M-5-11-6 & D-322391 & .1 & 3,200 & 210 & 6 \\
\hline M-5-23-3 & D-322392 & .1 & 9,200 & 120 & 3 \\
\hline M-5-23-6 & D-322393 & .1 & 2,000 & 230 & 4 \\
\hline$T-2-0-3$ & D-322394 & -- & - & $\ldots$ & -- \\
\hline$T-2-0-6$ & D-322395 & .1 & 1,000 & 470 & 5 \\
\hline $\mathrm{T}-2-11-3$ & D-322396 & .1 & 6,000 & 320 & 8 \\
\hline$T-2-11-6$ & D-322397 & .1 & 3,900 & 380 & 8 \\
\hline $\mathrm{T}-2-23-3$ & D-322398 & .1 & 6,000 & 310 & 6 \\
\hline$T-2-23-6$ & D-322399 & .1 & 2,300 & 400 & 10 \\
\hline H-8-0-3 duplicate & D-322400 & .3 & 6,300 & 180 & 19 \\
\hline Laboratory standard & D-322401 & .9 & 1,200 & 280 & 8 \\
\hline
\end{tabular}


Table 11. Selenium and water-extractable sulfate, chloride, and selenium concentrations in soils from 15 fields--Continued

\begin{tabular}{|c|c|c|c|c|c|}
\hline \multirow{2}{*}{$\begin{array}{c}\text { Soil } \\
\text { identifier }\end{array}$} & \multirow{2}{*}{$\begin{array}{l}\text { Laboratory } \\
\text { number }\end{array}$} & \multirow{2}{*}{$\frac{\text { Soil }}{\begin{array}{c}\text { Selenium } \\
(\mathrm{ppm})\end{array}}$} & \multicolumn{3}{|c|}{ Water extract } \\
\hline & & & $\begin{array}{l}\text { Sulfate } \\
(\mathrm{ppm})\end{array}$ & $\begin{array}{c}\text { Chloride } \\
\text { (ppm) }\end{array}$ & $\begin{array}{c}\text { Selenium } \\
\text { (ppb) }\end{array}$ \\
\hline \multicolumn{6}{|c|}{ Field at S-352 (Site 87) } \\
\hline H-10-0-3 & D-322542 & 0.1 & 5,200 & 1,100 & 14 \\
\hline H-10-0-6 & D-322543 & 1.2 & 6,400 & 1,700 & 52 \\
\hline H-10-50-3 & D-322544 & .1 & 5,100 & 980 & 15 \\
\hline H-10-50-6 & D-322545 & 1.3 & 12,000 & 2,000 & 75 \\
\hline $\mathrm{H}-10-100-3$ & D-322546 & .1 & 5,700 & 540 & 13 \\
\hline H-10-100-6 & D-322547 & 1.0 & 11,000 & 2,300 & 43 \\
\hline$M-8-0-3$ & D-322548 & .3 & 12,000 & 800 & 44 \\
\hline$M-8-0-6$ & D-322549 & .7 & 2,600 & 1,400 & 120 \\
\hline M-8-28-3 & D-322550 & .5 & 14,000 & 2,200 & 110 \\
\hline$M-8-28-6$ & $D-322551$ & .8 & 9,700 & 3,200 & 110 \\
\hline$M-8-57-3$ & D-322552 & .4 & 13,000 & 1,600 & 53 \\
\hline M-8-57-6 & D-322553 & .6 & 11,000 & 2,800 & 81 \\
\hline$T-4-0-3$ & D-322554 & .6 & 11,000 & 2,800 & 73 \\
\hline$T-4-0-6$ & D-322555 & .6 & 7,600 & 3,400 & 35 \\
\hline$T-4-14-3$ & D-322556 & .5 & 11,000 & 2,500 & 67 \\
\hline$T-4-14-6$ & $D-322557$ & .5 & 12,000 & 3,400 & 81 \\
\hline$T-4-28-3$ & D-322558 & .4 & 13,000 & 3,000 & 49 \\
\hline$T-4-28-6$ & D-322559 & .6 & 7,400 & 3,800 & 48 \\
\hline H-10-0-3 duplicate & D-322560 & .1 & 5,200 & 1,100 & 13 \\
\hline Laboratory standard & D-322561 & 1.1 & 1,200 & 290 & 10 \\
\hline \multicolumn{6}{|c|}{ Field at S-423 (Site 93) } \\
\hline $\mathrm{H}-2 \mathrm{C}-0-3$ & D-322442 & 0.3 & 2,300 & 190 & 5 \\
\hline H-2C-0-6 & D-322443 & .1 & 4,900 & 130 & 16 \\
\hline $\mathrm{H}-2 \mathrm{C}-26-3$ & D-322444 & .2 & 9,400 & 110 & 3 \\
\hline H-2C-26-6 & D-322445 & .1 & 5,800 & 610 & 12 \\
\hline H-2C-53-3 & D-322446 & .1 & 11,000 & 270 & 9 \\
\hline H-2C-53-6 & D-322447 & .1 & 1,500 & 350 & 12 \\
\hline$M-2 C-0-3$ & D-322448 & .1 & 9,400 & 110 & 6 \\
\hline$M-2 C-0-6$ & D-322449 & .4 & 450 & 430 & 35 \\
\hline$M-2 C-26-3$ & $D-322450$ & .1 & 9,600 & 310 & 7 \\
\hline$M-2 C-26-6$ & D-322451 & .3 & 2,600 & 430 & 16 \\
\hline M-2C-53-3 & D-322452 & .1 & 2,200 & 140 & 3 \\
\hline$M-2 C-53-6$ & D-322453 & .2 & 3,500 & 310 & 13 \\
\hline$T-2 C-0-3$ & $D-322454$ & .3 & 9,400 & 570 & 13 \\
\hline$T-2 C-0-6$ & D-322455 & .2 & 1,700 & 800 & 41 \\
\hline$T-2 C-26-3$ & D-322456 & .3 & 12,000 & 940 & 18 \\
\hline$T-2 C-26-6$ & D-322457 & .3 & 1,800 & 960 & 23 \\
\hline $\mathrm{T}-2 \mathrm{C}-53-3$ & D-322458 & .3 & 8,600 & 960 & 33 \\
\hline$T-2 C-53-6$ & D-322459 & .2 & 3,400 & 1,100 & 11 \\
\hline H-2C-0-3 duplicate & D-322460 & .2 & 2,700 & 190 & 3 \\
\hline Laboratory standard & D-322461 & .9 & 1,200 & 280 & 9 \\
\hline
\end{tabular}


Table 11. Selenium and water-extractable sulfate, chloride, and selenium concentrations in soils from 15 fields--Continued

\begin{tabular}{|c|c|c|c|c|c|}
\hline \multirow{2}{*}{$\begin{array}{c}\text { Soil } \\
\text { identifier }\end{array}$} & \multirow{2}{*}{$\begin{array}{l}\text { Laboratory } \\
\text { number }\end{array}$} & \multirow{2}{*}{$\frac{\text { Soil }}{\begin{array}{c}\text { Selenium } \\
\text { (ppm) }\end{array}}$} & \multicolumn{3}{|c|}{ Water extract } \\
\hline & & & $\begin{array}{l}\text { Sulfate } \\
(\mathrm{ppm})\end{array}$ & $\begin{array}{c}\text { Chloride } \\
\text { (ppm) }\end{array}$ & $\begin{array}{l}\text { Selenium } \\
\text { (ppb) }\end{array}$ \\
\hline \multicolumn{6}{|c|}{ Field at S-371 (Site 98) } \\
\hline $\mathrm{H}-4 \mathrm{~A}-0-3$ & D-322402 & 0.2 & 8,600 & 59 & 3 \\
\hline$H-4 A-0-6$ & D-322403 & .1 & 5,900 & 250 & 4 \\
\hline H-4A-25-3 & D-322404 & .2 & 1,400 & 110 & 3 \\
\hline H-4A-25-6 & D-322405 & .1 & 4,500 & 340 & 4 \\
\hline H-4A-53-3 & D-322406 & .2 & 9,800 & 38 & $<3$ \\
\hline H-4A-53-6 & D-322407 & .3 & 7,100 & 270 & $<3$ \\
\hline$M-7 A-0-3$ & D-322408 & .1 & 7,000 & 120 & $<3$ \\
\hline$M-7 A-0-6$ & D-322409 & .1 & 6,600 & 380 & $<3$ \\
\hline M-7A-25-3 & D-322410 & .1 & 7,400 & 120 & $<3$ \\
\hline$M-7 A-25-6$ & D-322411 & .1 & 5,000 & 470 & 3 \\
\hline M-7A-53-3 & D-322412 & .1 & 1,500 & 87 & $<3$ \\
\hline$M-7 A-53-6$ & D-322413 & $<.1$ & 4,000 & 560 & $<3$ \\
\hline$T-10 A-0-3$ & D-322414 & .1 & 1,400 & 110 & $<3$ \\
\hline $\mathrm{T}-10 \mathrm{~A}-0-6$ & D-322415 & .1 & 2,400 & 370 & 4 \\
\hline$T-10 A-25-3$ & D-322416 & .1 & 4,600 & 190 & 3 \\
\hline$T-10 A-25-6$ & D-322417 & .1 & 4,000 & 400 & 10 \\
\hline$T-10 A-53-3$ & D-322418 & .1 & 5,200 & 140 & $<3$ \\
\hline$T-10 A-53-6$ & D-322419 & .1 & 5,100 & 170 & 4 \\
\hline H-4A-0-3 duplicate & D-322420 & .2 & 9,100 & 61 & 3 \\
\hline Laboratory standard & D-322421 & 1.0 & 1,100 & 250 & 9 \\
\hline \multicolumn{6}{|c|}{ Field at S-176 (Site 104) } \\
\hline H-9-0-3 & D-322422 & 0.2 & 6,100 & 90 & 7 \\
\hline H-9-0-6 & D-322423 & .3 & 5,000 & 240 & 19 \\
\hline H-9-84-3 & D-322424 & .1 & 6,600 & 72 & 6 \\
\hline H-9-84-6 & D-322425 & .5 & 4,200 & 140 & 43 \\
\hline H-9-169-3 & D-322426 & .2 & 6,500 & 65 & 6 \\
\hline H-9-169-6 & D-322427 & .1 & 2,200 & 110 & 6 \\
\hline$M-8-0-3$ & D-322428 & .1 & 5,800 & 65 & 5 \\
\hline M-8-0-6 & D-322429 & .1 & 2,000 & 95 & 4 \\
\hline M-8-84-3 & D-322430 & .2 & 930 & 25 & 3 \\
\hline M-8-84-6 & D-322431 & .2 & 6,200 & 210 & 12 \\
\hline M-8-169-3 & D-322432 & .3 & 7,700 & 380 & 32 \\
\hline M-8-169-6 & D-322433 & .2 & 5,600 & 580 & 21 \\
\hline$T-6-0-3$ & D-322434 & .2 & 8,600 & 57 & 7 \\
\hline T-6-0-6 & D-322435 & .1 & 3,400 & 260 & 13 \\
\hline$T-6-84-3$ & D-322436 & .2 & 9,600 & 120 & 19 \\
\hline T-6-84-6 & D-322437 & .1 & 3,000 & 230 & 13 \\
\hline$T-6-169-3$ & D-322438 & .2 & 8,800 & 14 & 6 \\
\hline T-6-169-6 & D-322439 & $<.1$ & 1,200 & 1,600 & 5 \\
\hline H-9-0-3 duplicate & D-322440 & .1 & 5,400 & 80 & 6 \\
\hline Laboratory standard & D-322441 & .9 & 880 & 210 & 7 \\
\hline
\end{tabular}


Table 11. Selenium and water-extractable sulfate, chloride, and selenium concentrations in soils from 15 fields--Continued

\begin{tabular}{|c|c|c|c|c|c|}
\hline \multirow{2}{*}{$\begin{array}{c}\text { Soil } \\
\text { identifier }\end{array}$} & \multirow{2}{*}{$\begin{array}{l}\text { Laboratory } \\
\text { number }\end{array}$} & \multirow{2}{*}{$\frac{\text { Soil }}{\begin{array}{c}\text { Selenium } \\
\text { (ppm) }\end{array}}$} & \multicolumn{3}{|c|}{ Water extract } \\
\hline & & & $\begin{array}{c}\text { Sulfate } \\
\text { (ppm) }\end{array}$ & $\begin{array}{c}\text { Chloride } \\
\text { (ppm) }\end{array}$ & $\begin{array}{l}\text { Selenium } \\
(\mathrm{ppb})\end{array}$ \\
\hline \multicolumn{6}{|c|}{ Field at S-344 (Site 110) } \\
\hline H-10-0-3 & D-322362 & 0.2 & 7,300 & 210 & 22 \\
\hline H-10-0-6 & D-322363 & .2 & 6,700 & 770 & 19 \\
\hline H-10-83-3 & D-322364 & .2 & 8,800 & 420 & 8 \\
\hline H-10-83-6 & D-322365 & .2 & 6,400 & 980 & 18 \\
\hline H-10-166-3 & D-322366 & .3 & 6,400 & 520 & 16 \\
\hline$H-10-166-6$ & D-322367 & .2 & 3,700 & 1,300 & 16 \\
\hline M-8-0-3 & D-322368 & $<.1$ & 2,600 & 28 & 4 \\
\hline M-8-0-6 & D-322369 & .1 & 3,000 & 120 & 3 \\
\hline M-8-83-3 & D-322370 & .1 & 11,000 & 370 & 7 \\
\hline M-8-83-6 & D-322371 & .1 & 6,000 & 520 & 15 \\
\hline M-8-166-3 & D-322372 & .1 & 5,400 & 290 & 5 \\
\hline$M-8-166-6$ & D-322373 & .1 & 3,600 & 300 & 4 \\
\hline$T-2-0-3$ & D-322374 & .1 & 60,000 & 730 & 11 \\
\hline $\mathrm{T}-2-0-6$ & D-322375 & .2 & 8,000 & 1,800 & 11 \\
\hline$T-2-83-3$ & D-322376 & .1 & 13,000 & 1,400 & 17 \\
\hline$T-2-83-6$ & D-322377 & .2 & 1,400 & 2,900 & 20 \\
\hline$T-2-166-3$ & D-322378 & .2 & 8,400 & 510 & 14 \\
\hline$T-2-166-6$ & D-322379 & .2 & 6,600 & 1,900 & 20 \\
\hline H-10-0-3 duplicate & D-322380 & .2 & 7,100 & 220 & 13 \\
\hline Laboratory standard & D-322381 & .9 & 1,100 & 270 & 11 \\
\hline
\end{tabular}


Table 12. Arsenic, selenium, and boron, and water-extractable sulfate, chloride, and selenium concentrations in soils from 15 fields

[Soils data on dry-weight basis; aqueous data are from analysis of extract using 5 to 1 ratio of water to soil. Concentrations in ppm (parts per million) and ppb (parts per billion). See text (p. 12-13) for description of soil identifier; $D$ in soil identifier designates a duplicate analysis. Laboratory standard is a soil from the San Joaquin Valley]

\begin{tabular}{|c|c|c|c|c|c|c|c|c|}
\hline \multirow[b]{2}{*}{ Soil identifier } & \multicolumn{4}{|c|}{ Soil } & \multicolumn{4}{|c|}{ Water extract } \\
\hline & $\begin{array}{l}\text { Laboratory } \\
\text { number }\end{array}$ & $\begin{array}{c}\text { Arsenic } \\
(\mathrm{ppm})\end{array}$ & $\begin{array}{c}\text { Selenium } \\
(\mathrm{ppm})\end{array}$ & $\begin{array}{l}\text { Boron } \\
\text { (ppm) }\end{array}$ & $\begin{array}{l}\text { Laboratory } \\
\text { number }\end{array}$ & $\begin{array}{l}\text { Sulfate } \\
\text { (ppm) }\end{array}$ & $\begin{array}{l}\text { Chloride } \\
\text { (ppm) }\end{array}$ & $\begin{array}{l}\text { Selenium } \\
\text { (ppb) }\end{array}$ \\
\hline S-226-T-7-0-6 & D-317252 & 10 & 0.5 & 2.1 & D-318134 & 4,800 & 3,700 & 70 \\
\hline S-269-T-2A-0-6 & D-317253 & 8.8 & .2 & 2.7 & D-318135 & 5,700 & 1,100 & 42 \\
\hline S-417-T-18-0-6 & D-317247 & 9.1 & .3 & 2.3 & D-318129 & 11,000 & 740 & 40 \\
\hline S-94-T-9-0-6 & D-317254 & 8.9 & .3 & 2.2 & D-318136 & 5,300 & 1,100 & 20 \\
\hline S-142-T-2B-0-6 & D-317248 & 5.1 & .2 & 1.3 & D-318130 & 2,500 & 430 & 28 \\
\hline S-142-T-2B-0-6D & D-317256 & 5.0 & .1 & 1.3 & D-318138 & 2,200 & 430 & 24 \\
\hline S-241-T-14-0-6 & D-317255 & 8.3 & .3 & 2.4 & D-318137 & 11,000 & 1,400 & 34 \\
\hline S-154-H-23-0-3 & D-317221 & 8.3 & .5 & 1.6 & D-318143 & 560 & 61 & 10 \\
\hline S-154-H-23-0-6 & D-317222 & 8.5 & .4 & 1.4 & D-318144 & 850 & 190 & 6 \\
\hline S-154-H-23-44-3 & D-317223 & 8.0 & .4 & 2.1 & D-318145 & 9,900 & 400 & 12 \\
\hline S-154-H-23-44-6 & D-317224 & 8.8 & .5 & 2.2 & D-318146 & 3,300 & 1,900 & 12 \\
\hline S-154-H-23-89-3 & D-317225 & 8.1 & .4 & 1.9 & D-318147 & 2,500 & 400 & 7 \\
\hline S-154-H-23-89-3D & D-317239 & 8.2 & .3 & 1.8 & D-318161 & 2,400 & 360 & 10 \\
\hline S-154-H-23-89-6 & D-317226 & 10 & .6 & 1.9 & D-318148 & 4,600 & 2,100 & 53 \\
\hline S-154-M-17-0-3 & D-317227 & 7.4 & .4 & 1.8 & D-318149 & 4,800 & 390 & 7 \\
\hline S-154-M-17-0-6 & D-317228 & 9.1 & .5 & 1.7 & D-318150 & 2,200 & 2,600 & 16 \\
\hline S-154-M-17-49-3 & D-317229 & 8.8 & .3 & 1.8 & D-318151 & 4,300 & 510 & 13 \\
\hline S-154-M-17-49-6 & D-317230 & 9.7 & .8 & 1.7 & D-318152 & 4,700 & 3,200 & 31 \\
\hline S-154-M-17-98-3 & D-317231 & 8.2 & .4 & 2.0 & D-318153 & 9,000 & 670 & 17 \\
\hline S-154-M-17-98-6 & D-317232 & 10 & .5 & 1.4 & D-318154 & 5,600 & 3,600 & 23 \\
\hline S-154-T-5-0-3 & D-317233 & 8.8 & .4 & 2.2 & D-318155 & 9,900 & 1,200 & 16 \\
\hline S-154-T-5-0-6 & D-317234 & 10 & .6 & 1.5 & D-318156 & 1,500 & 3,700 & 13 \\
\hline S-154-T-5-0-6D & D-317244 & 10 & .5 & 1.4 & D-318126 & 1,500 & 3,800 & 14 \\
\hline S-154-T-5-49-3 & D-317235 & 9.1 & .4 & 2.0 & D-318157 & 9,800 & 1,300 & 20 \\
\hline S-154-T-5-49-3D & D-317240 & 9.5 & .3 & 1.9 & D-318162 & 9,900 & 1,300 & 19 \\
\hline S-154-T-5-49-6 & D-317236 & 10 & .5 & 1.4 & D-318158 & 5,400 & 3,200 & 21 \\
\hline S-154-T-5-98-3 & D-317237 & 11 & .5 & 2.2 & D-318159 & 9,200 & 1,500 & 26 \\
\hline S-154-T-5-98-6 & D-317238 & 10 & .5 & 1.4 & D-318160 & 2,500 & 3,500 & 22 \\
\hline S-265-T-30-0-6 & D-317250 & 6.2 & .1 & 1.4 & D-318132 & 2,100 & 160 & 12 \\
\hline S-4-T-6C-0-6 & D-317249 & 9.0 & .2 & 2.1 & D-318131 & 4,500 & 1,000 & 28 \\
\hline S-72-H-8-0-3 & D-317257 & 7.5 & .4 & 1.9 & D-318139 & 7,700 & 140 & 30 \\
\hline S-72-M-5-0-3 & D-317258 & 7.4 & .3 & 1.7 & D-318140 & 9,800 & 260 & 6 \\
\hline S-72-T-2-0-3 & D-317259 & 7.9 & .3 & 1.8 & D-318141 & 10,000 & 61 & 18 \\
\hline S-72-T-2-0-6 & D-317241 & 3.9 & .1 & 1.1 & D-318123 & 1,100 & 350 & 14 \\
\hline S-352-T-4-06 & D-317251 & 9.2 & .5 & 1.7 & D-318133 & 7,100 & 3,200 & 44 \\
\hline S-423-T-2C-0-6 & D-317246 & 8.4 & .2 & 1.3 & D-318128 & 1,800 & 860 & 60 \\
\hline S-371-T-10A-0-6 & D-317242 & 7.2 & .1 & 1.4 & D-318124 & 2,700 & 220 & 11 \\
\hline S-176-T-6-0-6 & D-317243 & 6.5 & .1 & 1.3 & D-318125 & 3,700 & 240 & 23 \\
\hline S-344-T-2-0-6 & D-317245 & 7.6 & .2 & 1.2 & D-318127 & 7,800 & 1,600 & 18 \\
\hline Laboratory Standard & D-317260 & 9.7 & 1.1 & 5.1 & D-318142 & 1,100 & 190 & 10 \\
\hline
\end{tabular}


Table 13. Concentrations of selected constituents in near-surface soils from 15 fields

[Concentrations from inductively coupled plasma analysis. ppm, parts per million; <, less than indicated reporting limit. See text (p. 12-13) for description of soil identifier; $D$ in soil identifier designates a duplicate analysis. Laboratory standard is a soil from the San Joaquin Valley. The analysis for each sample is displayed as one line on five consecutive pages]

\begin{tabular}{|c|c|c|c|c|c|c|c|c|c|}
\hline $\begin{array}{c}\text { Soil } \\
\text { identifier }\end{array}$ & $\begin{array}{l}\text { Lab. } \\
\text { No. }\end{array}$ & $\begin{array}{c}\text { Aluminum } \\
\text { (percent) }\end{array}$ & $\begin{array}{c}\text { Arsenic } \\
\text { (ppm) }\end{array}$ & $\begin{array}{c}\text { Barium } \\
(\mathrm{ppm})\end{array}$ & $\begin{array}{c}\text { Beryllium } \\
\text { (ppm) }\end{array}$ & $\begin{array}{c}\text { Bismuth } \\
\text { (ppm) }\end{array}$ & $\begin{array}{l}\text { Cadmium } \\
\text { (ppm) }\end{array}$ & $\begin{array}{c}\text { Calcium } \\
\text { (percent) }\end{array}$ & $\begin{array}{c}\text { Cerium } \\
\text { (ppm) }\end{array}$ \\
\hline S-226-T-7-0-6 & D-317252 & 7.9 & 10 & 420 & 2 & $<10$ & $<2$ & 5.1 & 66 \\
\hline S-269-T-2A-0-6 & D-317253 & 6.5 & $<10$ & 560 & 2 & $<10$ & $<2$ & 6.2 & 62 \\
\hline S-417-T-18-0-6. & D-317247 & 6.8 & $<10$ & 310 & 2 & $<10$ & $<2$ & 6.9 & 61 \\
\hline S-94-T-9-0-6 . & D-317254 & 7.8 & $<10$ & 460 & 2 & $<10$ & $<2$ & 4.4 & 65 \\
\hline S-142-T-2B-0-6 & D-317248 & 5.2 & $<10$ & 620 & 1 & $<10$ & $<2$ & 5.2 & 49 \\
\hline S-142-T-2B-0-6D & D-317256 & 5.2 & $<10$ & 630 & 1 & $<10$ & $<2$ & 5.2 & 51 \\
\hline S-241-T-14-0-6 . & D-317255 & 6.9 & $<10$ & 500 & 2 & $<10$ & $<2$ & 6.5 & 63 \\
\hline S-154-H-23-0-3 & D-317221 & 7.7 & $<10$ & 500 & 2 & $<10$ & $<2$ & 6.2 & 68 \\
\hline S-154-H-23-0-6 & D-317222 & 7.4 & $<10$ & 510 & 2 & $<10$ & $<2$ & 6.0 & 64 \\
\hline S-154-H-23-44-3 & D-317223 & 7.3 & $<10$ & 480 & 2 & $<10$ & $<2$ & 7.4 & 65 \\
\hline S-154-H-23-44-6 & D-317224 & 8.3 & $<10$ & 470 & 2 & $<10$ & $<2$ & 4.7 & 70 \\
\hline S-154-H-23-89-3 & D-317225 & 7.4 & $<10$ & 500 & 2 & $<10$ & $<2$ & 6.9 & 66 \\
\hline S-154-H-23-89-3D & D-317239 & 7.6 & $<10$ & 510 & 2 & $<10$ & $<2$ & 7.1 & 69 \\
\hline S-154-H-23-89-6 . & D-317226 & 8.2 & $<10$ & 470 & 2 & $<10$ & $<2$ & 5.0 & 70 \\
\hline$S-154-M-17-0-3$ & D-317227 & 7.1 & $<10$ & 500 & 2 & $<10$ & $<2$ & 7.4 & 64 \\
\hline S-154-M-17-0-6 & D-317228 & 7.9 & $<10$ & 460 & 2 & $<10$ & $<2$ & 5.0 & 70 \\
\hline S-154-M-17-49-3 & D-317229 & 7.5 & $<10$ & 480 & 2 & $<10$ & $<2$ & 6.8 & 64 \\
\hline S-154-M-17-49-6 & D-317230 & 7.9 & $<10$ & 430 & 2 & $<10$ & $<2$ & 5.2 & 70 \\
\hline S-154-M-17-98-3 & D-317231 & 7.3 & $<10$ & 510 & 2 & $<10$ & $<2$ & 8.1 & 64 \\
\hline S-154-M-17-98-6 & D-317232 & 7.8 & $<10$ & 450 & 2 & $<10$ & $<2$ & 5.2 & 68 \\
\hline S-154-T-5-0-3 & D-317233 & 7.5 & $<10$ & 460 & 2 & $<10$ & $<2$ & 6.9 & 66 \\
\hline S-154-T-5-0-6 & D-317234 & 7.4 & $<10$ & 390 & 2 & $<10$ & $<2$ & 5.0 & 67 \\
\hline S-154-T-5-0-6D & D-317244 & 8.1 & $<10$ & 480 & 2 & $<10$ & $<2$ & 5.0 & 70 \\
\hline S-154-T-5-49-3 . & D-317235 & 7.7 & $<10$ & 270 & 2 & $<10$ & $<2$ & 6.3 & 67 \\
\hline S-154-T-5-49-3D & D-317240 & 7.6 & $<10$ & 470 & 2 & $<10$ & $<2$ & 6.3 & 66 \\
\hline S-154-T-5-49-6. & D-317236 & 7.9 & $<10$ & 440 & 2 & $<10$ & $<2$ & 5.5 & 67 \\
\hline S-154-T-5-98-3 . & D-317237 & 8.3 & 10 & 200 & 2 & $<10$ & $<2$ & 5.2 & 67 \\
\hline S-154-T-5-98-6 . & D-317238 & 8.1 & $<10$ & 480 & 2 & $<10$ & $<2$ & 5.0 & 70 \\
\hline S-265-T-30-0-6 . . & D-317250 & 5.6 & $<10$ & 610 & 2 & $<10$ & $<2$ & 6.3 & 58 \\
\hline S-4-T-6C-0-6 . & D-317249 & 7.5 & $<10$ & 440 & 2 & $<10$ & $<2$ & 5.0 & 63 \\
\hline S-72-H-8-0-3 & D-317257 & 6.9 & $<10$ & 500 & 2 & $<10$ & $<2$ & 4.9 & 60 \\
\hline S-72-M-5-0-3 & D-317258 & 7.0 & $<10$ & 500 & 2 & $<10$ & $<2$ & 5.2 & 62 \\
\hline$S-72-T-2-0-3$ & D-317259 & 7.0 & $<10$ & 480 & 2 & $<10$ & $<2$ & 5.3 & 62 \\
\hline S-72-T-2-0-6 & D-317241 & 4.8 & $<10$ & 510 & 1 & $<10$ & $<2$ & 3.8 & 41 \\
\hline S-352-T-4-0-6 & D-317251 & 8.2 & $<10$ & 320 & 2 & $<10$ & $<2$ & 4.6 & 67 \\
\hline S-423-T-2C-0-6 & D-317246 & 7.9 & $<10$ & 520 & 2 & $<10$ & $<2$ & 4.4 & 65 \\
\hline S-371-T-10A-0-6 & D-317242 & 6.4 & $<10$ & 540 & 2 & $<10$ & $<2$ & 5.3 & 61 \\
\hline S-176-T-6-0-6 $\ldots$ & D-317243 & 6.0 & $<10$ & 550 & 2 & $<10$ & $<2$ & 4.8 & 57 \\
\hline S-344-T-2-0-6 $\ldots \ldots$ & D-317245 & 7.0 & $<10$ & 500 & 2 & $<10$ & $<2$ & 4.7 & 57 \\
\hline Laboratory standard & D-317260 & 7.9 & $<10$ & 860 & 2 & $<10$ & $<2$ & 2.2 & 43 \\
\hline
\end{tabular}


Table 13. Concentrations of selected constituents in near-surface soils from 15 fields--Continued

\begin{tabular}{|c|c|c|c|c|c|c|c|}
\hline $\begin{array}{l}\text { Chromium } \\
\text { (ppm) }\end{array}$ & $\begin{array}{l}\text { Cobalt } \\
\text { (ppm) }\end{array}$ & $\begin{array}{l}\text { Copper } \\
\text { (ppm) }\end{array}$ & $\begin{array}{l}\text { Europium } \\
\text { (ppm) }\end{array}$ & $\begin{array}{l}\text { Gallium } \\
\text { (ppm) }\end{array}$ & $\begin{array}{l}\text { Gold } \\
(\mathrm{ppm})\end{array}$ & $\begin{array}{l}\text { Holmium } \\
\text { (ppm) }\end{array}$ & $\begin{array}{c}\text { Iron } \\
\text { (percent) }\end{array}$ \\
\hline S-226-T-7-0-6 $\ldots \ldots \ldots \ldots 58$ & 13 & 29 & $<2$ & 18 & $<8$ & $<4$ & 3.3 \\
\hline S-269-T-2A-0-6 $\ldots \ldots \ldots \ldots 52$ & 12 & 24 & $<2$ & 15 & $<8$ & $<4$ & 2.8 \\
\hline S-417-T-18-0-6 . . . . . . . 5 54 & 12 & 25 & $<2$ & 17 & $<8$ & $<4$ & 2.9 \\
\hline S-94-T-9-0-6 . . . . . . . 6 60 & 12 & 26 & $<2$ & 19 & $<8$ & $<4$ & 3.3 \\
\hline S-142-T-2B-0-6 $\ldots \ldots \ldots \ldots 39$ & 10 & 18 & $<2$ & 12 & $<8$ & $<4$ & 2.0 \\
\hline S-142-T-2B-0-6D $\ldots \ldots \ldots 39$ & 10 & 20 & $<2$ & 13 & $<8$ & $<4$ & 2.0 \\
\hline 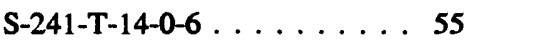 & 12 & 25 & $<2$ & 16 & $<8$ & $<4$ & 3.0 \\
\hline S-154-H-23-0-3 $\ldots \ldots \ldots \ldots 58$ & 13 & 28 & $<2$ & 19 & $<8$ & $<4$ & 3.3 \\
\hline S-154-H-23-0-6 $\ldots \ldots \ldots \ldots 56$ & 13 & 27 & $<2$ & 17 & $<8$ & $<4$ & 3.1 \\
\hline S-154-H-23-44-3 . . . . . . . 58 & 12 & 26 & $<2$ & 18 & $<8$ & $<4$ & 3.1 \\
\hline S-154-H-23-44-6 . . . . . . . . 64 & 13 & 31 & $<2$ & 21 & $<8$ & $<4$ & 3.5 \\
\hline S-154-H-23-89-3 . . . . . . . 59 & 12 & 27 & $<2$ & 18 & $<8$ & $<4$ & 3.1 \\
\hline S-154-H-23-89-3D $\ldots \ldots \ldots 62$ & 13 & 29 & $<2$ & 20 & $<8$ & $<4$ & 3.2 \\
\hline S-154-H-23-89-6 . . . . . . . 61 & 13 & 29 & $<2$ & 20 & $<8$ & $<4$ & 3.5 \\
\hline S-154-M-17-0-3 $\ldots \ldots \ldots \ldots 58$ & 12 & 26 & $<2$ & 18 & $<8$ & $<4$ & 3.0 \\
\hline 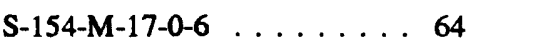 & 13 & 30 & $<2$ & 19 & $<8$ & $<4$ & 3.4 \\
\hline S-154-M-17-49-3 $\ldots \ldots \ldots 59$ & 12 & 28 & $<2$ & 19 & $<8$ & $<4$ & 3.1 \\
\hline S-154-M-17-49-6 $\ldots \ldots \ldots 63$ & 14 & 29 & $<2$ & 20 & $<8$ & $<4$ & 3.4 \\
\hline 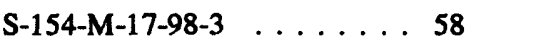 & 12 & 26 & $<2$ & 18 & $<8$ & $<4$ & 3.1 \\
\hline S-154-M-17-98-6 $\ldots \ldots \ldots 55$ & 13 & 29 & $<2$ & 19 & $<8$ & $<4$ & 3.4 \\
\hline S-154-T-5-0-3 $\ldots \ldots \ldots \ldots 49$ & 13 & 28 & $<2$ & 19 & $<8$ & $<4$ & 3.1 \\
\hline S-154-T-5-0-6 $\ldots \ldots \ldots \ldots 50$ & 14 & 25 & $<2$ & 19 & $<8$ & $<4$ & 3.5 \\
\hline S-154-T-5-0-6D $\ldots \ldots \ldots 62$ & 14 & 29 & $<2$ & 20 & $<8$ & $<4$ & 3.5 \\
\hline S-154-T-5-49-3 . . . . . . . 58 & 13 & 28 & $<2$ & 19 & $<8$ & $<4$ & 3.3 \\
\hline S-154-T-5-49-3D $\ldots \ldots \ldots 63$ & 13 & 28 & $<2$ & 19 & $<8$ & $<4$ & 3.3 \\
\hline S-154-T-5-49-6 . . . . . . 61 & 13 & 28 & $<2$ & 18 & $<8$ & $<4$ & 3.4 \\
\hline S-154-T-5-98-3 . . . . . . . . 61 & 13 & 28 & $<2$ & 20 & $<8$ & $<4$ & 3.4 \\
\hline S-154-T-5-98-6 . . . . . . . 60 & 14 & 30 & $<2$ & 21 & $<8$ & $<4$ & 3.5 \\
\hline S-265-T-30-0-6 . . . . . . . 46 & 11 & 20 & $<2$ & 13 & $<8$ & $<4$ & 2.3 \\
\hline S-4-T-6C-0-6 . . . . . . 55 & 13 & 26 & $<2$ & 18 & $<8$ & $<4$ & 3.1 \\
\hline 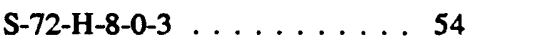 & 12 & 25 & $<2$ & 17 & $<8$ & $<4$ & 2.8 \\
\hline S-72-M-5-0-3 $\ldots \ldots \ldots \ldots$. . 56 & 12 & 24 & $<2$ & 16 & $<8$ & $<4$ & 2.9 \\
\hline S-72-T-2-0-3 $\ldots \ldots \ldots \ldots$. . 55 & 12 & 25 & $<2$ & 17 & $<8$ & $<4$ & 2.9 \\
\hline S-72-T-2-0-6 $\ldots \ldots \ldots \ldots .23$ & 7 & 14 & $<2$ & 10 & $<8$ & $<4$ & 1.7 \\
\hline S-352-T-4-0-6 $\ldots \ldots \ldots \ldots 63$ & 14 & 27 & $<2$ & 21 & $<8$ & $<4$ & 3.5 \\
\hline S-423-T-2C-0-6 $\ldots \ldots \ldots 64$ & 13 & 26 & $<2$ & 20 & $<8$ & $<4$ & 3.3 \\
\hline S-372-T-10A-0-6 $\ldots \ldots \ldots 47$ & 11 & 23 & $<2$ & 16 & $<8$ & $<4$ & 2.6 \\
\hline S-176-T-6-0-6 . . . . . . 52 & 11 & 22 & $<2$ & 14 & $<8$ & $<4$ & 2.6 \\
\hline S-344-T-2-0-6 $\ldots \ldots \ldots \ldots 52$ & 12 & 23 & $<2$ & 17 & $<8$ & $<4$ & 2.8 \\
\hline Laboratory standard $\ldots \ldots \ldots 12$ & 15 & 39 & $<2$ & 18 & $<8$ & $<4$ & 3.9 \\
\hline
\end{tabular}


Table 13. Concentrations of selected constituents in near-surface soils from 15 fields--Continued

\begin{tabular}{|c|c|c|c|c|c|c|c|c|}
\hline $\begin{array}{c}\text { Soil } \\
\text { identifier }\end{array}$ & $\begin{array}{l}\text { Lanthanum } \\
\text { (ppm) }\end{array}$ & $\begin{array}{l}\text { Lead } \\
\text { (ppm) }\end{array}$ & $\begin{array}{c}\text { Lithium } \\
\text { (ppm) }\end{array}$ & $\begin{array}{c}\text { Magnesium } \\
\text { (percent) }\end{array}$ & $\begin{array}{l}\text { Manganese } \\
(\mathrm{ppm})\end{array}$ & $\begin{array}{l}\text { Molybdenum } \\
\text { (ppm) }\end{array}$ & $\begin{array}{l}\text { Neodymium } \\
\quad(\mathrm{ppm})\end{array}$ & $\begin{array}{c}\text { Nickel } \\
(\mathrm{ppm})\end{array}$ \\
\hline S-226-T-7-0-6 $\ldots$ & 39 & 19 & 60 & 1.9 & 500 & $<2$ & 33 & 26 \\
\hline S-269-T-2A-0-6 & $\ldots 36$ & 19 & 49 & 1.9 & 500 & $<2$ & 33 & 22 \\
\hline S-417-T-18-0-6 $\ldots$ & $\ldots 35$ & 17 & 53 & 1.8 & 490 & $<2$ & 33 & 23 \\
\hline S-94-T-9-0-6 $\ldots$ & 38 & 20 & 57 & 1.8 & 480 & $<2$ & 33 & 26 \\
\hline S-142-T-2B-0-6 & . . 29 & 15 & 33 & 1.5 & 410 & $<2$ & 28 & 18 \\
\hline S-142-T-2B-0-6D & 29 & 16 & 33 & 1.4 & 410 & $<2$ & 28 & 18 \\
\hline S-241-T-14-0-6 . & 36 & 18 & 53 & 1.9 & 500 & $<2$ & 34 & 24 \\
\hline S-154-H-23-0-3 & 40 & 27 & 57 & 2.0 & 490 & $<2$ & 35 & 26 \\
\hline S-154-H-23-0-6 & 37 & 20 & 55 & 1.9 & 480 & $<2$ & 34 & 25 \\
\hline S-154-H-23-44-3 . & $\ldots 38$ & 20 & 56 & 2.0 & 480 & $<2$ & 35 & 25 \\
\hline S-154-H-23-44-6. & 41 & 19 & 64 & 1.9 & 490 & $<2$ & 36 & 29 \\
\hline S-154-H-23-89-3 . & 38 & 18 & 57 & 2.0 & 470 & $<2$ & 35 & 25 \\
\hline S-154-H-23-89-3D & 39 & 20 & 58 & 2.0 & 490 & $<2$ & 37 & 26 \\
\hline S-154-H-23-89-6 . & 40 & 18 & 62 & 2.0 & 520 & $<2$ & 35 & 28 \\
\hline S-154-M-17-0-3 . & 37 & 19 & 55 & 2.0 & 490 & $<2$ & 35 & 24 \\
\hline S-154-M-17-0-6 & 40 & 19 & 61 & 2.0 & 530 & $<2$ & 36 & 28 \\
\hline S-154-M-17-49-3 & 38 & 19 & 56 & 1.9 & 470 & $<2$ & 36 & 25 \\
\hline S-154-M-17-49-6 & 40 & 20 & 60 & 2.0 & 530 & $<2$ & 37 & 28 \\
\hline S-154-M-17-98-3 & 37 & 18 & 56 & 2.0 & 480 & $<2$ & 35 & 25 \\
\hline S-154-M-17-98-6 & 40 & 19 & 60 & 2.0 & 520 & $<2$ & 35 & 28 \\
\hline S-154-T-5-0-3 & 38 & 18 & 57 & 1.9 & 500 & $<2$ & 35 & 25 \\
\hline S-154-T-5-0-6 & 38 & 19 & 57 & 2.0 & 550 & $<2$ & 34 & 29 \\
\hline S-154-T-5-0-6D & 41 & 20 & 62 & 2.0 & 550 & $<2$ & 36 & 28 \\
\hline S-154-T-5-49-3 . & 39 & 18 & 58 & 1.9 & 500 & $<2$ & 36 & 27 \\
\hline S-154-T-5-49-3D & 39 & 21 & 57 & 1.9 & 500 & $<2$ & 34 & 26 \\
\hline S-154-T-5-49-6 . & 40 & 21 & 60 & 2.0 & 550 & $<2$ & 35 & 28 \\
\hline S-154-T-5-98-3 $\ldots$ & 39 & 19 & 62 & 2.0 & 520 & $<2$ & 35 & 28 \\
\hline S-154-T-5-98-6. & 41 & 21 & 62 & 2.0 & 540 & $<2$ & 38 & 29 \\
\hline S-265-T-30-0-6 . & 34 & 15 & 36 & 1.7 & 510 & $<2$ & 33 & 20 \\
\hline S-4-T-6C-0-6 $\ldots$ & 37 & 18 & 53 & 1.9 & 540 & $<2$ & 34 & 25 \\
\hline S-72-H-8-0-3 & 35 & 19 & 48 & 1.8 & 500 & $<2$ & 32 & 24 \\
\hline S-72-M-5-0-3 $\ldots$ & 37 & 19 & 49 & 1.8 & 500 & $<2$ & 33 & 23 \\
\hline S-72-T-2-0-3 $\ldots$ & . . 36 & 19 & 49 & 1.8 & 490 & $<2$ & 32 & 23 \\
\hline S-72-T-2-0-6 & $\ldots 25$ & 14 & 29 & 1.1 & 300 & $<2$ & 20 & 13 \\
\hline S-352-T-4-0-6 & . 39 & 18 & 63 & 1.9 & 560 & $<2$ & 35 & 28 \\
\hline S-423-T-2C-0-6 & . . 39 & 19 & 59 & 1.8 & 490 & $<2$ & 35 & 27 \\
\hline S-372-T-10A-0-6 & $\ldots 35$ & 19 & 43 & 1.7 & 520 & $<2$ & 32 & 22 \\
\hline S-176-T-6-0-6 $\ldots$ & . . 32 & 16 & 41 & 1.6 & 460 & $<2$ & 29 & 23 \\
\hline S-344-T-2-0-6 $\ldots$ & $\ldots 34$ & 16 & 50 & 1.6 & 480 & $<2$ & 30 & 24 \\
\hline Laboratory standar & 25 & 17 & 68 & 1.5 & 530 & $<2$ & 22 & 72 \\
\hline
\end{tabular}


Table 13. Concentrations of selected constituents in near-surface soils from 15 fields--Continued

\begin{tabular}{|c|c|c|c|c|c|c|c|c|}
\hline $\begin{array}{c}\text { Soil } \\
\text { identifier }\end{array}$ & $\begin{array}{c}\text { Niobium } \\
(\mathrm{ppm})\end{array}$ & $\begin{array}{c}\text { Phosphorus } \\
\text { (percent) }\end{array}$ & $\begin{array}{c}\text { Potassium } \\
\text { (percent) }\end{array}$ & $\begin{array}{l}\text { Scandium } \\
\text { (ppm) }\end{array}$ & $\begin{array}{l}\text { Silver } \\
\text { (ppm) }\end{array}$ & $\begin{array}{c}\text { Sodium } \\
(\mathrm{ppm})\end{array}$ & $\begin{array}{l}\text { Strontium } \\
\text { (ppm) }\end{array}$ & $\begin{array}{c}\text { Tantalum } \\
\text { (ppm) }\end{array}$ \\
\hline S-226-T-7-0-6 & $\ldots 9$ & 0.08 & 2.2 & 12 & $<2$ & 0.84 & 290 & $<40$ \\
\hline S-269-T-2A-0-6 & $\ldots<4$ & .07 & 2.1 & 9 & $<2$ & 1.0 & 330 & $<40$ \\
\hline S-417-T-18-0-6 . & $\ldots<4$ & .07 & 2.0 & 10 & $<2$ & .81 & 450 & $<40$ \\
\hline S-94-T-9-0-6 & . 8 & .08 & 2.1 & 11 & $<2$ & .78 & 260 & $<40$ \\
\hline S-142-T-2B-0-6 & $\ldots<4$ & .06 & 2.1 & 7 & $<2$ & 1.0 & 260 & $<40$ \\
\hline S-142-T-2B-0-6D & $\ldots<4$ & .06 & 2.0 & 7 & $<2$ & 1.0 & 260 & $<40$ \\
\hline S-241-T-14-0-6 $\ldots$ & $\ldots<4$ & .08 & 2.1 & 10 & $<2$ & .96 & 340 & $<40$ \\
\hline S-154-H-23-0-3 & . 10 & .08 & 2.2 & 11 & $<2$ & .48 & 360 & $<40$ \\
\hline S-154-H-23-0-6 & 7 & .08 & 2.2 & 11 & $<2$ & .52 & 350 & $<40$ \\
\hline S-154-H-23-44-3 & 4 & .08 & 2.1 & 11 & $<2$ & .55 & 390 & $<40$ \\
\hline S-154-H-23-44-6. & 10 & .08 & 2.3 & 12 & $<2$ & .61 & 280 & $<40$ \\
\hline S-154-H-23-89-3 . & . . 7 & .08 & 2.2 & 11 & $<2$ & .59 & 380 & $<40$ \\
\hline S-154-H-23-89-3D & $\ldots<4$ & .07 & 2.2 & 11 & $<2$ & .60 & 400 & $<40$ \\
\hline S-154-H-23-89-6 . & . . 9 & .08 & 2.2 & 12 & $<2$ & .63 & 300 & $<40$ \\
\hline S-154-M-17-0-3 & $\ldots<4$ & .08 & 2.1 & 10 & $<2$ & .58 & 410 & $<40$ \\
\hline S-154-M-17-0-6 & 10 & .08 & 2.2 & 12 & $<2$ & .65 & 270 & $<40$ \\
\hline S-154-M-17-49-3 & 6 & .08 & 2.1 & 11 & $<2$ & .53 & 370 & $<40$ \\
\hline S-154-M-17-49-6 & . . 10 & .08 & 2.2 & 12 & $<2$ & .69 & 290 & $<40$ \\
\hline S-154-M-17-98-3 & $\ldots<4$ & .07 & 2.1 & 11 & $<2$ & .60 & 450 & $<40$ \\
\hline S-154-M-17-98-6 & . . 9 & .08 & 2.2 & 12 & $<2$ & .68 & 290 & $<40$ \\
\hline S-154-T-5-0-3 & $<4$ & .07 & 2.1 & 11 & $<2$ & .59 & 390 & $<40$ \\
\hline S-154-T-5-0-6 & $<4$ & .08 & 1.8 & 11 & $<2$ & .46 & 270 & $<40$ \\
\hline S-154-T-5-0-6D & 9 & .08 & 2.2 & 12 & $<2$ & .66 & 290 & $<40$ \\
\hline S-154-T-5-49-3 . & 9 & .08 & 2.1 & 11 & $<2$ & .55 & 350 & $<40$ \\
\hline S-154-T-5-49-3D & 6 & .07 & 2.1 & 11 & $<2$ & .55 & 350 & $<40$ \\
\hline S-154-T-5-49-6 & 9 & .08 & 2.2 & 12 & $<2$ & .64 & 310 & $<40$ \\
\hline S-154-T-5-98-3 & 9 & .08 & 2.4 & 12 & $<2$ & .54 & 310 & $<40$ \\
\hline S-154-T-5-98-6 & 9 & .08 & 2.2 & 12 & $<2$ & .65 & 290 & $<40$ \\
\hline S-265-T-30-0-6 & $<4$ & .07 & 2.0 & 8 & $<2$ & 1.0 & 280 & $<40$ \\
\hline S-4-T-6C-0-6 . & 8 & .07 & 2.1 & 11 & $<2$ & .74 & 280 & $<40$ \\
\hline S-72-H-8-0-3 & $<4$ & .08 & 2.0 & 10 & $<2$ & .68 & 280 & $<40$ \\
\hline S-72-M-5-0-3 & 4 & .07 & 2.0 & 10 & $<2$ & .63 & 280 & $<40$ \\
\hline S-72-T-2-0-3 & $<4$ & .07 & 2.0 & 10 & $<2$ & .59 & 280 & $<40$ \\
\hline S-72-T-2-0-6 $\ldots$ & $<4$ & .04 & 2.0 & 6 & $<2$ & .78 & 210 & $<40$ \\
\hline S-352-T-4-0-6 $\ldots$ & 8 & .08 & 2.2 & 12 & $<2$ & .87 & 270 & $<40$ \\
\hline S-423-T-2C-0-6 & 9 & .08 & 2.2 & 11 & $<2$ & .69 & 250 & $<40$ \\
\hline S-372-T-10A-0-6 & $<4$ & .06 & 2.1 & 9 & $<2$ & .81 & 250 & $<40$ \\
\hline S-176-T-6-0-6 & $<4$ & .06 & 2.0 & 8 & $<2$ & .99 & 270 & $<40$ \\
\hline S-344-T-2-0-6 $\ldots$ & $<4$ & .07 & 2.1 & 10 & $<2$ & .80 & 270 & $<40$ \\
\hline Laboratory standarc & . . 7 & .06 & 1.9 & 13 & $<2$ & 1.1 & 230 & $<40$ \\
\hline
\end{tabular}


Table 13. Concentrations of selected constituents in near-surface soils from 15 fields--Continued

\begin{tabular}{|c|c|c|c|c|c|c|c|c|}
\hline $\begin{array}{c}\text { Soil } \\
\text { identifier }\end{array}$ & $\begin{array}{c}\text { Thorium } \\
\text { (ppm) }\end{array}$ & $\begin{array}{c}\text { Tin } \\
(\mathrm{ppm})\end{array}$ & $\begin{array}{l}\text { Titanium } \\
\text { (percent) }\end{array}$ & $\begin{array}{c}\text { Uranium } \\
\text { (ppm) }\end{array}$ & $\begin{array}{l}\text { Vanadium } \\
\text { (ppm) }\end{array}$ & $\begin{array}{l}\text { Ytterbium } \\
\text { (ppm) }\end{array}$ & $\begin{array}{l}\text { Yttrium } \\
\text { (ppm) }\end{array}$ & $\begin{array}{c}\text { Zinc } \\
(\mathrm{ppm})\end{array}$ \\
\hline S-226-T-7-0-6 & 11 & $<10$ & 0.33 & $<100$ & 110 & 3 & 22 & 86 \\
\hline S-269-T-2A-0-6 $\ldots \ldots \ldots$ & 9 & $<10$ & .30 & $<100$ & 79 & 2 & 20 & 70 \\
\hline S-417-T-18-0-6 $\ldots \ldots \ldots$ & 10 & $<10$ & .29 & $<100$ & 86 & 2 & 19 & 75 \\
\hline S-94-T-9-0-6 $\ldots \ldots \ldots$ & 11 & $<10$ & .32 & $<100$ & 100 & 3 & 21 & 82 \\
\hline S-142-T-2B-0-6 $\ldots \ldots$ & 7 & $<10$ & .22 & $<100$ & 55 & 2 & 16 & 51 \\
\hline S-142-T-2B-0-6D & 6 & $<10$ & .21 & $<100$ & 55 & 2 & 16 & 51 \\
\hline S-241-T-14-0-6 $\ldots$ & 11 & $<10$ & .30 & $<100$ & 88 & 2 & 20 & 77 \\
\hline S-154-H-23-0-3 $\ldots \ldots$ & 11 & $<10$ & .32 & $<100$ & 100 & 2 & 22 & 84 \\
\hline S-154-H-23-0-6 & 11 & $<10$ & .31 & $<100$ & 98 & 2 & 21 & 81 \\
\hline S-154-H-23-44-3 & 12 & $<10$ & .30 & $<100$ & 98 & 2 & 20 & 81 \\
\hline S-154-H-23-44-6 & 12 & $<10$ & .35 & $<100$ & 110 & 3 & 23 & 92 \\
\hline S-154-H-23-89-3 . & 10 & $<10$ & .32 & $<100$ & 99 & 2 & 21 & 82 \\
\hline S-154-H-23-89-3D & 11 & $<10$ & .31 & $<100$ & 100 & 3 & 22 & 85 \\
\hline S-154-H-23-89-6 . & 11 & $<10$ & .34 & $<100$ & 110 & 3 & 23 & 91 \\
\hline S-154-M-17-0-3 & 11 & $<10$ & .29 & $<100$ & 95 & 2 & 20 & 82 \\
\hline S-154-M-17-0-6 & 12 & $<10$ & .34 & $<100$ & 110 & 3 & 23 & 89 \\
\hline S-154-M-17-49-3 & 11 & $<10$ & .32 & $<100$ & 100 & 3 & 21 & 82 \\
\hline S-154-M-17-49-6 & 13 & $<10$ & .34 & $<100$ & 110 & 3 & 23 & 89 \\
\hline S-154-M-17-98-3 & 10 & $<10$ & .29 & $<100$ & 95 & 2 & 20 & 80 \\
\hline S-154-M-17-98-6 & 12 & $<10$ & .33 & $<100$ & 110 & 2 & 22 & 88 \\
\hline S-154-T-5-0-3 & 11 & $<10$ & .31 & $<100$ & 100 & 3 & 21 & 82 \\
\hline S-154-T-5-0-6 & 9 & $<10$ & .08 & $<100$ & 88 & 1 & 17 & 91 \\
\hline S-154-T-5-0-6D & 12 & $<10$ & .33 & $<100$ & 110 & 3 & 23 & 92 \\
\hline S-154-T-5-49-3 . & 11 & $<10$ & .33 & $<100$ & 110 & 3 & 22 & 86 \\
\hline S-154-T-5-49-3D & 11 & $<10$ & .33 & $<100$ & 100 & 3 & 21 & 86 \\
\hline S-154-T-5-49-6 . & 12 & $<10$ & .33 & $<100$ & 110 & 3 & 22 & 88 \\
\hline S-154-T-5-98-3 . & 11 & $<10$ & .33 & $<100$ & 110 & 3 & 22 & 88 \\
\hline S-154-T-5-98-6 . & 13 & $<10$ & .34 & $<100$ & 110 & 3 & 23 & 93 \\
\hline S-265-T-30-0-6 . & 8 & $<10$ & .26 & $<100$ & 65 & 2 & 19 & 55 \\
\hline S-4-T-6C-0-6 $\ldots \ldots \ldots$ & 11 & $<10$ & .31 & $<100$ & 97 & 2 & 21 & 77 \\
\hline S-72-H-8-0-3 & 10 & $<10$ & .28 & $<100$ & 86 & 2 & 20 & 73 \\
\hline S-72-M-5-0-3 & 9 & $<10$ & .30 & $<100$ & 86 & 3 & 21 & 74 \\
\hline S-72-T-2-0-3 & 10 & $<10$ & .30 & $<100$ & 88 & 2 & 20 & 73 \\
\hline S-72-T-2-0-6 $\ldots$ & 5 & $<10$ & .18 & $<100$ & 49 & 2 & 14 & 43 \\
\hline S-352-T-4-0-6 $\ldots$ & 12 & $<10$ & .33 & $<100$ & 110 & 3 & 22 & 91 \\
\hline S-423-T-2C-0-6 & 12 & $<10$ & .32 & $<100$ & 110 & 2 & 21 & 89 \\
\hline S-372-T-10A-0-6 & 9 & $<10$ & .25 & $<100$ & 80 & 2 & 19 & 68 \\
\hline S-176-T-6-0-6 & 8 & $<10$ & .26 & $<100$ & 71 & 2 & 19 & 57 \\
\hline S-344-T-2-0-6 $\ldots \ldots$ & 10 & $<10$ & .27 & $<100$ & 86 & 2 & 18 & 74 \\
\hline Laboratory standard & 11 & $<10$ & .36 & $<100$ & 130 & 2 & 16 & 110 \\
\hline
\end{tabular}


Table 14. Arsenic and selenium, and water-extractable sulfate, chloride, and selenium concentrations in cores

[Concentrations in parts per million (ppm) and in parts per billion (ppb). Aqueous data are from analysis of extract using 5 to 1 ratio of water to soil. Laboratory standard is a soil from the San Joaquin Valley. <, less than indicated reporting limit; $\mathrm{ft}$, foot]

\begin{tabular}{|c|c|c|c|c|c|c|c|}
\hline \multirow[b]{2}{*}{$\begin{array}{l}\text { Depth of core } \\
\text { (ft) }\end{array}$} & \multicolumn{3}{|c|}{ Soil } & \multicolumn{4}{|c|}{ Water extract } \\
\hline & $\begin{array}{l}\text { Laboratory } \\
\text { number }\end{array}$ & $\begin{array}{c}\text { Arsenic } \\
\text { (ppm) }\end{array}$ & $\begin{array}{l}\text { Selenium } \\
\text { (ppm) }\end{array}$ & $\begin{array}{l}\text { Laboratory } \\
\text { number }\end{array}$ & $\begin{array}{l}\text { Sulfate } \\
(\mathrm{ppb})\end{array}$ & $\begin{array}{l}\text { Chloride } \\
\text { (ppm) }\end{array}$ & $\begin{array}{l}\text { Selenium } \\
\text { (ppm) }\end{array}$ \\
\hline \multicolumn{8}{|c|}{ S-417 (Northern Site) Lysimeter Hole--(Site 8) } \\
\hline $\begin{array}{l}14.5-15 \\
20.5-21\end{array}$ & $\begin{array}{l}\text { D-328519 } \\
\text { D-328520 }\end{array}$ & $\begin{array}{l}8.2 \\
7.2\end{array}$ & $\begin{array}{r}0.4 \\
.3\end{array}$ & $\begin{array}{l}\text { D-328498 } \\
\text { D-328499 }\end{array}$ & $\begin{array}{l}4,000 \\
1,200\end{array}$ & $\begin{array}{l}5,700 \\
6,700\end{array}$ & $\begin{array}{l}95 \\
55\end{array}$ \\
\hline \multicolumn{8}{|c|}{ S-417 (Northern Site) Piezometer Hole } \\
\hline $\begin{array}{c}74.5-75 \\
141.5-142 \\
196.3-197\end{array}$ & $\begin{array}{l}D-328516 \\
D-328517 \\
D-328518\end{array}$ & $\begin{array}{c}5.0 \\
11 \\
3.6\end{array}$ & $\begin{array}{r}<0.2 \\
1.6 \\
<.2\end{array}$ & $\begin{array}{l}\text { D-328495 } \\
\text { D-328496 } \\
\text { D-328497 }\end{array}$ & $\begin{array}{r}1,300 \\
5,200 \\
180\end{array}$ & $\begin{array}{r}1,500 \\
2,100 \\
850\end{array}$ & $\begin{array}{r}25 \\
200 \\
14\end{array}$ \\
\hline \multicolumn{8}{|c|}{ S-154 (Middle Site) Lysimeter Hole--(Site 50) } \\
\hline $13.5-14$ & D-328525 & 9.0 & 0.3 & D-328504 & 10,000 & 5,500 & 55 \\
\hline \multicolumn{8}{|c|}{ S-154 (Middle Site) Piezometer Hole } \\
\hline $\begin{array}{c}26.5-27 \\
56.5-57 \\
71.5-72 \\
101.5-102\end{array}$ & $\begin{array}{l}\text { D-328521 } \\
\text { D-328522 } \\
\text { D-328523 } \\
\text { D-328524 }\end{array}$ & $\begin{array}{l}3.4 \\
5.9 \\
7.1 \\
7.1\end{array}$ & $\begin{array}{r}<0.2 \\
.3 \\
.3 \\
.3\end{array}$ & $\begin{array}{l}\text { D-328500 } \\
\text { D-328501 } \\
\text { D-328502 } \\
\text { D-328503 }\end{array}$ & $\begin{array}{r}1,100 \\
170 \\
490 \\
670\end{array}$ & $\begin{array}{l}3,900 \\
1,400 \\
1,600 \\
1,300\end{array}$ & $\begin{array}{l}5.5 \\
18 \\
14 \\
75\end{array}$ \\
\hline \multicolumn{8}{|c|}{ S-371 (Southern Site) Lysimeter Hole--(Site 98) } \\
\hline $\begin{array}{l}12.5-13 \\
18.5-19\end{array}$ & $\begin{array}{l}\text { D-328532 } \\
\text { D-328533 }\end{array}$ & $\begin{array}{l}5.0 \\
5.3\end{array}$ & $\begin{array}{r}<0.2 \\
<.2\end{array}$ & $\begin{array}{l}\text { D-328511 } \\
\text { D-328512 }\end{array}$ & $\begin{array}{l}220 \\
200\end{array}$ & $\begin{array}{l}83 \\
79\end{array}$ & $\begin{array}{l}14 \\
9.0\end{array}$ \\
\hline \multicolumn{8}{|c|}{ S-371 (Southern Site) Piezometer Hole } \\
\hline $\begin{array}{c}17.5-18 \\
23.5-24 \\
34.5-35 \\
65.5-65.8 \\
81.5-82 \\
106.5-107\end{array}$ & $\begin{array}{l}D-328526 \\
D-328527 \\
D-328528 \\
D-328529 \\
D-328530 \\
D-328531\end{array}$ & $\begin{array}{l}8.5 \\
5.5 \\
9.2 \\
9.2 \\
7.8 \\
2.4\end{array}$ & $\begin{array}{r}<0.2 \\
<.2 \\
.4 \\
.4 \\
<.2 \\
<.2\end{array}$ & $\begin{array}{l}\text { D-328505 } \\
\text { D-328506 } \\
\text { D-328507 } \\
\text { D-328508 } \\
\text { D-328509 } \\
\text { D-328510 }\end{array}$ & $\begin{array}{r}200 \\
150 \\
1,100 \\
460 \\
1,100 \\
130\end{array}$ & $\begin{array}{r}88 \\
120 \\
1,000 \\
960 \\
1,000 \\
730\end{array}$ & $\begin{array}{c}22 \\
9.0 \\
31 \\
14 \\
5.5 \\
<5.0\end{array}$ \\
\hline \multicolumn{8}{|c|}{ Duplicate at S-154 } \\
\hline $56.5-57$ & D-328534 & 5.7 & 0.2 & $\mathrm{D}-328513$ & 150 & 1,400 & 18 \\
\hline \multicolumn{8}{|c|}{ Duplicate at S-371 } \\
\hline $65.5-65.8$ & D-328535 & 9.2 & 0.5 & D-328514 & 420 & 920 & 14 \\
\hline \multicolumn{8}{|c|}{ Laboratory Standard } \\
\hline-- & D-328536 & 9.7 & 1.2 & D-328515 & 1,300 & 320 & 20 \\
\hline
\end{tabular}


Table 15. Concentrations of selected elements in cores

[Concentrations from inductively coupled plasma analysis. $\mathrm{ft}$, foot; <, less than indicated reporting limit. The analysis for each sample is displayed as one line on three consecutive pages]

\begin{tabular}{|c|c|c|c|c|c|c|c|c|c|c|c|c|}
\hline $\begin{array}{l}\text { Depth of } \\
\text { core (ft) }\end{array}$ & $\begin{array}{l}\text { Laboratory } \\
\text { number }\end{array}$ & $\begin{array}{c}\text { Alu- } \\
\text { mi- } \\
\text { num }\end{array}$ & $\begin{array}{l}\text { Cal- } \\
\text { cium }\end{array}$ & Iron & $\begin{array}{c}\text { Po } \\
\text { tas- } \\
\text { sium }\end{array}$ & $\begin{array}{c}\text { Mag- } \\
\text { ne- } \\
\text { sium }\end{array}$ & $\begin{array}{c}\text { So- } \\
\text { dium }\end{array}$ & $\begin{array}{l}\text { Phos- } \\
\text { pho- } \\
\text { rus }\end{array}$ & $\begin{array}{c}\text { Ti- } \\
\text { ta- } \\
\text { nium }\end{array}$ & $\begin{array}{l}\text { Sil- } \\
\text { ver }\end{array}$ & $\begin{array}{l}\text { Ar- } \\
\text { se- } \\
\text { nic }\end{array}$ & Gold \\
\hline & & \multicolumn{7}{|c|}{ (percent) } & & \multicolumn{3}{|c|}{ (parts per million) } \\
\hline \multicolumn{13}{|c|}{ S-417 (Northern Site) Lysimeter Hole-(Site 8) } \\
\hline $14.5-15$ & D-328519 & 7.41 & 6.57 & 3.15 & 2.02 & 2.11 & 0.86 & 0.07 & 0.31 & $<2$ & $<10$ & $<8$ \\
\hline 20.5-21 & D-328520 & 5.90 & 5.25 & 2.44 & 1.65 & 1.62 & 1.11 & .08 & .28 & $<2$ & $<10$ & $<8$ \\
\hline \multicolumn{13}{|c|}{ S-417 (Northern Site) Piezometer Hole } \\
\hline 74.5-75 & D-328516 & 3.66 & 2.81 & 1.09 & 1.67 & 0.75 & 0.96 & 0.04 & 0.17 & $<2$ & $<10$ & $<8$ \\
\hline $141.5-142$ & D-328517 & 7.44 & 8.03 & 3.09 & 1.92 & 1.89 & .72 & .07 & .30 & $<2$ & 10 & $<8$ \\
\hline 196.3-197 & D-328518 & 3.93 & 3.13 & 1.18 & 1.86 & .38 & 1.33 & .03 & .12 & $<2$ & $<10$ & $<8$ \\
\hline \multicolumn{13}{|c|}{ S-154 (Middle Site) Lysimeter Hole--(Site 50) } \\
\hline $13.5-14$ & D-328525 & 7.19 & 6.25 & 3.04 & 2.09 & 2.01 & 0.79 & 0.08 & 0.29 & $<2$ & $<10$ & $<8$ \\
\hline \multicolumn{13}{|c|}{ S-154 (Middle Site) Piezometer Hole } \\
\hline 26.5-27 & D-328521 & 2.95 & 2.22 & 0.79 & 1.72 & 0.51 & 0.85 & 0.03 & 0.09 & $<2$ & $<10$ & $<8$ \\
\hline $56.5-57$ & D-328522 & 6.14 & 5.09 & 2.54 & 1.78 & 1.66 & .90 & .08 & .27 & $<2$ & $<10$ & $<8$ \\
\hline $71.5-72$ & D-328523 & 5.69 & 5.70 & 2.30 & 1.98 & 1.66 & 1.03 & .07 & .27 & $<2$ & $<10$ & $<8$ \\
\hline $101.5-102$ & D-328524 & 8.03 & 4.73 & 3.33 & 2.11 & 1.95 & .54 & .08 & .33 & $<2$ & $<10$ & $<8$ \\
\hline \multicolumn{13}{|c|}{ S-371 (Southern Site) Lysimeter Hole--(Site 98) } \\
\hline $12.5-13$ & D-328532 & 4.56 & 4.56 & 1.67 & 1.68 & 1.23 & 0.88 & 0.06 & 0.19 & $<2$ & $<10$ & $<8$ \\
\hline $18.5-19$ & D-328533 & 5.28 & 6.27 & 2.12 & 1.92 & 1.69 & .86 & .08 & .27 & $<2$ & $<10$ & $<8$ \\
\hline \multicolumn{13}{|c|}{ S-371 (Southern Site) Piezometer Hole } \\
\hline $17.5-18$ & D-328526 & 7.47 & 5.35 & 3.21 & 1.87 & 1.92 & 0.50 & 0.08 & 0.30 & $<2$ & $<10$ & $<8$ \\
\hline 23.5-24 & D-328527 & 5.81 & 4.79 & 2.35 & 1.97 & 1.50 & .71 & .07 & .25 & $<2$ & $<10$ & $<8$ \\
\hline $34.5-35$ & D-328528 & 7.10 & 5.82 & 3.20 & 1.93 & 2.00 & .74 & .08 & .31 & $<2$ & $<10$ & $<8$ \\
\hline $65.5-65.8$ & D-328529 & 7.70 & 4.85 & 3.36 & 1.93 & 1.84 & .68 & .08 & .32 & $<2$ & 10 & $<8$ \\
\hline $81.5-82$ & D-328530 & 8.07 & 4.70 & 3.32 & 1.75 & 1.85 & .62 & .08 & .32 & $<2$ & $<10$ & $<8$ \\
\hline $106.5-107$ & D-328531 & 3.45 & 1.63 & .85 & 1.83 & .31 & 1.08 & .03 & .09 & $<2$ & $<10$ & $<8$ \\
\hline \multicolumn{13}{|c|}{ Duplicate at S-154 } \\
\hline $56.5-57$ & D-328534 & 6.19 & 5.15 & 2.75 & 1.58 & 1.67 & 0.89 & 0.08 & 0.28 & $<2$ & $<10$ & $<8$ \\
\hline \multicolumn{13}{|c|}{ Duplicate at S-371 } \\
\hline $65.5-65.8$ & D-328535 & 7.65 & 4.81 & 3.33 & 1.89 & 1.83 & 0.67 & 0.08 & 0.32 & $<2$ & $<10$ & $<8$ \\
\hline
\end{tabular}


Table 15. Concentrations of selected elements in cores--Continued

\begin{tabular}{|c|c|c|c|c|c|c|c|c|c|c|c|c|c|c|c|}
\hline $\begin{array}{l}\text { Depth of } \\
\text { core (ft) }\end{array}$ & $\begin{array}{c}\mathrm{Ba}- \\
\text { rium }\end{array}$ & $\begin{array}{l}\text { Be- } \\
\text { ryl- } \\
\text { lium }\end{array}$ & $\begin{array}{l}\text { Bis- } \\
\text { muth }\end{array}$ & $\begin{array}{l}\text { Cad- } \\
\text { mi- } \\
\text { um }\end{array}$ & $\begin{array}{l}\text { Ce- } \\
\text { ri- } \\
\text { um }\end{array}$ & $\begin{array}{l}\text { Co- } \\
\text { balt }\end{array}$ & $\begin{array}{l}\text { Chro- } \\
\text { mi- } \\
\text { um }\end{array}$ & $\begin{array}{l}\text { Cop- } \\
\text { per }\end{array}$ & $\begin{array}{l}\text { Eu- } \\
\text { ro- } \\
\text { pium }\end{array}$ & $\begin{array}{l}\text { Gal- } \\
\text { li- } \\
\text { um }\end{array}$ & $\begin{array}{l}\text { Hol- } \\
\text { mium }\end{array}$ & $\begin{array}{l}\text { Lan- } \\
\text { tha- } \\
\text { num }\end{array}$ & $\begin{array}{l}\mathrm{Li}- \\
\text { thi- } \\
\text { um }\end{array}$ & $\begin{array}{c}\text { Man- } \\
\text { ga- } \\
\text { nese }\end{array}$ & $\begin{array}{l}\text { Mo- } \\
\text { lyb- } \\
\text { denum }\end{array}$ \\
\hline \multicolumn{16}{|c|}{ (parts per million) } \\
\hline \multicolumn{16}{|c|}{ S-417 (Northern Site) Lysimeter Hole--(Site 8) } \\
\hline $14.5-15$ & 480 & 2 & $<10$ & $<2$ & 66 & 12 & 60 & 26 & $<2$ & 18 & $<4$ & 38 & 58 & 554 & $<2$ \\
\hline 20.5-21 & 592 & 2 & $<10$ & $<2$ & 58 & 10 & 45 & 18 & $<2$ & 14 & $<4$ & 34 & 40 & 465 & $<2$ \\
\hline \multicolumn{16}{|c|}{ S-417 (Northern Site) Piezometer Hole } \\
\hline 74.5-75 & 645 & $<1$ & $<10$ & $<2$ & 35 & 13 & 22 & 9 & $<2$ & 8 & $<4$ & 21 & 19 & 280 & $<2$ \\
\hline $141.5-142$ & 534 & 2 & $<10$ & $<2$ & 64 & 15 & 61 & 35 & $<2$ & 18 & $<4$ & 37 & 60 & 438 & $<2$ \\
\hline 196.3-197 & 696 & 1 & $<10$ & $<2$ & 25 & 4 & 13 & 4 & $<2$ & 8 & $<4$ & 17 & 16 & 178 & $<2$ \\
\hline \multicolumn{16}{|c|}{ S-154 (Middle Site) Lysimeter Hole--(Site 50) } \\
\hline $13.5-14$ & 474 & 2 & $<10$ & $<2$ & 63 & 12 & 59 & 26 & $<2$ & 17 & $<4$ & 36 & 55 & 513 & $<2$ \\
\hline \multicolumn{16}{|c|}{ S-154 (Middle Site) Piezometer Hole } \\
\hline 26.5-27 & 531 & $<1$ & $<10$ & $<2$ & 23 & 5 & 12 & 5 & $<2$ & 6 & $<4$ & 14 & 13 & 202 & $<2$ \\
\hline $56.5-57$ & 588 & 2 & $<10$ & $<2$ & 58 & 12 & 47 & 23 & $<2$ & 14 & $<4$ & 33 & 40 & 458 & $<2$ \\
\hline $71.5-72$ & 655 & 2 & $<10$ & $<2$ & 56 & 11 & 42 & 22 & $<2$ & 14 & $<4$ & 33 & 37 & 501 & $<2$ \\
\hline $101.5-102$ & 464 & 2 & $<10$ & $<2$ & 70 & 13 & 66 & 39 & $<2$ & 19 & $<4$ & 41 & 58 & 506 & $<2$ \\
\hline \multicolumn{16}{|c|}{ S-371 (Southern Site) Lysimeter Hole--(Site 98) } \\
\hline $12.5-13$ & 540 & 1 & $<10$ & $<2$ & 45 & 8 & 30 & 13 & $<2$ & 10 & $<4$ & 27 & 25 & 372 & $<2$ \\
\hline $18.5-19$ & 565 & 1 & $<10$ & $<2$ & 59 & 9 & 41 & 17 & $<2$ & 12 & $<4$ & 33 & 32 & 464 & $<2$ \\
\hline \multicolumn{16}{|c|}{ S-371 (Southern Site) Piezometer Hole } \\
\hline $17.5-18$ & 511 & 2 & $<10$ & $<2$ & 67 & 13 & 61 & 26 & $<2$ & 18 & $<4$ & 38 & 52 & 530 & $<2$ \\
\hline 23.5-24 & 547 & 2 & $<10$ & $<2$ & 55 & 10 & 43 & 18 & $<2$ & 13 & $<4$ & 33 & 36 & 440 & $<2$ \\
\hline $34.5-35$ & 560 & 2 & $<10$ & $<2$ & 70 & 13 & 58 & 27 & $<2$ & 17 & $<4$ & 40 & 51 & 580 & $<2$ \\
\hline $65.5-65.8$ & 509 & 2 & $<10$ & $<2$ & 70 & 14 & 66 & 28 & $<2$ & 18 & $<4$ & 40 & 56 & 574 & $<2$ \\
\hline $81.5-82$ & 452 & 2 & $<10$ & $<2$ & 71 & 13 & 66 & 26 & $<2$ & 20 & $<4$ & 41 & 58 & 571 & $<2$ \\
\hline $106.5-107$ & 627 & $<1$ & $<10$ & $<2$ & 25 & 4 & 12 & 5 & $<2$ & 7 & $<4$ & 16 & 11 & 165 & $<2$ \\
\hline \multicolumn{16}{|c|}{ Duplicate at S-154 } \\
\hline $56.5-57$ & 583 & 2 & $<10$ & $<2$ & 60 & 11 & 49 & 24 & $<2$ & 14 & $<4$ & 35 & 41 & 463 & $<2$ \\
\hline \multicolumn{16}{|c|}{ Duplicate at S-371 } \\
\hline $65.5-65.8$ & 505 & 2 & $<10$ & $<2$ & 70 & 15 & 64 & 27 & $<2$ & 19 & $<4$ & 40 & 56 & 566 & $<2$ \\
\hline
\end{tabular}


Table 15. Concentrations of selected elements in cores--Continued

\begin{tabular}{|c|c|c|c|c|c|c|c|c|c|c|c|c|c|c|}
\hline Depth of & $\begin{array}{l}\text { Nio- } \\
\text { bi- } \\
\text { um }\end{array}$ & $\begin{array}{l}\text { Neo- } \\
\text { dym- } \\
\text { ium }\end{array}$ & $\begin{array}{c}\text { Nick- } \\
\text { el }\end{array}$ & Lead & $\begin{array}{c}\text { Scan- } \\
\text { di- } \\
\text { um }\end{array}$ & Tin & $\begin{array}{l}\text { Stron- } \\
\text { ti- } \\
\text { um }\end{array}$ & $\begin{array}{l}\text { Tan- } \\
\text { ta- } \\
\text { lum }\end{array}$ & $\begin{array}{l}\text { Tho- } \\
\text { ri- } \\
\text { um }\end{array}$ & $\begin{array}{l}\text { Ura- } \\
\text { ni- } \\
\text { um }\end{array}$ & $\begin{array}{c}\text { Vana- } \\
\text { di- } \\
\text { um }\end{array}$ & $\begin{array}{l}\text { Yt- } \\
\text { tri- } \\
\text { um }\end{array}$ & $\begin{array}{l}\text { Yt- } \\
\text { terbi- } \\
\text { um }\end{array}$ & Zinc \\
\hline \multicolumn{15}{|c|}{ (parts per million) } \\
\hline \multicolumn{15}{|c|}{ S-417 (Northern Site) Lysimeter Hole-(Site 8) } \\
\hline $14.5-15$ & 8 & 34 & 25 & 19 & 11 & $<10$ & 468 & $<40$ & 12 & $<100$ & 94 & 22 & 2 & 77 \\
\hline 20.5-21 & 8 & 30 & 19 & 17 & 8 & $<10$ & 316 & $<40$ & 9 & $<100$ & 69 & 19 & 2 & 58 \\
\hline \multicolumn{15}{|c|}{ S-417 (Northern Site) Piezometer Hole } \\
\hline 74.5-75 & 4 & 17 & 26 & 13 & 4 & $<10$ & 212 & $<40$ & 6 & $<100$ & 32 & 11 & 1 & 54 \\
\hline $141.5-142$ & 9 & 32 & 33 & 21 & 11 & $<10$ & 534 & $<40$ & 10 & $<100$ & 106 & 21 & 2 & 80 \\
\hline 196.3-197 & $<4$ & 13 & 6 & 12 & 3 & $<10$ & 353 & $<40$ & 5 & $<100$ & 24 & 9 & $<1$ & 20 \\
\hline \multicolumn{15}{|c|}{ S-154 (Middle Site) Lysimeter Hole--(Site 50) } \\
\hline $13.5-14$ & 9 & 32 & 25 & 20 & 11 & $<10$ & 374 & $<40$ & 11 & $<100$ & 91 & 21 & 2 & 74 \\
\hline \multicolumn{15}{|c|}{ S-154 (Middle Site) Piezometer Hole } \\
\hline 26.5-27 & $<4$ & 12 & 5 & 10 & 2 & $<10$ & 173 & $<40$ & 5 & $<100$ & 19 & 8 & 1 & 15 \\
\hline 56.5-57 & 8 & 30 & 24 & 18 & 9 & $<10$ & 273 & $<40$ & 10 & $<100$ & 70 & 20 & 2 & 62 \\
\hline $71.5-72$ & 8 & 29 & 21 & 18 & 8 & $<10$ & 270 & $<40$ & 9 & $<100$ & 61 & 19 & 2 & 58 \\
\hline $101.5-102$ & 9 & 33 & 28 & 21 & 12 & $<10$ & 264 & $<40$ & 13 & $<100$ & 106 & 23 & 3 & 85 \\
\hline \multicolumn{15}{|c|}{ S-371 (Southern Site) Lysimeter Hole--(Site 98) } \\
\hline $12.5-13$ & 5 & 24 & 14 & 14 & 6 & $<10$ & 225 & $<40$ & 6 & $<100$ & 44 & 16 & 2 & 35 \\
\hline $18.5-19$ & 6 & 28 & 18 & 17 & 7 & $<10$ & 260 & $<40$ & 9 & $<100$ & 59 & 20 & 2 & 50 \\
\hline \multicolumn{15}{|c|}{ S-371 (Southern Site) Piezometer Hole } \\
\hline $17.5-18$ & 8 & 32 & 28 & 20 & 11 & $<10$ & 271 & $<40$ & 11 & $<100$ & 94 & 22 & 2 & 78 \\
\hline 23.5-24 & 7 & 27 & 18 & 18 & 8 & $<10$ & 239 & $<40$ & 8 & $<100$ & 66 & 18 & 2 & 55 \\
\hline $34.5-35$ & 9 & 33 & 26 & 21 & 11 & $<10$ & 286 & $<40$ & 12 & $<100$ & 91 & 23 & 2 & 80 \\
\hline $65.5-65.8$ & 9 & 32 & 29 & 22 & 11 & $<10$ & 263 & $<40$ & 10 & $<100$ & 104 & 23 & 3 & 84 \\
\hline $81.5-82$ & 10 & 34 & 29 & 22 & 12 & $<10$ & 242 & $<40$ & 12 & $<100$ & 107 & 24 & 3 & 85 \\
\hline $106.5-107$ & $<4$ & 12 & 5 & 11 & 2 & $<10$ & 194 & $<40$ & $<4$ & $<100$ & 20 & 7 & $<1$ & 16 \\
\hline \multicolumn{15}{|c|}{ Duplicate at S-154 } \\
\hline $56.5-57$ & 9 & 31 & 23 & 19 & 9 & $<10$ & 276 & $<40$ & 10 & $<100$ & 71 & 20 & 2 & 62 \\
\hline \multicolumn{15}{|c|}{ Duplicate at S-371 } \\
\hline $65.5-65.8$ & 10 & 33 & 28 & 23 & 11 & $<10$ & 260 & $<40$ & 12 & $<100$ & 103 & 23 & 2 & 83 \\
\hline
\end{tabular}


Table 16. Tritium concentration in soil moisture from eight fields in the Imperial Valley, August 1988 [Concentrations in picocuries per liter. See text (p. 12-13) for description of soil identifier]

\begin{tabular}{|c|c|c|c|c|c|c|c|}
\hline $\begin{array}{c}\text { Soil } \\
\text { identifier }\end{array}$ & Tritium & $\begin{array}{c}\text { Soil } \\
\text { identifier }\end{array}$ & Tritium & $\begin{array}{c}\text { Soil } \\
\text { identifier }\end{array}$ & Tritium & $\begin{array}{c}\text { Soil } \\
\text { identifier }\end{array}$ & Tritium \\
\hline \multicolumn{8}{|c|}{ Field at S-417 (Site 8) } \\
\hline H-32-0-3 & 121 & H-32-0-6 & 88 & $T-18-0-3$ & 118 & T-18-0-6 & 121 \\
\hline $\mathrm{H}-32-50-3$ & 146 & H-32-50-6 & 114 & $\mathrm{~T}-18-50-3$ & 143 & $\mathrm{~T}-18-50-6$ & 106 \\
\hline \multicolumn{8}{|c|}{ Field at S-94 (Site 30) } \\
\hline H-13-0-3 & 87 & H-13-0-6 & 156 & T-9-0-3 & 97 & $T-9-0-6$ & 202 \\
\hline H-13-46-3 & 106 & H-13-46-6 & 185 & $\mathrm{~T}-9-46-3$ & 95 & $T-9-46-6$ & 189 \\
\hline \multicolumn{8}{|c|}{ Field at S-154 (Site 50) } \\
\hline H-23-0-3 & 96 & H-23-0-6 & 96 & T-5-0-3 & 107 & $T-5-0-6$ & 65 \\
\hline H-23-89-3 & 111 & H-23-89-6 & 125 & $T-5-98-3$ & 117 & $T-5-98-06$ & 63 \\
\hline \multicolumn{8}{|c|}{ Field at S-265 (Site 67) } \\
\hline H-30-0-3 & 87 & H-30-0-6 & 140 & $T-30-0-3$ & 116 & T-30-0-6 & 160 \\
\hline H-30-50-3 & 112 & H-30-50-6 & 164 & $\mathrm{~T}-30-50-3$ & 103 & $\mathrm{~T}-30-50-6$ & 204 \\
\hline \multicolumn{8}{|c|}{ Field at S-72 (Site 79) } \\
\hline H-8-0-3 & 108 & H-8-0-6 & 203 & $T-2-0-3$ & 105 & $T-2-0-6$ & 103 \\
\hline H-8-11-3 & 95 & H-8-11-6 & 178 & $T-2-11-3$ & 114 & $T-2-11-6$ & 171 \\
\hline $\mathrm{H}-8-23-3$ & 84 & H-8-23-6 & 146 & $T-2-23-3$ & 102 & $T-2-23-6$ & 164 \\
\hline \multicolumn{8}{|c|}{ Field at S-423 (Site 93) } \\
\hline$H-2 C-0-3$ & 94 & $H-2 C-0-6$ & 149 & $\mathrm{~T}-2 \mathrm{C}-0-3$ & 132 & $T-2 C-0-6$ & 212 \\
\hline H-2C-53-3 & 135 & H-2C-53-6 & 159 & $T-2 C-53-3$ & 130 & $T-2 C-53-6$ & 205 \\
\hline \multicolumn{8}{|c|}{ Field at S-371 (Site 98) } \\
\hline $\mathrm{H}-4 \mathrm{~A}-0-3$ & 95 & H-4A-0-6 & 136 & T-10A-0-3 & 94 & $T-10 A-0-6$ & 148 \\
\hline$H-4 A-53-3$ & 111 & $H-4 A-53-6$ & 161 & $T-10 A-53-3$ & 95 & $T-10 A-53-6$ & 135 \\
\hline \multicolumn{8}{|c|}{ Field at S-176 (Site 104) } \\
\hline H-9-0-3 & 133 & H-9-0-6 & 195 & $\mathrm{~T}-6-0-3$ & 96 & $T-6-0-6$ & 206 \\
\hline H-9-169-3 & 103 & H-9-169-6 & 130 & T-6-169-3 & 102 & $T-6-169-6$ & 132 \\
\hline
\end{tabular}


Table 17. Analyses of untreated and acidified irrigation water from the East Highline Canal concentrated by

[Acidification by $\mathrm{HNO}_{3}$, except $\mathrm{HBr}$ used for January 1989 samples. Final volume of the most-concentrated solutions was $\mu \mathrm{S} / \mathrm{cm}$, microsiemen per centimeter at $25^{\circ} \mathrm{C} ;{ }^{\circ} \mathrm{C}$, degree Celsius; $\mathrm{mg} / \mathrm{L}$, milligram per liter, $\mathrm{mL}$, milliliter; $\mu \mathrm{g} / \mathrm{L}$, microgram

\begin{tabular}{|c|c|c|c|c|c|c|c|c|c|c|}
\hline Date & $\begin{array}{c}\text { Spe- } \\
\text { cific } \\
\text { con- } \\
\text { duct- } \\
\text { ance } \\
(\mu S / \mathrm{cm})\end{array}$ & $\begin{array}{c}\text { pH } \\
\text { (stand- } \\
\text { ard } \\
\text { units) }\end{array}$ & $\begin{array}{l}\text { Nitro- } \\
\text { gen, } \\
\text { Ammonia } \\
\text { (mg/L } \\
\text { as N) }\end{array}$ & $\begin{array}{c}\text { Alka- } \\
\text { linity, } \\
\text { lab } \\
(\mathrm{mg} / \mathrm{L} \\
\text { as } \\
\mathrm{CaCO}_{3} \text { ) }\end{array}$ & $\begin{array}{l}\text { Calcium } \\
\text { (mg/L } \\
\text { as } \mathrm{Ca})\end{array}$ & $\begin{array}{c}\text { Magne- } \\
\text { sium } \\
(\mathrm{mg} / \mathrm{L} \\
\text { as } \mathrm{Mg})\end{array}$ & $\begin{array}{l}\text { Sodium } \\
\text { (mg/L } \\
\text { as } \mathrm{Na} \text { ) }\end{array}$ & $\begin{array}{l}\text { Potas- } \\
\text { sium } \\
(\mathrm{mg} / \mathrm{L} \\
\text { as } \mathrm{K})\end{array}$ & $\begin{array}{c}\text { Sulfate } \\
(\mathrm{mg} / \mathrm{L} \\
\left.\text { as } \mathrm{SO}_{4}\right)\end{array}$ & $\begin{array}{l}\text { Chlo- } \\
\text { ride } \\
(\mathrm{mg} / \mathrm{L} \\
\text { as Cl) }\end{array}$ \\
\hline \multicolumn{11}{|c|}{ Evaporated Irrigation Water } \\
\hline 01-15-89 & $\ldots 1,250$ & 8.41 & -- & 164 & 84 & 32 & 140 & 4.4 & 320 & 120 \\
\hline 01-15-89 & $\ldots 3,750$ & 8.27 & -- & 109 & 140 & 120 & 540 & --- & 1,300 & -- \\
\hline 01-15-89 & $\ldots 7, \mathbf{7 3 3 0}$ & 8.19 & --- & 91 & 230 & 250 & 1,200 & --- & 2,900 & 1,100 \\
\hline 01-15-89 & . . 19,000 & 8.10 & --- & 109 & 610 & 780 & 3,200 & 95 & 7,900 & 3,200 \\
\hline 04-11-89 & $\ldots 14,100$ & 8.34 & -- & 101 & 540 & 600 & 2,300 & 92 & 6,000 & 2,100 \\
\hline $05-23-89$ & $\ldots 10,900$ & 8.12 & 0.20 & 105 & 440 & 470 & 1,700 & 79 & 4,800 & 1,500 \\
\hline $06-21-89$ & $\ldots 22,000$ & 8.22 & $\cdots$ & 127 & 940 & 1,100 & 3,800 & 4.3 & 11,000 & 3,200 \\
\hline $07-28-89$ & $\ldots \quad 28,400$ & 8.14 & --- & 147 & 640 & 1,400 & 5,800 & 150 & 13,000 & 4,300 \\
\hline $08-28-89$ & $\ldots 32,000$ & 8.0 & --- & 104 & 460 & 1,700 & 6,800 & 260 & 14,000 & 5,500 \\
\hline $10-04-89$ & $\ldots 3,780$ & 8.13 & -- & 85 & 140 & 120 & 510 & 20 & 1,400 & 430 \\
\hline $10-04-89$ & $\ldots 6,040$ & 8.13 & -- & 92 & 220 & 220 & 900 & 33 & 2,300 & 740 \\
\hline $10-04-89$ & $\ldots 12,500$ & 8.13 & -- & 107 & 450 & 460 & 2,000 & 76 & 5,200 & 1,800 \\
\hline $10-04-89$ & $\ldots 31,300$ & 8.17 & -- & 152 & 530 & 1,300 & 6,100 & 230 & 14,000 & 5,400 \\
\hline $11-01-89$ & . . 26,200 & 7.9 & -- & 145 & 490 & 1,500 & 6,600 & -- & 15,000 & 6,000 \\
\hline $12-27-89$ & $\ldots 1,200$ & 8.5 & -- & 113 & 81 & 30 & 120 & --- & 300 & 110 \\
\hline $12-27-89$ & $\ldots 33,000$ & 8.1 & -- & 114 & 680 & 1,300 & 5,300 & -- & 13,000 & 4,600 \\
\hline $01-26-90$ & $\ldots \quad 1,140$ & 8.4 & -- & 121 & 79 & 29 & 110 & $\cdots$ & 290 & 100 \\
\hline \multicolumn{11}{|c|}{ Evaporated, Acidified Irrigation Water } \\
\hline 01-15-89 & $\ldots \ldots \ldots$ & -- & - & --- & 340 & 130 & 550 & --- & 1,300 & 3,600 \\
\hline 01-15-89 & $\ldots \ldots \ldots$ & $\cdots$ & -- & -- & 810 & 730 & 3,200 & --- & 4,200 & 9,900 \\
\hline 04-11-89 & $\ldots 30,800$ & 1.4 & --- & -- & 1,400 & 530 & 4,600 & 63 & 4,500 & 1,700 \\
\hline 05-23-89 & $\ldots 50,900$ & 1.2 & -- & --- & 1,300 & 790 & 2,900 & 130 & 5,800 & 2,600 \\
\hline $06-21-89$ & $\ldots \quad 77,200$ & .9 & -- & --- & 1,800 & 1,400 & 4,500 & 200 & 8,000 & 4,200 \\
\hline $07-28-89$ & 52,000 & 1.5 & -- & -- & 800 & 1,700 & 5,600 & 320 & 8,900 & 5,700 \\
\hline $08-28-89$ & $\ldots 56,900$ & 1.6 & -- & -- & 5,200 & 11,000 & 7,800 & 250 & 9,000 & 4,800 \\
\hline $11-01-89$ & . . 39,500 & 1.7 & -- & --- & 910 & 1,500 & 5,100 & -- & 7,300 & 4,800 \\
\hline $12-27-89$ & ... 56,800 & 2.0 & -- & --- & 850 & 2,600 & 10,000 & -- & 12,000 & 6,400 \\
\hline $01-26-90$ & $\ldots 36,100$ & 1.6 & -- & -- & 950 & 2,200 & 8,500 & --- & 15,000 & 7,900 \\
\hline \multicolumn{11}{|c|}{ Acid-Soluble Precipitate from Evaporation } \\
\hline $01-15-89$ & $\ldots \ldots$ & -- & --- & --- & 3,400 & 140 & 13 & --- & 3.9 & 3.92 \\
\hline $05-23-89$ & $\ldots \ldots-$ & -- & $\ldots$ & -- & 4,400 & 240 & 300 & --- & -.. & --- \\
\hline $06-21-89$ & $\ldots \ldots-$ & -- & -- & -- & 1,100 & 110 & 150 & --- & --- & --- \\
\hline $07-28-89$ & $\ldots \ldots \ldots$ & - & -- & -- & 7,500 & 330 & 31 & --- & --- & -- \\
\hline $08-28-89$ & $\ldots \ldots$ & $\cdots$ & -- & -- & 1,500 & 100 & 370 & -- & --- & --- \\
\hline $10-04-89$ & $\ldots \ldots \ldots$ & -- & -- & --- & 16,000 & 730 & 540 & --- & -- & --- \\
\hline $11-01-89$ & $\ldots \ldots$ & --- & -- & --- & 7,100 & 330 & 370 & --- & --- & -- \\
\hline $12-27-89$ & $\ldots \ldots \ldots$ & --- & -- & -- & 9,900 & 420 & 18 & --- & -- & -- \\
\hline $01-26-90$ & $\ldots \ldots=$ & --- & -- & -- & -- & -- & -- & --- & --- & --- \\
\hline
\end{tabular}


evaporation in the laboratory

approximately $500 \mathrm{~mL}$. Acid-soluble part of precipitate was dissolved with a small quantity of nitric acid and diluted to $100 \mathrm{~mL}$. per liter; --, no data]

\begin{tabular}{|c|c|c|c|c|c|c|c|c|c|c|c|}
\hline $\begin{array}{l}\text { Fluo- } \\
\text { ride } \\
\text { (mg/L } \\
\text { as F) }\end{array}$ & $\begin{array}{c}\text { Bromide } \\
(\mathrm{mg} / \mathrm{L} \\
\text { as } \mathrm{Br})\end{array}$ & $\begin{array}{c}\text { Nitro- } \\
\text { gen, } \\
\mathrm{NO}_{2}+\mathrm{NO}_{3} \\
(\mathrm{mg} / \mathrm{L} \\
\text { as } \mathrm{N})\end{array}$ & $\begin{array}{c}\text { Arsenic } \\
(\mu \mathrm{g} / \mathrm{L} \\
\text { as As) }\end{array}$ & $\begin{array}{l}\text { Boron } \\
(\mu \mathrm{g} / \mathrm{L} \\
\text { as B })\end{array}$ & $\begin{array}{c}\text { Molyb- } \\
\text { denum } \\
(\mu \mathrm{g} / \mathrm{L} \\
\text { as Mo) }\end{array}$ & $\begin{array}{l}\text { Sele- } \\
\text { nium } \\
(\mu \mathrm{g} / \mathrm{L} \\
\text { as } \mathrm{Se})\end{array}$ & $\begin{array}{l}\text { Stron- } \\
\text { tium } \\
(\mu \mathrm{g} / \mathrm{L} \\
\text { as } \mathrm{Sr})\end{array}$ & $\frac{\text { Stable-i }}{{ }^{2} \mathrm{H} /{ }^{1} \mathrm{H}}$ & $\frac{\text { otope ratio }}{{ }^{18} \mathrm{O} /{ }^{16} \mathrm{O}}$ & $\frac{\text { (permil) }}{{ }^{34} \mathrm{~S} /{ }^{32} \mathrm{~S}}$ & $\begin{array}{c}\text { Selenium/ } \\
\text { chloride } \\
\text { (weight } \\
\text { ratio } \\
\times 10^{-5} \text { ) }\end{array}$ \\
\hline \multicolumn{12}{|c|}{ Evaporated Irrigation Water } \\
\hline -- & 0.073 & - & 2 & 180 & - & 2 & -- & --- & --- & -- & 1.7 \\
\hline-- & -- & -- & - & 200 & -- & 6 & -- & --- & --- & -- & -- \\
\hline --- & 1.0 & -- & -- & 2,100 & $\ldots$ & 13 & -. & --- & --- & -- & 1.2 \\
\hline-- & 3.6 & -- & 23 & 12,000 & - & 34 & - & -- & --- & --- & 1.1 \\
\hline-- & 1.3 & -- & -- & 4,300 & -- & 38 & -- & --- & - & -1.10 & 1.8 \\
\hline-- & .99 & 4.6 & -- & 3,200 & 72 & 25 & --- & 58.4 & 30.14 & -- & 1.7 \\
\hline 8.2 & 2.2 & $\ldots$ & 43 & 6,800 & 160 & 73 & 27,000 & --- & -- & -- & 2.3 \\
\hline 11 & 2.8 & -- & 39 & 9,700 & 300 & 86 & 26,000 & -- & -.- & -- & 2.0 \\
\hline -- & 4.4 & - & 97 & 11,000 & 280 & 89 & --. & - & -- & --- & 1.6 \\
\hline- & .28 & -- & 8 & 1,000 & 21 & 4 & -- & -5.4 & 15.15 & -- & .9 \\
\hline-- & .45 & -- & 12 & 1,700 & 40 & 10 & -- & 19.5 & 21.35 & -- & 1.4 \\
\hline- & 1.3 & -- & 18 & 4,000 & 100 & 20 & --- & 66.0 & 33.75 & -.. & 1.1 \\
\hline-- & 4.0 & -- & 55 & 12,000 & 340 & 77 & --- & 129.4 & 48.00 & --- & 1.4 \\
\hline- & 8.0 & 9.2 & 15 & 11,000 & --- & 87 & --- & --- & -- & --- & 1.4 \\
\hline-- & .080 & .18 & 2 & 180 & -.- & 2 & -.. & -- & - & $\ldots$ & 1.8 \\
\hline-- & 3.1 & 5.1 & 40 & 8,800 & --- & 67 & --- & -- & -- & --. & 1.5 \\
\hline- & .070 & .20 & 2 & 160 & -- & 2 & --- & -- & -- & --- & 2.0 \\
\hline \multicolumn{12}{|c|}{ Evaporated, Acidified Irrigation Water } \\
\hline-- & - & -- & -- & 1,200 & --- & 8 & -- & - & $\cdots$ & $\cdots$ & 0.2 \\
\hline - & -- & -- & --. & 6,500 & --- & 8 & --- & -- & --- & --- & .08 \\
\hline--- & 1.3 & -- & --- & 4,300 & --- & 38 & --- & -.- & -.. & -1.50 & 2.2 \\
\hline-- & 2.2 & - & 23 & 5,200 & 52 & 52 & --- & -. & $\ldots$ & -- & 2.0 \\
\hline 9.4 & 3.3 & - & 51 & 1,700 & 140 & 100 & 35,000 & -- & -- & - & 2.4 \\
\hline 17 & 5.6 & - & 39 & 5,000 & 330 & 150 & 39,000 & --- & --- & -- & 2.6 \\
\hline -- & .38 & $\ldots$ & 160 & 10,000 & 320 & 120 & --- & --- & --- & -.- & 2.5 \\
\hline--- & 3.8 & - & 86 & 9,800 & -- & 82 & -- & -- & -- & --- & 1.7 \\
\hline-- & 1.5 & -- & 110 & 17,000 & -- & 160 & --- & - & -- & $\cdots$ & 2.5 \\
\hline --- & 6.3 & - & 110 & 14,000 & -- & 150 & -- & -- & - & --- & 1.9 \\
\hline \multicolumn{12}{|c|}{ Acid-Soluble Precipitate from Evaporation } \\
\hline 130 & - & -- & - & 200 & -- & 11 & -- & --- & -- & -- & $\cdots$ \\
\hline-- & -- & - & - & 660 & -- & 25 & -- & --- & -- & --- & --- \\
\hline- & --- & -- & - & --- & -- & 8 & 7,400 & --- & --- & --- & --- \\
\hline-- & -- & -- & - & -- & --- & 31 & 30,000 & --- & --- & -- & $\cdots$ \\
\hline--- & 48 & -- & $<1$ & 2,500 & 20 & -- & -- & --- & - & -- & --- \\
\hline--- & --- & --- & -- & -- & --- & 5 & $\cdots$ & -- & --- & -- & -- \\
\hline--- & --- & -- & -- & $\cdots$ & --- & 27 & -- & -- & -- & --- & --- \\
\hline--- & --- & -- & --- & -- & -- & 14 & --- & --- & -- & -- & -- \\
\hline--- & - & -- & -- & -- & -- & --- & --- & -- & -- & $\cdots$ & --- \\
\hline
\end{tabular}


Table 18. Analyses of undiluted and diluted (with deionized water) water samples from the Salton Sea and the Pacific Ocean

[Seawater samples collected from end of pier at Scripps Institution of Oceanography in La Jolla, California; Salton Sea samples collected from center of south basin, except August 1988 samples are from the Niland boat ramp at North Shore, at Salton City, south basin, and between the deltas of the Alamo and New Rivers (in order listed). ${ }^{\circ} \mathrm{C}$, degree Celsius; $\mu \mathrm{S} / \mathrm{cm}$, microsiemen per centimeter at $25^{\circ} \mathrm{C} ; \mathrm{mg} / \mathrm{L}$, milligram per liter; $\mu \mathrm{g} / \mathrm{L}$, microgram per liter; $\mathrm{pCi} / \mathrm{L}$, picocurie per liter; $<$, less than indicated reporting limit; , approximate value; - no data. The analysis for each sample is displayed as one line on four consecutive pages]

\begin{tabular}{|c|c|c|c|c|c|c|c|c|}
\hline Date & $\begin{array}{c}\text { Spe- } \\
\text { cific } \\
\text { con- } \\
\text { duct- } \\
\text { ance } \\
(\mu S / c m)\end{array}$ & $\begin{array}{c}\text { pH } \\
\text { (stand- } \\
\text { ard } \\
\text { units) }\end{array}$ & $\begin{array}{c}\text { Temper- } \\
\text { ature, } \\
\text { water } \\
\left({ }^{\circ} \mathrm{C}\right)\end{array}$ & $\begin{array}{c}\text { Calcium } \\
\text { (mg/L } \\
\text { as } \mathrm{Ca})\end{array}$ & $\begin{array}{c}\text { Magne- } \\
\text { sium } \\
\text { (mg/L } \\
\text { as } \mathrm{Mg} \text { ) }\end{array}$ & $\begin{array}{c}\text { Sodium } \\
(\mathrm{mg} / \mathrm{L} \\
\text { as } \mathrm{Na})\end{array}$ & $\begin{array}{l}\text { Potas- } \\
\text { sium } \\
(\mathrm{mg} / \mathrm{L} \\
\text { as K) }\end{array}$ & $\begin{array}{c}\text { Alka- } \\
\text { linity, } \\
\text { lab } \\
(\mathrm{mg} / \mathrm{L} \\
\text { as } \\
\left(\mathrm{CaCO}_{3}\right)\end{array}$ \\
\hline \multicolumn{9}{|c|}{ Salton Sea } \\
\hline $8-17-88 \ldots$ & 48,200 & - & --- & --- & -- & -- & --- & -- \\
\hline 8-17-88 $\ldots$ & 52,200 & - & -- & -- & --- & 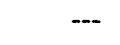 & --- & -- \\
\hline $8-17-88 \ldots$ & 53,200 & - & -- & --- & - & -- & $-\cdots$ & --- \\
\hline 8-24-88 $\ldots$ & 50,400 & -- & -- & --- & -- & --- & -- & --- \\
\hline 8-25-88 $\ldots$ & 46,200 & -- & - & -- & -- & --- & -- & --- \\
\hline $2-16-89 \ldots$ & 51,700 & - & -- & 1,000 & 1,300 & 11,000 & 180 & 199 \\
\hline $3-16-89 \ldots$ & 51,700 & - & -- & -- & --- & --- & --- & 187 \\
\hline $4-11-89 \ldots$ & 52,000 & -- & -- & 840 & 1,300 & 11,000 & --- & 192 \\
\hline $6-20-89 \ldots$ & 52,100 & - & -- & -- & --- & -- & -- & 178 \\
\hline 7-19-89 $\ldots$ & 52,300 & -- & -- & -- & $\cdots$ & -- & --- & 175 \\
\hline 10-11-89 . & 53,700 & 8.8 & 29.5 & 950 & 1,300 & 11,000 & 220 & 185 \\
\hline \multicolumn{9}{|c|}{ Salton Sea (diluted) } \\
\hline $2-16-89 \ldots$ & 6,990 & --- & --- & -- & --- & -- & -- & 23 \\
\hline $2-16-89 \ldots$ & 913 & -- & --- & --- & --- & -- & --- & 4.0 \\
\hline $3-16-89 \ldots$ & 4,960 & - & -- & -- & -- & -- & -- & 15 \\
\hline $4-11-89 \ldots$ & 7,740 & 7.7 &.-- & --- & 1,200 & 1,200 & -- & -- \\
\hline $6-20-89 \ldots$ & 10,000 & - & -- & -- & -- & -- & -- & 31 \\
\hline $6-20-89 \ldots$ & 1,870 & --- & -- & -- & --- & -- & -- & 6.0 \\
\hline 7-19-89 ... & 8,920 & --- & -- & --- & -- & -- & -- & 26 \\
\hline $7-19-89 \ldots$ & 1,380 & -- & - & -- & - & --- & -- & 5.0 \\
\hline \multicolumn{9}{|c|}{ Seawater } \\
\hline $1-01-89 \ldots$ & 51,700 & --- & -- & -- & - & -- & $\cdots$ & -- \\
\hline $2-16-89 \ldots$ & 50,700 & --- & --- & 360 & 1,200 & 10,000 & 290 & 112 \\
\hline $3-16-89 \ldots$ & 50,700 & --- & - & -- & --- & -- & --- & 111 \\
\hline $4-11-89 \ldots$ & 50,700 & 8.0 & -- & 380 & 1,200 & 9,000 & -- & 25 \\
\hline $6-20-89 \ldots$ & 51,100 & - & -- & -- & -- & -- & -- & 113 \\
\hline $7-19-89 \ldots$ & 51,200 & - & -- & -- & -- & -- & -- & 111 \\
\hline $10-12-89 \ldots$ & 51,200 & 8.1 & 20.0 & 360 & 1,200 & 10,000 & 300 & 111 \\
\hline Ocean average ${ }^{1}$ & 53,000 & -8 & -- & 403 & 1,260 & 10,500 & 390 & 120 \\
\hline \multicolumn{9}{|c|}{ Seawater (diluted) } \\
\hline $1-01-89$ & 4,460 & -- & --- & -- & -- & $\cdots$ & --- & -- \\
\hline $2-16-89 \ldots$ & 7,850 & -- & - & -- & -- & -- & --- & 16 \\
\hline $2-16-89 \ldots$ & 933 & -- & -- & -- & -- & -- & --- & 3.0 \\
\hline $3-16-89 \ldots$ & 5,480 & --- & -- & -- & -- & -- & -- & 9.0 \\
\hline 4-11-89 . & 5,600 & 7.7 & -- & $\cdots$ & 97 & 880 & -- & -- \\
\hline $6-20-89 \ldots$ & 8,080 & --- & -- & --- & - & -- & --- & 17 \\
\hline $6-20-89 \ldots$ & 1,530 & -- & --- & --- & -- & --- & -- & 4.0 \\
\hline 7-19-89 ... & 8,240 & -- & -- & -- & -- & -- & -- & 17 \\
\hline 7-19-89 ... & 1,220 & -- & -- & --- & -- & -- & --- & 4.0 \\
\hline
\end{tabular}

See footnote at end of table. 
Table 18. Analyses of undiluted and diluted (with deionized water) water samples from the Salton Sea and the Pacific Ocean--Continued

\begin{tabular}{|c|c|c|c|c|c|c|c|c|}
\hline Date & $\begin{array}{l}\text { Sulfate } \\
(\mathrm{mg} / \mathrm{L} \\
\left.\text { as } \mathrm{SO}_{4}\right)\end{array}$ & $\begin{array}{l}\text { Chlo- } \\
\text { ride } \\
(\mathrm{mg} / \mathrm{L} \\
\text { as } \mathrm{Cl})\end{array}$ & $\begin{array}{c}\text { Bromide } \\
(\mathrm{mg} / \mathrm{L} \\
\text { as } \mathrm{Br})\end{array}$ & $\begin{array}{c}\text { Silica } \\
(\mathrm{mg} / \mathrm{L} \\
\text { at } \\
\left.\mathrm{SiO}_{2}\right)\end{array}$ & $\begin{array}{c}\text { Solids, } \\
\text { residue } \\
\text { as } 180^{\circ} \mathrm{C} \\
(\mathrm{mg} / \mathrm{L})\end{array}$ & $\begin{array}{c}\text { Nitro- } \\
\text { gen, } \\
\mathrm{NO}_{2}+\mathrm{NO}_{3} \\
(\mathrm{mg} / \mathrm{L} \\
\text { as } \mathrm{N})\end{array}$ & $\begin{array}{c}\text { Nitro- } \\
\text { gen, } \\
\text { ammonia } \\
\text { (mg/L } \\
\text { as } \mathrm{N})\end{array}$ & $\begin{array}{c}\text { Nitro- } \\
\text { gen, am- } \\
\text { monia }+ \\
\text { organic } \\
(\mathrm{mg} / \mathrm{L} \\
\text { as } \mathrm{N})\end{array}$ \\
\hline \multicolumn{9}{|c|}{ Salton Sea } \\
\hline $8-17-88 \ldots$ & --- & -.. & -.. & -- & --- & -.. & $\ldots$ & -- \\
\hline 8-17-88. & -- & -- & -- & - & --- & --. & $\ldots$ & $\ldots$ \\
\hline $8-17-88 \ldots$ & --- & $\ldots$ & --. & - & --. & $\ldots$ & -.- & --- \\
\hline 8-24-88 & --- & -- & - & --- & -- & $-\cdots$ & --- & $-\cdots$ \\
\hline $8-25-88 \ldots$ & -- & -- & - & -- & -- & -- & --- & -- \\
\hline $2-16-89 \ldots$ & 10,000 & 17,000 & 13 & -- & 41,700 & - & -- & -.. \\
\hline 3-16-89 $\ldots$ & 9,600 & 17,000 & 12 & --- & -. & -.. & $\ldots$ & $\ldots$ \\
\hline $4-11-89$ & 9,600 & 16,000 & 12 & --- & -- & -- & -.. & -- \\
\hline 6-20-89 & 9,800 & 16,000 & 12 & --- & $\ldots$ & -.. & -- & -- \\
\hline $7-19-89$ & 9,800 & 15,000 & 13 & - & -- & -- & - & $\ldots$ \\
\hline $10-11-89 \ldots$ & 10,000 & 17,000 & 13 & 12 & 43,700 & $<0.100$ & 0.62 & 3.7 \\
\hline \multicolumn{9}{|c|}{ Salton Sea (diluted) } \\
\hline $2-16-89 \ldots$ & 1,100 & 1,700 & 1.3 & -- & -.- & $\ldots$ & -.- & $\ldots$ \\
\hline $2-16-89 \ldots$ & 110 & 190 & .15 & -- & -- & --- & --- & -- \\
\hline $3-16-89 \ldots$ & 730 & 1,200 & .84 & --- & -- &.- & -- & $\ldots$ \\
\hline $4-11-89 \ldots$ & 1,100 & 2,000 & .70 & -- & -- & --- & - & -- \\
\hline $6-20-89 \ldots$ & 1,500 & 2,600 & 1.9 & -- & --- & -- & --- & -- \\
\hline $6-20-89$ & 250 & 450 & $\ldots$ & -.. & -- & --. & --- & -- \\
\hline 7-19-89 $\ldots$ & 1,400 & 2,300 & 1.7 & -- & -- & --- & --- & -- \\
\hline $7-19-89 \ldots$ & 180 & 300 & .24 & -- & -- & --- & --- & -- \\
\hline \multicolumn{9}{|c|}{ Seawater } \\
\hline $1-01-89 \ldots$ & - & 21,000 & 67 & -.- & -- & --- & --- & -- \\
\hline $2-16-89 \ldots$ & 2,800 & 16,000 & 64 & -.. & 35,100 & --- & -- & $\ldots$ \\
\hline $3-16-89 \ldots$ & 2,700 & 20,000 & 59 & -.- & -- & --- & --- & -- \\
\hline $4-11-89 \ldots$ & 2,600 & 19,000 & 60 & -- & -- & --- & --- & -- \\
\hline $6-20-89 \ldots$ & 2,700 & 20,000 & 63 & - & -- & --- & -- & -- \\
\hline $7-19-89$ & 2,700 & 19,000 & 47 & - & --. & --- & --- & -- \\
\hline $10-12-89 \ldots$ & 2,300 & 18,000 & 64 & 0.40 & 35,000 & $<0.100$ & 0.24 & $<0.2$ \\
\hline Ocean average ${ }^{l}$ & 2,650 & 18,900 & 66 & 4 & 34,200 & {$[\quad-0$. } & as total nitr & ogen ] \\
\hline \multicolumn{9}{|c|}{ Seawater (diluted) } \\
\hline $1-01-89 \ldots$ & -- & 1,300 & 4.4 & -- & -- & -- & -. & --- \\
\hline $2-16-89 \ldots$ & 340 & 2,500 & 8.1 & -- & -. & -- & --- & --- \\
\hline $2-16-89 \ldots$ & 32 & 260 & .80 & -- & --- & - & -.- & --- \\
\hline 3-16-89 $\ldots$ & 230 & 1,600 & 5.3 & - & $\cdots$ & $\ldots$ & $\cdots$ & $\cdots$ \\
\hline $4-11-89$ & 220 & 1,700 & 5.2 & - & -- & -- & -- & -- \\
\hline $6-20-89$ & 350 & 2,400 & 8.4 & - & -- & -- & -- & -.. \\
\hline $6-20-89$ & 58 & 400 & 1.4 & $\ldots$ & $\ldots$ & -.. & -- & --- \\
\hline $7-19-89$ & 360 & 2,600 & 8.4 & $\ldots$ & - & --. & ... & -- \\
\hline $7-19-89$ & 45 & 320 & 1.0 & $\ldots$ & $-\ldots$ & -- & - & ... \\
\hline
\end{tabular}

See footnote at end of table. 
Table 18. Analyses of undiluted and diluted (with deionized water) water samples from the Salton Sea and the Pacific Ocean--Continued

\begin{tabular}{|c|c|c|c|c|c|c|c|c|}
\hline Date & $\begin{array}{c}\text { Phos- } \\
\text { phorus, } \\
\text { hydro. + } \\
\text { ortho } \\
\text { (mg/L } \\
\text { as P) }\end{array}$ & $\begin{array}{l}\text { Alumi- } \\
\text { num } \\
(\mu \mathrm{g} / \mathrm{L} \\
\text { as } \mathrm{Al})\end{array}$ & $\begin{array}{c}\text { Arsenic } \\
(\mu \mathrm{g} / \mathrm{L} \\
\text { as As) }\end{array}$ & $\begin{array}{l}\text { Boron } \\
(\mu \mathrm{g} / \mathrm{L} \\
\text { as B })\end{array}$ & $\begin{array}{c}\text { Iron } \\
(\mu \mathrm{g} / \mathrm{L} \\
\text { as } \mathrm{Fe})\end{array}$ & $\begin{array}{c}\text { Manga- } \\
\text { nese } \\
(\mu \mathrm{g} / \mathrm{L} \\
\text { as } \mathrm{Mn})\end{array}$ & $\begin{array}{l}\text { Molyb- } \\
\text { denum } \\
(\mu \mathrm{g} / \mathrm{L} \\
\text { as Mo) }\end{array}$ & $\begin{array}{l}\text { Sele- } \\
\text { nium } \\
(\mu \mathrm{g} / \mathrm{L} \\
\text { as } \mathrm{Se})\end{array}$ \\
\hline \multicolumn{9}{|c|}{ Salton Sea } \\
\hline $8-17-88 \ldots \ldots$ & $\ldots$ & -- & -. & - & -- & -- & --- & --- \\
\hline $8-17-88 \ldots \ldots$ & -- & -- & -- & -- & -- & -- & $\ldots$ & - \\
\hline $8-17-88 \ldots \ldots$ & --- & -.- & $\ldots$ & $\ldots$ & $\ldots$ & $\ldots$ & --- & $\ldots$ \\
\hline $8-24-88 \ldots \ldots$ & -- & - & - & - & -- & - & -- & --- \\
\hline $8-25-88 \ldots \ldots$ & -- & - & - & -- & -- & - & -- & -- \\
\hline $2-16-89 \ldots \ldots$ & -- & -- & -- & 11,000 & -- & -- & --- & -.. \\
\hline $3-16-89 \ldots \ldots$ & -- & -- & -- & 11,000 & --- & $\ldots$ & -.- & --- \\
\hline $4-11-89 \ldots \ldots$ & --. & --. & $\ldots$ & 11,000 & --- & $\ldots$ & --- & $\ldots$ \\
\hline $6-20-89 \ldots \ldots$ & -- & -- & -- & 12,000 & --- & -- & --- & --- \\
\hline 7-19-89 $\ldots \ldots$ & -- & --- & -- & 12,000 & --- & -- & --- & -- \\
\hline $10-11-89 \ldots \ldots$ & 0.06 & 50 & 9 & 12,000 & 340 & 80 & 6 & 2 \\
\hline \multicolumn{9}{|c|}{ Salton Sea (diluted) } \\
\hline $2-16-89$ & --- & --- & --- & 1,100 & --- & -- & -- & --- \\
\hline $2-16-89 \ldots \ldots$ & - & -.- & $\ldots$ & 120 & $\ldots$ & $\ldots$ & -.- & --- \\
\hline $3-16-89 \ldots \ldots$ & $\ldots$ & --- & -- & 800 & --- & - & $\ldots$ & -- \\
\hline $4-11-89 \ldots \ldots$ & -- & -- & -- & 1,400 & -- & - & - & --- \\
\hline $6-20-89$ & -- & -.- & - & 1,800 & --- & -- & --- & -.. \\
\hline $6-20-89 \ldots \ldots$ & $\ldots$ & $\ldots$ & --- & 280 & --- & -- & -- & --- \\
\hline 7-19-89 $\ldots \ldots$ & --- & - & - & 1,600 & --. & -- & --- & --- \\
\hline $7-19-89 \ldots \ldots$ & - & -- & -- & 210 & -- & --- & -- & --- \\
\hline \multicolumn{9}{|c|}{ Seawater } \\
\hline $1-01-89 \ldots \ldots$ & --- & -- & -- & 4,500 & -- & -- & -- & -- \\
\hline 2-16-89 $\ldots \ldots$ & --- & - & - & 4,200 & --- & -- & --- & --- \\
\hline $3-16-89$ & -- & --- &.-- & 4,700 & -- & $\ldots$ & $\ldots$ & --- \\
\hline $4-11-89$ & -. & - & - & 450 & --- & -.- & --- & -.- \\
\hline 6-20-89 $\ldots \ldots$ & - & -- & -- & 4,500 & --- & -- & --- & --- \\
\hline $7-19-89 \ldots \ldots$ & $\ldots$ & - & - & 5,000 & --- & $\ldots$ & --- & -.- \\
\hline $10-12-89 \ldots \ldots$ & 0.03 & 370 & 2 & 4,400 & 290 & 60 & 9 & $<1$ \\
\hline Ocean average ${ }^{1}$ & .06 & 2 & 3.7 & 4,400 & 2 & .2 & 10 & .2 \\
\hline \multicolumn{9}{|c|}{ Seawater (diluted) } \\
\hline $1-01-89$ & -- & - & $\ldots$ & 310 & --- & $\ldots$ & $\ldots$ & --- \\
\hline $2-16-89$ & --- & -- & $\cdots$ & 530 & --- & -- & --- & $\ldots$ \\
\hline $2-16-89$ & --- & -.. & $\ldots$ & 60 & --.- & $\ldots$ & --- & -.. \\
\hline $3-16-89 \ldots \ldots$ & -. & --- & --- & 380 & $\ldots$ & --- & --- & --- \\
\hline $4-11-89 \ldots \ldots$ & -- & -- & -- & 410 & -- & -- & --- & --- \\
\hline $6-20-89$ & --- & -- & - & 600 & --- & - & --- & --- \\
\hline $6-20-89$ & -- & -- & - & 100 & --- & -- & --- & --- \\
\hline $7-19-89$ & $-\cdots$ & -- & - & 610 & $\ldots$ & $\ldots$ & --- & --- \\
\hline $7-19-89 \ldots \ldots$ & $\ldots$ & -- & $\ldots$ & 790 & $\ldots$ & -- & --- & $\ldots$ \\
\hline
\end{tabular}

See footnote at end of table. 
Table 18. Analyses of undiluted and diluted (with deionized water) water samples from the Salton Sea and the Pacific Ocean--Continued

\begin{tabular}{|c|c|c|c|c|c|c|c|c|}
\hline \multirow{2}{*}{ Date } & \multirow{2}{*}{$\begin{array}{l}\text { Stron- } \\
\text { tium } \\
(\mu \mathrm{g} / \mathrm{L} \\
\text { as Sr) }\end{array}$} & \multirow{2}{*}{$\begin{array}{c}\text { Tri- } \\
\text { tium, } \\
\text { Total } \\
(\mathrm{pCi} / \mathrm{L})\end{array}$} & \multirow{2}{*}{$\begin{array}{c}\text { Uranium, } \\
\text { natural } \\
(\mu \mathrm{g} / \mathrm{L} \\
\text { as }(\mathrm{U})\end{array}$} & \multicolumn{3}{|c|}{ Stable-isotope ratio (permil) } & \multirow{2}{*}{$\begin{array}{c}\text { Carbon, } \\
\text { organic } \\
\text { total } \\
(\mathrm{mg} / \mathrm{L} \\
\text { as C) }\end{array}$} & \multirow{2}{*}{$\begin{array}{c}\text { Chloride/ } \\
\text { bromide } \\
\text { (weight } \\
\text { ratio) }\end{array}$} \\
\hline & & & & ${ }^{2} \mathbf{H} /{ }^{1} \mathbf{H}$ & ${ }^{18} \mathrm{O} /{ }^{16} \mathrm{O}$ & ${ }^{34} \mathrm{~S} /{ }^{32} \mathrm{~S}$ & & \\
\hline \multicolumn{9}{|c|}{ Salton Sea } \\
\hline $8-17-88 \ldots \ldots$ & -- & 101 & --- & -37.0 & -1.25 & --- & --- & --- \\
\hline $8-17-88 \ldots \ldots$ & - & 100 & -- & -29.5 & -.1 & -- & -- & $\ldots$ \\
\hline $8-17-88 \ldots \ldots$ & - & 108 & --- & -28.0 & .15 & --- & --- & --- \\
\hline $8-24-88 \ldots \ldots$ & --- & 103 & --- & -32.5 & -.74 & --. & --- & --- \\
\hline $8-25-88 \ldots \ldots$ & -- & 100 & -- & -37.5 & -2.05 & -- & --- & -- \\
\hline $2-16-89 \ldots \ldots$ & -- & --- & --- & -- & -- & 5.50 & -- & 1,308 \\
\hline $3-16-89 \ldots$ & --- & --- & --- & --- & --- & -- & --- & 1,417 \\
\hline $4-11-89 \ldots \ldots$ & --- & --- & --- & -- & --- & -- & --- & 1,333 \\
\hline $6-20-89 \ldots$ & -- & --- & -- & -- & -- & --- & --- & 1,333 \\
\hline $7-19-89 \ldots$ & -- & --- & -- & -- & --- & --- & --- & 1,154 \\
\hline $10-11-89 \ldots$ & 3,400 & --- & 5.2 & -29.5 & .05 & --- & 60 & 1,308 \\
\hline \multicolumn{9}{|c|}{ Salton Sea } \\
\hline 2-16-89 $\ldots$ & -- & --- & -- & -- & -- & --- & --- & 1,308 \\
\hline $2-16-89 \ldots$ & --- & --- & -- & $\ldots$ & --- & --- & --- & 1,267 \\
\hline 3-16-89 $\ldots$ & -- & -- & -- & --- & --- & --- & --- & 1,429 \\
\hline $4-11-89 \ldots$ & -- & -- & $\ldots$ & $-\ldots$ & -- & --- & $\ldots$ & 2,858 \\
\hline $6-20-89 \ldots \ldots$ & -- & -- & -- & -- & --- & --- & --- & 1,369 \\
\hline $6-20-89 \ldots \ldots$ & -- & --- & --- & --- & --- & $\ldots$ & --- & --- \\
\hline $7-19-89 \ldots$ & -- & -- & $\ldots$ & $-\cdots$ & --- & --- & --- & 1,353 \\
\hline $7-19-89 \ldots$ & -- & -- & -- & -- & -- & -- & --- & 1,250 \\
\hline \multicolumn{9}{|c|}{ Salton Sea (diluted) } \\
\hline $1-01-89 \ldots$ & -- & -- & -- & -- & --- & --- & --- & 313 \\
\hline 2-16-89 $\ldots$ & - & --- & -- & -- & --- & --- & -.- & 250 \\
\hline $3-16-89 \ldots$ & -- & --- & -- & -- & -- & 20.70 & --- & 339 \\
\hline $4-11-89 \ldots$ & - & -- & -- & -. & -- & --- & --- & 317 \\
\hline $6-20-89 \ldots$ & -- & --- & -- & -- & -- & --- & --- & 318 \\
\hline $7-19-89$ & - & -- & -- & -- & -- & --- & --- & 404 \\
\hline $10-12-89 \ldots \ldots$ & 7,800 & -- & 2.3 & -1.4 & -0.35 & --- & 1.1 & 281 \\
\hline Ocean average ${ }^{1}$ & 7,700 & -- & 3.2 & $\sim 0$ & $\sim 0$ & $\sim 21$ & 1 & 288 \\
\hline \multicolumn{9}{|c|}{ Seawater } \\
\hline $1-01-89$ & -- & -- & --- & -- & --- & --- & --- & 295 \\
\hline $2-16-89$ & - & -- & --- & --- & --- & -- & --- & 309 \\
\hline $2-16-89$ & - & -- & --- & -- & -- & --- & --- & 325 \\
\hline $3-16-89$ & -- & -- & --- & --- & --- & -- & -.. & 302 \\
\hline $4-11-89 \ldots$ & -- & -- & --- & -- & --- & $\cdots$ & --- & 327 \\
\hline $6-20-89$ & - & -- & - & $\ldots$ & -- & --- & --- & 286 \\
\hline $6-20-89 \ldots \ldots$ & -- & -- & - & $\cdots$ & - & --- & --- & 286 \\
\hline $7-19-89 \ldots$ & -- & - & - & -- & -- & --- & --- & 310 \\
\hline $7-19-89 \ldots$ & -- & -- & -- & $\ldots$ & --- & -- & --- & 320 \\
\hline
\end{tabular}

${ }^{\mathrm{I}}$ Values from Stumm and Morgan (1970), Riley and Skirrow (1975), and Fritz and Fontes (1980) are for seawater with a salinity of 35 parts per thousand. 
Table 19. Biological sampling sites for the detailed study of the Salton Sea area

[Location of sites shown in figure 4]

\begin{tabular}{|c|c|}
\hline Site number & Description \\
\hline $\begin{array}{l}\text { B1 } \\
\text { B2 } \\
\text { B3 } \\
\text { B4 } \\
\text { B5 } \\
\text { B6 } \\
\text { B7 } \\
\text { B8 } \\
\text { B9 } \\
\text { B10 } \\
\text { B11 } \\
\text { B12 } \\
\text { B13 } \\
\text { B14 } \\
\text { B15 } \\
\text { B16 }\end{array}$ & \begin{tabular}{l}
\multicolumn{1}{c}{ Salton Sea } \\
Salton Sea National Wildlife Refuge--Unit 1 \\
Poe Road \\
U.S. Navy test base \\
Salton City \\
Salton Sea Beach \\
Desert Shores \\
Desert Beach \\
Bob's Playa River Marina \\
Bombay Beach \\
S Lateral Drain outlet \\
Alamo River delta \\
Red Hill Marina \\
Obsidian Butte \\
Bowles Road \\
New River delta \\
Whitewater River delta
\end{tabular} \\
\hline $\begin{array}{l}\text { B17 } \\
\text { B18 } \\
\text { B19 } \\
\text { B20 } \\
\text { B21 }\end{array}$ & $\begin{array}{l}\quad \text { Rivers and Creeks } \\
\text { New River at Rio Bend } \\
\text { Alamo River at Garst Road } \\
\text { San Felipe Creek } \\
\text { Salt Creek } \\
\text { Colorado River at Palo Verde }\end{array}$ \\
\hline $\begin{array}{l}\text { B22 } \\
\text { B23 } \\
\text { B24 } \\
\text { B25 } \\
\text { B26 } \\
\text { B27 } \\
\text { B28 } \\
\text { B29 } \\
\text { B30 } \\
\text { B31 } \\
\text { B32 }\end{array}$ & $\begin{array}{l}\quad \text { Drainwater Ditches } \\
\text { Trifolium } 5 \\
\text { Trifolium } 13 \\
\text { Trifolium } 14 \\
\text { Vail Cutoff } \\
\text { Vail 4 } \\
\text { Vail 4A } \\
\text { Vail Drain at New River } \\
\text { S Lateral } \\
\text { Z Lateral } \\
\text { 81st Street } \\
\text { Johnson Street }\end{array}$ \\
\hline $\begin{array}{l}\text { B33 } \\
\text { B34 } \\
\text { B35 } \\
\text { B36 } \\
\text { B37 }\end{array}$ & $\begin{array}{l}\text { Freshwater Impoundments } \\
\text { Shady Acres Duck Club } \\
\text { RH Pond } \\
\text { HQ Pond } \\
\text { Reidman Pond } \\
\text { Hazard Pond }\end{array}$ \\
\hline $\begin{array}{l}\text { B38 } \\
\text { B39 }\end{array}$ & $\begin{array}{l}\quad \text { Imperial Valley } \\
\text { South Brawley } \\
\text { McKendry Road }\end{array}$ \\
\hline
\end{tabular}


Table 20. Biotic samples collected from sites in the study area

[Sites described in table 19]

$\begin{array}{lll}\text { Species } & \text { Sample Sites }\end{array}$

Blue-green algae (Myxophyceae)

Filamentous green algae (Chaetomorpha sp.)

Tubular green algae (Enteromorpha sp.)

Common cattail (Typha latifolia)

Periphyton

Asiatic river clam (Corbicula fluminea)

Crayfish (Procambarus clarki)

Pelagic invertebrate "mixture"

Pileworm (Nereis succinea)

Waterboatman (Corixidae)

Bairdiella (Bairdiella icistia)

Longjaw mudsucker (Gillichthys mirabilis)

Mosquitofish (Gambusia affinis)

Orangemouth corvina (Cynoscion xanthulus)

Redfin shiner (Lythrurus umbratilis)

Sailfin molly (Poecilia latipinna)

Tilapia (Tilapia zilli)

Bullfrog (Rana catesbeiana)

Spiny softshell turtle (Trionyx spiniferus)

American coot (Fulica americana)

Barn owl (Tyto alba)

Black-necked stilt (Himantopus mexicanus)

Cattle egret (Bubulcus ibis)

Double-crested cormorant (Phalacrocorax auritus)

Eared grebe (Podiceps nigricollis)

Great blue heron (Ardea herodias)

Herring gull (Larus argentatus)

Northern shoveler (Anas clypeata)

Ruddy duck (Oxyura jamaicensis)

White-faced ibis (Plegadis chihi)

Yuma clapper rail (Rallus longirostris yumanensis)

\section{Vegetation \\ Composite \\ Composite \\ Composite \\ Whole plant \\ Composite}

\section{Invertebrates}

Soft-tissue composite

\section{Whole body \\ Composite \\ Composite \\ Composite}

Fish

Whole body

Whole body

Whole body

Whole body

Whole body

Whole body

Whole body

Amphibians

Whole body

\section{Reptiles}

Fat/liver/egg

Birds

Liver

Muscle

Carcass/egg

Muscle

Muscle

Liver/muscle

Muscle

Muscle

Liver/muscle

Liver/muscle

Liver/muscle

Carcass

\section{B32}

$\mathrm{B} 1, \mathrm{~B} 3, \mathrm{~B} 4, \mathrm{~B} 5, \mathrm{~B} 6, \mathrm{~B} 7, \mathrm{~B} 8$, $\mathrm{B} 9, \mathrm{~B} 12, \mathrm{~B} 13, \mathrm{~B} 14, \mathrm{~B} 31$

$\mathrm{B} 1, \mathrm{~B} 2, \mathrm{~B} 3, \mathrm{~B} 4, \mathrm{~B} 5, \mathrm{~B} 6, \mathrm{~B} 7$, $\mathrm{B} 8, \mathrm{~B} 9, \mathrm{~B} 12, \mathrm{~B} 13, \mathrm{~B} 14, \mathrm{~B} 31$

$\mathrm{B} 19, \mathrm{~B} 20, \mathrm{~B} 30, \mathrm{~B} 31, \mathrm{~B} 32$

B1

B1, B15, B16, B17, B18, B21, $\mathrm{B} 23, \mathrm{~B} 24, \mathrm{~B} 25, \mathrm{~B} 26, \mathrm{~B} 28$, B30, B32

B1, B11, B15, B24, B37

$\mathrm{B} 1, \mathrm{~B} 23$

$\mathrm{B} 1, \mathrm{~B} 11, \mathrm{~B} 27$

B11, B15, B18

\section{B10}

B15

B15, B19, B20, B31, B32, B37

B1

B16

B1, B16, B17, B19, B20, B30, B31, B32, B37

B1, B15, B16, B17, B22, B37

B18

B26, B37

$\mathrm{B} 15, \mathrm{~B} 16, \mathrm{~B} 17, \mathrm{~B} 25, \mathrm{~B} 37$

B17

B1, B11, B15, B16, B17, B24, B25, B29, В34, B36, B37, В39

$\mathrm{B} 17, \mathrm{~B} 37$

B17, B37

B13, B15

B1

B37

B1, B11, B16, B33, B37

B1, B15, B17, B24, B26, B35, B37

B38

B29 
Table 21. Chemicals analyzed for in biota in the detailed study of the Salton Sea area

\begin{tabular}{|c|c|c|c|}
\hline \multicolumn{4}{|c|}{ Inorganic Analytes } \\
\hline $\begin{array}{l}\text { Aluminum (Al) } \\
\text { Antimony (Sb) } \\
\text { Arsenic (As) } \\
\text { Barium (Ba) } \\
\text { Beryllium (Be) } \\
\text { Boron (B) }\end{array}$ & $\begin{array}{l}\text { Cadmium }(\mathrm{Cd}) \\
\text { Chromium }(\mathrm{Cr}) \\
\text { Copper }(\mathrm{Cu}) \\
\text { Iron }(\mathrm{Fe}) \\
\text { Lead }(\mathrm{Pb}) \\
\text { Magnesium }(\mathrm{Mg})\end{array}$ & $\begin{array}{l}\text { Manganese (Mn) } \\
\text { Mercury (Hg) } \\
\text { Molybdenum (Mo) } \\
\text { Nickel (Ni) } \\
\text { Selenium (Se) } \\
\text { Silver (Ag) }\end{array}$ & $\begin{array}{l}\text { Strontium (Sr) } \\
\text { Thallium (Tl) } \\
\text { Tin (Sn) } \\
\text { Vanadium (V) } \\
\text { Zinc (Zn) }\end{array}$ \\
\hline \multicolumn{4}{|c|}{ Organic Analytes } \\
\hline $\begin{array}{l}\text { Aldrin } \\
\text { Benzene hexachloride (BHC) } \\
\text { beta-BHC } \\
\text { delta-BHC } \\
\text { gamma-BHC } \\
\text { alpha-Chlordane } \\
\text { cis-Chlordane } \\
\text { gamma-Chlordane } \\
\text { trans-Chlordane } \\
\text { Chlorthal-dimethyl (DCPA) } \\
\text { o,p'-DDD } \\
\text { p,p'-DDD } \\
\text { o, p'-DDD } \\
\text { p,p'-DDE } \\
\text { o,p'-DDT } \\
\text { p, p'-DDT } \\
\text { Total DDT }\end{array}$ & $\begin{array}{l}\text { Dicofol } \\
\text { Dieldrin } \\
\text { Endosulfan I } \\
\text { Endosulfan II } \\
\text { Endosulfan sulfate } \\
\text { Endrin } \\
\text { Heptachlor } \\
\text { Heptachlor epoxide } \\
\text { Hexachlorobenzene } \\
\text { Lindane } \\
\text { Methoxychlor } \\
\text { Mirex } \\
\text { cis-Nonachlor } \\
\text { trans-Nonachlor } \\
\text { Oxychlordane } \\
\text { Biphenyl CL-2 } \\
\text { Biphenyl CL-3 }\end{array}$ & $\begin{array}{l}\text { Biphenyl CL-4 } \\
\text { Biphenyl CL-5 } \\
\text { Biphenyl CL-6 } \\
\text { Biphenyl CL-7 } \\
\text { Biphenyl CL-8 } \\
\text { Biphenyl CL-9 } \\
\text { PCB } 1016 \\
\text { PCB } 1221 \\
\text { PCB } 1232 \\
\text { PCB } 1242 \\
\text { PCB } 1248 \\
\text { PCB } 1254 \\
\text { PCB 1260 } \\
\text { Total PCB } \\
\text { Tetradifon } \\
\text { Toxaphene }\end{array}$ & $\begin{array}{l}\text { Naphthalene } \\
\text { Acenapthene } \\
\text { Fluorene } \\
\text { Phenanthrene } \\
\text { Anthracene } \\
\text { Fluoranthene } \\
\text { Pyrene } \\
\text { Benzo (a) anthracene } \\
\text { Chrysene } \\
\text { Benzo (b) fluoranthene } \\
\text { Benzo(k) fluoranthene } \\
\text { Benzo (a) pyrene } \\
\text { Benzo (e) pyrene } \\
\text { Perylene } \\
\text { Indeno (1,2,3-cd)pyrene } \\
\text { Dibenz (ah) anthracene } \\
\text { Benzo (ghi) perylene }\end{array}$ \\
\hline
\end{tabular}


Table 22. Inorganic chemical analysis and moisture content for biotic samples collected during 1988-90 from the Salton Sea and associated drainwaters and rivers

[All chemical data reported in micrograms per gram, dry weight. <, less than indicated reporting limit; ---, not determined. The analysis for each sample is displayed as one line on three consecutive pages. NWR, National Wildlife Refuge]

\begin{tabular}{|c|c|c|c|c|c|c|c|}
\hline $\begin{array}{l}\text { Record } \\
\text { number }\end{array}$ & $\begin{array}{l}\text { Sample } \\
\text { number }\end{array}$ & Species & Matrix & $\begin{array}{l}\text { Percent } \\
\text { moisture }\end{array}$ & $\begin{array}{l}\text { Sil- } \\
\text { ver }\end{array}$ & $\begin{array}{c}\text { Alu- } \\
\text { mi- } \\
\text { num }\end{array}$ & $\begin{array}{c}\text { Arse- } \\
\text { nic }\end{array}$ \\
\hline \multicolumn{8}{|c|}{ Site B1 (Salton Sea NWR--Unit 1) } \\
\hline . . & LNSS88-22 & Ruddy duck & Liver & 71.9 & $<2$ & 5 & $<0.2$ \\
\hline 2 & LNSS88-42 & Ruddy duck & Liver & 69.1 & $<2$ & 6 & $<.2$ \\
\hline $3 \ldots$ & LNSS88-44 & Ruddy duck & Liver & 69.4 & $<2$ & 6 & $<.2$ \\
\hline $4 \ldots$ & LNSS88-46 & Ruddy duck & Liver & 71.2 & 3 & 7 & $<.2$ \\
\hline $5 \ldots$ & LNSS88-48 & Ruddy duck & Liver & 68.4 & $<2$ & 6 & .3 \\
\hline $6 \ldots$ & LNSS88-50 & Ruddy duck & Liver & 69.1 & $<2$ & $<3$ & $<.2$ \\
\hline $7 \ldots$ & LNSS88-21 & Ruddy duck & Breast muscle & 69.6 & $<2$ & 4 & .3 \\
\hline $8 \ldots$ & LNSS88-43 & Ruddy duck & Breast muscle & 70.5 & $<2$ & 5 & $<.2$ \\
\hline $9 \ldots$ & LNSS88-45 & Ruddy duck & Breast muscle & 69.0 & $<2$ & $<3$ & $<.2$ \\
\hline $10 \ldots$ & LNSS88-49 & Ruddy duck & Breast muscle & 69.7 & $<2$ & $<3$ & $<.2$ \\
\hline $11 \ldots$ & LNSS89-33 & Periphyton & Composite & 64.7 & $<2$ & 24,100 & 2.5 \\
\hline $12 \ldots$ & LNSS89-34 & Pileworm & Composite & 80.9 & $<2$ & 24,300 & 4.1 \\
\hline $13 \ldots$ & LNSS89-35 & Pileworm & Composite & 81.9 & $<2$ & 16,000 & 2.9 \\
\hline $14 \ldots$ & LNSS89-36 & Pileworm & Composite & 84.1 & $<2$ & 37,800 & 9.0 \\
\hline $15 \ldots$ & LNSS89-37 & Pileworm & Composite & 86.2 & $<2$ & 17,200 & 4.4 \\
\hline $16 \ldots$ & LNSS89-38 & Pileworm & Composite & 83.5 & $<2$ & 18,300 & 4.0 \\
\hline $17 \ldots$ & SS89-62 & Invertebrates & Composite & 81.1 & $<2$ & 4,350 & 2.9 \\
\hline $18 \ldots$ & SS89-35 & Northern shoveler & Liver & 71.8 & $<2$ & $<3$ & .2 \\
\hline $19 \ldots$ & SS89-36 & Northern shoveler & Liver & 70.6 & $<2$ & 6 & $<.1$ \\
\hline $20 \ldots$ & SS89-37 & Northern shoveler & Liver & 70.5 & $<2$ & $<3$ & $<.1$ \\
\hline $21 \ldots$ & SS89-40 & Northern shoveler & Liver & 70.5 & $<2$ & 3 & .3 \\
\hline $22 \ldots$ & SS89-51 & Northern shoveler & Liver & 74.4 & $<2$ & 13 & .34 \\
\hline $23 \ldots$ & SS89-56 & Northern shoveler & Liver & 70.8 & $<2$ & 29 & $<.1$ \\
\hline $24 \ldots$ & LNSS89-02 & Ruddy duck & Liver & 69.2 & $<2$ & 8 & .35 \\
\hline $25 \ldots$ & LNSS89-03 & Ruddy duck & Liver & 72.1 & $<2$ & $<3$ & .37 \\
\hline $26 \ldots$ & LNSS89-05 & Ruddy duck & Liver & 72.8 & $<2$ & $<3$ & .51 \\
\hline $27 \ldots$ & LNSS89-07 & Ruddy duck & Liver & 70.7 & $<2$ & $<3$ & .30 \\
\hline $28 \ldots$ & LNSS89-09 & Ruddy duck & Liver & 70.1 & $<2$ & 3 & .84 \\
\hline $29 \ldots$ & LNSS89-11 & Ruddy duck & Liver & 70.3 & $<2$ & $<3$ & .52 \\
\hline $30 \ldots$ & LNSS89-13 & Ruddy duck & Liver & 70.3 & $<2$ & 4 & .64 \\
\hline $31 \ldots$ & LNSS89-15 & Ruddy duck & Liver & 71.7 & $<2$ & $<3$ & .53 \\
\hline $32 \ldots$ & LNSS89-17 & Ruddy duck & Liver & 69.3 & $<2$ & $<3$ & .40 \\
\hline $33 \ldots$ & LNSS89-19 & Ruddy duck & Liver & 70.5 & $<2$ & 9 & .50 \\
\hline $34 \ldots$ & LNSS89-21 & Ruddy duck & Liver & 71.3 & $<2$ & $<3$ & .2 \\
\hline $35 \ldots$ & LNSS89-23 & Ruddy duck & Liver & 71.7 & $<2$ & $<3$ & .58 \\
\hline $36 \ldots$ & LNSS89-24 & Ruddy duck & Liver & 70.9 & $<2$ & 6 & .44 \\
\hline $37 \ldots$ & LNSS89-25 & Ruddy duck & Liver & 71.2 & $<2$ & $<3$ & .3 \\
\hline $38 \ldots$ & LNSS89-26 & Ruddy duck & Liver & 70.1 & $<2$ & $<3$ & .2 \\
\hline $39 \ldots$ & LNSS89-27 & Ruddy duck & Liver & 71.1 & $<2$ & $<3$ & .38 \\
\hline
\end{tabular}


Table 22. Inorganic chemical analysis and moisture content for biotic samples collected during 1988-90 from the Salton Sea and associated drainwaters and rivers--Continued

\begin{tabular}{|c|c|c|c|c|c|c|c|c|c|c|c|}
\hline $\begin{array}{l}\text { Record } \\
\text { number }\end{array}$ & $\begin{array}{l}\text { Sample } \\
\text { number }\end{array}$ & $\begin{array}{l}\text { Bo- } \\
\text { ron }\end{array}$ & $\begin{array}{l}\mathrm{Ba-} \\
\text { rium }\end{array}$ & $\begin{array}{l}\text { Be- } \\
\text { ryl- } \\
\text { lium }\end{array}$ & $\begin{array}{l}\text { Cad- } \\
\text { mium }\end{array}$ & $\begin{array}{l}\text { Chro- } \\
\text { mi- } \\
\text { um }\end{array}$ & $\begin{array}{l}\text { Cop- } \\
\text { per }\end{array}$ & Iron & $\begin{array}{l}\text { Mer- } \\
\text { cury }\end{array}$ & $\begin{array}{l}\text { Mag- } \\
\text { ne- } \\
\text { sium }\end{array}$ & $\begin{array}{l}\text { Man- } \\
\text { ga- } \\
\text { nese }\end{array}$ \\
\hline \multicolumn{12}{|c|}{ Site B1 (Salton Sea NWR--Unit 1) } \\
\hline 1 & LNSS88-22 & 6.5 & 0.1 & $<0.1$ & 0.99 & $<1$ & 14 & 8,810 & 0.056 & 765 & 11 \\
\hline 2 & LNSS88-42 & 2 & $<.1$ & $<.1$ & .2 & $<1$ & 129 & 4,460 & 1.5 & 786 & 23.2 \\
\hline 3 & LNSS88-44 & 4 & .1 & $<.1$ & $<.2$ & 2 & 21.3 & 3,290 & .937 & 763 & 24.4 \\
\hline 4 & LNSS88-46 & 3 & $<.1$ & $<.1$ & 2.8 & 3.0 & 35.4 & 2,070 & .752 & 808 & 17 \\
\hline 5 & LNSS88-48 & 4 & $<.1$ & $<.1$ & 2.3 & 2 & 42.9 & 1,570 & .23 & 651 & 12 \\
\hline 6 & LNSS88-50 & 4 & $<.1$ & $<.1$ & $<.2$ & $<1$ & 42.4 & 1,210 & .21 & 769 & 15 \\
\hline 7 & LNSS88-21 & 2 & $<.1$ & $<.1$ & $<.2$ & $<1$ & 26.8 & 407 & .022 & 1,010 & 1.8 \\
\hline 8 & LNSS88-43 & 2 & $<.1$ & $<.1$ & $<.2$ & $<1$ & 30.1 & 471 & .24 & 1,020 & 2.7 \\
\hline 9 & LNSS88-45 & $<2$ & $<.1$ & $<.1$ & $<.2$ & $<1$ & 24.4 & 324 & .20 & 1,030 & 2.4 \\
\hline 10 & LNSS88-49 & 2 & $<.1$ & $<.1$ & $<.2$ & $<1$ & 27.6 & 368 & .033 & 1,100 & 2.3 \\
\hline 11 & LNSS89-33 & 40 & 180 & .47 & $<.8$ & 18 & 11 & 13,300 & .016 & 12,000 & 247 \\
\hline 12 & LNSS89-34 & 140 & 162 & .3 & $<.8$ & 18 & 13 & 13,100 & .021 & 18,900 & 242 \\
\hline 13 & LNSS89-35 & 83 & 117 & $<.1$ & $<.8$ & 12 & 11 & 8,270 & .032 & 12,800 & 215 \\
\hline 14 & LNSS89-36 & 94 & 230 & .59 & $<.8$ & 27 & 20 & 19,000 & .025 & 20,600 & 418 \\
\hline 15 & LNSS89-37 & 160 & 112 & $<.1$ & $<.8$ & 15 & 8.5 & 9,830 & .019 & 16,900 & 336 \\
\hline 16 & LNSS89-38 & 150 & 142 & $<.1$ & $<.8$ & 12 & 8.9 & 10,100 & .033 & 16,500 & 381 \\
\hline 17 & SS89-62 & 20 & 44.2 & $<.1$ & .3 & 6.2 & 21.1 & 2,850 & .047 & 4,040 & 54.2 \\
\hline 18 & SS89-35 & 5.8 & $<.1$ & $<.1$ & 1.1 & 1 & 26.5 & 4,100 & 1.10 & 772 & 12 \\
\hline 19 & SS89-36 & 5.8 & .1 & $<.1$ & .89 & 1 & 29.9 & 5,070 & 4.3 & 780 & 11 \\
\hline 20 & SS89-37 & 5 & $<.1$ & $<.1$ & 1.3 & $<1$ & 19 & 1,920 & .777 & 701 & 8.1 \\
\hline 21 & SS89-40 & 5 & $<.1$ & $<.1$ & 1.4 & 2 & 16 & 5,210 & .502 & 701 & 13 \\
\hline 22 & SS89-51 & 4 & .2 & $<.1$ & 2.3 & $<1$ & 47.5 & 3,850 & .558 & 707 & 11 \\
\hline 23 & SS89-56 & 3 & .2 & $<.1$ & 2.1 & $<1$ & 38.1 & 2,850 & .978 & 687 & 16 \\
\hline 24 & LNSS89-02 & 4.0 & .2 & $<.1$ & $<.8$ & $<1$ & 37.5 & 1,390 & .12 & 760 & 11.0 \\
\hline 25 & LNSS89-03 & $<3$ & .2 & $<.1$ & 1 & $<1$ & 79.2 & 2,740 & 2.09 & 741 & 13 \\
\hline 26 & LNSS89-05 & $<3$ & .1 & $<.1$ & $<.8$ & $<1$ & 140 & 2,660 & 2.0 & 810 & 10 \\
\hline 27 & LNSS89-07 & $<3$ & .1 & $<.1$ & .8 & $<1$ & 67.5 & 1,270 & .513 & 807 & 14 \\
\hline 28 & LNSS89-09 & $<3$ & .2 & $<.1$ & $<.8$ & $<1$ & 53.9 & 1,880 & .962 & 743 & 13 \\
\hline 29 & LNSS89-11 & $<3$ & .1 & $<.1$ & 1 & $<1$ & 64.1 & 1,310 & .16 & 711 & 13 \\
\hline 30 & LNSS89-13 & $<3$ & .2 & $<.1$ & .8 & $<1$ & 76.0 & 2,770 & .17 & 847 & 13 \\
\hline 31 & LNSS89-15 & $<3$ & .31 & $<.1$ & 1 & $<2$ & 59.0 & 3,080 & .43 & 817 & 13 \\
\hline 32 & LNSS89-17 & $<3$ & .1 & $<.1$ & $<.8$ & $<2$ & 63.8 & 3,280 & .13 & 774 & 17 \\
\hline 33 & LNSS89-19 & $<3$ & .32 & $<.1$ & 1 & $<2$ & 125 & 2,790 & .30 & 954 & 13 \\
\hline 34 & LNSS89-21 & $<3$ & .1 & $<.1$ & $<.8$ & $<1$ & 47.9 & 1,700 & .18 & 856 & 16 \\
\hline 35 & LNSS89-23 & $<3$ & .2 & $<.1$ & $<.8$ & $<1$ & 140 & 3,680 & .23 & 924 & 14 \\
\hline 36 & LNSS89-24 & $<3$ & .3 & $<.1$ & 2 & $<1$ & 104 & 4,010 & .21 & 896 & 13 \\
\hline 37 & LNSS89-25 & $<3$ & .1 & $<.1$ & $<.8$ & $<1$ & 51.4 & 1,560 & .15 & 700 & 15 \\
\hline 38 & LNSS89-26 & $<3$ & $<.1$ & $<.1$ & $<.8$ & $<1$ & 213 & 1,960 & .25 & 761 & 16 \\
\hline 39 & LNSS89-27 & $<3$ & $<.1$ & $<.1$ & $<.8$ & $<1$ & 66.1 & 1,420 & .19 & 694 & 12 \\
\hline
\end{tabular}


Table 22. Inorganic chemical analysis and moisture content for biotic samples collected during 1988-90 from the Salton Sea and associated drainwaters and rivers--Continued

\begin{tabular}{|c|c|c|c|c|c|c|c|c|c|c|c|}
\hline $\begin{array}{l}\text { Record } \\
\text { number }\end{array}$ & $\begin{array}{l}\text { Sample } \\
\text { number }\end{array}$ & $\begin{array}{c}\text { Molyb- } \\
\text { de- } \\
\text { num }\end{array}$ & $\begin{array}{l}\text { Nick- } \\
\text { el }\end{array}$ & Lead & $\begin{array}{c}\text { An- } \\
\text { timo- } \\
\text { ny }\end{array}$ & $\begin{array}{l}\text { Sele- } \\
\text { ni- } \\
\text { um }\end{array}$ & Tin & $\begin{array}{c}\text { Stron- } \\
\text { ti- } \\
\text { um }\end{array}$ & $\begin{array}{l}\text { Thal- } \\
\text { li- } \\
\text { um }\end{array}$ & $\begin{array}{c}\text { Va- } \\
\text { nadi- } \\
\text { um }\end{array}$ & Zinc \\
\hline \multicolumn{12}{|c|}{ Site B1 (Salton Sea NWR--Unit 1) } \\
\hline 1 & LNSS88-22 & 4 & $<1$ & $<4$ & --- & 7.6 & -- & 0.50 & $<5$ & 0.6 & 115 \\
\hline 2 & LNSS88-42 & 4 & $<1$ & $<4$ & --- & 17.0 & -- & 1.0 & $<5$ & $<.3$ & 154 \\
\hline 3 & LNSS88-44 & 3 & 2 & $<4$ & --- & 10.9 & -- & 1.1 & $<5$ & $<.3$ & 135 \\
\hline 4 & LNSS88-46 & 3 & 2 & $<4$ & --- & 10.4 & --- & .61 & $<5$ & 1.1 & 118 \\
\hline 5 & LNSS88-48 & 3 & 2 & $<4$ & --- & 19.9 & --- & .59 & $<5$ & .5 & 147 \\
\hline 6 & LNSS88-50 & 3 & $<1$ & $<4$ & -- & 13.3 & -.- & .65 & $<5$ & .6 & 134 \\
\hline 7 & LNSS88-21 & $<1$ & $<1$ & $<4$ & -- & 2.8 & -- & .2 & $<5$ & $<.3$ & 39.1 \\
\hline 8 & LNSS88-43 & $<1$ & $<1$ & $<4$ & -- & 3.6 & --- & .51 & $<5$ & $<.3$ & 40.4 \\
\hline 9 & LNSS88-45 & $<1$ & $<1$ & $<4$ & --- & 2.7 & --- & .38 & $<5$ & $<.3$ & 35.8 \\
\hline 10 & LNSS88-49 & $<1$ & $<1$ & $<4$ & -- & 5.5 & --- & .43 & $<5$ & $<.3$ & 35.3 \\
\hline 11 & LNSS89-33 & $<2$ & 12 & 10 & -- & .66 & --- & 242 & $<4$ & 32 & 43 \\
\hline 12 & LNSS89-34 & $<2$ & 14 & 10 & -- & .82 & $\cdots$ & 376 & $<4$ & 32 & 40 \\
\hline 13 & LNSS89-35 & $<2$ & 11 & 9 & -- & 3.4 & --- & 459 & $<4$ & 22 & 42 \\
\hline 14 & LNSS89-36 & $<3$ & 18 & 16 & -- & 1.3 & --- & 463 & $<4$ & 49 & 61 \\
\hline 15 & LNSS89-37 & $<2$ & 9 & 10 & - & 1.8 & --- & 343 & $<4$ & 23 & 32 \\
\hline 16 & LNSS89-38 & $<2$ & 11 & 9 & -- & 1.6 & --- & 450 & $<4$ & 24 & 35 \\
\hline 17 & SS89-62 & $<1$ & 2 & $<4$ & -- & 3.1 & -- & 292 & $<4$ & 7.9 & 100 \\
\hline 18 & SS89-35 & 3 & $<1$ & $<4$ & -- & 9.1 & -- & .84 & $<5$ & .9 & 114 \\
\hline 19 & SS89-36 & 3 & $<1$ & $<4$ & -- & 12 & -- & 1.3 & $<5$ & $<.3$ & 101 \\
\hline 20 & SS89-37 & 2 & $<1$ & $<4$ & -- & 12 & -- & 2.1 & $<5$ & $<.3$ & 88.9 \\
\hline 21 & SS89-40 & 3 & $<1$ & $<4$ & -- & 16 & -- & .99 & $<6$ & $<.3$ & 120 \\
\hline 22 & SS89-51 & 2 & $<1$ & $<4$ & -- & 25.9 & -- & 3.1 & $<5$ & $<.3$ & 123 \\
\hline 23 & SS89-56 & 3 & $<1$ & $<4$ & $\cdots$ & 23.7 & --- & 1.1 & $<5$ & $<.3$ & 99.1 \\
\hline 24 & LNSS89-02 & 3.0 & $<3$ & $<4$ & - & 12 & --- & 1.3 & $<4$ & $<.7$ & 120 \\
\hline 25 & LNSS89-03 & 4.2 & $<3$ & $<4$ & - & 12 & --- & .71 & $<4$ & $<.7$ & 127 \\
\hline 26 & LNSS89-05 & 3 & $<3$ & $<4$ & - & 18 & --- & .63 & $<4$ & $<.7$ & 117 \\
\hline 27 & LNSS89-07 & 3 & $<3$ & $<4$ & -- & 14.5 & --- & .56 & $<4$ & $<.7$ & 110 \\
\hline 28 & LNSS89-09 & 3 & $<3$ & $<4$ & - & 20.4 & --- & 1.9 & $<4$ & $<.7$ & 106 \\
\hline 29 & LNSS89-11 & 3.4 & 4 & $<4$ & -- & 11 & -- & .68 & $<4$ & $<.7$ & 97.1 \\
\hline 30 & LNSS89-13 & 4 & $<3$ & $<4$ & -- & 9.6 & --- & 1.4 & $<4$ & $<.7$ & 139 \\
\hline 31 & LNSS89-15 & 3 & $<3$ & $<4$ & --- & 11 & -- & .72 & $<4$ & .9 & 94.3 \\
\hline 32 & LNSS89-17 & 4.5 & $<3$ & $<4$ & --- & 12 & --- & .65 & $<4$ & $<.7$ & 142 \\
\hline 33 & LNSS89-19 & 4 & $<4$ & $<4$ & --- & 13 & --- & 4.4 & $<4$ & $<.7$ & 181 \\
\hline 34 & LNSS89-21 & 4 & $<3$ & $<4$ & -- & 12 & -- & .58 & $<4$ & $<.7$ & 114 \\
\hline 35 & LNSS89-23 & 5.4 & $<3$ & $<4$ & -- & 11 & --- & 1.4 & $<4$ & $<.7$ & 122 \\
\hline 36 & LNSS89-24 & 4.8 & $<3$ & $<4$ & -- & 10 & --- & 2.0 & $<4$ & $<.7$ & 162 \\
\hline 37 & LNSS89-25 & 3.6 & $<3$ & $<4$ & $\cdots$ & 13 & --- & .64 & $<4$ & $<.7$ & 109 \\
\hline 38 & LNSS89-26 & 3.8 & $<3$ & $<4$ & -- & 18.6 & -- & .2 & $<4$ & $<.7$ & 141 \\
\hline 39 & LNSS89-27 & 3 & $<3$ & $<4$ & -- & 12 & --- & .3 & $<4$ & $<.7$ & 131 \\
\hline
\end{tabular}


Table 22. Inorganic chemical analysis and moisture content for biotic samples collected during 1988-90 from the Salton Sea and associated drainwaters and rivers--Continued

\begin{tabular}{|c|c|c|c|c|c|c|c|}
\hline $\begin{array}{l}\text { Recor } \\
\text { numbe }\end{array}$ & $\begin{array}{l}\text { Sample } \\
\text { number }\end{array}$ & Species & Matrix & $\begin{array}{l}\text { Percent } \\
\text { moisture }\end{array}$ & $\begin{array}{l}\text { Sil- } \\
\text { ver }\end{array}$ & $\begin{array}{l}\text { Alu- } \\
\text { mi- } \\
\text { num }\end{array}$ & $\begin{array}{c}\text { Arse- } \\
\text { nic }\end{array}$ \\
\hline \multicolumn{8}{|c|}{ Site B1 (Salton Sea NWR--Unit 1)-Continued } \\
\hline 40 & . . . . LNSS89-28 & Ruddy duck & Liver & 70.0 & $<2$ & $<3$ & 0.65 \\
\hline 41 & . . . . LNSS89-29 & Ruddy duck & Liver & 71.9 & $<2$ & $<3$ & .1 \\
\hline 42 & . . . . LNSS89-30 & Ruddy duck & Liver & 71.7 & $<2$ & $<3$ & .2 \\
\hline 43 & . . . . LNSS89-31 & Ruddy duck & Liver & 69.4 & $<2$ & $<3$ & .48 \\
\hline 44 & . . . . LNSS89-32 & Ruddy duck & Liver & 68.8 & $<2$ & $<3$ & .84 \\
\hline 45 & $\ldots \ldots$ SS89-33 & Ruddy duck & Liver & 74.6 & $<2$ & 5 & .30 \\
\hline 46 & . . . . SS889-34 & Ruddy duck & Liver & 70.8 & $<2$ & $<3$ & .2 \\
\hline 47 & . . . . . SS89-41 & Ruddy duck & Liver & 70.4 & $<2$ & 7 & .2 \\
\hline 48 & . . . . SS899-42 & Ruddy duck & Liver & 72.6 & $<2$ & 19 & .2 \\
\hline 49 & . . . . SS89-43 & Ruddy duck & Liver & 70.6 & $<2$ & $<3$ & .3 \\
\hline 50 & . . . . SS89-44 & Ruddy duck & Liver & 69.2 & $<2$ & $<3$ & .1 \\
\hline 51 & . . . . . SS89-47 & Ruddy duck & Liver & 72.4 & $<2$ & $<3$ & .53 \\
\hline 52 & . . . . SSS89-48 & Ruddy duck & Liver & 71.4 & $<2$ & $<3$ & .84 \\
\hline 53 & . . . . . SS89-49 & Ruddy duck & Liver & 71.0 & $<2$ & $<3$ & .3 \\
\hline 54 & . . . . L LNSS89-01 & Ruddy duck & Breast muscle & 70.4 & $<2$ & $<3$ & .1 \\
\hline 55 & . . . . . LNSS89-04 & Ruddy duck & Breast muscle & 70.9 & $<2$ & $<3$ & .3 \\
\hline 56 & . . . . LNSS89-06 & Ruddy duck & Breast muscle & 72.3 & $<2$ & $<3$ & .2 \\
\hline 57 & . . . . L LNSS89-08 & Ruddy duck & Breast muscle & 69.1 & $<2$ & $<3$ & .3 \\
\hline 58 & . . . . LNSS89-10 & Ruddy duck & Breast muscle & 70.4 & $<2$ & $<3$ & .2 \\
\hline 59 & . . . . L LNSS89-12 & Ruddy duck & Breast muscle & 71.8 & $<2$ & $<3$ & .40 \\
\hline 60 & . . . . LNSS89-14 & Ruddy duck & Breast muscle & 70.7 & $<2$ & $<3$ & .48 \\
\hline 61 & . . . . L LNSS89-16 & Ruddy duck & Breast muscle & 69.3 & $<2$ & $<3$ & .31 \\
\hline 62 & . . . . LNSS89-18 & Ruddy duck & Breast muscle & 70.1 & $<2$ & $<3$ & $<.2$ \\
\hline 63 & . . . . L LNSS89-22 & Ruddy duck & Breast muscle & 70.2 & $<2$ & $<3$ & .5 \\
\hline 64 & . . . . . LNSS89-22 & Ruddy duck & Breast muscle & 67.3 & $<2$ & $<3$ & $<.2$ \\
\hline 65 & . . . . SS89-112 & Black-necked stilt & Egg & 75.1 & $<2$ & $<3$ & $<.1$ \\
\hline 66 & . . . . SSS89-113 & Black-necked stilt & Egg & 74.3 & $<2$ & $<3$ & $<.1$ \\
\hline 67 & . . . . SS89-114 & Black-necked stilt & Egg & 71.3 & $<2$ & $<3$ & $<.1$ \\
\hline 68 & . . . . SS89-115 & Black-necked stilt & Egg & 73.4 & $<2$ & $<3$ & .1 \\
\hline 69 & . . . . SS89-116 & Black-necked stilt & Egg & 74.4 & $<2$ & $<3$ & $<.1$ \\
\hline 70 & $\ldots \ldots$ SS90a-d & Filamentous green algae & Vegetation & 89.1 & --- & 860 & $<.87$ \\
\hline 71 & $\ldots \ldots$ SS90el & Tubular green algae & Vegetation & 89.6 & -- & 1,500 & 6.6 \\
\hline \multicolumn{8}{|c|}{ Site B2 (Poe Road) } \\
\hline 72 & $\ldots . . . \mathrm{SS} 90 \mathrm{e} 2$ & Tubular green algae & Vegetation & 89.9 & -- & 1,100 & 4.7 \\
\hline \multicolumn{8}{|c|}{ Site B3 (U.S. Navy Test Base) } \\
\hline 73 & $\ldots$. . SS90a1 & Filamentous green algae & Vegetation & 86.7 & -- & 230 & 15 \\
\hline 74 & $\ldots . . . \mathrm{ss} 90 \mathrm{e} 3$ & Tubular green algae & Vegetation & 87.9 & --- & 530 & 5.0 \\
\hline \multicolumn{8}{|c|}{ Site B4 (Salton City) } \\
\hline 75 & $\ldots . . . S S 90 a 2$ & Filamentous green algae & Vegetation & 83.7 & -- & 230 & 4.4 \\
\hline 76 & .... SS9004 & Tubular green algae & Vegetation & 91.5 & -- & 1,500 & $<1.1$ \\
\hline \multicolumn{8}{|c|}{ Site B5 (Salton Sea Beach) } \\
\hline 77 & $\ldots . . . \mathrm{SS} 90 \mathrm{a} 3$ & Filamentous green algae & Vegetation & 88.1 & -- & 1,800 & 14 \\
\hline
\end{tabular}


Table 22. Inorganic chemical analysis and moisture content for biotic samples collected during 1988-90 from the Salton Sea and associated drainwaters and rivers--Continued

\begin{tabular}{|c|c|c|c|c|c|c|c|c|c|c|c|}
\hline $\begin{array}{l}\text { Record } \\
\text { number }\end{array}$ & $\begin{array}{l}\text { Sample } \\
\text { number }\end{array}$ & $\begin{array}{l}\text { Bo- } \\
\text { ron }\end{array}$ & $\begin{array}{l}\text { Ba- } \\
\text { rium }\end{array}$ & $\begin{array}{l}\text { Be- } \\
\text { ryl- } \\
\text { lium }\end{array}$ & $\begin{array}{l}\text { Cad- } \\
\text { mium }\end{array}$ & $\begin{array}{c}\text { Chro- } \\
\text { mi- } \\
\text { um }\end{array}$ & $\begin{array}{l}\text { Cop- } \\
\text { per }\end{array}$ & Iron & $\begin{array}{l}\text { Mer- } \\
\text { cury }\end{array}$ & $\begin{array}{c}\text { Mag- } \\
\text { ne- } \\
\text { sium }\end{array}$ & $\begin{array}{c}\text { Man- } \\
\text { ga- } \\
\text { nese }\end{array}$ \\
\hline \multicolumn{12}{|c|}{ Site B1 (Salton Sea NWR--Unit 1)--Continued } \\
\hline 40 & LNSS89-28 & $<3$ & $<0.1$ & $<0.1$ & $<0.8$ & $<1$ & 46.6 & 1,550 & 0.23 & 699 & 13 \\
\hline 41 & LNSS89-29 & $<3$ & $<.1$ & $<.1$ & $<.8$ & $<1$ & 114 & 2,570 & 5.5 & 781 & 15 \\
\hline 42 & LNSS89-30 & $<3$ & $<.1$ & $<.1$ & $<.8$ & $<1$ & 36.3 & 2,370 & .19 & 800 & 12 \\
\hline 43 & LNSS89-31 & $<3$ & $<.1$ & $<.1$ & $<.8$ & $<1$ & 41.2 & 956 & .18 & 727 & 11 \\
\hline 44 & LNSS89-32 & $<3$ & $<.1$ & $<.1$ & $<.8$ & $<1$ & 52.8 & 3,570 & .45 & 762 & 12 \\
\hline 45 & SS89-33 & 2 & .1 & $<.1$ & 2.3 & 2 & 167 & 6,330 & .606 & 769 & 11 \\
\hline 46 & SS89-34 & 3 & $<.1$ & $<.1$ & .5 & 1 & 29.1 & 3,470 & .14 & 734 & 12 \\
\hline 47 & SS89-41 & 3 & $<.1$ & $<.1$ & 2.1 & $<1$ & 45.7 & 2,560 & 1.4 & 689 & 14 \\
\hline 48 & SS89-42 & 4 & .2 & $<.1$ & 1.1 & 1 & 54.4 & 1,090 & .526 & 779 & 11 \\
\hline 49 & SS89-43 & 4 & $<.1$ & $<.1$ & .5 & 2 & 40.2 & 6,100 & .19 & 670 & 16 \\
\hline 50 & SS89-44 & 3 & $<.1$ & $<.1$ & .3 & $<1$ & 71.4 & 2,550 & .23 & 655 & 9.4 \\
\hline 51 & SS89-47 & 4 & $<.1$ & $<.1$ & .8 & $<1$ & 49.1 & 3,030 & .17 & 649 & 11 \\
\hline 52 & SS89-48 & 6.6 & $<.1$ & $<.1$ & .3 & 1 & 28.5 & 4,020 & .22 & 707 & 11 \\
\hline 53 & SS89-49 & 6.2 & $<.1$ & $<.1$ & 1.5 & $<1$ & 62.7 & 2,230 & .846 & 788 & 11 \\
\hline 54 & LNSS89-01 & $<3$ & $<.1$ & $<.1$ & $<.8$ & $<1$ & 52.6 & 464 & .039 & 1,090 & 2.0 \\
\hline 55 & LNSS89-04 & 4 & $<.1$ & $<.1$ & $<.7$ & $<1$ & 33.9 & 427 & .44 & 1,020 & 1.5 \\
\hline 56 & LNSS89-06 & $<3$ & $<.1$ & $<.1$ & $<.8$ & $<1$ & 32.8 & 447 & .522 & 1,160 & 2.5 \\
\hline 57 & LNSS89-08 & $<3$ & $<.1$ & $<.1$ & $<.8$ & $<1$ & 30.0 & 379 & .078 & 1,080 & 2.8 \\
\hline 58 & LNSS89-10 & 5 & $<.1$ & $<.1$ & $<.8$ & $<1$ & 33.4 & 398 & .19 & 1,050 & 2.5 \\
\hline 59 & LNSS89-12 & $<3$ & $<.1$ & $<.1$ & $<.8$ & $<1$ & 31.4 & 451 & .049 & 1,070 & 1.2 \\
\hline 60 & LNSS89-14 & $<3$ & $<.1$ & $<.1$ & $<.8$ & $<1$ & 59.1 & 476 & .049 & 1,070 & 1.6 \\
\hline 61 & LNSS89-16 & $<3$ & $<.1$ & $<.1$ & $<.8$ & $<1$ & 24.5 & 418 & .047 & 1,120 & 2.0 \\
\hline 62 & LNSS89-18 & $<3$ & $<.1$ & $<.1$ & $<.8$ & $<1$ & 56.7 & 557 & .027 & 1,040 & 2.6 \\
\hline 63 & LNSS89-20 & $<3$ & .1 & $<.1$ & $<.8$ & $<1$ & 28.7 & 384 & .036 & 1,040 & 1.1 \\
\hline 64 & LNSS89-22 & $<3$ & $<.1$ & $<.1$ & $<.8$ & $<1$ & 32.5 & 406 & .024 & 1,080 & 2.8 \\
\hline 65 & SS89-112 & $<3$ & .66 & $<.1$ & $<.5$ & $<2$ & 3.3 & 101 & .46 & 387 & 1.3 \\
\hline 66 & SS89-113 & $<3$ & .60 & $<.1$ & $<.5$ & $<2$ & 3.8 & 96 & .542 & 329 & 1.1 \\
\hline 67 & SS89-114 & $<3$ & 1.5 & $<.1$ & $<.5$ & $<2$ & 3.4 & 96 & .14 & 327 & 2.8 \\
\hline 68 & SS89-115 & 3 & .75 & $<.1$ & $<.5$ & $<2$ & 3.6 & 129 & 1.6 & 388 & 1.4 \\
\hline 69 & SS89-116 & $<3$ & 4.1 & $<.1$ & $<.5$ & $<2$ & 4.7 & 140 & .736 & 634 & 1.4 \\
\hline 70 & SS90a-d & 180 & 37 & $<4.4$ & $<4.4$ & 120 & 11 & 1,800 & $\ldots$ & 9,500 & 130 \\
\hline 71 & SS80e1 & 160 & 38 & $<4.1$ & $<4.1$ & $<120$ & $<9.1$ & 3,100 & -- & 12,000 & 83 \\
\hline \multicolumn{12}{|c|}{ Site B2 (Poe Road) } \\
\hline 72 & SS90e 2 & 170 & 36 & $<4.6$ & $<4.6$ & $<130$ & $<10$ & 2,900 & -- & 12,000 & 74 \\
\hline \multicolumn{12}{|c|}{ Site B3 (U.S. Navy Test Base) } \\
\hline 73 & SS90a1 & 220 & 6.3 & $<3.5$ & $<3.5$ & $<98$ & $<7.7$ & 960 & --- & 6,100 & $<35$ \\
\hline 74 & SS90e3 & 150 & 18 & $<3.3$ & $<3.3$ & $<92$ & $<7.2$ & 1,400 & -- & 11,000 & 42 \\
\hline \multicolumn{12}{|c|}{ Site B4 (Salton City) } \\
\hline 75 & SS90a 2 & 170 & 6.4 & $<2.6$ & $<2.6$ & $<72$ & $<5.7$ & 510 & -- & 5,600 & 95 \\
\hline 76 & SS90e4 & 130 & 17 & $<5.6$ & $<5.6$ & $<160$ & $<12$ & 1,800 & $\cdots$ & 25,000 & 74 \\
\hline \multicolumn{12}{|c|}{ Site B5 (Salton Sea Beach) } \\
\hline 77 & SS90a3 & 290 & 46 & $<3.7$ & $<3.7$ & $<100$ & $<8.1$ & 3,700 & -- & 2,900 & 180 \\
\hline
\end{tabular}


Table 22. Inorganic chemical analysis and moisture content for biotic samples collected during 1988-90 from the Salton Sea and associated drainwaters and rivers--Continued

\begin{tabular}{|c|c|c|c|c|c|c|c|c|c|c|c|}
\hline $\begin{array}{l}\text { Record } \\
\text { number }\end{array}$ & $\begin{array}{l}\text { Sample } \\
\text { number }\end{array}$ & $\begin{array}{c}\text { Molyb- } \\
\text { de- } \\
\text { num }\end{array}$ & $\begin{array}{c}\text { Nick- } \\
\text { el }\end{array}$ & Lead & $\begin{array}{l}\text { Anti- } \\
\text { mo- } \\
\text { ny }\end{array}$ & $\begin{array}{c}\text { Sele- } \\
\text { ni- } \\
\text { um }\end{array}$ & Tin & $\begin{array}{l}\text { Stron- } \\
\text { ti- } \\
\text { um }\end{array}$ & $\begin{array}{c}\text { Thal- } \\
\text { li- } \\
\text { um }\end{array}$ & $\begin{array}{c}\text { Va- } \\
\text { nadi- } \\
\text { um }\end{array}$ & Zinc \\
\hline \multicolumn{12}{|c|}{ Site B1 (Salton Sea NWR--Unit 1)--Continued } \\
\hline 40 & LNSS89-28 & 3 & $<3$ & $<4$ & -- & 13 & - & 0.62 & $<4$ & $<0.7$ & 120 \\
\hline 41 & LNSS89-29 & 4.1 & $<3$ & $<4$ & -- & 25.6 & - & .3 & $<4$ & $<.7$ & 114 \\
\hline 42 & LNSS89-30 & 3.7 & $<3$ & $<4$ & -- & 11 & - & .3 & $<4$ & $<.7$ & 107 \\
\hline 43 & LNSS89-31 & 2 & $<3$ & $<4$ & - & 13 & -- & .3 & $<4$ & $<.7$ & 111 \\
\hline 44 & LNSS89-32 & 3 & $<3$ & $<4$ & - & 11 & -- & .60 & $<4$ & $<.7$ & 102 \\
\hline 45 & SS89-33 & 4 & $<1$ & $<4$ & -- & 5.2 & - & 1.2 & $<5$ & .91 & 171 \\
\hline 46 & SS89-34 & 3 & $<1$ & $<4$ & $\ldots$ & 16 & -- & .51 & $<5$ & .3 & 113 \\
\hline 47 & SS89-41 & 3 & $<1$ & $<4$ & -- & 7.4 &.-- & 1 & $<5$ & .6 & 135 \\
\hline 48 & SS89-42 & 2 & $<1$ & $<4$ & -- & 14 & -- & 3.1 & $<5$ & .3 & 134 \\
\hline 49 & SS89-43 & 4.8 & $<1$ & $<4$ & -- & 13 & -- & .51 & $<5$ & .4 & 119 \\
\hline 50 & SS89-44 & 2 & $<1$ & $<4$ & -- & 16 & -- & .65 & $<5$ & $<.3$ & 140 \\
\hline 51 & SS89-47 & 2 & $<1$ & $<4$ & -- & 8.8 &.-- & .33 & $<5$ & $<.3$ & 110 \\
\hline 52 & SS89-48 & 2 & $<1$ & $<4$ & -- & 13 & - & 1.6 & $<5$ & $<.3$ & 106 \\
\hline 53 & SS89-49 & 2 & $<1$ & $<4$ & -- & 9.8 & -- & 2.0 & $<5$ & .7 & 128 \\
\hline 54 & LNSS89-01 & $<1$ & $<3$ & $<4$ & -- & 4.5 & - & .44 & $<4$ & $<.7$ & 36 \\
\hline 55 & LNSS89-04 & $<1$ & $<3$ & $<4$ & -- & 4.5 & - & .31 & $<4$ & $<.6$ & 33 \\
\hline 56 & LNSS89-06 & $<1$ & $<3$ & $<4$ & - & 5.8 & -- & .67 & $<4$ & $<.7$ & 39 \\
\hline 57 & LNSS89-08 & $<1$ & $<3$ & $<4$ & -- & 4.8 & - & .54 & $<4$ & $<.7$ & 33 \\
\hline 58 & LNSS89-10 & $<1$ & $<3$ & $<4$ & -- & 7.2 & - & .79 & $<4$ & $<.6$ & 30 \\
\hline 59 & LNSS89-12 & $<1$ & $<3$ & $<4$ & -- & 6.5 & -- & .55 & $<4$ & $<.7$ & 30 \\
\hline 60 & LNSS89-14 & $<1$ & $<3$ & $<4$ & -- & 6.0 & -- & .71 & $<4$ & $<.7$ & 44 \\
\hline 61 & LNSS89-16 & $<1$ & $<3$ & $<4$ & -- & 5.6 & -- & .47 & $<4$ & $<.7$ & 36 \\
\hline 62 & LNSS89-18 & $<1$ & $<3$ & $<4$ & -- & 6.7 & -- & .67 & $<4$ & $<.7$ & 41 \\
\hline 63 & LNSS89-20 & $<1$ & $<3$ & $<4$ & -- & 6.6 & -- & 1.0 & $<4$ & $<.7$ & 30 \\
\hline 64 & LNSS89-22 & $<1$ & $<3$ & $<4$ & -- & 4.3 & -- & .63 & $<4$ & $<.7$ & 35 \\
\hline 65 & SS89-112 & $<1$ & $<3$ & $<4$ & --- & 4.3 & - & 17.5 & $<4$ & $<.7$ & 40 \\
\hline 66 & SS89-113 & $<1$ & $<3$ & $<4$ & -- & 3.6 & -- & 22.5 & $<4$ & $<.6$ & 41 \\
\hline 67 & SS89-114 & $<1$ & $<3$ & $<4$ & --- & 4.9 & -- & 21.8 & $<4$ & $<.6$ & 53.4 \\
\hline 68 & SS89-115 & $<1$ & $<3$ & $<4$ & -- & 5.0 & -- & 23.7 & $<4$ & $<.6$ & 50.0 \\
\hline 69 & SS89-116 & $<1$ & 7 & $<4$ & --- & 6.8 & -- & 49.3 & $<4$ & $<.7$ & 55.2 \\
\hline 70 & SS90a-d & $<34$ & 13 & $<35$ & --- & 1.3 & $<44$ & 1,300 & -- & $<11$ & $<240$ \\
\hline 71 & SS80e1 & $<32$ & 6.9 & $<33$ & -- & $<.87$ & $<41$ & 610 & $\cdots$ & $<10$ & $<220$ \\
\hline \multicolumn{12}{|c|}{ Site B2 (Poe Road) } \\
\hline 72 & SS90e 2 & $<36$ & $<7.4$ & $<37$ & - & 1.3 & $<46$ & 320 & -- & $<12$ & $<250$ \\
\hline \multicolumn{12}{|c|}{ Site B3 (U.S. Navy Test Base) } \\
\hline 73 & SS90a1 & $<27$ & 5.8 & $<28$ & -- & 1.0 & $<35$ & 180 & $\cdots$ & $<8.8$ & $<190$ \\
\hline 74 & SS90e3 & $<25$ & 7.4 & $<26$ & -- & $<.75$ & $<33$ & 470 & -- & $<8.2$ & $<180$ \\
\hline \multicolumn{12}{|c|}{ Site B4 (Salton City) } \\
\hline 75 & SS90a2 & $<20$ & 6.5 & $<20$ & --- & 1.1 & $<26$ & 220 & -- & $<6.4$ & $<140$ \\
\hline 76 & SS90e4 & $<43$ & $<9.0$ & $<44$ & --- & $<1.1$ & $<56$ & 290 & -- & $<14$ & $<300$ \\
\hline \multicolumn{12}{|c|}{ Site B5 (Salton Sea Beach) } \\
\hline 77 & SS90a3 & $<28$ & 25 & $<29$ & -- & 1.7 & $<37$ & 1,100 & -- & $<9.2$ & $<200$ \\
\hline
\end{tabular}


Table 22. Inorganic chemical analysis and moisture content for biotic samples collected during 1988-90 from the Salton Sea and associated drainwaters and rivers--Continued

\begin{tabular}{|c|c|c|c|c|c|c|c|}
\hline $\begin{array}{l}\text { Recorc } \\
\text { numbe }\end{array}$ & $\begin{array}{l}\text { Sample } \\
\text { number }\end{array}$ & Species & Matrix & $\begin{array}{l}\text { Percent } \\
\text { moisture }\end{array}$ & $\begin{array}{l}\text { Sil- } \\
\text { ver }\end{array}$ & $\begin{array}{c}\text { Alu- } \\
\text { mi- } \\
\text { num }\end{array}$ & $\begin{array}{l}\text { Arse- } \\
\text { nic }\end{array}$ \\
\hline \multicolumn{8}{|c|}{ Site B5 (Salton Sea Beach)--Continued } \\
\hline 78 & $\ldots . . . \mathrm{sS} 90 \mathrm{es}$ & Tubular green algae & Vegetation & 86.5 & --- & 3,500 & $<0.59$ \\
\hline \multicolumn{8}{|c|}{ Site B6 (Desert Shores) } \\
\hline 79 & . . . . SS90a4 & Filamentous green algae & Vegetation & 87.6 & --- & 220 & $<0.76$ \\
\hline 80 & . . . . SS90e6 & Tubular green algae & Vegetation & 90.8 & --- & 220 & 8.0 \\
\hline \multicolumn{8}{|c|}{ Site B7 (Desert Beach) } \\
\hline 81 & $\ldots .$. SS90e10 & Tubular green algae & Vegetation & 91.2 & --- & 97 & $<1.1$ \\
\hline 82 & $\ldots .$. SS90f10 & Filamentous green algae & Vegetation & 87.3 & $\cdots$ & 140 & 5.6 \\
\hline \multicolumn{8}{|c|}{ Site B8 (Bob's Playa River Marina) } \\
\hline 83 & . . . . SS90e11 & Tubular green algae & Vegetation & 84.7 & -- & 970 & 3.6 \\
\hline 84 & $\ldots .$. SS90f11 & Filamentous green algae & Vegetation & 86.5 & -- & 140 & 16 \\
\hline \multicolumn{8}{|c|}{ Site B9 (Bombay Beach) } \\
\hline 85 & .....sS90e12 & Tubular green algae & Vegetation & 86.6 & -- & 1,100 & $<0.68$ \\
\hline 86 & $\ldots .$. SS90f12 & Filamentous green algae & Vegetation & 84.0 & --- & 210 & 2.5 \\
\hline \multicolumn{8}{|c|}{ Site B10 (S Drain Outlet) } \\
\hline 87 & . . . . SS89-170 & Bairdiella & Whole body & 71.1 & $<2$ & 200 & 12 \\
\hline 88 & . . . . SS89-171 & Bairdiella & Whole body & 73.9 & $<2$ & 140 & 10 \\
\hline 89 & $\ldots \ldots$ SS89-172 & Bairdiella & Whole body & 76.1 & $<2$ & 27 & 8.4 \\
\hline 90 & $\ldots \ldots$ SS89-173 & Bairdiella & Whole body & 73.4 & $<2$ & 76 & 4.5 \\
\hline 91 & $\ldots \ldots$ SS89-174 & Bairdiella & Whole body & 75.7 & $<2$ & 100 & 4.5 \\
\hline \multicolumn{8}{|c|}{ Site B11 (Alamo River Delta) } \\
\hline 92 & . . . . LNSS88-72 & Waterboatman & Composite & 75.7 & $<2.06$ & 1,330 & 0.295 \\
\hline 93 & . . . . . LNSS88-93 & Pileworm & Composite & 93.4 & $<7.58$ & 333 & 22.0 \\
\hline 94 & . . . . . LNSS88-61 & Crayfish & Whole body & 79.1 & $<2.40$ & 360 & .458 \\
\hline 95 & . . . . . LNSS88-28 & Northern shoveler & Liver & 69.6 & $<2$ & 4 & $<.2$ \\
\hline 96 & . . . . L LNSS88-30 & Northern shoveler & Liver & 70.9 & $<2$ & 9.8 & $<.2$ \\
\hline 97 & . . . . L LNSS88-27 & Northern shoveler & Breast muscle & 72.6 & $<2$ & 6 & $<.2$ \\
\hline 98 & $\ldots \ldots$. . SS89-53 & Northern shoveler & Liver & 70.3 & $<2$ & 7 & .2 \\
\hline 99 & . . . . SS899-128 & Black-necked stilt & Carcass & 66.4 & $<2$ & 140 & .3 \\
\hline 100 & . . . . . SS89-129 & Black-necked stilt & Carcass & 66.2 & $<2$ & 373 & .3 \\
\hline 101 & . . . . SS89-130 & Black-necked stilt & Carcass & 63.7 & $<2$ & 280 & .30 \\
\hline 102 & . . . . SS889-131 & Black-necked stilt & Carcass & 67.7 & $<2$ & 170 & .40 \\
\hline 103 & . . . . SS89-132 & Black-necked stilt & Carcass & 63.8 & $<2$ & 190 & .42 \\
\hline \multicolumn{8}{|c|}{ Site B12 (Red Hill Marina) } \\
\hline 104 & $\ldots . . . s 990 \mathrm{e} 13$ & Tubular green algae & Vegetation & 89.1 & $-\cdots$ & 1,400 & $<0.77$ \\
\hline 105 & $\ldots . . . \mathrm{sS90f13}$ & Filamentous green algae & Vegetation & 81.7 & --- & 330 & $<.46$ \\
\hline \multicolumn{8}{|c|}{ Site B13 (Obsidian Butte) } \\
\hline 106 & . . . . SS899-143 & Eared grebe & Liver & 67.0 & $<2$ & $<3$ & $<0.1$ \\
\hline
\end{tabular}


Table 22. Inorganic chemical analysis and moisture content for biotic samples collected during 1988-90 from the Salton Sea and associated drainwaters and rivers--Continued

\begin{tabular}{|c|c|c|c|c|c|c|c|c|c|c|c|}
\hline $\begin{array}{l}\text { Record } \\
\text { number }\end{array}$ & $\begin{array}{l}\text { Sample } \\
\text { number }\end{array}$ & $\begin{array}{l}\text { Bo- } \\
\text { ron }\end{array}$ & $\begin{array}{l}\mathbf{B a -} \\
\text { rium }\end{array}$ & $\begin{array}{l}\text { Be- } \\
\text { ryl- } \\
\text { lium }\end{array}$ & $\begin{array}{l}\text { Cad- } \\
\text { mium }\end{array}$ & $\begin{array}{c}\text { Chro- } \\
\text { mi- } \\
\text { um }\end{array}$ & $\begin{array}{l}\text { Cop- } \\
\text { per }\end{array}$ & Iron & $\begin{array}{l}\text { Mer- } \\
\text { cury }\end{array}$ & $\begin{array}{c}\text { Mag- } \\
\text { ne- } \\
\text { sium }\end{array}$ & $\begin{array}{c}\text { Man- } \\
\text { ga- } \\
\text { nese }\end{array}$ \\
\hline \multicolumn{12}{|c|}{ Site B5 (Salton Sea Beach)--Continued } \\
\hline 78 & SS90e5 & 160 & 58 & $<3.3$ & 28 & $<93$ & 8.7 & 4,100 & - & 14,000 & 85 \\
\hline \multicolumn{12}{|c|}{ Site B6 (Desert Shores) } \\
\hline 79 & SS90a4 & 390 & 9.4 & $<3.7$ & 5.3 & $<100$ & $<8.2$ & 600 & -- & 8,800 & 140 \\
\hline 80 & SS90e6 & 220 & 6.2 & $<5.4$ & $<5.4$ & 200 & 12 & 380 & -- & 18,000 & 120 \\
\hline \multicolumn{12}{|c|}{ Site B7 (Desert Beach) } \\
\hline 81 & SS90e10 & 230 & 7.6 & $<4.2$ & $<4.2$ & $<120$ & 14 & 610 & -- & 23,000 & $<42$ \\
\hline 82 & SS90f10 & 170 & 4.5 & $<3.2$ & $<3.2$ & $<91$ & $<7.1$ & 240 & -- & 5,600 & 52 \\
\hline \multicolumn{12}{|c|}{ Site B8 (Bob's Playa River Marina) } \\
\hline 83 & SS90e11 & $<55$ & 31 & $<2.5$ & $<2.5$ & $<09$ & $<5.5$ & 2,300 & -- & 8,400 & 79 \\
\hline 84 & SS90f11 & 300 & $<3.6$ & $<3.6$ & $<3.6$ & $<100$ & $<7.9$ & 410 & -- & 5,900 & $<36$ \\
\hline \multicolumn{12}{|c|}{ Site B9 (Bombay Beach) } \\
\hline 85 & SS90e12 & 160 & 38 & $<3.7$ & $<3.7$ & $<100$ & $<8.2$ & 2,800 & -- & 11,000 & 61 \\
\hline 86 & SS90f12 & 380 & 6.6 & $<2.8$ & $<2.8$ & $<78$ & $<6.1$ & 1,400 & -- & 5,700 & $<28$ \\
\hline \multicolumn{12}{|c|}{ Site B10 (S Drain Outlet) } \\
\hline 87 & SS89-170 & 5 & 4.5 & $<0.1$ & $<0.2$ & $<1$ & 1.8 & 316 & 0.057 & 1,080 & 7.0 \\
\hline 88 & SS89-171 & 6 & 5.3 & $<.1$ & $<.3$ & 7.0 & 2.2 & 297 & .045 & 1,270 & 6.6 \\
\hline 89 & SS89-172 & 6.3 & .85 & $<.1$ & $<.3$ & 1 & 1.7 & 189 & .049 & 1,260 & 1.7 \\
\hline 90 & SS89-173 & 6.0 & 1.7 & $<.1$ & $<.2$ & $<1$ & 1.5 & 143 & .048 & 1,190 & 2.5 \\
\hline 91 & SS89-174 & 8.3 & 1.8 & $<.1$ & $<.3$ & 3 & 2.1 & 188 & .045 & 1,470 & 2.7 \\
\hline \multicolumn{12}{|c|}{ Site B11 (Alamo River Delta) } \\
\hline 92 & LNSS88-72 & $<20.6$ & 29.1 & $<0.206$ & $<0.206$ & 2.47 & 11.9 & 1,450 & $<0.103$ & 3,230 & 45.7 \\
\hline 93 & LNSS88-93 & $<75.8$ & $<7.58$ & $<.758$ & $<.758$ & 1.67 & 18.0 & 874 & $<.379$ & 2,800 & 28.5 \\
\hline 94 & LNSS88-61 & 23.9 & 51.7 & $<.240$ & $<.240$ & $<.478$ & 75.6 & 383 & $<.120$ & 4,000 & 190 \\
\hline 95 & LNSS88-28 & 4 & $<.1$ & $<.1$ & .78 & 1 & 17 & 5,030 & 26.7 & 789 & 12 \\
\hline 96 & LNSS88-30 & 4 & $<.1$ & $<.1$ & 2.1 & $<1$ & 13 & 7,810 & .48 & 860 & 10 \\
\hline 97 & LNSS88-27 & 2 & $<.1$ & $<.1$ & $<.2$ & $<1$ & 16 & 217 & 2.3 & 1,170 & 1.2 \\
\hline 98 & SS89-53 & 3 & $<.1$ & $<.1$ & 5.5 & 1 & 20 & 5,160 & .846 & 664 & 11 \\
\hline 99 & SS89-128 & 5 & 4.7 & $<.1$ & $<.3$ & 8.6 & 6.5 & 273 & .17 & 1,300 & 6.3 \\
\hline 100 & SS89-129 & 6.6 & 6.4 & $<.1$ & $<.2$ & 9.8 & 6.6 & 420 & .28 & 1,440 & 10 \\
\hline 101 & SS89-130 & 3 & 7.6 & $<.1$ & $<.4$ & 5.3 & 11 & 285 & .062 & 1,650 & 8.1 \\
\hline 102 & SS89-131 & 6.4 & 4.2 & $<.1$ & $<.2$ & 3 & 7.2 & 291 & .26 & 1,440 & 6.3 \\
\hline 103 & SS89-132 & 6.3 & 3.4 & $<.1$ & $<.3$ & 3 & 6.8 & 269 & .25 & 1,510 & 6.3 \\
\hline \multicolumn{12}{|c|}{ Site B12 (Red Hill Marina) } \\
\hline 104 & SS90e13 & 130 & 36 & $<4.5$ & $<4.5$ & $<130$ & $<10$ & 3,100 & --- & 9,100 & 120 \\
\hline 105 & SS90f13 & 70 & 6.3 & $<2.4$ & $<2.4$ & $<66$ & $<5.2$ & 540 & -- & 3,800 & 27 \\
\hline \multicolumn{12}{|c|}{ Site B13 (Obsidian Butte) } \\
\hline 106 & SS89-143 & 2 & $<0.1$ & $<0.1$ & 3.8 & 1 & 14 & 4,420 & 13 & 616 & 13 \\
\hline
\end{tabular}


Table 22. Inorganic chemical analysis and moisture content for biotic samples collected during 1988-90 from the Salton Sea and associated drainwaters and rivers--Continued

\begin{tabular}{|c|c|c|c|c|c|c|c|c|c|c|c|}
\hline $\begin{array}{l}\text { Record } \\
\text { number }\end{array}$ & $\begin{array}{l}\text { Sample } \\
\text { number }\end{array}$ & $\begin{array}{c}\text { Molyb- } \\
\text { de- } \\
\text { num }\end{array}$ & $\begin{array}{c}\text { Nick- } \\
\text { el }\end{array}$ & Lead & $\begin{array}{l}\text { Anti- } \\
\text { mo- } \\
\text { ny }\end{array}$ & $\begin{array}{l}\text { Sele- } \\
\text { ni- } \\
\text { um }\end{array}$ & Tin & $\begin{array}{c}\text { Stron- } \\
\text { ti- } \\
\text { um }\end{array}$ & $\begin{array}{c}\text { Thal- } \\
\text { li- } \\
\text { um }\end{array}$ & $\begin{array}{c}\text { Va- } \\
\text { nadi- } \\
\text { um }\end{array}$ & Zinc \\
\hline \multicolumn{12}{|c|}{ Site B5 (Salton Sea Beach)--Continued } \\
\hline 78 & SS90es & $<26$ & 7.9 & $<26$ & -- & 0.79 & 57 & 1,400 & -- & 14 & 450 \\
\hline \multicolumn{12}{|c|}{ Site B6 (Desert Shores) } \\
\hline 79 & SS90a4 & $<29$ & $<6.0$ & $<30$ & $\ldots$ & 0.80 & $<37$ & 490 & $\cdots$ & $<9.3$ & $<200$ \\
\hline 80 & SS90e6 & $<41$ & $<8.6$ & $<42$ & - & 1.3 & $<54$ & 260 & - & $<13$ & 660 \\
\hline \multicolumn{12}{|c|}{ Site B7 (Desert Beach) } \\
\hline 81 & SS90e10 & $<32$ & 17 & $<33$ & $\ldots$ & $<1.1$ & $<42$ & 500 & - & 15 & $<230$ \\
\hline 82 & SS90f10 & $<25$ & 5.3 & $<26$ & -- & 1.7 & $<32$ & 170 & -- & $<8.1$ & $<170$ \\
\hline \multicolumn{12}{|c|}{ Site B8 (Bob's Playa River Marina) } \\
\hline 83 & SS90e11 & $<19$ & 7.3 & $<20$ & -- & 0.56 & $<25$ & 620 & -- & $<6.2$ & $<130$ \\
\hline 84 & SS90f11 & $<28$ & 9.6 & 34 & -- & .62 & $<36$ & 130 & -- & $<9.0$ & $<190$ \\
\hline \multicolumn{12}{|c|}{ Site B9 (Bombay Beach) } \\
\hline 85 & SS90e 12 & $<29$ & $<6.0$ & $<29$ & - & 0.78 & $<37$ & 520 & -- & $<9.3$ & $<200$ \\
\hline 86 & SS90f12 & $<21$ & $<4.5$ & $<22$ & -- & 1.1 & $<28$ & 360 & -- & $<7.0$ & $<150$ \\
\hline \multicolumn{12}{|c|}{ Site B10 (S Drain Outlet) } \\
\hline 87 & SS89-170 & $<1$ & $<2$ & $<4$ & -- & 12 & -- & 104 & $<4$ & 0.4 & 44.2 \\
\hline 88 & SS89-171 & $<1$ & 3 & $<5$ & $\ldots$ & 12 & -- & 148 & $<4$ & .4 & 51.7 \\
\hline 89 & SS89-172 & $<1$ & $<2$ & $<4$ & -- & 16 & -- & 44.3 & $<4$ & .4 & 29.3 \\
\hline 90 & SS89-173 & $<1$ & $<2$ & $<4$ & --- & 12 & -- & 74.3 & $<4$ & $<.3$ & 50.8 \\
\hline 91 & SS89-174 & $<1$ & $<2$ & $<4$ & --- & 13 & --- & 51.4 & $<4$ & $<.3$ & 56.9 \\
\hline
\end{tabular}

\begin{tabular}{|c|c|c|c|c|c|c|c|c|c|c|c|}
\hline \multicolumn{12}{|c|}{ Site B11 (Alamo River Delta) } \\
\hline 92 & LNSS88-72 & 2.55 & 2.92 & 6.21 & $<0.103$ & 3.3 & 9.01 & 145 & $<0.42$ & 4.48 & 112 \\
\hline 93 & LNSS88-93 & $<7.58$ & $<6.06$ & $<15.2$ & $<.379$ & 12.1 & $<7.58$ & 47.4 & $<1.6$ & $<7.58$ & 164 \\
\hline 94 & LNSS88-61 & $<2.40$ & $<1.91$ & $<4.79$ & $<.120$ & 3.3 & 5.22 & 866 & $<.48$ & $<2.40$ & 84.0 \\
\hline 95 & LNSS88-28 & 4 & $<1$ & $<4$ & --- & 17.1 & -- & .49 & $<5$ & .6 & 107 \\
\hline 96 & LNSS88-30 & 4 & $<1$ & $<4$ & $\cdots$ & 12.4 & - & .60 & $<5$ & 1 & 141 \\
\hline 97 & LNSS88-27 & $<1$ & $<1$ & $<4$ & --- & 6.0 & - & .37 & $<5$ & $<.3$ & 39.2 \\
\hline 98 & SS89-53 & 2 & $<1$ & $<4$ & -- & 24.3 & -- & .61 & $<5$ & .3 & 108 \\
\hline 99 & SS89-128 & $<1$ & 4 & $<4$ & --. & 8.6 & -.. & 145 & $<4$ & .98 & 87.9 \\
\hline 100 & SS89-129 & $<1$ & 4 & $<4$ & -- & 4.9 & $\sim$ & 226 & $<4$ & .5 & 89.3 \\
\hline 101 & SS89-130 & 1 & 4 & $<6$ & - & 4.7 & -- & 248 & $<4$ & .7 & 101 \\
\hline 102 & SS89-131 & $<1$ & $<2$ & $<4$ & --- & 5.7 & $\sim$ & 161 & $<4$ & .4 & 102 \\
\hline 103 & SS89-132 & $<1$ & $<2$ & $<5$ & -- & 5.3 & -- & 228 & $<4$ & $<.3$ & 105 \\
\hline \multicolumn{12}{|c|}{ Site B12 (Red Hill Marina) } \\
\hline 104 & SS90e13 & $<35$ & $<7.2$ & $<36$ & --- & 1.4 & $<45$ & 470 & -- & $<11$ & $<240$ \\
\hline 105 & SS90f13 & $<18$ & $<3.8$ & $<19$ & --- & .81 & $<24$ & 110 & -- & $<5.9$ & $<130$ \\
\hline \multicolumn{12}{|c|}{ Site B13 (Obsidian Butte) } \\
\hline 106 & SS89-143 & $<1$ & $<1$ & $<4$ & $\cdots$ & 33.1 & -- & 0.77 & $<6$ & $<.3$ & 116 \\
\hline
\end{tabular}


Table 22. Inorganic chemical analysis and moisture content for biotic samples collected during 1988-90 from the Salton Sea and associated drainwaters and rivers--Continued

\begin{tabular}{|c|c|c|c|c|c|c|c|}
\hline $\begin{array}{l}\text { Record } \\
\text { number }\end{array}$ & $\begin{array}{l}\text { Sample } \\
\text { number }\end{array}$ & Species & Matrix & $\begin{array}{l}\text { Percent } \\
\text { moisture }\end{array}$ & $\begin{array}{l}\text { Sil- } \\
\text { ver }\end{array}$ & $\begin{array}{l}\text { Alu- } \\
\text { mi- } \\
\text { num }\end{array}$ & $\begin{array}{l}\text { Arse- } \\
\text { nic }\end{array}$ \\
\hline \multicolumn{8}{|c|}{ Site B13 (Obsidian Butte)--Continued } \\
\hline $107 \ldots \ldots$ & SS89-145 & Eared grebe & Liver & 69.5 & $<2$ & $<3$ & 0.1 \\
\hline $108 \ldots \ldots$ & SS89-147 & Eared grebe & Liver & 77.5 & $<2$ & $<4$ & $<.1$ \\
\hline $109 \ldots \ldots$ & SS90e14 & Tubular green algae & Vegetation & 87.6 & --- & 530 & $<.71$ \\
\hline $110 \ldots \ldots$ & SS90f14 & Filamentous green algae & Vegetation & 86.9 & -- & 82 & 4.4 \\
\hline \multicolumn{8}{|c|}{ Site B14 (Bowles Road) } \\
\hline $111 \ldots$ & SS90e15 & Tubular green algae & Vegetation & 90.1 & -- & 490 & $<1.0$ \\
\hline $112 \ldots \ldots$ & SS90f15 & Filamentous green algae & Vegetation & 86.2 & - & 360 & 77 \\
\hline \multicolumn{8}{|c|}{ Site B15 (New River Delta) } \\
\hline $113 \ldots \ldots$ & LNSS88-91 & Waterboatman & Composite & 74.9 & $<2.00$ & 51.4 & 0.709 \\
\hline $114 \ldots \ldots$ & LNSS88-85 & Asiatic river clam & Soft tissue & 82.9 & $<2.93$ & 130 & 1.80 \\
\hline$\ldots$ & LNSS88-63 & Crayfish & Whole body & 76.2 & 3.70 & 550 & .685 \\
\hline$\ldots$ & LNSS88-64 & Longjaw mudsucker & Whole body & 80.2 & $<2.53$ & 224 & .312 \\
\hline $117 \ldots \ldots$ & LNSS88-66 & Mosquitofish & Whole body & 77.4 & $<2.22$ & 145 & .165 \\
\hline $118 \ldots \ldots$ & SS89-76 & Waterboatman & Composite & 81.3 & $<2$ & 3,220 & 2.0 \\
\hline $119 \ldots \ldots$ & SS89-149 & Eared grebe & Liver & 70.4 & $<2$ & 4 & .1 \\
\hline $120 \ldots \ldots$ & SS89-151 & Eared grebe & Liver & 74.2 & $<2$ & 4 & $<.1$ \\
\hline $121 \ldots \ldots$ & SS89-93 & Black-necked stilt & $\mathrm{Egg}$ & 76.5 & $<2$ & $<3$ & $<.1$ \\
\hline $122 \ldots \ldots$ & SS89-94 & Black-necked stilt & Egg & 73.3 & $<2$ & $<3$ & $<.1$ \\
\hline $123 \ldots \ldots$ & SS89-95 & Black-necked stilt & $\mathrm{Egg}$ & 74.0 & $<2$ & $<3$ & $<.1$ \\
\hline $124 \ldots \ldots$ & SS89-96 & Black-necked stilt & Egg & 73.8 & $<2$ & $<3$ & $<.1$ \\
\hline $125 \ldots \ldots$ & SS89-97 & Black-necked stilt & Egg & 71.3 & $<2$ & $<3$ & $<.1$ \\
\hline $126 \ldots \ldots$ & SS89-98 & Black-necked stilt & $\mathrm{Egg}$ & 79.1 & $<2$ & $<3$ & $<.1$ \\
\hline$\ldots$ & SS89-99 & Black-necked stilt & Egg & 74.9 & $<2$ & $<3$ & .1 \\
\hline $128 \ldots \ldots$ & SS89-100 & Black-necked stilt & Egg & 75.3 & $<2$ & $<3$ & $<.1$ \\
\hline $129 \ldots \ldots$ & SS89-101 & Black-necked stilt & Egg & 73.8 & $<2$ & $<3$ & $<.1$ \\
\hline $130 \ldots \ldots$ & SS89-102 & Black-necked stilt & Egg & 72.7 & $<2$ & $<3$ & $<.1$ \\
\hline $131 \ldots \ldots$ & SS89-133 & Black-necked stilt & Carcass & 68.4 & $<2$ & 240 & .2 \\
\hline $132 \ldots \ldots$ & SS89-134 & Black-necked stilt & Carcass & 66.2 & $<2$ & 210 & .2 \\
\hline $133 \ldots \ldots$ & SS89-135 & Black-necked stilt & Carcass & 66.6 & $<2$ & 150 & .2 \\
\hline $134 \ldots \ldots$ & SS89-136 & Black-necked stilt & Carcass & 65.5 & $<2$ & 302 & .1 \\
\hline $135 \ldots \ldots$ & SS89-137 & Black-necked stilt & Carcass & 64.2 & $<2$ & 230 & $<.1$ \\
\hline \multicolumn{8}{|c|}{ Site B17 (New River at Rio Bend) } \\
\hline $136 \ldots \ldots$ & LNSS88-68 & Sailfin molly & Whole body & 72.6 & $<1.83$ & 135 & 0.265 \\
\hline $137 \ldots \ldots$ & LNSS88-96 & Black-necked stilt & Egg & 69.4 & --- & 1.5 & $<.2$ \\
\hline $138 \ldots \ldots$ & LNSS88-138 & Black-necked stilt & Egg & 70.0 & --- & 1.5 & $<.2$ \\
\hline $139 \ldots \ldots$ & LNSS88-139 & Black-necked stilt & Egg & 74.5 & $<2$ & 5 & $<.2$ \\
\hline $140 \ldots \ldots$ & LNSS88-141 & Black-necked stilt & Egg & 74.0 & $-\ldots$ & 1.7 & $<.2$ \\
\hline $141 \ldots \ldots$ & LNSS88-142 & Black-necked stilt & Egg & 72.3 & $<2$ & 5 & $<.2$ \\
\hline $142 \ldots \ldots$ & LNSS88-144 & Black-necked stilt & Egg & 72.4 & $-\cdots$ & .91 & $<.2$ \\
\hline $143 \ldots \ldots$ & LNSS88-145 & Black-necked stilt & Egg & 68.8 & $<2$ & 5 & $<.2$ \\
\hline $144 \ldots \ldots$ & LNSS88-147 & Black-necked stilt & Egg & 73.4 & -.. & 1.4 & $<.2$ \\
\hline $145 \ldots \ldots$ & LNSS88-148 & Black-necked stilt & Egg & 73.4 & $<2$ & 4 & $<.2$ \\
\hline $146 \ldots \ldots$ & LNSS88-150 & Black-necked stilt & Egg & 75.2 & --- & 1.1 & $<.2$ \\
\hline
\end{tabular}


Table 22. Inorganic chemical analysis and moisture content for biotic samples collected during 1988-90 from the Salton Sea and associated drainwaters and rivers--Continued

\begin{tabular}{|c|c|c|c|c|c|c|c|c|c|c|c|}
\hline $\begin{array}{l}\text { Record } \\
\text { number }\end{array}$ & $\begin{array}{l}\text { Sample } \\
\text { number }\end{array}$ & $\begin{array}{l}\text { Bo- } \\
\text { ron }\end{array}$ & $\begin{array}{l}\mathrm{Ba}- \\
\text { rium }\end{array}$ & $\begin{array}{c}\text { Be- } \\
\text { ryl- } \\
\text { lium }\end{array}$ & $\begin{array}{l}\text { Cad- } \\
\text { mium }\end{array}$ & $\begin{array}{c}\text { Chro- } \\
\text { mi- } \\
\text { um }\end{array}$ & $\begin{array}{c}\text { Cop- } \\
\text { per }\end{array}$ & Iron & $\begin{array}{l}\text { Mer- } \\
\text { cury }\end{array}$ & $\begin{array}{c}\text { Mag- } \\
\text { ne- } \\
\text { sium }\end{array}$ & $\begin{array}{c}\text { Man- } \\
\text { ga- } \\
\text { nese }\end{array}$ \\
\hline \multicolumn{12}{|c|}{ Site B13 (Obsidian Butte)--Continued } \\
\hline 107 & SS89-145 & $<2$ & 0.2 & $<.1$ & 1.5 & $<1$ & 14 & 2,980 & 12 & 654 & 11 \\
\hline 108 & SS89-147 & $<2$ & $<.1$ & $<.1$ & 6.7 & $<1$ & 12 & 877 & 3 & 659 & 8.9 \\
\hline 109 & SS90e14 & $<85$ & 13 & $<3.9$ & $<3.9$ & $<110$ & $<8.5$ & 1,000 & -- & 7,000 & $<39$ \\
\hline 110 & SS90f14 & 110 & 5.8 & $<3.2$ & $<3.2$ & $<91$ & $<7.1$ & 230 & --- & 4,900 & $<32$ \\
\hline \multicolumn{12}{|c|}{ Site B14 (Bowles Road) } \\
\hline 111 & SS90e15 & $<99$ & 15 & $<4.5$ & $<4.5$ & $<130$ & $<9.9$ & 1,000 & -- & 10,000 & 170 \\
\hline 112 & SS90f15 & $<65$ & 34 & $<3.0$ & $<3.0$ & $<83$ & 7.7 & 22,000 & -- & 5,400 & 10,000 \\
\hline \multicolumn{12}{|c|}{ Site B15 (New River Delta) } \\
\hline 113 & LNSS88-91 & $<19.9$ & 5.62 & $<0.200$ & $<0.200$ & $<0.399$ & 10.8 & 174 & $<0.100$ & 1,520 & 10.0 \\
\hline 114 & LNSS88-85 & $<29.2$ & 7.31 & $<.293$ & .526 & $<.585$ & 35.6 & 284 & $<.147$ & 1,510 & 26.1 \\
\hline 115 & LNSS88-63 & $<21.0$ & 37.8 & $<.211$ & 1.39 & $<.421$ & 99.67 & 655 & $<.106$ & 3,500 & 257 \\
\hline 116 & LNSS88-64 & $<25.3$ & 6.11 & $<.253$ & .303 & 1.31 & 4.19 & 260 & $<.127$ & 1,949 & 35.9 \\
\hline 117 & LNSS88-66 & $<22.1$ & 8.67 & $<.222$ & $<.222$ & .619 & 7.04 & 184 & .181 & 1,940 & 34.6 \\
\hline 118 & SS89-76 & 10 & 30.2 & $<.2$ & $<.7$ & 2 & 8.4 & 2,450 & .12 & 2,650 & 55.5 \\
\hline 119 & SS89-149 & $<2$ & .1 & $<.1$ & 1.8 & 1 & 71.9 & 3,440 & 7.32 & 754 & 13 \\
\hline 120 & SS89-151 & $<2$ & $<.1$ & $<.1$ & 1.4 & $<1$ & 9.6 & 904 & 1.02 & 787 & 9.3 \\
\hline 121 & SS89-93 & $<3$ & 1.4 & $<.1$ & $<.5$ & $<2$ & 4 & 68 & .667 & 285 & 1.2 \\
\hline 122 & SS89-94 & $<3$ & 2.3 & $<.1$ & $<.5$ & $<2$ & 2.9 & 109 & .15 & 380 & 1.5 \\
\hline 123 & SS89-95 & $<3$ & 1.2 & $<.1$ & $<.5$ & $<2$ & 1.7 & 111 & .47 & 459 & .87 \\
\hline 124 & SS89-96 & $<3$ & 3.0 & $<.1$ & $<.5$ & $<2$ & 2.2 & 103 & .22 & 404 & 1.5 \\
\hline 125 & SS89-97 & $<3$ & 0.76 & $<.1$ & $<.5$ & $<2$ & 2.5 & 91 & .34 & 391 & 1.2 \\
\hline 126 & SS89-98 & $<3$ & 2.3 & $<.1$ & $<.5$ & $<2$ & 2.6 & 82 & .26 & 434 & 1.2 \\
\hline 127 & SS89-99 & $<3$ & 1.1 & $<.1$ & $<.5$ & $<2$ & 3.1 & 119 & .41 & 396 & 1.2 \\
\hline 128 & SS89-100 & $<3$ & 1.8 & $<.1$ & $<.5$ & $<2$ & 2.8 & 91 & 1.02 & 442 & 2.2 \\
\hline 129 & SS89-101 & $<3$ & 5.4 & $<.1$ & $<.5$ & $<2$ & 3.0 & 105 & .565 & 395 & 1.3 \\
\hline 130 & SS89-102 & $<3$ & .70 & $<.1$ & $<.5$ & $<2$ & 1.6 & 78 & .39 & 217 & 1.6 \\
\hline 131 & SS89-133 & 2 & 8.7 & $<.1$ & $<.3$ & 7.9 & 30.3 & 331 & .35 & 1,320 & 9.6 \\
\hline 132 & SS89-134 & 2 & 7.3 & $<.1$ & $<.2$ & 7.6 & 10 & 291 & .43 & 1,300 & 8.4 \\
\hline 133 & SS89-135 & 3 & 6.2 & $<.1$ & $<.3$ & 5.8 & 9.1 & 347 & .39 & 1,240 & 7.8 \\
\hline 134 & SS89-136 & 2 & 7.1 & $<.1$ & $<.3$ & 11 & 13 & 426 & .28 & 1,260 & 7.8 \\
\hline 135 & SS89-137 & 2 & 5.8 & $<.1$ & $<.2$ & 6 & 5.9 & 303 & .26 & 1,100 & 5.6 \\
\hline \multicolumn{12}{|c|}{ Site B17 (New River at Rio Bend) } \\
\hline 136 & LNSS88-68 & $<18.2$ & 10.4 & $<0.183$ & $<0.183$ & 0.839 & 6.97 & 196 & 0.095 & 1,500 & 39.4 \\
\hline 137 & LNSS88-96 & -- & --- & $<.01$ & $<.03$ & .3 & 3.19 & 115 & .67 & - & 1.3 \\
\hline 138 & LNSS88-138 & -- & - & $<.01$ & $<.03$ & .2 & 3.34 & 109 & .29 & -- & 1.9 \\
\hline 139 & LNSS88-139 & $<2$ & 3.3 & $<.1$ & $<.4$ & $<1$ & 1.8 & 120 & .14 & 419 & .9 \\
\hline 140 & LNSS88-141 & - & -- & $<.01$ & $<.03$ & 1.4 & 4.17 & 124 & .56 & -- & 1.8 \\
\hline 141 & LNSS88-142 & 4 & 1.3 & $<.1$ & $<.4$ & $<1$ & 2.5 & 71 & .26 & 447 & 1.3 \\
\hline 142 & LNSS88-144 & -- & -- & $<.009$ & $<.03$ & .2 & 3.47 & 93.8 & .25 & -.- & 1.4 \\
\hline 143 & LNSS88-145 & 3 & 2.3 & $<.1$ & $<.4$ & $<1$ & 3.1 & 84 & .44 & 423 & .5 \\
\hline 144 & LNSS88-147 & -- & -- & $<.01$ & $<.03$ & .2 & 4.00 & 116 & .38 & --- & 2.94 \\
\hline 145 & LNSS88-148 & 3 & 1.4 & $<.1$ & $<.4$ & $<1$ & 3.5 & 110 & .25 & 541 & 1.4 \\
\hline 146 & LNSS88-150 & -- &.- & $<.01$ & $<.03$ & .2 & 3.59 & 117 & .099 & -- & 2.69 \\
\hline
\end{tabular}


Table 22. Inorganic chemical analysis and moisture content for biotic samples collected during 1988-90 from the Salton Sea and associated drainwaters and rivers--Continued

\begin{tabular}{|c|c|c|c|c|c|c|c|c|c|c|c|}
\hline $\begin{array}{l}\text { Record } \\
\text { number }\end{array}$ & $\begin{array}{l}\text { Sample } \\
\text { number }\end{array}$ & $\begin{array}{c}\text { Molyb- } \\
\text { de- } \\
\text { num }\end{array}$ & $\begin{array}{c}\text { Nick- } \\
\text { el }\end{array}$ & Lead & $\begin{array}{l}\text { Anti- } \\
\text { mo- } \\
\text { ny }\end{array}$ & $\begin{array}{c}\text { Sele- } \\
\text { ni- } \\
\text { um }\end{array}$ & Tin & $\begin{array}{l}\text { Stron- } \\
\text { ti- } \\
\text { um }\end{array}$ & $\begin{array}{c}\text { Thal- } \\
\text { li- } \\
\text { um }\end{array}$ & $\begin{array}{l}\text { Va- } \\
\text { nadi- } \\
\text { um }\end{array}$ & Zinc \\
\hline \multicolumn{12}{|c|}{ Site B13 (Obsidian Butte)-Continued } \\
\hline 107 & SS89-145 & $<1$ & $<1$ & $<4$ & - & 35.1 & -- & 0.74 & $<5$ & $<0.3$ & 102 \\
\hline 108 & SS89-147 & $<1$ & $<2$ & $<4$ & - & 32.3 & - & 1.1 & $<4$ & $<.3$ & 99.0 \\
\hline 109 & SS90e14 & $<30$ & $<6.2$ & $<31$ & - & $<.71$ & $<39$ & 250 & -- & 9.6 & $<210$ \\
\hline 110 & SS90f14 & $<25$ & $<5.2$ & $<26$ & - & $<.75$ & $<32$ & 250 & - & $<8.1$ & $<170$ \\
\hline \multicolumn{12}{|c|}{ Site B14 (Bowles Road) } \\
\hline 111 & SS90e15 & $<35$ & $<7.2$ & $<36$ & -- & $<1.0$ & $<45$ & 270 & -- & $<11$ & $<240$ \\
\hline 112 & SS90f15 & $<23$ & 7.8 & $<23$ & - & $<.58$ & $<30$ & 390 & -- & 26 & $<160$ \\
\hline \multicolumn{12}{|c|}{ Site B15 (New River Delta) } \\
\hline 113 & LNSS88-91 & $<2.00$ & $<1.60$ & $<3.99$ & $<0.100$ & 2.0 & 4.38 & 106 & $<0.40$ & $<2.00$ & 90.4 \\
\hline 114 & LNSS88-85 & $<2.93$ & 2.57 & $<5.85$ & $<.147$ & 6.4 & 5.32 & 90.1 & $<.59$ & $<2.93$ & 217 \\
\hline 115 & LNSS88-63 & $<2.11$ & 2.02 & $<4.21$ & $<.106$ & 2.9 & 4.16 & 752 & $<.42$ & $<2.11$ & 73.1 \\
\hline 116 & LNSS88-64 & $<2.53$ & $<2.03$ & $<5.06$ & $<.127$ & 6.1 & 5.15 & 227 & $<.51$ & $<2.53$ & 111 \\
\hline 117 & LNSS88-66 & $<2.22$ & 2.30 & $<4.43$ & $<.111$ & 3.5 & 5.18 & 181 & $<.45$ & $<2.22$ & 137 \\
\hline 118 & SS89-76 & $<1$ & $<4$ & $<4$ & -- & 1.4 & - & 71.9 & $<4$ & 5.6 & 121 \\
\hline 119 & SS89-149 & $<1$ & $<2$ & $<4$ & -- & 3.3 & --- & 1.6 & $<4$ & $<.3$ & 97.2 \\
\hline 120 & SS89-151 & $<1$ & $<2$ & $<4$ & -- & 2.7 & --- & 2.0 & $<4$ & $<.3$ & 75.6 \\
\hline 121 & SS89-93 & $<1$ & $<3$ & $<4$ & --- & 2.7 & -- & 17.2 & $<4$ & $<.6$ & 30 \\
\hline 122 & SS89-94 & $<1$ & $<3$ & $<4$ & --- & 3.2 & -- & 25.3 & $<4$ & $<.7$ & 39 \\
\hline 123 & SS89-95 & $<1$ & $<3$ & $<4$ & -- & 2.6 & -- & 26.9 & $<4$ & $<.7$ & 50.8 \\
\hline 124 & SS89-96 & $<1$ & $<3$ & $<4$ & -- & 2.4 & $\cdots$ & 71.4 & $<4$ & $<.7$ & 44 \\
\hline 125 & SS89-97 & $<1$ & $<3$ & $<4$ & -- & 3.1 & -- & 27.3 & $<4$ & $<.6$ & 49.6 \\
\hline 126 & SS89-98 & $<1$ & $<3$ & $<4$ & -- & 3.7 & -- & 42.6 & $<4$ & $<.6$ & 39 \\
\hline 127 & SS89-99 & $<1$ & $<3$ & $<4$ & -- & 3.1 & -- & 32.7 & $<4$ & $<.6$ & 45 \\
\hline 128 & SS89-100 & $<1$ & $<3$ & $<4$ & -- & 3.3 & -- & 25.1 & $<4$ & $<.7$ & 42 \\
\hline 129 & SS89-101 & $<1$ & $<3$ & $<4$ & -- & 2.1 & -- & 24.2 & $<4$ & $<.7$ & 39 \\
\hline 130 & SS89-102 & $<1$ & $<3$ & $<4$ & -- & 1.9 & -- & 13.7 & $<4$ & $<.7$ & 26 \\
\hline 131 & SS89-133 & $<1$ & 3 & $<4$ & -- & 7.1 & -- & 190 & $<4$ & .4 & 88.6 \\
\hline 132 & SS89-134 & $<1$ & 3 & $<4$ & -- & 7.1 & -- & 194 & $<4$ & .4 & 93.3 \\
\hline 133 & SS89-135 & $<1$ & $<2$ & $<4$ & --- & 7.2 & -- & 164 & $<4$ & $<.3$ & 87.2 \\
\hline 134 & SS89-136 & $<1$ & 5 & $<5$ & -- & 4.1 & --- & 174 & $<4$ & .6 & 84.7 \\
\hline 135 & SS89-137 & $<1$ & 2 & $<4$ & - & 3.9 & -- & 151 & $<4$ & .5 & 87.4 \\
\hline \multicolumn{12}{|c|}{ Site B17 (New River at Rio Bend) } \\
\hline 136 & LNSS88-68 & $<1.83$ & $<1.46$ & $<3.65$ & $<0.092$ & 2.9 & 5.40 & 289 & $<0.37$ & $<1.83$ & 105 \\
\hline 137 & LNSS88-96 & -- & $<.2$ & $<.5$ & -- & 2.8 & -- & -- & $<.5$ & --- & 42 \\
\hline 138 & LNSS88-138 & - & .2 & 1 & --- & 5.6 & -- & -- & $<.5$ & -.. & 45.7 \\
\hline 139 & LNSS88-139 & $<1$ & $<2$ & $<4$ & --- & 3.6 & -- & 45.7 & $<4$ & $<4$ & 55.1 \\
\hline 140 & LNSS88-141 & - & 1.3 & .7 & --- & 5.7 & --- & -- & $<.5$ & --- & 50.9 \\
\hline 141 & LNSS88-142 & $<1$ & $<2$ & $<4$ & -- & 3.6 & -- & 38.8 & $<4$ & $<4$ & 46.5 \\
\hline 142 & LNSS88-144 & -- & $<.2$ & .8 & --- & 2.8 & -- & -- & $<.4$ & -.. & 50.7 \\
\hline 143 & LNSS88-145 & $<1$ & $<2$ & $<4$ & -- & 5.0 & -- & 25.4 & $<4$ & $<4$ & 48.8 \\
\hline 144 & LNSS88-147 & - & $<.2$ & $<.5$ & -- & 2.6 & -- & -- & $<.5$ & --- & 46.2 \\
\hline 145 & LNSS88-148 & $<1$ & $<2$ & $<4$ & -- & 3.5 & -- & 29.9 & $<4$ & $<4$ & 47.4 \\
\hline 146 & LNSS88-150 & --- & .4 & .6 & -- & 4.5 & -- & --- & $<.5$ & --- & 57.2 \\
\hline
\end{tabular}


Table 22. Inorganic chemical analysis and moisture content for biotic samples collected during 1988-90 from the Salton Sea and associated drainwaters and rivers--Continued

\begin{tabular}{|c|c|c|c|c|c|c|c|}
\hline $\begin{array}{l}\text { Record } \\
\text { number }\end{array}$ & $\begin{array}{l}\text { Sample } \\
\text { number }\end{array}$ & Species & Matrix & $\begin{array}{l}\text { Percent } \\
\text { moisture }\end{array}$ & $\begin{array}{l}\text { Sil- } \\
\text { ver }\end{array}$ & $\begin{array}{c}\text { Alu- } \\
\text { mi- } \\
\text { num }\end{array}$ & $\begin{array}{c}\text { Arse- } \\
\text { nic }\end{array}$ \\
\hline \multicolumn{8}{|c|}{ Site B17 (New River at Rio Bend)--Continued } \\
\hline 147 & . . . LNSS88-151 & Black-necked stilt & Egg & 70.9 & $<2$ & 5 & $<0.2$ \\
\hline 148 & . . . L LNSS88-153 & Black-necked stilt & Egg & 72.5 & -.. & 1.3 & $<.2$ \\
\hline 149 & . . . L LNSS88-154 & Black-necked stilt & Egg & 72.6 & $<2$ & 4 & $<.2$ \\
\hline 150 & . . . LNSS88-156 & Black-necked stilt & Egg & 73.5 & -- & .8 & $<.2$ \\
\hline 151 & . . . LNSS88-157 & Black-necked stilt & Egg & 75.5 & $<2$ & $<3$ & $<.2$ \\
\hline 152 & . . . . LNSS88-159 & Black-necked stilt & Egg & 73.3 & -.. & .7 & $<.2$ \\
\hline 153 & . . . . LNSS88-160 & Black-necked stilt & Egg & 70.6 & $<2$ & $<3$ & $<.2$ \\
\hline 154 & . . . L LNSS88-162 & Black-necked stilt & Egg & 71.7 & -- & 1.2 & $<.2$ \\
\hline 155 & . . . L LNSS88-163 & Black-necked stilt & Egg & 72.5 & $<2$ & $<3$ & $<.2$ \\
\hline 156 & . . . . LNSS88-165 & Black-necked stilt & Egg & 73.6 & $-\cdots$ & 1.1 & $<.2$ \\
\hline 157 & . . . . LNSS88-166 & Black-necked stilt & Egg & 73.6 & $<2$ & $<3$ & $<.2$ \\
\hline 158 & . . . . LNSS88-168 & Black-necked stilt & Egg & 73.4 & --- & .6 & $<.2$ \\
\hline 159 & . . . . LNSS88-169 & Black-necked stilt & Egg & 72.7 & $<2$ & $<6$ & $<.2$ \\
\hline 160 & . . . LNSS88-171 & Black-necked stilt & Egg & 74.4 & -- & .7 & $<.2$ \\
\hline 161 & . . . . LNSS88-174 & Black-necked stilt & Egg & 73.6 & -- & .8 & $<.2$ \\
\hline 162 & . . . . LNSS88-177 & Black-necked stilt & Egg & 73.1 & --- & 1.4 & $<.2$ \\
\hline 163 & . . . . LNSS88-181 & Black-necked stilt & Egg & 73.1 & $<2$ & $<3$ & $<.2$ \\
\hline 164 & . . . L LNSS88-183 & Black-necked stilt & Egg & 71.4 & $\ldots$ & 2.6 & .3 \\
\hline 165 & . . . L LNSS88-186 & Black-necked stilt & Egg & 73.1 & -- & 1 & $<.2$ \\
\hline 166 & . . . LNSS88-187 & Black-necked stilt & Egg & 64.4 & $<2$ & 5 & $<.2$ \\
\hline 167 & . . . . LNSS88-191 & Black-necked stilt & Egg & 73.4 & $<2$ & $<3$ & $<.2$ \\
\hline 168 & . . . . LNSS88-193 & Black-necked stilt & Egg & 73.7 & --- & 1.1 & $<.2$ \\
\hline 169 & . . . . LNSS88-202 & Black-necked stilt & Egg & 73.2 & -- & .8 & $<.2$ \\
\hline 170 & . . . L LNSS88-205 & Black-necked stilt & Egg & 73.0 & - & 2.1 & $<.2$ \\
\hline 171 & . . . . LNSS88-211 & Black-necked stilt & Egg & 73.2 & $-\cdots$ & 1.2 & $<.2$ \\
\hline 172 & . . . L LNSS88-212 & Black-necked stilt & Egg & 73.0 & $<2$ & $<3$ & $<.2$ \\
\hline 173 & . . . . LNSS88-214 & Black-necked stilt & Egg & 73.4 & -.. & .8 & $<.2$ \\
\hline 174 & . . . L LNSS88-229 & Black-necked stilt & Egg & 70.8 & -- & 1.3 & $<.2$ \\
\hline 175 & $\ldots \ldots$ SS89-121 & Asiatic river clam & Soft tissue & 86.6 & $<2$ & 110 & 11 \\
\hline 176 & . . . . SS899-85 & Black-necked stilt & Egg & 68.9 & $<2$ & $<3$ & $<.1$ \\
\hline 177 & . . . . SS89-86 & Black-necked stilt & Egg & 75.6 & $<2$ & $<3$ & $<.1$ \\
\hline 178 & . . . . SS89-87 & Black-necked stilt & Egg & 73.3 & $<2$ & $<3$ & $<.1$ \\
\hline 179 & . . . . SS89-88 & Black-necked stilt & Egg & 73.8 & $<2$ & $<3$ & $<.1$ \\
\hline 180 & . . . . SS89-89 & Black-necked stilt & Egg & 72.7 & $<2$ & $<3$ & $<.1$ \\
\hline 181 & . . . S SS89-90 & Black-necked stilt & Egg & 73.5 & $<2$ & $<3$ & $<.1$ \\
\hline 182 & . . . S SS89-91 & Black-necked stilt & Egg & 75.1 & $<2$ & $<3$ & $<.1$ \\
\hline 183 & . . . . SS89-92 & Black-necked stilt & Egg & 73.8 & $<2$ & $<3$ & $<.2$ \\
\hline 184 & . . . . SS89-138 & Black-necked stilt & Carcass & 66.2 & $<2$ & 523 & .2 \\
\hline 185 & . . . . SS89-139 & Black-necked stilt & Carcass & 68.6 & $<2$ & 95 & .1 \\
\hline 186 & . . . . SS89-140 & Black-necked stilt & Carcass & 63.9 & $<2$ & 170 & .2 \\
\hline 187 & . . . . SS89-141 & Black-necked stilt & Carcass & 53.8 & $<2$ & 100 & $<.1$ \\
\hline \multicolumn{8}{|c|}{ Site B18 (Alamo River at Garst Road) } \\
\hline 188 & . . . . SS89-64 & Waterboatman & Composite & 86.6 & $<2$ & 789 & 1.0 \\
\hline 189 & . . . . SS89-120 & Asiatic river clam & Soft tissue & 84.2 & $<2$ & 862 & 10 \\
\hline 190 & .... SS89-165 & Asiatic river clam & Soft tissue & 81.1 & $<2$ & 10,700 & 10 \\
\hline
\end{tabular}


Table 22. Inorganic chemical analysis and moisture content for biotic samples collected during 1988-90 from the Salton Sea and associated drainwaters and rivers--Continued

\begin{tabular}{|c|c|c|c|c|c|c|c|c|c|c|c|}
\hline $\begin{array}{l}\text { Record } \\
\text { number }\end{array}$ & $\begin{array}{l}\text { Sample } \\
\text { number }\end{array}$ & $\begin{array}{l}\text { Bo- } \\
\text { ron }\end{array}$ & $\begin{array}{l}\mathrm{Ba-} \\
\text { rium }\end{array}$ & $\begin{array}{l}\text { Be- } \\
\text { ryl- } \\
\text { lium }\end{array}$ & $\begin{array}{l}\text { Cad- } \\
\text { mium }\end{array}$ & $\begin{array}{c}\text { Chro- } \\
\text { mi- } \\
\text { um }\end{array}$ & $\begin{array}{l}\text { Cop- } \\
\text { per }\end{array}$ & Iron & $\begin{array}{l}\text { Mer- } \\
\text { cury }\end{array}$ & $\begin{array}{c}\text { Mag- } \\
\text { ne- } \\
\text { sium }\end{array}$ & $\begin{array}{c}\text { Man- } \\
\text { ga- } \\
\text { nese }\end{array}$ \\
\hline \multicolumn{12}{|c|}{ Site B17 (New River at Rio Bend)--Continued } \\
\hline 147 & LNSS88-151 & $<2$ & 2.3 & $<0.1$ & $<0.4$ & $<1$ & 3.5 & 110 & 0.22 & 437 & 1.9 \\
\hline 148 & LNSS88-153 & - & - & $<.01$ & $<.03$ & .2 & 3.46 & 125 & .23 & - & 1.2 \\
\hline 149 & LNSS88-154 & 3 & .88 & $<.1$ & $<.4$ & $<1$ & 3.8 & 99 & .24 & 401 & .9 \\
\hline 150 & LNSS88-156 & -- & $\ldots$ & $<.01$ & $<.03$ & $<.1$ & 3.76 & 93.7 & .44 & $\ldots$ & 1.6 \\
\hline 151 & LNSS88-157 & $<2$ & 2.6 & $<.1$ & $<.4$ & $<1$ & 3.6 & 98 & .11 & 426 & 1.2 \\
\hline 152 & LNSS88-159 & --- & --- & $<.01$ & $<.03$ & $<.1$ & 3.11 & 164 & .48 & $\ldots$ & 2.5 \\
\hline 153 & LNSS88-160 & $<2$ & 3.2 & $<.1$ & $<.4$ & $<1$ & 2.8 & 110 & .768 & 358 & 1.8 \\
\hline 154 & LNSS88-162 & $\ldots$ & $\ldots$ & $<.01$ & $<.03$ & $<.1$ & 2.97 & 130 & .097 & --. & 1.4 \\
\hline 155 & LNSS88-163 & $<2$ & 1.4 & $<.1$ & $<.4$ & $<1$ & 3.7 & 86 & .45 & 447 & 1.3 \\
\hline 156 & LNSS88-165 & $\ldots$ & $\ldots$ & $<.01$ & $<.03$ & $<.1$ & 2.81 & 90.4 & .37 & - & 1.9 \\
\hline 157 & LNSS88-166 & $<2$ & 1.2 & $<.1$ & $<.4$ & $<1$ & 3.2 & 130 & .18 & 478 & .98 \\
\hline 158 & LNSS88-168 & -- & - & $<.01$ & $<.03$ & $<.1$ & 3.74 & 95.3 & .28 & - & 1.6 \\
\hline 159 & LNSS88-169 & $<2$ & .68 & $<.1$ & $<.4$ & $<1$ & 3.6 & 81 & .19 & 378 & 1.4 \\
\hline 160 & LNSS88-171 & - & - & $<.01$ & $<.03$ & $<.1$ & 3.48 & 98.5 & .25 & -- & 1.9 \\
\hline 161 & LNSS88-174 & - & $\ldots$ & $<.01$ & $<.03$ & $<.1$ & 3.10 & 103 & .29 & --- & 2.91 \\
\hline 162 & LNSS88-177 & -- & $\ldots$ & $<.01$ & $<.03$ & $<.1$ & 3.08 & 111 & .30 & $\ldots$ & 2.3 \\
\hline 163 & LNSS88-181 & $<2$ & .67 & $<.1$ & $<.4$ & $<1$ & 1.5 & 74 & .10 & 284 & .5 \\
\hline 164 & LNSS88-183 & $\ldots$ & - & $<.01$ & $<.03$ & $<.1$ & 3.37 & 117 & .16 & --. & 2.3 \\
\hline 165 & LNSS88-186 & - & -- & .01 & $<.03$ & $<.1$ & 2.1 & 128 & .093 & -.- & 1.5 \\
\hline 166 & LNSS88-187 & 4 & 5.8 & $<.1$ & $<.4$ & $<1$ & 31.8 & 143 & 2.1 & 604 & 1.8 \\
\hline 167 & LNSS88-191 & 3 & 1.8 & $<.1$ & $<.4$ & $<1$ & 3.3 & 97 & .11 & 439 & 1.1 \\
\hline 168 & LNSS88-193 & $\ldots$ & $\ldots$ & $<.01$ & $<.03$ & $<.1$ & 2.56 & 108 & .24 & --. & 1.3 \\
\hline 169 & LNSS88-202 & - & --. & $<.01$ & $<.03$ & $<.1$ & 3.11 & 125 & .48 & -.. & 2.4 \\
\hline 170 & LNSS88-205 & --- & -- & $<.01$ & .04 & $<.1$ & 3.47 & 111 & .19 & -- & 1.1 \\
\hline 171 & LNSS88-211 & - & -- & $<.01$ & $<.03$ & $<.1$ & 2.3 & 98.6 & .14 & -- & 1.2 \\
\hline 172 & LNSS88-212 & 3 & .79 & $<.1$ & $<.4$ & $<1$ & 2.4 & 110 & .24 & 435 & .96 \\
\hline 173 & LNSS88-214 & - & --- & $<.01$ & $<.03$ & $<.1$ & 3.08 & 98.9 & .13 & -- & .84 \\
\hline 174 & LNSS88-229 & - & -- & $<.01$ & $<.03$ & .2 & 3.23 & 120 & .49 & --- & 1.4 \\
\hline 175 & SS89-121 & $<3$ & 95.3 & $<.1$ & .6 & $<2$ & 44.5 & 285 & .19 & 967 & 16 \\
\hline 176 & SS89-85 & $<3$ & .97 & $<.1$ & $<.5$ & $<2$ & 1.9 & 124 & .25 & 387 & 3.3 \\
\hline 177 & SS89-86 & $<3$ & 1.1 & $<.1$ & $<.5$ & $<2$ & 2.6 & 97 & .528 & 418 & 1.3 \\
\hline 178 & SS89-87 & $<3$ & 1.7 & $<.1$ & $<.5$ & $<2$ & 3.2 & 104 & .29 & 420 & 2.1 \\
\hline 179 & SS89-88 & $<3$ & 2.4 & $<.1$ & $<.5$ & $<2$ & 2.2 & 100 & .16 & 413 & 2.2 \\
\hline 180 & SS89-89 & $<3$ & 2.4 & $<.1$ & $<.5$ & $<2$ & 3.3 & 114 & 2.8 & 484 & 2.8 \\
\hline 181 & SS89-90 & $<3$ & .63 & $<.1$ & $<.5$ & $<2$ & 3.5 & 100 & .38 & 384 & 2.6 \\
\hline 182 & SS89-91 & $<3$ & 1.2 & $<.1$ & $<.5$ & $<2$ & 3.5 & 69 & .34 & 399 & 2.8 \\
\hline 183 & SS89-92 & $<3$ & 1.6 & $<.1$ & $<.5$ & $<2$ & 3.0 & 110 & .081 & 355 & 2.7 \\
\hline 184 & SS89-138 & 3 & 7.4 & $<.1$ & $<.2$ & 7.3 & 13 & 515 & .12 & 1,300 & 15 \\
\hline 185 & SS89-139 & 2 & 8.0 & $<.1$ & $<.2$ & 3.0 & 7.2 & 255 & .071 & 1,200 & 26 \\
\hline 186 & SS89-140 & 4 & 7.0 & $<.1$ & $<.3$ & 5.5 & 26.3 & 265 & .086 & 1,410 & 27 \\
\hline 187 & SS89-141 & $<2$ & 2.5 & $<.1$ & $<.3$ & 3 & 6.9 & 195 & .20 & 870 & 4.0 \\
\hline \multicolumn{12}{|c|}{ Site B18 (Alamo River at Garst Road) } \\
\hline 188 & SS89-64 & 21 & 11.6 & $<0.2$ & $<0.7$ & $<2$ & 12 & 610 & 0.036 & 3,960 & 24.2 \\
\hline 189 & SS89-120 & $<3$ & 21.4 & $<.1$ & .6 & $<2$ & 31.3 & 781 & .14 & 1,230 & 24.4 \\
\hline 190 & SS89-165 & 13 & 129 & .35 & .7 & 14 & 44.0 & 6,080 & .15 & 4,180 & 150 \\
\hline
\end{tabular}


Table 22. Inorganic chemical analysis and moisture content for biotic samples collected during 1988-90 from the Salton Sea and associated drainwaters and rivers--Continued

\begin{tabular}{|c|c|c|c|c|c|c|c|c|c|c|c|}
\hline $\begin{array}{l}\text { Record } \\
\text { number }\end{array}$ & $\begin{array}{l}\text { Sample } \\
\text { number }\end{array}$ & $\begin{array}{c}\text { Molyb- } \\
\text { de- } \\
\text { num }\end{array}$ & $\begin{array}{l}\text { Nick- } \\
\text { el }\end{array}$ & Lead & $\begin{array}{l}\text { Anti- } \\
\text { mo- } \\
\text { ny }\end{array}$ & $\begin{array}{l}\text { Sele- } \\
\text { ni- } \\
\text { um }\end{array}$ & Tin & $\begin{array}{l}\text { Stron- } \\
\text { ti- } \\
\text { um }\end{array}$ & $\begin{array}{c}\text { Thal- } \\
\text { li- } \\
\text { um }\end{array}$ & $\begin{array}{l}\text { Va- } \\
\text { nadi- } \\
\text { um }\end{array}$ & Zinc \\
\hline & & & \multicolumn{9}{|c|}{ Site B17 (New River at Rio Bend)--Continued } \\
\hline 147 & LNSS88-151 & $<1$ & $<2$ & $<4$ & -.- & 4.4 & --- & 28.3 & $<4$ & $<4$ & 48.5 \\
\hline 148 & LNSS88-153 & - & .2 & $<.5$ & -- & 3.6 & -- & -- & $<.5$ & --- & 47.4 \\
\hline 149 & LNSS88-154 & $<1$ & $<2$ & $<4$ & -- & 5.6 & -- & 21.6 & $<4$ & $<4$ & 41 \\
\hline 150 & LNSS88-156 & -- & $<.2$ & 2.4 & -- & 4.5 & --- & -- & $<.7$ & -- & 37.8 \\
\hline 151 & LNSS88-157 & $<1$ & $<2$ & $<4$ & --- & 6.1 & --- & 27 & $<4$ & $<4$ & 48.4 \\
\hline 152 & LNSS88-159 & -- & $<.2$ & 1.7 & -- & 3.5 & -- & -- & $<.7$ & -- & 62.5 \\
\hline 153 & LNSS88-160 & $<1$ & $<2$ & $<4$ & -- & 6.3 & -- & 17.3 & $<4$ & $<4$ & 50.9 \\
\hline 154 & LNSS88-162 & - & $<.2$ & 1.6 & -- & 3.8 & -- & - & $<.7$ & - & 49.1 \\
\hline 155 & LNSS88-163 & $<1$ & $<2$ & $<4$ & -- & 3.9 & --. & 17.1 & $<4$ & $<4$ & 42.9 \\
\hline 156 & LNSS88-165 & $\cdots$ & $<.2$ & 1.7 & - & 2.9 & -- & -- & $<.7$ & --- & 35.1 \\
\hline 157 & LNSS88-166 & $<1$ & $<2$ & $<4$ & -- & 4.7 & --- & 32.5 & $<4$ & $<4$ & 49.9 \\
\hline 158 & LNSS88-168 & - & $<.2$ & 3.4 & --- & 5.4 & --- & --- & $<.7$ & --- & 43.5 \\
\hline 159 & LNSS88-169 & $<1$ & $<2$ & $<4$ & --- & 4.5 & --- & 17.9 & $<4$ & $<4$ & 42.5 \\
\hline 160 & LNSS88-171 & -- & $<.2$ & $<.5$ & --- & 3.3 & --- & --- & $<.7$ & --- & 45.8 \\
\hline 161 & LNSS88-174 & $\ldots$ & $<.2$ & $<.5$ & --- & 2.3 & --- & --- & $<.7$ & --- & 45.1 \\
\hline 162 & LNSS88-177 & -- & $<.2$ & 1 & --- & 2.6 & --- & --- & $<.6$ & -.. & 49.0 \\
\hline 163 & LNSS88-181 & $<1$ & $<2$ & $<4$ & $-\ldots$ & 1.7 & $\cdots$ & 27.7 & $<4$ & $<4$ & 30.6 \\
\hline 164 & LNSS88-183 & -- & $<.2$ & $<.5$ & --- & 4.0 & --- & --- & $<.7$ & -- & 45.4 \\
\hline 165 & LNSS88-186 & $\cdots$ & $<.2$ & $<.5$ & -- & 2.8 & -- & -- & $<1$ & --- & 51.0 \\
\hline 166 & LNSS88-187 & $<1$ & $<2$ & $<4$ & --- & 6.6 & -- & 41.7 & $<4$ & $<4$ & 57.0 \\
\hline 167 & LNSS88-191 & $<1$ & $<2$ & $<4$ & $\ldots$ & 5.7 & --- & 34.7 & $<4$ & $<4$ & 48.1 \\
\hline 168 & LNSS88-193 & - & $<.2$ & $<.5$ & -- & 3.0 & --. & $\ldots$ & $<.7$ & $\cdots$ & 40.5 \\
\hline 169 & LNSS88-202 & - & $<.2$ & $<.5$ & --- & 3.5 & --- & --- & $<.7$ & --- & 54.9 \\
\hline 170 & LNSS88-205 & -- & $<.2$ & $<.5$ & $\ldots$ & 4.5 & --- & --- & $<.6$ & --- & 47.8 \\
\hline 171 & LNSS88-211 & -- & $<.2$ & $<.5$ & --- & 4.9 & -.- & $\ldots$ & $<.7$ & $-\ldots$ & 44.5 \\
\hline 172 & LNSS88-212 & $<1$ & $<2$ & $<4$ & --- & 4.6 & $-\ldots$ & 27.4 & $<4$ & $<4$ & 43.9 \\
\hline 173 & LNSS88-214 & -- & $<.2$ & $<.5$ & -- & 2.2 & $\cdots$ & -- & $<.7$ & --- & 47.7 \\
\hline 174 & LNSS88-229 & --. & $<.2$ & $<.5$ & $\ldots$ & 5.0 & --- & --- & $<.7$ & --- & 46.1 \\
\hline 175 & SS89-121 & $<1$ & $<3$ & $<4$ & - & 5.9 & -- & 67.1 & $<4$ & .7 & 105 \\
\hline 176 & SS89-85 & $<1$ & $<3$ & $<4$ & --- & 3.3 & --- & 25.3 & $<4$ & $<.6$ & 49.4 \\
\hline 177 & SS89-86 & $<1$ & $<3$ & $<4$ & --- & 2.6 & --- & 24.1 & $<4$ & $<.7$ & 43 \\
\hline 178 & SS89-87 & $<1$ & $<3$ & $<4$ & --- & 5.7 & -- & 25.4 & $<4$ & $<.7$ & 42 \\
\hline 179 & SS89-88 & $<1$ & $<3$ & $<4$ & --- & 2.9 & --. & 40.3 & $<4$ & $<.6$ & 46 \\
\hline 180 & SS89-89 & $<1$ & 6 & $<4$ & $\cdots$ & 3.0 & -- & 37.8 & $<4$ & $<.6$ & 44 \\
\hline 181 & SS89-90 & $<1$ & $<3$ & $<4$ & --- & 3.5 & --- & 18.3 & $<4$ & $<.6$ & 35 \\
\hline 182 & SS89-91 & $<1$ & $<3$ & $<4$ & -- & 10 & --- & 20.6 & $<4$ & $<.7$ & 41 \\
\hline 183 & SS89-92 & $<1$ & $<3$ & $<4$ & --- & 6.7 & --- & 24.4 & $<4$ & $<.6$ & 44 \\
\hline 184 & SS89-13 & $<1$ & 3 & $<4$ & --- & 11.3 & --- & 210 & $<4$ & .94 & 96.0 \\
\hline 185 & SS89-139 & $<1$ & $<2$ & $<4$ & -- & 7.6 & $\ldots$ & 203 & $<4$ & $<.3$ & 95.2 \\
\hline 186 & SS89-140 & $<1$ & $<2$ & $<4$ & -- & 7.3 & --- & 262 & $<4$ & $<.3$ & 102 \\
\hline 187 & SS89-141 & $<1$ & $<2$ & $<4$ & -- & 3.4 & -- & 108 & $<4$ & $<.3$ & 73.3 \\
\hline \multicolumn{12}{|c|}{ Site B18 (Alamo River at Garst Road) } \\
\hline 188 & SS89-64 & $<1$ & $<4$ & $<4$ & -- & 2.6 & -- & 265 & $<4$ & 2.8 & 99.5 \\
\hline 189 & SS89-120 & $<1$ & $<3$ & $<4$ & --- & 5.4 & -.- & 28.1 & $<4$ & 2 & 107 \\
\hline 190 & SS89-165 & $<1$ & 7.6 & 5 & -.. & 5.2 & --- & 117 & $<4$ & 15 & 97.2 \\
\hline
\end{tabular}


Table 22. Inorganic chemical analysis and moisture content for biotic samples collected during 1988-90 from the Salton Sea and associated drainwaters and rivers--Continued

\begin{tabular}{|c|c|c|c|c|c|c|c|}
\hline $\begin{array}{l}\text { Record } \\
\text { number }\end{array}$ & $\begin{array}{l}\text { Sample } \\
\text { number }\end{array}$ & Species & Matrix & $\begin{array}{l}\text { Percent } \\
\text { moisture }\end{array}$ & $\begin{array}{l}\text { Sil- } \\
\text { ver }\end{array}$ & $\begin{array}{l}\text { Alu- } \\
\text { mi- } \\
\text { num }\end{array}$ & $\begin{array}{l}\text { Arse- } \\
\text { nic }\end{array}$ \\
\hline \multicolumn{8}{|c|}{ Site B18 (Alamo River at Garst Road)--Continued } \\
\hline 191 & . . . SS89-167 & Asiatic river clam & Soft tissue & 80.6 & $<2$ & 5,320 & 8.4 \\
\hline 192 & $\ldots$ SS89-175 & Bullfrog & Whole body & 72.5 & $<2$ & 336 & $<.2$ \\
\hline 193 & . . . SS89-176 & Bullfrog & Whole body & 80.1 & $<2$ & 890 & .3 \\
\hline \multicolumn{8}{|c|}{ Site B19 (San Felipe Creek) } \\
\hline 194 & $\ldots$....SS90-s1 & Sediment & Sediment & 30.5 & -- & 4,500 & 1.4 \\
\hline 195 & $\ldots$...SS90v1 & Common cattail & Vegetation & 87.7 & -- & 230 & $<.78$ \\
\hline 196 & $\ldots$. . Ss90f1 & Mosquitofish & Whole body & 76.8 & -- & 110 & $<.41$ \\
\hline 197 & $\ldots$. . SS90f1a & Sailfin molly & Whole body & 66.4 & $\cdots$ & 160 & 1.0 \\
\hline \multicolumn{8}{|c|}{ Site B20 (Salt Creek) } \\
\hline 198 & $\ldots .$. ss90-s4 & Sediment & Sediment & 52.2 & --- & 12,000 & 6.4 \\
\hline 199 & $\ldots .$. ss90v4 & Common cattail & Vegetation & 84.0 & --- & 510 & $<.62$ \\
\hline 200 & $\ldots$. . SS90f4 & Mosquitofish & Whole body & 78.0 & -- & 63 & $<.41$ \\
\hline 201 & $\ldots$. . SS90f4 & Sailfin molly & Whole body & 74.4 & -- & 980 & 12 \\
\hline \multicolumn{8}{|c|}{ Site B21 (Colorado River at Palo Verde) } \\
\hline 202 & $\ldots$. . SS89-117 & Asiatic river clam & Soft tissue & 85.1 & $<2$ & 140 & 7.5 \\
\hline 203 & $\ldots$. . SS89-118 & Asiatic river clam & Soft tissue & 88.9 & $<2$ & 260 & 9.5 \\
\hline 204 . & $\ldots$. . SS89-119 & Asiatic river clam & Soft tissue & 86.9 & $<2$ & 230 & 8.7 \\
\hline \multicolumn{8}{|c|}{ Site B23 (Trifolium 13 Drain) } \\
\hline 205 & $\ldots$. . SS89-122 & Asiatic river clam & Soft tissue & 89.8 & $<2$ & 130 & 12 \\
\hline 206 & $\ldots$. . SS89-166 & Asiatic river clam & Soft tissue & 83.0 & $<2$ & 3,640 & 9.7 \\
\hline 207 & $\ldots$. . SS89-168 & Asiatic river clam & Soft tissue & 86.4 & $<2$ & 2,740 & 12 \\
\hline \multicolumn{8}{|c|}{ Site B24 (Trifolium 14 Drain) } \\
\hline 208 & . . . . LNSS88-180 & Black-necked stilt & Egg & 70.8 & --- & 0.7 & $<0.2$ \\
\hline 209 & . . . . LNSS88-194 & Black-necked stilt & Egg & 69.4 & $<2$ & $<3$ & $<.2$ \\
\hline 210 & . . . L LNSS88-206 & Black-necked stilt & Egg & 69.7 & $<2$ & $<3$ & $<.2$ \\
\hline 211 & . . . . LNSS88-208 & Black-necked stilt & Egg & 71.9 & -- & 1.3 & $<.2$ \\
\hline 212 . & . . . LNSS88-215 & Black-necked stilt & Egg & 72.5 & $<2$ & 6 & $<.2$ \\
\hline 213 & . . . L LNSS88-217 & Black-necked stilt & Egg & 72.6 & -- & 1.6 & $<.2$ \\
\hline 214 & . . . L LNSS88-218 & Black-necked stilt & Egg & 70.5 & $<2$ & $<3$ & $<.2$ \\
\hline 215 & . . . . LNSS88-223 & Black-necked stilt & Egg & 72.4 & --- & .9 & $<.2$ \\
\hline 216 . & . . . LNSS88-224 & Black-necked stilt & Egg & 68.0 & $<2$ & $<3$ & $<.2$ \\
\hline 217 . & . . . L LNSS88-226 & Black-necked stilt & Egg & 73.1 & --- & .8 & $<.2$ \\
\hline 218 . & . . . . LNSS88-227 & Black-necked stilt & Egg & 62.5 & $<2$ & $<3$ & $<.2$ \\
\hline 219 . & $\ldots \ldots$ SS89-45 & Ruddy duck & Liver & 71.2 & $<2$ & $<3$ & $<.1$ \\
\hline 220 & . . . . SS89-46 & Ruddy duck & Liver & 71.7 & $<2$ & $<3$ & $<.1$ \\
\hline 221 & . . . . SS89-50 & Ruddy duck & Liver & 71.7 & $<2$ & $<3$ & .65 \\
\hline 222 & $\ldots . .$. SS90c1 & Asiatic river clam & Soft tissue & 61.5 & $\cdots$ & 56 & 2.9 \\
\hline 223 . & $\ldots \mathrm{ss} 90 \mathrm{c} 2$ & Asiatic river clam & Soft tissue & 82.5 & --- & 150 & 4.9 \\
\hline \multicolumn{8}{|c|}{ Site B25 (Vail Cutoff Drain) } \\
\hline 224 . & $\ldots .$. SS89-71 & American coot & Liver & 72.5 & $<2$ & 9.7 & 0.36 \\
\hline
\end{tabular}


Table 22. Inorganic chemical analysis and moisture content for biotic samples collected during 1988-90 from the Salton Sea and associated drainwaters and rivers--Continued

\begin{tabular}{|c|c|c|c|c|c|c|c|}
\hline $\begin{array}{l}\text { Record } \\
\text { number }\end{array}$ & $\begin{array}{l}\text { Sample } \\
\text { number }\end{array}$ & Species & Matrix & $\begin{array}{l}\text { Percent } \\
\text { moisture }\end{array}$ & $\begin{array}{l}\text { Sil- } \\
\text { ver }\end{array}$ & $\begin{array}{l}\text { Alu- } \\
\text { mi- } \\
\text { num }\end{array}$ & $\begin{array}{c}\text { Arse- } \\
\text { nic }\end{array}$ \\
\hline \multicolumn{8}{|c|}{ Site B25 (Vail Cutoff Drain)--Continued } \\
\hline 225 & SS89-72 & American coot & Liver & 71.6 & $<2$ & 3 & 0.3 \\
\hline 226 & SS89-73 & American coot & Liver & 74.7 & $<2$ & 16 & .2 \\
\hline 227 & SS89-66 & Black-necked stilt & Egg & 72.3 & $<2$ & $<3$ & $<.1$ \\
\hline 228 & SS89-67 & Black-necked stilt & Egg & 72.5 & $<2$ & $<3$ & $<.1$ \\
\hline 229 & SS89-68 & Black-necked stilt & Egg & 72.0 & $<2$ & $<3$ & $<.1$ \\
\hline 230 & SS89-69 & Black-necked stilt & Egg & 73.0 & $<2$ & $<3$ & .1 \\
\hline 231 & SS89-70 & Black-necked stilt & Egg & 72.5 & $<2$ & $<3$ & .1 \\
\hline 232 & SS90c3 & Asiatic river clam & Soft tissue & 82.7 & --- & 790 & 6.0 \\
\hline \multicolumn{8}{|c|}{ Site B26 (Vail 4 Drain) } \\
\hline 233 & LNSS88-32 & Ruddy duck & Liver & 69.0 & $<2$ & $<3$ & $<0.2$ \\
\hline 234 & SS89-160 & Spiny softshell turtle & Liver & 78.5 & $<2$ & 15 & $<.1$ \\
\hline 235 & SS89-162 & Spiny softshell turtle & Liver & 79.5 & $<2$ & 14 & $<.1$ \\
\hline 236 & SS89-164 & Spiny softshell turtle & Liver & 81.2 & $<2$ & 24 & $<.1$ \\
\hline \multicolumn{8}{|c|}{ Site B27 (Vail 4A Drain) } \\
\hline 237 & SS89-63 & Pileworm & Whole body & 85.0 & $<2$ & 2,410 & 3.3 \\
\hline 238 & SS89-65 & Pileworm & Whole body & 85.8 & $<2$ & 4,180 & 3.3 \\
\hline \multicolumn{8}{|c|}{ Site B29 (S Lateral Drain) } \\
\hline 239 & SS89-169 & Yuma clapper rail & Carcass & 6.3 & $<2$ & 11,100 & 2.9 \\
\hline 240 & SS89-123 & Black-necked stilt & Carcass & 59.7 & $<2$ & 210 & .3 \\
\hline 241 & SS89-124 & Black-necked stilt & Carcass & 67.6 & $<2$ & 210 & .2 \\
\hline 242 & SS89-125 & Black-necked stilt & Carcass & 66.5 & $<2$ & 260 & .2 \\
\hline 243 & SS89-126 & Black-necked stilt & Carcass & 67.4 & $<2$ & 230 & .3 \\
\hline 244 & SS89-127 & Black-necked stilt & Carcass & 62.6 & $<2$ & 330 & .2 \\
\hline \multicolumn{8}{|c|}{ Site B30 (Z Lateral Drain) } \\
\hline 245 & SS90-s5 & Sediment & Sediment & 66.2 & $-\ldots$ & 20,000 & 10 \\
\hline 246 & SS90v5 & Common cattail & Vegetation & 82.2 & -.. & 61 & $<.46$ \\
\hline 247 & SS90c5 & Asiatic river clam & Soft tissue & 70.5 & -.. & 1,300 & 2.3 \\
\hline 248 & SS90f5 & Sailfin molly & Whole body & 76.2 & --- & 910 & $<.40$ \\
\hline \multicolumn{8}{|c|}{ Site B31 (81st Street Drain) } \\
\hline 249 & SS90-s2 & Sediment & Sediment & 63.6 & $\cdots$ & 14,000 & 6.2 \\
\hline 250 & SS90a5 & Filamentous green algae & Vegetation & 78.5 & --- & 4,600 & 8.0 \\
\hline 251 & SS90e7 & Tubular green algae & Vegetation & 87.5 & $\cdots$ & 3,200 & 2.4 \\
\hline 252 & SS90v2 & Common cattail & Vegetation & 85.0 & -.- & 84 & $<.63$ \\
\hline 253 & SS90f2 & Mosquitofish & Whole body & 77.3 & -. & 51 & $<.38$ \\
\hline 254 & SS9012a & Sailfin molly & Whole body & 79.6 & -- & 950 & 3.8 \\
\hline \multicolumn{8}{|c|}{ Site B32 (Johnson Street Drain) } \\
\hline 255 & SS90-s3 & Sediment & Sediment & 38.1 & -.. & 12,00 & 4.5 \\
\hline 256 & SS90b9 & Blue-green algae & Vegetation & 92.2 & $\ldots$ & 5,600 & 7.4 \\
\hline 257 & SS90v3 & Common cattail & Vegetation & 85.3 & --- & 140 & $<.64$ \\
\hline 258 & SS90c4 & Asiatic river clam & Soft tissue & 76.5 & --- & 240 & 7.3 \\
\hline
\end{tabular}


Table 22. Inorganic chemical analysis and moisture content for biotic samples collected during 1988-90 from the Salton Sea and associated drainwaters and rivers--Continued

\begin{tabular}{|c|c|c|c|c|c|c|c|c|c|c|c|}
\hline $\begin{array}{l}\text { Record } \\
\text { number }\end{array}$ & $\begin{array}{l}\text { Sample } \\
\text { number }\end{array}$ & $\begin{array}{l}\text { Bo- } \\
\text { ron }\end{array}$ & $\begin{array}{l}\text { Ba- } \\
\text { rium }\end{array}$ & $\begin{array}{l}\text { Be- } \\
\text { ryl- } \\
\text { lium }\end{array}$ & $\begin{array}{l}\text { Cad- } \\
\text { mium }\end{array}$ & $\begin{array}{c}\text { Chro- } \\
\text { mi- } \\
\text { um }\end{array}$ & $\begin{array}{l}\text { Cop- } \\
\text { per }\end{array}$ & Iron & $\begin{array}{l}\text { Mer- } \\
\text { cury }\end{array}$ & $\begin{array}{c}\text { Mag- } \\
\text { ne- } \\
\text { sium }\end{array}$ & $\begin{array}{c}\text { Man- } \\
\text { ga- } \\
\text { nese }\end{array}$ \\
\hline \multicolumn{12}{|c|}{ Site B25 (Vail Cutoff Drain)--Continued } \\
\hline 225 & SS89-72 & $<3$ & $<0.1$ & $<0.2$ & 1 & $<2$ & 19.9 & 2,080 & 0.52 & 763 & 12 \\
\hline 226 & SS89-73 & 4 & $<.1$ & $<.2$ & $<.7$ & $<2$ & 23.7 & 2,500 & .46 & 753 & 7.1 \\
\hline 227 & SS89-66 & $<3$ & .98 & $<.1$ & $<.5$ & $<2$ & 3.6 & 98.7 & .35 & 411 & 2.8 \\
\hline 228 & SS89-67 & $<3$ & .80 & $<.1$ & $<.5$ & $<2$ & 1.9 & 108 & .091 & 372 & 1.8 \\
\hline 229 & SS89-68 & $<3$ & .78 & $<.1$ & $<.6$ & $<2$ & 3.2 & 108 & .34 & 385 & 1.3 \\
\hline 230 & SS89-69 & $<3$ & .47 & $<.1$ & $<.5$ & $<2$ & 3.2 & 97 & .25 & 390 & 1.4 \\
\hline 231 & SS89-70 & $<3$ & .78 & $<.1$ & $<.5$ & $<2$ & 2.9 & 107 & 1.6 & 433 & 1.2 \\
\hline 232 & SS90c3 & $<18$ & 8.9 & $<.57$ & $<1.1$ & $<2.1$ & 47 & 710 & $<.23$ & 1,200 & 39 \\
\hline \multicolumn{12}{|c|}{ Site B26 (Vail 4 Drain) } \\
\hline 233 & LNSS88-32 & 5 & $<0.1$ & $<0.1$ & $<0.2$ & $<1$ & 22.2 & 2,430 & 0.15 & 706 & 12 \\
\hline 234 & SS89-160 & 2 & .1 & $<.1$ & .3 & $<1$ & 41.7 & 1,150 & .20 & 650 & 6.2 \\
\hline 235 & SS89-162 & 3 & .2 & $<.1$ & .3 & $<1$ & 63.2 & 1,480 & .12 & 665 & 5.0 \\
\hline 236 & SS89-164 & 5 & .2 & $<.1$ & $<.3$ & $<1$ & 22.8 & 1,470 & .26 & 770 & 5.4 \\
\hline \multicolumn{12}{|c|}{ Site B27 (Vail 4A Drain) } \\
\hline 237 & SS89-63 & 27 & 24.8 & $<0.2$ & $<0.7$ & 2 & 9.9 & 1,920 & 0.069 & 5,180 & 48.9 \\
\hline 238 & SS89-65 & 22 & 36.4 & $<.2$ & $<.7$ & 3 & 20.7 & 2,920 & .030 & 4,980 & 72.5 \\
\hline \multicolumn{12}{|c|}{ Site B29 (S Lateral Drain) } \\
\hline 239 & SS89-169 & 14 & 105 & 0.38 & 0.2 & 28 & 18 & 6,560 & 0.28 & 9,930 & 126 \\
\hline 240 & SS89-123 & 4 & 5.0 & $<.1$ & $<.4$ & 12 & 24.3 & 322 & .21 & 1,420 & 9.4 \\
\hline 241 & SS89-124 & 3 & 4.8 & $<.1$ & $<.2$ & 3.4 & 10 & 301 & .16 & 1,490 & 16 \\
\hline 242 & SS89-125 & 3 & 4.5 & $<.1$ & $<.3$ & 8.6 & 7.8 & 347 & .21 & 1,370 & 7.2 \\
\hline 243 & SS89-126 & 2 & 3.7 & $<.1$ & $<.2$ & 12 & 6.7 & 325 & .21 & 1,390 & 12 \\
\hline 244 & SS89-127 & 4 & 8.4 & $<.1$ & $<.3$ & 14 & 35.0 & 554 & .689 & 1,250 & 11 \\
\hline \multicolumn{12}{|c|}{ Site B30 (Z Lateral Drain) } \\
\hline 245 & SS90-s5 & $<13$ & 370 & $<1.3$ & $<1.3$ & 15 & 32 & 35,000 & $<0.12$ & 22,000 & 650 \\
\hline 246 & SS90v5 & $<51$ & 6.2 & $<2.3$ & $<2.3$ & $<64$ & $<5.1$ & 170 & $<.19$ & 2,000 & 150 \\
\hline 247 & SS90c5 & $<9.0$ & 18 & $<.29$ & 1.2 & $<1.1$ & 15 & 1,200 & $<.14$ & 1,000 & 34 \\
\hline 248 & SS90f5 & $<11$ & 13 & $<.36$ & $<.68$ & 44 & 16 & 980 & $<.17$ & 1,500 & 32 \\
\hline \multicolumn{12}{|c|}{ Site B31 (81st Street Drain) } \\
\hline 249 & SS90-s2 & $<13$ & 190 & $<1.3$ & $<1.3$ & 14 & 26 & 22,000 & $<0.11$ & 17,000 & 380 \\
\hline 250 & SS90a5 & 180 & 100 & $<2.3$ & $<2.3$ & 69 & 14 & 9,400 & --- & 8,900 & 180 \\
\hline 251 & SS90e7 & 130 & 71 & $<3.3$ & $<3.3$ & $<92$ & $<7.2$ & 5,300 & -- & 12,000 & 96 \\
\hline 252 & SS90v2 & $<68$ & 6.8 & $<3.1$ & $<3.1$ & $<87$ & $<6.8$ & 200 & $<.26$ & 1,800 & 140 \\
\hline 253 & SS90P2 & $<13$ & 5.1 & $<.44$ & $<.83$ & 8.5 & 13 & 160 & $<.16$ & 1,400 & 20 \\
\hline 254 & SS90f2a & $<15$ & 29 & $<.47$ & $<.90$ & 27 & 27 & 1,400 & $<.17$ & 1,800 & 47 \\
\hline \multicolumn{12}{|c|}{ Site B32 (Johnson Street Drain) } \\
\hline 255 & SS90-s3 & $<7.8$ & 90 & $<0.78$ & $<0.78$ & 7.1 & 15 & 20,000 & $<0.062$ & 8,400 & 290 \\
\hline 256 & SS90b9 & 270 & 83 & $<6.1$ & 8.0 & $<170$ & 13 & 8,400 & $<.1$ & 25,000 & 180 \\
\hline 257 & SS90v3 & $<75$ & 8.6 & $<3.4$ & $<3.4$ & $<95$ & $<7.5$ & 310 & $<.26$ & 2,200 & 160 \\
\hline 258 & SS90c4 & $<13$ & 3.8 & $<.41$ & $<.78$ & $<1.5$ & 30 & 260 & $<.14$ & 700 & 12 \\
\hline
\end{tabular}


Table 22. Inorganic chemical analysis and moisture content for biotic samples collected during $1988-90$ from the Salton Sea and associated drainwaters and rivers--Continued

\begin{tabular}{|c|c|c|c|c|c|c|c|c|c|c|c|}
\hline $\begin{array}{l}\text { Record } \\
\text { number }\end{array}$ & $\begin{array}{l}\text { Sample } \\
\text { number }\end{array}$ & $\begin{array}{l}\text { Bo- } \\
\text { ron }\end{array}$ & $\begin{array}{l}\mathrm{Ba}- \\
\text { rium }\end{array}$ & $\begin{array}{l}\text { Be- } \\
\text { ryl- } \\
\text { lium }\end{array}$ & $\begin{array}{l}\text { Cad- } \\
\text { mium }\end{array}$ & $\begin{array}{c}\text { Chro- } \\
\text { mi- } \\
\text { um }\end{array}$ & $\begin{array}{l}\text { Cop- } \\
\text { per }\end{array}$ & Iron & $\begin{array}{l}\text { Mer- } \\
\text { cury }\end{array}$ & $\begin{array}{c}\text { Mag- } \\
\text { ne- } \\
\text { sium }\end{array}$ & $\begin{array}{c}\text { Man- } \\
\text { ga- } \\
\text { nese }\end{array}$ \\
\hline \multicolumn{12}{|c|}{ Site B18 (Alamo River at Garst Road)--Continued } \\
\hline 191 & SS89-167 & 7.5 & 86.0 & 0.2 & 0.6 & 12 & 65.7 & 3,470 & 0.16 & 2,700 & 93.1 \\
\hline 192 & SS89-175 & 3 & 10.8 & $<.1$ & $<.2$ & 1 & 16 & 341 & .13 & 1,260 & 11 \\
\hline 193 & SS89-176 & 5 & 12.5 & $<.1$ & $<.2$ & 2 & 20.5 & 610 & .17 & 2,120 & 18 \\
\hline \multicolumn{12}{|c|}{ Site B19 (San Felipe Creek) } \\
\hline 194 & SS90-s 1 & $<5.8$ & 83 & $<0.58$ & $<0.58$ & 6.9 & 9.4 & 7,700 & $<0.055$ & 3,900 & 200 \\
\hline 195 & SS90v1 & 110 & 11 & $<4.1$ & $<4.1$ & $<110$ & 190 & 880 & .38 & 2,800 & 230 \\
\hline 196 & SS90f1 & $<45$ & $<4.3$ & $<1.4$ & $<2.8$ & 6.4 & 11 & 140 & .23 & 1,700 & 20 \\
\hline 197 & SS90f1a & $<9.1$ & 3.9 & $<.29$ & $<.56$ & 510 & 21 & 2,700 & .12 & 670 & 55 \\
\hline \multicolumn{12}{|c|}{ Site B20 (Salt Creek) } \\
\hline 198 & SS90-s4 & $<8.6$ & 240 & $<0.86$ & $<0.86$ & 11 & 28 & 19,000 & $<0.080$ & 12,000 & 380 \\
\hline 199 & SS90v4 & 99 & 23 & $<2.9$ & $<2.9$ & $<81$ & $<6.4$ & 1,300 & $<.23$ & 3,700 & 140 \\
\hline 200 & SS90f4 & $<14$ & 4.6 & $<.44$ & $<.84$ & 7.1 & 4.5 & 120 & $<.17$ & 560 & 11 \\
\hline 201 & SS90f4a & $<11$ & 14 & $<.36$ & $<.69$ & 48 & 12 & 1,100 & $<.15$ & 1,800 & 38 \\
\hline \multicolumn{12}{|c|}{ Site B21 (Colorado River at Palo Verde) } \\
\hline 202 & SS89-117 & $<3$ & 22.6 & $<0.1$ & $<0.6$ & $<2$ & 27.0 & 213 & 0.072 & 915 & 10 \\
\hline 203 & SS89-118 & $<3$ & 25.5 & $<.1$ & $<.5$ & $<2$ & 48.8 & 535 & .10 & 1,050 & 20.9 \\
\hline 204 & SS89-119 & $<3$ & 19.6 & $<.1$ & $<.6$ & $<2$ & 24.2 & 313 & .096 & 1,050 & 14 \\
\hline \multicolumn{12}{|c|}{ Site B23 (Trifolium 13 Drain) } \\
\hline 205 & SS89-122 & 5 & 30 & $<0.1$ & 1 & $<2$ & 58.5 & 492 & 0.17 & 1,390 & 17 \\
\hline 206 & SS89-166 & 8.4 & 130 & .1 & .7 & 8.8 & 68.1 & 2,510 & .20 & 1,980 & 88.2 \\
\hline 207 & SS89-168 & 6.9 & 105 & .1 & .80 & 8.3 & 90.1 & 2,360 & .22 & 2,090 & 69.7 \\
\hline \multicolumn{12}{|c|}{ Site B24 (Trifolium 14 Drain) } \\
\hline 208 & LNSS88-180 & -- & -- & $<0.01$ & $<0.03$ & $<0.1$ & 3.44 & 123 & 0.21 & -- & 1.4 \\
\hline 209 & LNSS88-194 & 3 & .79 & $<.1$ & $<.4$ & $<1$ & 3.8 & 93 & .36 & 355 & .96 \\
\hline 210 & LNSS88-206 & 3 & 1.2 & $<.1$ & $<.4$ & $<1$ & 3.4 & 110 & .30 & 471 & 1.1 \\
\hline 211 & LNSS88-208 & -- & $\cdots$ & $<.01$ & $<.03$ & $<.1$ & 3.07 & 117 & 1.5 & -- & 1.1 \\
\hline 212 & LNSS88-215 & $<2$ & 1.4 & $<.1$ & $<.4$ & $<1$ & 3.5 & 120 & .27 & 427 & .98 \\
\hline 213 & LNSS88-217 & - & -- & $<.009$ & $<.03$ & 1.9 & 3.20 & 119 & .15 & -- & 1.2 \\
\hline 214 & LNSS88-218 & $<2$ & .42 & $<.1$ & $<.4$ & $<1$ & 2.0 & 48 & .40 & 208 & $<.3$ \\
\hline 215 & LNSS88-223 & - & - & $<.01$ & $<.1$ & $<.4$ & 2.90 & 83.6 & .28 & -- & .52 \\
\hline 216 & LNSS88-224 & 3 & .91 & $<.1$ & $<.4$ & $<1$ & 4.8 & 110 & .13 & 499 & .9 \\
\hline 217 & LNSS88-226 & -- & -- & $<.01$ & $<.03$ & $<.1$ & 3.02 & 98.0 & .26 & -- & 1.1 \\
\hline 218 & LNSS88-227 & 4 & 1.4 & $<.1$ & $<.4$ & $<1$ & 3.1 & 120 & .11 & 543 & .9 \\
\hline 219 & SS89-45 & $<2$ & $<.1$ & $<.1$ & .4 & 1 & 40.1 & 2,750 & .30 & 639 & 13 \\
\hline 220 & SS89-46 & 2 & $<.1$ & $<.1$ & .7 & $<1$ & 240 & 2,760 & .518 & 754 & 16 \\
\hline 221 & SS89-50 & 5 & $<.1$ & $<.1$ & .6 & $<1$ & 27.1 & 870 & 1.09 & 637 & 8.4 \\
\hline 222 & SS90c1 & $<25$ & 3.3 & $<.88$ & $<1.7$ & $<2.9$ & 33 & 290 & $<.11$ & 440 & $<8.8$ \\
\hline 223 & SS90c2 & $<18$ & 5.6 & $<.57$ & $<1.1$ & $<2.1$ & 35 & 240 & $<.27$ & 910 & 22 \\
\hline \multicolumn{12}{|c|}{ Site B25 (Vail Cutoff Drain) } \\
\hline 224 & SS89-71 & $<3$ & 0.1 & $<0.2$ & $<0.7$ & $<2$ & 16 & 5,120 & 0.34 & 828 & 12 \\
\hline
\end{tabular}


Table 22. Inorganic chemical analysis and moisture content for biotic samples collected during 1988-90 from the Salton Sea and associated drainwaters and rivers--Continued

\begin{tabular}{|c|c|c|c|c|c|c|c|c|c|c|c|}
\hline $\begin{array}{l}\text { Record } \\
\text { number }\end{array}$ & $\begin{array}{l}\text { Sample } \\
\text { number }\end{array}$ & $\begin{array}{l}\text { Molyb- } \\
\text { de- } \\
\text { num }\end{array}$ & $\begin{array}{l}\text { Nick- } \\
\text { el }\end{array}$ & Lead & $\begin{array}{l}\text { Anti- } \\
\text { mo- } \\
\text { ny }\end{array}$ & $\begin{array}{l}\text { Sele- } \\
\text { ni- } \\
\text { um }\end{array}$ & Tin & $\begin{array}{l}\text { Stron- } \\
\text { ti- } \\
\text { um }\end{array}$ & $\begin{array}{l}\text { Thal- } \\
\text { li- } \\
\text { um }\end{array}$ & $\begin{array}{c}\text { Va- } \\
\text { nadi- } \\
\text { um }\end{array}$ & Zinc \\
\hline \multicolumn{12}{|c|}{ Site B18 (Alamo River at Garst Road)--Continued } \\
\hline 191 & SS89-167 & $<1$ & 6.1 & $<4$ & -- & 5.7 & -- & 100 & $<4$ & 8.8 & 111 \\
\hline 192 & SS89-175 & $<1$ & $<2$ & $<4$ & --- & 5.4 & --- & 72.0 & $<4$ & .7 & 220 \\
\hline 193 & SS89-176 & $<1$ & $<2$ & $<4$ & --- & 3.6 & --- & 183 & $<4$ & 1.4 & 114 \\
\hline \multicolumn{12}{|c|}{ Site B19 (San Felipe Creek) } \\
\hline 194 & SS90-s1 & $<1.8$ & 4.6 & 5.8 & - & 0.31 & $<5.8$ & 82 & - & 12 & 48 \\
\hline 195 & SS90v1 & $<31$ & 9.2 & $<32$ & - & 1.1 & $<41$ & 160 & --- & $<10$ & $<220$ \\
\hline 196 & SS90f1 & $<3.5$ & 3.5 & $<3.0$ & - & 7.4 & $<14$ & 190 & --- & $<1.4$ & 210 \\
\hline 197 & SS90f1a & 4.8 & 280 & $<.62$ & -- & 7.4 & 3.8 & 87 & --- & .88 & 43 \\
\hline \multicolumn{12}{|c|}{ Site B20 (Salt Creek) } \\
\hline 198 & SS90-s4 & $<2.8$ & 13 & 13 & --- & 0.89 & $<8.6$ & 370 & --- & 20 & 53 \\
\hline 199 & SS90v4 & $<22$ & $<4.6$ & $<23$ & $\ldots$ & $<.62$ & $<29$ & 310 & --- & $<7.2$ & $<160$ \\
\hline 200 & SS90f4 & $<1.1$ & 5.5 & $<.92$ & $-\ldots$ & 6.4 & $<4.4$ & 200 & --- & $<.44$ & 92 \\
\hline 201 & SS90f4a & 1.0 & 19 & $<.76$ & -- & 5.5 & $<3.6$ & 370 & -- & 1.3 & 82 \\
\hline \multicolumn{12}{|c|}{ Site B21 (Colorado River at Palo Verde) } \\
\hline 202 & SS89-117 & $<1$ & $<3$ & $<4$ & -- & 5.2 & -- & 40.8 & $<4$ & $<0.7$ & 101 \\
\hline 203 & SS89-118 & $<1$ & $<3$ & $<4$ & - & 6.2 & -- & 49.3 & $<4$ & $<.6$ & 144 \\
\hline 204 & SS89-119 & $<1$ & $<3$ & $<4$ & - & 4.6 & -- & 47.5 & $<4$ & $<.7$ & 120 \\
\hline \multicolumn{12}{|c|}{ Site B23 (Trifolium 13 Drain) } \\
\hline 205 & SS89-122 & $<1$ & $<3$ & $<4$ & -- & 7.5 & -- & 50.7 & $<4$ & 0.8 & 124 \\
\hline 206 & SS89-166 & $<1$ & 5.4 & $<5$ & -- & 6.2 & --- & 132 & $<4$ & 5.2 & 87.2 \\
\hline 207 & SS89-168 & $<1$ & 4 & $<4$ & -- & 6.6 & -- & 219 & $<4$ & 4.7 & 86.3 \\
\hline \multicolumn{12}{|c|}{ Site B24 (Trifolium 14 Drain) } \\
\hline 208 & LNSS88-180 & -- & $<0.2$ & $<0.5$ & -- & 5.7 & -- & - & $<0.7$ & --- & 52.1 \\
\hline 209 & LNSS88-194 & $<1$ & $<2$ & $<4$ & --- & 7.0 & --- & 37.9 & $<4$ & $<4$ & 46.4 \\
\hline 210 & LNSS88-206 & $<1$ & $<2$ & $<4$ & --- & 4.8 & --- & 40.4 & $<4$ & $<4$ & 48.9 \\
\hline 211 & LNSS88-208 & -- & $<.2$ & $<.5$ & --- & 5.9 & $\cdots$ & - & $<.7$ & -- & 48.8 \\
\hline 212 & LNSS88-215 & $<1$ & $<2$ & $<4$ & -- & 4.6 & $\ldots$ & 41.6 & $<4$ & $<4$ & 51.2 \\
\hline 213 & LNSS88-217 & - & $<.1$ & $<.4$ & -- & 3.8 & $\cdots$ & -- & $<.5$ & --- & 47.1 \\
\hline 214 & LNSS88-218 & $<1$ & $<2$ & $<4$ & --- & 1.6 & --- & 13.2 & $<4$ & $<4$ & 21 \\
\hline 215 & LNSS88-223 & - & $<.4$ & $<.5$ & - & 4.5 & --- & -- & $<.5$ & --- & 43.7 \\
\hline 216 & LNSS88-224 & $<1$ & $<2$ & $<4$ & - & 6.6 & -- & 30.4 & $<4$ & $<4$ & 53.7 \\
\hline 217 & LNSS88-226 & - & $<.2$ & $<.5$ & - & 3.7 & -- & -- & $<.7$ & --- & 42.1 \\
\hline 218 & LNSS88-227 & $<1$ & $<2$ & $<4$ & -. & 6.0 & -- & 38.2 & $<4$ & $<4$ & 56.8 \\
\hline 219 & SS89-45 & 2 & $<1$ & $<4$ & -- & 7.7 &.- & .64 & $<5$ & $<.3$ & 119 \\
\hline 220 & SS89-46 & 3 & $<1$ & $<4$ & -- & 21.7 & --- & .2 & $<5$ & $<.3$ & 164 \\
\hline 221 & SS89-50 & 1 & $<1$ & $<4$ & -- & 7.1 & - & 1.0 & $<5$ & $<.3$ & 90.0 \\
\hline 222 & SS90c1 & 2.1 & $<.88$ & $<1.7$ & -- & 4.4 & $<8.8$ & 33 & -- & $<.79$ & 45 \\
\hline 223 & SS90c2 & $<1.4$ & $<.57$ & 1.4 & -- & 6.3 & $<5.7$ & 30 & --- & $<.57$ & 96 \\
\hline \multicolumn{12}{|c|}{ Site B25 (Vail Cutoff Drain) } \\
\hline 224 & SS89-71 & 3 & $<4$ & $<4$ & -- & 7.9 & -- & 2.4 & $<4$ & $<0.8$ & 128 \\
\hline
\end{tabular}


Table 22. Inorganic chemical analysis and moisture content for biotic samples collected during $1988-90$ from the Salton Sea and associated drainwaters and rivers--Continued

\begin{tabular}{|c|c|c|c|c|c|c|c|c|c|c|c|}
\hline $\begin{array}{l}\text { Record } \\
\text { number }\end{array}$ & $\begin{array}{l}\text { Sample } \\
\text { number }\end{array}$ & $\begin{array}{c}\text { Molyb- } \\
\text { de- } \\
\text { num }\end{array}$ & $\begin{array}{l}\text { Nick- } \\
\text { el }\end{array}$ & Lead & $\begin{array}{l}\text { Anti- } \\
\text { mo- } \\
\text { ny }\end{array}$ & $\begin{array}{c}\text { Sele- } \\
\text { ni- } \\
\text { um }\end{array}$ & Tin & $\begin{array}{c}\text { Stron- } \\
\text { ti- } \\
\text { um }\end{array}$ & $\begin{array}{c}\text { Thal- } \\
\text { li- } \\
\text { um }\end{array}$ & $\begin{array}{c}\text { Va- } \\
\text { nadi- } \\
\text { um }\end{array}$ & Zinc \\
\hline \multicolumn{12}{|c|}{ Site B25 (Vail Cutoff Drain)--Continued } \\
\hline 225 & SS89-72 & 5.4 & $<4$ & $<4$ & --- & 16.3 & --- & 1.3 & $<4$ & $<0.8$ & 121 \\
\hline 226 & SS89-73 & 4 & $<4$ & $<4$ & -- & 8.4 & -- & 1.5 & $<4$ & $<.8$ & 120 \\
\hline 227 & SS89-66 & $<1$ & $<3$ & 12 & -- & 4.1 & --- & 41 & $<4$ & $<.6$ & 48.0 \\
\hline 228 & SS89-67 & $<1$ & $<3$ & $<4$ & $\ldots$ & 4.2 & -- & 15.5 & $<4$ & $<.7$ & 41 \\
\hline 229 & SS89-68 & $<1$ & $<3$ & $<4$ & - & 3.7 & -- & 25.3 & $<4$ & $<.7$ & 48 \\
\hline 230 & SS89-69 & $<1$ & $<3$ & $<4$ & - & 3.7 & - & 23.9 & $<4$ & $<.7$ & 42 \\
\hline 231 & SS89-70 & $<1$ & $<3$ & $<4$ & -- & 3.8 & -- & 34.2 & $<4$ & $<.6$ & 48.7 \\
\hline 232 & SS90c3 & $<1.4$ & $<.57$ & $<1.2$ & -- & 5.6 & $<5.7$ & 29 & -- & 1.1 & 93 \\
\hline \multicolumn{12}{|c|}{ Site B26 (Vail 4 Drain) } \\
\hline 233 & LNSS88-32 & 2 & $<1$ & $<4$ & - & 6.4 & -- & 0.50 & $<5$ & 0.5 & 175 \\
\hline 234 & SS89-160 & $<1$ & $<1$ & $<4$ & -- & 10 & $\ldots$ & 2.5 & $<5$ & $<.3$ & 93.6 \\
\hline 235 & SS89-162 & 1 & $<1$ & $<4$ & -- & 14 & -- & 5.8 & $<5$ & $<.3$ & 85.6 \\
\hline 236 & SS89-164 & $<1$ & $<1$ & $<4$ & -- & 12 & - & 5.5 & $<5$ & $<.3$ & 90.0 \\
\hline \multicolumn{12}{|c|}{ Site B27 (Vail 4A Drain) } \\
\hline 237 & SS89-63 & $<1$ & $<4$ & $<4$ & $\ldots$ & 8.6 & - & 301 & $<4$ & 4.3 & 89.7 \\
\hline 238 & SS89-65 & $<1$ & $<4$ & 5 & - & 7.2 & -- & 378 & $<4$ & 7.4 & 76.1 \\
\hline \multicolumn{12}{|c|}{ Site B29 (S Lateral Drain) } \\
\hline 239 & SS89-169 & $<1$ & 12 & 6 & - & 4.8 & -- & 375 & $<4$ & 16 & 101 \\
\hline 240 & SS89-123 & $<1$ & 6.2 & $<6$ & -- & 3.6 & -- & 205 & $<4$ & .8 & 94.4 \\
\hline 241 & SS89-124 & $<1$ & $<2$ & $<4$ & - & 6.7 & -- & 142 & $<4$ & .4 & 102 \\
\hline 242 & SS89-125 & $<1$ & 4 & $<4$ & -- & 3.5 & - & 188 & $<4$ & .4 & 91.0 \\
\hline 243 & SS89-126 & $<1$ & 5.9 & $<4$ & -- & 3.2 & -- & 172 & $<4$ & .4 & 97.5 \\
\hline 244 & SS89-127 & 1 & 3 & $<5$ & -- & 4.2 & - & 126 & $<4$ & .7 & 94.7 \\
\hline \multicolumn{12}{|c|}{ Site B30 (Z Lateral Drain) } \\
\hline 245 & SS90-s5 & $<4.2$ & 23 & 21 & --- & 1.6 & 13 & 460 & -- & 33 & 98 \\
\hline 246 & SS90v5 & $<18$ & $<3.7$ & $<18$ & -- & $<.46$ & $<23$ & 98 & -- & $<5.7$ & $<120$ \\
\hline 247 & SS90c5 & $<.70$ & 1.1 & $<.61$ & --- & 2.9 & $<2.9$ & 33 & -- & 2.1 & 52 \\
\hline 248 & SS90f5 & $<.86$ & 19 & $<.75$ & -- & 5.3 & $<3.6$ & 180 & --- & 1.4 & 76 \\
\hline \multicolumn{12}{|c|}{ Site B31 (81st Street Drain) } \\
\hline 249 & SS90-s2 & $<4.2$ & 10 & 6.3 & --- & 3.8 & $<13$ & 1,200 & -- & 29 & 65 \\
\hline 250 & SS90a5 & $<17$ & 48 & $<18$ & --- & .98 & $<23$ & 520 &.- & 17 & $<120$ \\
\hline 251 & SS90e7 & $<25$ & 12 & $<26$ & -- & $<.73$ & $<33$ & 830 & --- & 17 & $<180$ \\
\hline 252 & SS90v2 & $<24$ & $<5.0$ & $<25$ & --- & $<.63$ & $<31$ & 140 & --- & $<7.8$ & $<170$ \\
\hline 253 & SS90P2 & $<1.0$ & 4.7 & $<.91$ & --- & 4.7 & $<4.4$ & 180 & --- & 1.2 & 160 \\
\hline 254 & SS90f $2 a$ & 1.6 & 9.3 & $<1.0$ & --- & 5.8 & $<4.7$ & 310 & -- & 3.5 & 100 \\
\hline \multicolumn{12}{|c|}{ Site B32 (Johnson Street Drain) } \\
\hline 255 & SS90-s3 & $<2.5$ & 4.9 & 1.6 & --- & 0.55 & $<7.8$ & 160 & --- & 27 & 61 \\
\hline 256 & SS90b9 & $<47$ & 12 & $<48$ & --- & 1.8 & $<61$ & 650 & --- & $<15$ & $<330$ \\
\hline 257 & SS90v3 & $<26$ & $<5.4$ & $<27$ & -- & $<.64$ & $<34$ & 200 & -.- & $<8.5$ & $<180$ \\
\hline 258 & SS90c4 & $<.98$ & $<.41$ & $<.86$ & -- & 2.6 & $<4.1$ & 26 & --- & .49 & 89 \\
\hline
\end{tabular}


Table 22. Inorganic chemical analysis and moisture content for biotic samples collected during 1988-90 from the Salton Sea and associated drainwaters and rivers--Continued

\begin{tabular}{|c|c|c|c|c|c|c|c|}
\hline $\begin{array}{l}\text { Record } \\
\text { number }\end{array}$ & $\begin{array}{l}\text { Sample } \\
\text { number }\end{array}$ & Species & Matrix & $\begin{array}{l}\text { Percent } \\
\text { moisture }\end{array}$ & $\begin{array}{l}\text { Sil- } \\
\text { ver }\end{array}$ & $\begin{array}{c}\text { Alu- } \\
\text { mi- } \\
\text { num }\end{array}$ & $\begin{array}{l}\text { Arse- } \\
\text { nic }\end{array}$ \\
\hline \multicolumn{8}{|c|}{ Site B32 (Johnson Street Drain)--Continued } \\
\hline 259 & SS90ß3 & Mosquitofish & Whole body & 75.2 & --- & 2,000 & 23 \\
\hline 260 & SS90ß3a & Sailfin molly & Whole body & 75.4 & --- & 1,600 & $<0.36$ \\
\hline \multicolumn{8}{|c|}{ Site B33 (Shady Acres Duck Club) } \\
\hline 261 & LNSS88-54 & Northern shoveler & Liver & 70.5 & $<2$ & 6 & 0.2 \\
\hline 262 & LNSS88-58 & Northern shoveler & Liver & 71.0 & $<2$ & 20 & .3 \\
\hline 263 & LNSS88-60 & Northern shoveler & Liver & 71.1 & $<2$ & 15 & .4 \\
\hline 264 & LNSS88-53 & Northern shoveler & Breast Muscle & 73.0 & $<2$ & 70 & $<.2$ \\
\hline 265 & LNSS88-57 & Northern shoveler & Breast muscle & 72.3 & $<2$ & 7 & $<.2$ \\
\hline \multicolumn{8}{|c|}{ Site B34 (RH Pond) } \\
\hline 266 & LNSS88-97 & Black-necked stilt & Egg & 68.9 & $<2$ & $<3$ & $<0.2$ \\
\hline 267 & LNSS88-99 & Black-necked stilt & Egg & 72.5 & --- & 1.2 & $<.2$ \\
\hline 268 & LNSS88-100 & Black-necked stilt & Egg & 71.7 & $<2$ & $<3$ & $<.2$ \\
\hline 269 & LNSS88-102 & Black-necked stilt & Egg & 71.4 & -- & 1 & $<.2$ \\
\hline 270 & LNSS88-103 & Black-necked stilt & Egg & 71.5 & $<2$ & 4 & .4 \\
\hline 271 & LNSS88-105 & Black-necked stilt & Egg & 62.5 & -- & 1.1 & $<.2$ \\
\hline 272 & LNSS88-106 & Black-necked stilt & Egg & 72.7 & $<2$ & $<3$ & $<.2$ \\
\hline 273 & LNSS88-108 & Black-necked stilt & Egg & 68.6 & -- & 1.1 & $<.2$ \\
\hline 274 & LNSS88-109 & Black-necked stilt & Egg & 71.1 & $<2$ & 3 & $<.2$ \\
\hline 275 & LNSS88-127 & Black-necked stilt & Egg & 73.7 & $<2$ & 5 & $<.2$ \\
\hline 276 & LNSS88-133 & Black-necked stilt & Egg & 72.1 & $<2$ & 4 & $<.2$ \\
\hline \multicolumn{8}{|c|}{ Site B35 (HQ Pond) } \\
\hline 277 & LNSS88-34 & Ruddy duck & Liver & 71.1 & $<2$ & $<3$ & $<0.2$ \\
\hline 278 & LNSS88-36 & Ruddy duck & Liver & 70.0 & $<2$ & 6 & $<.2$ \\
\hline 279 & LNSS88-38 & Ruddy duck & Liver & 74.1 & 6.4 & 4 & $<.2$ \\
\hline 280 & LNSS88-40 & Ruddy duck & Liver & 69.3 & $<2$ & 7 & $<.2$ \\
\hline 281 & LNSS88-35 & Ruddy duck & Breast muscle & 70.3 & $<2$ & $<3$ & $<.2$ \\
\hline 282 & LNSS88-39 & Ruddy duck & Breast muscle & 71.3 & $<2$ & $<3$ & $<.2$ \\
\hline \multicolumn{8}{|c|}{ Site B36 (Reidman Pond) } \\
\hline 283 & LNSS88-172 & Black-necked stilt & Egg & 71.6 & $<2$ & $<3$ & $<0.2$ \\
\hline 284 & LNSS88-175 & Black-necked stilt & Egg & 73.7 & $<2$ & $<3$ & $<.2$ \\
\hline 285 & LNSS88-178 & Black-necked stilt & Egg & 74.1 & $<2$ & $<3$ & $<.2$ \\
\hline 286 & LNSS88-184 & Black-necked stilt & Egg & 73.5 & $<2$ & $<3$ & $<.2$ \\
\hline 287 & LNSS88-189 & Black-necked stilt & Egg & 74.0 & --- & 1.9 & $<.2$ \\
\hline 288 & LNSS88-196 & Black-necked stilt & Egg & 74.3 & --- & 1.7 & $<.2$ \\
\hline 289 & LNSS88-197 & Black-necked stilt & Egg & 73.2 & $<2$ & 6 & $<.2$ \\
\hline 290 & LNSS88-199 & Black-necked stilt & Egg & 72.3 & --. & 3.2 & $<.2$ \\
\hline 291 & LNSS88-200 & Black-necked stilt & Egg & 72.3 & $<2$ & $<3$ & $<.2$ \\
\hline 292 & LNSS88-203 & Black-necked stilt & Egg & 72.5 & $<2$ & 5 & $<.2$ \\
\hline 293 & LNSS88-209 & Black-necked stilt & Egg & 71.1 & $<2$ & $<3$ & $<.2$ \\
\hline 294 & LNSS88-220 & Black-necked stilt & Egg & 73.1 & $\ldots$ & 1.5 & $<.2$ \\
\hline 295 & LNSS88-221 & Black-necked stilt & Egg & 70.3 & $<2$ & $<3$ & $<.2$ \\
\hline 296 & LNSS88-230 & Black-necked stilt & Egg & 72.2 & $<2$ & $<3$ & $<.2$ \\
\hline
\end{tabular}


Table 22. Inorganic chemical analysis and moisture content for biotic samples collected during 1988-90 from the Salton Sea and associated drainwaters and rivers--Continued

\begin{tabular}{|c|c|c|c|c|c|c|c|c|c|c|c|}
\hline $\begin{array}{l}\text { Record } \\
\text { number }\end{array}$ & $\begin{array}{l}\text { Sample } \\
\text { number }\end{array}$ & $\begin{array}{l}\text { Bo- } \\
\text { ron }\end{array}$ & $\begin{array}{l}\text { Ba- } \\
\text { rium }\end{array}$ & $\begin{array}{l}\text { Be- } \\
\text { ryl- } \\
\text { lium }\end{array}$ & $\begin{array}{l}\text { Cad- } \\
\text { mium }\end{array}$ & $\begin{array}{l}\text { Chro- } \\
\text { mi- } \\
\text { um }\end{array}$ & $\begin{array}{c}\text { Cop- } \\
\text { per }\end{array}$ & Iron & $\begin{array}{l}\text { Mer- } \\
\text { cury }\end{array}$ & $\begin{array}{c}\text { Mag- } \\
\text { ne- } \\
\text { sium }\end{array}$ & $\begin{array}{c}\text { Man- } \\
\text { ga- } \\
\text { nese }\end{array}$ \\
\hline \multicolumn{12}{|c|}{ Site B32 (Johnson Street Drain)--Continued } \\
\hline 259 & SS90f3 & $<12$ & 23 & $<0.39$ & $<0.74$ & 55 & 7.7 & 2,600 & $<0.15$ & 2,200 & 55 \\
\hline 260 & SS90f3a & $<14$ & 34 & $<.45$ & $<.85$ & 13 & 23 & 1,700 & $<.18$ & 2,100 & 47 \\
\hline \multicolumn{12}{|c|}{ Site B33 (Shady Acres Duck Club) } \\
\hline 261 & LNSS88-54 & 4 & 0.3 & $<0.1$ & 1.8 & 2 & 59.4 & 9,500 & 0.681 & 778 & 16 \\
\hline 262 & LNSS88-58 & 5 & .3 & $<.1$ & .82 & 2 & 20.9 & 6,340 & .970 & 737 & 7.9 \\
\hline 263 & LNSS88-60 & 6.3 & .2 & $<.1$ & 1.3 & 2 & 84.9 & 4,810 & 7.96 & 711 & 10 \\
\hline 264 & LNSS88-53 & $<2$ & .54 & $<.1$ & $<.2$ & $<1$ & 22.6 & 316 & .16 & 1,150 & 3.1 \\
\hline 265 & LNSS88-57 & 3 & $<.1$ & $<.1$ & $<.2$ & $<1$ & 20.2 & 302 & .20 & 1,090 & 1.4 \\
\hline \multicolumn{12}{|c|}{ Site B34 (RH Pond) } \\
\hline 266 & LNSS88-97 & 5 & 0.50 & $<0.1$ & $<0.4$ & $<1$ & 3.7 & 80 & 0.45 & 433 & 0.99 \\
\hline 267 & LNSS88-99 & -- & -- & $<.01$ & $<.03$ & .2 & 3.45 & 112 & .077 & -- & 1.2 \\
\hline 268 & LNSS88-100 & 4 & .72 & $<.1$ & $<.4$ & $<1$ & 3.6 & 110 & .074 & 457 & 1.3 \\
\hline 269 & LNSS88-102 & -- & $\cdots$ & $<.01$ & $<.03$ & .3 & 3.08 & 91.7 & .44 & --- & 1.8 \\
\hline 270 & LNSS88-103 & 6 & .2 & $<.1$ & $<.4$ & $<1$ & 3.7 & 100 & .33 & 403 & 4.5 \\
\hline 271 & LNSS88-105 & -- & $\cdots$ & $<.01$ & $<.03$ & $<.1$ & 3.17 & 113 & .10 & --- & 1.9 \\
\hline 272 & LNSS88-106 & 4 & .81 & $<.1$ & $<.4$ & $<1$ & 2.9 & 87 & .094 & 412 & 1.4 \\
\hline 273 & LNSS88-108 & $\cdots$ & -- & $<.01$ & $<.03$ & .3 & 3.22 & 112 & .75 & --. & 2.2 \\
\hline 274 & LNSS88-109 & 4 & .2 & $<.1$ & $<.4$ & $<1$ & 3.2 & 100 & .746 & 421 & 2.7 \\
\hline 275 & LNSS88-127 & 2 & .38 & $<.1$ & $<.4$ & $<1$ & 4.5 & 76 & 2.7 & 448 & 3.5 \\
\hline 276 & LNSS88-133 & 2 & .78 & $<.1$ & $<.4$ & $<1$ & 3.3 & 100 & .905 & 483 & 1.9 \\
\hline \multicolumn{12}{|c|}{ Site B35 (HQ Pond) } \\
\hline 277 & LNSS88-34 & 3 & $<0.1$ & $<0.1$ & $<0.2$ & $<1$ & 66.6 & 1,100 & 0.835 & 785 & 14 \\
\hline 278 & LNSS88-36 & 5 & .1 & $<.1$ & $<.2$ & $<1$ & 13 & 5,680 & .16 & 832 & 12 \\
\hline 279 & LNSS88-38 & 5 & $<.1$ & $<.1$ & .5 & $<1$ & 118 & 811 & .13 & 796 & 11 \\
\hline 280 & LNSS88-40 & 4 & .2 & $<.1$ & 2.0 & $<1$ & 30.6 & 1,480 & 1.3 & 802 & 22.4 \\
\hline 281 & LNSS88-35 & 3 & $<.1$ & $<.1$ & $<.2$ & $<1$ & 28.8 & 589 & .046 & 1,040 & 2.0 \\
\hline 282 & LNSS88-39 & $<2$ & $<.1$ & $<.1$ & $<.2$ & $<1$ & 32.5 & 436 & .24 & 1,040 & 2.2 \\
\hline \multicolumn{12}{|c|}{ Site B36 (Reidman Pond) } \\
\hline 283 & LNSS88-172 & 3 & 2.9 & $<0.1$ & $<0.4$ & $<1$ & 3.2 & 93 & 0.44 & 410 & 1.8 \\
\hline 284 & LNSS88-175 & $<2$ & 1.2 & $<.1$ & $<.4$ & $<1$ & 4.4 & 110 & .616 & 391 & .5 \\
\hline 285 & LNSS88-178 & $<2$ & 1.6 & $<.1$ & $<.4$ & $<1$ & 3.5 & 90 & .46 & 480 & .5 \\
\hline 286 & LNSS88-184 & 4 & 1.4 & $<.1$ & $<.4$ & $<1$ & 3.4 & 97 & .47 & 435 & .9 \\
\hline 287 & LNSS88-189 & -- & $\ldots$ & $<.01$ & $<.03$ & $<.1$ & 3.32 & 111 & .35 & -- & 1.2 \\
\hline 288 & LNSS88-196 & $\cdots$ & -- & $<.01$ & .35 & $<.1$ & 3.46 & 131 & .34 & -- & 1.1 \\
\hline 289 & LNSS88-197 & 3 & .61 & $<.1$ & $<.4$ & $<1$ & 4.3 & 86 & .702 & 433 & .96 \\
\hline 290 & LNSS88-199 & $\cdots$ & -- & $<.01$ & $<.03$ & $<.1$ & 3.50 & 104 & .36 & -- & 1.4 \\
\hline 291 & LNSS88-200 & 3 & 1.3 & $<.1$ & $<.4$ & $<1$ & 3.5 & 95 & .25 & 463 & 1.0 \\
\hline 292 & LNSS88-203 & 4 & 1.8 & $<.1$ & $<.4$ & $<1$ & 3.8 & 97 & .39 & 550 & .9 \\
\hline 293 & LNSS88-209 & 2 & 1.8 & $<.1$ & $<.4$ & $<1$ & 3.5 & 100 & .16 & 426 & .9 \\
\hline 294 & LNSS88-220 &.-- & --- & $<.01$ & $<.03$ & .80 & 3.61 & 118 & .41 & -- & 1.4 \\
\hline 295 & LNSS88-221 & 2 & 1.4 & $<.1$ & $<.4$ & $<1$ & 4.0 & 97 & .40 & 507 & .9 \\
\hline 296 & LNSS88-230 & $<2$ & 1.2 & $<.1$ & $<.4$ & $<1$ & 3.5 & 74 & .565 & 379 & .9 \\
\hline
\end{tabular}


Table 22. Inorganic chemical analysis and moisture content for biotic samples collected during 1988-90 from the Salton Sea and associated drainwaters and rivers--Continued

\begin{tabular}{|c|c|c|c|c|c|c|c|c|c|c|c|}
\hline $\begin{array}{l}\text { Record } \\
\text { number }\end{array}$ & $\begin{array}{l}\text { Sample } \\
\text { number }\end{array}$ & $\begin{array}{c}\text { Molyb- } \\
\text { de- } \\
\text { num }\end{array}$ & $\begin{array}{l}\text { Nick- } \\
\text { el }\end{array}$ & Lead & $\begin{array}{c}\text { Anti- } \\
\text { mo- } \\
\text { ny }\end{array}$ & $\begin{array}{c}\text { Sele- } \\
\text { ni- } \\
\text { um }\end{array}$ & Tin & $\begin{array}{c}\text { Stron- } \\
\text { ti- } \\
\text { um }\end{array}$ & $\begin{array}{c}\text { Thal- } \\
\text { li- } \\
\text { um }\end{array}$ & $\begin{array}{c}\text { Va- } \\
\text { nadi- } \\
\text { um }\end{array}$ & Zinc \\
\hline \multicolumn{12}{|c|}{ Site B32 (Johnson Street Drain)--Continued } \\
\hline 259 & SS90f3 & 1.3 & 18 & $<0.82$ & $-\ldots$ & 2.6 & $<3.9$ & 200 & -.. & 2.9 & 74 \\
\hline 260 & SS90f3a & $<1.1$ & 6.0 & $<.94$ & -- & 2.5 & $<4.5$ & 240 & - & 2.3 & 73 \\
\hline \multicolumn{12}{|c|}{ Site B33 (Shady Acres Duck Club) } \\
\hline 261 & LNSS88-54 & 4 & $<1$ & $<4$ & -- & 9.98 & -- & 2.1 & $<5$ & 0.4 & 121 \\
\hline 262 & LNSS88-58 & 4 & 2 & $<4$ & --- & 11 & -.. & 2.1 & $<5$ & .99 & 108 \\
\hline 263 & LNSS88-60 & 5.3 & 1 & $<4$ & -- & 20 & -.- & 2.1 & $<5$ & .8 & 113 \\
\hline 264 & LNSS88-53 & $<1$ & $<1$ & $<4$ & - & 4.0 & --- & 2.9 & $<5$ & .4 & 42.1 \\
\hline 265 & LNSS88-57 & $<1$ & $<1$ & $<4$ & -- & 3.9 & -- & .62 & $<5$ & $<.3$ & 45.6 \\
\hline \multicolumn{12}{|c|}{ Site B34 (RH Pond) } \\
\hline 266 & LNSS88-97 & $<1$ & $<2$ & $<4$ & --- & 4.0 & $\ldots$ & 26.3 & $<4$ & $<4$ & 43.5 \\
\hline 267 & LNSS88-99 & -- & .3 & $<.5$ & --- & 4.3 & -- & -- & $<.5$ & --- & 45.3 \\
\hline 268 & LNSS88-100 & $<1$ & $<2$ & $<4$ & -.- & 4.7 & --- & 22.9 & $<4$ & $<4$ & 52.2 \\
\hline 269 & LNSS88-102 & -- & .3 & $<.9$ & - & 2.9 & -- & - & $<.5$ & --- & 47.0 \\
\hline 270 & LNSS88-103 & $<1$ & $<2$ & $<4$ & -- & 2.6 & $\ldots$ & 30.5 & $<4$ & $<4$ & 45.3 \\
\hline 271 & LNSS88-105 & -- & $<.2$ & $<.5$ & --- & 4.4 & -- & -- & $<.4$ & --- & 52.2 \\
\hline 272 & LNSS88-106 & $<1$ & $<2$ & $<4$ & -- & 3.4 & -- & 24.3 & $<4$ & $<4$ & 44.8 \\
\hline 273 & LNSS88-108 & $\ldots$ & .3 & .6 & $\ldots$ & 4.1 &.- & -- & $<.5$ & --- & 49.7 \\
\hline 274 & LNSS88-109 & $<1$ & $<2$ & $<4$ & -- & 3.2 & -- & 43.8 & $<4$ & $<4$ & 40.4 \\
\hline 275 & LNSS88-127 & $<1$ & $<2$ & $<4$ & --- & 5.5 & --- & 25.2 & $<4$ & $<4$ & 38.5 \\
\hline 276 & LNSS88-133 & $<1$ & $<2$ & $<4$ & -- & 5.4 & -- & 74.7 & $<4$ & $<4$ & 42.8 \\
\hline \multicolumn{12}{|c|}{ Site B35 (HQ Pond) } \\
\hline 277 & LNSS88-34 & 2 & $<1$ & $<4$ & --- & 9.8 & $\ldots$ & 0.59 & $<5$ & 0.3 & 165 \\
\hline 278 & LNSS88-36 & 3 & $<1$ & $<4$ & --- & 7.9 & -- & 2.9 & $<5$ & .4 & 162 \\
\hline 279 & LNSS88-38 & 2 & $<1$ & $<4$ & -.- & 26 & -- & 1.6 & $<5$ & .5 & 148 \\
\hline 280 & LNSS88-40 & 2 & $<1$ & $<4$ & - & 16 & -_ & 6.9 & $<5$ & $<.3$ & 147 \\
\hline 281 & LNSS88-35 & $<1$ & $<1$ & $<4$ & -- & 3.1 & -- & .61 & $<5$ & $<.3$ & 37.9 \\
\hline 282 & LNSS88-39 & $<1$ & $<1$ & $<4$ & -- & 5.4 & -- & .52 & $<5$ & $<.3$ & 40.4 \\
\hline \multicolumn{12}{|c|}{ Site B36 (Reidman Pond) } \\
\hline 283 & LNSS88-172 & $<1$ & $<2$ & $<4$ & $\ldots$ & 3.9 & $\ldots$ & 16.0 & $<4$ & $<4$ & 45.9 \\
\hline 284 & LNSS88-175 & $<1$ & $<2$ & $<4$ & --. & 5.3 & -.- & 29.5 & $<4$ & $<4$ & 45.8 \\
\hline 285 & LNSS88-178 & $<1$ & $<2$ & $<4$ & -- & 6.0 & --- & 30.1 & $<4$ & $<4$ & 46.2 \\
\hline 286 & LNSS88-184 & $<1$ & $<2$ & $<4$ & -_- & 13.5 & -.- & 23.8 & $<4$ & $<4$ & 44.9 \\
\hline 287 & LNSS88-189 & -- & $<.2$ & .5 & -- & 5.6 & -- & -- & $<.6$ & --- & 47.8 \\
\hline 288 & LNSS88-196 & -- & $<.2$ & $<.5$ & -- & 4.0 & --- & - & $<.7$ & --- & 48.2 \\
\hline 289 & LNSS88-197 & $<1$ & $<2$ & $<4$ & -- & 35 & --- & 23.4 & $<4$ & $<4$ & 41 \\
\hline 290 & LNSS88-199 & --. & $<.2$ & .6 & --- & 4.7 & $-\ldots$ & -- & $<.7$ & --. & 48.0 \\
\hline 291 & LNSS88-200 & $<1$ & $<2$ & $<4$ & -.- & 6.1 & -- & 21.1 & $<4$ & $<4$ & 48.2 \\
\hline 292 & LNSS88-203 & $<1$ & $<2$ & $<4$ & -- & 5.2 & -- & 25.8 & $<4$ & $<4$ & 45.8 \\
\hline 293 & LNSS88-209 & $<1$ & $<2$ & $<4$ & -- & 4.3 & --- & 26.2 & $<4$ & $<4$ & 49.0 \\
\hline 294 & LNSS88-220 & --- & $<.2$ & $<.5$ & --- & 5.1 & $\ldots$ & -- & $<.7$ & --- & 48.9 \\
\hline 295 & LNSS88-221 & $<1$ & $<2$ & $<4$ & - & 5.9 & --- & 24.4 & $<4$ & $<4$ & 49 \\
\hline 296 & LNSS88-230 & $<1$ & $<4$ & $<5$ & -- & 5.2 & -- & 11 & $<4$ & $<.3$ & 40.1 \\
\hline
\end{tabular}


Table 22. Inorganic chemical analysis and moisture content for biotic samples collected during 1988-90 from the Salton Sea and associated drainwaters and rivers--Continued

\begin{tabular}{|c|c|c|c|c|c|c|c|}
\hline $\begin{array}{l}\text { Record } \\
\text { number }\end{array}$ & $\begin{array}{l}\text { Sample } \\
\text { number }\end{array}$ & Species & Matrix & $\begin{array}{l}\text { Percent } \\
\text { moisture }\end{array}$ & $\begin{array}{l}\text { Sil- } \\
\text { ver }\end{array}$ & $\begin{array}{l}\text { Alu- } \\
\text { mi- } \\
\text { num }\end{array}$ & $\begin{array}{c}\text { Arse- } \\
\text { nic }\end{array}$ \\
\hline \multicolumn{8}{|c|}{ Site B37 (Hazard Pond) } \\
\hline 297 & LNSS88-24 & Northern shoveler & Liver & 71.4 & $<2$ & 89 & $<0.2$ \\
\hline 298 & LNSS88-26 & Northern shoveler & Liver & 68.7 & $<2$ & 51 & $<.2$ \\
\hline 299 & LNSS88-52 & Northern shoveler & Liver & 69.9 & $<2$ & 10 & $<.2$ \\
\hline 300 & LNSS88-56 & Northern shoveler & Liver & 68.9 & $<2$ & $<3$ & .2 \\
\hline 301 & LNSS88-23 & Northern shoveler & Breast muscle & 72.9 & $<2$ & 15 & $<.2$ \\
\hline 302 & LNSS88-51 & Northern shoveler & Breast muscle & 72.3 & $<2$ & 50 & $<.2$ \\
\hline 303 & LNSS88-55 & Northern shoveler & Breast muscle & 71.5 & $<2$ & 6 & $<.2$ \\
\hline 304 & LNSS88-02 & Ruddy duck & Liver & 70.7 & $<2$ & 5 & .4 \\
\hline 305 & LNSS88-04 & Ruddy duck & Liver & 73.9 & $<2$ & 5 & .5 \\
\hline 306 & LNSS88-06 & Ruddy duck & Liver & 72.2 & $<2$ & 10 & .2 \\
\hline 307 & LNSS88-08 & Ruddy duck & Liver & 70.0 & $<2$ & 14 & .4 \\
\hline 308 & LNSS88-10 & Ruddy duck & Liver & 71.2 & 2 & 34 & .67 \\
\hline 309 & LNSS88-12 & Ruddy duck & Liver & 71.2 & $<2$ & 12 & .4 \\
\hline 310 & LNSS88-14 & Ruddy duck & Liver & 73.3 & 2 & 5 & .3 \\
\hline 311 & LNSS88-16 & Ruddy duck & Liver & 71.9 & $<2$ & 3 & .5 \\
\hline 312 & LNSS88-18 & Ruddy duck & Liver & 69.9 & $<2$ & $<3$ & .4 \\
\hline 313 & LNSS88-20 & Ruddy duck & Liver & 70.6 & $<2$ & 23 & .4 \\
\hline 314 & LNSS88-111 & Black-necked stilt & Egg & 73.6 & --- & .8 & $<.2$ \\
\hline 315 & LNSS88-112 & Black-necked stilt & Egg & 72.6 & $<2$ & 3 & $<.2$ \\
\hline 316 & LNSS88-114 & Black-necked stilt & Egg & 74.8 & --- & 3.3 & $<.2$ \\
\hline 317 & LNSS88-115 & Black-necked stilt & Egg & 75.1 & $<2$ & 5 & $<.2$ \\
\hline 318 & LNSS88-117 & Black-necked stilt & Egg & 73.1 & --- & 1.3 & $<.2$ \\
\hline 319 & LNSS88-118 & Black-necked stilt & Egg & 70.4 & $<2$ & 5 & $<.2$ \\
\hline 320 & LNSS88-120 & Black-necked stilt & Egg & 74.3 & --- & .7 & $<.2$ \\
\hline 321 & LNSS88-121 & Black-necked stilt & Egg & 67.5 & $<2$ & 4 & $<.2$ \\
\hline 322 & LNSS88-123 & Black-necked stilt & Egg & 72.6 & --- & 5.2 & $<.2$ \\
\hline 323 & LNSS88-124 & Black-necked stilt & Egg & 72.8 & $<2$ & 4 & $<.2$ \\
\hline 324 & LNSS88-126 & Black-necked stilt & Egg & 72.3 & --- & 2.4 & $<.2$ \\
\hline 325 & LNSS88-129 & Black-necked stilt & Egg & 72.9 & --- & .8 & $<.2$ \\
\hline 326 & LNSS88-130 & Black-necked stilt & Egg & 72.0 & $<2$ & 5 & $<.2$ \\
\hline 327 & LNSS88-132 & Black-necked stilt & Egg & 74.8 & --- & 1.4 & $<.2$ \\
\hline 328 & LNSS88-135 & Black-necked stilt & Egg & 73.5 & -- & 2.4 & $<.2$ \\
\hline 329 & LNSS88-136 & Black-necked stilt & Egg & 71.7 & $<2$ & 4 & .61 \\
\hline 330 & SS89-154 & Spiny softshell turtle & Liver & 74.9 & $<2$ & 26 & $<.1$ \\
\hline 331 & SS89-156 & Spiny softshell turtle & Liver & 72.6 & $<2$ & 12 & $<.1$ \\
\hline 332 & SS89-158 & Spiny softshell turtle & Liver & 78.2 & $<2$ & 9.8 & $<.1$ \\
\hline 333 & SS89-52 & Northern shoveler & Liver & 72.4 & $<2$ & $<3$ & $<.1$ \\
\hline 334 & SS89-54 & Northern shoveler & Liver & 73.3 & $<2$ & 9.6 & $<.1$ \\
\hline 335 & SS89-55 & Northern shoveler & Liver & 71.6 & $<2$ & $<3$ & .2 \\
\hline 336 & SS89-74 & Black-necked stilt & Egg & 74.6 & $<2$ & $<3$ & $<.1$ \\
\hline 337 & SS89-75 & Black-necked stilt & Egg & 71.5 & $<2$ & $<3$ & .1 \\
\hline 338 & SS89-77 & Black-necked stilt & Egg & 67.7 & $<2$ & $<3$ & $<.1$ \\
\hline 339 & SS89-83 & Black-necked stilt & Egg & 76.2 & $<2$ & $<3$ & .2 \\
\hline 340 & SS89-84 & Black-necked stilt & Egg & 75.0 & $<2$ & $<3$ & .2 \\
\hline
\end{tabular}


Table 22. Inorganic chemical analysis and moisture content for biotic samples collected during 1988-90 from the Salton Sea and associated drainwaters and rivers--Continued

\begin{tabular}{|c|c|c|c|c|c|c|c|c|c|c|c|}
\hline $\begin{array}{l}\text { Record } \\
\text { number }\end{array}$ & $\begin{array}{l}\text { Sample } \\
\text { number }\end{array}$ & $\begin{array}{l}\text { Bo- } \\
\text { ron }\end{array}$ & $\begin{array}{l}\mathrm{Ba-} \\
\text { rium }\end{array}$ & $\begin{array}{l}\text { Be- } \\
\text { ryl- } \\
\text { lium }\end{array}$ & $\begin{array}{l}\text { Cad- } \\
\text { mium }\end{array}$ & $\begin{array}{l}\text { Chro- } \\
\text { mi- } \\
\text { um }\end{array}$ & $\begin{array}{l}\text { Cop- } \\
\text { per }\end{array}$ & Iron & $\begin{array}{l}\text { Mer- } \\
\text { cury }\end{array}$ & $\begin{array}{l}\text { Mag- } \\
\text { ne- } \\
\text { sium }\end{array}$ & $\begin{array}{c}\text { Man- } \\
\text { ga- } \\
\text { nese }\end{array}$ \\
\hline \multicolumn{12}{|c|}{ Site B37 (Hazard Pond) } \\
\hline 297 & LNSS88-24 & 6 & 0.61 & $<0.1$ & 2.6 & $<1$ & 91.2 & 3,510 & 1.07 & 874 & 19 \\
\hline 298 & LNSS88-26 & 3 & .2 & $<.1$ & 2.9 & $<1$ & 57.5 & 4,220 & 1.5 & 799 & 15 \\
\hline 299 & LNSS88-52 & $<2$ & $<.1$ & $<.1$ & 1.6 & 1 & 35.5 & 647 & 2.7 & 789 & 11 \\
\hline 300 & LNSS88-56 & 3 & $<.1$ & $<.1$ & 1.1 & 2 & 58.2 & 1,670 & 3.6 & 772 & 17 \\
\hline 301 & LNSS88-23 & $<2$ & .1 & $<.1$ & $<.2$ & $<1$ & 25.4 & 278 & .22 & 1,090 & 1.9 \\
\hline 302 & LNSS88-51 & $<2$ & .31 & $<.1$ & $<.2$ & $<1$ & 24.1 & 295 & .695 & 1,080 & 2.1 \\
\hline 303 & LNSS88-55 & $<2$ & $<.1$ & $<.1$ & $<.2$ & $<1$ & 20.6 & 208 & .27 & 1,080 & 1.9 \\
\hline 304 & LNSS88-02 & 5 & .77 & $<.1$ & $<.2$ & $<1$ & 37.8 & 1,810 & .13 & 915 & 17 \\
\hline 305 & LNSS88-04 & 7.3 & 1.3 & $<.1$ & $<.2$ & $<1$ & 26.9 & 2,180 & 1.02 & 967 & 17 \\
\hline 306 & LNSS88-06 & 4 & .85 & $<.1$ & 1.5 & $<1$ & 163 & 2,560 & .650 & 898 & 18 \\
\hline 307 & LNSS88-08 & 6 & 1.0 & $<.1$ & 5.0 & $<1$ & 66.5 & 1,380 & .23 & 902 & 15 \\
\hline 308 & LNSS88-10 & 5 & 1.4 & $<.1$ & $<.2$ & $<1$ & 205 & 1,380 & 4.6 & 885 & 14 \\
\hline 309 & LNSS88-12 & 5.9 & .70 & $<.1$ & $<.2$ & $<1$ & 115 & 3,770 & .507 & 865 & 16 \\
\hline 310 & LNSS88-14 & 3 & .82 & $<.1$ & .6 & $<1$ & 191 & 4,680 & 2.3 & 946 & 15 \\
\hline 311 & LNSS88-16 & 5 & .63 & $<.1$ & 2.7 & $<1$ & 91.4 & 3,060 & .29 & 751 & 11 \\
\hline 312 & LNSS88-18 & 5 & .61 & $<.1$ & .5 & $<1$ & 51.4 & 1,990 & .16 & 739 & 12 \\
\hline 313 & LNSS88-20 & 3 & .2 & $<.1$ & .81 & $<1$ & 48.6 & 1,370 & .548 & 820 & 14 \\
\hline 314 & LNSS88-111 & -- & -- & $<.01$ & $<.03$ & $<.1$ & 3.12 & 110 & .22 & - & 1.8 \\
\hline 315 & LNSS88-112 & 3 & .40 & $<.1$ & $<.4$ & $<1$ & 3.2 & 93 & .30 & 469 & 1.7 \\
\hline 316 & LNSS88-114 & - & $-\ldots$ & $<.01$ & $<.03$ & .2 & 6.22 & 130 & .21 & - & 2.36 \\
\hline 317 & LNSS88-115 & 3 & 1.3 & $<.1$ & $<.4$ & $<1$ & 3.7 & 110 & .16 & 518 & .8 \\
\hline 318 & LNSS88-117 & -- & - & $<.01$ & $<.03$ & .3 & 3.22 & 104 & .13 & $\ldots$ & 1.2 \\
\hline 319 & LNSS88-118 & $<2$ & .90 & $<.1$ & $<.4$ & $<1$ & 3.5 & 84 & .37 & 374 & 2.4 \\
\hline 320 & LNSS88-120 & -- & -- & $<.01$ & $<.03$ & .2 & 46.0 & 94.3 & .29 & --- & 1.2 \\
\hline 321 & LNSS88-121 & $<2$ & .31 & $<.1$ & $<.4$ & $<1$ & 3.2 & 90 & .26 & 478 & 1.9 \\
\hline 322 & LNSS88-123 & -- & --- & $<.01$ & $<.03$ & .2 & 3.46 & 109 & .086 & --- & 2.2 \\
\hline 323 & LNSS88-124 & $<2$ & .60 & $<.1$ & $<.4$ & $<1$ & 3.7 & 81 & .839 & 564 & 2.0 \\
\hline 324 & LNSS88-126 & $\ldots$ & --- & $<.01$ & $<.03$ & .4 & 3.99 & 105 & .14 & --- & 1.1 \\
\hline 325 & LNSS88-129 & -- & -- & $<.01$ & $<.03$ & .2 & 3.60 & 90.4 & .012 & --- & 2.1 \\
\hline 326 & LNSS88-130 & $<2$ & 2.4 & $<.1$ & $<.4$ & $<1$ & 3.8 & 110 & .17 & 524 & .9 \\
\hline 327 & LNSS88-132 & - & -- & $<.01$ & $<.03$ & .2 & 3.53 & 128 & .19 & -- & 1.1 \\
\hline 328 & LNSS88-135 & $\ldots$ & -- & $<.01$ & $<.03$ & .2 & 3.35 & 92.9 & .69 & $\ldots$ & 2.74 \\
\hline 329 & LNSS88-136 & $<2$ & .52 & $<.1$ & $<.4$ & $<1$ & 3.5 & 94 & .20 & 376 & 1.8 \\
\hline 330 & SS89-154 & 2 & .78 & $<.1$ & $<.3$ & $<1$ & 25.9 & 1,940 & .20 & 502 & 6.9 \\
\hline 331 & SS89-156 & 2 & .1 & $<.1$ & $<.3$ & $<1$ & 19 & 605 & .23 & 423 & 5.2 \\
\hline 332 & SS89-158 & 3 & .3 & $<.1$ & $<.3$ & $<1$ & 24.6 & 1,150 & .20 & 651 & 4.4 \\
\hline 333 & SS89-52 & 4 & $<.1$ & $<.1$ & 2.0 & $<1$ & 83.5 & 3,220 & .645 & 736 & 14 \\
\hline 334 & SS89-54 & 4 & .1 & $<.1$ & 1.3 & 1 & 69.4 & 3,640 & .986 & 719 & 12 \\
\hline 335 & SS89-55 & 4 & $<.1$ & $<.1$ & 2.4 & $<1$ & 37.3 & 2,870 & 1.2 & 756 & 16 \\
\hline 336 & SS89-74 & $<3$ & 2.2 & $<.1$ & $<.6$ & $<2$ & 2.9 & 103 & .48 & 390 & 1.5 \\
\hline 337 & SS89-75 & 3 & .46 & $<.1$ & $<.5$ & $<2$ & 3.0 & 90 & .781 & 321 & .69 \\
\hline 338 & SS89-77 & $<3$ & .48 & $<.1$ & $<.5$ & $<2$ & 2.4 & 67 & 1.8 & 255 & 1.1 \\
\hline 339 & SS89-83 & $<3$ & .62 & $<.1$ & $<.5$ & $<2$ & 2.8 & 81 & .68 & 350 & 1.3 \\
\hline 340 & SS89-84 & 3 & .45 & $<.1$ & $<.5$ & $<2$ & 3.2 & 70 & .626 & 372 & 2.8 \\
\hline
\end{tabular}


Table 22. Inorganic chemical analysis and moisture content for biotic samples collected during 1988-90 from the Salton Sea and associated drainwaters and rivers--Continued

\begin{tabular}{|c|c|c|c|c|c|c|c|c|c|c|c|}
\hline $\begin{array}{l}\text { Record } \\
\text { number }\end{array}$ & $\begin{array}{l}\text { Sample } \\
\text { number }\end{array}$ & $\begin{array}{c}\text { Molyb- } \\
\text { de- } \\
\text { num }\end{array}$ & $\begin{array}{c}\text { Nick- } \\
\text { el }\end{array}$ & Lead & $\begin{array}{l}\text { Anti- } \\
\text { mo- } \\
\text { ny }\end{array}$ & $\begin{array}{c}\text { Sele- } \\
\text { ni- } \\
\text { um }\end{array}$ & Tin & $\begin{array}{l}\text { Stron- } \\
\text { ti- } \\
\text { um }\end{array}$ & $\begin{array}{l}\text { Thal- } \\
\text { li- } \\
\text { um }\end{array}$ & $\begin{array}{c}\text { Va- } \\
\text { nadi- } \\
\text { um }\end{array}$ & Zinc \\
\hline \multicolumn{12}{|c|}{ Site B37 (Hazard Pond) } \\
\hline 297 & LNSS88-24 & 3 & $<1$ & $<4$ & -- & 47.0 & -- & 3.5 & $<5$ & $<0.3$ & 156 \\
\hline 298 & LNSS88-26 & 4.4 & $<1$ & $<4$ & -- & 44.0 & -- & .53 & $<5$ & 1.1 & 146 \\
\hline 299 & LNSS88-52 & 3 & $<1$ & $<4$ & -- & 17.2 & -- & .81 & $<5$ & $<.3$ & 105 \\
\hline 300 & LNSS88-56 & 3.4 & $<1$ & $<4$ & -- & 17.0 & -- & .48 & $<5$ & .6 & 142 \\
\hline 301 & LNSS88-23 & $<1$ & $<1$ & $<4$ & -- & 12.0 & -- & 1.2 & $<5$ & $<.3$ & 35.1 \\
\hline 302 & LNSS88-51 & $<1$ & $<1$ & $<4$ & -- & 3.8 & -- & 1.5 & $<5$ & $<.3$ & 41.2 \\
\hline 303 & LNSS88-55 & $<1$ & $<1$ & $<4$ & -- & 4.7 & -- & .46 & $<5$ & $<.3$ & 41.2 \\
\hline 304 & LNSS88-02 & 2 & $<1$ & $<4$ & -- & 11.1 & -- & .88 & $<5$ & .4 & 162 \\
\hline 305 & LNSS88-04 & 2 & $<1$ & $<4$ & -- & 8.5 & --- & 1 & $<5$ & $<.3$ & 180 \\
\hline 306 & LNSS88-06 & 3 & $<1$ & $<4$ & -- & 9.0 & -- & 1.6 & $<5$ & $<.3$ & 202 \\
\hline 307 & LNSS88-08 & 2 & $<1$ & $<4$ & -- & 8.3 & -- & 1.1 & $<5$ & .4 & 142 \\
\hline 308 & LNSS88-10 & 3 & $<1$ & $<4$ & -- & 26.0 & --- & 2.1 & $<5$ & $<.3$ & 144 \\
\hline 309 & LNSS88-12 & 3 & $<1$ & $<4$ & -- & 10.2 & -- & 1.0 & $<5$ & .5 & 195 \\
\hline 310 & LNSS88-14 & 3 & $<1$ & $<4$ & -- & 7.7 & -- & .98 & $<5$ & $<.3$ & 261 \\
\hline 311 & LNSS88-16 & 2 & $<1$ & $<4$ & -- & 6.7 & -- & .82 & $<5$ & .7 & 153 \\
\hline 312 & LNSS88-18 & 2 & $<1$ & $<4$ & -- & 14.0 & -- & .45 & $<5$ & $<.3$ & 145 \\
\hline 313 & LNSS88-20 & 3 & $<1$ & $<4$ & --- & 13.1 & -- & 1.2 & $<5$ & .6 & 145 \\
\hline 314 & LNSS88-111 & -- & .3 & $<.4$ & -- & 4.6 & --- & --- & $<.4$ & --- & 46.3 \\
\hline 315 & LNSS88-112 & $<1$ & $<2$ & $<4$ & -- & 4.5 & --- & 30.6 & $<4$ & $<4$ & 44.7 \\
\hline 316 & LNSS88-114 & -- & .4 & 1.9 & -- & 6.0 & --. & --- & $<.5$ & -. & 50.7 \\
\hline 317 & LNSS88-115 & $<1$ & $<2$ & $<4$ & -- & 4.7 & -- & 28.5 & $<4$ & $<4$ & 46.2 \\
\hline 318 & LNSS88-117 & -- & .3 & $<.5$ & -- & 3.7 & --- & -- & $<.5$ & -- & 47.9 \\
\hline 319 & LNSS88-118 & $<1$ & $<2$ & $<4$ & -- & 4.9 & -- & 14.9 & $<4$ & $<4$ & 39.4 \\
\hline 320 & LNSS88-120 & -- & $<.2$ & $<.5$ & $\cdots$ & 4.5 & --- & $\ldots$ & $<.5$ & -- & 44.7 \\
\hline 321 & LNSS88-121 & $<1$ & $<2$ & $<4$ & -- & 3.7 & $\cdots$ & 19.8 & $<4$ & $<4$ & 49.2 \\
\hline 322 & LNSS88-123 & -- & $<.2$ & $<.5$ & -- & 3.6 & --- & -- & $<.5$ & -- & 48.6 \\
\hline 323 & LNSS88-124 & $<1$ & $<2$ & $<4$ & -- & 4.7 & --- & 29.2 & $<4$ & $<4$ & 49 \\
\hline 324 & LNSS88-126 & -- & $<.2$ & $<.4$ & -- & 4.6 & $\cdots$ & --- & $<.4$ & -- & 48.2 \\
\hline 325 & LNSS88-129 & -- & .3 & $<.5$ & --- & 3.5 & --- & -- & $<.5$ & -- & 40.4 \\
\hline 326 & LNSS88-130 & $<1$ & $<2$ & $<4$ & --- & 5.2 & --- & 36.8 & $<4$ & $<4$ & 52.6 \\
\hline 327 & LNSS88-132 & -- & .3 & 1 & --- & 4.7 & -- & -- & $<.5$ & -- & 49.8 \\
\hline 328 & LNSS88-135 & -- & .2 & 1 & - & 4.6 & -- & --- & $<.5$ & -- & 51.7 \\
\hline 329 & LNSS88-136 & $<1$ & $<2$ & $<4$ & -- & 5.1 & -- & 19.3 & $<4$ & $<4$ & 43.9 \\
\hline 330 & SS89-154 & $<1$ & $<1$ & $<4$ & --- & 9.1 & -- & 7.3 & $<5$ & .5 & 67.1 \\
\hline 331 & SS89-156 & $<1$ & $<1$ & $<4$ & --- & 8.0 & --- & 2.1 & $<5$ & $<.3$ & 65.4 \\
\hline 332 & SS89-158 & $<1$ & $<1$ & $<4$ & --- & 9.6 & -- & 6.1 & $<5$ & $<.3$ & 80 \\
\hline 333 & SS89-52 & 2 & $<1$ & $<4$ & -- & 37.1 & --- & 1.2 & $<5$ & $<.3$ & 122 \\
\hline 334 & SS89-54 & 3 & $<1$ & $<4$ & --- & 22.5 & --- & 1.0 & $<6$ & .3 & 118 \\
\hline 335 & SS89-55 & 3 & $<1$ & $<4$ & -- & 28.6 & -- & 1.0 & $<5$ & $<.3$ & 127 \\
\hline 336 & SS89-74 & $<1$ & $<3$ & $<4$ & -- & 6.3 & -- & 27.3 & $<4$ & $<.7$ & 45 \\
\hline 337 & SS89-75 & $<1$ & $<3$ & $<4$ & -- & 7.0 & --- & 16.8 & $<4$ & $<.7$ & 43 \\
\hline 338 & SS89-77 & $<1$ & $<3$ & $<4$ & -- & 5.1 & -- & 12.3 & $<4$ & $<.6$ & 40 \\
\hline 339 & SS89-83 & $<1$ & $<3$ & $<4$ & -- & 6.0 & --- & 19.2 & $<4$ & $<.7$ & 37 \\
\hline 340 & SS89-84 & $<1$ & $<3$ & $<4$ & -- & 9.6 & --- & 22.9 & $<4$ & $<.6$ & 39 \\
\hline
\end{tabular}


Table 22. Inorganic chemical analysis and moisture content for biotic samples collected during 1988-90 from the Salton Sea and associated drainwaters and rivers--Continued

\begin{tabular}{|c|c|c|c|c|c|c|c|}
\hline $\begin{array}{l}\text { Record } \\
\text { number }\end{array}$ & $\begin{array}{l}\text { Sample } \\
\text { number }\end{array}$ & Species & Matrix & $\begin{array}{l}\text { Percent } \\
\text { moisture }\end{array}$ & $\begin{array}{l}\text { Sil- } \\
\text { ver }\end{array}$ & $\begin{array}{l}\text { Alu- } \\
\text { mi- } \\
\text { num }\end{array}$ & $\begin{array}{c}\text { Arse- } \\
\text { nic }\end{array}$ \\
\hline \multicolumn{8}{|c|}{ Site B38 (South Brawley) } \\
\hline 341 & $89-030$ & White-faced ibis & Liver & 67.6 & $<1.54$ & 52.1 & $<0.31$ \\
\hline 342 . & $89-031$ & White-faced ibis & Liver & 69.2 & $<1.62$ & 11.3 & $<.32$ \\
\hline 343 . & $89-032$ & White-faced ibis & Liver & 68.7 & $<1.60$ & 32.3 & $<.32$ \\
\hline 344 . & 89-033 & White-faced ibis & Liver & 69.3 & $<1.63$ & 4.33 & $<.33$ \\
\hline 345 . & 89-034 & White-faced ibis & Liver & 67.1 & $<1.52$ & 5.93 & $<.30$ \\
\hline 346 . & 89-035 & White-faced ibis & Liver & 69.9 & $<1.66$ & 3.65 & $<.33$ \\
\hline 347 . & $89-036$ & White-faced ibis & Liver & 69.8 & $<1.66$ & 8.21 & $<.33$ \\
\hline 348 . & $89-037$ & White-faced ibis & Liver & 66.7 & $<1.50$ & $<3.00$ & $<.30$ \\
\hline 349 . & $89-038$ & White-faced ibis & Liver & 68.1 & $<1.54$ & 7.87 & $<.31$ \\
\hline 350 . & SS89-103 & White-faced ibis & Breast muscle & 70.9 & $<2$ & 50 & $<.1$ \\
\hline 351 . & SS89-104 & White-faced ibis & Breast muscle & 73.0 & $<2$ & 17 & $<.1$ \\
\hline 352 . & SS89-105 & White-faced ibis & Breast muscle & 72.3 & $<2$ & 13 & $<.1$ \\
\hline 353 . & SS89-106 & White-faced ibis & Breast muscle & 73.8 & $<2$ & 13 & $<.1$ \\
\hline 354 . & SS89-107 & White-faced ibis & Breast muscle & 73.2 & $<2$ & 19 & $<.1$ \\
\hline 355 . & SS89-108 & White-faced ibis & Breast muscle & 72.8 & $<2$ & 40 & $<.1$ \\
\hline 356 . & SS89-109 & White-faced ibis & Breast muscle & 71.4 & $<2$ & 17 & $<.1$ \\
\hline 357 . & SS89-110 & White-faced ibis & Breast muscle & 72.0 & $<2$ & 20 & $<.1$ \\
\hline 358 . & SS89-111 & White-faced ibis & Breast muscle & 72.6 & $<2$ & 16 & $<.1$ \\
\hline \multicolumn{8}{|c|}{ Site B39 (McKendry Road) } \\
\hline 359 . & SS89-78 & Black-necked stilt & Egg & 68.6 & $<2$ & $<3$ & $<0.1$ \\
\hline 360. & SS89-79 & Black-necked stilt & Egg & 74.3 & $<2$ & $<3$ & .1 \\
\hline 361 . & SS89-80 & Black-necked stilt & Egg & 67.9 & $<2$ & $<3$ & .1 \\
\hline 362 . & SS89-81 & Black-necked stilt & Egg & 74.0 & $<2$ & $<3$ & $<.1$ \\
\hline 363. & SS89-82 & Black-necked stilt & Egg & 73.9 & $<2$ & $<3$ & .1 \\
\hline
\end{tabular}


Table 22. Inorganic chemical analysis and moisture content for biotic samples collected during 1988-90 from the Salton Sea and associated drainwaters and rivers--Continued

\begin{tabular}{|c|c|c|c|c|c|c|c|c|c|c|c|}
\hline $\begin{array}{l}\text { Record } \\
\text { number }\end{array}$ & $\begin{array}{l}\text { Sample } \\
\text { number }\end{array}$ & $\begin{array}{l}\text { Bo- } \\
\text { ron }\end{array}$ & $\begin{array}{l}\mathrm{Ba}- \\
\text { rium }\end{array}$ & $\begin{array}{l}\text { Be- } \\
\text { ryl- } \\
\text { lium }\end{array}$ & $\begin{array}{l}\text { Cad- } \\
\text { mium }\end{array}$ & $\begin{array}{c}\text { Chro- } \\
\text { mi- } \\
\text { um }\end{array}$ & $\begin{array}{l}\text { Cop- } \\
\text { per }\end{array}$ & Iron & $\begin{array}{l}\text { Mer- } \\
\text { cury }\end{array}$ & $\begin{array}{c}\text { Mag- } \\
\text { ne- } \\
\text { sium }\end{array}$ & $\begin{array}{c}\text { Man- } \\
\text { ga- } \\
\text { nese }\end{array}$ \\
\hline \multicolumn{12}{|c|}{ Site B38 (South Brawley) } \\
\hline 341 & $89-030$ & $<1.54$ & 1.54 & $<0.15$ & 0.77 & 0.46 & 12.7 & 1,100 & 0.284 & 512 & 7.16 \\
\hline 342 & $89-031$ & $<1.62$ & $<1.62$ & $<.16$ & 2.86 & .42 & 19.7 & 1,840 & .273 & 584 & 9.03 \\
\hline 343 & $89-032$ & $<1.60$ & $<1.60$ & $<.16$ & 1.95 & .51 & 11.4 & 1,780 & .208 & 585 & 7.60 \\
\hline 344 & $89-033$ & $<1.63$ & $<1.63$ & $<.16$ & 2.05 & .65 & 20.3 & 2,730 & .176 & 668 & 15.4 \\
\hline 345 & $89-034$ & $<1.52$ & $<1.52$ & $<.15$ & 3.10 & .49 & 16.6 & 1,840 & .343 & 602 & 8.85 \\
\hline 346 & 89-035 & $<1.66$ & $<1.66$ & $<.17$ & 2.23 & .56 & 13.2 & 2,040 & .246 & 588 & 10.4 \\
\hline 347 & $89-036$ & $<1.66$ & $<1.66$ & $<.17$ & .96 & $<.33$ & 6.99 & 621 & .285 & 351 & 4.80 \\
\hline 348 & $89-037$ & $<1.50$ & $<1.50$ & $<.15$ & 2.67 & .57 & 17.3 & 2,740 & .300 & 700 & 16.9 \\
\hline 349 & 89-038 & $<.5$ & $<.5$ & $<.05$ & .59 & .10 & 3.51 & 492 & .313 & 170 & 2.33 \\
\hline 350 & SS89-103 & $<3$ & .35 & $<.2$ & $<.7$ & $<2$ & 14 & 239 & .13 & 1,140 & 2.6 \\
\hline 351 & SS89-104 & $<3$ & .2 & .58 & $<.7$ & $<2$ & 16 & 249 & .13 & 1,160 & 1.9 \\
\hline 352 & SS89-105 & $<3$ & $<.1$ & .66 & $<.7$ & $<2$ & 16 & 292 & .17 & 1,080 & 1.3 \\
\hline 353 & SS89-106 & $<3$ & $<.1$ & .68 & $<.7$ & $<2$ & 16 & 217 & .16 & 1,190 & 1.4 \\
\hline 354 & SS89-107 & $<3$ & .1 & .67 & $<.7$ & $<2$ & 15 & 239 & .14 & 1,160 & 1.2 \\
\hline 355 & SS89-108 & $<3$ & .2 & .68 & $<.7$ & $<2$ & 13 & 195 & .18 & 1,160 & 1.6 \\
\hline 356 & SS89-109 & $<3$ & $<.1$ & .66 & $<.7$ & $<2$ & 14 & 226 & .13 & 1,080 & 1.4 \\
\hline 357 & SS89-110 & $<3$ & .2 & .69 & $<.7$ & $<2$ & 15 & 266 & .36 & 1,060 & 1.5 \\
\hline 358 & SS89-111 & 3 & $<.1$ & .71 & $<.7$ & $<2$ & 11 & 289 & .14 & 1,110 & 1.4 \\
\hline \multicolumn{12}{|c|}{ Site B39 (McKendry Road) } \\
\hline 359 & SS89-78 & $<3$ & 1.5 & $<0.1$ & $<0.5$ & $<2$ & 3.2 & 96 & 0.11 & 328 & 1.2 \\
\hline 360 & SS89-79 & $<3$ & 1.7 & $<.1$ & $<.5$ & $<2$ & 2.9 & 87 & .685 & 409 & 1.5 \\
\hline 361 & SS89-80 & $<3$ & .97 & $<.1$ & $<.5$ & $<2$ & 2.6 & 98 & .099 & 385 & 2.2 \\
\hline 362 & SS89-81 & 3 & 2.2 & $<.1$ & $<.5$ & $<2$ & 3.1 & 119 & .626 & 420 & 2.6 \\
\hline 363 & SS89-82 & $<3$ & .51 & $<.1$ & $<.5$ & $<2$ & 3.3 & 93 & .20 & 396 & 2.3 \\
\hline
\end{tabular}


Table 22. Inorganic chemical analysis and moisture content for biotic samples collected during 1988-90 from the Salton Sea and associated drainwaters and rivers--Continued

\begin{tabular}{|c|c|c|c|c|c|c|c|c|c|c|c|}
\hline $\begin{array}{l}\text { Record } \\
\text { number }\end{array}$ & $\begin{array}{l}\text { Sample } \\
\text { number }\end{array}$ & $\begin{array}{c}\text { Molyb- } \\
\text { de- } \\
\text { num }\end{array}$ & $\begin{array}{c}\text { Nick- } \\
\text { el }\end{array}$ & Lead & $\begin{array}{c}\text { Anti- } \\
\text { mo- } \\
\text { ny }\end{array}$ & $\begin{array}{c}\text { Sele- } \\
\text { ni- } \\
\text { um }\end{array}$ & Tin & $\begin{array}{c}\text { Stron- } \\
\text { ti- } \\
\text { um }\end{array}$ & $\begin{array}{l}\text { Thal- } \\
\text { li- } \\
\text { um }\end{array}$ & $\begin{array}{c}\text { Va- } \\
\text { nadi- } \\
\text { um }\end{array}$ & Zinc \\
\hline \multicolumn{12}{|c|}{ Site B38 (South Brawley) } \\
\hline 341 & $89-030$ & $<1.54$ & $<1.23$ & $<0.93$ & $<3$ & 5.2 & 8.43 & 1.27 & $<6.17$ & $<1.54$ & 52.8 \\
\hline 342 & $89-031$ & 2.08 & $<1.3$ & $<.97$ & $<3.25$ & 9.4 & 8.41 & .94 & $<6.49$ & $<1.62$ & 70.1 \\
\hline 343 & $89-032$ & $<1.60$ & $<1.28$ & $<.96$ & $<3.19$ & 5.1 & 7.38 & 1.57 & $<6.39$ & $<1.60$ & 52.4 \\
\hline 344 & $89-033$ & 2.21 & $<1.3$ & $<.98$ & $<3.26$ & 10.7 & 8.93 & 4.07 & $<6.51$ & $<1.63$ & 91.5 \\
\hline 345 & $89-034$ & 1.73 & $<1.22$ & $<.91$ & $<3.04$ & 6.7 & 6.02 & 1.79 & $<6.08$ & $<1.52$ & 71.4 \\
\hline 346 & $89-035$ & 1.83 & $<1.33$ & $<1.00$ & $<3.32$ & 5.0 & 7.64 & 1.10 & $<6.64$ & $<1.66$ & 65.2 \\
\hline 347 & $89-036$ & $<1.66$ & $<1.32$ & $<.99$ & $<3.31$ & 10.6 & 4.30 & 1.42 & $<6.62$ & $<1.66$ & 34 \\
\hline 348 & $89-037$ & 2.40 & $<1.20$ & $<.90$ & $<3.00$ & 13.2 & 8.08 & 1.68 & $<6.00$ & $<1.50$ & 99.2 \\
\hline 349 & $89-038$ & $<.5$ & $<.4$ & $<.3$ & $<1$ & 5.3 & 1.94 & .42 & $<2$ & $<.5$ & 16.0 \\
\hline 350 & SS89-103 & $<1$ & $<4$ & $<4$ & --. & 5.1 & --. & .75 & $<4$ & $<.8$ & 34 \\
\hline 351 & SS89-104 & $<1$ & $<4$ & $<4$ & -.- & 5.6 & -- & .3 & $<4$ & $<.8$ & 38 \\
\hline 352 & SS89-105 & $<1$ & $<4$ & $<4$ & -- & 6.6 & -- & .48 & $<4$ & $<.8$ & 34 \\
\hline 353 & SS89-106 & $<1$ & $<4$ & $<4$ & -- & 6.0 & -.. & .2 & $<4$ & $<.8$ & 37 \\
\hline 354 & SS89-107 & $<1$ & $<4$ & $<4$ & -- & 6.4 & -- & .33 & $<4$ & $<.8$ & 29 \\
\hline 355 & SS89-108 & $<1$ & $<4$ & $<4$ & -.. & 5.6 & -. & .57 & $<4$ & $<.8$ & 30 \\
\hline 356 & SS89-109 & $<1$ & $<4$ & $<4$ & $\ldots$ & 4.7 & --. & .1 & $<4$ & $<.8$ & 30 \\
\hline 357 & SS89-110 & $<1$ & $<4$ & $<4$ & -- & 4.4 & --. & .57 & $<4$ & $<.8$ & 29 \\
\hline 358 & SS89-111 & $<1$ & $<4$ & $<4$ & -- & 3.9 & -- & .56 & $<4$ & $<.8$ & 28 \\
\hline \multicolumn{12}{|c|}{ Site B39 (McKendry Road) } \\
\hline 359 & SS89-78 & $<1$ & $<3$ & $<4$ & $\ldots$ & 5.9 & $\ldots$ & 21.2 & $<4$ & $<0.6$ & 45 \\
\hline 360 & SS89-79 & $<1$ & $<3$ & $<4$ & -- & 14.2 & -- & 26.5 & $<4$ & $<.6$ & 55.3 \\
\hline 361 & SS89-80 & $<1$ & $<3$ & $<4$ & - & 3.7 & - & 61.5 & $<4$ & $<.6$ & 63.2 \\
\hline 362 & SS89-81 & $<1$ & $<3$ & $<4$ & -- & 5.8 & -- & 34.3 & $<4$ & $<.6$ & 52.4 \\
\hline 363 & SS89-82 & $<1$ & $<3$ & $<4$ & -- & 4.3 & - & 22.2 & $<4$ & $<.7$ & 47 \\
\hline
\end{tabular}


Table 23. Organic chemical analysis, moisture content, and lipid content for biotic samples collected during 1986-90 from the Salton Sea and associated drainwaters and rivers

[All chemical data reported in micrograms per gram, wet weight. DDD, dichlorodiphenyldichloroethane; DDE, dichlorodiphenylethylene; DDT, dichlorodiphenyltrichloroethane; $o, p^{\prime}$, ortho, para prime; $p, p^{\prime}$ para,para prime; BHC, benzene hexachloride; DCPA, dimethyltetrachlorophthalate; BI PH CL, biphenyl chemical structure with number of chlorine substituents designated; PCB, polychlorinated biphenyls with Arochlor numbers; <, less than indicated reporting limit; ---, not determined. The analysis for each sample is displayed as one line on six consecutive pages. NWR, National Wildlife Refuge]

\begin{tabular}{|c|c|c|c|c|c|c|c|c|}
\hline $\begin{array}{l}\text { Record } \\
\text { number }\end{array}$ & $\begin{array}{l}\text { Sample } \\
\text { number }\end{array}$ & Species & Matrix & $\begin{array}{l}\text { Percent } \\
\text { moisture }\end{array}$ & $\begin{array}{c}\text { Percent } \\
\text { lipid }\end{array}$ & $\begin{array}{l}\text { Oxychlor- } \\
\text { dane }\end{array}$ & $\begin{array}{l}\text { alpha- } \\
\text { Chlordane }\end{array}$ & $\begin{array}{c}\text { cis- } \\
\text { Chlordane }\end{array}$ \\
\hline
\end{tabular}

\begin{tabular}{|c|c|c|c|c|c|c|c|c|}
\hline \multirow[b]{2}{*}{1} & \multicolumn{8}{|c|}{ Site B1 (Salton Sea NWR--Unit 1) } \\
\hline & . . . LNSS86-04 & Sailfin molly & Whole body & 73.0 & 0.60 & $<0.0099$ & --- & $<0.0099$ \\
\hline 2 & ... LNSS86-01B & Tilapia & Whole body & 76.1 & 1.45 & $<.10$ & -- & $<.010$ \\
\hline 3 & . . L LNSS86-05B & Tilapia & Whole body & 74.1 & 1.02 & $<.0099$ & --- & $<.0099$ \\
\hline 4 & $\ldots$ LNSS86-40 & Tilapia & Whole body & 71.9 & 6.88 & $<.0099$ & -- & $<.0099$ \\
\hline 5 & . . LNSS86-41B & Orangemouth corvina & Whole body & 74.6 & 1.37 & $<.010$ & --- & $<.010$ \\
\hline 6 & . . . LNSS86-23 & Northern shoveler & Breast muscle & 70.6 & 3.60 & $<.0099$ & -- & $<.0099$ \\
\hline 7 & . . L LNSS86-25 & Ruddy duck & Breast muscle & 70.4 & 4.53 & $<.010$ & --. & $<.010$ \\
\hline 8 & . . . LNSS86-27 & Black-necked stilt & Breast muscle & 69.7 & 8.18 & $<.010$ & -.. & $<.010$ \\
\hline 9 & . . LNSS87-20 & Crayfish & Whole body & 72.2 & .69 & $\ldots$ & --- & $\ldots$ \\
\hline 10 & . . L LNSS87-10 & Asiatic river clam & Soft tissue & 28.4 & .40 & $<.01$ & $\ldots$ & $<.01$ \\
\hline 11 & . . L LNSS87-12 & Asiatic river clam & Soft tissue & 30.4 & .30 & $<.01$ & --- & $<.01$ \\
\hline 12 & . . L LNSS87-22 & Great blue heron & Breast muscle & 68.6 & 2.60 & .02 & -- & $<.01$ \\
\hline 13 & . . L LNSS88-41 & Ruddy duck & Breast muscle & 71.0 & 8.56 & $<.01$ & $<0.01$ & -- \\
\hline 14 & . . L LNSS88-47 & Ruddy duck & Breast muscle & 70.8 & 5.65 & $<.01$ & $<.01$ & -- \\
\hline 15 & . . LNSS89-01 & Ruddy duck & Breast muscle & 70.2 & 3.96 & $<.01$ & $<.01$ & -- \\
\hline 16 & . . LNSS89-04 & Ruddy duck & Breast muscle & 71.0 & 2.68 & $<.01$ & $<.01$ & -- \\
\hline 17 & . . . LNSS89-06 & Ruddy duck & Breast muscle & 71.2 & 2.20 & $<.01$ & $<.01$ & -- \\
\hline 18 & . . L LNSS89-08 & Ruddy duck & Breast muscle & 68.2 & 5.24 & $<.01$ & $<.01$ & $-\ldots$ \\
\hline 19 & . . L LNSS89-10 & Ruddy duck & Breast muscle & 70.0 & 3.60 & $<.01$ & $<.01$ & $-\ldots$ \\
\hline 20 & . . L LNSS89-12 & Ruddy duck & Breast muscle & 72.0 & 4.62 & $<.01$ & $<.01$ & -- \\
\hline 21 & . . LNSS89-14 & Ruddy duck & Breast muscle & 69.5 & 3.64 & $<.01$ & $<.01$ & -- \\
\hline 22 & . . L LNSS89-16 & Ruddy duck & Breast muscle & 68.5 & 4.26 & $<.01$ & $<.01$ & $\ldots$ \\
\hline 23 & . . L LNSS89-18 & Ruddy duck & Breast muscle & 70.0 & 5.18 & $<.01$ & $<.01$ & --- \\
\hline 24 & . . L LNSS89-20 & Ruddy duck & Breast muscle & 71.5 & 3.24 & $<.01$ & $<.01$ & --- \\
\hline 25 & . . L LNSS89-22 & Ruddy duck & Breast muscle & 68.0 & 6.04 & $<.01$ & $<.01$ & --- \\
\hline 26 & . . . SS89-112 & Black-necked stilt & Egg & 75.0 & 12.5 & .02 & .01 & -- \\
\hline 27 & $\ldots$. . ss89-113 & Black-necked stilt & Egg & 73.0 & 12.4 & .01 & .01 & $\ldots$ \\
\hline 28 & $\ldots$ ss89-114 & Black-necked stilt & Egg & 76.0 & 10.7 & $<.01$ & $<.01$ & $\ldots$ \\
\hline 29 & . . Ss889-115 & Black-necked stilt & Egg & 75.0 & 13.6 & $<.01$ & $<.01$ & -.. \\
\hline 30 & $\ldots$. SS89-116 & Black-necked stilt & Egg & 74.0 & 10.5 & .03 & .01 & -- \\
\hline \multicolumn{9}{|c|}{ Site B10 (S Drain Outlet) } \\
\hline 31 & . . ss89-170 & Bairdiella & Whole body & 70.5 & 8.20 & $<0.01$ & $<0.01$ & --- \\
\hline 32 & . . ss89-171 & Bairdiella & Whole body & 71.5 & 5.34 & $<.01$ & $<.01$ & $-\cdots$ \\
\hline 33 & . . . SS89-172 & Bairdiella & Whole body & 74.5 & 5.32 & $<.01$ & $<.01$ & $-\cdots$ \\
\hline 34 & $\ldots$. . ss89-173 & Bairdiella & Whole body & 72.5 & 6.64 & $<.01$ & $<.01$ & -- \\
\hline 35 & ... SS89-174 & Bairdiella & Whole body & 76.0 & 4.30 & $<.01$ & $<.01$ & -- \\
\hline \multicolumn{9}{|c|}{ Site B11 (Alamo River Delta) } \\
\hline 36 & . . . LNSS86-67 & Northern shoveler & Breast muscle & 71.7 & 3.44 & $<0.0099$ & -- & $<0.0099$ \\
\hline 37 & . . LNSS88-73 & Waterboatman & Composite & 73.2 & 1.59 & $<.05$ & $<0.05$ & -- \\
\hline 38 & . . LNSS88-62 & Cray fish & Whole body & 78.5 & 1.25 & $<.05$ & $<.05$ & --- \\
\hline 39 & . . . LNSS88-29 & Northern shoveler & Breast muscle & 74.0 & 3.08 & $<.01$ & $<.01$ & -- \\
\hline 40 & . . LNSS88-74 & Black-necked stilt & Carcass & 77.2 & 5.02 & $<.05$ & $<.05$ & -- \\
\hline 41 & . . LNSS88-75 & Black-necked stilt & Carcass & 79.7 & 5.98 & $<.05$ & $<.05$ & -- \\
\hline 42 & . . LNSS88-76 & Black-necked stilt & Carcass & 82.0 & 3.51 & $<.05$ & $<.05$ & --. \\
\hline
\end{tabular}


Table 23. Organic chemical analysis, moisture content, and lipid content for biotic samples collected during 1986-90 from the Salton Sea and associated drainwaters and rivers--Continued

\begin{tabular}{|c|c|c|c|c|c|c|c|c|c|}
\hline $\begin{array}{l}\text { Record } \\
\text { number }\end{array}$ & $\begin{array}{l}\text { Sample } \\
\text { number }\end{array}$ & $\begin{array}{l}\text { gamma- } \\
\text { Chlordane }\end{array}$ & $\begin{array}{c}\text { trans- } \\
\text { Chlordane }\end{array}$ & $\begin{array}{l}\text { cis-Nona- } \\
\text { chlor }\end{array}$ & $\begin{array}{c}\text { trans- } \\
\text { Nonachlor }\end{array}$ & $\begin{array}{l}\text { Hepta- } \\
\text { chlor }\end{array}$ & $\begin{array}{l}\text { Heptachlor } \\
\text { epoxide }\end{array}$ & $\begin{array}{l}\text { Methyoxy- } \\
\text { chlor }\end{array}$ & $o, p^{\prime}-D D E$ \\
\hline \multicolumn{10}{|c|}{ Site B1 (Salton Sea NWR--Unit 1) } \\
\hline 1 & LNSS86-04 & -- & $<0.0099$ & $<0.0099$ & $<0.0099$ & --- & $<0.0099$ & -- & - \\
\hline 2 & LNSS86-01B & -- & $<.010$ & $<.010$ & $<.010$ & $-\ldots$ & $<.010$ & $\ldots$ &.-- \\
\hline 3 & LNSS86-05B & $\ldots$ & $<.0099$ & $<.0099$ & $<.0099$ & -- & $<.0099$ & $\ldots$ & $\ldots$ \\
\hline 4 & LNSS86-40 & -- & $<.0099$ & $<.0099$ & $<.0099$ & -- & $<.0099$ & $\ldots$ & $-\ldots$ \\
\hline 5 & LNSS86-41B & --- & $<.010$ & $<.010$ & $<.010$ & --- & $<.010$ & -- & - \\
\hline 6 & LNSS86-23 & - & $<.0099$ & $<.0099$ & $<.0099$ & - & $<.0099$ & $\ldots$ & --. \\
\hline 7 & LNSS86-25 & -- & $<.010$ & $<.010$ & $<.010$ & -.- & $<.010$ & $\ldots$ & - \\
\hline 8 & LNSS86-27 & -- & $<.010$ & $<.010$ & $<.010$ & - & $<.010$ & -- & -- \\
\hline 9 & LNSS87-20 & -- & -- & --- & - & --- & - & -- & $\ldots$ \\
\hline 10 & LNSS87-10 & - & $<.01$ & $<.01$ & $<.01$ & $<0.01$ & $<.01$ & $<0.01$ & 0.01 \\
\hline 11 & LNSS87-12 & -- & $<.01$ & $<.01$ & $<.01$ & $<.01$ & $<.01$ & $<.01$ & .01 \\
\hline 12 & LNSS87-22 & - & $<.01$ & $<.01$ & .05 & $<.01$ & .01 & .03 & $<.01$ \\
\hline 13 & LNSS88-41 & $<0.01$ & - & $<.01$ & $<.01$ & -- & $<.01$ & - & $<.01$ \\
\hline 14 & LNSS88-47 & $<.01$ & -- & $<.01$ & $<.01$ & -- & $<.01$ & -- & $<.01$ \\
\hline 15 & LNSS89-01 & $<.01$ & -- & $<.01$ & $<.01$ & $\ldots$ & $<.01$ & - & $<.01$ \\
\hline 16 & LNSS89-04 & $<.01$ & -- & $<.01$ & $<.01$ & -- & $<.01$ & - & $<.01$ \\
\hline 17 & LNSS89-06 & $<.01$ & - & $<.01$ & $<.01$ & -- & $<.01$ & --- & $<.01$ \\
\hline 18 & LNSS89-08 & $<.01$ & - & $<.01$ & $<.01$ & $\ldots$ & $<.01$ & -- & $<.01$ \\
\hline 19 & LNSS89-10 & $<.01$ & - & $<.01$ & $<.01$ & - & $<.01$ & $\ldots$ & $<.01$ \\
\hline 20 & LNSS89-12 & $<.01$ & $-\ldots$ & $<.01$ & $<.01$ & -- & $<.01$ & -- & $<.01$ \\
\hline 21 & LNSS89-14 & $<.01$ & -- & $<.01$ & $<.01$ & -- & $<.01$ &.-- & $<.01$ \\
\hline 22 & LNSS89-16 & $<.01$ & - & $<.01$ & $<.01$ & - & $<.01$ & --- & $<.01$ \\
\hline 23 & LNSS89-18 & $<.01$ & -- & $<.01$ & $<.01$ & -- & $<.01$ & -- & $<.01$ \\
\hline 24 & LNSS89-20 & $<.01$ & - & $<.01$ & $<.01$ & - & $<.01$ & -- & $<.01$ \\
\hline 25 & LNSS89-22 & $<.01$ & -- & $<.01$ & $<.01$ & -- & $<.01$ & -- & $<.01$ \\
\hline 26 & SS89-112 & $<.01$ & - & $<.01$ & $<.01$ & - & .01 & -- & $<.01$ \\
\hline 27 & SS89-113 & $<.01$ & -- & $<.01$ & $<.01$ & - & $<.01$ & - & $<.01$ \\
\hline 28 & SS89-114 & $<.01$ & -- & $<.01$ & $<.01$ & - & $<.01$ & --- & $<.01$ \\
\hline 29 & SS89-115 & $<.01$ & $\ldots$ & $<.01$ & $<.01$ & $\ldots$ & $<.01$ & --- & $<.01$ \\
\hline 30 & SS89-116 & $<.01$ & -- & $<.01$ & .01 & -- & .01 & -- & $<.01$ \\
\hline \multicolumn{10}{|c|}{ Site B10 (S Drain Outlet) } \\
\hline 31 & SS89-170 & $<0.01$ & $-\ldots$ & $<0.01$ & $<0.01$ & --- & $<0.01$ & --- & $<0.01$ \\
\hline 32 & Ss89-171 & $<.01$ & - & $<.01$ & $<.01$ & -- & $<.01$ & --- & $<.01$ \\
\hline 33 & SS89-172 & $<.01$ & - & $<.01$ & $<.01$ & - & $<.01$ & --- & $<.01$ \\
\hline 34 & SS89-173 & $<.01$ & - & $<.01$ & $<.01$ & - & $<.01$ & ... & $<.01$ \\
\hline 35 & SS89-174 & $<.01$ & -- & $<.01$ & $<.01$ & -- & $<.01$ & $\cdots$ & $<.01$ \\
\hline \multicolumn{10}{|c|}{ Site B11 (Alamo River Delta) } \\
\hline 36 & LNSS86-67 & $\ldots$ & $<0.0099$ & $<0.0099$ & $<0.0099$ & -- & $<0.0099$ & -- & -- \\
\hline 37 & LNSS88-73 & $<0.05$ & - & $<.05$ & $<.05$ & $<0.05$ & $<.05$ & --- & $<0.05$ \\
\hline 38 & LNSS88-62 & $<.05$ & --- & $<.05$ & $<.05$ & $<.05$ & $<.05$ & -.. & $<.05$ \\
\hline 39 & LNSS88-29 & $<.01$ & --- & $<.01$ & $<.01$ & $\ldots$ & $<.01$ &.-- & $<.01$ \\
\hline 40 & LNSS88.74 & $<.05$ & -- & $<.05$ & $<.05$ & $<.05$ & $<.05$ & -- & $<.05$ \\
\hline 41 & LNSS88-75 & $<.05$ & -- & $<.05$ & $<.05$ & $<.05$ & $<.05$ & --- & $<.05$ \\
\hline 42 & LNSS88-76 & $<.05$ & --- & $<.05$ & $<.05$ & $<.05$ & $<.05$ & -- & $<.05$ \\
\hline
\end{tabular}


Table 23. Organic chemical analysis, moisture content, and lipid content for biotic samples collected during 1986-90 from the Salton Sea and associated drainwaters and rivers--Continued

\begin{tabular}{|c|c|c|c|c|c|c|c|c|c|c|}
\hline $\begin{array}{l}\text { Record } \\
\text { number }\end{array}$ & $\begin{array}{l}\text { Sample } \\
\text { number }\end{array}$ & p,p'-DDE & $o, p^{\prime}-D D D$ & $p, p^{\prime}-D D D$ & $0, \mathbf{p}^{\prime}$-DDT & p,p'-DDT & $\begin{array}{l}\text { Total } \\
\text { DDT }\end{array}$ & Endrin & Dieldrin & Aldrin \\
\hline \multicolumn{11}{|c|}{ Site B1 (Salton Sea NWR--Unit 1) } \\
\hline 1 & LNSS86-04 & 0.18 & -- & $<0.0099$ & -- & $<0.0099$ & 0.180 & $<0.0099$ & $<0.0099$ & -- \\
\hline 2 & LNSS86-01B & .35 & -- & $<.010$ & -- & $<.010$ & .350 & $<.010$ & $<.010$ & $\ldots$ \\
\hline 3 & LNSS86-05B & .20 & -- & $<.0099$ & -- & $<.0099$ & .200 & $<.0099$ & $<.0099$ & -- \\
\hline 4 & LNSS86-40 & .37 & -- & 0.36 &.- & $<.0099$ & .406 & $<.0099$ & $<.0099$ & -- \\
\hline 5 & LNSS86-41 B & .087 & -- & $<.010$ & -- & $<.010$ & .087 & $<.010$ & $<.010$ & -- \\
\hline 6 & LNSS86-23 & .24 & -- & $<.0099$ & -- & $<.0099$ & .240 & $<.0099$ & $<.0099$ & -- \\
\hline 7 & LNSS86-25 & .096 & -- & $<.010$ & -- & $<.010$ & .096 & $<.010$ & $<.010$ & - \\
\hline 8 & LNSS86-27 & 4.8 & -- & $<.010$ & -- & .062 & 4.862 & $<.010$ & .038 & 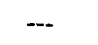 \\
\hline 9 & LNSS87-20 & -- & -- & -- & --- & -- & -- & - & --- & --- \\
\hline 10 & LNSS87-10 & .19 & $<0.01$ & $<.01$ & $<0.01$ & $<.01$ & .200 & $<.01$ & $<.01$ & 0.01 \\
\hline 11 & LNSS87-12 & .19 & $<.01$ & $<.01$ & $<.01$ & $<.01$ & .200 & $<.01$ & $<.01$ & $<.01$ \\
\hline 12 & LNSS87-22 & 13 & $<.01$ & .02 & .03 & .01 & 13.060 & $<.01$ & .11 & $<.01$ \\
\hline 13 & LNSS88-41 & .56 & $<.01$ & $<.01$ & $<.01$ & $<.01$ & .560 & $<.01$ & .01 & -- \\
\hline 14 & LNSS88-47 & 1.5 & $<.01$ & .03 & $<.01$ & .02 & 1.550 & $<.01$ & .02 & - \\
\hline 15 & LNSS89-01 & .26 & $<.01$ & $<.01$ & $<.01$ & $<.01$ & .260 & $<.01$ & $<.01$ & - \\
\hline 16 & LNSS89-04 & .19 & $<.01$ & $<.01$ & $<.01$ & $<.01$ & .190 & $<.01$ & $<.01$ & -- \\
\hline 17 & LNSS89-06 & 1.0 & $<.01$ & $<.01$ & $<.01$ & $<.01$ & 1.000 & $<.01$ & $<.01$ & -- \\
\hline 18 & LNSS89-08 & .36 & $<.01$ & $<.01$ & $<.01$ & $<.01$ & .360 & $<.01$ & $<.01$ & -- \\
\hline 19 & LNSS89-10 & .24 & $<.01$ & $<.01$ & $<.01$ & $<.01$ & .240 & $<.01$ & $<.01$ & $\cdots$ \\
\hline 20 & LNSS89-12 & .51 & $<.01$ & $<.01$ & $<.01$ & $<.01$ & .510 & $<.01$ & $<.01$ & $\ldots$ \\
\hline 21 & LNSS89-14 & .28 & $<.01$ & $<.01$ & $<.01$ & $<.01$ & .280 & $<.01$ & $<.01$ & $\cdots$ \\
\hline 22 & LNSS89-16 & .46 & $<.01$ & .02 & $<.01$ & .02 & .500 & $<.01$ & .01 & -- \\
\hline 23 & LNSS89-18 & .37 & $<.01$ & $<.01$ & $<.01$ & $<.01$ & .370 & $<.01$ & $<.01$ & -- \\
\hline 24 & LNSS89-20 & .16 & $<.01$ & $<.01$ & $<.01$ & $<.01$ & .160 & $<.01$ & $<.01$ & $\cdots$ \\
\hline 25 & LNSS89-22 & .17 & $<.01$ & $<.01$ & $<.01$ & $<.01$ & .170 & $<.01$ & $<.01$ & -- \\
\hline 26 & SS89-112 & 2.0 & $<.01$ & $<.01$ & $<.01$ & .07 & 2.070 & $<.01$ & .01 & -- \\
\hline 27 & SS89-113 & 2.1 & $<.01$ & $<.01$ & $<.01$ & .03 & 2.130 & $<.01$ & .02 &.-- \\
\hline 28 & SS89-114 & .64 & $<.01$ & $<.01$ & $<.01$ & $<.01$ & .640 & $<.01$ & .01 & --- \\
\hline 29 & SS89-115 & .86 & $<.01$ & $<.01$ & $<.01$ & $<.01$ & .860 & $<.01$ & .02 & --- \\
\hline 30 & SS89-116 & 1.6 & $<.01$ & $<.01$ & $<.01$ & .05 & 1.650 & $<.01$ & .03 & --- \\
\hline \multicolumn{11}{|c|}{ Site B10 (S Drain Outlet) } \\
\hline 31 & SS89-170 & 0.09 & $<0.01$ & $<0.01$ & $<0.01$ & $<0.01$ & 0.090 & $<0.01$ & $<0.01$ & --- \\
\hline 32 & SS89-171 & .08 & $<.01$ & $<.01$ & $<.01$ & $<.01$ & .080 & $<.01$ & $<.01$ & --- \\
\hline 33 & SS89-172 & .12 & $<.01$ & $<.01$ & $<.01$ & $<.01$ & .120 & $<.01$ & $<.01$ & --- \\
\hline 34 & SS89-173 & .12 & $<.01$ & $<.01$ & $<.01$ & $<.01$ & .120 & $<.01$ & $<.01$ & $\cdots$ \\
\hline 35 & SS89-174 & .10 & $<.01$ & $<.01$ & $<.01$ & $<.01$ & .100 & $<.01$ & $<.01$ & -- \\
\hline \multicolumn{11}{|c|}{ Site B11 (Alamo River Delta) } \\
\hline 36 & LNSS86-67 & 0.63 & -- & 0.021 & $\ldots$ & $<0.0099$ & 0.840 & $<0.0099$ & 0.012 & -- \\
\hline 37 & LNSS88-73 & .07 & $<0.05$ & $<.05$ & $<0.05$ & $<.05$ & .07 & $<.05$ & $<.05$ & $<0.05$ \\
\hline 38 & LNSS88-62 & .10 & $<.05$ & $<.05$ & $<.05$ & $<.05$ & 1.09 & $<.05$ & $<.05$ & $<.05$ \\
\hline 39 & LNSS88-29 & .89 & $<.01$ & .01 & $<.01$ & .01 & .910 & $<.01$ & .02 & --- \\
\hline 40 & LNSS88-74 & .39 & $<.05$ & $<.05$ & $<.05$ & $<.05$ & $<.39$ & $<.05$ & .05 & $<.05$ \\
\hline 41 & LNSS88-75 & 1.0 & $<.05$ & $<.05$ & $<.05$ & $<.05$ & 9.97 & $<.05$ & .07 & $<.05$ \\
\hline 42 & LNSS88-76 & 1.42 & $<.05$ & $<.05$ & $<.05$ & $<.05$ & 1.42 & $<.05$ & $<.05$ & $<.05$ \\
\hline
\end{tabular}


Table 23. Organic chemical analysis, moisture content, and lipid content for biotic samples collected during 1986-90 from the Salton Sea and associated drainwaters and rivers--Continued

\begin{tabular}{|c|c|c|c|c|c|c|c|c|c|c|c|c|c|}
\hline $\begin{array}{l}\text { Record } \\
\text { number }\end{array}$ & $\begin{array}{l}\text { Sample } \\
\text { number }\end{array}$ & $\begin{array}{l}\text { alpha- } \\
\text { BHC }\end{array}$ & $\begin{array}{l}\text { beta- } \\
\text { BHC }\end{array}$ & $\begin{array}{l}\text { delta- } \\
\text { BHC }\end{array}$ & $\begin{array}{c}\text { gamma- } \\
\text { BHC }\end{array}$ & $\begin{array}{l}\text { Lin- } \\
\text { dane }\end{array}$ & $\begin{array}{c}\text { Hexa- } \\
\text { chloro- } \\
\text { ben- } \\
\text { zene }\end{array}$ & $\begin{array}{c}\text { Endo- } \\
\text { sul- } \\
\text { fan I }\end{array}$ & $\begin{array}{c}\text { Endo- } \\
\text { sul- } \\
\text { fan II }\end{array}$ & $\begin{array}{l}\text { Endo- } \\
\text { sulfan } \\
\text { sulfate }\end{array}$ & Mirex & DCPA & Dicofol \\
\hline
\end{tabular}

Site B1 (Salton Sea NWR--Unit 1)

\begin{tabular}{|c|c|c|c|c|c|c|c|c|c|c|c|c|c|}
\hline 1 & LNSS86-04 & -- & - & $\cdots$ & $\ldots$ & $\cdots$ & - & $\ldots$ & $\ldots$ & $\ldots$ & -- & $\cdots$ &.-- \\
\hline 2 & LNSS86-01B & - & -- & --- & --- & $\ldots$ & - & $-\ldots$ & 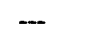 & $\ldots$ & -- & $\ldots$ & --. \\
\hline 3 & LNSS86-05B & -- & - & -- & - & -- & -- & - & -- & - & -- & $-\cdots$ & -- \\
\hline 4 & LNSS86-40 & - & $\ldots$ & -- & -- & -- & -- & -- & - & - & - & --- & -- \\
\hline 5 & LNSS86-41B & - & -- & $\cdots$ & -- & -- & -- & - & $\cdots$ & - & - & --- & -- \\
\hline 6 & LNSS86-23 & - & - & - & - & - & - & - & - & - & -- & $\cdots$ & -- \\
\hline 7 & LNSS86-25 & -- & -- & - & -- & - & -- & -- & -- & -- & -- & $\cdots$ & --- \\
\hline 8 & LNSS86-27 & - & -- & - & -- & - & - & -- & - & -- & -- & --- & $\cdots$ \\
\hline 9 & LNSS87-20 & - & - & - & -- & -- & -- & -- & - & -- & --- & -- & -.. \\
\hline 10 & LNSS87-10 & $<0.01$ & $<0.01$ & $<0.01$ & $<0.01$ & -- & $<0.01$ & $<0.01$ & $<0.01$ & $<0.01$ & $<0.01$ & 0.24 & $<0.01$ \\
\hline 11 & LNSS87-12 & $<.01$ & $<.01$ & $<.01$ & $<.01$ & --- & $<.01$ & $<.01$ & $<.01$ & $<.01$ & $<.01$ & .32 & $<.01$ \\
\hline 12 & LNSS87-22 & $<.01$ & $<.01$ & $<.01$ & $<.01$ & -- & .02 & .01 & .02 & $<.01$ & $<.01$ & $<.01$ & $<.01$ \\
\hline 13 & LNSS88-41 & $<.01$ & $<.01$ & $<.01$ & $<.01$ & -- & $<.01$ & -- & -- & - & $<.01$ & -- & - \\
\hline 14 & LNSS88-47 & $<.01$ & $<.01$ & $<.01$ & $<.01$ & -- & .01 & - & -- & - & $<.01$ & -- & -- \\
\hline 15 & LNSS89-01 & $<.01$ & $<.01$ & $<.01$ & $<.01$ & -- & $<.01$ & $<.01$ & $<.01$ & - & $<.01$ & --- & $\cdots$ \\
\hline 16 & LNSS89-04 & $<.01$ & $<.01$ & $<.01$ & $<.01$ & -- & $<.01$ & $<.01$ & $<.01$ & $\ldots$ & $<.01$ & --- & - \\
\hline 17 & LNSS89-06 & $<.01$ & $<.01$ & $<.01$ & $<.01$ & -- & $<.01$ & $<.01$ & $<.01$ & - & $<.01$ & -- & -- \\
\hline 18 & LNSS89-08 & $<.01$ & $<.01$ & $<.01$ & $<.01$ & -.. & $<.01$ & $<.01$ & $<.01$ & - & $<.01$ & --- & -- \\
\hline 19 & LNSS89-10 & $<.01$ & $<.01$ & $<.01$ & $<.01$ & -- & $<.01$ & $<.01$ & $<.01$ & $\ldots$ & $<.01$ & --. & -- \\
\hline 20 & LNSS89-12 & $<.01$ & $<.01$ & $<.01$ & $<.01$ & --- & $<.01$ & $<.01$ & $<.01$ & -- & $<.01$ & --- & -- \\
\hline 21 & LNSS89-14 & $<.01$ & $<.01$ & $<.01$ & $<.01$ & -- & $<.01$ & $<.01$ & $<.01$ & -- & $<.01$ & --- & - \\
\hline 22 & LNSS89-16 & $<.01$ & $<.01$ & $<.01$ & $<.01$ & -- & $<.01$ & $<.01$ & $<.01$ & -- & $<.01$ & --- & -- \\
\hline 23 & LNSS89-18 & $<.01$ & $<.01$ & $<.01$ & $<.01$ & -- & $<.01$ & $<.01$ & $<.01$ & -- & $<.01$ & --- & -- \\
\hline 24 & LNSS89-20 & $<.01$ & $<.01$ & $<.01$ & $<.01$ & -- & $<.01$ & $<.01$ & $<.01$ & -- & $<.01$ & -.- & -- \\
\hline 25 & LNSS89-22 & $<.01$ & $<.01$ & $<.01$ & $<.01$ & -- & $<.01$ & $<.01$ & $<.01$ & --- & $<.01$ &.-- & --- \\
\hline 26 & SS89-112 & $<.01$ & .09 & $<.01$ & $<.01$ & -- & .01 & -- & -- & - & $<.01$ & --- & -- \\
\hline 27 & SS89-113 & $<.01$ & .04 & $<.01$ & $<.01$ & -- & .01 & - & - & -- & $<.01$ & --- & -- \\
\hline 28 & SS89-114 & $<.01$ & $<.01$ & $<.01$ & $<.01$ & --. & $<.01$ & -- & -- & -. & $<.01$ & --- & -- \\
\hline 29 & SS89-115 & $<.01$ & .05 & $<.01$ & $<.01$ & -- & $<.01$ & - & --- & -- & $<.01$ & --- & -- \\
\hline 30 & SS89-116 & $<.01$ & .16 & $<.01$ & $<.01$ & --- & .01 & -- & -- & -- & $<.01$ & --- & -- \\
\hline \multicolumn{14}{|c|}{ Site B10 (S Drain Outlet) } \\
\hline 31 & SS89-170 & $<0.01$ & $<0.01$ & $<0.01$ & $<0.01$ & -- & $<0.01$ & $<0.01$ & $<0.01$ & $\cdots$ & $<0.01$ & --- & -- \\
\hline 32 & SS89-171 & $<.01$ & $<.01$ & $<.01$ & $<.01$ & -- & $<.01$ & $<.01$ & $<.01$ & -- & $<.01$ & $\ldots$ & --- \\
\hline 33 & SS89-172 & $<.01$ & $<.01$ & $<.01$ & $<.01$ & -- & $<.01$ & $<.01$ & $<.01$ & --- & $<.01$ & -- & - \\
\hline 34 & SS89-173 & $<.01$ & $<.01$ & $<.01$ & $<.01$ & -- & $<.01$ & $<.01$ & $<.01$ & - & $<.01$ & -- & -- \\
\hline 35 & SS89-174 & $<.01$ & $<.01$ & $<.01$ & $<.01$ & -- & $<.01$ & $<.01$ & $<.01$ & -- & $<.01$ & -- & --- \\
\hline
\end{tabular}

\section{Site B11 (Alamo River Delta)}

\begin{tabular}{|c|c|c|c|c|c|c|c|c|c|c|c|c|c|}
\hline 36 & LNSS86-67 & - & - & - & -- & - & $\ldots$ & - & -- & -- & -- & -- & -- \\
\hline 37 & LNSS88-73 & $<0.05$ & $<0.05$ & $<0.05$ & - & $<0.05$ & $<0.05$ & -- & -. & -- & $<0.05$ & -- & -- \\
\hline 38 & LNSS88-62 & $<.05$ & $<.05$ & $<.05$ & - & $<.05$ & $<.05$ & $\ldots$ & -- & - & $<.05$ &.-- & -- \\
\hline 39 & LNSS88-29 & $<.01$ & $<.01$ & $<.01$ & $<0.01$ & $\ldots$ & $<.01$ & $\cdots$ &.- &.- & $<.01$ & $\ldots$ & -- \\
\hline 40 & LNSS88-74 & $<.05$ & $<.05$ & $<.05$ & -- & $<.05$ & $<.05$ & $\ldots$ & -- & -- & $<.05$ & -- & -- \\
\hline 41 & LNSS88-75 & $<.05$ & $<.05$ & $<.05$ & $\ldots$ & $<.05$ & $<.05$ & -. & -- &.- & $<.05$ & --- & $\cdots$ \\
\hline 42 & LNSS88-76 & $<.05$ & $<.05$ & $<.05$ & - & $<.05$ & $<.05$ & -.. & -- & --. & $<.05$ &..- & $\cdots$ \\
\hline
\end{tabular}


Table 23. Organic chemical analysis, moisture content, and lipid content for biotic samples collected during 1986-90 from the Salton Sea and associated drainwaters and rivers--Continued

\begin{tabular}{lllllllllllllll}
\hline Record & Sample & Tetra- & BI PH & BI PH & BI PH & BI PH & BI PH & BI PH & BI PH & BI PH & PCB & PCB & PCB & PCB \\
number & number & difon & CL-2 & CL-3 & CL-4 & CL-5 & CL-6 & CL-7 & CL-8 & CL-9 & 1016 & 1221 & 1232 & 1242
\end{tabular}

\begin{tabular}{|c|c|c|c|c|c|c|c|c|c|c|c|c|c|c|}
\hline \multirow[b]{2}{*}{1} & \multirow[b]{2}{*}{ LNSS86-04 } & \multirow[b]{2}{*}{--} & \multirow[b]{2}{*}{--} & \multicolumn{6}{|c|}{ Site B1 (Salton Sea NWR--Unit 1) } & \multirow[b]{2}{*}{--} & \multirow[b]{2}{*}{--} & \multirow[b]{2}{*}{---} & \multirow[b]{2}{*}{--} & \multirow[b]{2}{*}{--} \\
\hline & & & & - & -- & -- & - & -- & -- & & & & & \\
\hline 2 & LNSS86-01B & -- & -- & -- & -- & - & - & -- & -- & -- & --- & --- & --- & --- \\
\hline 3 & LNSS86-05B & -- & -- & -- & -- & --- & --- & -- & -- & $-\cdots$ & -- &.-- & --- & -- \\
\hline 4 & LNSS86-40 & -- & - & - & -- & - & -- & --- & -- & -- & -- & -- & --- & - \\
\hline 5 & LNSS86-41B & - & - & - & -- & -- & - & -- & - & - & -- & -- & --- &.-- \\
\hline 6 & LNSS86-23 & -- & -- & - & - & -- & --- & -- & -- & - & -- & --- & --- & -- \\
\hline 7 & LNSS86-25 & -- & -- & -- & -- & - & -- & --- & - & - & -- & -- & --- & -- \\
\hline 8 & LNSS86-27 & -- & -- & - & $\cdots$ & -- & -- & -- & -- & -- & -- & --- & --- & --- \\
\hline 9 & LNSS87-20 & -- & --- & - & -- & -- & -- & - & -- & - & $\ldots$ & -.- & -- & -- \\
\hline 10 & LNSS87-10 & $<0.01$ & - & - & -- & --- & $\ldots$ & -- & $\ldots$ & $\cdots$ & $<0.05$ & $<0.50$ & $<0.05$ & $<0.05$ \\
\hline 11 & LNSS87-12 & $<.01$ & -- & -- & -- & -- & -- & --- & -- & -- & $<.05$ & $<.50$ & $<.05$ & $<.05$ \\
\hline 12 & LNSS87-22 & $<.01$ & -- & - & -- & - & - & -- & -- & -- & $<.05$ & $<.50$ & $<.05$ & $<.05$ \\
\hline 13 & LNSS88-41 & -- & -- & - & -- & -- & -- & -- & -- & --- & --- & --- & --- & -- \\
\hline 14 & LNSS88-47 & -- & -- & -- & -- & -- & -- & --- & --- & -- & -- &.-- & --- & -- \\
\hline 15 & LNSS89-01 & - & -- & -- & --- & -- & -- & -- & --- & -- & --- & --- & --- & $\cdots$ \\
\hline 16 & LNSS89-04 & -- & --- & -- & -- & --- & --- & --- &.-- & --- & --- & -- & --- & -- \\
\hline 17 & LNSS89-06 & - & - & -- & -- & -- & -- & -- & --- & --. & --- & --- & --- & -- \\
\hline 18 & LNSS89-08 & -- & -- & - & $-\cdots$ & $\cdots$ & -- & --- & --- & $--\cdot$ & -- & -- & --- & - \\
\hline 19 & LNSS89-10 & -- & - & -- & --- & --- & -- & -.. & $\ldots$ & - & -- & --- & -- & - \\
\hline 20 & LNSS89-12 & -- & -- & -- & -- & -- & --- & -- & $-\cdots$ & $\ldots$ & --- & --- & --- & --- \\
\hline 21 & LNSS89-14 & -- & -- & - & -- & -- & -- & --- & --- & -- & -- & -- & -- & -- \\
\hline 22 & LNSS89-16 & -- & - & -- & -- & -- & --- & -- & --- & -- & -- & --- & --- & --- \\
\hline 23 & LNSS89-18 & -- & -- & -- & -- & --- & --- & --- & --- & -- & --- & --- & -- & --- \\
\hline 24 & LNSS89-20 & - & - & --- & -- & -- & --- & -- & --- & -- & $\ldots$ & - & -- & -- \\
\hline 25 & LNSS89-22 & -- & -- & -- & -- & -- & --- & --- & -- & -.. & -- & --- & -.. & $\ldots$ \\
\hline 26 & SS89-112 & -- & - & -- & --- & -- & --- & -- & --- & -- & --- & --- & $-m$ & $\cdots$ \\
\hline 27 & SS89-113 & -- & - & $\cdots$ & - & 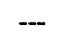 & --- & --- & --- & - & -- & --- & --. & $\cdots$ \\
\hline 28 & SS89-114 & -- & - & -- & -- & -- & -- & --- & --- & -- & -- & --- & --- & $\cdots$ \\
\hline 29 & SS89-115 & -- & -- & - & - & -- & -- & --- & --- & - & -- & --- & --- & $\cdots$ \\
\hline 30 & SS89-116 & -- & -- & -- & - & -- & -- & --- & -- & - & -- & -- & -- & -- \\
\hline
\end{tabular}

Site B10 (S Drain Outlet)

\begin{tabular}{|c|c|c|c|c|c|c|c|c|c|c|c|c|c|c|}
\hline & & & & & & 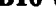 & - & & & & & & & \\
\hline 31 & SS89-170 & -- & -- & --- & - & --- & -- & -- & $\cdots$ & $\cdots$ & -- & $\cdots$ & --- & -- \\
\hline 32 & SS89-171 & - & -- & - & -- & -- & --- & -- & --- & - & -- & $\cdots$ & -- & $\cdots$ \\
\hline 33 & SS89-172 & - & -- & -- & -- & --- & -- & --- & -- & $\cdots$ & -- & --- & -- & -- \\
\hline 34 & SS89-173 & -- & -- & - & - & --- & -- & -- & -- & - & -- & --- & $-\cdots$ & -- \\
\hline 35 & SS89-174 & - & --- & -- & -- & --- & -- & $\cdots$ & -... & -- & -.- & --. & -.- & $\cdots$ \\
\hline
\end{tabular}

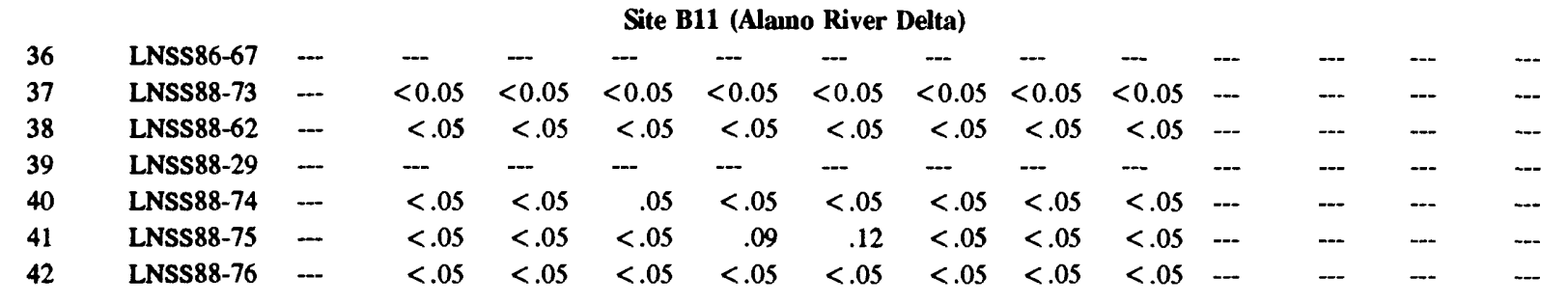


Table 23. Organic chemical analysis, moisture content, and lipid content for biotic samples collected during 1986-90 from the Salton Sea and associated drainwaters and rivers--Continued

\begin{tabular}{|c|c|c|c|c|c|c|}
\hline $\begin{array}{l}\text { Record } \\
\text { number }\end{array}$ & $\begin{array}{l}\text { Sample } \\
\text { number }\end{array}$ & $\begin{array}{l}\text { PCB } \\
1248\end{array}$ & $\begin{array}{l}\text { PCB } \\
1254\end{array}$ & $\begin{array}{l}\text { PCB } \\
1260\end{array}$ & $\begin{array}{l}\text { Total } \\
\text { PCB }\end{array}$ & Toxaphene \\
\hline \multicolumn{7}{|c|}{ Site B1 (Salton Sea NWR-Unit 1) } \\
\hline 1 & LNSS86-04 & -- & $<0.099$ & $<0.099$ & - & $<0.50$ \\
\hline 2 & LNSS86-01B & - & $<.10$ & $<.10$ & - & $<.50$ \\
\hline 3 & LNSS86-05B & -- & $<.099$ & $<.099$ & -- & $<.49$ \\
\hline 4 & LNSS86-40 & -- & $<.099$ & $<.099$ & $\ldots$ & $<.50$ \\
\hline 5 & LNSS86-41B & - & $<.10$ & $<.10$ & -- & $<.50$ \\
\hline 6 & LNSS86-23 & -- & $<.099$ & $<.099$ & - & $<.50$ \\
\hline 7 & LNSS86-25 & -- & $<.10$ & $<.10$ & -- & $<.50$ \\
\hline 8 & LNSS86-27 & -- & $<.10$ & $<.10$ & - & $<.50$ \\
\hline 9 & LNSS87-20 & -- & - & -- & - & --- \\
\hline 10 & LNSS87-10 & $<0.05$ & $<.05$ & $<.05$ & -- & $<.50$ \\
\hline 11 & LNSS87-12 & $<.05$ & $<.05$ & $<.05$ & $\cdots$ & $<.50$ \\
\hline 12 & LNSS87-22 & $<.05$ & $<.05$ & $<.05$ & -- & $<.50$ \\
\hline 13 & LNSS88-41 & -- & -- & -- & $<0.05$ & $<.05$ \\
\hline 14 & LNSS88-47 & -- & $\cdots$ & -- & $<.05$ & $<.05$ \\
\hline 15 & LNSS89-01 & -- & - & - & $<.05$ & $<.05$ \\
\hline 16 & LNSS89-04 & -- & -- & $\cdots$ & $<.05$ & $<.05$ \\
\hline 17 & LNSS89-06 & - & -- & -- & $<.05$ & $<.05$ \\
\hline 18 & LNSS89-08 & -- & --- & --. & $<.05$ & $<.05$ \\
\hline 19 & LNSS89-10 & $\cdots$ & -- & -- & $<.05$ & $<.05$ \\
\hline 20 & LNSS89-12 & -- & -.- & - & $<.05$ & $<.05$ \\
\hline 21 & LNSS89-14 & -- & $\cdots$ & - & $<.05$ & $<.05$ \\
\hline 22 & LNSS89-16 & -- & -- & -- & $<.05$ & $<.05$ \\
\hline 23 & LNSS89-18 & -- & $\cdots$ & - & $<.05$ & $<.05$ \\
\hline 24 & LNSS89-20 & - & -- & -- & $<.05$ & $<.05$ \\
\hline 25 & LNSS89-22 & $\ldots$ & -- & -- & $<.05$ & $<.05$ \\
\hline 26 & SS89-112 & -- & -- & -- & $<.05$ & $<.05$ \\
\hline 27 & SS89-113 & - & -- & - & $<.05$ & $<.05$ \\
\hline 28 & SS89-114 & -- & -.- & -- & $<.05$ & $<.05$ \\
\hline 29 & SS89-115 & -- & --- & -- & $<.05$ & $<.05$ \\
\hline 30 & SS89-116 & - & - & - & $<.05$ & $<.05$ \\
\hline \multicolumn{7}{|c|}{ Site B10 (S Drain Outlet) } \\
\hline 31 & SS89-170 & - & -- & -- & $<0.05$ & $<0.05$ \\
\hline 32 & SS89-171 & -- & - & - & $<.05$ & $<.05$ \\
\hline 33 & SS89-172 & - & - & -- & $<.05$ & $<.05$ \\
\hline 34 & SS89-173 & -- & -- & -- & $<.05$ & $<.05$ \\
\hline 35 & SS89-174 & - & -- & -- & $<.05$ & $<.05$ \\
\hline \multicolumn{7}{|c|}{ Site B11 (Alamo River Delta) } \\
\hline 36 & LNSS86-67 & -- & $<0.099$ & $<0.099$ & - & $<0.50$ \\
\hline 37 & LNSS88-73 & -- & --- & --- & - & $<.50$ \\
\hline 38 & LNSS88-62 & - & -- & $\ldots$ & +- & $<.50$ \\
\hline 39 & LNSS88-29 & $\cdots$ & - & -- & $<0.05$ & $<.05$ \\
\hline 40 & LNSS88-74 & - & -- & - & - & $<.50$ \\
\hline 41 & LNSS88-75 & -- & - & - & -- & $<.50$ \\
\hline 42 & LNSS88-76 & -- & - & -- & -- & $<.50$ \\
\hline
\end{tabular}


Table 23. Organic chemical analysis, moisture content, and lipid content for biotic samples collected during 1986-90 from the Salton Sea and associated drainwaters and rivers--Continued

\begin{tabular}{|c|c|c|c|c|c|c|c|c|}
\hline $\begin{array}{l}\text { Record } \\
\text { number }\end{array}$ & $\begin{array}{l}\text { Sample } \\
\text { number }\end{array}$ & Species & Matrix & $\begin{array}{l}\text { Percent } \\
\text { moisture }\end{array}$ & $\begin{array}{c}\text { Percent } \\
\text { lipid }\end{array}$ & $\begin{array}{l}\text { Oxychlor- } \\
\text { dane }\end{array}$ & $\begin{array}{c}\text { alpha- } \\
\text { Chlordane }\end{array}$ & $\begin{array}{c}\text { cis- } \\
\text { Chlordane }\end{array}$ \\
\hline
\end{tabular}

$\begin{array}{lll}43 & \ldots & \text { LNSS88-77 } \\ 44 & \ldots & \text { LNSS88-78 } \\ 45 & \ldots & \text { SS89-128 } \\ 46 & \ldots & \text { SS89-129 } \\ 47 & \ldots & \text { SS89-130 } \\ 48 & \ldots & \text { SS89-131 } \\ 49 & \ldots & \text { SS89-132 }\end{array}$

$50 \ldots$. $\$ \$ 89-142$

51 ... Ss89-144

$52 \ldots$. SS89-146

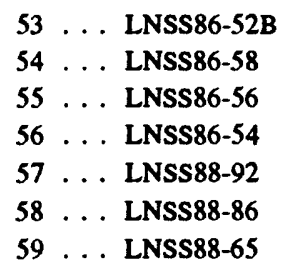

$60 \ldots$ LNSS88-67

$61 \ldots$ LNSS88-79

$62 \ldots$ LNSS88-80

$63 \ldots$ LNSS88-81

$64 \ldots$ LNSS88-82

65 ... LNSS88-83

$66 \ldots$. . SS89-76

$67 \ldots$. . SS89-148

$68 \ldots . \$ \$ 89-150$

$69 \ldots$. . SS89-133

70 ... ss89-134

$71 \ldots$. . SS89-135

$72 \ldots \mathbf{s} 889-136$

$73 \ldots$. . S\$89-137

74 ... SS89-93

$75 \ldots$. . SS89-94

$76 \ldots$. . s $\$ 89-95$

77 . . . s\$89-96

$78 \ldots$. . s $589-97$

79 . . . S\$89-98

$80 \ldots$. . S\$89-99

$81 \ldots$. . \$S89-100

$82 \ldots$. . SS89-101

83 ... SS89-102

$84 \ldots$ LNSS86-68B

85 ... LNSS86-51

86 ... LNSS86-48B

87 ... LNSS86-49B

\begin{abstract}
Black-necked stilt
Black-necked stilt

Black-necked stilt

Black-necked stilt

Black-necked stilt

Black-necked stilt

Black-necked stilt
\end{abstract}

\section{Eared grebe \\ Eared grebe \\ Eared grebe}

Tilapia
Ruddy duck
American coot
Black-necked stilt

Waterboatman

Asiatic river clam

Crayfish

Mosquitofish

Black-necked stilt

Black-necked stilt

Black-necked stilt

Black-necked stilt

Black-necked stilt

Waterboatman

Eared grebe

Eared grebe

Black-necked stilt

Black-necked stilt

Black-necked stilt

Black-necked stilt

Black-necked stilt

Black-necked stilt

Black-necked stilt

Black-necked stilt

Black-necked stilt

Black-necked stilt

Black-necked stilt

Black-necked stilt

Black-necked stilt

Black-necked stilt

Black-necked stilt

Asiatic river clam

Sailfin molly

Tilapia

Tilapia
Site B11 (Alamo River Delta)--Continued

$\begin{array}{lrrrrr}\text { Carcass } & 79.0 & 3.74 & <0.05 & <0.05 & \ldots \\ \text { Carcass } & 80.6 & 4.25 & <.05 & <.05 & \ldots \\ \text { Carcass } & 66.4 & 16.4 & .05 & <.01 & \ldots \\ \text { Carcass } & 66.2 & 15.4 & .02 & <.01 & -. \\ \text { Carcass } & 63.7 & 12.6 & .02 & <.01 & \ldots- \\ \text { Carcass } & 67.7 & 13.1 & .02 & <.01 & \ldots \\ \text { Carcass } & 63.8 & 11.9 & <.01 & <.01 & -\end{array}$

Site B13 (Obsidian Butte)

$\begin{array}{lllrrr}\text { Breast muscle } & 72.0 & 3.46 & <0.01 & <0.01 & -- \\ \text { Breast muscle } & 70.0 & 6.20 & <.01 & <.01 & -- \\ \text { Breast muscle } & 74.0 & 3.00 & <.01 & <.01 & --\end{array}$

Site B15 (New River Delta)

$\begin{array}{cc}\text { Whole body } & 72.1 \\ \text { Breast muscle } & 70.2 \\ \text { Breast muscle } & 72.0 \\ \text { Breast muscle } & 70.0 \\ \text { Composite } & 74.6 \\ \text { Soft tissue } & 80.0 \\ \text { Whole body } & 80.1 \\ \text { Whole body } & 78.2 \\ \text { Carcass } & 74.0 \\ \text { Carcass } & 74.4 \\ \text { Carcass } & 79.9 \\ \text { Carcass } & 83.6 \\ \text { Carcass } & 86.2 \\ \text { Composite } & 83.0 \\ \text { Breast muscle } & 74.0 \\ \text { Breast muscle } & 75.0 \\ \text { Carcass } & 68.4 \\ \text { Carcass } & 66.2 \\ \text { Carcass } & 66.6 \\ \text { Carcass } & 65.5 \\ \text { Carcass } & 64.2 \\ \text { Egg } & 73.0 \\ \text { Egg } & 74.0 \\ \text { Egg } & 72.0 \\ \text { Egg } & 76.0 \\ \text { Egg } & 76.0 \\ \text { Egg } & 63.0 \\ \text { Egg } & 76.0 \\ \text { Egg } & 76.0 \\ \text { Egg } & 76.0 \\ \text { Egg } & 71.0\end{array}$

\begin{tabular}{|c|c|c|c|}
\hline 1.24 & $<0.0099$ & $\cdots$ & $<0.0099$ \\
\hline 3.59 & $<.010$ & --- & $<.010$ \\
\hline 4.00 & $<.010$ & --. & $<.010$ \\
\hline 5.61 & $<.0099$ & --. & $<.0099$ \\
\hline 3.19 & $<.05$ & $<0.05$ & -- \\
\hline 1.53 & $<.05$ & $<.05$ & $\ldots$ \\
\hline 1.09 & $<.05$ & $<.05$ & - \\
\hline 2.60 & $<.05$ & $<.05$ & -- \\
\hline 3.66 & $<.05$ & $<.05$ & -- \\
\hline 11.12 & $<.05$ & $<.05$ & - \\
\hline 7.41 & $<.05$ & $<.05$ & - \\
\hline 3.38 & $<.05$ & $<.05$ & $-\cdots$ \\
\hline 2.10 & $<.05$ & $<.05$ & -- \\
\hline 1.3 & $<.01$ & $<.01$ & - \\
\hline 2.45 & $<.01$ & $<.01$ & --- \\
\hline 6.08 & $<.01$ & $<.01$ & - \\
\hline 11.3 & $<.01$ & $<.01$ & -- \\
\hline 14.1 & $<.01$ & $<.01$ & -- \\
\hline 16.9 & $<.01$ & $<.01$ & -- \\
\hline 20.2 & .05 & $<.01$ & $\cdots$ \\
\hline 22.7 & .03 & $<.01$ & $-\cdots$ \\
\hline 14.0 & .06 & .05 & $\ldots$ \\
\hline 12.7 & $<.01$ & .01 & $\cdots$ \\
\hline 11.2 & $<.01$ & $<.01$ & $\cdots$ \\
\hline 11.3 & .03 & .02 & -- \\
\hline 12.0 & $<.01$ & $<.01$ & -- \\
\hline 14.8 & .04 & .02 & --- \\
\hline 14.7 & .02 & .02 & -- \\
\hline 10.8 & .01 & .01 & -- \\
\hline 9.05 & $<.01$ & $<.01$ & -- \\
\hline 14.0 & $<.01$ & .01 & - \\
\hline
\end{tabular}

Site B16 (Whitewater River Delta)

$\begin{array}{cccccc}\text { Soft tissue } & 91.3 & 0.20 & <0.0099 & -- & <0.0099 \\ \text { Whole body } & 75.5 & 2.11 & <.010 & \ldots & <.010 \\ \text { Whole body } & 75.8 & 1.32 & <.0099 & \ldots & <.0099 \\ \text { Whole body } & 73.5 & 1.53 & <.0099 & \ldots & <.0099\end{array}$


Table 23. Organic chemical analysis, moisture content, and lipid content for biotic samples collected during 1986-90 from the Salton Sea and associated drainwaters and rivers--Continued

\begin{tabular}{|c|c|c|c|c|c|c|c|c|c|}
\hline $\begin{array}{l}\text { Record } \\
\text { number }\end{array}$ & $\begin{array}{l}\text { Sample } \\
\text { number }\end{array}$ & $\begin{array}{l}\text { gamma- } \\
\text { Chlordane }\end{array}$ & $\begin{array}{c}\text { trans- } \\
\text { Chlordane }\end{array}$ & $\begin{array}{l}\text { cis-Nona- } \\
\text { chlor }\end{array}$ & $\begin{array}{c}\text { trans- } \\
\text { Nonachlor }\end{array}$ & $\begin{array}{l}\text { Hepta- } \\
\text { chlor }\end{array}$ & $\begin{array}{l}\text { Heptachlor } \\
\text { epoxide }\end{array}$ & $\begin{array}{l}\text { Methyoxy- } \\
\text { chlor }\end{array}$ & $o, p^{\prime}-D D E$ \\
\hline \multicolumn{10}{|c|}{ Site B11 (Alamo River Delta)--Continued } \\
\hline 43 & LNSS88-77 & $<0.05$ & $\cdots$ & $<0.05$ & $<0.05$ & $<0.05$ & $<0.05$ & -- & $<0.05$ \\
\hline 44 & LNSS88-78 & $<.05$ & $\ldots$ & $<.05$ & $<.05$ & $<.05$ & $<.05$ & --- & $<.05$ \\
\hline 45 & SS89-128 & $<.01$ & $\ldots$ & $<.01$ & $<.01$ & -- & $<.01$ & --- & $<.01$ \\
\hline 46 & SS89-129 & $<.01$ & -- & $<.01$ & $<.01$ & --- & $<.01$ & --- & $<.01$ \\
\hline 47 & SS89-130 & $<.01$ & -- & $<.01$ & $<.01$ & -- & $<.01$ & -- & $<.01$ \\
\hline 48 & SS89-131 & $<.01$ & - & $<.01$ & $<.01$ & - & .01 & -- & $<.01$ \\
\hline 49 & SS89-132 & $<.01$ & - & $<.01$ & $<.01$ & -- & $<.01$ & -- & $<.01$ \\
\hline \multicolumn{10}{|c|}{ Site B13 (Obsidian Butte) } \\
\hline 50 & SS89-142 & $<0.01$ & -- & $<0.01$ & $<0.01$ & -- & $<0.01$ & --- & $<0.01$ \\
\hline 51 & SS89-144 & $<.01$ & -- & $<.01$ & $<.01$ & $\cdots$ & $<.01$ & --- & $<.01$ \\
\hline 52 & SS89-146 & $<.01$ & -- & $<.01$ & $<.01$ & -- & $<.01$ & -- & $<.01$ \\
\hline \multicolumn{10}{|c|}{ Site B15 (New River Delta) } \\
\hline 53 & LNSS86-52B & - & $<0.0099$ & $<0.0099$ & $<0.0099$ & -- & $<0.0099$ & --- & -- \\
\hline 54 & LNSS86-58 & $\ldots$ & $<.010$ & $<.010$ & $<.010$ & -.-. & $<.010$ & -- & --- \\
\hline 55 & LNSS86-56 & - & $<.010$ & $<.010$ & $<.010$ & -- & $<.010$ & --- & --- \\
\hline 56 & LNSS86-54 & $\cdots$ & $<.0099$ & $<.0099$ & $<.0099$ & -- & $<.0099$ & - & --- \\
\hline 57 & LNSS88-92 & $<0.05$ & - & $<.05$ & $<.05$ & $<0.05$ & $<.05$ & -- & $<0.05$ \\
\hline 58 & LNSS88-86 & $<.05$ & $\ldots$ & $<.05$ & $<.05$ & $<.05$ & $<.05$ & -- & $<.05$ \\
\hline 59 & LNSS88-65 & $<.05$ & - & $<.05$ & $<.05$ & $<.05$ & $<.05$ & -- & $<.05$ \\
\hline 60 & LNSS88-67 & $<.05$ & - & $<.05$ & $<.05$ & $<.05$ & $<.05$ & -- & $<.05$ \\
\hline 61 & LNSS88-79 & $<.05$ & - & $<.05$ & $<.05$ & $<.05$ & $<.05$ & $\ldots$ & $<.05$ \\
\hline 62 & LNSS88-80 & $<.05$ & -- & $<.05$ & $<.05$ & $<.05$ & $<.05$ & --. & $<.05$ \\
\hline 63 & LNSS88-81 & $<.05$ & - & $<.05$ & $<.05$ & $<.05$ & $<.05$ & --. & $<.05$ \\
\hline 64 & LNSS88-82 & $<.05$ & -- & $<.05$ & $<.05$ & $<.05$ & $<.05$ & $\ldots$ & $<.05$ \\
\hline 65 & LNSS88-83 & $<.05$ & - & $<.05$ & $<.05$ & $<.05$ & $<.05$ & --- & $<.05$ \\
\hline 66 & SS89-76 & $<.01$ & -- & $<.01$ & $<.01$ & $\ldots$ & $<.01$ & --- & $<.01$ \\
\hline 67 & SS89-148 & $<.01$ & - & $<.01$ & $<.01$ & -- & $<.01$ & --- & $<.01$ \\
\hline 68 & SS89-150 & $<.01$ & -- & $<.01$ & $<.01$ & -- & $<.01$ & --- & $<.01$ \\
\hline 69 & SS89-133 & $<.01$ & - & $<.01$ & $<.01$ & $\ldots$ & $<.01$ & -- & $<.01$ \\
\hline 70 & SS89-134 & $<.01$ & $\ldots$ & $<.01$ & $<.01$ & $\ldots$ & $<.01$ & $\ldots$ & $<.01$ \\
\hline 71 & SS89-135 & $<.01$ & - & $<.01$ & $<.01$ & -- & $<.01$ & --- & $<.01$ \\
\hline 72 & SS89-136 & $<.01$ & - & $<.01$ & $<.01$ & -- & $<.01$ & -.- & $<.01$ \\
\hline 73 & SS89-137 & $<.01$ & - & $<.01$ & $<.01$ & -- & $<.01$ & -- & $<.01$ \\
\hline 74 & SS89-93 & $<.01$ & $-\ldots$ & $<.01$ & .03 & ... & .03 & $\cdots$ & $<.01$ \\
\hline 75 & SS89-94 & $<.01$ & - & $<.01$ & $<.01$ & -- & $<.01$ & --- & $<.01$ \\
\hline 76 & SS89-95 & $<.01$ & -- & $<.01$ & $<.01$ & -.. & $<.01$ & --- & $<.01$ \\
\hline 77 & SS89-96 & $<.01$ & $\ldots$ & $<.01$ & .01 & --- & .02 & --- & $<.01$ \\
\hline 78 & SS89-97 & $<.01$ & $\ldots$ & $<.01$ & $<.01$ & - & $<.01$ & --- & $<.01$ \\
\hline 79 & SS89-98 & $<.01$ & $\ldots$ & $<.01$ & $<.01$ & $\ldots$ & .01 & -- & $<.01$ \\
\hline 80 & SS89-99 & $<.01$ & - & $<.01$ & $<.01$ & -- & $<.01$ & --- & $<.01$ \\
\hline 81 & SS89-100 & $<.01$ & $-\ldots$ & $<.01$ & $<.01$ & $\ldots$ & $<.01$ & $-\cdots$ & $<.01$ \\
\hline 82 & SS89-101 & $<.01$ &.- & $<.01$ & $<.01$ & - & .03 & --- & $<.01$ \\
\hline 83 & SS89-102 & $<.01$ & $\ldots$ & $<.01$ & $<.01$ & -- & $<.01$ & --- & $<.01$ \\
\hline \multicolumn{10}{|c|}{ Site B16 (Whitewater River Delta) } \\
\hline 84 & LNSS86-68B & - & $<0.0099$ & $<0.0099$ & $<0.0099$ & -- & $<0.0099$ & 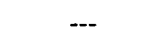 & --- \\
\hline 85 & LNSS86-51 & - & $<.010$ & $<.010$ & $<.010$ & $\ldots$ & $<.010$ & --- & --- \\
\hline 86 & LNSS86-48B & -- & $<.0099$ & $<.0099$ & $<.0099$ & $\cdots$ & $<.0099$ & -- & --- \\
\hline 87 & LNSS86-49B & - & $<.0099$ & $<.0099$ & $<.0099$ &.-- & $<.0099$ & -- & -.. \\
\hline
\end{tabular}


Table 23. Organic chemical analysis, moisture content, and lipid content for biotic samples collected during 1986-90 from the Salton Sea and associated drainwaters and rivers--Continued

\begin{tabular}{|c|c|c|c|c|c|c|c|c|c|c|}
\hline $\begin{array}{l}\text { Record } \\
\text { number }\end{array}$ & $\begin{array}{l}\text { Sample } \\
\text { number }\end{array}$ & $\mathrm{p}, \mathrm{p}^{\prime}-\mathrm{DDE}$ & $o, \mathbf{p}^{\prime}$-DDD & $\mathbf{p}, \mathbf{p}^{\prime}-\mathrm{DDD}$ & $o, p^{\prime}-\mathrm{DDT}$ & $\mathbf{p}, \mathbf{p}^{\prime}-\mathrm{DDT}$ & $\begin{array}{l}\text { Total } \\
\text { DDT }\end{array}$ & Endrin & Dieldrin & Aldrin \\
\hline \multicolumn{11}{|c|}{ Site B11 (Alamo River Delta)--Continued } \\
\hline 43 & LNSS88-77 & 0.74 & $<0.05$ & $<0.05$ & $<0.05$ & $<0.05$ & 0.74 & $<0.05$ & $<0.05$ & $<0.05$ \\
\hline 44 & LNSS88-78 & .53 & $<.05$ & $<.05$ & $<.05$ & $<.05$ & .53 & $<.05$ & $<.05$ & $<.05$ \\
\hline 45 & SS89-128 & 1.9 & $<.01$ & $<.01$ & $<.01$ & $<.01$ & 1.900 & $<.01$ & $<.01$ & --- \\
\hline 46 & SS89-129 & 3.1 & $<.01$ & $<.01$ & $<.01$ & $<.01$ & 3.100 & $<.01$ & .02 & --- \\
\hline 47 & SS89-130 & 4.0 & $<.01$ & $<.01$ & $<.01$ & $<.01$ & 4.000 & $<.01$ & .06 & --- \\
\hline 48 & SS89-131 & 2.4 & $<.01$ & $<.01$ & $<.01$ & $<.01$ & 2.400 & $<.01$ & .14 & --- \\
\hline 49 & SS89-132 & 2.6 & $<.01$ & $<.01$ & $<.01$ & $<.01$ & 2.600 & $<.01$ & .10 & -- \\
\hline \multicolumn{11}{|c|}{ Site B13 (Obsidian Butte) } \\
\hline 50 & SS89-142 & 0.61 & $<0.01$ & $<0.01$ & $<0.01$ & $<0.01$ & 0.610 & $<0.01$ & $<0.01$ & - \\
\hline 51 & SS89-144 & 1.1 & $<.01$ & $<.01$ & $<.01$ & $<.01$ & 1.100 & $<.01$ & $<.01$ & --- \\
\hline 52 & SS89-146 & .27 & $<.01$ & $<.01$ & $<.01$ & $<.01$ & .270 & $<.01$ & $<.01$ & $\cdots$ \\
\hline \multicolumn{11}{|c|}{ Site B15 (New River Delta) } \\
\hline 53 & LNSS86-52B & 0.23 & -- & 0.082 & -- & 0.029 & 0.341 & $<0.0099$ & $<0.0099$ & -- \\
\hline 54 & LNSS86-58 & .13 & --- & $<.010$ & - & $<.010$ & .130 & $<.010$ & $<.010$ & -- \\
\hline 55 & LNSS86-56 & .090 & -- & $<.010$ & -- & $<.010$ & .090 & $<.010$ & $<.010$ & --- \\
\hline 56 & LNSS86-54 & .90 & $\ldots$ & $<.0099$ & -- & $<.0099$ & .900 & $<.0099$ & .33 & --- \\
\hline 57 & LNSS88-92 & .07 & $<0.05$ & $<.05$ & $<0.05$ & $<.05$ & .07 & $<.05$ & $<.05$ & $<0.05$ \\
\hline 58 & LNSS88-86 & .47 & $<.05$ & $<.05$ & $<.05$ & .05 & .47 & $<.05$ & $<.05$ & $<.05$ \\
\hline 59 & LNSS88-65 & .68 & $<.05$ & $<.05$ & $<.05$ & $<.05$ & .68 & $<.05$ & $<.05$ & $<.05$ \\
\hline 60 & LNSS88-67 & .60 & $<.05$ & $<.05$ & $<.05$ & $<.05$ & .60 & $<.05$ & $<.05$ & $<.05$ \\
\hline 61 & LNSS88-79 & .82 & $<.05$ & $<.05$ & $<.05$ & $<.05$ & $<.05$ & $<.05$ & $<.05$ & $<.05$ \\
\hline 62 & LNSS88-80 & 1.67 & $<.05$ & $<.05$ & $<.05$ & $<.05$ & 12.11 & $<.05$ & .05 & $<.05$ \\
\hline 63 & LNSS88-81 & .78 & $<.05$ & $<.05$ & $<.05$ & $<.05$ & .78 & $<.05$ & $<.05$ & $<.05$ \\
\hline 64 & LNSS88-82 & .85 & $<.05$ & $<.05$ & $<.05$ & $<.05$ & .85 & $<.05$ & $<.05$ & $<.05$ \\
\hline 65 & LNSS88-83 & .80 & $<.05$ & $<.05$ & $<.05$ & $<.05$ & .8 & $<.05$ & $<.05$ & $<.05$ \\
\hline 66 & SS89-76 & .07 & $<.01$ & $<.01$ & $<.01$ & $<.01$ & .070 & $<.01$ & $<.01$ & -- \\
\hline 67 & SS89-148 & .18 & $<.01$ & $<.01$ & $<.01$ & $<.01$ & .180 & $<.01$ & $<.01$ & --- \\
\hline 68 & SS89-150 & .18 & $<.01$ & $<.01$ & $<.01$ & $<.01$ & .180 & $<.01$ & $<.01$ & --- \\
\hline 69 & SS89-133 & 1.5 & $<.01$ & $<.01$ & $<.01$ & $<.01$ & 1.500 & $<.01$ & .06 & --- \\
\hline 70 & SS89-134 & .05 & $<.01$ & $<.01$ & $<.01$ & $<.01$ & .050 & $<.01$ & .06 & --- \\
\hline 71 & SS89-135 & 1.7 & $<.01$ & $<.01$ & $<.01$ & $<.01$ & 1.700 & $<.01$ & .02 & -- \\
\hline 72 & SS89-136 & 2.3 & $<.01$ & $<.01$ & $<.01$ & $<.01$ & 2.300 & $<.01$ & .06 & -- \\
\hline 73 & SS89-137 & 2.0 & $<.01$ & $<.01$ & $<.01$ & $<.01$ & 2.000 & $<.01$ & .09 & -- \\
\hline 74 & SS89-93 & 4.1 & $<.01$ & $<.01$ & $<.01$ & $<.01$ & 4.100 & $<.01$ & .15 & --- \\
\hline 75 & SS89-94 & .64 & $<.01$ & $<.01$ & $<.01$ & .02 & .660 & $<.01$ & $<.01$ & --- \\
\hline 76 & SS89-95 & 1.7 & $<.01$ & $<.01$ & $<.01$ & $<.01$ & 1.700 & $<.01$ & .02 &.-- \\
\hline 77 & SS89-96 & 1.9 & $<.01$ & $<.01$ & $<.01$ & .03 & 1.930 & $<.01$ & .04 & --- \\
\hline 78 & SS89-97 & .46 & $<.01$ & $<.01$ & $<.01$ & $<.01$ & .460 & $<.01$ & .01 & -- \\
\hline 79 & SS89-98 & 2.7 & $<.01$ & $<.01$ & $<.01$ & .03 & 2.730 & $<.01$ & .05 & -- \\
\hline 80 & SS89-99 & 2.3 & $<.01$ & $<.01$ & $<.01$ & .03 & 2.330 & $<.01$ & .03 & --- \\
\hline 81 & SS89-100 & 4.0 & $<.01$ & .04 & $<.01$ & .31 & 4.350 & $<.01$ & .01 & $\ldots$ \\
\hline 82 & SS89-101 & 1.1 & $<.01$ & $<.01$ & $<.01$ & .01 & 1.110 & $<.01$ & $<.01$ & -- \\
\hline 83 & SS89-102 & .82 & $<.01$ & $<.01$ & $<.01$ & .02 & .840 & $<.01$ & .01 & $\cdots$ \\
\hline \multicolumn{11}{|c|}{ Site B16 (Whitewater River Delta) } \\
\hline 84 & LNSS86-68B & 0.35 & -- & $<0.0099$ & -- & $<0.0099$ & 0.350 & $<0.0099$ & $<0.0099$ & $-\ldots$ \\
\hline 85 & LNSS86-51 & .26 & --- & .028 & -- & $<.010$ & .288 & $<.010$ & $<.010$ & -- \\
\hline 86 & LNSS86-48B & .35 & -- & .035 & -- & $<.0099$ & .385 & $<.0099$ & $<.0099$ & --- \\
\hline 87 & LNSS86-49B & .28 & -- & $<.0099$ & $\ldots$ & $<.0099$ & .280 & $<.0099$ & $<.0099$ & -- \\
\hline
\end{tabular}


Table 23. Organic chemical analysis, moisture content, and lipid content for biotic samples collected during 1986-90 from the Salton Sea and associated drainwaters and rivers--Continued

\begin{tabular}{llllllllllll}
\hline Record & Sample & alpha- & beta- & delta- & gamma- & Hexa- & Endo- & Endo- & Endo- \\
chloro- & sul- & sul- & sulfan \\
number & number & BHC & BHC & BHC & BHC & dane & $\begin{array}{c}\text { ben- } \\
\text { zene }\end{array}$ &
\end{tabular}

\begin{tabular}{|c|c|c|c|c|c|c|c|c|c|c|c|c|c|}
\hline \multirow[b]{2}{*}{43} & \multicolumn{13}{|c|}{ Site B11 (Alamo River Delta)--Continued } \\
\hline & LNSS88-77 & $<0.05$ & $<0.05$ & $<0.05$ & - & $<0.05$ & $<0.05$ & -- & --- & --- & $<0.05$ & -- & -- \\
\hline 44 & LNSS88-78 & $<.05$ & $<.05$ & $<.05$ & $\ldots$ & $<.05$ & $<.05$ & --- & --- & -- & $<.05$ & -- & -- \\
\hline 45 & SS89-128 & $<.01$ & .02 & $<.01$ & $<0.01$ & -- & $<.01$ & $<0.01$ & $<0.01$ & -- & $<.01$ & -- & --- \\
\hline 46 & SS89-129 & $<.01$ & .02 & $<.01$ & $<.01$ & - & $<.01$ & $<.01$ & $<.01$ & --- & $<.01$ & -- & --- \\
\hline 47 & SS89-130 & $<.01$ & $<.01$ & $<.01$ & $<.01$ & - & $<.01$ & $<.01$ & $<.01$ & --- & $<.01$ & - & -- \\
\hline 48 & SS89-131 & $<.01$ & $<.01$ & $<.01$ & $<.01$ & - & $<.01$ & $<.01$ & $<.01$ & - & $<.01$ & -- & -- \\
\hline 49 & SS89-132 & $<.01$ & $<.01$ & $<.01$ & $<.01$ & --- & $<.01$ & $<.01$ & $<.01$ & -- & $<.01$ & -- & -- \\
\hline
\end{tabular}

\begin{tabular}{|c|c|c|c|c|c|c|c|c|c|c|c|c|c|}
\hline \multirow[b]{2}{*}{50} & \multicolumn{13}{|c|}{ Site B13 (Obsidian Butte) } \\
\hline & SS89-142 & $<0.01$ & $<0.01$ & $<0.01$ & $<0.01$ & $\cdots$ & $<0.01$ & $<0.01$ & $<0.01$ & --- & $<0.01$ & --- & --- \\
\hline 51 & SS89-144 & $<.01$ & $<.01$ & $<.01$ & $<.01$ & $\ldots$ & $<.01$ & $<.01$ & $<.01$ & -- & $<.01$ & $\ldots$ & $-\cdots$ \\
\hline 52 & SS89-146 & $<.01$ & $<.01$ & $<.01$ & $<.01$ & --- & $<.01$ & $<.01$ & $<.01$ & --- & $<.01$ & -- & $-\ldots$ \\
\hline
\end{tabular}

Site B15 (New River Delta)

\begin{tabular}{|c|c|c|c|c|c|c|c|c|c|c|c|c|c|}
\hline \multirow[b]{2}{*}{53} & \multirow[b]{2}{*}{ LNSS86-52B } & \\
\hline & & $\cdots$ & $\cdots$ & -- & -- & --- & --- & --- & --- & --- & --- & --- & --- \\
\hline 54 & LNSS86-58 & - & -- & - & -- & --- & --- & --- & --- & --- & -- & -- & $\cdots$ \\
\hline 55 & LNSS86-56 & - & -- & $\cdots$ & --- & --- & --- & - & - & --- & $\cdots$ & -- & -- \\
\hline 56 & LNSS86-54 & -- & --- & -- & -- & - & $\cdots$ & -- & -- & -- & --- & --- & -- \\
\hline 57 & LNSS88-92 & $<0.05$ & $<0.05$ & $<0.05$ & -- & $<0.05$ & $<0.05$ & --- & $\cdots$ & --- & $<0.05$ & $\ldots$ & -- \\
\hline 58 & LNSS88-86 & $<.05$ & $<.05$ & $<.05$ & $\cdots$ & $<.05$ & $<.05$ & $\cdots$ & -- & --- & $<.05$ & --- & $\cdots$ \\
\hline 59 & LNSS88-65 & $<.05$ & $<.05$ & $<.05$ & --- & $<.05$ & $<.05$ & -- & -- & --- & $<.05$ & -.. & $\ldots$ \\
\hline 60 & LNSS88-67 & $<.05$ & $<.05$ & $<.05$ & $\ldots$ & $<.05$ & $<.05$ & $\cdots$ & --- & --- & $<.05$ & $\cdots$ & - \\
\hline 61 & LNSS88-79 & $<.05$ & $<.05$ & $<.05$ & -- & $<.05$ & $<.05$ & - & -- & $\cdots$ & $<.05$ & -- & -- \\
\hline 62 & LNSS88-80 & $<.05$ & $<.05$ & $<.05$ & - & $<.05$ & $<.05$ & -- & -- & --- & $<.05$ & --- & -- \\
\hline 63 & LNSS88-81 & $<.05$ & $<.05$ & $<.05$ & $\cdots$ & $<.05$ & $<.05$ & $\cdots$ & --- & -- & $<.05$ & -- & $\cdots$ \\
\hline 64 & LNSS88-82 & $<.05$ & $<.05$ & $<.05$ & $\cdots$ & $<.05$ & $<.05$ & -- & --- & --- & $<.05$ & $\cdots$ & -- \\
\hline 65 & LNSS88-83 & $<.05$ & $<.05$ & $<.05$ & $\cdots$ & $<.05$ & $<.05$ & - & --- & --- & $<.05$ & -- & -- \\
\hline 66 & SS89-76 & $<.01$ & -- & $<.01$ & $<0.01$ & $<.01$ & $<.01$ & -- & --- & -- & $<.01$ & --- & --- \\
\hline 67 & SS89-148 & $<.01$ & $<.01$ & $<.01$ & $<.01$ & -- & $<.01$ & $<0.01$ & $<0.01$ & -- & $<.01$ & -- & --- \\
\hline 68 & SS89-150 & $<.01$ & $<.01$ & $<.01$ & $<.01$ & --- & $<.01$ & $<.01$ & $<.01$ & -- & $<.01$ & -- & -- \\
\hline 69 & SS89-133 & $<.01$ & $<.01$ & $<.01$ & $<.01$ & -- & .02 & $<.01$ & $<.01$ & --- & $<.01$ & --- & $\cdots$ \\
\hline 70 & SS89-134 & $<.01$ & $<.01$ & $<.01$ & $<.01$ & -- & $<.01$ & $<.01$ & $<.01$ & -- & $<.01$ & -- & -- \\
\hline 71 & SS89-135 & $<.01$ & $<.01$ & $<.01$ & $<.0 \mathrm{i}$ & -- & $<.01$ & $<.01$ & $<.01$ & -- & $<.01$ & --- & -- \\
\hline 72 & SS89-136 & $<.01$ & $<.01$ & $<.01$ & $<.01$ & -- & $<.01$ & $<.01$ & $<.01$ & --- & $<.01$ & --- & -- \\
\hline 73 & SS89-137 & $<.01$ & $<.01$ & $<.01$ & $<.01$ & -- & $<.01$ & $<.01$ & $<.01$ & --- & $<.01$ & --- & $\cdots$ \\
\hline 74 & SS89-93 & $<.01$ & $<.01$ & $<.01$ & $<.01$ & -- & .05 & --- & $\ldots$ & --- & $<.01$ & --- & $\cdots$ \\
\hline 75 & SS89-94 & $<.01$ & .01 & $<.01$ & $<.01$ & $\cdots$ & .02 & -- & -.. & --- & $<.01$ & --- & -- \\
\hline 76 & SS89-95 & $<.01$ & $<.01$ & $<.01$ & $<.01$ & -- & .01 & -- & -- & $\cdots$ & $<.01$ & -- & -- \\
\hline 77 & SS89-96 & $<.01$ & .04 & $<.01$ & $<.01$ & -- & .01 & -- & --- & -- & $<.01$ & -- & -- \\
\hline 78 & S\$89-97 & $<.01$ & .01 & $<.01$ & $<.01$ & --- & .01 & -- & -- & --- & $<.01$ & $\cdots$ & --- \\
\hline 79 & S\$89-98 & $<.01$ & .02 & $<.01$ & $<.01$ & --- & .03 & --- & --- & $-\cdots$ & $<.01$ & $\cdots$ & -- \\
\hline 80 & SS89-99 & $<.01$ & .01 & $<.01$ & $<.01$ & -- & .01 & --- & $\cdots$ & --- & $<.01$ & -- & - \\
\hline 81 & SS89-100 & $<.01$ & .01 & $<.01$ & $<.01$ & $\cdots$ & .01 & -- & $\cdots$ & -- & $<.01$ & -- & $\cdots$ \\
\hline 82 & SS89-101 & $<.01$ & .05 & $<.01$ & $<.01$ & -- & $<.01$ & -- & --- & --- & $<.01$ & -- & --- \\
\hline 83 & SS89-102 & $<.01$ & .06 & $<.01$ & $<.01$ & -- & .02 & -- & -- & -- & $<.01$ & $\cdots$ & -- \\
\hline
\end{tabular}

\section{Site B16 (Whitewater River Delta)}

\begin{tabular}{|c|c|c|c|c|c|c|c|c|c|c|c|c|c|}
\hline 84 & LNSS86-68B & -- & -- & $\cdots$ & --. & - & $\cdots$ & --- & --. & $\cdots$ & -- & -- & --- \\
\hline 85 & LNSS86-51 & - & - & -- & $\ldots$ & $\cdots$ & -.- & -- & -- & $\cdots$ & -- & -- & $\cdots$ \\
\hline 86 & LNSS86-48B & - & -- & - & -- & --- & -- & -- & $\ldots$ & $\cdots$ & -- & -- & -- \\
\hline 87 & LNSS86-49B & -- & -- & -- & - & --- & -- & -- & $\cdots$ & $\cdots$ & --- & --- & --- \\
\hline
\end{tabular}


Table 23. Organic chemical analysis, moisture content, and lipid content for biotic samples collected during 1986-90 from the Salton Sea and associated drainwaters and rivers--Continued

\begin{tabular}{|c|c|c|c|c|c|c|c|c|c|c|c|c|c|c|}
\hline $\begin{array}{l}\text { Record } \\
\text { number }\end{array}$ & $\begin{array}{l}\text { Sample } \\
\text { number }\end{array}$ & $\begin{array}{l}\text { Tetra- } \\
\text { difon }\end{array}$ & $\begin{array}{c}\text { BI PH } \\
\text { CL-2 }\end{array}$ & $\begin{array}{c}\text { BI PH } \\
\text { CL-3 }\end{array}$ & $\begin{array}{c}\text { BI PH } \\
\text { CL-4 }\end{array}$ & $\begin{array}{c}\text { BI PH } \\
\text { CL-5 }\end{array}$ & $\begin{array}{c}\text { BI PH } \\
\text { CL-6 }\end{array}$ & $\begin{array}{c}\text { BI PH } \\
\text { CL-7 }\end{array}$ & $\begin{array}{c}\text { BI PH } \\
\text { CL-8 }\end{array}$ & $\begin{array}{c}\text { BI PH } \\
\text { CL-9 }\end{array}$ & $\begin{array}{l}\text { PCB } \\
1016\end{array}$ & $\begin{array}{l}\text { PCB } \\
1221\end{array}$ & $\begin{array}{l}\text { PCB } \\
1232\end{array}$ & $\begin{array}{l}\text { PCB } \\
1242\end{array}$ \\
\hline
\end{tabular}

\begin{tabular}{|c|c|c|c|c|c|c|c|c|c|c|c|c|c|c|}
\hline \multicolumn{15}{|c|}{ Site B11 (Alamo River Delta)--Continued } \\
\hline 43 & LNSS88-77 & - & $<0.05$ & $<0.05$ & $<0.05$ & $<0.05$ & $<0.05$ & $<0.05$ & $<0.05$ & $<0.05$ & -- & --- & -- & $\cdots$ \\
\hline 44 & LNSS88-78 & -- & $<.05$ & $<.05$ & $<.05$ & $<.05$ & $<.05$ & $<.05$ & $<.05$ & $<.05$ & -- & -- & -- & $-\cdots$ \\
\hline 45 & SS89-128 & - & -- & --- & -- & -- & -- & -- & $\cdots$ & -- & -- & $\cdots$ & -- & - \\
\hline 46 & SS89-129 & -- & -- & - & -- & - & - & -- & -- & --- & -- & --- & $\cdots$ & $\cdots$ \\
\hline 47 & SS89-130 & -- & -- & -- & -- & - & - & -- & --- & -- & -- & --- & - & - \\
\hline 48 & SS89-131 & - & -- & -- & --- & -- & - & $\cdots$ & -- & -- & -- & $\cdots$ & -- & -- \\
\hline 49 & SS89-132 & - & - & - & -- & -- & -- & -- & - & -- & -- & -- & -- & - \\
\hline
\end{tabular}

$\begin{array}{lllll}50 & \text { SS89-142 } & -- & - & -- \\ 51 & \text { SS89-144 } & -- & - & - \\ 52 & \text { SS89-146 } & -- & - & -\end{array}$

Site B13 (Obsidian Butte)

$\begin{array}{llllllllll}-- & -- & -- & -- & -- & -- & -- & -- & -- & - \\ -- & -- & -- & -- & -- & -- & -- & -- & -- & -- \\ -- & -- & -- & -- & -- & -- & -- & -- & -- & --\end{array}$

\begin{tabular}{|c|c|c|c|c|c|c|c|c|c|c|c|c|c|c|}
\hline \multicolumn{15}{|c|}{ Site B15 (New River Delta) } \\
\hline 53 & LNSS86-52B & --- & --- & --- & -- & --- & --- & --- & -- & -- & --- & -- & -- & $\cdots$ \\
\hline 54 & LNSS86-58 & -- & -- & -- & $\cdots$ & $\ldots$ & --- & --- & --- & $\ldots$ & -- & --- & --- & - \\
\hline 55 & LNSS86-56 & --- & -- & -- & --- & -- & -- & -- & --- & -- & 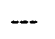 & --- & $\ldots$ & -.. \\
\hline 56 & LNSS86-54 & -- & - & -- & -- & -- & -- & $\cdots$ & -- & --- & -- & -.- &.-- & $\ldots$ \\
\hline 57 & LNSS88-92 & -- & $<0.05$ & $<0.05$ & $<0.05$ & $<0.05$ & $<0.05$ & $<0.05$ & $<0.05$ & $<.05$ & $\ldots$ & $-\ldots$ & $\ldots$ & -- \\
\hline 58 & LNSS88-86 & - & $<.05$ & $<.05$ & $<.05$ & $<.05$ & $<.05$ & $<.05$ & $<.05$ & $<.05$ & -.- & --- & -- & -.. \\
\hline 59 & LNSS88-65 & -- & $<.05$ & $<.05$ & $<.05$ & $<.05$ & $<.05$ & $<.05$ & $<.05$ & $<.05$ & --- & --- & --- & -- \\
\hline 60 & LNSS88-67 & -- & $<.05$ & $<.05$ & $<.05$ & $<.05$ & $<.05$ & $<.05$ & $<.05$ & $<.05$ & -- & -- & --- & -- \\
\hline 61 & LNSS88-79 & -- & $<.05$ & $<.05$ & $<.05$ & $<.05$ & .05 & $<.05$ & $<.05$ & $<.05$ & - & -- & -- & -- \\
\hline 62 & LNSS88-80 & $\cdots$ & $<.05$ & $<.05$ & .06 & $<.05$ & $<.05$ & $<.05$ & $<.05$ & $<.05$ & - & -- & -- & -- \\
\hline 63 & LNSS88-81 & -- & $<.05$ & $<.05$ & $<.05$ & $<.05$ & $<.05$ & $<.05$ & $<.05$ & $<.05$ & -- & --- & - &.- \\
\hline 64 & LNSS88-82 & -- & $<.05$ & $<.05$ & $<.05$ & $<.05$ & $<.05$ & $<.05$ & $<.05$ & $<.05$ & -- & --- & --- & - \\
\hline 65 & LNSS88-83 & -- & $<.05$ & $<.05$ & $<.05$ & $<.05$ & $<.05$ & $<.05$ & $<.05$ & $<.05$ & -- & -- & --- & - \\
\hline 66 & SS89-76 & -- & -- & -.- & -- & -- & -- & -- & -- & $\cdots$ & $\cdots$ &.- & -- & -- \\
\hline 67 & SS89-148 & -- & -- & -- & -- & --- & -- & - & -- & $\cdots$ & - & $-\cdots$ & -- & -- \\
\hline 68 & SS89-150 & -- & -- & -- & -- & -.- & --- & -- & -- & -- & -- & -- & -- & -- \\
\hline 69 & SS89-133 & -- & $\ldots$ & - & - & $\ldots$ & --- & --- & --- & $\cdots$ & -- & --- & -- & -- \\
\hline 70 & SS89-134 & -- & -- & -- & -- & --- & --- & --- & -- & --- & -- & --- & -- & - \\
\hline 71 & SS89-135 & --- & -- & --- & - & --- & -- & -- & --- & -- & -- & --- & --- & - \\
\hline 72 & SS89-136 & -- & - & --- & --- & $--\cdot$ & -- & --- & --- & -- & -- & -- & -- & -. \\
\hline 73 & SS89-137 & -- & -- & -- & -- & --- & -- & --- & --- & -- & -- & -- & -- & - \\
\hline 74 & SS89-93 & -- & --- & --- & -- & -- & -- & -- & --- & --- & -- & -- & -- & - \\
\hline 75 & SS89-94 & -- & -- & -- & -- & -- & -- & -- & -- & --- & --- & --- & -- & - \\
\hline 76 & SS89-95 & -- & --- & --- & - & $\ldots$ & --- & --- & --- & --- & - & -- & -- & $\ldots$ \\
\hline 77 & SS89-96 & -- & - & - & -- & -- & -- & -- & --- & -- & -- & -- & -- & - \\
\hline 78 & SS89-97 & -- & -- & -- & --- & --- & --- & -- & --- & -- & --- & --- & -- & -- \\
\hline 79 & SS89-98 & -- & -- & --- & -- & -- & -- & --- & -- & -- & -- & -- & -- & $\cdots$ \\
\hline 80 & SS89-99 & $\cdots$ & -- & $\cdots$ & -- & -- & --- & -- & --- & --- & -- & -- & -- & - \\
\hline 81 & SS89-100 & -- & -- & --- & --- & --- & --- & $\ldots$ & - & --- & --- & -- & -- & - \\
\hline 82 & SS89-101 & --- & -- & -- & -- & --- & --- & -- & -- & -- & --- & --- & $-\cdots$ & $\cdots$ \\
\hline 83 & SS89-102 & --- & - & --- & -- & -- & -- & -- & --- &.- & $\ldots$ & --- & --- & - \\
\hline
\end{tabular}

Site B16 (Whitewater River Delta)

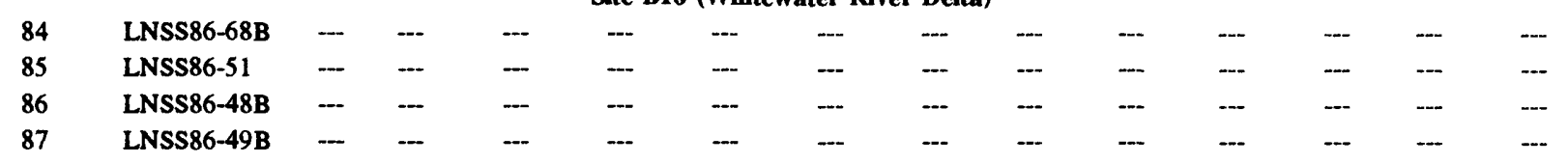


Table 23. Organic chemical analysis, moisture content, and lipid content for biotic samples collected during 1986-90 from the Salton Sea and associated drainwaters and rivers--Continued

\begin{tabular}{|c|c|c|c|c|c|c|}
\hline $\begin{array}{l}\text { Record } \\
\text { number }\end{array}$ & $\begin{array}{l}\text { Sample } \\
\text { number }\end{array}$ & $\begin{array}{l}\text { PCB } \\
1248\end{array}$ & $\begin{array}{l}\text { PCB } \\
1254\end{array}$ & $\begin{array}{l}\text { PCB } \\
1260\end{array}$ & $\begin{array}{l}\text { Total } \\
\text { PCB }\end{array}$ & Toxaphene \\
\hline \multicolumn{7}{|c|}{ Site B11 (Alamo River Delta)--Continued } \\
\hline 43 & LNSS88-77 & - & - & - & - & $<0.50$ \\
\hline 44 & LNSS88-78 & -- & -- & - & -- & $<.50$ \\
\hline 45 & SS89-128 &.- & -- & -- & $<0.05$ & $<.05$ \\
\hline 46 & SS89-129 & $\ldots$ & -- & $-\ldots$ & $<.05$ & $<.05$ \\
\hline 47 & SS89-130 & - & -- & $-\ldots$ & $<.05$ & $<.05$ \\
\hline 48 & SS89-131 & - & - & -- & $<.05$ & $<.05$ \\
\hline 49 & SS89-132 & - & - & -- & $<.05$ & $<.05$ \\
\hline \multicolumn{7}{|c|}{ Site B13 (Obsidian Butte) } \\
\hline 50 & SS89-142 & $\cdots$ & -- & - & $<0.05$ & $<0.05$ \\
\hline 51 & SS89-144 & -- & -- & -- & $<.05$ & $<.05$ \\
\hline 52 & SS89-146 & -- & - & -- & $<.05$ & $<.05$ \\
\hline \multicolumn{7}{|c|}{ Site B15 (New River Delta) } \\
\hline 53 & LNSS86-52B & -- & $<0.099$ & 0.20 & -- & $<0.49$ \\
\hline 54 & LNSS86-58 & -- & $<.10$ & $<.10$ & -- & $<.50$ \\
\hline 55 & LNSS86-56 & - & $<.10$ & .17 & - & $<.50$ \\
\hline 56 & LNSS86-54 & - & $<.099$ & $<.099$ & -- & $<.50$ \\
\hline 57 & LNSS88-92 & -- & -- & -- & -- & $<.50$ \\
\hline 58 & LNSS88-86 & - & -- & --- & - & $<.50$ \\
\hline 59 & LNSS88-65 & -- & -- & -- & -- & $<.50$ \\
\hline 60 & LNSS88-67 & - & $\ldots$ & -- & -- & $<.50$ \\
\hline 61 & LNSS88-79 & $\ldots$ & $\cdots$ & -- & -- & $<.50$ \\
\hline 62 & LNSS88-80 & -- & -- & -- & -- & $<.50$ \\
\hline 63 & LNSS88-81 & -- & -- & -- & -- & $<.50$ \\
\hline 64 & LNSS88-82 & - & $\ldots$ & -- & $\ldots$ & $<.50$ \\
\hline 65 & LNSS88-83 & - & $\cdots$ & -- & $\cdots$ & $<.50$ \\
\hline 66 & SS89-76 & - & -- & - & $<0.05$ & $<.05$ \\
\hline 67 & SS89-148 & -- & - & - & $<.05$ & $<.05$ \\
\hline 68 & SS89-150 & $\ldots$ & $\ldots$ & -- & $<.05$ & $<.05$ \\
\hline 69 & SS89-133 & -- & -- & -- & $<.05$ & $<.05$ \\
\hline 70 & SS89-134 & - & - & -- & $<.05$ & $<.05$ \\
\hline 71 & SS89-135 & --- & $\ldots$ & -- & $<.05$ & $<.05$ \\
\hline 72 & SS89-136 & --- & - & $\ldots$ & $<.05$ & $<.05$ \\
\hline 73 & SS89-137 & -- & - & $\ldots$ & $<.05$ & $<.05$ \\
\hline 74 & SS89-93 & - & - & $\ldots$ & $<.05$ & $<.05$ \\
\hline 75 & SS89-94 & -- & -- & - & $<.05$ & $<.05$ \\
\hline 76 & SS89-95 & -- & - & - & $<.05$ & $<.05$ \\
\hline 77 & S\$89-96 & -- & - & $\ldots$ & $<.05$ & $<.05$ \\
\hline 78 & S\$89-97 & $\cdots$ & $\cdots$ & - & $<.05$ & $<.05$ \\
\hline 79 & SS89-98 & -- & -- & - & $<.05$ & $<.05$ \\
\hline 80 & SS89-99 & - & - & - & $<.05$ & $<.05$ \\
\hline 81 & SS89-100 & -- & - & - & $<.05$ & $<.05$ \\
\hline 82 & SS89-101 & $\cdots$ & - & -- & $<.05$ & $<.05$ \\
\hline 83 & SS89-102 & -- & -- & - & $<.05$ & $<.05$ \\
\hline \multicolumn{7}{|c|}{ Site B16 (Whitewater River Delta) } \\
\hline 84 & LNSS86-68B & - & $<0.099$ & $<0.099$ & - & $<0.50$ \\
\hline 85 & LNSS86-51 & -- & $<.10$ & $<.10$ & --- & $<.50$ \\
\hline 86 & LNSS86-48B & -- & $<.099$ & $<.099$ & - & $<.50$ \\
\hline 87 & LNSS86-49B & -- & $<.099$ & .18 & -- & $<.49$ \\
\hline
\end{tabular}


Table 23. Organic chemical analysis, moisture content, and lipid content for biotic samples collected during 1986-90 from the Salton Sea and associated drainwaters and rivers--Continued

\begin{tabular}{|c|c|c|c|c|c|c|c|c|}
\hline $\begin{array}{l}\text { Record } \\
\text { number }\end{array}$ & $\begin{array}{l}\text { Sample } \\
\text { number }\end{array}$ & Species & Matrix & $\begin{array}{l}\text { Percent } \\
\text { moisture }\end{array}$ & $\begin{array}{c}\text { Percent } \\
\text { lipid }\end{array}$ & $\begin{array}{l}\text { Oxychlor- } \\
\text { dane }\end{array}$ & $\begin{array}{l}\text { alpha- } \\
\text { Chlordane }\end{array}$ & $\begin{array}{l}\text { cis- } \\
\text { Chlordane }\end{array}$ \\
\hline \multicolumn{9}{|c|}{ Site B16 (Whitewater River Delta)--Continued } \\
\hline 88 & LNSS86-43 & Northern shoveler & Breast muscle & 70.0 & 4.69 & $<0.010$ & -- & $<0.010$ \\
\hline $89 \ldots$ & LNSS86-47 & American coot & Breast muscle & 71.5 & 2.17 & $<.0099$ & -- & $<.0099$ \\
\hline $90 \ldots$ & LNSS86-45 & Black-necked stilt & Breast muscle & 71.1 & 3.88 & $<.010$ & --- & $<.010$ \\
\hline $91 \ldots$ & LNSS87-06 & Redfin shiner & Whole body & 73.1 & 6.30 & $<.01$ & --- & $<.01$ \\
\hline \multicolumn{9}{|c|}{ Site B17 (New River at Rio Bend) } \\
\hline $92 \ldots$ & LNSS86-28B & Sailfin molly & Whole body & 74.5 & 3.01 & $<0.013$ & -- & $<0.013$ \\
\hline $93 \ldots$ & LNSS86-31B & Sailfin molly & Whole body & 73.2 & 2.38 & $<.010$ & -- & $<.010$ \\
\hline $94 \ldots$ & LNSS86-29B & Tilapia & Whole body & 70.3 & 6.75 & $<.010$ & -- & $<.010$ \\
\hline $95 \ldots$ & LNSS86-30B & Tilapia & Whole body & 73.5 & 5.00 & $<.0099$ & -- & $<.0099$ \\
\hline $96 \ldots$ & LNSS86-38 & Double-crested cormorant & Breast muscle & 72.2 & 5.18 & $<.0098$ & -- & $<.0098$ \\
\hline $97 \ldots$ & LNSS86-34 & Ruddy duck & Breast muscle & 68.6 & 4.99 & $<.0099$ & $\cdots$ & $<.0099$ \\
\hline 98 & LNSS86-36 & American coot & Breast muscle & 70.1 & 6.62 & $<.010$ & -- & $<.010$ \\
\hline 99 . & LNSS87-15 & Cattle egret & Breast muscle & 70.8 & 3.7 & $<.01$ & --- & $<.01$ \\
\hline 100 & LNSS87-05 & Barn owl & Breast muscle & 63.2 & 11.5 & .03 & -- & $<.01$ \\
\hline 101 & LNSS88-87 & Black-necked stilt & Carcass & 79.4 & 3.10 & $<.05$ & $<0.05$ & -- \\
\hline 102 & LNSS88-69 & Black-necked stilt & Carcass & 80.7 & 4.58 & $<.05$ & $<.05$ & --- \\
\hline 103 & LNSS88-70 & Black-necked stilt & Carcass & 78.4 & 6.29 & $<.05$ & $<.05$ & -- \\
\hline 104 & LNSS88-71 & Black-necked stilt & Carcass & 76.9 & 6.62 & $<.05$ & $<.05$ & --- \\
\hline 105 & LNSS88-88 & Black-necked stilt & Carcass & 73.5 & 2.07 & $<.05$ & $<.05$ & -- \\
\hline 106 & $88-137$ & Black-necked stilt & Egg & 74.0 & 12.7 & $<.01$ & $<.01$ & --- \\
\hline 107 & $88-140$ & Black-necked stilt & Egg & 73.0 & 12.3 & .01 & $<.01$ & --- \\
\hline 108 & $88-143$ & Black-necked stilt & Egg & 74.5 & 12.2 & $<.01$ & $<.01$ & -- \\
\hline 109 & $88-146$ & Black-necked stilt & Egg & 76.5 & 8.66 & .01 & $<.01$ & - \\
\hline 110 & $88-149$ & Black-necked stilt & Egg & 73.5 & 20.2 & $<.01$ & $<.01$ & -- \\
\hline 111 & $88-152$ & Black-necked stilt & Egg & 74.0 & 10.7 & .08 & $<.01$ & -- \\
\hline 112 & $88-155$ & Black-necked stilt & Egg & 71.0 & 15.7 & .02 & $<.01$ & -- \\
\hline 113 & $88-158$ & Black-necked stilt & Egg & 73.0 & 14.3 & .01 & $<.01$ & -- \\
\hline 114 & $88-161$ & Black-necked stilt & Egg & 70.0 & 11.7 & .01 & $<.01$ & -- \\
\hline 115 & $88-164$ & Black-necked stilt & Egg & 72.5 & 12.9 & .02 & $<.01$ & -- \\
\hline 116 & $88-167$ & Black-necked stilt & Egg & 73.5 & 21.9 & .01 & $<.01$ & -- \\
\hline 117. & $88-170$ & Black-necked stilt & Egg & 69.0 & 15.3 & .01 & $<.01$ & -- \\
\hline 118 & $88-173$ & Black-necked stilt & Egg & 71.5 & 14.1 & .01 & $<.01$ & --- \\
\hline 119 & $88-176$ & Black-necked stilt & Egg & 76.5 & 14.9 & .01 & $<.01$ & -- \\
\hline 120 & $88-185$ & Black-necked stilt & Egg & 64.0 & 19.1 & .01 & $<.01$ & -- \\
\hline 121 & $88-188$ & Black-necked stilt & Egg & 66.5 & 20.2 & .01 & $<.01$ & -- \\
\hline 122 & $88-195$ & Black-necked stilt & Egg & 69.0 & 15.0 & .09 & $<.01$ & -- \\
\hline 123 & $88-198$ & Black-necked stilt & Egg & 66.5 & 12.8 & .01 & $<.01$ & $\cdots$ \\
\hline 124 & $88-201$ & Black-necked stilt & Egg & 69.0 & 14.2 & .01 & $<.01$ & $\cdots$ \\
\hline 125 & SS89-121 & Asiatic river clam & Carcass & 86.5 & 1.76 & $<.01$ & .02 & $\cdots$ \\
\hline 126 & SS89-138 & Black-necked stilt & Carcass & 66.2 & 12.6 & .02 & $<.01$ & $\cdots$ \\
\hline 127 & SS89-139 & Black-necked stilt & Carcass & 68.6 & 6.95 & $<.01$ & $<.01$ & $\cdots$ \\
\hline 128 & SS89-140 & Black-necked stilt & Carcass & 63.9 & 6.85 & $<.01$ & $<.01$ & -- \\
\hline 129 & SS89-141 & Black-necked stilt & Carcass & 53.8 & 30.7 & $<.01$ & $<.01$ & -- \\
\hline 130 & SS89-85 & Black-necked stilt & Egg & 78.0 & 20.3 & .01 & $<.01$ & -- \\
\hline 131 & SS89-86 & Black-necked stilt & Egg & 80.0 & 11.0 & .03 & .06 & -- \\
\hline 132 & SS89-87 & Black-necked atilt & Egg & 71.0 & 12.3 & .03 & .02 & -- \\
\hline 133 & SS89-88 & Black-necked stilt & Egg & 74.0 & 10.2 & .03 & .02 & $\cdots$ \\
\hline 134 & SS89-89 & Black-necked stilt & Egg & 71.0 & 12.5 & .02 & .03 & $\cdots$ \\
\hline 135 & SS89-90 & Black-necked stilt & Egg & 73.0 & 12.9 & .01 & $<.01$ & -- \\
\hline 136 & SS89-91 & Black-necked stilt & Egg & 67.0 & 16.0 & .01 & .01 & -- \\
\hline 137 & SS89-92 & Black-necked stilt & Egg & 73.0 & 13.3 & $<.01$ & $<.01$ &.- \\
\hline
\end{tabular}


Table 23. Organic chemical analysis, moisture content, and lipid content for biotic samples collected during 1986-90 from the Salton Sea and associated drainwaters and rivers--Continued

\begin{tabular}{|c|c|c|c|c|c|c|c|c|c|}
\hline $\begin{array}{l}\text { Record } \\
\text { number }\end{array}$ & $\begin{array}{l}\text { Sample } \\
\text { number }\end{array}$ & $\begin{array}{l}\text { gamma- } \\
\text { Chlondane }\end{array}$ & $\begin{array}{l}\text { trans- } \\
\text { Chlondane }\end{array}$ & $\begin{array}{l}\text { cis-Nona- } \\
\text { chlor }\end{array}$ & $\begin{array}{c}\text { trans- } \\
\text { Nonachlor }\end{array}$ & $\begin{array}{l}\text { Hepta- } \\
\text { chlor }\end{array}$ & $\begin{array}{l}\text { Heptachlor } \\
\text { epoxide }\end{array}$ & $\begin{array}{l}\text { Methyoxy- } \\
\text { chlor }\end{array}$ & o,p'-DDE \\
\hline \multicolumn{10}{|c|}{ Site B16 (Whitewater River Delta)--Continued } \\
\hline 88 & LNSS86-43 & -- & $<0.010$ & $<0.010$ & $<0.010$ & --- & $<0.010$ & --- & $-\ldots$ \\
\hline 89 & LNSS86-47 & -- & $<.0099$ & $<.0099$ & $<.0099$ & -- & $<.0099$ & --- & --- \\
\hline 90 & LNSS86-45 & -- & $<.010$ & $<.010$ & $<.010$ & --- & $<.010$ & -.. & $-\ldots$ \\
\hline 91 & LNSS87-06 & -- & $<.01$ & $<.01$ & $<.01$ & $<0.01$ & $<.01$ & $<0.01$ & $<0.02$ \\
\hline \multicolumn{10}{|c|}{ Site B17 (New River at Rio Bend) } \\
\hline 92 & LNSS86-28B & - & $<0.013$ & $<0.013$ & $<0.013$ & -- & $<0.013$ & - & -- \\
\hline 93 & LNSS86-31B & -- & $<.010$ & $<.010$ & $<.010$ & $\ldots$ & $<.010$ & - & -- \\
\hline 94 & LNSS86-29B & -- & $<.010$ & $<.010$ & $<.010$ & - & $<.010$ & - & $\ldots$ \\
\hline 95 & LNSS86-30B & -- & $<.0099$ & $<.0099$ & $<.0099$ & - & $<.0099$ & -.. & -- \\
\hline 96 & LNSS86-38 & -- & $<.0098$ & $<.0098$ & $<.0098$ & $\ldots$ & $<.0098$ & -- & $\cdots$ \\
\hline 97 & LNSS86-34 & -- & $<.0099$ & $<.0099$ & $<.0099$ & $\ldots$ & $<.0099$ & $\ldots$ &.- \\
\hline 98 & LNSS86-36 & -- & $<.010$ & $<.010$ & $<.010$ & $\ldots$ & $<.010$ & -- & $\ldots$ \\
\hline 99 & LNSS87-15 & -- & $<.01$ & $<.01$ & .01 & $<0.01$ & $<.01$ & $<0.01$ & $<0.01$ \\
\hline 100 & LNSS87-05 & -- & $<.01$ & $<.01$ & .02 & $<.01$ & $<.01$ & $<.01$ & $<.01$ \\
\hline 101 & LNSS88-87 & $<0.05$ & $\ldots$ & $<.05$ & $<.05$ & $<.05$ & $<.05$ & --- & $<.05$ \\
\hline 102 & LNSS88-69 & $<.05$ & -- & $<.05$ & $<.05$ & $<.05$ & $<.05$ & -- & $<.05$ \\
\hline 103 & LNSS88-70 & $<.05$ & --- & $<.05$ & $<.05$ & $<.05$ & $<.05$ & -- & $<.05$ \\
\hline 104 & LNSS88-71 & $<.05$ & $\ldots$ & $<.05$ & $<.05$ & $<.05$ & $<.05$ & -- & $<.05$ \\
\hline 105 & LNSS88-88 & $<.05$ & $-\cdots$ & $<.05$ & $<.05$ & $<.05$ & $<.05$ & -- & $<.05$ \\
\hline 106 & $88-137$ & $<.01$ & -- & $<.01$ & $<.01$ & - & $<.01$ & $\ldots$ & $<.01$ \\
\hline 107 & $88-140$ & $<.01$ & -- & $<.01$ & $<.01$ & -- & .01 & -- & $<.01$ \\
\hline 108 & $88-143$ & $<.01$ & $\ldots$ & $<.01$ & $<.01$ & --- & $<.01$ & -- & $<.01$ \\
\hline 109 & $88-146$ & $<.01$ & $\ldots$ & $<.01$ & $<.01$ & --- & $<.01$ & - & $<.01$ \\
\hline 110 & $88-149$ & $<.01$ & - & $<.01$ & $<.01$ & --- & $<.01$ & - & $<.01$ \\
\hline 111 & $88-152$ & $<.01$ & - & $<.01$ & .02 & -- & .06 & $\ldots$ & $<.01$ \\
\hline 112 & $88-155$ & $<.01$ & -- & $<.01$ & .01 & -- & .02 & --- & $<.01$ \\
\hline 113 & $88-158$ & $<.01$ & -- & $<.01$ & .01 & -- & $<.01$ & -- & $<.01$ \\
\hline 114 & $88-161$ & $<.01$ & -- & $<.01$ & $<.01$ & - & $<.01$ & -- & $<.01$ \\
\hline 115 & $88-164$ & $<.01$ & -- & $<.01$ & $<.01$ & -- & $<.01$ & - & $<.01$ \\
\hline 116 & $88-167$ & $<.01$ & -- & $<.01$ & $<.01$ & --. & $<.01$ & - & $<.01$ \\
\hline 117 & $88-170$ & $<.01$ & - & $<.01$ & $<.01$ & -- & $<.01$ & - & $<.01$ \\
\hline 118 & $88-173$ & $<.01$ & -- & $<.01$ & $<.01$ & --- & $<.01$ & -- & $<.01$ \\
\hline 119 & $88-176$ & $<.01$ & -- & $<.01$ & $<.01$ & -- & $<.01$ & -- & $<.01$ \\
\hline 120 & $88-185$ & $<.01$ & - & $<.01$ & .02 & - & $<.01$ & $\ldots$ & $<.01$ \\
\hline 121 & $88-188$ & $<.01$ & -- & $<.01$ & .01 & -- & $<.01$ & -- & $<.01$ \\
\hline 122 & $88-195$ & $<.01$ & -- & $<.01$ & .01 & -- & .06 & -- & $<.01$ \\
\hline 123 & $88-198$ & $<.01$ & -- & $<.01$ & $<.01$ & -- & $<.01$ & -.. & $<.01$ \\
\hline 124 & 88-201 & $<.01$ & -- & $<.01$ & $<.01$ & -- & $<.01$ & -- & $<.01$ \\
\hline 125 & SS89-121 & $<.01$ & -- & $<.01$ & .01 & -- & $<.01$ & -.. & .03 \\
\hline 126 & SS89-138 & $<.01$ & -- & $<.01$ & $<.01$ & --- & $<.01$ & -- & $<.01$ \\
\hline 127 & SS89-139 & $<.01$ & -- & $<.01$ & $<.01$ & -- & $<.01$ & - & $<.01$ \\
\hline 128 & SS89-140 & $<.01$ & -- & $<.01$ & $<.01$ & -- & $<.01$ & -.. & $<.01$ \\
\hline 129 & SS89-141 & $<.01$ & -- & $<.01$ & $<.01$ & $\ldots$ & $<.01$ & -- & $<.01$ \\
\hline 130 & SS89-85 & $<.01$ & $\ldots$ & $<.01$ & $<.01$ & --- & $<.01$ & --- & $<.01$ \\
\hline 131 & SS89-86 & $<.01$ & -- & $<.01$ & $<.01$ & -- & .01 & -- & $<.01$ \\
\hline 132 & SS89-87 & $<.01$ & - & $<.01$ & .02 & -- & .01 & --- & $<.01$ \\
\hline 133 & SS89-88 & $<.01$ & -- & $<.01$ & .01 & -.. & .01 & --- & $<.01$ \\
\hline 134 & SS89-89 & $<.01$ & -- & $<.01$ & .02 & $\ldots$ & .01 & --- & $<.01$ \\
\hline 135 & SS89-90 & $<.01$ & -- & $<.01$ & $<.01$ & $\ldots$ & $<.01$ & -- & $<.01$ \\
\hline 136 & SS89-91 & $<.01$ & -- & $<.01$ & $<.01$ & - & $<.01$ & -- & $<.01$ \\
\hline 137 & S\$89-92 & $<.01$ & -- & $<.01$ & $<.01$ & -- & $<.01$ & -- & $<.01$ \\
\hline
\end{tabular}


Table 23. Organic chemical analysis, moisture content, and lipid content for biotic samples collected during 1986-90 from the Salton Sea and associated drainwaters and rivers--Continued

\begin{tabular}{|c|c|c|c|c|c|c|c|c|c|c|}
\hline $\begin{array}{l}\text { Record } \\
\text { number }\end{array}$ & $\begin{array}{l}\text { Sample } \\
\text { number }\end{array}$ & $\mathbf{p}, \mathbf{p}^{\prime}-\mathrm{DDE}$ & $0, p^{\prime}-D D D$ & p,p'-DDD & o,p'-DDT & p,p'-DDT & $\begin{array}{l}\text { Total } \\
\text { DDT }\end{array}$ & Endrin & Dieldrin & Aldrin \\
\hline \multicolumn{11}{|c|}{ Site B16 (Whitewater River Delta)--Continued } \\
\hline 88 & LNSS86-43 & 0.17 & $\cdots$ & $<0.010$ & -.- & $<0.010$ & 0.170 & $<0.010$ & $<0.010$ & --- \\
\hline 89 & LNSS86-47 & .31 & -- & $<.0099$ & -- & $<.0099$ & .310 & $<.0099$ & $<.0099$ & $\cdots$ \\
\hline 90 & LNSS86-45 & .89 & -- & $<.010$ & - & $<.010$ & .890 & $<.010$ & .0222 &.- \\
\hline 91 & LNSS87-06 & 5.7 & 0.04 & .09 & 0.03 & .03 & 5.910 & $<.01$ & .02 & $<0.01$ \\
\hline \multicolumn{11}{|c|}{ Site B17 (New River at Rio Bend) } \\
\hline 92 & LNSS86-28B & 0.18 & - & 0.36 & -.. & 0.033 & 0.249 & $<0.013$ & $<0.013$ & $\ldots$ \\
\hline 93 & LNSS86-31B & .14 & - & $<.010$ & --- & $<.010$ & .140 & $<.010$ & $<.010$ & $\cdots$ \\
\hline 94 & LNSS86-29B & .073 & $\cdots$ & $<.010$ & -- & $<.010$ & .073 & $<.010$ & $<.010$ & $\ldots$ \\
\hline 95 & LNSS86-30B & .11 & - & $<.0099$ & -- & $<.0099$ & .110 & $<.0099$ & $<.0099$ & -- \\
\hline 96 & LNSS86-38 & .38 & - & $<.0098$ & -- & $<.0098$ & .380 & $<.0098$ & $<.0098$ & $\cdots$ \\
\hline 97 & LNSS86-34 & .17 & - & $<.0099$ & --- & $<.0099$ & .170 & $<.0099$ & $<.0099$ & -- \\
\hline 98 & LNSS86-36 & .45 & - & $<.010$ & -- & $<.010$ & .450 & $<.010$ & $<.010$ & $\ldots$ \\
\hline 99 & LNSS87-15 & 2.2 & $<0.01$ & .01 & $<0.01$ & $<.01$ & 2.210 & .01 & .03 & $<0.01$ \\
\hline 100 & LNSS87-05 & 2.7 & $<.01$ & .01 & $<.01$ & $<.01$ & 2.710 & $<.01$ & .09 & $<.01$ \\
\hline 101 & LNSS88-87 & .78 & $<.05$ & $<.05$ & $<.05$ & $<.05$ & $<.05$ & $<.05$ & $<.05$ & $<.05$ \\
\hline 102 & LNSS88-69 & .94 & $<.05$ & $<.05$ & $<.05$ & $<.05$ & .94 & $<.05$ & $<.05$ & $<.05$ \\
\hline 103 & LNSS88-70 & 2.76 & $<.05$ & $<.05$ & $<.05$ & $<.05$ & 2.76 & $<.05$ & .05 & $<.05$ \\
\hline 104 & LNSS88-71 & 2.76 & $<.05$ & $<.05$ & $<.05$ & $<.05$ & 2.76 & $<.05$ & $<.05$ & $<.05$ \\
\hline 105 & LNSS88-88 & .75 & $<.05$ & $<.05$ & $<.05$ & $<.05$ & $<.05$ & $<.05$ & $<.05$ & $<.05$ \\
\hline 106 & $88-137$ & 1.7 & $<.01$ & $<.01$ & $<.01$ & $<.01$ & 1.700 & $<.01$ & .01 & -- \\
\hline 107 & $88-140$ & 4.3 & $<.01$ & $<.01$ & $<.01$ & .02 & 4.320 & $<.01$ & .06 & -- \\
\hline 108 & $88-143$ & 3.8 & $<.01$ & $<.01$ & $<.01$ & .03 & 3.830 & $<.01$ & .02 & $\cdots$ \\
\hline 109 & $88-146$ & 1.1 & $<.01$ & .02 & $<.01$ & .01 & 1.130 & $<.01$ & .01 & -- \\
\hline 110 & $88-149$ & 1.7 & $<.01$ & $<.01$ & $<.01$ & $<.01$ & 1.700 & $<.01$ & .02 & -- \\
\hline 111 & $88-152$ & 7.4 & $<.01$ & .04 & $<.01$ & .12 & 7.560 & $<.01$ & .11 & -- \\
\hline 112 & $88-155$ & 2.0 & $<.01$ & $<.01$ & $<.01$ & $<.01$ & 2.000 & $<.01$ & .06 & -- \\
\hline 113 & $88-158$ & 6.5 & $<.01$ & .03 & $<.01$ & 0.21 & 6.740 & $<.01$ & .03 & -- \\
\hline 114 & $88-161$ & 1.9 & $<.01$ & .02 & $<.01$ & .02 & 1.940 & $<.01$ & .01 & -- \\
\hline 115 & $88-164$ & 4.0 & $<.01$ & $<.01$ & $<.01$ & .02 & 4.020 & $<.01$ & .04 & $\cdots$ \\
\hline 116 & $88-167$ & 3.4 & $<.01$ & $<.01$ & $<.01$ & .02 & 3.420 & $<.01$ & .04 & -- \\
\hline 117 & $88-170$ & 1.9 & $<.01$ & $<.01$ & $<.01$ & .01 & 1.910 & $<.01$ & .02 & $\ldots$ \\
\hline 118 & $88-173$ & 3.6 & $<.01$ & $<.01$ & $<.01$ & .02 & 3.620 & $<.01$ & .02 & -- \\
\hline 119 & $88-176$ & .94 & $<.01$ & $<.01$ & $<.01$ & .04 & .980 & $<.01$ & .02 & -- \\
\hline 120 & $88-185$ & 3.5 & $<.01$ & .02 & $<.01$ & .02 & 3.540 & $<.01$ & .10 & -- \\
\hline 121 & $88-188$ & 2.3 & $<.01$ & $<.01$ & $<.01$ & $<.01$ & 2.300 & $<.01$ & .04 & -- \\
\hline 122 & $88-195$ & 8.1 & $<.01$ & .02 & $<.01$ & .06 & 8.180 & $<.01$ & .11 & -- \\
\hline 123 & $88-198$ & 3.1 & $<.01$ & $<.01$ & $<.01$ & .02 & 3.120 & $<.01$ & .01 & - \\
\hline 124 & $88-201$ & 3.0 & $<.01$ & .01 & $<.01$ & .03 & 3.040 & $<.01$ & .03 & -- \\
\hline 125 & SS89-121 & .62 & .13 & .04 & .02 & .05 & .840 & $<.01$ & .01 & -- \\
\hline 126 & SS89-138 & 1.3 & $<.01$ & $<.01$ & $<.01$ & $<.01$ & 1.300 & $<.01$ & .03 & -- \\
\hline 127 & SS89-139 & .85 & $<.01$ & $<.01$ & $<.01$ & $<.01$ & .850 & $<.01$ & .01 & -- \\
\hline 128 & SS89-140 & .83 & $<.01$ & $<.01$ & $<.01$ & $<.01$ & .830 & $<.01$ & $<.01$ & -- \\
\hline 129 & SS89-141 & 3.0 & $<.01$ & $<.01$ & $<.01$ & $<.01$ & 3.000 & $<.01$ & $<.01$ & -- \\
\hline 130 & SS89-85 & 1.1 & $<.01$ & $<.01$ & $<.01$ & $<.01$ & 1.100 & $<.01$ & .02 & -- \\
\hline 131 & SS89-86 & 5.7 & $<.01$ & $<.01$ & $<.01$ & $<.01$ & 5.700 & $<.01$ & .10 & -- \\
\hline 132 & SS89-87 & 5.9 & $<.01$ & $<.01$ & $<.01$ & .07 & 5.970 & $<.01$ & .14 & -- \\
\hline 133 & SS89-88 & 2.4 & $<.01$ & $<.01$ & $<.01$ & .05 & 2.450 & $<.01$ & .03 & -- \\
\hline 134 & SS89-89 & 4.3 & $<.01$ & $<.01$ & $<.01$ & $<.01$ & 4.300 & $<.01$ & .15 & --- \\
\hline 135 & SS89-90 & 2.2 & $<.01$ & $<.01$ & $<.01$ & .03 & 2.230 & $<.01$ & .02 & -- \\
\hline 136 & SS89-91 & 3.3 & $<.01$ & $<.01$ & $<.01$ & .19 & 3.490 & $<.01$ & .04 & -- \\
\hline 137 & SS89-92 & 0.87 & $<.01$ & $<.01$ & $<.01$ & $<.01$ & 0.870 & $<.01$ & .01 & --- \\
\hline
\end{tabular}


Table 23. Organic chemical analysis, moisture content, and lipid content for biotic samples collected during 1986-90 from the Salton Sea and associated drainwaters and rivers--Continued

\begin{tabular}{|c|c|c|c|c|c|c|c|c|c|c|c|c|c|}
\hline $\begin{array}{l}\text { Record } \\
\text { number }\end{array}$ & $\begin{array}{l}\text { Sample } \\
\text { number }\end{array}$ & $\begin{array}{l}\text { alpha- } \\
\text { BHC }\end{array}$ & $\begin{array}{l}\text { beta- } \\
\text { BHC }\end{array}$ & $\begin{array}{l}\text { delta- } \\
\text { BHC }\end{array}$ & $\begin{array}{c}\text { gamma- } \\
\text { BHC }\end{array}$ & $\begin{array}{l}\text { Lin- } \\
\text { dane }\end{array}$ & $\begin{array}{l}\text { Hexa- } \\
\text { chloro- } \\
\text { ben- } \\
\text { zene }\end{array}$ & $\begin{array}{c}\text { Endo- } \\
\text { sul- } \\
\text { fan I }\end{array}$ & $\begin{array}{l}\text { Endo- } \\
\text { sul- } \\
\text { fan II }\end{array}$ & $\begin{array}{l}\text { Endo- } \\
\text { sulfan } \\
\text { sulfate }\end{array}$ & Mirex & DCPA & Dicofol \\
\hline
\end{tabular}

\begin{tabular}{|c|c|c|c|c|c|c|c|c|c|c|c|c|c|}
\hline \multicolumn{14}{|c|}{ Site B16 (Whitewater River Delta)--Continued } \\
\hline 88 & LNSS86-43 & -- & -- & - & -- & -- & -- & $-\ldots$ & -- & -- & -- & --- & $-\ldots$ \\
\hline 89 & LNSS86-47 & -- & -- & -- & -- & -- & -- & -- & - & -- & -- & -- & -- \\
\hline 90 & LNSS86-45 & -- & -- & -- & -- & -- & -- & -- & - & $-\cdots$ & -- & -- & -. \\
\hline 91 & LNSS87-06 & $<0.01$ & $<0.01$ & $<0.01$ & $<0.01$ & - & $<0.01$ & $<0.01$ & $<0.01$ & $<0.01$ & $<0.01$ & 0.03 & $<0.01$ \\
\hline
\end{tabular}

Site B17 (New River at Rio Bend)

\begin{tabular}{|c|c|c|c|c|c|c|c|c|c|c|c|c|c|}
\hline 92 & LNSS86-28B & -- & - & - & - & -- & $\ldots$ & -.. & - & ... & $\ldots$ & -- & ... \\
\hline 93 & LNSS86-31B & - & -- & - & - & - & $\ldots$ & - & - & $\ldots$ & - & $\ldots$ & $\ldots$ \\
\hline 94 & LNSS86-29B & - & - & - & - & - & $\ldots$ & - & $\ldots$ & $\ldots$ & - & $\ldots$ & - \\
\hline 95 & LNSS86-30B & - & - & - & - & - & - & -- & - & -- & -- & - & -- \\
\hline 96 & LNSS86-38 & - & - & - & -- & --- & - & -- & -- & -- & -- & -- & --- \\
\hline 97 & LNSS86-34 & - & -- & - & - & -- & -- & -- & -- & --- & --- & -- & - \\
\hline 98 & LNSS86-36 & - & - & -- & -- & -.. & -- & $-\ldots$ & -- & --- & --- & -- & --- \\
\hline 99 & LNSS87-15 & $<0.01$ & $<0.01$ & $<0.01$ & $<0.01$ & -- & 0.25 & $<0.01$ & $<0.01$ & $<0.01$ & $<0.01$ & 0.01 & $<0.01$ \\
\hline 100 & LNSS87-05 & $<.01$ & $<.01$ & $<.01$ & $<.01$ & -- & 1.30 & $<.01$ & $<.01$ & $<.01$ & $<.01$ & $<.01$ & $<.01$ \\
\hline 101 & LNSS88-87 & $<.05$ & $<.05$ & $<.05$ & -- & $<0.05$ & $<.05$ & -- & $\ldots$ & $\ldots$ & $<.05$ & --- & $\ldots$ \\
\hline 102 & LNSS88-69 & $<.05$ & $<.05$ & $<.05$ & -- & $<.05$ & $<.05$ & --- & - & $\ldots$ & $<.05$ & -.- & --- \\
\hline 103 & LNSS88-70 & $<.05$ & $<.05$ & $<.05$ & -- & $<.05$ & $<.05$ & -- & -. & --- & $<.05$ & --- & -.- \\
\hline 104 & LNSS88-71 & $<.05$ & $<.05$ & $<.05$ & $\ldots$ & $<.05$ & $<.05$ & -- & - & -- & $<.05$ & --- & $\ldots$ \\
\hline 105 & LNSS88-88 & $<.05$ & $<.05$ & $<.05$ & -- & $<.05$ & $<.05$ & -- & -- & -- & $<.05$ & $\ldots$ & -.. \\
\hline 106 & $88-137$ & $<.01$ & .01 & $<.01$ & $<.01$ &.- & $<.01$ & --- & - & $\ldots$ & $<.01$ & -- & -.. \\
\hline 107 & $88-140$ & $<.01$ & .02 & $<.01$ & $<.01$ & -- & .02 & -- & -- & -.. & $<.01$ & $\ldots$ & -.. \\
\hline 108 & $88-143$ & $<.01$ & .03 & $<.01$ & $<.01$ & --- & .01 & -- & -- & -.. & $<.01$ & -- & $\ldots$ \\
\hline 109 & $88-146$ & $<.01$ & $<.01$ & $<.01$ & $<.01$ & -- & .03 & -- & -- & -- & $<.01$ & --- & -- \\
\hline 110 & 88-149 & $<.01$ & .01 & $<.01$ & $<.01$ & $\ldots$ & $<.01$ & -.. & $\ldots$ & --- & $<.01$ & --- & -- \\
\hline 111 & $88-152$ & $<.01$ & .16 & $<.01$ & $<.01$ & -.- & .01 & - &.- & $\ldots$ & $<.01$ & -.. & --- \\
\hline 112 & $88-155$ & $<.01$ & .02 & $<.01$ & $<.01$ & --- & .01 & - & -- & -- & $<.01$ & --. & - \\
\hline 113 & $88-158$ & $<.01$ & .02 & $<.01$ & $<.01$ & -- & .02 & -- & --- & -- & $<.01$ & -- & --- \\
\hline 114 & $88-161$ & $<.01$ & $<.01$ & $<.01$ & $<.01$ & -- & .04 & $-\cdots$ & $\ldots$ & -- & $<.01$ & - & -- \\
\hline 115 & $88-164$ & $<.01$ & .02 & $<.01$ & $<.01$ & --- & .05 & -- & - & --- & $<.01$ & $\ldots$ & -.. \\
\hline 116 & $88-167$ & $<.01$ & .01 & $<.01$ & $<.01$ & -- & .01 & -- & $\ldots$ & $\ldots$ & $<.01$ & $\ldots$ &.- \\
\hline 117 & $88-170$ & $<.01$ & .01 & $<.01$ & $<.01$ & $\ldots$ & .01 & -- & -- & -- & $<.01$ & -- & -- \\
\hline 118 & $88-173$ & $<.01$ & .02 & $<.01$ & $<.01$ & -- & $<.01$ & -- & $\ldots$ & -- & $<.01$ & -- & -- \\
\hline 119 & $88-176$ & $<.01$ & .04 & $<.01$ & $<.01$ & $\ldots$ & $<.01$ & -- & -- & -- & $<.01$ & $\ldots$ & - \\
\hline 120 & $88-185$ & $<.01$ & .01 & $<.01$ & $<.01$ & - & .01 & -- & -- & -- & $<.01$ & -- & -- \\
\hline 121 & $88-188$ & $<.01$ & .01 & $<.01$ & $<.01$ & --. & $<.01$ & --- & -- & -- & $<.01$ & -- & -- \\
\hline 122 & $88-195$ & $<.01$ & .36 & $<.01$ & $<.01$ & -.. & .04 & $\ldots$ & -- & -- & $<.01$ & --- & -- \\
\hline 123 & $88-198$ & $<.01$ & .08 & $<.01$ & $<.01$ & -- & .03 & -- & -- & -- & $<.01$ & --- & - \\
\hline 124 & $88-201$ & $<.01$ & .02 & $<.01$ & $<.01$ & $\cdots$ & .01 & -- & -- & $\cdots$ & $<.01$ & $\ldots$ & $\ldots$ \\
\hline 125 & SS89-121 & $<.01$ & $<.01$ & $<.01$ & $<.01$ & -- & $<.01$ & $\ldots$ & -- & -- & $<.01$ & -- & -- \\
\hline 126 & SS89-138 & $<.01$ & $<.01$ & $<.01$ & $<.01$ & -- & $<.01$ & $<.01$ & $<.01$ & -- & $<.01$ & -- & -- \\
\hline 127 & SS89-139 & $<.01$ & $<.01$ & $<.01$ & $<.01$ &.-- & $<.01$ & $<.01$ & $<.01$ & -- & $<.01$ & -- & -- \\
\hline 128 & SS89-140 & $<.01$ & $<.01$ & $<.01$ & $<.01$ & -.. & $<.01$ & $<.01$ & $<.01$ & -.- & $<.01$ & -- & $\ldots$ \\
\hline 129 & SS89-141 & $<.01$ & $<.01$ & $<.01$ & $<.01$ & -.. & $<.01$ & $<.01$ & $<.01$ & --- & $<.01$ & -- & -- \\
\hline 130 & SS89-85 & $<.01$ & .01 & $<.01$ & $<.01$ & -.. & $<.01$ & $\ldots$ & --- & $\ldots$ & $<.01$ & $\ldots$ & -- \\
\hline 131 & SS89-86 & $<.01$ & .01 & $<.01$ & $<.01$ & ... & .01 & -.. & -- & $\ldots$ & $<.01$ & -.- & -- \\
\hline 132 & SS89-87 & $<.01$ & .02 & $<.01$ & $<.01$ & $\ldots$ & .77 & - & -- & $\ldots$ & $<.01$ & --- & - \\
\hline 133 & SS89-88 & $<.01$ & .03 & $<.01$ & $<.01$ & $\ldots$ & .02 & $\ldots$ & $\ldots$ & -- & $<.01$ & -- & -- \\
\hline 134 & SS89-89 & $<.01$ & .13 & $<.01$ & $<.01$ & - & .03 & -.. & - & $\ldots$ & $<.01$ & -- & -- \\
\hline 135 & SS89-90 & $<.01$ & .01 & $<.01$ & $<.01$ & ... & .01 & -- & -.. & -- & $<.01$ & -- & -- \\
\hline 136 & SS89-91 & $<.01$ & $<.01$ & $<.01$ & $<.01$ & -.. & $<.01$ & -.. & $\ldots$ & --- & $<.01$ & $\ldots$ & -- \\
\hline 137 & SS89-92 & $<.01$ & .04 & $<.01$ & $<.01$ & -- & $<.01$ & -. & --- & -- & $<.01$ & $\ldots$ & -- \\
\hline
\end{tabular}


Table 23. Organic chemical analysis, moisture content, and lipid content for biotic samples collected during 1986-90 from the Salton Sea and associated drainwaters and rivers--Continued

\begin{tabular}{|c|c|c|c|c|c|c|c|c|c|c|c|c|c|c|}
\hline $\begin{array}{l}\text { Record } \\
\text { number }\end{array}$ & $\begin{array}{l}\text { Sample } \\
\text { number }\end{array}$ & $\begin{array}{l}\text { Tetra- } \\
\text { difon }\end{array}$ & $\begin{array}{c}\text { BI PH } \\
\text { CL-2 }\end{array}$ & $\begin{array}{c}\text { BI PH } \\
\text { CL-3 }\end{array}$ & $\begin{array}{c}\text { BI PH } \\
\text { CL-4 }\end{array}$ & $\begin{array}{c}\text { BI PH } \\
\text { CL-5 }\end{array}$ & $\begin{array}{c}\text { BI PH } \\
\text { CL-6 }\end{array}$ & $\begin{array}{c}\text { BI PH } \\
\text { CL-7 }\end{array}$ & $\begin{array}{c}\text { BI PH } \\
\text { CL-8 }\end{array}$ & $\begin{array}{c}\text { BI PH } \\
\text { CL-9 }\end{array}$ & $\begin{array}{l}\text { PCB } \\
1016\end{array}$ & $\begin{array}{l}\text { PCB } \\
1221\end{array}$ & $\begin{array}{l}\text { PCB } \\
1232\end{array}$ & $\begin{array}{l}\text { PCB } \\
1242\end{array}$ \\
\hline
\end{tabular}

\begin{tabular}{|c|c|c|c|c|c|c|c|c|c|c|c|c|c|c|}
\hline \multirow[b]{2}{*}{88} & \multicolumn{14}{|c|}{ Site B16 (Whitewater River Delta)--Continued } \\
\hline & LNSS86-43 & -- & - & - & - & -- & - & -- & -- & $\cdots$ & - & -- & --- & - \\
\hline 89 & LNSS86-47 & - & - & - & -- & --- & - & $\ldots$ & --- & -- & -- & -- & -- & -- \\
\hline 90 & LNSS86-45 & -- & - & -- & - & -- & -- & - & -- & -- & -- & --- & - & -- \\
\hline 91 & LNSS87-06 & $<0.01$ & - & -- & - & -- & -- & $\ldots$ & - & $\ldots$ & $<0.05$ & $<0.50$ & $<0.05$ & $<0.05$ \\
\hline
\end{tabular}

\section{Site B17 (New River at Rio Bend)}

\begin{tabular}{|c|c|c|c|c|c|c|c|c|c|c|c|c|c|c|}
\hline 92 & LNSS86-28B & -- & - & -- & - & - & -.- & -- & - & $\cdots$ & -.. & - & - & -- \\
\hline 93 & LNSS86-31B & -- & -- & - & - & -- & - & $\ldots$ & -- & -- & --- & -- & $\ldots$ & $-\ldots$ \\
\hline 94 & LNSS86-29B & -- & -- & -- & - & -- & -- & -- & $\ldots$ & -- & $\cdots$ & $\cdots$ & $\cdots$ & $\cdots$ \\
\hline 95 & LNSS86-30B & -- & -- & $\ldots$ & - & - & -- & - & -.. & $\ldots$ & $\ldots$ & - & - & - \\
\hline 96 & LNSS86-38 & - & -- & - & - & $\cdots$ & $\cdots$ & - & $\cdots$ & $\cdots$ & $\cdots$ & - & -- & - \\
\hline 97 & LNSS86-34 & -- & $\ldots$ & $\ldots$ & $\ldots$ & -.. & $\ldots$ & -- & $\ldots$ & -- & $\ldots$ & $\ldots$ & -- & $\ldots$ \\
\hline 98 & LNSS86-36 & -- & $\cdots$ & $\ldots$ & -- & $\cdots$ & - & -- & -- & -- &.-- & - & $\cdots$ & --- \\
\hline 99 & LNSS87-15 & $<0.01$ & -- & $\ldots$ & -- & $\ldots$ & $\ldots$ & $\ldots$ & -- & $\ldots$ & $<0.05$ & $<0.50$ & $<0.05$ & $<0.0$ \\
\hline 100 & LNSS87-05 & $<.01$ & -- & --- & $\ldots$ & - & --- & -- & $\ldots$ & $\ldots$ & $<.05$ & $<.50$ & $<.05$ & $<.0$ \\
\hline 101 & LNSS88-87 & -- & $<0.05$ & $<0.05$ & $<0.05$ & $<0.05$ & $<0.05$ & $<0.05$ & $<0.05$ & $<0.05$ & $\cdots$ & - & -- & -- \\
\hline 102 & LNSS88-69 & -- & $<.05$ & $<.05$ & $<.05$ & $<.05$ & $<.05$ & $<.05$ & $<.05$ & $<.05$ & -- & -- & $\ldots$ & $\cdots$ \\
\hline 103 & LNSS88-70 & -- & $<.05$ & $<.05$ & .05 & $<.05$ & $<.05$ & $<.05$ & $<.05$ & $<.05$ &.- & -- & -- & $\ldots$ \\
\hline 104 & LNSS88-71 & -- & $<.05$ & $<.05$ & $<.05$ & .06 & .08 & $<.05$ & $<.05$ & $<.05$ & -- & - & - & -- \\
\hline 105 & LNSS88-88 & -- & $<.05$ & $<.05$ & $<.05$ & $<.05$ & .11 & $<.05$ & $<.05$ & $<.05$ & -- & - & -- & -- \\
\hline 106 & $88-137$ & -- & $\ldots$ & $\ldots$ & $\ldots$ & $\ldots$ & -- & $\ldots$ & -- & -- & -- & $\ldots$ & - & - \\
\hline 107 & $88-140$ & -- & -- & -- & --. & - & -- & -- & -- & - & $\ldots$ & $\ldots$ & -- & $\ldots$ \\
\hline 108 & $88-143$ & -- & - & - & - & - & -- & -- & -- & -- & - & $-\ldots$ & -- & -- \\
\hline 109 & $88-146$ & -- & --. & - & -- & - & -- & -- & -- & -- & - & $-\ldots$ & -- & - \\
\hline 110 & $88-149$ & - & $\cdots$ & $\cdots$ & $\cdots$ & -- & - & -- & -- & -- & -- & $\cdots$ & -- & --- \\
\hline 111 & $88-152$ & $\cdots$ & - & $\ldots$ & $\ldots$ & -- & -. & -- & --. & - & -- & $\ldots$ & $\ldots$ & --. \\
\hline 112 & $88-155$ & - & -.- & $\ldots$ & - & -- & -- & $\ldots$ & -. & -- & --. & $\ldots$ & $\ldots$ & -.. \\
\hline 113 & $88-158$ & -- & -- & -- & -.. & --. & -.. & -.. & ... & $\ldots$ & -. & $\ldots$ & $\ldots$ & --- \\
\hline 114 & $88-161$ & -- & --- & -- & - & -- & - & --- & -. & - & -- & $\cdots$ & $\ldots$ & $\ldots$ \\
\hline 115 & $88-164$ & -- & - & --- & -- & - & -- & -- & -- & -- & -- & --- &.- & -- \\
\hline 116 & $88-167$ & -- & --- & -- & -- & - & -- & -- & -- & -- & -- & -- & -- & -- \\
\hline 117 & $88-170$ & - & -- & - & -- & -- & -- & -- & -- & --- & -- & $\cdots$ & -- & -- \\
\hline 118 & $88-173$ & -- & - & -- & -- & -- & - &.- & -- & -- & -- & $\cdots$ & --- & - \\
\hline 119 & $88-176$ & -- & -.- & -- & -- & - & - & -. & -- & - & -- & $\cdots$ & -. & - \\
\hline 120 & $88-185$ & - & $\cdots$ & - & $\cdots$ & $\ldots$ & $\ldots$ & --- &.- & -- & $\ldots$ & $\cdots$ & -- & -- \\
\hline 121 & $88-188$ & -- & - & $\cdots$ & -- & - & - & $\cdots$ & - & - & $\cdots$ & - & -- & - \\
\hline 122 & $88-195$ & - & $\ldots$ & $\ldots$ & --- & -.. & -. & ... & $\ldots$ & -.. & $\ldots$ & $\ldots$ & --- & $\ldots$ \\
\hline 123 & $88-198$ & -. & - & - & -- & -- & -- & -- & -- & -- & -- & -- & -- & -- \\
\hline 124 & $88-201$ & -- & -- & --- & --- & - & -- & -- & -- & --- & -- & $\cdots$ & $\ldots$ & -- \\
\hline 125 & SS89-121 & -- & -- & -- & -- & -- & -- & -. & -- & $\ldots$ & -- & -.. & -.. & -- \\
\hline 126 & SS89-138 & - & - & -- & - & $\ldots$ & -- & -- & - & $\ldots$ & -- & $\cdots$ & - & - \\
\hline 127 & SS89-139 & -- & $\ldots$ & -- & - & -- & $\ldots$ & $\ldots$ & -- & -- & $\ldots$ & $\ldots$ & $\ldots$ & - \\
\hline 128 & SS89-140 & - & $\cdots$ & -- & $\cdots$ & -- & -- & $\ldots$ & - & -.- & --. & -- & $\ldots$ & $\cdots$ \\
\hline 129 & SS89-141 & - & - & $\ldots$ & $\ldots$ & -- & -- & $\ldots$ & -- & -.- & - & $\cdots$ & -- & -- \\
\hline 130 & SS89-85 & -- & -- & - & $\ldots$ & - &.- & - & -- & --- & -- & $\ldots$ & -- & - \\
\hline 131 & SS89-86 & - & $\ldots$ & - & -- & $\ldots$ & -- & - & -- & --. & -- & $\ldots$ & - & -- \\
\hline 132 & SS89-87 & -- & - & - & -- & $\ldots$ & $\ldots$ & -- & $\ldots$ & -- & - & $\cdots$ & -.. & -- \\
\hline 133 & SS89-88 & -- & - & - & - & - & - & -- & -- & -- & -- & $\ldots$ & $\ldots$ & -.. \\
\hline 134 & SS89-89 & -- & - & -- & -- & -- & -- & -- & -- & -- & - & $-\cdots$ & -- & -- \\
\hline 135 & SS89-90 & -- & - & - & -- & -- & --- & -- & - & --- & -- & -- & $\ldots$ & - \\
\hline 136 & SS89-91 & -- & - & -- & -- & -- & -- & -- & - & -- & -- & -- & -- & - \\
\hline 137 & SS89-92 & -- & $\ldots$ & -.. & -- & - & $\ldots$ & -.- & -- & - & --- & --. & --- & - \\
\hline
\end{tabular}


Table 23. Organic chemical analysis, moisture content, and lipid content for biotic samples collected during 1986-90 from the Salton Sea and associated drainwaters and rivers--Continued

\begin{tabular}{|c|c|c|c|c|c|c|}
\hline $\begin{array}{l}\text { Record } \\
\text { number }\end{array}$ & $\begin{array}{l}\text { Sample } \\
\text { number }\end{array}$ & $\begin{array}{l}\text { PCB } \\
1248\end{array}$ & $\begin{array}{l}\text { PCB } \\
1254\end{array}$ & $\begin{array}{l}\text { PCB } \\
1260\end{array}$ & $\begin{array}{l}\text { Total } \\
\text { PCB }\end{array}$ & Toxaphene \\
\hline \multicolumn{7}{|c|}{ Site B16 (Whitewater River Delta)--Continued } \\
\hline 88 & LNSS86-43 & -- & $<0.01$ & $<0.10$ & - & $<0.50$ \\
\hline 89 & LNSS86-47 & $\cdots$ & $<.099$ & $<.099$ & - & $<.50$ \\
\hline 90 & LNSS86-45 & - & $<.10$ & $<.10$ & -- & $<.50$ \\
\hline 91 & LNSS87-06 & $<0.05$ & $<.05$ & $<.05$ & -- & $<.50$ \\
\hline \multicolumn{7}{|c|}{ Site B17 (New River at Rio Bend) } \\
\hline 92 & LNSS86-28B & -- & $<0.13$ & 0.26 & -- & $<0.63$ \\
\hline 93 & LNSS86-31B & -- & $<.10$ & $<.10$ & -- & $<.50$ \\
\hline 94 & LNSS86-29B & -- & $<.10$ & $<.10$ & $-\cdots$ & $<.50$ \\
\hline 95 & LNSS86-30B & -- & $<.099$ & $<.099$ & -- & $<.50$ \\
\hline 96 & LNSS86-38 & -- & $<.098$ & $<.098$ & -- & $<.49$ \\
\hline 97 & LNSS86-34 & $\cdots$ & $<.099$ & .18 & $\sim-$ & $<.49$ \\
\hline 98 & LNSS86-36 & -- & $<.10$ & $<.10$ & $\cdots$ & $<.50$ \\
\hline 99 & LNSS87-15 & $<0.05$ & $<.05$ & $<.05$ & -- & $<.50$ \\
\hline 100 & LNSS87-05 & $<.05$ & $<.05$ & $<.05$ &..- & $<.50$ \\
\hline 101 & LNSS88-87 & -- & -- & 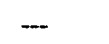 & $\cdots$ & $<.50$ \\
\hline 102 & LNSS88-69 & - & --- & - & -- & $<.50$ \\
\hline 104 & LNSS88-71 & -- & - & -- & - & $<.50$ \\
\hline 105 & LNSS88-88 & - & - & -- & -- & $<.50$ \\
\hline 106 & $88-137$ & - & -- & - & $<0.05$ & $<.05$ \\
\hline 107 & $88-140$ & - & $-\cdots$ & - & $<.05$ & $<.05$ \\
\hline 108 & $88-143$ & - & -- & -- & $<.05$ & $<.05$ \\
\hline 109 & $88-146$ & - & -- & - & $<.05$ & $<.05$ \\
\hline 110 & $88-149$ & - & - & - & $<.05$ & $<.05$ \\
\hline 111 & $88-152$ & - & -- & - & $<.05$ & $<.05$ \\
\hline 112 & $88-155$ & -- & - & - & $<.05$ & $<.05$ \\
\hline 113 & $88-158$ & -- & $\ldots$ & -- & $<.05$ & $<.05$ \\
\hline 114 & $88-161$ & -- & -- & - & $<.05$ & $<.05$ \\
\hline 115 & $88-164$ & -- & - & -- & $<.05$ & $<.05$ \\
\hline 116 & $88-167$ & - & -- & - & $<.05$ & $<.05$ \\
\hline 117 & $88-170$ & -. & -- & -- & $<.05$ & $<.05$ \\
\hline 118 & $88-173$ & -- & -- & $\ldots$ & $<.05$ & $<.05$ \\
\hline 119 & $88-176$ & - & - & - & $<.05$ & $<.05$ \\
\hline 120 & $88-185$ & - & -- & -- & $<.05$ & $<.05$ \\
\hline 121 & $88-188$ & - & - & - & $<.05$ & $<.05$ \\
\hline 122 & 88-195 & -- & $\ldots$ & -- & $<.05$ & $<.05$ \\
\hline 123 & $88-198$ & -- & -- & -- & $<.05$ & $<.05$ \\
\hline 124 & $88-201$ & -- & -- & -- & $<.05$ & $<.05$ \\
\hline 125 & SS89-121 & - & -- & -- & $<.05$ & $<.05$ \\
\hline 126 & SS89-138 & - & -- & -- & $<.05$ & $<.05$ \\
\hline 127 & SS89-139 & - & - & -- & $<.05$ & $<.05$ \\
\hline 128 & SS89-140 & - & --- &.- & $<.05$ & $<.05$ \\
\hline 129 & SS89-141 & $\cdots$ & --- & -- & $<.05$ & $<.05$ \\
\hline 130 & SS89-85 & $\cdots$ & $\cdots$ & $\ldots$ & $<.05$ & $<.05$ \\
\hline 131 & SS89-86 & - & -- & -- & $<.05$ & $<.05$ \\
\hline 132 & SS89-87 & -- & - & - & $<.05$ & $<.05$ \\
\hline 133 & SS89-88 & - & - & -- & $<.05$ & $<.05$ \\
\hline 134 & SS89-89 & $\ldots$ & -- & $\cdots$ & $<.05$ & $<.05$ \\
\hline 135 & SS89-90 & - & -- & - & $<.05$ & $<.05$ \\
\hline 136 & SS89-91 & - & -- & - & $<.05$ & $<.05$ \\
\hline 137 & SS89-92 & -- & - & - & $<.05$ & $<.05$ \\
\hline
\end{tabular}


Table 23. Organic chemical analysis, moisture content, and lipid content for biotic samples collected during 1986-90 from the Salton Sea and associated drainwaters and rivers--Continued

\begin{tabular}{|c|c|c|c|c|c|c|c|c|}
\hline $\begin{array}{l}\text { Record } \\
\text { number }\end{array}$ & $\begin{array}{l}\text { Sample } \\
\text { number }\end{array}$ & Species & Matrix & $\begin{array}{l}\text { Percent } \\
\text { moisture }\end{array}$ & $\begin{array}{c}\text { Percent } \\
\text { lipid }\end{array}$ & $\begin{array}{l}\text { Oxychlor- } \\
\text { dane }\end{array}$ & $\begin{array}{l}\text { alpha- } \\
\text { Chlordane }\end{array}$ & $\begin{array}{c}\text { cis- } \\
\text { Chlordane }\end{array}$ \\
\hline \multicolumn{9}{|c|}{ Site B18 (Alamo River at Garst Road) } \\
\hline 138 & . . ss89-64 & Waterboatman & Composite & 86.5 & 86.5 & $<0.01$ & $<0.01$ & $-\ldots$ \\
\hline 139 & . . SS89-120 & Asiatic river clam & Soft tissue & 86.0 & 86.0 & $<.01$ & .06 & -- \\
\hline 140 & . . SS89-165 & Asiatic river clam & Soft tissue & 81.1 & 81.1 & $<.01$ & $<.01$ & -- \\
\hline 141 & . . SS89-167 & Asiatic river clam & Soft tissue & 80.6 & 80.6 & $<.01$ & .12 & - \\
\hline 142 & . . SS89-175 & Bullfrog & Whole body & 76.0 & 76.0 & .01 & $<.01$ & -- \\
\hline 143 & . . SS89-176 & Bullfrog & Whole body & 79.5 & .480 & $<.01$ & $<.01$ & -- \\
\hline \multicolumn{9}{|c|}{ Site B19 (San Felipe Creek) } \\
\hline 144 & $\ldots$ ss90s1 & Sediment & Sediment & 30.5 & -- & $<0.009$ & $<0.009$ & -- \\
\hline \multicolumn{9}{|c|}{ Site B20 (Salt Creek) } \\
\hline 145 & $\ldots$ S\$90S4 & Sediment & Sediment & 52.2 & -- & $<0.0099$ & $<0.0099$ & --- \\
\hline \multicolumn{9}{|c|}{ Site B21 (Colorado River at Palo Verde) } \\
\hline 146 & . . S\$89-117 & Asiatic river clam & Soft tissue & 87.0 & 1.38 & $<0.01$ & $<0.01$ & -- \\
\hline 147 & . . S\$89-118 & Asiatic river clam & Soft tissue & 92.5 & .82 & $<.01$ & $<.01$ & --- \\
\hline 148 & . . ss89-119 & Asiatic river clam & Soft tissue & 87.5 & 1.78 & $<.01$ & $<.01$ & - \\
\hline \multicolumn{9}{|c|}{ Site B22 (Trifolium 5 Drain) } \\
\hline 149 & . . LNSS86-59B & Tilapia & Whole body & 76.3 & 2.27 & $<0.0099$ & $\cdots$ & $<0.0099$ \\
\hline \multicolumn{9}{|c|}{ Site B23 (Trifolium 13 Drain) } \\
\hline 150 & $\ldots \operatorname{ss89-62}$ & Invertebrates & Composite & 81.1 & 3.08 & $<0.01$ & $<0.01$ & --- \\
\hline 151 & . . SS89-168 & Asiatic river clam & Soft tissue & 86.4 & 8.62 & $<.01$ & $<.01$ & -- \\
\hline \multicolumn{9}{|c|}{ Site B24 (Trifolium 14 Drain) } \\
\hline 152 & . . LNSS88-95 & Crayfish & Whole body & 76.9 & 7.61 & $<0.05$ & $<0.05$ & -- \\
\hline 153 & . . LNSS88-94 & Black-necked stilt & Carcass & 76.7 & 7.60 & $<.05$ & $<.05$ & --- \\
\hline 154 & . . LNSS88-89 & Black-necked stilt & Carcass & 74.0 & 7.21 & $<.05$ & $<.05$ & - \\
\hline 155 & . . LNSS88-90 & Black-necked stilt & Carcass & 72.1 & 11.07 & $<.05$ & $<.05$ & $\ldots$ \\
\hline 156 & . . 88-192 & Black-necked stilt & Egg & 72.5 & 10.6 & .01 & $<.01$ & -- \\
\hline 157 & . 8 88-204 & Black-necked stilt & Egg & 64.0 & 17.2 & .01 & $<.01$ & --- \\
\hline 158 & . 88-207 & Black-necked stilt & Egg & 72.5 & 12.7 & $<.01$ & $<.01$ & -- \\
\hline 159 & . . 88-210 & Black-necked stilt & Egg & 71.0 & 15.0 & $<.01$ & $<.01$ & -- \\
\hline 160 & . . 88-213 & Black-necked stilt & Egg & 72.0 & 13.1 & .01 & $<.01$ & --- \\
\hline 161 & . . 88-216 & Black-necked stilt & Egg & 73.0 & 14.4 & $<.01$ & $<.01$ & -- \\
\hline 162 & . 88-225 & Black-necked stilt & Egg & 71.5 & 10.5 & .02 & $<.01$ & -- \\
\hline 163 & . 8 88-228 & Black-necked stilt & Egg & 71.5 & 14.2 & .01 & $<.01$ & --- \\
\hline 164 & . . 88-231 & Black-necked stilt & Egg & 74.0 & 12.9 & .02 & $<.01$ & --- \\
\hline 165 & . . 88-232 & Black-necked stilt & Egg & 72.0 & 13.9 & .01 & $<.01$ & --- \\
\hline 166 & . . SS89-166 & Asiatic river clam & Soft tissue & 83.0 & 13.1 & $<.01$ & $<.01$ & -- \\
\hline 167 & . . ss89-122 & Asiatic river clam & Soft tissue & 90.0 & 1.00 & $<.01$ & .01 & -- \\
\hline 168 & $\ldots$ ss90C1 & Asiatic river clam & Soft tissue & 61.5 & 3.00 & $<.049$ & $<.049$ & --- \\
\hline 169 & $\ldots \mathrm{ss} 90 \mathrm{C} 2$ & Asiatic river clam & Soft tissue & 82.5 & 4.80 & $<.049$ & $<.049$ & -- \\
\hline \multicolumn{9}{|c|}{ Site B25 (Vail Cutoff Drain) } \\
\hline 170 & . . SS89-71 & American coot & Liver & 75.0 & 3.20 & $<0.01$ & $<0.01$ & -- \\
\hline 171 & . s\$89-72 & American coot & Liver & 75.0 & 3.70 & $<.01$ & $<.01$ & -- \\
\hline 172 & . s ss89-73 & American coot & Liver & 76.0 & 3.40 & $<.01$ & $<.01$ & --- \\
\hline 173 & . . SS89-66 & Black-necked stilt & Egg & 74.0 & 12.4 & $<.01$ & $<.01$ & $-\ldots$ \\
\hline 174 & . . ss89-67 & Black-necked stilt & Egg & 76.0 & 11.0 & $<.01$ & $<.01$ & --- \\
\hline 175 & . . $\$ \$ S 89-68$ & Black-necked stilt & Egg & 73.0 & 13.7 & .03 & $<.01$ & --- \\
\hline 176 & . . Ss89-69 & Black-necked stilt & Egg & 71.0 & 5.22 & $<.01$ & $<.01$ & --- \\
\hline 177 & . s $\mathbf{s} 89-70$ & Black-necked stilt & Egg & 74.0 & 4.96 & $<.01$ & $<.01$ & --- \\
\hline
\end{tabular}


Table 23. Organic chemical analysis, moisture content, and lipid content for biotic samples collected during 1986-90 from the Salton Sea and associated drainwaters and rivers--Continued

\begin{tabular}{|c|c|c|c|c|c|c|c|c|c|}
\hline $\begin{array}{l}\text { Record } \\
\text { number }\end{array}$ & $\begin{array}{l}\text { Sample } \\
\text { number }\end{array}$ & $\begin{array}{l}\text { gamma- } \\
\text { Chlordane }\end{array}$ & $\begin{array}{c}\text { trans- } \\
\text { Chlordane }\end{array}$ & $\begin{array}{l}\text { cis-Nona- } \\
\text { chlor }\end{array}$ & $\begin{array}{l}\text { trans- } \\
\text { Nonachlor }\end{array}$ & $\begin{array}{l}\text { Hepta- } \\
\text { chlor }\end{array}$ & $\begin{array}{l}\text { Heptachlor } \\
\text { epoxide }\end{array}$ & $\begin{array}{l}\text { Methyoxy- } \\
\text { chlor }\end{array}$ & o,p'-DDE \\
\hline \multicolumn{10}{|c|}{ Site B18 (Alamo River at Garst Road) } \\
\hline 138 & S\$89-64 & $<0.01$ & $\ldots$ & $<0.01$ & $<0.01$ & - & $<0.01$ & --- & $<0.01$ \\
\hline 139 & SS89-120 & $<.01$ & - & $<.01$ & .02 & -- & $<.01$ & $\ldots$ & .12 \\
\hline 140 & S\$89-165 & $<.01$ & - & $<.01$ & $<.01$ & -- & $<.01$ & $-\ldots$ & $<.01$ \\
\hline 141 & S\$89-167 & .02 & $\cdots$ & $<.01$ & .04 & - & $<.01$ & -- & $<.01$ \\
\hline 142 & SS89-175 & $<.01$ & - & $<.01$ & $<.01$ & -- & $<.01$ & -- & $<.01$ \\
\hline 143 & SS89-176 & $<.01$ & - & $<.01$ & $<.01$ & -- & $<.01$ & - & $<.01$ \\
\hline \multicolumn{10}{|c|}{ Site B19 (San Felipe Creek) } \\
\hline 144 & SS90S1 & - & $<0.009$ & $<0.009$ & $<0.009$ & - & $<0.009$ & - & $<0.01$ \\
\hline \multicolumn{10}{|c|}{ Site B20 (Salt Creek) } \\
\hline 145 & SS90S4 & $\cdots$ & $<0.0099$ & $<0.0099$ & $<0.0099$ & -- & $<0.0099$ & -- & $<0.01$ \\
\hline \multicolumn{10}{|c|}{ Site B21 (Colorado River at Palo Verde) } \\
\hline 146 & SS89-117 & $<0.01$ & - & $<0.01$ & $<0.01$ & -- & $<0.01$ & -- & $<0.01$ \\
\hline 147 & S\$89-118 & $<.01$ & -- & $<.01$ & $<.01$ & -- & $<.01$ & $\cdots$ & $<.01$ \\
\hline 148 & SS89-119 & $<.01$ & -- & $<.01$ & $<.01$ & -- & $<.01$ & - & $<.01$ \\
\hline \multicolumn{10}{|c|}{ Site B22 (Trifolium 5 Drain) } \\
\hline 149 & LNSS86-59B & - & $<0.0099$ & $<0.0099$ & $<0.0099$ & -- & $<0.0099$ & -- & - \\
\hline \multicolumn{10}{|c|}{ Site B23 (Trifolium 13 Drain) } \\
\hline 150 & SS89-62 & $<0.01$ & - & $<0.01$ & $<0.01$ & - & $<0.01$ & -- & $<0.01$ \\
\hline 151 & SS89-168 & $<.01$ & $\ldots$ & $<.01$ & $<.01$ & -- & $<.01$ & -- & $<.01$ \\
\hline \multicolumn{10}{|c|}{ Site B24 (Trifolium 14 Drain) } \\
\hline 152 & LNSS88-95 & $<0.05$ & $\ldots$ & $<0.05$ & $<0.05$ & $<0.05$ & $<0.05$ & -- & $<0.05$ \\
\hline 153 & LNSS88-94 & $<.05$ & - & $<.05$ & $<.05$ & $<.05$ & $<.05$ & -- & $<.05$ \\
\hline 154 & LNSS88-89 & $<.05$ & -- & $<.05$ & $<.05$ & $<.05$ & $<.05$ & --- & $<.05$ \\
\hline 155 & LNSS88-90 & $<.05$ & - & $<.05$ & $<.05$ & $<.05$ & $<.05$ & -- & $<.05$ \\
\hline 156 & 88-192 & $<.01$ & -- & $<.01$ & $<.01$ & -- & $<.01$ & --- & $<.01$ \\
\hline 157 & $88-204$ & $<.01$ & -- & $<.01$ & .01 & - & $<.01$ & -- & $<.01$ \\
\hline 158 & $88-207$ & $<.01$ & - & $<.01$ & .01 & - & $<.01$ & --- & $<.01$ \\
\hline 159 & $88-210$ & $<.01$ & -- & $<.01$ & $<.01$ & -- & $<.01$ & --- & $<.01$ \\
\hline 160 & $88-213$ & $<.01$ & - & $<.01$ & $<.01$ & - & $<.01$ & --- & $<.01$ \\
\hline 161 & $88-216$ & $<.01$ & -- & $<.01$ & $<.01$ & - & $<.01$ & --- & $<.01$ \\
\hline 162 & $88-225$ & $<.01$ & - & $<.01$ & $<.01$ & -- & $<.01$ & -- & $<.01$ \\
\hline 163 & $88-228$ & $<.01$ & - & $<.01$ & $<.01$ & -- & $<.01$ & -- & $<.01$ \\
\hline 164 & $88-231$ & $<.01$ & - & $<.01$ & $<.01$ & -- & .01 & -- & $<.01$ \\
\hline 165 & $88-232$ & $<.01$ & - & $<.01$ & .01 & - & $<.01$ & -.- & $<.01$ \\
\hline 166 & SS89-166 & $<.01$ & -- & $<.01$ & $<.01$ & - & $<.01$ & -- & $<.01$ \\
\hline 167 & SS89-122 & $<.01$ & - & $<.01$ & $<.01$ & -- & $<.01$ & - & .02 \\
\hline 168 & Ss90C1 & - & $<0.049$ & $<.049$ & $<.049$ & - & $<.049$ & -- & $<.01$ \\
\hline 169 & SS90C2 & -- & $<.049$ & $<.049$ & $<.049$ & $\cdots$ & $<.049$ & $\cdots$ & $<.01$ \\
\hline \multicolumn{10}{|c|}{ Site B25 (Vail Cutoff Drain) } \\
\hline 170 & SS89-71 & $<0.01$ & -- & $<0.01$ & $<0.01$ & -- & $<0.01$ & -- & $<0.01$ \\
\hline 171 & SS89-72 & $<.01$ & - & $<.01$ & $<.01$ & -- & $<.01$ & - & $<.01$ \\
\hline 172 & SS89-73 & $<.01$ & - & $<.01$ & $<.01$ & -- & $<.01$ & $\ldots$ & $<.01$ \\
\hline 173 & SS89-66 & $<.01$ & -- & $<.01$ & $<.01$ & -- & $<.01$ & -- & $<.01$ \\
\hline 174 & SS89-67 & $<.01$ & - & $<.01$ & $<.01$ & --- & $<.01$ & -- & $<.01$ \\
\hline 175 & SS89-68 & $<.01$ & -- & $<.01$ & $<.01$ & -- & .01 & --- & $<.01$ \\
\hline 176 & SS89-69 & $<.01$ & -- & $<.01$ & $<.01$ & -- & $<.01$ & -- & $<.01$ \\
\hline 177 & SS89-70 & $<.01$ & -- & $<.01$ & $<.01$ & -- & $<.01$ & -- & $<.01$ \\
\hline
\end{tabular}


Table 23. Organic chemical analysis, moisture content, and lipid content for biotic samples collected during 1986-90 from the Salton Sea and associated drainwaters and rivers--Continued

\begin{tabular}{|c|c|c|c|c|c|c|c|c|c|c|}
\hline $\begin{array}{l}\text { Record } \\
\text { number }\end{array}$ & $\begin{array}{l}\text { Sample } \\
\text { number }\end{array}$ & $p, p^{\prime}-D D E$ & $o, p^{\prime}-D D D$ & p,p'-DDD & $o, p^{\prime}-$ DDT & $\mathrm{p}, \mathrm{p}^{\prime}-\mathrm{DDT}$ & $\begin{array}{l}\text { Total } \\
\text { DDT }\end{array}$ & Endrin & Dieldrin & Aldrin \\
\hline \multicolumn{11}{|c|}{ Site B18 (Alamo River at Garst Road) } \\
\hline 138 & SS89-64 & 0.01 & $<0.01$ & $<0.01$ & $<0.01$ & $<0.01$ & 0.010 & $<0.01$ & $<0.01$ & -- \\
\hline 139 & SS89-120 & 2.9 & .09 & .14 & .25 & .26 & 3.500 & $<.01$ & .01 & -- \\
\hline 140 & SS89-165 & .71 & $<.01$ & .10 & $<.01$ & $<.01$ & .810 & $<.01$ & .09 & -- \\
\hline 141 & SS89-167 & 3.0 & $<.01$ & $<.01$ & $<.01$ & $<.01$ & 3.350 & $<.01$ & .24 & -- \\
\hline 142 & SS89-175 & .38 & $<.01$ & $<.01$ & $<.01$ & $<.01$ & .380 & $<.01$ & .01 & -- \\
\hline 143 & SS89-176 & .01 & $<.01$ & $<.01$ & $<.01$ & $<.01$ & .010 & $<.01$ & $<.01$ & - \\
\hline \multicolumn{11}{|c|}{ Site B19 (San Felipe Creek) } \\
\hline 144 & SS90S1 & 0.025 & $<0.01$ & $<0.009$ & $<0.01$ & $<0.009$ & -- & $<0.009$ & $<0.009$ & -- \\
\hline \multicolumn{11}{|c|}{ Site B20 (Salt Creek) } \\
\hline 145 & SS90S4 & 0.053 & $<0.01$ & $<0.0099$ & $<0.01$ & $<0.0099$ & -- & $<0.0099$ & $<0.0099$ & - \\
\hline \multicolumn{11}{|c|}{ Site B21 (Colorado River at Palo Verde) } \\
\hline 146 & SS89-117 & 0.02 & $<0.01$ & $<0.01$ & $<0.01$ & $<0.01$ & 0.020 & $<0.01$ & $<0.01$ & --- \\
\hline 147 & SS89-118 & .01 & $<.01$ & $<.01$ & $<.01$ & $<.01$ & .010 & $<.01$ & $<.01$ & -- \\
\hline 148 & SS89-119 & .02 & $<.01$ & $<.01$ & $<.01$ & $<.01$ & .020 & $<.01$ & $<.01$ & -- \\
\hline \multicolumn{11}{|c|}{ Site B22 (Trifolium 5 Drain) } \\
\hline 149 & LNSS86-59B & 0.25 & - & $<0.0099$ & -- & 0.014 & 0.264 & $<0.0099$ & $<0.0099$ & --- \\
\hline \multicolumn{11}{|c|}{ Site B23 (Trifolium 13 Drain) } \\
\hline 150 & SS89-62 & 0.08 & $<0.01$ & $<0.01$ & $<0.01$ & $<0.01$ & 0.080 & $<0.01$ & $<0.01$ &.-- \\
\hline 151 & SS89-168 & .37 & $<.01$ & $<.01$ & $<.01$ & $<.01$ & .370 & $<.01$ & $<.01$ & -- \\
\hline \multicolumn{11}{|c|}{ Site B24 (Trifolium 14 Drain) } \\
\hline 152 & LNSS88-95 & 0.55 & $<0.05$ & $<0.05$ & $<0.05$ & $<0.05$ & $<0.05$ & $<0.05$ & $<0.05$ & $<0.05$ \\
\hline 153 & LNSS88-94 & 1.32 & $<.05$ & $<.05$ & $<.05$ & $<.05$ & $<.05$ & $<.05$ & $<.05$ & $<.05$ \\
\hline 154 & LNSS88-89 & .67 & $<.05$ & $<.05$ & $<.05$ & $<.05$ & $<.05$ & $<.05$ & $<.05$ & $<.05$ \\
\hline 155 & LNSS88-90 & .64 & $<.05$ & $<.05$ & $<.05$ & $<.05$ & $<.05$ & $<.05$ & $<.05$ & $<.05$ \\
\hline 156 & $88-192$ & 3.7 & $<.01$ & .02 & $<.01$ & .17 & 3.890 & $<.01$ & .05 & --- \\
\hline 157 & $88-204$ & 6.5 & $<.01$ & .02 & $<.01$ & .02 & 6.540 & $<.01$ & .06 & $\cdots$ \\
\hline 158 & $88-207$ & 2.7 & $<.01$ & $<.01$ & $<.01$ & $<.01$ & 2.700 & $<.01$ & .02 & --- \\
\hline 159 & $88-210$ & 1.9 & $<.01$ & $<.01$ & $<.01$ & $<.01$ & 1.900 & $<.01$ & .01 & -- \\
\hline 160 & $88-213$ & 2.1 & $<.01$ & $<.01$ & $<.01$ & $<.01$ & 2.100 & $<.01$ & .01 & -- \\
\hline 161 & $88-216$ & .26 & $<.01$ & $<.01$ & $<.01$ & $<.01$ & .260 & $<.01$ & $<.01$ & $\ldots$ \\
\hline 162 & $88-225$ & 2.6 & $<.01$ & .02 & $<.01$ & .12 & 2.740 & $<.01$ & .03 & --- \\
\hline 163 & $88-228$ & 4.8 & $<.01$ & .02 & $<.01$ & .02 & 4.840 & $<.01$ & .03 & --- \\
\hline 164 & $88-231$ & 4.6 & $<.01$ & $<.01$ & $<.01$ & .04 & 4.640 & $<.01$ & .02 & -- \\
\hline 165 & $88-232$ & 2.2 & $<.01$ & .01 & $<.01$ & .02 & 2.230 & $<.01$ & $<.01$ & -.- \\
\hline 166 & SS89-166 & .27 & $<.01$ & $<.01$ & $<.01$ & $<.01$ & .270 & $<.01$ & $<.01$ & - \\
\hline 167 & SS89-122 & .83 & .02 & $<.01$ & .03 & .05 & .900 & $<.01$ & $<.01$ & -.- \\
\hline 168 & SS90Cl & .49 & $<.01$ & .064 & $<.01$ & .075 & .629 & $<.049$ & $<.049$ & -- \\
\hline 169 & SS90C2 & .31 & $<.01$ & .070 & $<.01$ & .079 & .459 & $<.049$ & .059 & --- \\
\hline \multicolumn{11}{|c|}{ Site B25 (Vail Cutoff Drain) } \\
\hline 170 & SS89-71 & 0.01 & $<0.01$ & $<0.01$ & $<0.01$ & $<0.01$ & 0.010 & $<0.01$ & $<0.01$ & --- \\
\hline 171 & SS89-72 & .01 & $<.01$ & $<.01$ & $<.01$ & $<.01$ & .010 & $<.01$ & $<.01$ & -- \\
\hline 172 & SS89-73 & .03 & $<.01$ & $<.01$ & $<.01$ & $<.01$ & .030 & $<.01$ & $<.01$ & -- \\
\hline 173 & SS89-66 & .71 & $<.01$ & $<.01$ & $<.01$ & $<.01$ & .710 & $<.01$ & $<.01$ & -- \\
\hline 174 & SS89-67 & .55 & $<.01$ & $<.01$ & $<.01$ & $<.01$ & .550 & $<.01$ & $<.01$ & $\ldots$ \\
\hline 175 & SS89-68 & 2.8 & $<.01$ & $<.01$ & $<.01$ & .06 & 2.860 & $<.01$ & .14 & --- \\
\hline 176 & SS89-69 & .9 & $<.01$ & $<.01$ & $<.01$ & $<.01$ & .900 & $<.01$ & .02 & -- \\
\hline 177 & SS89-70 & .05 & $<.01$ & $<.01$ & $<.01$ & $<.01$ & .050 & $<.01$ & $<.01$ & - \\
\hline
\end{tabular}


Table 23. Organic chemical analysis, moisture content, and lipid content for biotic samples collected during 1986-90 from the Salton Sea and associated drainwaters and rivers--Continued

\begin{tabular}{lllllllllllll}
\hline Record & Sample & alpha- & beta- & delta- & gamma- & Lin- & $\begin{array}{c}\text { Hexa- } \\
\text { chloro- }\end{array}$ & Endo- & Endo- & Endo- \\
number & number & BHC & BHC & BHC & BHC & dane & $\begin{array}{c}\text { sul- } \\
\text { ben- } \\
\text { zan I }\end{array}$ & fan II & sulfate & Mirex & DCPA Dicofol
\end{tabular}

\begin{tabular}{|c|c|c|c|c|c|c|c|c|c|c|c|c|c|}
\hline \multirow[b]{2}{*}{138} & \multicolumn{13}{|c|}{ Site B18 (Alamo River at Garst Road) } \\
\hline & SS89-64 & $<0.01$ & $<0.01$ & $<0.01$ & $<0.01$ & -- & $<0.01$ & - & -- & --- & $<0.01$ & -- & -- \\
\hline 139 & SS89-120 & $<.01$ & $<.01$ & $<.01$ & $<.01$ & $\ldots$ & $<.01$ & -- & -- & --- & $<.01$ & -- & - \\
\hline 140 & SS89-165 & $<.01$ & $<.01$ & $<.01$ & $<.01$ & -- & .01 & $<0.01$ & $<0.01$ & -- & $<.01$ & -- & $-\cdots$ \\
\hline 141 & SS89-167 & $<.01$ & $<.01$ & $<.01$ & $<.01$ & - & $<.01$ & $<.01$ & $<.01$ & -- & $<.01$ & - & $\cdots$ \\
\hline 142 & SS89-175 & $<.01$ & $<.01$ & $<.01$ & $<.01$ & -- & $<.01$ & $<.01$ & $<.01$ & -- & $<.01$ & - & -- \\
\hline 143 & ss89-176 & $<.01$ & $<.01$ & $<.01$ & $<.01$ & -- & $<.01$ & $<.01$ & $<.01$ & $\ldots$ & $<.01$ & -.. & -- \\
\hline
\end{tabular}

144 SS90S1

Site B19 (San Felipe Creek)

Site B20 (Salt Creek)

$145 \quad$ SS90S4

146

147

148

SS89-117

SS89-118

SS89-119

$<0.01$

$<$.01

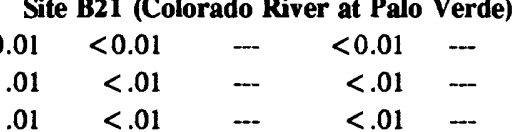

\section{Site B22 (Trifolium 5 Drain)}

149

LNSS86-59B --

150

SS89-62

SS89-168

$<0.01<0.01<0.01$

Site B23 (Trifolium 13 Drain)

$<0.01 \quad--\quad<0.01<0.01<0.01 \quad--<<0.01$

$<.01$

$<.0$

\section{Site B24 (Trifolium 14 Drain)}

\begin{tabular}{|c|c|c|c|c|c|c|c|c|c|c|c|c|c|}
\hline 152 & LNSS88-95 & $<0.05$ & $<0.05$ & $<0.05$ & -- & $<0.05$ & $<0.05$ & -- & -- & -- & $<0.05$ & -- & -- \\
\hline 153 & LNSS88-94 & $<.05$ & $<.05$ & $<.05$ & -- & $<.05$ & $<.05$ & -- & - & -- & $<.05$ & -- & -- \\
\hline 154 & LNSS88-89 & $<.05$ & $<.05$ & $<.05$ &.-- & $<.05$ & $<.05$ & $\ldots$ & -- & -- & $<.05$ & -- & -- \\
\hline 155 & LNSS88-90 & $<.05$ & $<.05$ & $<.05$ & -- & $<.05$ & $<.05$ & -- & -- & -- & $<.05$ &.-- & -- \\
\hline 156 & $88-192$ & $<.01$ & .15 & $<.01$ & $<0.01$ & -- & .01 & -- & -- & -- & $<.01$ & -- & -- \\
\hline 157 & 88-204 & $<.01$ & .01 & $<.01$ & $<.01$ & $\cdots$ & .02 & -- & -- & $\cdots$ & $<.01$ & -- & -- \\
\hline 158 & $88-207$ & $<.01$ & $<.01$ & $<.01$ & $<.01$ & $\cdots$ & $<.01$ & -- & -- & -- & $<.01$ & --- & --- \\
\hline 159 & $88-210$ & $<.01$ & $<.01$ & $<.01$ & $<.01$ & $\cdots$ & .01 & - & $\cdots$ & -- & $<.01$ & -- & $\cdots$ \\
\hline 160 & $88-213$ & $<.01$ & $<.01$ & $<.01$ & $<.01$ & -- & .01 & -- & -- & -- & $<.01$ & $\cdots$ & -- \\
\hline 161 & $88-216$ & $<.01$ & $<.01$ & $<.01$ & $<.01$ & -- & .01 & $\cdots$ & $\cdots$ & -- & $<.01$ & -- & -- \\
\hline 162 & $88-225$ & $<.01$ & .10 & $<.01$ & $<.01$ & -. & .01 & -- & -- & -- & .01 & -- & --- \\
\hline 163 & $88-228$ & $<.01$ & .01 & $<.01$ & $<.01$ & -- & .01 & -- & $\cdots$ & -- & .01 & --- & -- \\
\hline 164 & $88-231$ & $<.01$ & .16 & $<.01$ & $<.01$ & -- & .01 & -- & -- & -- & .01 & $\cdots$ & --- \\
\hline 165 & $88-232$ & $<.01$ & .04 & $<.01$ & $<.01$ & -- & .01 & -- & -- & -- & $<.01$ & --- & -- \\
\hline 166 & SS89-166 & $<.01$ & $<.01$ & $<.01$ & $<.01$ & --- & $<.01$ & $<0.01$ & $<0.01$ & -- & $<.01$ & --- & -- \\
\hline 167 & SS89-122 & $<.01$ & $<.01$ & $<.01$ & $<.01$ & -- & $<.01$ & -- & -- & -- & $<.01$ & +- & $\cdots$ \\
\hline 168 & SS90C1 & --- & -- & $\cdots$ & +- & -- & -- & $\cdots$ & $\cdots$ & -- & --- & $\cdots$ & -- \\
\hline 169 & SS90C2 & -- & $\ldots$ & -- & -- & -- & -- & -- & -- & $\ldots$ & $-\cdots$ & -- & \\
\hline
\end{tabular}

\begin{tabular}{|c|c|c|c|c|}
\hline 170 & SS89-71 & $<0.01$ & $<0.01$ & $<0.01$ \\
\hline 171 & SS89-72 & $<.01$ & $<.01$ & $<.01$ \\
\hline 172 & SS89-73 & $<.01$ & $<.01$ & $<.01$ \\
\hline 173 & SS89-66 & $<.01$ & $<.01$ & $<.01$ \\
\hline 174 & SS89-67 & $<.01$ & .04 & $<.01$ \\
\hline 175 & SS89-68 & $<.01$ & .03 & $<.01$ \\
\hline 176 & SS89-69 & $<.01$ & $<.01$ & $<.01$ \\
\hline 177 & SS89-70 & $<.01$ & $<.01$ & $<.01$ \\
\hline
\end{tabular}

Site B25 (Vail Cutoff Drain)

$<0.01$

$<.01$

$<.01 \quad-$.

$<.01$

$<.01$

$<.01$

$<.01$

--

\begin{tabular}{|c|c|}
\hline \multicolumn{2}{|l|}{$<0.01$} \\
\hline$<.01$ & \\
\hline .01 & \\
\hline .01 & \\
\hline .11 & \\
\hline .01 & \\
\hline$<.01$ & \\
\hline
\end{tabular}

$\begin{array}{lll}<0.01 & -- & -- \\ <.01 & -- & -- \\ <.01 & --- & -- \\ <.01 & --- & -- \\ <.01 & -- & -- \\ <.01 & -- & - \\ <.01 & -- & - \\ <.01 & -- & --\end{array}$ 
Table 23. Organic chemical analysis, moisture content, and lipid content for biotic samples collected during 1986-90 from the Salton Sea and associated drainwaters and rivers--Continued

\begin{tabular}{|c|c|c|c|c|c|c|c|c|c|c|c|c|c|c|}
\hline $\begin{array}{l}\text { Record } \\
\text { number }\end{array}$ & $\begin{array}{l}\text { Sample } \\
\text { number }\end{array}$ & $\begin{array}{l}\text { Tetra- } \\
\text { difon }\end{array}$ & $\begin{array}{l}\text { BI PH } \\
\text { CL-2 }\end{array}$ & $\begin{array}{l}\text { BI PH } \\
\text { CL-3 }\end{array}$ & $\begin{array}{c}\text { BI PH } \\
\text { CL-4 }\end{array}$ & $\begin{array}{c}\text { BI PH } \\
\text { CL-5 }\end{array}$ & $\begin{array}{c}\text { BI PH } \\
\text { CL-6 }\end{array}$ & $\begin{array}{c}\text { BI PH } \\
\text { CL-7 }\end{array}$ & $\begin{array}{c}\text { BI PH } \\
\text { CL-8 }\end{array}$ & $\begin{array}{c}\text { BI PH } \\
\text { CL-9 }\end{array}$ & $\begin{array}{l}\text { PCB } \\
1016\end{array}$ & $\begin{array}{l}\text { PCB } \\
1221\end{array}$ & $\begin{array}{l}\text { PCB } \\
1232\end{array}$ & $\begin{array}{l}\text { PCB } \\
1242\end{array}$ \\
\hline
\end{tabular}

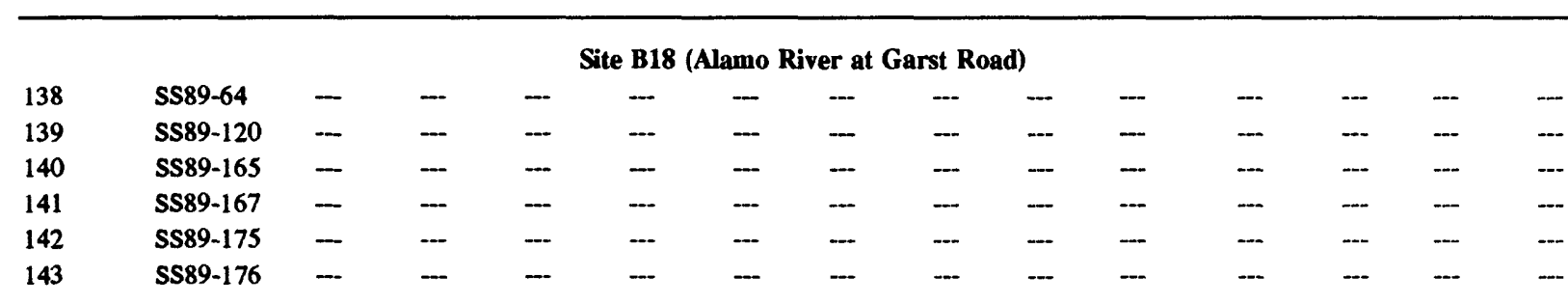

\section{Site B19 (San Felipe Creek)}

\begin{tabular}{|c|c|c|c|c|c|c|c|c|c|c|c|c|c|c|}
\hline \multirow{2}{*}{\multicolumn{15}{|c|}{ ssoos 1}} \\
\hline & SS90S1 & - & -- & - & -- & - & - & - & -- & -- & -- & -- & - & -- \\
\hline \multicolumn{15}{|c|}{ Site B20 (Salt Creek) } \\
\hline 145 & SS90S4 & - & -- & -- & - & -- & -- & -- & -- & -- & -- & -- & -- & --- \\
\hline \multicolumn{15}{|c|}{ Site B21 (Colorado River at Palo Verde) } \\
\hline 146 & SS89-117 & - & -- & -- & - & -- & -- & -- & $-\ldots$ & -- & -- & -- & -- & - \\
\hline 147 & SS89-118 & - & - & - & $\ldots$ & -- & $\cdots$ & $\ldots$ & -- & $\cdots$ & -- & $\ldots$ & $\ldots$ & -- \\
\hline 148 & SS89-119 & - & -- & -- & - & -- & -- & - & -- & - & --- & - & - & -- \\
\hline \multicolumn{15}{|c|}{ Site B22 (Trifolium 5 Drain) } \\
\hline 149 & LNSS86-59B & - & - & -- & - & $-\cdots$ & -- & -- & -- & - & -- & -- & $\cdots$ & -- \\
\hline \multicolumn{15}{|c|}{ Site B23 (Trifolium 13 Drain) } \\
\hline 150 & SS89-62 & - & $-\cdots$ & -- & -- & -.. & - & -- & $\ldots$ & -- & -- & --- & -- & -- \\
\hline 151 & SS89-168 & -- & -- & -- & -- & -- & -- & -- & -- & -- & -- & -- & -- & -- \\
\hline \multicolumn{15}{|c|}{ Site B24 (Trifolium 14 Drain) } \\
\hline 152 & LNSS88-95 & - & $<0.05$ & $<0.05$ & $<0.05$ & $<0.05$ & 0.12 & $<0.05$ & $<0.05$ & $<0.05$ & $\ldots$ & $\cdots$ & -- & -- \\
\hline 153 & LNSS88-94 & -- & $<.05$ & $<.05$ & .06 & $<.05$ & .05 & .07 & $<.05$ & $<.05$ & -- & $-\ldots$ & - & $\ldots$ \\
\hline 154 & LNSS88-89 & - & $<.05$ & $<.05$ & .07 & $<.05$ & .05 & .05 & $<.05$ & $<.05$ & -- & -- & -- & $\ldots$ \\
\hline 155 & LNSS88-90 & $\ldots$ & $<.05$ & $<.05$ & $<.05$ & $<.05$ & .48 & $<.05$ & $<.05$ & $<.05$ & -. & -- & -- & -- \\
\hline 156 & $88-192$ & -- & $\ldots$ & -- & -- & --- & --- & -- & --- & -- & --- & -- & -- & $\cdots$ \\
\hline 157 & $88-204$ & - & - & - & $-\ldots$ & --- & -- & -- & -- & - & -- & -- & -- & $\cdots$ \\
\hline 158 & $88-207$ & - & $-\cdots$ & - & -- & -- & -- & -- & --- & - & -- & -- & -- & -- \\
\hline 159 & $88-210$ & - & - & - & $\ldots$ & -.. &.- & - & -. & -- & -- & -- & -- & - \\
\hline 160 & $88-213$ & -- & $\cdots$ & - & -- & -- & $\cdots$ & $\ldots$ & -- & - & - & $\cdots$ & -- & $\ldots$ \\
\hline 161 & $88-216$ & -- & - & -- & -- & -- & -- & -- & -- & -- & -- & -- & -- & - \\
\hline 162 & $88-225$ & - & - & - & - & $\cdots$ & - & -.- & -- & -.- & -- & --- & -- & $\cdots$ \\
\hline 163 & $88-228$ & -- & -- & - & -- & - & -- & $\ldots$ & --- & --- & $\cdots$ & - & $\cdots$ & $\cdots$ \\
\hline 164 & $88-231$ & - & $\cdots$ & - & -- & -- & $\cdots$ & -- & -- & $\cdots$ & $\cdots$ & -- & - & $\ldots$ \\
\hline 165 & $88-232$ & -- & - & - & --- & -- & -- & -- & -- & -- & -- & -- & -- & - \\
\hline 166 & SS89-166 & - & - & -- & - & $\ldots$ & -- & -- & -- & -- & $-\ldots$ & -.- & -- & $\ldots$ \\
\hline 167 & SS89-122 & - & -- & -- & $\cdots$ & $\cdots$ & $\cdots$ & -- & -- & -- & -- & --- & -- & $\ldots$ \\
\hline 168 & SS90C1 & - & - & $\cdots$ & --- & -- & -- & -- & --- & -- & -- & -- & -- & $\ldots$ \\
\hline 169 & SS90C2 & - & $\cdots$ & $\cdots$ & -- & -- & -- & -- & --- & $\cdots$ & -- & --- & -- & $\ldots$ \\
\hline
\end{tabular}

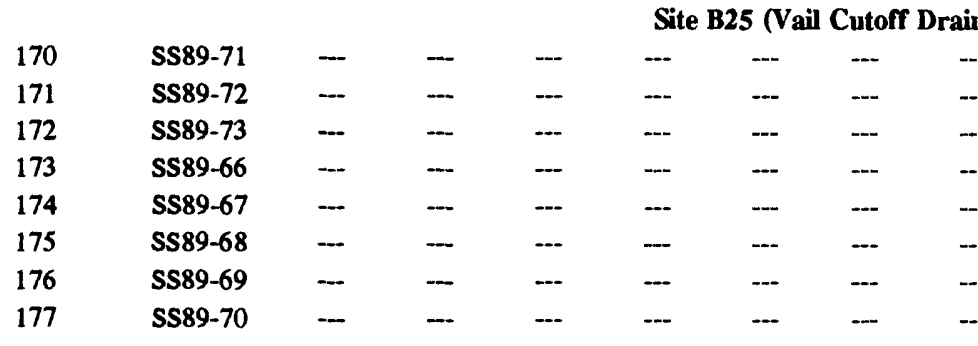


Table 23. Organic chemical analysis, moisture content, and lipid content for biotic samples collected during 1986-90 from the Salton Sea and associated drainwaters and rivers--Continued

\begin{tabular}{|c|c|c|c|c|c|c|}
\hline $\begin{array}{l}\text { Record } \\
\text { number }\end{array}$ & $\begin{array}{l}\text { Sample } \\
\text { number }\end{array}$ & $\begin{array}{l}\text { PCB } \\
1248\end{array}$ & $\begin{array}{l}\text { PCB } \\
1254\end{array}$ & $\begin{array}{l}\text { PCB } \\
1260\end{array}$ & $\begin{array}{l}\text { Total } \\
\text { PCB }\end{array}$ & Toxaphene \\
\hline \multicolumn{7}{|c|}{ Site B18 (Alamo River at Garst Road) } \\
\hline 138 & SS89-64 & -- & - & - & $<0.05$ & $<0.05$ \\
\hline 139 & SS89-120 & -- & -- & -- & $<.05$ & $<.05$ \\
\hline 140 & SS89-165 & -- & -- & -- & $<.05$ & $<.05$ \\
\hline 141 & SS89-167 & - & - & -- & $<.05$ & $<.05$ \\
\hline 142 & SS89-175 & -- & -- & -- & $<.05$ & $<.05$ \\
\hline 143 & SS89-176 & -- & -- & - & $<.05$ & $<.05$ \\
\hline \multicolumn{7}{|c|}{ Site B19 (San Felipe Creek) } \\
\hline 144 & SS90S1 & - & $<0.049$ & - & -- & -- \\
\hline \multicolumn{7}{|c|}{ Site B20 (Salt Creek) } \\
\hline 145 & SS90S4 & - & $<0.049$ & - & -- & -- \\
\hline \multicolumn{7}{|c|}{ Site B21 (Colorado River at Palo Verde) } \\
\hline 146 & SS89-117 & -- & -- & -- & $<0.05$ & $<0.05$ \\
\hline 147 & SS89-118 & - & - & -- & $<.05$ & $<.05$ \\
\hline 148 & SS89-119 & -- & - & - & $<.05$ & $<.05$ \\
\hline \multicolumn{7}{|c|}{ Site B22 (Trifolium 5 Drain) } \\
\hline 149 & LNSS86-59B & -- & $<0.099$ & $<0.099$ & -- & $<0.49$ \\
\hline \multicolumn{7}{|c|}{ Site B23 (Trifolium 13 Drain) } \\
\hline 150 & SS89-62 & -- & - & - & $<0.05$ & $<0.05$ \\
\hline 151 & SS89-168 & $\cdots$ & - & -- & $<.05$ & $<.05$ \\
\hline \multicolumn{7}{|c|}{ Site B24 (Trifolium 14 Drain) } \\
\hline 152 & LNSS88-95 & -- & - & - & - & $<0.50$ \\
\hline 153 & LNSS88-94 & -- & - & -- & - & $<.50$ \\
\hline 154 & LNSS88-89 & - & - & - & -- & $<.50$ \\
\hline 155 & LNSS88-90 & - & - & -- & -- & $<.50$ \\
\hline 156 & $88-192$ & -- & - & $\cdots$ & $<0.05$ & $<.05$ \\
\hline 157 & $88-204$ & - & -- & -- & $<.05$ & $<.05$ \\
\hline 158 & $88-207$ & - & -- & -- & $<.05$ & $<.05$ \\
\hline 159 & $88-210$ & -- & -- & -- & $<.05$ & $<.05$ \\
\hline 160 & $88-213$ & -- & -- & - & $<.05$ & $<.05$ \\
\hline 161 & $88-216$ & - & -- & -- & $<.05$ & $<.05$ \\
\hline 162 & $88-225$ & -- & -- & $\ldots$ & $<.05$ & $<.05$ \\
\hline 163 & $88-228$ & - & - & -- & $<.05$ & $<.05$ \\
\hline 164 & $88-231$ & - & -- & --- & $<.05$ & $<.05$ \\
\hline 165 & $88-232$ & - & $\ldots$ & $\ldots$ & $<.05$ & $<.05$ \\
\hline 166 & SS89-166 & - & $\cdots$ & -- & $<.05$ & $<.05$ \\
\hline 167 & SS89-122 & -- & -- & -- & $<.05$ & $<.05$ \\
\hline 168 & SS90C1 & - & $<0.25$ & -- & $\cdots$ & $<2.5$ \\
\hline 169 & SS90C2 & - & $<.25$ & -- & - & $<2.5$ \\
\hline \multicolumn{7}{|c|}{ Site B25 (Vail Cutoff Drain) } \\
\hline 170 & SS89-71 & -- & -- & -- & $<0.05$ & $<0.05$ \\
\hline 171 & SS89-72 & - & - & $\cdots$ & $<.05$ & $<.05$ \\
\hline 172 & SS89-73 & - & -- & - & $<.05$ & $<.05$ \\
\hline 173 & SS89-66 & - & - & -- & $<.05$ & $<.05$ \\
\hline 174 & SS89-67 & $\ldots$ & --- & -- & $<.05$ & $<.05$ \\
\hline 175 & SS89-68 & -- & - & -- & $<.05$ & $<.05$ \\
\hline 176 & SS89-69 & - & -- & $\cdots$ & $<.05$ & $<.05$ \\
\hline 177 & SS89-70 & -- & -- & -- & $<.05$ & $<.05$ \\
\hline
\end{tabular}


Table 23. Organic chemical analysis, moisture content, and lipid content for biotic samples collected during 1986-90 from the Salton Sea and associated drainwaters and rivers--Continued

\begin{tabular}{|c|c|c|c|c|c|c|c|c|}
\hline $\begin{array}{l}\text { Recond } \\
\text { number }\end{array}$ & $\begin{array}{l}\text { Sample } \\
\text { number }\end{array}$ & Species & Matrix & $\begin{array}{l}\text { Percent } \\
\text { moisture }\end{array}$ & $\begin{array}{l}\text { Percent } \\
\text { lipid }\end{array}$ & $\begin{array}{l}\text { Oxychlor- } \\
\text { dane }\end{array}$ & $\begin{array}{c}\text { alpha- } \\
\text { Chlordane }\end{array}$ & $\begin{array}{c}\text { cis- } \\
\text { Chlordane }\end{array}$ \\
\hline \multicolumn{9}{|c|}{ Site B26 (Vail 4 Drain) } \\
\hline 178 & LNSS88-31 & Ruddy duck & Breast muscle & 74.4 & 2.17 & $<0.01$ & $<0.01$ & --- \\
\hline 179 & SS89-159 & Spiny softshell turtle & Fat & 25.0 & 74.8 & .64 & .13 & -- \\
\hline 180 & SS89-161 & Spiny softshell turtle & Fat & 26.0 & 74.5 & .05 & .13 & -- \\
\hline 181 & SS89-163 & Spiny softshell turtle & Fat & 35.8 & 56.8 & .05 & .14 & -.- \\
\hline 182 & Ss90C3 & Asiatic river clam & Soft tissue & 82.7 & 3.85 & $<.049$ & $<.049$ & - \\
\hline \multicolumn{9}{|c|}{ Site B27 (Vail 4A Drain) } \\
\hline 183 & SS89-63 & Pileworms & Composite & 84.0 & 1.02 & $<0.01$ & $<0.01$ & $\cdots$ \\
\hline 184 & SS89-65 & Pileworms & Composite & 85.0 & 1.35 & $<.01$ & $<.01$ & -- \\
\hline \multicolumn{9}{|c|}{ Site B28 (Vail Drain at New River) } \\
\hline 185 & LNSS86-06B & Asiatic river clam & Soft tissue & 93.2 & 0.25 & $<0.010$ & --- & $<0.010$ \\
\hline 186 & LNSS86-07B & Asiatic river clam & Soft tissue & 93.8 & .28 & $<.0099$ & -- & $<.0099$ \\
\hline 187 & LNSS86-08B & Asiatic river clam & Soft tissue & 93.1 & .41 & $<.0099$ & --- & $<.0099$ \\
\hline \multicolumn{9}{|c|}{ Site B29 (S Lateral Drain) } \\
\hline 188 & SS89-123 & Black-necked stilt & Carcass & 59.7 & 9.00 & $<0.01$ & $<0.01$ & -- \\
\hline 189 & SS89-124 & Black-necked stilt & Carcass & 67.6 & 7.95 & .03 & $<.01$ & -- \\
\hline 190 & SS89-125 & Black-necked stilt & Carcass & 66.5 & 14.5 & .01 & $<.01$ & -- \\
\hline 191 & SS89-126 & Black-necked stilt & Carcass & 67.4 & 16.0 & $<.01$ & $<.01$ & --- \\
\hline 192 & SS89-127 & Black-necked stilt & Carcass & 62.6 & 12.9 & .03 & $<.01$ & -- \\
\hline \multicolumn{9}{|c|}{ Site B30 (Z Lateral Drain) } \\
\hline 193 & SS90S5 & Sediment & Sediment & 66.2 & -- & $<0.0095$ & $<0.0095$ & $\cdots$ \\
\hline 194 & SS90C5 & Asiatic river clam & Soft tissue & 70.6 & 4.00 & $<.049$ & $<.049$ & -- \\
\hline \multicolumn{9}{|c|}{ Site B31 (81st Street Drain) } \\
\hline 195 & Ss90S2 & Sediment & Sediment & 63.6 & -- & $<0.009$ & $<0.009$ & -- \\
\hline \multicolumn{9}{|c|}{ Site B32 (Johnson Street Drain) } \\
\hline 196 & ss90s3 & Sediment & Sediment & 38.1 & -- & $<0.0098$ & $<0.0098$ & $\cdots$ \\
\hline 197 & SS90C4 & Asiatic river clam & Soft tissue & 76.5 & 2.68 & $<.019$ & $<.019$ & -- \\
\hline \multicolumn{9}{|c|}{ Site B33 (Shady Acres Duck Club) } \\
\hline 198 & LNSS88-59 & Northern shoveler & Breast muscle & 75.2 & 2.68 & $<0.01$ & $<0.01$ &.- \\
\hline \multicolumn{9}{|c|}{ Site B34 (RH Pond) } \\
\hline 199 & 88-98 & Black-necked stilt & Egg & 70.5 & 16.0 & 0.04 & $<0.01$ &.- \\
\hline 200 & $88-101$ & Black-necked stilt & Egg & 69.0 & 15.5 & .04 & $<.01$ & -- \\
\hline 201 & 88-104 & Black-necked stilt & Egg & 71.5 & 16.8 & .04 & $<.01$ & --- \\
\hline 202 & $88-107$ & Black-necked stilt & Egg & 70.5 & 13.1 & .01 & $<.01$ & $\cdots$ \\
\hline 203 & $88-110$ & Black-necked stilt & Egg & 71.0 & 15.6 & .01 & $<.01$ & $\ldots$ \\
\hline 204 & $88-116$ & Black-necked stilt & Egg & 71.0 & 15.1 & .01 & $<.01$ & $\ldots$ \\
\hline 205 & $88-119$ & Black-necked stilt & Egg & 64.5 & 16.7 & .01 & $<.01$ & -- \\
\hline 206 & $88-128$ & Black-necked stilt & Egg & 73.0 & 15.3 & .01 & $<.01$ & -- \\
\hline 207 & $88-131$ & Black-necked stilt & Egg & 72.0 & 15.7 & .02 & $<.01$ & -- \\
\hline \multicolumn{9}{|c|}{ Site B35 (HQ Pond) } \\
\hline 208 & LNSS88-33 & Ruddy duck & Breast muscle & 72.8 & 2.16 & $<0.01$ & $<0.01$ & $\ldots$ \\
\hline 209 & LNSS88-37 & Ruddy duck & Breast muscle & 73.6 & 4.82 & $<.01$ & $<.01$ & -- \\
\hline
\end{tabular}


Table 23. Organic chemical analysis, moisture content, and lipid content for biotic samples collected during 1986-90 from the Salton Sea and associated drainwaters and rivers--Continued

\begin{tabular}{|c|c|c|c|c|c|c|c|c|c|}
\hline $\begin{array}{l}\text { Recond } \\
\text { number }\end{array}$ & $\begin{array}{l}\text { Sample } \\
\text { number }\end{array}$ & $\begin{array}{l}\text { gamma- } \\
\text { Chlordane }\end{array}$ & $\begin{array}{c}\text { trans- } \\
\text { Chlordane }\end{array}$ & $\begin{array}{l}\text { cis-Nona- } \\
\text { chlor }\end{array}$ & $\begin{array}{c}\text { trans- } \\
\text { Nonachlor }\end{array}$ & $\begin{array}{l}\text { Hepta- } \\
\text { chlor }\end{array}$ & $\begin{array}{l}\text { Heptachlor } \\
\text { epoxide }\end{array}$ & $\begin{array}{l}\text { Methyoxy- } \\
\text { chlor }\end{array}$ & $o, p^{\prime}-D D E$ \\
\hline \multicolumn{10}{|c|}{ Site B26 (Vail 4 Drain) } \\
\hline 178 & LNSS88-31 & $<0.01$ & - & $<0.01$ & $<0.01$ & - & $<0.01$ & -- & $<0.01$ \\
\hline 179 & SS89-159 & $<.01$ & -- & .05 & .16 & - & .02 & - & $<.01$ \\
\hline 180 & SS89-161 & .02 & -- & .02 & .14 & - & .03 & -- & $<.01$ \\
\hline 181 & SS89-163 & .02 & - & .03 & .14 & - & .03 & -- & $<.01$ \\
\hline 182 & SS90C3 & - & $<0.049$ & .05 & $<.049$ & - & $<.049$ & -- & $<.01$ \\
\hline \multicolumn{10}{|c|}{ Site B27 (Vail 4A Drain) } \\
\hline 183 & SS89-63 & $<0.01$ & -- & $<0.01$ & $<0.01$ & -- & $<0.01$ & -- & $<0.01$ \\
\hline 184 & SS89-65 & $<.01$ & - & $<.01$ & $<.01$ & - & $<.01$ & -- & $<.01$ \\
\hline \multicolumn{10}{|c|}{ Site B28 (Vail Drain at New River) } \\
\hline 185 & LNSS86-06B & - & $<0.010$ & $<0.010$ & $<0.010$ & -- & $<0.010$ & -- & -- \\
\hline 186 & LNSS86-07B & - & $<.0099$ & $<.0099$ & $<.0099$ & - & $<.0099$ & --- & -- \\
\hline 187 & LNSS86-08B & -- & $<.0099$ & $<.0099$ & $<.0099$ & -- & $<.0099$ & -- & -- \\
\hline \multicolumn{10}{|c|}{ Site B29 (S Lateral Drain) } \\
\hline 188 & SS89-123 & $<0.01$ & -- & $<0.01$ & $<0.01$ & - & $<0.01$ & -- & $<0.01$ \\
\hline 189 & SS89-124 & $<.01$ & - & $<.01$ & $<.01$ & -- & $<.01$ & -- & $<.01$ \\
\hline 190 & SS89-125 & $<.01$ & - & $<.01$ & $<.01$ & $\rightarrow$ & $<.01$ & -- & $<.01$ \\
\hline 191 & SS89-126 & $<.01$ & -- & $<.01$ & $<.01$ &.-- & $<.01$ & -- & $<.01$ \\
\hline 192 & SS89-127 & $<.01$ & -- & $<.01$ & $<.01$ & -- & $<.01$ & $\cdots$ & $<.01$ \\
\hline \multicolumn{10}{|c|}{ Site B30 (Z Lateral Drain) } \\
\hline 193 & SS90S5 & -- & $<0.0095$ & $<0.0095$ & $<0.0095$ & -- & $<0.0095$ & -- & $<0.01$ \\
\hline 194 & SS90C5 & - & $<.049$ & $<.049$ & $<.049$ & - & $<.049$ & -- & $<.01$ \\
\hline \multicolumn{10}{|c|}{ Site B31 (81st Street Drain) } \\
\hline 195 & SS90S2 & - & $<0.009$ & $<0.009$ & $<0.009$ & -- & $<0.009$ & -- & $<0.01$ \\
\hline \multicolumn{10}{|c|}{ Site B32 (Johnson Street Drain) } \\
\hline 196 & SS90S3 & -- & $<0.0098$ & $<0.0098$ & $<0.0098$ & -- & $<0.0098$ & --- & $<0.01$ \\
\hline 197 & SS90C4 & - & $<.019$ & $<.019$ & $<.019$ & -- & $<.019$ & --- & $<.01$ \\
\hline \multicolumn{10}{|c|}{ Site B33 (Shady Acres Duck Club) } \\
\hline 198 & LNSS88-59 & $<0.01$ & -- & $<0.01$ & $<0.01$ & -- & $<0.01$ & - & $<0.01$ \\
\hline \multicolumn{10}{|c|}{ Site B34 (RH Pond) } \\
\hline 199 & 88-98 & $<0.01$ & -- & $<0.01$ & 0.01 & - & 0.01 &.- & $<0.01$ \\
\hline 200 & $88-101$ & $<.01$ & $\cdots$ & $<.01$ & .01 & -- & .02 & -- & $<.01$ \\
\hline 201 & 88-104 & $<.01$ & -- & $<.01$ & .01 & -- & .02 & --- & $<.01$ \\
\hline 202 & 88-107 & $<.01$ & -- & $<.01$ & .01 & -- & .01 & -- & $<.01$ \\
\hline 203 & $88-110$ & $<.01$ & -- & $<.01$ & $<.01$ & -- & $<.01$ & --- & $<.01$ \\
\hline 204 & $88-116$ & $<.01$ & -- & $<.01$ & .01 & -- & $<.01$ & -- & $<.01$ \\
\hline 205 & $88-119$ & $<.01$ & - & $<.01$ & .01 & -- & $<.01$ & -- & $<.01$ \\
\hline 206 & $88-128$ & $<.01$ & --- & $<.01$ & .01 & -- & $<.01$ & - & $<.01$ \\
\hline 207 & 88-131 & $<.01$ & -- & $<.01$ & $<.01$ & -- & $<.01$ & $\cdots$ & $<.01$ \\
\hline \multicolumn{10}{|c|}{ Site B35 (HQ Pond) } \\
\hline 208 & LNSS88-33 & $<0.01$ & -- & $<0.01$ & $<0.01$ & -- & $<0.01$ & -- & $<0.01$ \\
\hline 209 & LNSS88-37 & $<.01$ & -- & $<.01$ & $<.01$ & - & $<.01$ & -- & $<.01$ \\
\hline
\end{tabular}


Table 23. Organic chemical analysis, moisture content, and lipid content for biotic samples collected during 1986-90 from the Salton Sea and associated drainwaters and rivers--Continued

\begin{tabular}{|c|c|c|c|c|c|c|c|c|c|c|}
\hline $\begin{array}{l}\text { Record } \\
\text { number }\end{array}$ & $\begin{array}{l}\text { Sample } \\
\text { number }\end{array}$ & p,p'-DDE & $o, p^{\prime}-D D D$ & p,p'-DDD & $o, p^{\prime}$-DDT & $\mathrm{p}, \mathrm{p}^{\prime}-\mathrm{DDT}$ & $\begin{array}{l}\text { Total } \\
\text { DDT }\end{array}$ & Endrin & Dieldrin & Aldrin \\
\hline \multicolumn{11}{|c|}{ Site B26 (Vail 4 Drain) } \\
\hline 178 & LNSS88-31 & 0.19 & $<0.01$ & 0.01 & $<0.01$ & $<0.01$ & 0.200 & $<0.01$ & $<0.01$ & -- \\
\hline 179 & SS89-159 & 12 & $<.01$ & .16 & $<.01$ & $<.01$ & 12.160 & $<.01$ & .43 & -- \\
\hline 180 & SS89-161 & 17 & $<.01$ & .45 & $<.01$ & $<.01$ & 17.450 & $<.01$ & .71 & -- \\
\hline 181 & SS89-163 & 14 & $<.01$ & .25 & $<.01$ & $<.01$ & 14.250 & $<.01$ & .85 & -- \\
\hline 182 & SS90C3 & 5.5 & $<.01$ & .12 & $<.01$ & .12 & 5.740 & $<.049$ & .12 & -- \\
\hline \multicolumn{11}{|c|}{ Site B27 (Vail 4A Drain) } \\
\hline 183 & SS89-63 & 0.04 & $<0.01$ & $<0.01$ & $<0.01$ & $<0.01$ & 0.040 & $<0.01$ & $<0.01$ & -- \\
\hline 184 & SS89-65 & .03 & $<.01$ & $<.01$ & $<.01$ & $<.01$ & .030 & $<.01$ & $<.01$ & -- \\
\hline \multicolumn{11}{|c|}{ Site B28 (Vail Drain at New River) } \\
\hline 185 & LNSS86-06B & 0.13 & - & $<0.010$ & $\cdots$ & $<0.010$ & 0.130 & $<0.010$ & $<0.010$ & -- \\
\hline 186 & LNSS86-07B & .16 & - & $<.0099$ & -- & $<.0099$ & .160 & $<.0099$ & $<.0099$ & -- \\
\hline 187 & LNSS86-08B & .24 & - & $<.0099$ & -- & $<.0099$ & .240 & $<.0099$ & $<.0099$ & --- \\
\hline \multicolumn{11}{|c|}{ Site B29 (S Lateral Drain) } \\
\hline 188 & SS89-123 & 0.75 & $<0.01$ & $<0.01$ & $<0.01$ & $<0.01$ & 0.750 & $<0.01$ & 0.01 & -- \\
\hline 189 & SS89-124 & 3.1 & $<.01$ & $<.01$ & $<.01$ & $<.01$ & 3.100 & $<.01$ & .09 & $\ldots$ \\
\hline 190 & SS89-125 & 1.2 & $<.01$ & $<.01$ & $<.01$ & $<.01$ & 1.200 & $<.01$ & .02 & --- \\
\hline 191 & SS89-126 & 2.7 & $<.01$ & $<.01$ & $<.01$ & $<.01$ & 2.700 & $<.01$ & .11 & --- \\
\hline 192 & SS89-127 & 5.8 & $<.01$ & $<.01$ & $<.01$ & $<.01$ & 5.800 & $<.01$ & .15 & -- \\
\hline \multicolumn{11}{|c|}{ Site B30 (Z Lateral Drain) } \\
\hline 193 & SS90s5 & 0.10 & $<0.01$ & $<0.0095$ & $<0.01$ & $<0.0095$ & -- & $<0.0095$ & $<0.0095$ & -- \\
\hline 194 & SS90C5 & $<.049$ & $<.01$ & $<.049$ & $<.01$ & $<.049$ & 0.000 & $<.049$ & $<.049$ & -- \\
\hline \multicolumn{11}{|c|}{ Site B31 (81st Street Drain) } \\
\hline 195 & SS90S2 & 0.066 & $<0.01$ & $<0.009$ & $<0.01$ & $<0.009$ & - & $<0.009$ & $<0.009$ & -- \\
\hline \multicolumn{11}{|c|}{ Site B32 (Johnson Street Drain) } \\
\hline 196 & ss90s3 & 0.023 & $<0.01$ & $<0.0098$ & $<0.01$ & $<0.0098$ & - & $<0.0098$ & $<0.0098$ & --- \\
\hline 197 & SS90C4 & .16 & $<.01$ & .025 & $<.01$ & $<.019$ & 0.185 & $<.019$ & .070 & --- \\
\hline \multicolumn{11}{|c|}{ Site B33 (Shady Acres Duck Club) } \\
\hline 198 & LNSS88-59 & 0.57 & $<0.01$ & 0.01 & $<0.01$ & 0.01 & 0.590 & $<0.01$ & 0.01 & -- \\
\hline \multicolumn{11}{|c|}{ Site B34 (RH Pond) } \\
\hline 199 & $88-98$ & 7.4 & $<.01$ & 0.09 & $<0.01$ & $<0.01$ & 7.490 & $<0.01$ & 0.10 & --- \\
\hline 200 & $88-101$ & 7.7 & $<.01$ & $<.01$ & $<.01$ & .08 & 7.780 & $<.01$ & .11 & --- \\
\hline 201 & $88-104$ & 7.7 & $<.01$ & $<.01$ & $<.01$ & .08 & 7.780 & $<.01$ & .11 & $-\cdots$ \\
\hline 202 & $88-107$ & 6.9 & $<.01$ & .02 & $<.01$ & .03 & 6.950 & $<.01$ & .06 & -- \\
\hline 203 & $88-110$ & 4.7 & $<.01$ & .01 & $<.01$ & .16 & 4.870 & $<.01$ & .03 & -- \\
\hline 204 & $88-116$ & 4.9 & $<.01$ & .01 & $<.01$ & .02 & 4.930 & $<.01$ & .07 & --- \\
\hline 205 & $88-119$ & 5.4 & $<.01$ & .01 & $<.01$ & .03 & 5.440 & $<.01$ & .08 & $\cdots$ \\
\hline 206 & $88-128$ & 4.5 & $<.01$ & .01 & $<.01$ & .02 & 4.530 & $<.01$ & .09 & --- \\
\hline 207 & $88-131$ & 8.9 & $<.01$ & .01 & $<.01$ & .02 & 8.930 & $<.01$ & .06 & $\cdots$ \\
\hline \multicolumn{11}{|c|}{ Site B35 (HQ Pond) } \\
\hline 208 & LNSS88-33 & 0.56 & $<0.01$ & $<0.01$ & $<0.01$ & 0.01 & 0.570 & $<0.01$ & 0.01 & --- \\
\hline 209 & LNSS88-37 & .64 & $<.01$ & .01 & $<.01$ & .01 & .660 & $<.01$ & .01 & --- \\
\hline
\end{tabular}


Table 23. Organic chemical analysis, moisture content, and lipid content for biotic samples collected during 1986-90 from the Salton Sea and associated drainwaters and rivers--Continued

\begin{tabular}{|c|c|c|c|c|c|c|c|c|c|c|c|c|c|}
\hline $\begin{array}{l}\text { Record } \\
\text { number }\end{array}$ & $\begin{array}{l}\text { Sample } \\
\text { number }\end{array}$ & $\begin{array}{l}\text { alpha- } \\
\text { BHC }\end{array}$ & $\begin{array}{l}\text { beta- } \\
\text { BHC }\end{array}$ & $\begin{array}{l}\text { delta- } \\
\text { BHC }\end{array}$ & $\begin{array}{c}\text { gamma- } \\
\text { BHC }\end{array}$ & $\begin{array}{l}\text { Lin- } \\
\text { dane }\end{array}$ & $\begin{array}{c}\text { Hexa- } \\
\text { chloro- } \\
\text { ben- } \\
\text { zene }\end{array}$ & $\begin{array}{l}\text { Endo- } \\
\text { sul- } \\
\text { fan I }\end{array}$ & $\begin{array}{l}\text { Endo- } \\
\text { sul- } \\
\text { fan II }\end{array}$ & $\begin{array}{l}\text { Endo- } \\
\text { sulfan } \\
\text { sulfate }\end{array}$ & Mirex & DCPA & Dicofol \\
\hline \multicolumn{14}{|c|}{ Site B26 (Vail 4 Drain) } \\
\hline 178 & LNSS88-31 & $<0.01$ & $<0.01$ & $<0.01$ & $<0.01$ & -- & $<0.01$ & - & -- & - & $<0.01$ & -- & -- \\
\hline 179 & SS89-159 & $<.01$ & .02 & $<.01$ & $<.01$ & - & .12 & $<0.01$ & $<0.01$ & - & $<.01$ & -- & --- \\
\hline 180 & SS89-161 & $<.01$ & .01 & $<.01$ & $<.01$ & -- & .01 & $<.01$ & $<.01$ & - & $<.01$ & -- & --- \\
\hline 181 & SS89-163 & $<.01$ & .01 & $<.01$ & $<.01$ & -- & .02 & $<.01$ & $<.01$ & - & $<.01$ & -- & -- \\
\hline 182 & ss90C3 & -- & -- & -- & - & -- & --- & $\cdots$ & - & -- & -- & - & --- \\
\hline \multicolumn{14}{|c|}{ Site B27 (Vail 4A Drain) } \\
\hline 183 & SS89-63 & $<0.01$ & $<0.01$ & $<0.01$ & $<0.01$ & - & $<0.01$ & - & - & - & $<0.01$ & - & $\ldots$ \\
\hline 184 & SS89-65 & $<.01$ & $<.01$ & $<.01$ & $<.01$ & -- & $<.01$ & -- & -- & -- & $<.01$ & - & -- \\
\hline
\end{tabular}

Site B28 (Vail Drain at New River)

\begin{tabular}{|c|c|c|c|c|c|c|c|c|c|c|c|c|}
\hline 185 & LNSS86-06B & --- & - & $\cdots$ & -- & $\cdots$ & -- & -- & -- & $\cdots$ & -- & -- \\
\hline 186 & LNSS86-07B & -- & - & - & $\ldots$ & - & $\cdots$ & -- & $\ldots$ & - & -- & -- \\
\hline 187 & LNSS86-08B & --. & - & -- & $\ldots$ & -- & -- & -- & -- & -- & -- & --. \\
\hline
\end{tabular}

\begin{tabular}{|c|c|c|c|c|c|c|c|c|c|c|c|c|c|}
\hline \multirow[b]{2}{*}{188} & \multicolumn{13}{|c|}{ Site B29 (S Lateral Drain) } \\
\hline & SS89-123 & $<0.01$ & $<0.01$ & $<0.01$ & $<0.01$ & -- & $<0.01$ & $<0.01$ & $<0.01$ & -- & $<0.01$ & --- & --- \\
\hline 189 & SS89-124 & $<.01$ & $<.01$ & $<.01$ & $<.01$ & -- & $<.01$ & $<.01$ & $<.01$ & - & $<.01$ & - & -- \\
\hline 190 & SS89-125 & $<.01$ & $<.01$ & $<.01$ & $<.01$ & -- & .01 & $<.01$ & $<.01$ & - & $<.01$ & -- & -- \\
\hline 191 & SS89-126 & $<.01$ & $<.01$ & $<.01$ & $<.01$ & --- & $<.01$ & $<.01$ & $<.01$ & -- & $<.01$ & $\ldots$ & --- \\
\hline 192 & SS89-127 & $<.01$ & .13 & $<.01$ & $<.01$ & --. & .02 & $<.01$ & $<.01$ & - & $<.01$ & $\ldots$ & \\
\hline
\end{tabular}

Site B30 ( $Z$ Lateral Drain)

\begin{tabular}{|c|c|c|c|c|c|c|c|c|c|c|c|c|c|}
\hline \multirow[b]{2}{*}{193} & \\
\hline & SS90S5 & - & -- & -- & -- & -- & - & -- & - & $\cdots$ & --- & --- & -- \\
\hline 194 & SS90C5 & -- & $\cdots$ & $\cdots$ & $\ldots$ & -- & - & $\cdots$ & $\cdots$ & -- & --- & --- & -- \\
\hline \multicolumn{14}{|c|}{ Site B31 (81st Street Drain) } \\
\hline 195 & SS90S2 & -- & -- & -- & $\cdots$ & --- & -- & --- & -- & -- & -- & -- & - \\
\hline \multicolumn{14}{|c|}{ Site B32 (Johnson Street Drain) } \\
\hline 196 & SS90S3 & - & -- & -- & $\ldots$ & -- & --- & $\cdots$ & --- & $\cdots$ & --- & --- & -- \\
\hline 197 & SS90C4 & -- & $\cdots$ & $\cdots$ & -- & $\cdots$ & --- & --- & -- & --- & -- & --- & -- \\
\hline \multicolumn{14}{|c|}{ Site B33 (Shady Acres Duck Club) } \\
\hline 198 & LNSS88-59 & $<0.01$ & $<0.01$ & $<0.01$ & $<0.01$ & -- & 0.01 & -- & -- & $\cdots$ & $<0.01$ & -- & -- \\
\hline \multicolumn{14}{|c|}{ Site B34 (RH Pond) } \\
\hline 199 & $88-98$ & $<0.01$ & 0.25 & $<0.01$ & $<0.01$ & -- & 0.01 & -- & --- & $\cdots$ & $<0.01$ & --- & -- \\
\hline 200 & 88-101 & $<.01$ & .14 & $<.01$ & $<.01$ & $-\cdots$ & .01 & --- & $-\cdots$ & --- & $<.01$ &.-- & -- \\
\hline 201 & 88-104 & $<.01$ & .15 & $<.01$ & $<.01$ & -- & .01 & --- & $\cdots$ & -- & $<.01$ & $\cdots$ & -- \\
\hline 202 & $88-107$ & $<.01$ & .01 & $<.01$ & $<.01$ & -- & .01 & --- & -- & --- & $<.01$ & --- & -- \\
\hline 203 & $88-110$ & $<.01$ & .05 & $<.01$ & $<.01$ & -- & .01 & --- & -- & $-\cdots$ & $<.01$ & --- & $\cdots$ \\
\hline 204 & $88-116$ & $<.01$ & .03 & $<.01$ & $<.01$ & - & .01 & -- & --- & -- & $<.01$ & $-\cdots$ & - \\
\hline 205 & $88-119$ & $<.01$ & .04 & $<.01$ & $<.01$ & -- & .01 & -- & $-\ldots$ & -- & $<.01$ & --. & - \\
\hline 206 & $88-128$ & $<.01$ & .01 & $<.01$ & $<.01$ & -- & .01 & -- & -- & $-\cdots$ & $<.01$ & --- & $\cdots$ \\
\hline 207 & $88-131$ & $<.01$ & .02 & $<.01$ & $<.01$ & - & $<.01$ & $\cdots$ & -- & --- & $<.01$ & $\cdots$ & $\cdots$ \\
\hline \multicolumn{14}{|c|}{ Site B35 (HQ Pond) } \\
\hline 208 & LNSS88-33 & $<0.01$ & $<0.01$ & $<0.01$ & $<0.01$ & - & 0.01 & $\cdots$ & -- & $\cdots$ & $<0.01$ & $\cdots$ & - \\
\hline 209 & LNSS88-37 & $<.01$ & $<.01$ & $<.01$ & $<.01$ & -- & .01 & -- & -- & -- & $<.01$ & -- & $\ldots$ \\
\hline
\end{tabular}


Table 23. Organic chemical analysis, moisture content, and lipid content for biotic samples collected during 1986-90 from the Salton Sea and associated drainwaters and rivers--Continued

\begin{tabular}{|c|c|c|c|c|c|c|c|c|c|c|c|c|c|c|}
\hline $\begin{array}{l}\text { Record } \\
\text { number }\end{array}$ & $\begin{array}{l}\text { Sample } \\
\text { number }\end{array}$ & $\begin{array}{l}\text { Tetra- } \\
\text { difon }\end{array}$ & $\begin{array}{c}\text { BI PH } \\
\text { CL-2 }\end{array}$ & $\begin{array}{c}\text { BI PH } \\
\text { CL-3 }\end{array}$ & $\begin{array}{l}\text { BI PF } \\
\text { CL-4 }\end{array}$ & $\begin{array}{c}\text { BI PH } \\
\text { CL-5 }\end{array}$ & $\begin{array}{l}\text { BI PH } \\
\text { CL-6 }\end{array}$ & $\begin{array}{c}\text { BI PH } \\
\text { CL-7 }\end{array}$ & BI PF & BI PH & PC & PC & $\begin{array}{l}\text { PCB } \\
1232\end{array}$ & PCB \\
\hline
\end{tabular}

\begin{tabular}{|c|c|c|c|c|c|c|c|c|c|c|c|c|c|c|}
\hline \multicolumn{15}{|c|}{ Site B26 (Vail 4 Drain) } \\
\hline 178 & LNSS88-31 & - & - & -- & --- & -- & -- & -- & -- & - & -- & -- & --- & --- \\
\hline 179 & SS89-159 & - & - & --- & -- & --- & - & -- & - & -- & -- & -- & --- & -- \\
\hline 180 & SS89-161 & - & - & - & - & - & - & -- & -- & -- & -- & -- & -- & -- \\
\hline 181 & SS89-163 & -- & - & -- & -- & - & -- & -- & -- & -- & -- & $\cdots$ & -- & -- \\
\hline 182 & ss90C3 & -- & - & -- & -- & -- & -- & $\cdots$ & --- & -- & $\cdots$ & - & --- & --- \\
\hline \multicolumn{15}{|c|}{ Site B27 (Vail 4A Drain) } \\
\hline 183 & SS89-63 & - & - & -- & - & -- & -- & - & --- & -- & -- & -- & -- & $\ldots$ \\
\hline 184 & SS89-65 & - & $\cdots$ & -- & -- & $\cdots$ & - & -- & -- & -- & -- & -- & --- & -- \\
\hline \multicolumn{15}{|c|}{ Site B28 (Vail Drain at New River) } \\
\hline 185 & LNSS86-06B & -- & - & --- & --- & -- & $\ldots$ & $\cdots$ & --- & $\cdots$ & --- & -- & --- & -- \\
\hline 186 & LNSS86-07B & - & - & - & - & -- & $\ldots$ & -- & -- & -- & --- & -- & --- & $\cdots$ \\
\hline 187 & LNSS86-08B & -- & - & -- & -- & -- & - & --- & -- & -- & --- & -- & $\cdots$ & --- \\
\hline \multicolumn{15}{|c|}{ Site B29 (S Lateral Drain) } \\
\hline 188 & SS89-123 & -- & -- & --- & -- & -- & -- & -- & -- & -- & --- & -- & --- & - \\
\hline 189 & SS89-124 & -- & $\cdots$ & - & - & -- & -- & - & -- & -- & --- & -- & --- & -- \\
\hline 190 & SS89-125 & $\ldots$ & $\cdots$ & $-\cdots$ & -- & $\ldots$ & --- & $\cdots$ & -- & --- & --- & --- & - & -- \\
\hline 191 & SS89-126 & - & - & $\cdots$ & -- & - & $\cdots$ & $\ldots$ & -- & -- & -- & --- & - & -- \\
\hline 192 & SS89-127 & - & -- & -- & - & -- & - & $\cdots$ & -- & -- & --- & --- & --- & --- \\
\hline \multicolumn{15}{|c|}{ Site B30 (Z Lateral Drain) } \\
\hline 193 & SS90S5 & - & -- & -- & $\cdots$ & -- & -- & $\cdots$ & -- & -- & -- & -- & -- & -- \\
\hline 194 & SS90C5 & -- & -- & - & -- & $\cdots$ & -- & -- & $\cdots$ & -- & - & --- & --- & -- \\
\hline \multicolumn{15}{|c|}{ Site B31 (81st Street Drain) } \\
\hline 195 & SS90S2 & -- & -- & -- & - & -- & -- & -- & - & $\cdots$ & $\cdots$ & --- & --- & -- \\
\hline \multicolumn{15}{|c|}{ Site B32 (Johnson Street Drain) } \\
\hline 196 & S\$90S3 & -- & -- & - & -- & - & -- & -- & --- & --- & --- & -- & -- & -- \\
\hline 197 & SS90C4 & -- & -- & -- & $\cdots$ & $\cdots$ & -- & $-\cdots$ & -- & --- & -- & --- & -- & -- \\
\hline \multicolumn{15}{|c|}{ Site B33 (Shady Acres Duck Club) } \\
\hline 198 & LNSS88-59 & - & - & $\cdots$ & $\cdots$ & $\cdots$ & -- & $\cdots$ & --- & --- & --- & -- & -- & -- \\
\hline \multicolumn{15}{|c|}{ Site B34 (RH Pond) } \\
\hline 199 & $88-98$ & -- & -- & -- & $\ldots$ & -- & -- & --- & -- & -- & --- & --- & -- & -- \\
\hline 200 & $88-101$ & -- & --- & -- & --- & $\cdots$ & -- & -- & -- & -- & -- & -- & --- & -- \\
\hline 201 & $88-104$ & -- & - & -- & $\cdots$ & --- & -- & $-\cdots$ & -- & - & --- & --- & --- & -- \\
\hline 202 & $88-107$ & - & -- & -- & -- & - & -- & - & --- & --- & --- & --- & $\cdots$ & -- \\
\hline 203 & $88-110$ & - & -- & - & $-\cdots$ & --- & -- & -- & -- & -- & --- & --- & --- & $\cdots$ \\
\hline 204 & $88-116$ & - & - & --- & -- & - & -- & $-\cdots$ & $\cdots$ & - & --- & --- & --. & - \\
\hline 205 & $88-119$ & -- & -- & -- & -- & - & -- & $\cdots$ & -.- & -- & --- & -- & --- & - \\
\hline 206 & $88-128$ & $\ldots$ & --- & --- & - & - & - & - & -- & -.- &.-- & $\ldots$ & $\ldots$ & -- \\
\hline 207 & $88-131$ & - & -- & --- & -- & --- & --- & --- & -- & -.- & --. & --- & --- & -- \\
\hline
\end{tabular}


Table 23. Organic chemical analysis, moisture content, and lipid content for biotic samples collected during 1986-90 from the Salton Sea and associated drainwaters and rivers--Continued

\begin{tabular}{|c|c|c|c|c|c|c|}
\hline $\begin{array}{l}\text { Record } \\
\text { number }\end{array}$ & $\begin{array}{l}\text { Sample } \\
\text { number }\end{array}$ & $\begin{array}{l}\text { PCB } \\
1248\end{array}$ & $\begin{array}{l}\text { PCB } \\
1254\end{array}$ & $\begin{array}{l}\text { PCB } \\
1260\end{array}$ & $\begin{array}{l}\text { Total } \\
\text { PCB }\end{array}$ & Toxaphene \\
\hline \multicolumn{7}{|c|}{ Site B26 (Vail 4 Drain) } \\
\hline 178 & LNSS88-31 & - & - & -- & $<0.05$ & $<0.05$ \\
\hline 179 & SS89-159 & - & $-\cdots$ & - & $<.05$ & 3.6 \\
\hline 180 & SS89-161 & - & - & - & $<.05$ & 5.6 \\
\hline 181 & SS89-163 & - & - & - & $<.05$ & 4.3 \\
\hline 182 & ss90c3 & - & $<0.25$ & - & - & 7.0 \\
\hline \multicolumn{7}{|c|}{ Site B27 (Vail 4A Drain) } \\
\hline 183 & SS89-63 & - & - & -- & $<0.05$ & $<0.05$ \\
\hline 184 & SS89-65 & - & - & - & $<.05$ & $<.05$ \\
\hline \multicolumn{7}{|c|}{ Site B28 (Vail Drain at New River) } \\
\hline 185 & LNSS86-06B & $\cdots$ & $<0.01$ & $<0.10$ & -- & $<0.50$ \\
\hline 186 & LNSS86-07B & - & $<.099$ & $<.099$ & - & $<.50$ \\
\hline 187 & LNSS86-08B & - & $<.099$ & $<.099$ & - & $<.49$ \\
\hline \multicolumn{7}{|c|}{ Site B29 (S Lateral Drain) } \\
\hline 188 & SS89-123 & - & -- & - & $<0.05$ & $<0.05$ \\
\hline 189 & SS89-124 & -- & - & -- & $<.05$ & $<.05$ \\
\hline 190 & SS89-125 & -- & - & - & $<.05$ & $<.05$ \\
\hline 191 & SS89-126 &.- & -- & -- & $<.05$ & $<.05$ \\
\hline 192 & SS89-127 & - & -- & -- & $<.05$ & $<.05$ \\
\hline \multicolumn{7}{|c|}{ Site B30 (Z Lateral Drain) } \\
\hline 193 & Ss90S5 & - & $<0.047$ & - & -- & -- \\
\hline 194 & SS90C5 & - & $<.25$ & - & - & $<2.5$ \\
\hline \multicolumn{7}{|c|}{ Site B31 (81st Street Drain) } \\
\hline 195 & SS90S2 & -- & $<0.050$ & - & -- & -- \\
\hline \multicolumn{7}{|c|}{ Site B32 (Johnson Street Drain) } \\
\hline 196 & ss90s3 & - & $<0.049$ & - & $\ldots$ & $-\ldots$ \\
\hline 197 & Ss9oC4 & - & $<.01$ & - & - & 1.1 \\
\hline \multicolumn{7}{|c|}{ Site B33 (Shady Acres Duck Club) } \\
\hline 198 & LNSS88-59 & -- & - & - & $<0.05$ & $<0.05$ \\
\hline \multicolumn{7}{|c|}{ Site B34 (RH Pond) } \\
\hline 199 & 88-98 & - & - & - & $<0.05$ & 0.52 \\
\hline 200 & $88-101$ & -- & - & -- & $<.05$ & .70 \\
\hline 201 & 88-104 & - & - & - & $<.05$ & .81 \\
\hline 202 & $88-107$ & -- & $\ldots$ &..- & $<.05$ & .71 \\
\hline 203 & $88-110$ & - & $\ldots$ & $\ldots$ & $<.05$ & .29 \\
\hline 204 & $88-116$ & -- & - & -- & $<.05$ & .45 \\
\hline 205 & $88-119$ & -- & - & - & $<.05$ & .49 \\
\hline 206 & $88-128$ & -- & -- & - & $<.05$ & .64 \\
\hline 207 & $88-131$ & -- & -- & - & $<.05$ & $<.05$ \\
\hline \multicolumn{7}{|c|}{ Site B35 (HQ Pond) } \\
\hline 208 & LNSS88-33 & - & - & - & $<0.05$ & $<0.05$ \\
\hline 209 & LNSS88-37 & $\cdots$ & - & - & $<.05$ & $<.05$ \\
\hline
\end{tabular}


Table 23. Organic chemical analysis, moisture content, and lipid content for biotic samples collected during 1986-90 from the Salton Sea and associated drainwaters and rivers--Continued

\begin{tabular}{|c|c|c|c|c|c|c|c|c|}
\hline $\begin{array}{l}\text { Record } \\
\text { number }\end{array}$ & $\begin{array}{l}\text { Sample } \\
\text { number }\end{array}$ & Species & Matrix & $\begin{array}{l}\text { Percent } \\
\text { moisture }\end{array}$ & $\begin{array}{c}\text { Percent } \\
\text { lipid }\end{array}$ & $\begin{array}{l}\text { Oxychlor- } \\
\text { dane }\end{array}$ & $\begin{array}{l}\text { alpha- } \\
\text { Chlordane }\end{array}$ & $\begin{array}{c}\text { cis- } \\
\text { Chlordane }\end{array}$ \\
\hline \multicolumn{9}{|c|}{ Site B36 (Reidman Pond) } \\
\hline 210 . & . $88-179$ & Black-necked stilt & Egg & 71.0 & 16.3 & $<0.01$ & $<0.01$ & -- \\
\hline 211 & . $88-182$ & Black-necked stilt & Egg & 65.0 & 18.7 & .02 & $<.01$ & -- \\
\hline 212 & . LNSS88-84 & Black-necked stilt & Carcass & 84.0 & 3.83 & $<.05$ & $<.05$ & $-\cdots$ \\
\hline 213 & . $88-219$ & Black-necked stilt & Egg & 74.0 & 11.9 & $<.01$ & $<.01$ & -- \\
\hline 214 & - $88-222$ & Black-necked stilt & Egg & 70.5 & 16.7 & .02 & $<.01$ & -- \\
\hline \multicolumn{9}{|c|}{ Site B37 (Hazard Pond) } \\
\hline 215 & - LNSS86-09B & Mosquitofish & Whole body & 75.1 & 3.68 & $<0.0099$ & --- & $<0.0099$ \\
\hline 216 & . LNSS86-10B & Mosquitofish & Whole body & 75.1 & 3.49 & $<.0098$ & -- & $<.0098$ \\
\hline 217 & - LNSS86-11B & Sailfin molly & Whole body & 79.3 & 2.14 & $<.010$ & --- & $<.010$ \\
\hline 218 & . LNSS86-12B & Tilapia & Whole body & 77.2 & 1.77 & $<.010$ & --- & $<.010$ \\
\hline 219 & . LNSS86-13B & Tilapia & Whole body & 75.9 & 1.13 & $<.010$ & --- & $<.010$ \\
\hline 220 & . LNSS86-21 & Double-crested cormorant & Breast muscle & 69.2 & 5.44 & $<.010$ & --- & $<.010$ \\
\hline 221 & . LNSS86-19 & Ruddy duck & Breast muscle & 72.0 & 2.71 & $<.0099$ & $\ldots$ & $<.0099$ \\
\hline 222 & . LNSS86-17 & American coot & Breast muscle & 73.0 & 5.09 & $<.010$ & --- & $<.010$ \\
\hline 223 & LNSS86-15 & Black-necked stilt & Breast muscle & 64.1 & 6.10 & $<.010$ & $--\cdot$ & $<.010$ \\
\hline 224 & . LNSS87-19 & Crayfish & Whole body & 68.9 & 1.0 & $<.01$ & -- & $<.01$ \\
\hline 225 & . LNSS87-25 & Tilapia & Whole body & 65.4 & 11.2 & -- & --- & --- \\
\hline 226 & . LNSS87-26 & Tilapia & Whole body & 68.6 & 2.6 & .01 & -- & $<.01$ \\
\hline 227 & . LNSS87-03 & Double-crested cormorant & Breast muscle & 70.6 & 4.7 & $<.01$ & -- & $<.01$ \\
\hline 228 & - LNSS87-17 & Cattle egret & Breast muscle & 69.3 & 3.5 & $<.01$ & -- & $<.01$ \\
\hline 229 & - LNSS87-01 & Herring gull & Breast muscle & 66.1 & 11.5 & .02 & -- & $<.01$ \\
\hline 230 & - LNSS88-25 & Northern shoveler & Breast muscle & 73.4 & 4.38 & $<.01$ & $<0.01$ & -- \\
\hline 231 & - LNSS88-01 & Ruddy duck & Breast muscle & 69.4 & 3.70 & $<.01$ & $<.01$ & -- \\
\hline 232 & . LNSS88-03 & Ruddy duck & Breast muscle & 71.6 & 2.67 & $<.01$ & $<.01$ & -- \\
\hline 233 & . LNSS88-05 & Ruddy duck & Breast muscle & 70.8 & 3.64 & $<.01$ & $<.01$ & --- \\
\hline 234 & . LNSS88-07 & Ruddy duck & Breast muscle & 70.8 & 2.53 & $<.01$ & $<.01$ & -- \\
\hline 235 & . LNSS88-09 & Ruddy duck & Breast muscle & 71.0 & 2.27 & $<.01$ & $<.01$ & --- \\
\hline 236 & · LNSS88-11 & Ruddy duck & Breast muscle & 71.2 & 2.43 & $<.01$ & $<.01$ & --- \\
\hline 237 & . LNSS88-13 & Ruddy duck & Breast muscle & 74.8 & 2.15 & .04 & $<.01$ & -- \\
\hline 238 & . LNSS88-15 & Ruddy duck & Breast muscle & 72.4 & 3.64 & .01 & $<.01$ & -- \\
\hline 239 & - LNSS88-17 & Ruddy duck & Breast muscle & 69.0 & 5.84 & $<.01$ & $<.01$ & -- \\
\hline 240 & . LNSS88-19 & Ruddy duck & Breast muscle & 71.0 & 3.21 & $<.01$ & $<.01$ & --- \\
\hline 241 & . $88-113$ & Black-necked stilt & Egg & 65.0 & 14.9 & .03 & $<.01$ & $-\cdots$ \\
\hline 242 & . $88-122$ & Black-necked stilt & Egg & 64.0 & 20.0 & .03 & $<.01$ & - \\
\hline 243 & . $88-125$ & Black-necked stilt & Egg & 67.0 & 16.1 & .04 & $<.01$ & - \\
\hline 244 & . $88-134$ & Black-necked stilt & Egg & 73.0 & 12.8 & .01 & $<.01$ & - \\
\hline 245 & . $\mathbf{S S 8 9 - 1 5 3}$ & Spiny softshell turtle & Egg & 58.0 & 14.3 & .01 & .03 & --- \\
\hline 246 & . $\quad$ SS89-152 & Spiny softshell turtle & Fat & 19.0 & 78.5 & .05 & .16 & -- \\
\hline 247 & . SS89-155 & Spiny softshell turtle & Fat & 18.0 & 83.9 & .07 & .24 & - \\
\hline 248 & . SS89-157 & Spiny softshell turtle & Fat & 23.0 & 76.5 & .07 & .09 & --- \\
\hline 249 & . SS89-74 & Black-necked stilt & Egg & 74.0 & 12.7 & .02 & $<.01$ & -.- \\
\hline 250 & . $5 S 89-75$ & Black-necked stilt & Egg & 74.0 & 13.5 & .02 & $<.01$ & -- \\
\hline 251 & - $\quad$ SS89-77 & Black-necked stilt & Egg & 80.0 & 9.60 & .02 & $<.01$ & -- \\
\hline 252 & . $\mathbf{S S 8 9 - 8 3}$ & Black-necked stilt & Egg & 75.0 & 13.3 & .01 & .01 & $\ldots$ \\
\hline 253 & . $\mathbf{S S 8 9 - 8 4}$ & Black-necked stilt & Egg & 76.0 & 11.5 & .02 & $<.01$ & -- \\
\hline \multicolumn{9}{|c|}{ Site B38 (South Brawley) } \\
\hline 254 & . $89-030$ & White-faced ibis & Liver & 70.5 & 4.30 & $<0.01$ & $<0.01$ &.-- \\
\hline 255 & . $89-031$ & White-faced ibis & Liver & 71.5 & 4.90 & $<.01$ & $<.01$ & -- \\
\hline 256 & . $89-032$ & White-faced ibis & Liver & 71.2 & 3.60 & $<.01$ & $<.01$ & -- \\
\hline 257 & . $89-033$ & White-faced ibis & Liver & 72.9 & -- & $<.01$ & $<.01$ & --- \\
\hline 258 & . $89-034$ & White-faced ibis & Liver & 70.0 & 7.20 & $<.01$ & $<.01$ & --- \\
\hline
\end{tabular}


Table 23. Organic chemical analysis, moisture content, and lipid content for biotic samples collected during 1986-90 from the Salton Sea and associated drainwaters and rivers--Continued

\begin{tabular}{|c|c|c|c|c|c|c|c|c|c|}
\hline $\begin{array}{l}\text { Record } \\
\text { number }\end{array}$ & $\begin{array}{l}\text { Sample } \\
\text { number }\end{array}$ & $\begin{array}{l}\text { gamma- } \\
\text { Chlordane }\end{array}$ & $\begin{array}{c}\text { trans- } \\
\text { Chlordane }\end{array}$ & $\begin{array}{l}\text { cis-Nona- } \\
\text { chlor }\end{array}$ & $\begin{array}{l}\text { trans- } \\
\text { Nonachlor }\end{array}$ & $\begin{array}{l}\text { Hepta- } \\
\text { chlor }\end{array}$ & $\begin{array}{l}\text { Heptachlor } \\
\text { epoxide }\end{array}$ & $\begin{array}{l}\text { Methyoxy- } \\
\text { chlor }\end{array}$ & $0, p^{\prime}-D D E$ \\
\hline \multicolumn{10}{|c|}{ Site B36 (Reidman Pond) } \\
\hline 210 & $88-179$ & 0.02 & - & $<0.01$ & $<0.01$ & -- & 0.05 & --- & $<0.01$ \\
\hline 211 & $88-182$ & $<.01$ & - & $<.01$ & .01 & - & .01 & -.. & $<.01$ \\
\hline 212 & LNSS88-84 & $<.05$ & - & $<.05$ & $<.05$ & $<0.05$ & $<.05$ & -- & $<.05$ \\
\hline 213 & $88-219$ & $<.01$ & -- & $<.01$ & $<.01$ & -- & .01 & -- & $<.01$ \\
\hline 214 & $88-222$ & $<.01$ & - & $<.01$ & .01 & - & $<.01$ & - & $<.01$ \\
\hline \multicolumn{10}{|c|}{ Site B37 (Hazard Pond) } \\
\hline 215 & LNSS86-09B & - & $<0.0099$ & $<0.0099$ & $<0.0099$ & - & $<0.0099$ & -- & - \\
\hline 216 & LNSS86-10B & - & $<.0098$ & $<.0098$ & $<.0098$ & -- & $<.0098$ & -- & -- \\
\hline 217 & LNSS86-11B & - & $<.010$ & $<.010$ & $<.010$ & - & $<.010$ & -- & - \\
\hline 218 & LNSS86-12B & -- & $<.010$ & $<.010$ & $<.010$ & -- & $<.010$ & -- & - \\
\hline 219 & LNSS86-13B & $\cdots$ & $<.010$ & $<.010$ & $<.010$ & - & $<.010$ & -- & -- \\
\hline 220 & LNSS86-21 & - & $<.010$ & $<.010$ & $<.010$ & - & $<.010$ & -- & - \\
\hline 221 & LNSS86-19 & - & $<.0099$ & $<.0099$ & $<.0099$ & -- & $<.0099$ & --- & -- \\
\hline 222 & LNSS86-17 & - & $<.010$ & $<.010$ & $<.010$ & -- & $<.010$ & -- & --- \\
\hline 223 & LNSS86-15 & - & $<.010$ & $<.010$ & $<.010$ & -- & $<.010$ & --- & --- \\
\hline 224 & LNSS87-19 & - & $<.01$ & $<.01$ & $<.01$ & $<0.01$ & $<.01$ & $<0.01$ & $<0.01$ \\
\hline 225 & LNSS87-25 & - & - & - & -- & -- & -- & -- & --- \\
\hline 226 & LNSS87-26 & - & $<.01$ & $<.01$ & .01 & $<.01$ & $<.01$ & $<.01$ & .02 \\
\hline 227 & LNSS87-03 & - & $<.01$ & $<.01$ & $<.01$ & $<.01$ & $<.01$ & .01 & $<.01$ \\
\hline 228 & LNSS87-17 & - & $<.01$ & $<.01$ & $<.01$ & $<.01$ & $<.01$ & $<.01$ & $<.01$ \\
\hline 229 & LNSS87-01 & - & $<.01$ & $<.01$ & $<.01$ & $<.01$ & .02 & .02 & $<.01$ \\
\hline 230 & LNSS88-25 & $<0.01$ & -- & $<.01$ & $<.01$ & -- & $<.01$ & --- & $<.01$ \\
\hline 231 & LNSS88-01 & $<.01$ & - & $<.01$ & $<.01$ & -- & $<.01$ & --- & $<.01$ \\
\hline 232 & LNSS88-03 & $<.01$ & - & $<.01$ & $<.01$ & -- & $<.01$ & --- & $<.01$ \\
\hline 233 & LNSS88-05 & $<.01$ & -- & $<.01$ & $<.01$ & -- & $<.01$ & --- & $<.01$ \\
\hline 234 & LNSS88-07 & $<.01$ & - & $<.01$ & $<.01$ & -- & $<.01$ & -- & $<.01$ \\
\hline 235 & LNSS88-09 & $<.01$ & - & $<.01$ & $<.01$ & - & $<.01$ & --- & $<.01$ \\
\hline 236 & LNSS88-11 & $<.01$ & $\cdots$ & $<.01$ & $<.01$ & - & $<.01$ & --- & $<.01$ \\
\hline 237 & LNSS88-13 & $<.01$ & $\cdots$ & $<.01$ & $<.01$ & -- & .02 & --- & $<.01$ \\
\hline 238 & LNSS88-15 & $<.01$ & -- & $<.01$ & $<.01$ & -- & $<.01$ & -- & $<.01$ \\
\hline 239 & LNSS88-17 & $<.01$ & -- & $<.01$ & $<.01$ & $\ldots$ & $<.01$ & --- & $<.01$ \\
\hline 240 & LNSS88-19 & $<.01$ & -- & $<.01$ & $<.01$ & --- & $<.01$ & -. & $<.01$ \\
\hline 241 & $88-113$ & $<.01$ & - & $<.01$ & .01 & -- & .01 & --- & $<.01$ \\
\hline 242 & $88-122$ & $<.01$ & -- & $<.01$ & .01 & - & .01 & --- & $<.01$ \\
\hline 243 & $88-125$ & $<.01$ & -- & $<.01$ & .01 & - & .01 & -- & $<.01$ \\
\hline 244 & 88-134 & $<.01$ & - & $<.01$ & .01 & - & $<.01$ & -- & $<.01$ \\
\hline 245 & SS89-153 & .02 & -- & .02 & .05 & - & .01 & $\cdots$ & $<.01$ \\
\hline 246 & SS89-152 & .05 & - & .11 & .23 & -- & .04 & -- & $<.01$ \\
\hline 247 & SS89-155 & .08 & - & .11 & .29 & -- & .04 &.- & $<.01$ \\
\hline 248 & SS89-157 & .02 & -- & .02 & .10 & - & .04 & --- & $<.01$ \\
\hline 249 & SS89-74 & $<.01$ & - & $<.01$ & $<.01$ & - & .01 & --- & $<.01$ \\
\hline 250 & SS89-75 & $<.01$ & - & $<.01$ & $<.01$ & - & .02 & --- & $<.01$ \\
\hline 251 & SS89-77 & $<.01$ & - & $<.01$ & $<.01$ & -- & .01 & -- & $<.01$ \\
\hline 252 & SS89-83 & $<.01$ & -- & $<.01$ & $<.01$ & -- & $<.01$ & --- & $<.01$ \\
\hline 253 & SS89-84 & $<.01$ & -- & $<.01$ & $<.01$ & -- & .01 & -- & $<.01$ \\
\hline \multicolumn{10}{|c|}{ Site B38 (South Brawley) } \\
\hline 254 & $89-030$ & $<0.01$ & -- & $<0.01$ & $<0.01$ & -- & $<0.01$ & --- & $<0.01$ \\
\hline 255 & $89-031$ & $<.01$ & -- & $<.01$ & .03 & -- & $<.01$ & --- & $<.01$ \\
\hline 256 & $89-032$ & $<.01$ & - & $<.01$ & $<.01$ & --- & $<.01$ & --- & $<.01$ \\
\hline 257 & $89-033$ & $<.01$ & -- & $<.01$ & $<.01$ & -- & $<.01$ & --- & $<.01$ \\
\hline 258 & 89-034 & $<.01$ & -- & $<.01$ & $<.01$ & -- & $<.01$ & --- & $<.01$ \\
\hline
\end{tabular}


Table 23. Organic chemical analysis, moisture content, and lipid content for biotic samples collected during 1986-90 from the Salton Sea and associated drainwaters and rivers--Continued

\begin{tabular}{|c|c|c|c|c|c|c|c|c|c|c|}
\hline $\begin{array}{l}\text { Record } \\
\text { number }\end{array}$ & $\begin{array}{l}\text { Sample } \\
\text { number }\end{array}$ & p,p'-DDE & o,p'-DDD & p,p'-DDD & o,p'-DDT & $\mathbf{p}, \mathbf{p}^{\prime}-\mathrm{DDT}$ & $\begin{array}{l}\text { Total } \\
\text { DDT }\end{array}$ & Endrin & Dieldrin & Aldrin \\
\hline \multicolumn{11}{|c|}{ Site B36 (Reidman Pond) } \\
\hline 210 & $88-179$ & 10 & $<0.01$ & 0.06 & $<0.01$ & 1.2 & 11.260 & $<0.01$ & 0.10 & -- \\
\hline 211 & $88-182$ & 6.2 & $<.01$ & .01 & $<.01$ & .06 & 6.270 & $<.01$ & .10 & -- \\
\hline 212 & LNSS88-84 & .75 & $<.05$ & $<.05$ & $<.05$ & $<.05$ & $<.05$ & $<.05$ & $<.05$ & $<0.05$ \\
\hline 213 & $88-219$ & 1.6 & $<.01$ & $<.01$ & $<.01$ & $<.01$ & 1.600 & $<.01$ & $<.01$ & $\ldots$ \\
\hline 214 & $88-222$ & 6.6 & $<.01$ & .01 & $<.01$ & .03 & 6.640 & $<.01$ & .08 & - \\
\hline \multicolumn{11}{|c|}{ Site B37 (Hazard Pond) } \\
\hline 215 & LNSS86-09B & 0.61 & - & $<0.0099$ & $\ldots$ & $<0.0099$ & 0.610 & $<0.0099$ & $<0.0099$ & $\ldots$ \\
\hline 216 & LNSS86-10B & .54 & -- & .030 & -- & $<.0098$ & .570 & $<.0098$ & .024 & $\ldots$ \\
\hline 217 & LNSS86-11B & .35 & - & .018 & $\ldots$ & $<.010$ & .368 & $<.010$ & .016 & --- \\
\hline 218 & LNSS86-12B & .37 & - & $<.010$ & -- & $<.010$ & .370 & $<.010$ & $<.010$ & -- \\
\hline 219 & LNSS86-13B & .23 & - & $<.010$ & -- & $<.010$ & .230 & $<.010$ & $<.010$ & --- \\
\hline 220 & LNSS86-21 & .78 & -- & $<.010$ & $\ldots$ & $<.010$ & .780 & $<.010$ & $<.010$ & -- \\
\hline 221 & LNSS86-19 & .30 & -- & $<.0099$ & --- & $<.0099$ & .300 & $<.0099$ & $<.0099$ & -- \\
\hline 222 & LNSS86-17 & .17 & $\cdots$ & $<.010$ & --- & $<.010$ & .170 & $<.010$ & $<.010$ & --- \\
\hline 223 & LNSS86-15 & 4.2 & -- & $<.010$ & -- & $<.010$ & 4.200 & $<.010$ & .098 & --- \\
\hline 224 & LNSS87-19 & .25 & $<0.01$ & $<.01$ & $<0.01$ & $<.01$ & .250 & $<.01$ & $<.01$ & $<0.01$ \\
\hline 225 & LNSS87-25 & -- & --- & - & -- & -- & - & -- & -- & --- \\
\hline 226 & LNSS87-26 & .30 & $<.01$ & .02 & .01 & .01 & .360 & $<.01$ & .02 & $<.01$ \\
\hline 227 & LNSS87-03 & 4.9 & $<.01$ & .02 & $<.01$ & $<.01$ & 4.920 & $<.01$ & .02 & $<.01$ \\
\hline 228 & LNSS87-17 & 2.4 & $<.01$ & .02 & $<.01$ & .01 & 2.430 & $<.01$ & .03 & $<.01$ \\
\hline 229 & LNSS87-01 & 2.8 & .01 & .01 & $<.01$ & $<.01$ & 2.820 & $<.01$ & .03 & $<.01$ \\
\hline 230 & LNSS88-25 & 2.1 & $<.01$ & .03 & $<.01$ & .01 & 2.140 & $<.01$ & .01 & $\ldots$ \\
\hline 231 & LNSS88-01 & .14 & $<.01$ & $<.01$ & $<.01$ & $<.01$ & .140 & $<.01$ & .01 & -- \\
\hline 232 & LNSS88-03 & .19 & $<.01$ & .01 & $<.01$ & $<.01$ & .200 & $<.01$ & .01 & --- \\
\hline 233 & LNSS88-05 & .26 & $<.01$ & .01 & $<.01$ & $<.01$ & .270 & $<.01$ & .01 & -- \\
\hline 234 & LNSS88-07 & .49 & $<.01$ & $<.01$ & $<.01$ & $<.01$ & .490 & $<.01$ & .01 & --- \\
\hline 235 & LNSS88-09 & .1 & $<.01$ & $<.01$ & $<.01$ & $<.01$ & .100 & $<.01$ & .01 & --- \\
\hline 236 & LNSS88-11 & .13 & $<.01$ & $<.01$ & $<.01$ & $<.01$ & .130 & $<.01$ & .01 & --- \\
\hline 237 & LNSS88-13 & .28 & $<.01$ & $<.01$ & $<.01$ & $<.01$ & .280 & $<.01$ & .01 &.- \\
\hline 238 & LNSS88-15 & .22 & $<.01$ & $<.01$ & $<.01$ & $<.01$ & .220 & $<.01$ & .01 & --- \\
\hline 239 & LNSS88-17 & .12 & $<.01$ & .01 & $<.01$ & $<.01$ & .130 & $<.01$ & .01 & --- \\
\hline 240 & LNSS88-19 & .12 & $<.01$ & .01 & $<.01$ & $<.01$ & .130 & $<.01$ & .01 & --- \\
\hline 241 & $88-113$ & 6.0 & $<.01$ & .02 & $<.01$ & .03 & 6.050 & $<.01$ & .05 & --- \\
\hline 242 & $88-122$ & 5.5 & $<.01$ & .02 & $<.01$ & .02 & 5.540 & $<.01$ & .03 & --- \\
\hline 243 & $88-125$ & 12 & $<.01$ & .01 & $<.01$ & .05 & 12.060 & .03 & .10 &.-- \\
\hline 244 & $88-134$ & 3.6 & $<.01$ & .02 & $<.01$ & .03 & 3.650 & $<.01$ & .04 & --- \\
\hline 245 & SS89-153 & 7.8 & $<.01$ & .16 & $<.01$ & $<.01$ & 7.960 & $<.01$ & .13 & --- \\
\hline 246 & SS89-152 & 21 & $<.01$ & .89 & $<.01$ & $<.01$ & 21.890 & $<.01$ & .68 & -- \\
\hline 247 & SS89-155 & 16 & $<.01$ & .74 & $<.01$ & $<.01$ & 16.740 & $<.01$ & .81 & $\ldots$ \\
\hline 248 & SS89-157 & 11 & $<.01$ & .21 & $<.01$ & $<.01$ & 11.210 & $<.01$ & .25 & $\cdots$ \\
\hline 249 & SS89-74 & 3.4 & $<.01$ & $<.01$ & $<.01$ & .03 & 3.430 & $<.01$ & .05 & --- \\
\hline 250 & SS89-75 & 3.2 & $<.01$ & $<.01$ & $<.01$ & .02 & 3.220 & $<.01$ & .07 & --- \\
\hline 251 & SS89-77 & 1.8 & $<.01$ & $<.01$ & $<.01$ & $<.01$ & 1.800 & $<.01$ & .03 &.-- \\
\hline 252 & SS89-83 & 2.7 & $<.01$ & $<.01$ & $<.01$ & .09 & 2.790 & $<.01$ & .05 & --. \\
\hline 253 & SS89-84 & 3.2 & $<.01$ & $<.01$ & $<.01$ & .13 & 3.330 & $<.01$ & .03 & -- \\
\hline \multicolumn{11}{|c|}{ Site B38 (South Brawley) } \\
\hline 254 & 89-030 & 5.6 & $<0.01$ & 0.22 & $<0.01$ & $<0.01$ & 5.820 & $<0.01$ & 0.05 & $\cdots$ \\
\hline 255 & $89-031$ & 4.6 & $<.01$ & .06 & $<.01$ & $<.01$ & 4.660 & $<.01$ & .15 & -- \\
\hline 256 & $89-032$ & 4.5 & $<.01$ & .20 & $<.01$ & $<.01$ & 4.700 & $<.01$ & .12 & --- \\
\hline 257 & $89-033$ & 7.9 & $<.01$ & .19 & $<.01$ & $<.01$ & 8.090 & $<.01$ & .08 & --- \\
\hline 258 & 89-034 & 5.6 & $<.01$ & .07 & $<.01$ & $<.01$ & 5.670 & $<.01$ & .08 & -- \\
\hline
\end{tabular}


Table 23. Organic chemical analysis, moisture content, and lipid content for biotic samples collected during 1986-90 from the Salton Sea and associated drainwaters and rivers--Continued

\begin{tabular}{|c|c|c|c|c|c|c|c|c|c|c|c|c|c|}
\hline $\begin{array}{l}\text { Record } \\
\text { number }\end{array}$ & $\begin{array}{l}\text { Sample } \\
\text { number }\end{array}$ & $\begin{array}{l}\text { alpha- } \\
\text { BHC }\end{array}$ & $\begin{array}{l}\text { beta- } \\
\text { BHC }\end{array}$ & $\begin{array}{l}\text { delta- } \\
\text { BHC }\end{array}$ & $\begin{array}{l}\text { gamma- } \\
\text { BHC }\end{array}$ & $\begin{array}{l}\text { Lin- } \\
\text { dane }\end{array}$ & $\begin{array}{l}\text { Hexa- } \\
\text { chloro- } \\
\text { ben- } \\
\text { zene }\end{array}$ & $\begin{array}{l}\text { Endo- } \\
\text { sul- } \\
\text { fan I }\end{array}$ & $\begin{array}{c}\text { Endo- } \\
\text { sul- } \\
\text { fan II }\end{array}$ & $\begin{array}{l}\text { Endo- } \\
\text { sulfan } \\
\text { sulfate }\end{array}$ & Mirex & DCPA & Dicofol \\
\hline \multicolumn{14}{|c|}{ Site B36 (Reidman Pond) } \\
\hline 210 & $88-179$ & $<0.01$ & $<0.01$ & 0.05 & $<0.01$ & - & 0.02 & -- & -- & -- & $<0.01$ & - & -- \\
\hline 211 & $88-182$ & $<.01$ & .01 & $<.01$ & $<.01$ & - & .01 & - & - & - & $<.01$ & - & $\cdots$ \\
\hline 212 & LNSS88-84 & $<.05$ & $<.05$ & $<.05$ & - & $<0.05$ & $<.05$ & - & - & - & $<.05$ & -- & $-\cdots$ \\
\hline 213 & $88-219$ & $<.01$ & $<.01$ & .02 & $<.01$ & - & .01 & - & - & - & $<.01$ & -- & -- \\
\hline 214 & $88-222$ & $<.01$ & .01 & $<.01$ & $<.01$ & -- & .02 & -- & - & - & $<.01$ & -- & -- \\
\hline
\end{tabular}

\begin{tabular}{|c|c|c|c|c|c|c|c|c|c|c|c|c|c|}
\hline \multicolumn{14}{|c|}{ Site B37 (Hazard Pond) } \\
\hline 215 & LNSS86-09B & -- & - & $\cdots$ & -- & - & - & - & - & - & -- & -- & --- \\
\hline 216 & LNSS86-10B & - & - & -- & -- & -- & -- & - & $\cdots$ & - & -- & - & -- \\
\hline 217 & LNSS86-11B & -- & - & - & - & - & -- & - & -- & - & - & - & -- \\
\hline 218 & LNSS86-12B & - & - & -- & - & -- & - & - & -- & -- & -- & -- & --- \\
\hline 219 & LNSS86-13B & -- & --- & - & -- & - & - & -- & - & -- & -- & -- & -- \\
\hline 220 & LNSS86-21 & - & -- & -- & -- & - &.-- & - & - & --- & - & - & -- \\
\hline 221 & LNSS86-19 & -- & - & - & -- & - & - & - & - & -- & -- & --- & -- \\
\hline 222 & LNSS86-17 & - & -- & -- & $\ldots$ & -- & - & -- & - & -- & -- & $\ldots$ & - \\
\hline 223 & LNSS86-15 & - & -- & -- & $-\ldots$ & - & -- & -- & - & -- & --- & -- & -- \\
\hline 224 & LNSS87-19 & $<0.01$ & 0.01 & $<0.01$ & $<0.01$ & -- & $<0.01$ & $<0.01$ & $<0.01$ & $<0.01$ & $<0.01$ & 0.02 & $<0.01$ \\
\hline 225 & LNSS87-25 & -- & - & -- & --- & -- & -- & -- & - & --- & -- & -- & -- \\
\hline 226 & LNSS87-26 & $<.01$ & .03 & $<.01$ & $<.01$ & -- & $<.01$ & $<.01$ & $<.01$ & .02 & $<.01$ & .14 & $<.01$ \\
\hline 227 & LNSS87-03 & $<.01$ & $<.01$ & $<.01$ & $<.01$ & - & $<.01$ & $<.01$ & $<.01$ & $<.01$ & $<.01$ & .03 & $<.01$ \\
\hline 228 & LNSS87-17 & $<.01$ & .01 & $<.01$ & $<.01$ & - & .06 & $<.01$ & $<.01$ & $<.01$ & $<.01$ & $<.01$ & $<.01$ \\
\hline 229 & LNSS87-01 & $<.01$ & $<.01$ & $<.01$ & $<.01$ & - & .01 & $<.01$ & $<.01$ & $<.01$ & $<.01$ & $<.01$ & $<.01$ \\
\hline 230 & LNSS88-25 & $<.01$ & $<.01$ & $<.01$ & $<.01$ & - & $<.01$ & -- & -- & -- & $<.01$ & $\ldots$ & --- \\
\hline 231 & LNSS88-01 & $<.01$ & $<.01$ & $<.01$ & $<.01$ & -- & $<.01$ & -- & --- & -- & $<.01$ & -- & -- \\
\hline 232 & LNSS88-03 & $<.01$ & $<.01$ & $<.01$ & $<.01$ & -- & $<.01$ & -- & -- & -- & $<.01$ & - & - \\
\hline 233 & LNSS88-05 & $<.01$ & $<.01$ & $<.01$ & $<.01$ & -- & $<.01$ & -- & - & -- & $<.01$ & --- & -- \\
\hline 234 & LNSS88-07 & $<.01$ & $<.01$ & $<.01$ & $<.01$ & -- & $<.01$ & -- & -- & $-\ldots$ & $<.01$ & - & - \\
\hline 235 & LNSS88-09 & $<.01$ & $<.01$ & $<.01$ & $<.01$ & - & $<.01$ & -- & $\ldots$ & -- & $<.01$ & -- & - \\
\hline 236 & LNSS88-11 & $<.01$ & $<.01$ & $<.01$ & $<.01$ & -- & $<.01$ & -- & -- & --- & $<.01$ & - & -- \\
\hline 237 & LNSS88-13 & $<.01$ & $<.01$ & $<.01$ & $<.01$ & -- & $<.01$ & -- & -- & --- & $<.01$ & -- & - \\
\hline 238 & LNSS88-15 & $<.01$ & $<.01$ & $<.01$ & $<.01$ & -- & $<.01$ & -- & -.- & -- & $<.01$ & --- & -- \\
\hline 239 & LNSS88-17 & $<.01$ & $<.01$ & $<.01$ & $<.01$ & - & $<.01$ & $\cdots$ & -- & $\ldots$ & $<.01$ & -- & -- \\
\hline 240 & LNSS88-19 & $<.01$ & $<.01$ & $<.01$ & $<.01$ & $\cdots$ & $<.01$ & -- & -- & -- & $<.01$ & -- & -- \\
\hline 241 & $88-113$ & $<.01$ & .03 & $<.01$ & $<.01$ & -- & .02 & -- & --- & --- & $<.01$ & -- & -.. \\
\hline 242 & $88-122$ & $<.01$ & .03 & $<.01$ & $<.01$ & -- & .02 & $\cdots$ & $\cdots$ & -- & $<.01$ & -- & -- \\
\hline 243 & $88-125$ & $<.01$ & .03 & $<.01$ & $<.01$ & -- & .05 & -- & --- & -- & $<.01$ & -- & -- \\
\hline 244 & 88-134 & $<.01$ & .03 & $<.01$ & $<.01$ & -- & .01 & -- & -- & -- & $<.01$ & -- & -- \\
\hline 245 & SS89-153 & $<.01$ & .01 & $<.01$ & $<.01$ & - & $<.01$ & $<.01$ & $<.01$ & -- & $<.01$ & -- & -- \\
\hline 246 & SS89-152 & $<.01$ & .02 & $<.01$ & $<.01$ & --- & $<.01$ & $<.01$ & $<.01$ & - & $<.01$ & $\ldots$ & -.. \\
\hline 247 & SS89-155 & $<.01$ & .02 & $<.01$ & $<.01$ & -- & $<.01$ & $<.01$ & $<.01$ & - & $<.01$ & -- & -- \\
\hline 248 & SS89-157 & $<.01$ & .01 & $<.01$ & $<.01$ & -- & $<.01$ & $<.01$ & $<.01$ & -- & $<.01$ & -- & $-\ldots$ \\
\hline 249 & SS89-74 & $<.01$ & .05 & $<.01$ & $<.01$ & --- & .01 & - & -- & -- & $<.01$ & --- & -- \\
\hline 250 & SS89-75 & $<.01$ & .12 & $<.01$ & $<.01$ & - & .01 & $\ldots$ & -- & --- & $<.01$ & -- & -- \\
\hline 251 & SS89-77 & $<.01$ & .09 & $<.01$ & $<.01$ & - & .01 & -- & -- & -- & $<.01$ & $\cdots$ & -- \\
\hline 252 & SS89-83 & $<.01$ & .03 & $<.01$ & $<.01$ & -- & .01 & -- & -- & $\cdots$ & $<.01$ & -- & -- \\
\hline 253 & SS89-84 & $<.01$ & .03 & $<.01$ & $<.01$ & - & $<.01$ & -- & -- & - & $<.01$ & -- & -- \\
\hline \multicolumn{14}{|c|}{ Site B38 (South Brawley) } \\
\hline 254 & $89-030$ & $<0.01$ & $<0.01$ & $<0.01$ & $<0.01$ & - & 2.9 & -- & -- & -- & $<0.01$ & --- & -- \\
\hline 255 & $89-031$ & $<.01$ & $<.01$ & $<.01$ & $<.01$ & --- & .04 & $\ldots$ & $-\ldots$ & --- & $<.01$ & -.. & -- \\
\hline 256 & $89-032$ & $<.01$ & $<.01$ & $<.01$ & $<.01$ & -- & 1.5 & - & -- & -- & $<.01$ & -- & - \\
\hline 257 & $89-033$ & $<.01$ & $<.01$ & $<.01$ & $<.01$ & - & 2.7 & -- &.- &.- & $<.01$ & -.. & -- \\
\hline 258 & $89-034$ & $<.01$ & $<.01$ & $<.01$ & $<.01$ & -- & .02 & - & -- & -- & $<.01$ & -- & -- \\
\hline
\end{tabular}


Table 23. Organic chemical analysis, moisture content, and lipid content for biotic samples collected during 1986-90 from the Salton Sea and associated drainwaters and rivers--Continued

\begin{tabular}{|c|c|c|c|c|c|c|c|c|c|c|c|c|c|c|}
\hline econd & $\begin{array}{l}\text { Sample } \\
\text { number }\end{array}$ & $\begin{array}{l}\text { Tetra- } \\
\text { difon }\end{array}$ & $\begin{array}{l}\text { BI PH } \\
\text { CL-2 }\end{array}$ & $\begin{array}{l}\text { BI PH } \\
\text { CL-3 }\end{array}$ & $\begin{array}{c}\text { BI PH } \\
\text { CL-4 }\end{array}$ & $\begin{array}{c}\text { BI PH } \\
\text { CL-5 }\end{array}$ & $\begin{array}{l}\text { BI PH } \\
\text { CL-6 }\end{array}$ & $\begin{array}{c}\text { BI PH } \\
\text { CL-7 }\end{array}$ & $\begin{array}{l}\text { BI PH } \\
\text { CL-8 }\end{array}$ & $\begin{array}{c}\text { BI PH } \\
\text { CL-9 }\end{array}$ & $\begin{array}{l}\text { PCB } \\
1016\end{array}$ & $\begin{array}{l}\text { PCB } \\
1221\end{array}$ & $\begin{array}{l}\text { PCB } \\
1232\end{array}$ & $\begin{array}{l}\text { PCB } \\
1242\end{array}$ \\
\hline
\end{tabular}

\begin{tabular}{|c|c|c|c|c|c|c|c|c|c|c|c|c|c|c|}
\hline \multicolumn{15}{|c|}{ Site B36 (Reidman Pond) } \\
\hline 210 & $88-179$ & - & - & - & - & - & - & - & -- & -- & - & - & - & - \\
\hline 211 & $88-182$ & - & - & - & - & - & - & - & - & -- & - & - & -- & -- \\
\hline 212 & LNSS88-84 & -- & $<0.05$ & $<0.05$ & 0.05 & 0.06 & 0.07 & $<0.05$ & $<0.05$ & $<0.05$ & $\cdots$ & - & -- & - \\
\hline 213 & $88-219$ & $\ldots$ & -- & -- & -- & -- & - & - & -- & -- & -- & - & -- & - \\
\hline 214 & $88-222$ & -- & - & -- & - & -- & - & -- & -- & -- & $-\cdots$ & ... & -- & -- \\
\hline
\end{tabular}

\begin{tabular}{|c|c|c|c|c|c|c|c|c|c|c|c|c|c|c|}
\hline \multicolumn{15}{|c|}{ Site B37 (Hazard Pond) } \\
\hline 215 & LNSS86-09B & - & $\ldots$ & - & -- & -- & $\ldots$ & -. & - & $\ldots$ & - & -.. & ... & -- \\
\hline 216 & LNSS86-10B & - & - & -- & -.. & -. & - & $\ldots$ & - & -- & $\ldots$ & $\ldots$ & -- & - \\
\hline 217 & LNSS86-11B & $\ldots$ & - & - & $\ldots$ & -. & - & - & -- & - & -- & -.. & -- & -- \\
\hline 218 & LNSS86-12B & - & -- & -- & -- & -- & - & - & -.. & $\ldots$ & - & $\ldots$ & -.. & -. \\
\hline 219 & LNSS86-13B & - & - & -- & $\ldots$ & -.. & -- & $\ldots$ & -- & -- & $\ldots$ & -. & -- & -.. \\
\hline 220 & LNSS86-21 & -.. & -- & -.- & $\ldots$ & -- & -.. & -- & -_ & - & -- & -.- & -- & -- \\
\hline 221 & LNSS86-19 & - & - & - & - & - & -.. & - & -.. & $\ldots$ & - & ... & $\ldots$ & --- \\
\hline 222 & LNSS86-17 & - & - & -- & - & - & - & --- & -- & - & $\ldots$ & -- & -. & $\ldots$ \\
\hline 223 & LNSS86-15 & - & -- & - & - & -- & -- & -. & - & $-\ldots$ & - & -- & -.- & $\ldots$ \\
\hline 224 & LNSS87-19 & $<0.01$ & - & -- & - & - & - & -. & - & -.- & $<0.05$ & $<0.50$ & $<0.05$ & $<0.05$ \\
\hline 225 & LNSS87-25 & - & - & - & - & -- & - & -. & - & --- & -- & -- & $\ldots$ & -- \\
\hline 226 & LNSS87-26 & $<.01$ & - & - & - & -- & -- & -- & - & -- & $<.05$ & $<.50$ & $<.05$ & $<.05$ \\
\hline 227 & LNSS87-03 & $<.01$ & - & -- & - & - & $\ldots$ & -- & - & -.- & $<.05$ & $<.50$ & $<.05$ & $<.05$ \\
\hline 228 & LNSS87-17 & $<.01$ & - & -- & - & -- & - & -.. & -- & - & $<.05$ & $<.50$ & $<.05$ & $<.05$ \\
\hline 229 & LNSS87-01 & $<.01$ & - & $\ldots$ & $\ldots$ & $\ldots$ & $\ldots$ & -.- & $\ldots$ & -.- & $<.05$ & $<.50$ & $<.05$ & $<.05$ \\
\hline 230 & LNSS88-25 & -- & -- & -- & -. & - & -. & $\ldots$ & $\ldots$ & -- & $\ldots$ & $\ldots$ & $\ldots$ & $\ldots$ \\
\hline 231 & LNSS88-01 & - & - & - & -- & -- & - & -.- & -. & $\ldots$ & $\ldots$ & -.- & $\ldots$ & --- \\
\hline 232 & LNSS88-03 & - & - & -- & - & -- & -- & -- & - & -- & -.. & --- & -- & -. \\
\hline 233 & LNSS88-05 & $\ldots$ & - & - & - & -. & -.. & .-. & -.- & - & - & -.. & $\ldots$ & -.. \\
\hline 234 & LNSS88-07 & - & - & $\ldots$ & -- & - & -. & -.. & $\ldots$ & -. & $\ldots$ & -.- & -.- & -.- \\
\hline 235 & LNSS88-09 & - & - & $-\ldots$ & - & --. & --- & $\ldots$ & -- & - & $\ldots$ & --. & -- & -.- \\
\hline 236 & LNSS88-11 & - & - & - & -- & -.. & - & -- & - & -- & $\ldots$ & $-\ldots$ & --- & -- \\
\hline 237 & LNSS88-13 & $\ldots$ & -. & -. & $\ldots$ & -.. & $\ldots$ & -.- & $\ldots$ & -.. & $\ldots$ & -.- & $\ldots$ & -.. \\
\hline 238 & LNSS88-15 & $\ldots$ & - & -- & - & -. & - & $\ldots$ & $\ldots$ & --- & $\ldots$ & --. & $\ldots$ & -- \\
\hline 239 & LNSS88-17 & - & $\ldots$ & -.. & $\ldots$ & -. & -.. & -.. & -- & -- & -- & --. & $\ldots$ & ... \\
\hline 240 & LNSS88-19 & - & - & -- & -- & - & $-\ldots$ & -- & $\ldots$ & $\ldots$ & $\ldots$ & --- & -- & $\ldots$ \\
\hline 241 & $88-113$ & $\ldots$ & - & -- & -- & -- & -.- & --- & -- & -.- & $\ldots$ & --- & ... & --- \\
\hline 242 & $88-122$ & $\ldots$ & $\ldots$ & - & -- & - & -- & -- & -.. & -.. & $\ldots$ & -.. & -- & -.. \\
\hline 243 & $88-125$ & - & $\ldots$ & -. & - & -- & --. & --- & $\ldots$ & --- & -.. & --- & ... & $\ldots$ \\
\hline 244 & $88-134$ & $\ldots$ & $\ldots$ & -- & -. & -- & --. & --. & - & -. & -.. & --- & -- & -- \\
\hline 245 & SS89-153 & -- & - & - & - & -- & -- & -- & -- & - & - & --- & $\ldots$ & -- \\
\hline 246 & SS89-152 & - & - & -- & -- & --. & -. & $\ldots$ & -- & $\ldots$ & $\ldots$ & ... & -.. & -.. \\
\hline 247 & SS89-155 & - & - & -- & - & -- & - & $\ldots$ & -- & $\ldots$ & $\ldots$ & $\ldots$ & $\ldots$ & -.. \\
\hline 248 & SS89-157 & - & $\ldots$ & -- & - & -- & - & -- & - & $\ldots$ & $\ldots$ & -- & -- & -.. \\
\hline 249 & SS89-74 & - & - & - & - & - & - & -.. & - & -.- & $\ldots$ & --- & -. & -- \\
\hline 250 & SS89-75 & -- & - & $\ldots$ & - & $\ldots$ & $\ldots$ & -- & $\ldots$ & -.. & $\ldots$ & $\therefore$ & -.. & -.. \\
\hline 251 & SS89-77 & - & - & - & - & -. & - & - & - & --. & - & -- & --- & -- \\
\hline 252 & SS89-83 & $\ldots$ & $\ldots$ & - & -.. & $\ldots$ & - & $\ldots$ & - & --. & -- & ... & --- & $\ldots$ \\
\hline 253 & SS89-84 & - & - & - & - & $\ldots$ & -. & $\ldots$ & -. & ... & -.. & --. & $\ldots$ & -- \\
\hline
\end{tabular}

Site B38 (South Brawley)

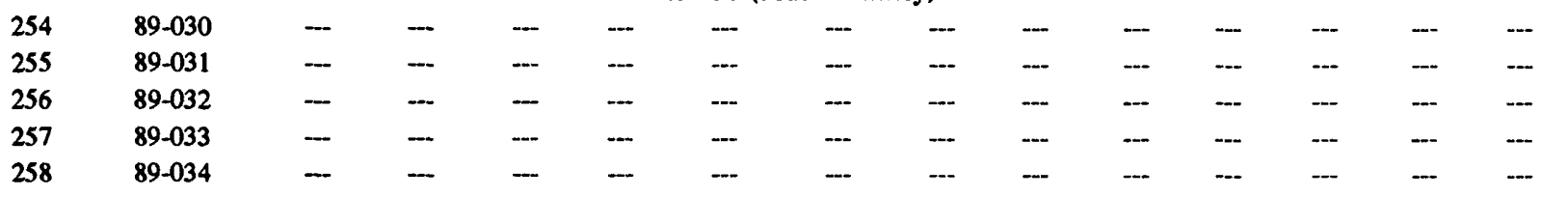


Table 23. Organic chemical analysis, moisture content, and lipid content for biotic samples collected during 1986-90 from the Salton Sea and associated drainwaters and rivers--Continued

\begin{tabular}{|c|c|c|c|c|c|c|}
\hline $\begin{array}{l}\text { Record } \\
\text { number }\end{array}$ & $\begin{array}{l}\text { Sample } \\
\text { number }\end{array}$ & $\begin{array}{l}\text { PCB } \\
1248\end{array}$ & $\begin{array}{l}\text { PCB } \\
1254\end{array}$ & $\begin{array}{l}\text { PCB } \\
1260\end{array}$ & $\begin{array}{l}\text { Total } \\
\text { PCB }\end{array}$ & Toxaphene \\
\hline \multicolumn{7}{|c|}{ Site B36 (Reidman Pond) } \\
\hline 210 & 88-179 & - & $\ldots$ & -- & $<0.05$ & 0.01 \\
\hline 211 & $88-182$ & - & -- & -- & $<.05$ & $<.05$ \\
\hline 212 & LNSS88-84 & - & -- & - & $<.50$ & $<.50$ \\
\hline 213 & $88-219$ & - & $\ldots$ & $\ldots$ & $<.05$ & $<.05$ \\
\hline 214 & $88-222$ & - & - & - & $<.05$ & $<.05$ \\
\hline \multicolumn{7}{|c|}{ Site B37 (Hazard Pond) } \\
\hline 215 & LNSS86-09B & - & $<0.099$ & $<0.099$ & - & $<0.49$ \\
\hline 216 & LNSS86-10B & - & $<.098$ & $<.098$ & - & $<.49$ \\
\hline 217 & LNSS86-11B & $\ldots$ & $<.10$ & $<.10$ & $\ldots$ & $<.50$ \\
\hline 218 & LNSS86-12B & -- & $<.10$ & $<.10$ & -- & $<.50$ \\
\hline 219 & LNSS86-13B & - & $<.10$ & $<.10$ & $\ldots$ & $<.50$ \\
\hline 220 & LNSS86-21 & -- & $<.10$ & $<.10$ & -- & $<.50$ \\
\hline 221 & LNSS86-19 & $-\ldots$ & $<.099$ & $<.099$ & - & $<.50$ \\
\hline 222 & LNSS86-17 & $\cdots$ & $<.10$ & $<.10$ & -- & $<.50$ \\
\hline 223 & LNSS86-15 & $\ldots$ & $<.10$ & $<.10$ & - & $<.50$ \\
\hline 224 & LNSS87-19 & $<0.05$ & $<.05$ & $<.05$ & $\ldots$ & $<.50$ \\
\hline 225 & LNSS87-25 & -- & $\ldots$ & $\ldots$ & - & -- \\
\hline 226 & LNSS87-26 & $<.05$ & $<.05$ & $<.05$ & -- & $<.50$ \\
\hline 227 & LNSS87-03 & $<.05$ & $<.05$ & $<.05$ & - & $<.50$ \\
\hline 228 & LNSS87-17 & $<.05$ & $<.05$ & $<.05$ & - & $<.50$ \\
\hline 229 & LNSS87-01 & $<.05$ & $<.05$ & $<.05$ & - & $<.50$ \\
\hline 230 & LNSS88-25 & $\ldots$ & -- & -- & $<0.05$ & $<0.05$ \\
\hline 231 & LNSS88-01 & -- & -- & - & $<.05$ & $<.05$ \\
\hline 232 & LNSS88-03 & -- & -- & -.. & .05 & $<.05$ \\
\hline 233 & LNSS88-05 & -- & -- & -- & .15 & $<.05$ \\
\hline 234 & LNSS88-07 & -- & -- & $-\cdots$ & $<.05$ & $<.05$ \\
\hline 235 & LNSS88-09 & -- & -- & - & $<.05$ & $<.05$ \\
\hline 236 & LNSS88-11 & - & -- & -- & $<.05$ & $<.05$ \\
\hline 237 & LNSS88-13 & - & -- & -- & .30 & $<.05$ \\
\hline 238 & LNSS88-15 & $\ldots$ & $\ldots$ & - & .26 & $<.05$ \\
\hline 239 & LNSS88-17 & - & -- & -- & $<.05$ & $<.05$ \\
\hline 240 & LNSS88-19 & -- & - & - & $<.05$ & $<.05$ \\
\hline 241 & $88-113$ & - & -- & -- & $<.05$ & .41 \\
\hline 242 & $88-122$ & -- & -- & -- & $<.05$ & $<.05$ \\
\hline 243 & $88-125$ & - & $\ldots$ & - & $<.05$ & .95 \\
\hline 244 & $88-134$ & - & - & -- & $<.05$ & $<.05$ \\
\hline 245 & SS89-153 & -- & - & - & $<.05$ & .99 \\
\hline 246 & SS89-152 & -- & -- & - & $<.05$ & 5.9 \\
\hline 247 & SS89-155 & - & -- & -- & $<.05$ & 4.9 \\
\hline 248 & SS89-157 & -- & -- & - & $<.05$ & 2.3 \\
\hline 249 & SS89-74 & -- & - & -- & $<.05$ & $<.05$ \\
\hline 250 & SS89-75 & - & - & -- & $<.05$ & $<.05$ \\
\hline 251 & SS89-77 & -- & - & -- & $<.05$ & $<.05$ \\
\hline 252 & SS89-83 & -- & $\ldots$ & -- & $<.05$ & $<.05$ \\
\hline 253 & SS89-84 & - & - & -- & $<.05$ & $<.05$ \\
\hline \multicolumn{7}{|c|}{ Site B38 (South Brawley) } \\
\hline 254 & $89-030$ & - & - & -- & $<0.05$ & $<0.05$ \\
\hline 255 & $89-031$ & - & -- & - & $<.05$ & $<.05$ \\
\hline 256 & $89-032$ & - & - & - & $<.05$ & $<.05$ \\
\hline 257 & 89-033 & -- & - & - & $<.05$ & $<.05$ \\
\hline 258 & 89-034 & - & - & - & $<.05$ & $<.05$ \\
\hline
\end{tabular}


Table 23. Organic chemical analysis, moisture content, and lipid content for biotic samples collected during 1986-90 from the Salton Sea and associated drainwaters and rivers--Continued

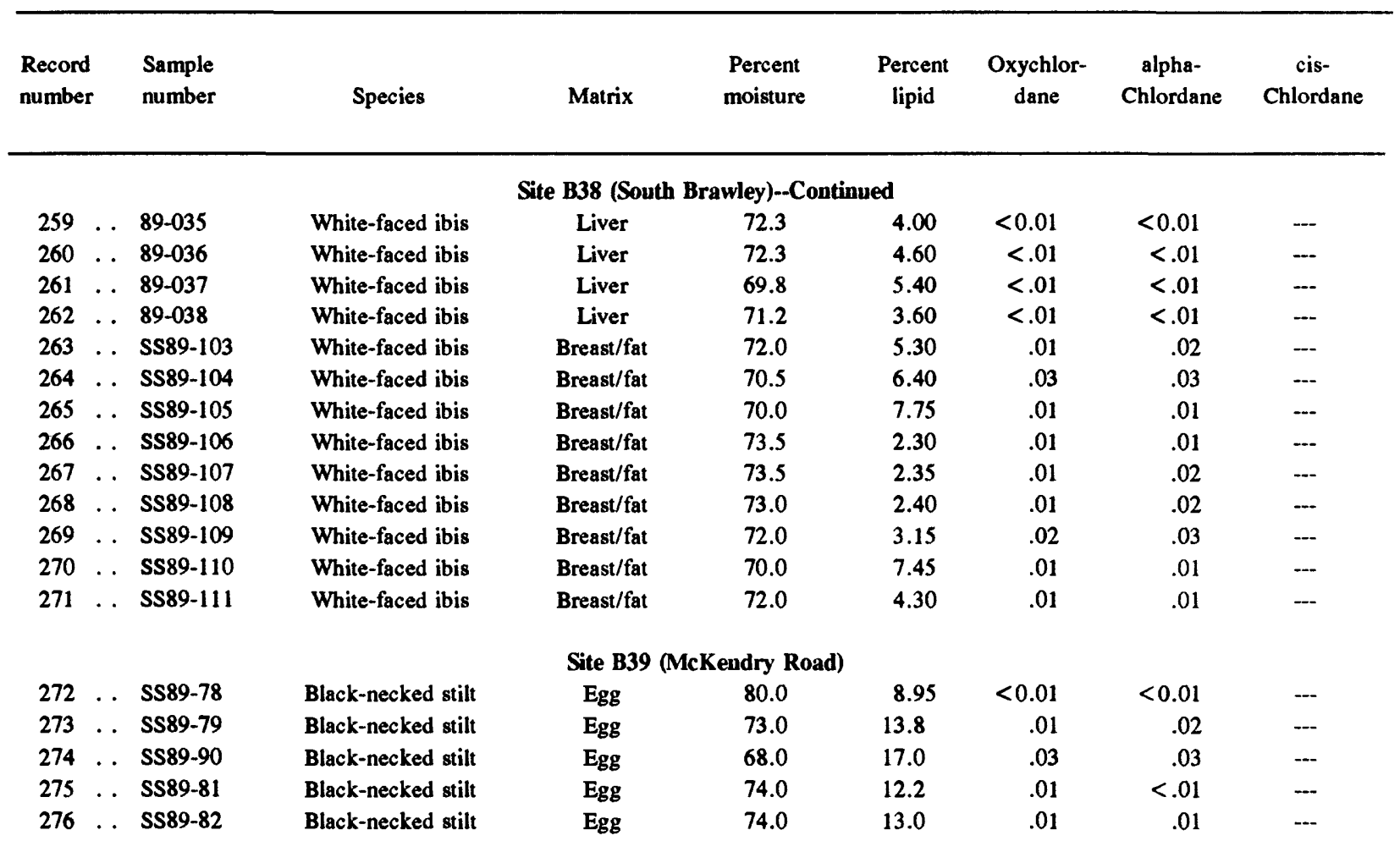


Table 23. Organic chemical analysis, moisture content, and lipid content for biotic samples collected during 1986-90 from the Salton Sea and associated drainwaters and rivers--Continued

\begin{tabular}{|c|c|c|c|c|c|c|c|c|c|}
\hline $\begin{array}{l}\text { Record } \\
\text { number }\end{array}$ & $\begin{array}{l}\text { Sample } \\
\text { number }\end{array}$ & $\begin{array}{l}\text { gamma- } \\
\text { Chlordane }\end{array}$ & $\begin{array}{c}\text { trans- } \\
\text { Chlordane }\end{array}$ & $\begin{array}{l}\text { cis-Nona- } \\
\text { chlor }\end{array}$ & $\begin{array}{c}\text { trans- } \\
\text { Nonachlor }\end{array}$ & $\begin{array}{l}\text { Hepta- } \\
\text { chlor }\end{array}$ & $\begin{array}{l}\text { Heptachlor } \\
\text { epoxide }\end{array}$ & $\begin{array}{l}\text { Methyoxy- } \\
\text { chlor }\end{array}$ & $o, p^{\prime}-D D E$ \\
\hline \multicolumn{10}{|c|}{ Site B38 (South Brawley)--Continued } \\
\hline 259 & $89-035$ & $<0.01$ & -- & $<0.01$ & $<0.01$ & -- & $<0.01$ & -- & $<0.01$ \\
\hline 260 & $89-036$ & $<.01$ & - & $<.01$ & .03 & - & $<.01$ & - & $<.01$ \\
\hline 261 & $89-037$ & $<.01$ & - & $<.01$ & .04 & -- & $<.01$ & -- & $<.01$ \\
\hline 262 & $89-038$ & $<.01$ & - & $<.01$ & $<.01$ & - & $<.01$ & -- & $<.01$ \\
\hline 263 & SS89-103 & $<.01$ & -- & $<.01$ & $<.01$ & -- & $<.01$ & -- & $<.01$ \\
\hline 264 & SS89-104 & $<.01$ & -- & $<.01$ & .07 & -- & .03 & - & $<.01$ \\
\hline 265 & SS89-105 & $<.01$ & - & $<.01$ & .03 & - & .01 & -- & $<.01$ \\
\hline 266 & SS89-106 & $<.01$ & - & $<.01$ & .02 & -- & .01 & -- & $<.01$ \\
\hline 267 & SS89-107 & $<.01$ & - & $<.01$ & .02 & -- & .01 & -- & $<.01$ \\
\hline 268 & SS89-108 & $<.01$ & - & $<.01$ & .02 & -- & $<.01$ & - & $<.01$ \\
\hline 269 & SS89-109 & $<.01$ & -- & $<.01$ & .03 & - & .01 & - & $<.01$ \\
\hline 270 & SS89-110 & $<.01$ & - & $<.01$ & .03 & -- & .01 & -- & $<.01$ \\
\hline 271 & SS89-111 & $<.01$ & -- & $<.01$ & .03 & -- & .01 & $\cdots$ & $<.01$ \\
\hline \multicolumn{10}{|c|}{ Site B39 (McKendry Road) } \\
\hline 272 & SS89-78 & $<0.01$ & - & $<0.01$ & $<0.01$ & - & $<0.01$ & - & $<0.01$ \\
\hline 273 & SS89-79 & $<.01$ & - & $<.01$ & .02 & -- & .01 & - & $<.01$ \\
\hline 274 & SS89-80 & $<.01$ & - & $<.01$ & .01 & -- & .01 & -- & $<.01$ \\
\hline 275 & S\$89-81 & $<.01$ & - & $<.01$ & $<.01$ & -- & $<.01$ & -- & $<.01$ \\
\hline 276 & S\$89-82 & $<.01$ & -- & $<.01$ & $<.01$ & -- & $<.01$ & -- & $<.01$ \\
\hline
\end{tabular}


Table 23. Organic chemical analysis, moisture content, and lipid content for biotic samples collected during 1986-90 from the Salton Sea and associated drainwaters and rivers--Continued

\begin{tabular}{|c|c|c|c|c|c|c|c|c|c|c|}
\hline $\begin{array}{l}\text { Record } \\
\text { number }\end{array}$ & $\begin{array}{l}\text { Sample } \\
\text { number }\end{array}$ & p,p'-DDE & o,p'-DDD & p,p'-DDD & o,p'-DDT & p,p'-DDT & $\begin{array}{l}\text { Total } \\
\text { DDT }\end{array}$ & Endrin & Dieldrin & Aldrin \\
\hline \multicolumn{11}{|c|}{ Site B38 (South Brawley)--Continued } \\
\hline 259 & $89-035$ & 8.2 & $<0.01$ & 0.13 & $<0.01$ & $<0.01$ & 8.330 & $<0.01$ & 0.11 & -- \\
\hline 260 & $89-036$ & 9.6 & $<.01$ & .21 & $<.01$ & .04 & 9.850 & $<.01$ & .17 & - \\
\hline 261 & $89-037$ & 7.3 & $<.01$ & .06 & $<.01$ & $<.01$ & 7.360 & $<.01$ & .21 & -- \\
\hline 262 & $89-038$ & 3.1 & $<.01$ & .04 & $<.01$ & $<.01$ & 3.140 & $<.01$ & .15 & -- \\
\hline 263 & SS89-103 & 5.9 & $<.01$ & $<.01$ & $<.01$ & .09 & 5.990 & .03 & .13 & $\ldots$ \\
\hline 264 & SS89-104 & 9.2 & $<.01$ & .07 & $<.01$ & .30 & 9.570 & .02 & .22 & $\ldots$ \\
\hline 265 & SS89-105 & 11 & $<.01$ & .18 & $<.01$ & .54 & 11.720 & .02 & .08 & - \\
\hline 266 & SS89-106 & 4.4 & $<.01$ & .09 & $<.01$ & .08 & 4.570 & .01 & .06 & - \\
\hline 267 & SS89-107 & 4.6 & $<.01$ & .08 & $<.01$ & .07 & 4.750 & .01 & .05 & - \\
\hline 268 & SS89-108 & 3.7 & $<.01$ & .10 & $<.01$ & .10 & 3.900 & $<.01$ & .02 & - \\
\hline 269 & SS89-109 & 4.6 & $<.01$ & .10 & $<.01$ & .10 & 4.800 & .02 & .08 & - \\
\hline 270 & SS89-110 & 6.1 & $<.01$ & .11 & $<.01$ & .04 & 6.250 & .01 & .05 & - \\
\hline 271 & SS89-111 & 4.1 & $<.01$ & .03 & $<.01$ & .15 & 4.280 & $<.01$ & .15 & - \\
\hline \multicolumn{11}{|c|}{ Site B39 (McKendry Road) } \\
\hline 272 & SS89-78 & 0.6 & $<0.01$ & $<0.01$ & $<0.01$ & $<0.01$ & 0.600 & $<0.01$ & $<0.01$ & -- \\
\hline 273 & SS89-79 & 4.3 & $<.01$ & $<.01$ & $<.01$ & .05 & 4.350 & $<.01$ & .15 & - \\
\hline 274 & SS89-80 & 2.5 & $<.01$ & $<.01$ & $<.01$ & .05 & 2.550 & $<.01$ & .10 & - \\
\hline 275 & SS89-81 & .95 & $<.01$ & $<.01$ & $<.01$ & $<.01$ & .950 & $<.01$ & .02 & - \\
\hline 276 & SS89-82 & 3.7 & $<.01$ & $<.01$ & $<.01$ & $<.01$ & 3.700 & $<.01$ & .08 & - \\
\hline
\end{tabular}


Table 23. Organic chemical analysis, moisture content, and lipid content for biotic samples collected during 1986-90 from the Salton Sea and associated drainwaters and rivers--Continued

\begin{tabular}{|c|c|c|c|c|c|c|c|c|c|c|c|c|c|}
\hline $\begin{array}{l}\text { Recond } \\
\text { number }\end{array}$ & $\begin{array}{l}\text { Sample } \\
\text { number }\end{array}$ & $\begin{array}{l}\text { alpha- } \\
\text { BHC }\end{array}$ & $\begin{array}{l}\text { beta- } \\
\text { BHC }\end{array}$ & $\begin{array}{l}\text { delta- } \\
\text { BHC }\end{array}$ & $\begin{array}{l}\text { gamma- } \\
\text { BHC }\end{array}$ & $\begin{array}{l}\text { Lin- } \\
\text { dane }\end{array}$ & $\begin{array}{c}\text { Hexa- } \\
\text { chloro- } \\
\text { ben- } \\
\text { zene }\end{array}$ & $\begin{array}{c}\text { Endo- } \\
\text { sul- } \\
\text { fan I }\end{array}$ & $\begin{array}{c}\text { Endo- } \\
\text { sul- } \\
\text { fan II }\end{array}$ & $\begin{array}{l}\text { Endo- } \\
\text { sulfan } \\
\text { sulfate }\end{array}$ & Mirex & DCPA & Dicofol \\
\hline
\end{tabular}

\begin{tabular}{|c|c|c|c|c|c|c|c|c|c|c|c|c|c|}
\hline \multicolumn{14}{|c|}{ Site B38 (South Brawley)--Continued } \\
\hline 259 & 89-035 & $<0.01$ & $<0.01$ & $<0.01$ & $<0.01$ & - & 1.6 & -- & - & -- & $<0.01$ & -- & -- \\
\hline 260 & 89-036 & $<.01$ & $<.01$ & $<.01$ & $<.01$ & -- & 1.5 & - & - & -- & $<.01$ & - & - \\
\hline 261 & 89-037 & $<.01$ & $<.01$ & $<.01$ & $<.01$ & $\ldots$ & .04 & - & $\ldots$ & --- & $<.01$ & $\ldots$ & --- \\
\hline 262 & $89-038$ & $<.01$ & $<.01$ & $<.01$ & $<.01$ & - & .01 & - & - & - & $<.01$ & - & -- \\
\hline 263 & SS89-103 & $<.01$ & $<.01$ & $<.01$ & $<.01$ & -- & .01 & - & -- & -- & $<.01$ & --- & --- \\
\hline 264 & SS89-104 & $<.01$ & $<.01$ & $<.01$ & $<.01$ & - & .05 & -- & -- & -- & $<.01$ & $\cdots$ & $\cdots$ \\
\hline 265 & SS89-105 & $<.01$ & $<.01$ & $<.01$ & $<.01$ & - & 2.4 & - & $\ldots$ & -- & $<.01$ & -- & - \\
\hline 266 & SS89-106 & $<.01$ & $<.01$ & $<.01$ & $<.01$ & - & .67 & --- & - & -- & $<.01$ & -- & -- \\
\hline 267 & SS89-107 & $<.01$ & $<.01$ & $<.01$ & $<.01$ & - & .74 & -- & -- & - & $<.01$ & -- & $\cdots$ \\
\hline 268 & SS89-108 & $<.01$ & $<.01$ & $<.01$ & $<.01$ & -- & 1.3 & - & -- & --- & $<.01$ & --- & - \\
\hline 269 & S\$89-109 & $<.01$ & $<.01$ & $<.01$ & $<.01$ & -- & 1.0 & --- & --- & --- & $<.01$ & -- & - \\
\hline 270 & SS89-110 & $<.01$ & $<.01$ & $<.01$ & $<.01$ & -- & .01 & --- & -- & -- & $<.01$ & --- & - \\
\hline 271 & SS89-111 & $<.01$ & $<.01$ & $<.01$ & $<.01$ & - & .01 & --- & -- & --- & $<.01$ & --- & -- \\
\hline \multicolumn{14}{|c|}{ Site B39 (McKendry Road) } \\
\hline 272 & SS89-78 & $<0.01$ & 0.04 & $<0.01$ & $<0.01$ & -.- & $<0.01$ & -- & -- & -- & $<0.01$ & --- & -- \\
\hline 273 & SS89-79 & $<.01$ & .09 & $<.01$ & $<.01$ & - & .02 & $\ldots$ & $\ldots$ & -- & $<.01$ & --- & -- \\
\hline 274 & SS89-80 & $<.01$ & .02 & $<.01$ & $<.01$ & -- & .01 & -- & $\cdots$ & -- & $<.01$ & -- & $\cdots$ \\
\hline 275 & SS89-81 & $<.01$ & $<.01$ & $<.01$ & $<.01$ & -- & $<.01$ & -- & -- & -- & $<.01$ & -- & - \\
\hline 276 & SS89-82 & $<.01$ & $<.01$ & $<.01$ & $<.01$ & -- & .01 & -- & $\cdots$ & -- & $<.01$ & -.- & -- \\
\hline
\end{tabular}


Table 23. Organic chemical analysis, moisture content, and lipid content for biotic samples collected during 1986-90 from the Salton Sea and associated drainwaters and rivers--Continued

\begin{tabular}{lllllllllllllll}
\hline Record & Sample & Tetra- & BI PH & BI PH & BI PH & BI PH & BI PH & BI PH & BI PH & BI PH & PCB & PCB & PCB & PCB \\
number & number & difon & CL-2 & CL-3 & CL-4 & CL-5 & CL-6 & CL-7 & CL-8 & CL-9 & 1016 & 1221 & 1232 & 1242 \\
& & & & & 0 & & & & & & & & &
\end{tabular}

\begin{tabular}{|c|c|c|c|c|c|c|c|c|c|c|c|c|c|c|}
\hline \multirow[b]{2}{*}{259} & \multicolumn{14}{|c|}{ Site B38 (South Brawley)--Continued } \\
\hline & $89-035$ & $\ldots$ & -- & $\cdots$ & - & - & $\ldots$ & $\ldots$ & $\ldots$ & -. & --- & $\ldots$ & $\ldots$ & --- \\
\hline 260 & $89-036$ & - & - & --- & --- & $\ldots$ & -.- & -.. & -- & -- & --. & --- & $\ldots$ & -- \\
\hline 261 & $89-037$ & - & - & -- & --- & - & -- & --- & --- & --. & --- & -- & -.. & -- \\
\hline 262 & $89-038$ & - & - & -- & $\ldots$ & - & -. & - & -- & --. & $\ldots$ & $-\ldots$ & $\ldots$ & $\ldots$ \\
\hline 263 & SS89-103 & - & --. & -_ & --- & -.. & --- & -- & -.- & --- & -..- & ... & ... & -.. \\
\hline 264 & SS89-104 & -- & -- & $\ldots$ & -.. & - & -- & -.- & -. & -.. & -.- & --- & --- & -- \\
\hline 265 & SS89-105 & - & $\ldots$ & -- & -.- & -.. & $\ldots$ & --- & $\ldots$ & -.. & $\ldots$ & $\ldots$ & $\ldots$ & $\ldots$ \\
\hline 266 & SS89-106 & - & - & -. & - & - & -- & $\ldots$ & $\ldots$ & -.. & --- & --. & -.- & -- \\
\hline 267 & SS89-107 & - & - & -- & -.. & -. & --. & -.- & - & - & $\ldots$ & -- & -.. & -- \\
\hline 268 & SS89-108 & - & -- & - & - & - & --. & -- & - & -- & $\ldots$ & $\ldots$ & $\ldots$ & --- \\
\hline 269 & SS89-109 & - & -- & --- & -- & -.. & --- & --. & -- & -.. & $\ldots$ & -.- & $\ldots$ & $\ldots$ \\
\hline 270 & SS89-110 & - & - & -- & -- & - & -- & --- & - & -- & --- & --- & -- & --- \\
\hline 271 & SS89-111 & - & - & - & -- & -- & --- & - & --- & -. & -- & --- & -- & - \\
\hline \multicolumn{15}{|c|}{ Site B39 (McKendry Road) } \\
\hline 272 & SS89-78 & - & -- & - & -- & $\ldots$ & $\ldots$ & -.. & $\ldots$ & -.. & -- & --- & --- & $\cdots$ \\
\hline 273 & SS89-79 & - & -- & - & $\ldots$ & -.. & $\ldots$ & $\ldots$ & - &.- & -..- & $\ldots$ & -.. &.- \\
\hline 274 & SS89-80 & - & - & - & -- & ... & -- & -- & - & -.. & $\ldots$ & $\ldots$ & $\ldots$ & $\ldots$ \\
\hline 275 & SS89-81 & - & -- & -- & $\ldots$ & ... & -.. & ... & - & -- & --. & ... & --- & -.. \\
\hline 276 & SS89-82 & - & - & - & -- & -- & - & --- & --. & - & $\ldots$ & $\ldots$ & -. & --- \\
\hline
\end{tabular}


Table 23. Organic chemical analysis, moisture content, and lipid content for biotic samples collected during 1986-90 from the Salton Sea and associated drainwaters and rivers--Continued

\begin{tabular}{|c|c|c|c|c|c|c|}
\hline $\begin{array}{l}\text { Record } \\
\text { number }\end{array}$ & $\begin{array}{l}\text { Sample } \\
\text { number }\end{array}$ & $\begin{array}{l}\text { PCB } \\
1248\end{array}$ & $\begin{array}{l}\text { PCB } \\
1254\end{array}$ & $\begin{array}{l}\text { PCB } \\
1260\end{array}$ & $\begin{array}{l}\text { Total } \\
\text { PCB }\end{array}$ & Toxaphene \\
\hline \multicolumn{7}{|c|}{ Site B38 (South Brawley)--Continued } \\
\hline 259 & $89-035$ & -- & -- & $\cdots$ & $<0.05$ & $<0.05$ \\
\hline 260 & 89-036 & $\ldots$ & $\cdots$ & - & $<.05$ & $<.05$ \\
\hline 261 & 89-037 & -- & - & - & $<.05$ & $<.05$ \\
\hline 262 & 89-038 & - & -- & -- & $<.05$ & $<.05$ \\
\hline 263 & SS89-103 & -- & - & -- & $<.05$ & $<.05$ \\
\hline 264 & SS89-104 & $\ldots$ & -- & - & $<.05$ & $<.05$ \\
\hline 265 & SS89-105 & -- & - & $\cdots$ & $<.05$ & $<.05$ \\
\hline 266 & SS89-106 & - & - & -- & $<.05$ & $<.05$ \\
\hline 267 & SS89-107 & - & - & -- & $<.05$ & $<.05$ \\
\hline 268 & SS89-108 & -- & - & -- & $<.05$ & $<.05$ \\
\hline 269 & S\$89-109 & $\ldots$ & -- & $\ldots$ & $<.05$ & $<.05$ \\
\hline 270 & SS89-110 & $-\cdots$ & -- & - & $<.05$ & $<.05$ \\
\hline 271 & SS89-111 & -- & $\cdots$ & --- & $<.05$ & $<.05$ \\
\hline \multicolumn{7}{|c|}{ Site B39 (McKendry Road) } \\
\hline 272 & SS89-78 & $\cdots$ & $-\cdots$ & $\ldots$ & $<0.05$ & $<0.05$ \\
\hline 273 & SS89-79 & - & -- & -- & $<.05$ & $<.05$ \\
\hline 274 & SS89-80 & -- & -- & -- & $<.05$ & $<.05$ \\
\hline 275 & SS89-81 & -- & -- & -- & $<.05$ & $<.05$ \\
\hline 276 & SS89-82 & -- & -- & -- & $<.05$ & $<.05$ \\
\hline
\end{tabular}


Table 24. Polycyclic aromatic hydrocarbon concentration, moisture content, and lipid content for single samples of crayfish and tilapia collected in the study area in 1987

[All chemical data reported in micrograms per gram, wet weight; NWR, National Wildlife Refuge. $<$, less than indicated reporting limit]

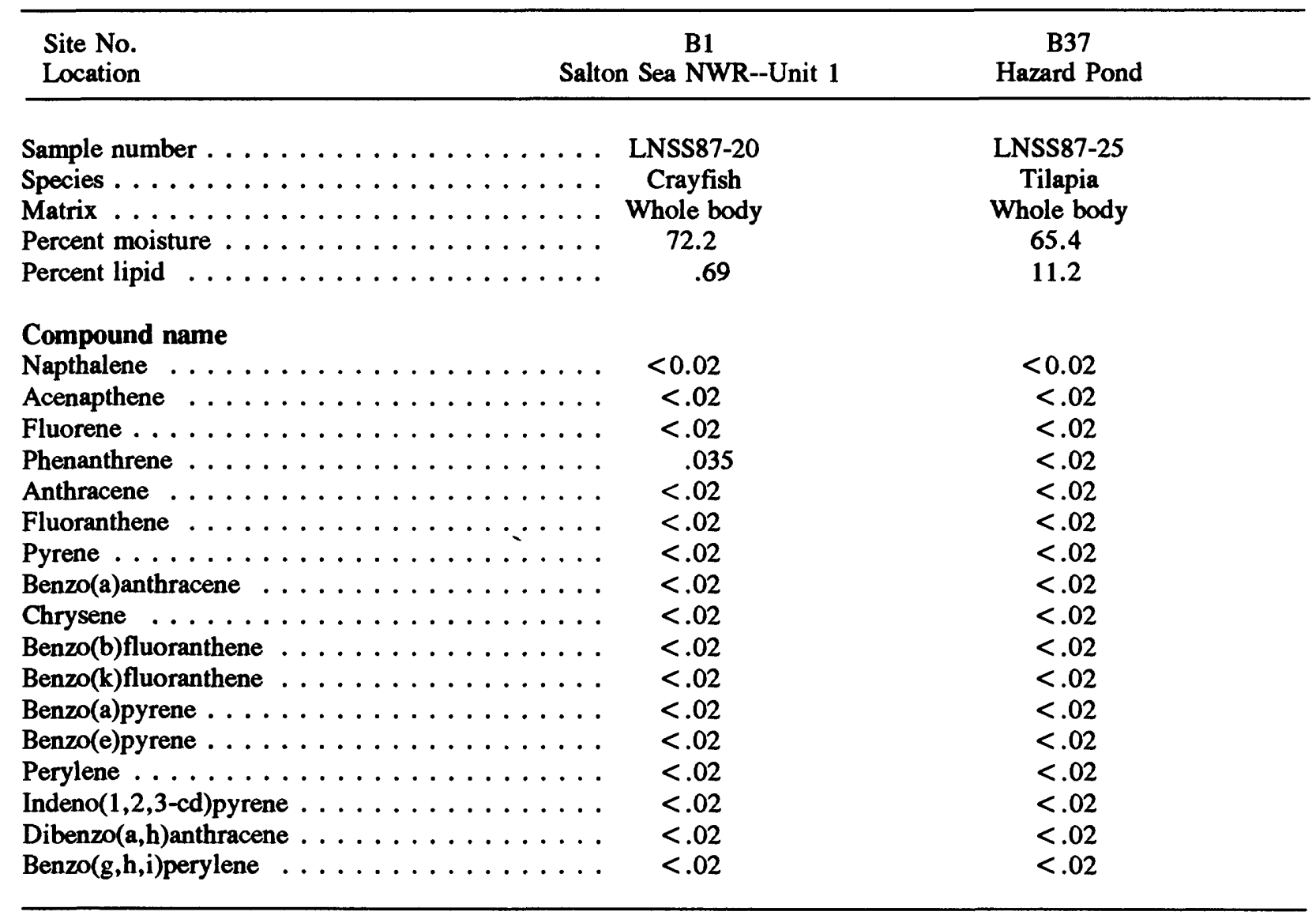

